\title{
DUSTY, RADIATION PRESSURE-DOMINATED PHOTOIONIZATION. I. MODEL DESCRIPTION, STRUCTURE, AND GRIDS
}

\author{
Brent A. Groves, Michael A. Dopita, and Ralph S. Sutherland \\ Research School of Astronomy and Astrophysics, Institute of Advanced Studies, The Australian National University, Cotter Road, \\ Weston Creek, ACT 2611, Australia; bgroves@mso.anu.edu.au, michael.dopita@anu.edu.au,ralph@mso.anu.edu.au \\ Received 2003 December 3; accepted 2004 March 19
}

\begin{abstract}
We present the implementation of dusty, radiation pressure-dominated photoionization models applicable to the narrow-line regions (NLRs) of active galactic nuclei, using the MAPPINGS III code. We give a grid of the predicted intensities of the most commonly used diagnostic spectral lines in the UV, optical, and IR, covering a wide range of density, metallicity, the power-law index characterizing the photoionizing source, and photoionization parameter, for use in the diagnosis of NLRs. We examine the temperature, density, and ionization structure of these models, investigating the effect of variation of these parameters in order to gain a better understanding of NLR clouds themselves.
\end{abstract}

Subject headings: galaxies: active — galaxies: Seyfert — ISM: general — line: formation

On-line material: color figures

\section{INTRODUCTION}

Emission line spectroscopy combined with photoionization or shock modeling codes has proved to be an important tool in determining the physical properties and chemical abundances of astrophysical nebulae. The narrow-line regions (NLRs) within active galaxies represent one of the more energetic types of ionized nebulae, and they are characterized by emission lines across a wide range of ionization states. In addition, these regions are often spatially resolved in both nearby and high-redshift galaxies as extended narrow-line regions (ENLRs). Resolved NLRs are excellent laboratories to study the detailed physics of active galactic nucleus (AGN) nebulae and allow strong tests of the various ionization models that have been developed to explain their properties.

Photoionization models invoke a nuclear source of highenergy photons to provide the dominant mechanism of ionization within a nebulae. These models can be characterized by the dimensionless ionization parameter $U=S_{*} / n_{\mathrm{H}} c$, where $S_{*}$ is the number of ionizing photons passing through a unit area control surface per unit time, $n_{\mathrm{H}}$ is the hydrogen density, and $c$ is the speed of light. The parameter $U$ is therefore the ratio of the photon density to the atomic density. For active galaxies, the "unified model" holds that an optically thick torus confines the UV ionizing photons originating from the central source (the AGN) to a cone-shaped region along the polar axis, and it is these photons that excite the NLR. The exact production mechanism of these photons remains unclear, but to match the models to the observations the ionizing spectrum is required to be a smooth, featureless power law or broken power law (Koski 1978; Stasińska 1984; Osterbrock 1989; Laor et al. 1997; Alexander et al. 2000; Prieto \& Viegas 2000; Sazonov et al. 2004) with $U \sim 0.01$. This spectrum and the low densities $\left(n_{\mathrm{H}} \sim 10^{2}-10^{4} \mathrm{~cm}^{-3}\right)$ result in forbidden line emission over a wide range of ionization states. Penetrating soft X-ray photons also ensure the existence of an extensive partially ionized region in which the lines of low-ionization species such as $\left[\mathrm{O}_{\mathrm{I}}\right]$ are strong. Both of these properties are required by the observational constraints from diagnostic emission line ratios involving low- and intermediate-ionization states (Veilleux \& Osterbrock 1987; Osterbrock et al. 1992).

However, simple constant-density photoionization models have difficulty in explaining the existence of the strong coronal line intensities relative to $\mathrm{H} \beta$, which are found in a number of Seyfert galaxy NLR regions (Oliva et al. 1994; Moorwood et al. 1996; Kraemer \& Crenshaw 2000). The existence of coronal gas requires that both the ionization parameter and the radiation pressure are very large (Binette et al. 1996). This is in contradiction to the constraints on the ionization parameter provided by the lower ionization lines, which require $U \sim 0.01$ (Veilleux \& Osterbrock 1987; Osterbrock et al. 1992; Véron-Cetty \& Véron 2000).

This problem can be alleviated by abandoning the single density (isochoric) photoionization models and invoking multicomponent models of varying degrees of complexity, such as the $A_{M / I}$ models of Binette et al. $(1996,1997)$ or the integrated emission models of Komossa \& Schulz (1997) and Ferguson et al. (1997). An unsatisfactory aspect of such models is that the geometry of the absorbers with respect to the ionization sources is a free parameter, so that the predictive nature of the photoionization models is, to an extent, lost. Hubble Space Telescope Space Telescope Imaging Spectrograph (HST STIS) observations have been able to resolve several Seyfert galaxies down to a few tens of parsecs (e.g., Kraemer et al. 2000; Bradley et al. 2004) and thus place some constraints upon the geometry of these models, but not enough to completely substantiate these models.

None of these photoionization models address the issue of the gasdynamics of the photoionized region, which often display evidence of nongravitational motions (Whittle 1992). Examples of this can be found amongst the more radioluminous galaxies including Mrk 78 (Pedlar et al. 1989), NGC 2992 (Allen et al. 1999), or Mrk 1066 (Bower et al. 1995). Detailed HST STIS observations of NLR also show strong evidence of nongravitational motions (e.g., Crenshaw \& Kraemer 2000; Kaiser et al. 2000; Cecil et al. 2002). These observations provide the motivation to consider excitation by fast radiative shocks (Dopita \& Sutherland 1995, 1996). These 
are successful in reproducing many of the observed diagnostic line ratios. In practice, these models are also dominated by photoionization effects because shocks with velocities $V_{S} \geq$ $200 \mathrm{~km} \mathrm{~s}^{-1}$ generate a strong internal photoionizing field as the shocked gas cools, which can ionize both the material ahead of the shock as well as the cooler gas behind the shock. However, for Seyfert galaxies at least, recent X-ray data (Brinkman et al. 2002; Ogle et al. 2003) provide powerful evidence for the existence of central photoionizing fields with very high ionization parameters, and so makes such models less viable, insofar as it is shocks that are the strongest source of ionizing photons. Shocks may be still necessary to provide the observed dynamical structure of many sources.

In an attempt to self-consistently address both the problems of the gasdynamics and of the wide range in observed excitation conditions that exist within the NLR, a new paradigm of dusty, radiation pressure-dominated photoionization was developed (Dopita et al. 2002, hereafter DG02). This model is based upon the standard photoionization paradigm, but it also includes the physics of dust, known to exist in these regions, and radiation pressure. These provide the physical basis needed to explain the observed emission line ratios from NLRs, especially the combination of high- and low- ionization lines within one physical structure, and can also allow for the possibility of fast, radiatively driven outflows in Seyfert galaxies.

Here we continue the work of DG02 and explain how this model is implemented in the photoionization \& shock code MAPPINGS III. The majority of the code has been discussed previously (Dopita et al. 1982; Sutherland \& Dopita 1993), and a general overview of the photoionization calculations as implemented in the current code is given in B. A. Groves et al. (2004, in preparation). Here we discuss only our implementation of dust and radiation pressure within the code.

We also extend the previous work (DG02) by presenting the results of a grid of both the standard photoionization models (with no dust or radiation pressure, one set allowing for depletion of heavy elements from the gas phase, and one set nondepleted) and our dusty, radiation pressure-dominated models. These models cover a wide range in metallicity, density, photoionization power-law index, and ionization parameter $U$.

The purpose of this paper is twofold. The first purpose is to present the grid of models and the resulting line intensities, since complete FUV-FIR diagnostics have not been previously presented, even for the standard photoionization models. The increasing availability of emission line data, and, in particular, the launch of Spitzer makes such a set of models timely as a diagnostic tool for observers. Second, we aim to provide insight into the physical structure of the models. In association with the emission lines this assists in understanding of how variation in the parameters results in the observed ratios. The full discussion of the emission line ratios, and a detailed comparison of the models with available observations, is given in the following paper in this series (Groves et al. 2004).

\section{DUST MODELS}

Dust is almost ubiquitous in the interstellar medium (ISM). It makes its presence felt directly through its absorption, scattering, and polarization of incident light. Warm dust can be seen directly through its IR re-emission and its electric and magnetic dipole emission at sub-mm wavelengths (Draine \& Lazarian 1998). Its existence can also be indirectly inferred through the measurement of atomic depletion of refractory species from the gas phase along various sight lines through the ISM. These observations provide a wide number of constraints on size distributions, chemical compositions, and physical state of the dust particles, yet even with these constraints, there remains considerable uncertainty as to its form, physical structure, and chemical composition.

There are two main problems with distinguishing the general properties of dust. First, dust properties are seen to vary between different sight lines and within different phases of the interstellar medium. Such variation is a natural result of the combination of grain growth (within dense dust clouds, or in the stellar outflows and ejecta) and a wide variety of grain destruction or shattering mechanisms. Second, several of the observable properties of dust appear to be degenerate in that they can be equally well reproduced by different theoretical grain size distributions and compositions.

In the light of this complexity, we have elected to use in our photoionization modeling the simplest grain composition and grain size distribution model that is consistent with the known properties of the dust in the solar neighborhood. This model is almost certainly not applicable in detail to the environment of AGNs, but it at least allows us to evaluate the (often fairly gross) effects that dust will have on the emission line spectrum of photoionized NLR. Additionally, the results we have obtained and presented below should be easily reproducible by other modeling codes.

It might be naively expected that the energetic environment and powerful radiation fields known to exist in the vicinity of AGNs would be inimical to the survival of dust. However, dust is known to exist within AGNs, and spectral constraints on both its form and composition have been derived (Laor \& Draine 1993).

Most investigations of the dust content have concentrated upon the dusty tori; however, there is also evidence that dust exists in the NLR of AGNs (Tomono et al. 2001; Radomski et al. 2003). As discussed in Dopita et al. (2002), dust survives in the dense molecular clouds and is not exposed the radiation field from the AGN until it passes through the ionization front surrounding these photoablating clouds. Despite the energetic EUV field, refractory grain species are not primarily destroyed by sublimation, but rather, by grain shattering followed by sublimation of the smallest grains. The larger dust grains should be able to survive their crossing of the high-emissivity regions close to the surface of NLR clouds where most of the UV, optical, and IR lines are produced. However, the coronal species are produced further out in the ionized flow, and dust is likely to be destroyed in these regions. We therefore caution the reader against the use of coronal line intensities predicted in the models presented in this paper, in which grain destruction mechanisms are not taken into account.

\subsection{Dust Composition}

Through observations of the IR emission bands or UV absorption features associated with dust, several particular dust species have been identified in the ISM. These include diamond (Van Kerckhoven et al. 2002), crystalline silicates (such as enstatite $\mathrm{MgSiO}_{3}$; Honda et al. 2003), water ice (Alexander et al. 2003), carbides ( $\mathrm{SiC}, \mathrm{MgC}, \mathrm{TiC}$; Hony et al. 2003), sulfides (FeS, MgS, $\mathrm{SiS}_{2}$; Hony et al. 2002), and carbonates $\left(\mathrm{CaCO}_{3}\right.$; Onaka \& Okada 2003). Another important dust component that is believed to play a major part in the physics of the neutral medium are polycyclic aromatic hydrocarbons (PAHs). Their effect is felt through processes such as photoelectric heating, and they are believed to be the source of the "unidentified bands" (UIBs) in the infrared. 
However, the existence and relative abundance of all these components in the photoionized plasma around AGNs is entirely unknown. Therefore, for simplicity, we use only two types of dust, amorphous "astronomical" silicates (such as forsterite or fayalite) and amorphous carbon grains. Both are observed in the interstellar medium through absorption, and together they can reproduce most of the spectral properties of dust, including both the observed IR continuum emission and the UV extinction (Draine 2003). It is these two components that are the basis for most previous dust modeling, and for which a large set of optical data exist.

The "astronomical" amorphous silicate grains are generally described as an olivine $\left[(\mathrm{Mg}, \mathrm{Fe})_{2} \mathrm{SiO}_{4}\right]$ ranging somewhere between fayalite $\left(\mathrm{Fe}_{2} \mathrm{SiO}_{4}\right)$ and forsterite $\left(\mathrm{Mg}_{2} \mathrm{SiO}_{4}\right)$, although some form of enstatite has also been used. All of these have been observed in crystalline form, but the amorphous form allows for a random structure, with possible vacuum spaces within the grain. This can provide a broader emission without production of the crystalline features, which in any case are not observed in the ISM at large (Jäger et al. 2003).

The amorphous carbon component is believed to range somewhere between the less saturated aromatic forms characteristic of PAHs and a saturated hydrocarbon form. Dust of such a composition is absolutely necessary if we are to successfully reproduce the both the extinction and emission data. This component is believed to be the source of the $2200 \AA$ absorption band in the UV.

Although we have assumed only these two types of dust grain, we deplete all elements that have gas-phase condensation temperatures above a few hundred degrees. The choice of the depletion factors is based upon observations of the diffuse ISM (e.g., Savage \& Sembach 1996).

\subsection{Dust Abundance}

Dust formation depletes the heavy elements from the surrounding gas. Those that have a higher condensation temperature are observed to be more depleted, while the noble gases such as $\mathrm{He}, \mathrm{Ne}$, and Ar remain in the gaseous phase. Although there is clear evidence that the depletion of metals from the gas onto dust is strongly dependent on the local conditions (Savage \& Sembach 1996), for simplicity we assume that the fractional depletion of dust from the gas is the same as found within the local interstellar medium. We also assume that this fractional depletion does not vary with changes in the metallicity of the gas, so that the gas-to-dust mass ratio is simply proportional to metallicity.

Such assumptions are a gross oversimplification and cannot be justified. We adopt them simply because, faced with our minimal knowledge of dust depletion in active galaxies, they represent the simplest and best understood grain model we can test, and they minimize the abundance-dependent effects of dust changing with metallicity.

The adopted depletion factors are listed in Table 1. These values are those used by Dopita et al. (2000) for starburst and active galaxy photoionization modeling and are similar to those found by Jenkins (1987) and Savage \& Sembach (1996) in the local ISM using the UV absorption lines to probe various local lines of sight.

The heavy element abundances and their depletion factors then determine the total gas-to-dust ratio. The total mass of carbon depleted from the gas phase determines the amount of carbonaceous dust, while the remainder of the heavy elements locked up in dust (mainly $\mathrm{Si}$ and $\mathrm{O}$, but also including $\mathrm{Fe}, \mathrm{Ca}$, and $\mathrm{Mg}$ ) is assumed to have gone into forming the silicates.
TABLE 1

DePLETION FACTORS

\begin{tabular}{|c|c|}
\hline Element & Depletion $^{\mathrm{a}}$ \\
\hline Н & 0.00 \\
\hline$\ldots$ & 0.00 \\
\hline С & -0.30 \\
\hline N & -0.22 \\
\hline 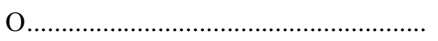 & -0.22 \\
\hline $\mathrm{Ne}$ & 0.00 \\
\hline $\mathrm{Na}$ & -0.60 \\
\hline $\mathrm{Mg} \ldots \ldots \ldots \ldots \ldots . . . . . .$. & -0.70 \\
\hline 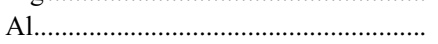 & -1.70 \\
\hline 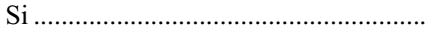 & -1.00 \\
\hline S & 0.00 \\
\hline $\mathrm{Cl}$ & -0.30 \\
\hline $\operatorname{Ar}$ & 0.00 \\
\hline $\mathrm{Ca}$ & -2.52 \\
\hline $\mathrm{Fe}$ & -2.00 \\
\hline 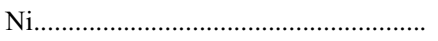 & -1.40 \\
\hline
\end{tabular}

Although again, this is an oversimplification, nonetheless we find that our model gives both a reasonable ratio of carbon to silicates and an extinction generally similar to that found within our galaxy. The total amount of dust is then distributed across a range of sizes determined by the grain size distribution.

\subsection{Grain Size Distribution}

The grain size distribution and the grain composition are the most important factors determining the wavelengthdependence of the absorption and scattering processes. The grain size distribution in the ISM results from the balance between formation and destruction processes. In general, it can be reasonably well represented by a power law over a wide range of sizes, $a$ (Mathis et al. 1977, hereafter MRN):

$$
d N(a) / d a=k a^{-\alpha}, \quad a_{\min } \leq a \leq a_{\max } .
$$

More complex distributions have been suggested on physical grounds (Weingartner \& Draine 2001a), but even these can be approximated by power laws over a wide range of radii. Grain shattering has been shown to lead naturally to the formation of a power-law size distribution of grains with $\alpha \sim 3.3$ (Jones et al. 1996), observationally indistinguishable from the MRN value, $\alpha \sim 3.5$.

We have adopted MRN grain size distribution in our models with $\alpha \sim 3.5$ and the factor $k$ being determined by the gas-todust mass ratio. For silicate grains we take $a_{\min }=100 \AA$ and $a_{\max }=2500 \AA$, while for carbonaceous grains we have $a_{\min }=$ $50 \AA$ and $a_{\max }=2500 \AA$. These lower limits are considerably larger than might be used in the local ISM, since we expect that in AGN environments small grains are much more likely to be destroyed. This absence of small grains reduces both the total absorption and the photoelectric heating by grains in the models. The assumption of a large minimum grain size is supported by the relative absence of the $2200 \AA$ dust feature in AGN spectra (Pitman et al. 2000). This feature is believed to be caused by graphite dust and is dominated by the smallest grain sizes. So an absence of this feature indicates that the smallest of grains are insignificant in quantity.

Within the MAPPINGS III code, we divide the dust grains into 80 bins spaced logarithmically between 0.001 and $10 \mu \mathrm{m}$. The number in each bin is then determined by the distribution. 


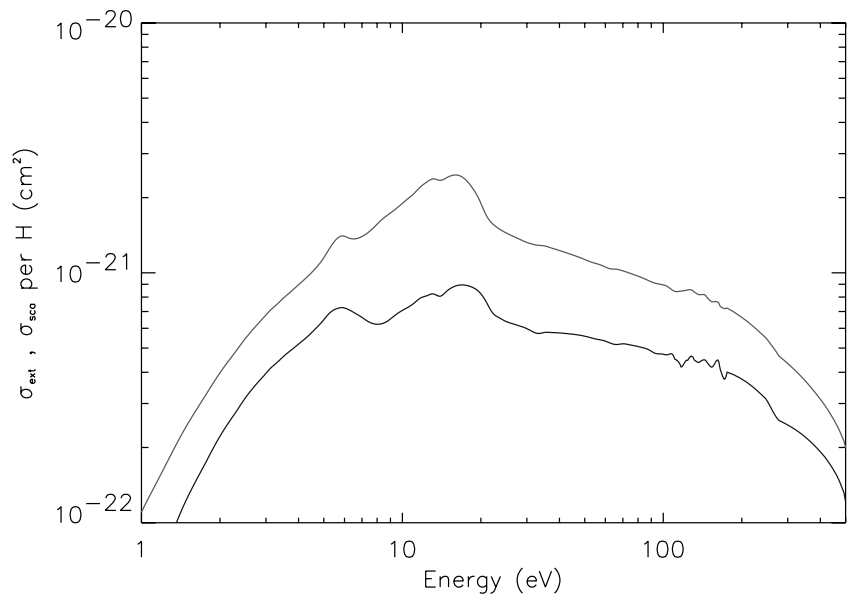

FIG. 1.-Extinction and scattering cross section of dust per $\mathrm{H}$ atom in a $1 Z_{\odot}$ metallicity model. Note the peak in extinction curve around the Lyman limit (13.6 eV).

The absorption, scattering, and photoelectric heating within the code is then calculated for each size bin.

\subsection{Extinction and Scattering by Dust}

For the optical properties of the two compositions of dust we use the data created by Draine and coauthors using calculated dielectric functions and a combination of Mie and Rayleigh-Gans theory.

To represent the carbon grains we have used the graphite optical data (Draine \& Lee 1984; Laor \& Draine 1993) and for the silicate grains the "smoothed astronomical silicate" data (Draine \& Lee 1984; Laor \& Draine 1993; Weingartner \& Draine 2001a). These data ${ }^{1}$ include the scattering and absorption cross sections for grains ranging from 0.001 to $10 \mu \mathrm{m}$. A full description of the data and its construction can be found in the cited papers.

In MAPPINGS III the data is read in as a table and applied to each relevant grain size bin for both the graphitic and silicaceous grains, allowing the absorption and scattering cross section to be calculated for each bin. The final total extinction and scattering cross section of dust within the models is shown in Figure 1.

\subsection{Photoelectric Heating}

Photoelectric heating of the gas by dust has been shown to be an important factor in influencing the temperature structure of both H II regions (Maciel \& Pottasch 1982) and planetary nebulae (Borkowski \& Harrington 1991). This process was first computed quantitatively by Draine (1978), and this work has been followed by more accurate calculations that explore both the physics of the process (Weingartner \& Draine 2001b) and explore the importance of the photoelectric (PE) effect in nebular modeling (Dopita \& Sutherland 2000).

The charge of dust grains is determined by the balance between the PE effect and the collisional "sticking" of charged particles to the grain. In highly ionized nebulae with strong radiation fields, the $\mathrm{PE}$ effect dominates and grains are positively charged, but when the radiation field is weak, electron sticking dominates and the grains are negatively charged.

\footnotetext{
1 The optical data can be downloaded at http://www.astro.princeton .edu/ draine/dust/dust.diel.html.
}

The implementation of the photoelectric effect on dust in MAPPINGS III has already been discussed previously in Dopita \& Sutherland (2000). This implementation has since been altered so that the heating is calculated for the individual grain size bins rather than for the dust as a whole. This allows different grain sizes to have different charges.

The photoelectric heating turns out to be important in our NLR models as it strongly heats and excites the highionization gas and helps address the temperature problem of these regions. Charged grains are locked to the ionized plasma (drift velocities are only a few $\mathrm{cm} \mathrm{s}^{-1}$ ), so the charge serves to couple the radiation pressure (acting on dust) directly to the gas (Dopita et al. 2002).

\subsection{Radiation Pressure}

The importance of radiation pressure acting on dust in high ionization parameter photoionized regions was demonstrated in DG02. In an ionized plasma the radiation pressure forces can be separated into two components: the force exerted by the photoionization process and the component due to the absorption by dust,

$$
\begin{aligned}
F_{\text {rad }}(x)= & \sum_{m=1} \sum_{i=0} n_{i}\left(X_{m}^{+i}\right) \int_{\nu_{m}^{i}}^{\infty} \frac{\varphi(\nu)}{c} a_{\nu}\left(X_{m}^{+i}\right) d \nu \\
& +n_{\mathrm{H}} \int_{0}^{\infty}\left\{\kappa_{\mathrm{abs}}(\nu)+\kappa_{\mathrm{sca}}(\nu)[1-g(\nu)]\right\} \frac{\varphi(\nu)}{c} d \nu,
\end{aligned}
$$

where $\varphi(\nu)$ is the local radiation flux at frequency $\nu, n_{i}\left(X_{m}^{+i}\right)$ is the number density of ion $+i$ of atomic species $m$, and $a_{\nu}\left(X_{m}^{+i}\right)$ is the corresponding photoionization cross section with threshold $\nu_{m}^{i}$. In the second part, the dust term, $\kappa_{\text {abs }}(\nu)$ and $\kappa_{\text {sca }}(\nu)$ are the dust absorption cross section and scattering cross section, respectively, both normalized to the hydrogen density, and $g(\nu)=\left\langle\cos \theta_{\nu}\right\rangle$ is the mean scattering angle of the dust grains at this frequency. Note that the major component of the radiation pressure on dust comes from the farUV region of the spectrum, where the dust opacity is greatest and the opacity due to hydrogen is effectively zero. The radiation force acting directly onto the gas can be considered solely due to photoionization, as the bound-bound transitions contribute a relatively negligible force due to the stationary nature of the NLR cloud gas along the stagnation point.

Equation (2) contains the implicit assumption that the gas and the dust are closely coupled by Coulomb forces, and hence any force acting upon the dust can be considered as imparted to the system as a whole.

In the case of an ionization front facing the source of ionizing photons, the ionized plasma can be approximated as being in hydrostatic equilibrium (which is assumed in our isobaric models), and in this case the gas pressure gradient must match the local radiative volume force,

$$
\frac{d P_{\mathrm{gas}}}{d x}=F_{\mathrm{rad}}(x) .
$$

This gives the gas pressure structure of the nebulae, from which the density structure can be determined, assuming equilibrium conditions. For ionization fronts that are oblique to the ionizing source, the radiation pressure provides a shear force that serves to accelerate the flow away from the source of UV photons. In this case, a hydrodynamic photoionization model would be required to describe the flow. This is beyond the scope of this paper. 


\subsection{Grain Destruction}

The assumption of close coupling between gas and dust breaks down at a single point in the flow from the ionization front at which the collisional charging and the photoelectric charging rates of a grain balance, and the grain consequently has zero charge. As these processes are size dependent, this point occurs at a different part of the flow for each grain size. At this point, the full radiation pressure force acts on the grain, and it is rapidly accelerated toward its terminal velocity with respect to the gas and other grain sizes. The terminal velocity is determined by the kinetic friction forces exerted on it by the gas. This phenomenon is almost certainly unimportant in affecting the ionized gas flow as a whole, but it could be important in providing a means of shattering grains by highvelocity grain-grain collisions.

As this process occurs at different points for each grain size, with the largest grains becoming neutral first in the flow, what can occur is that the large grains become neutral, dissociate from the gas, and are shattered to small grains that become charged again. These then become neutral further down in the photoablated flow from the NLR cloud, and the process is repeated. This will reoccur until the grains are small enough to be destroyed through stochastic heating. Thus, through this process we have a mechanism to destroy dust grains and return the metals in dust to the gas in the coronal region around NLR clouds. Modeling this destruction, however, is beyond the scope of this work.

\section{CHEMICAL ABUNDANCES}

The major parameters that influence the spectrum of a photoionized plasma are the ionization parameter and the chemical abundances. To investigate the influence of abundance, we use five abundance sets: $4 Z_{\odot}, 2 Z_{\odot}, Z_{\odot}, 0.5 Z_{\odot}$, and $0.25 Z_{\odot}$, where $Z_{\odot}$ represents solar metallicity.

\subsection{The Solar Abundance Set}

The last few years have seen a more accurate determination of the solar abundances of several chemical species, including carbon, nitrogen, and oxygen. These papers by Asplund and his collaborators (Asplund et al. 2000; Asplund 2000, 2003; Allende Prieto et al. 2001, 2002) use three-dimensional hydrodynamical modeling of the solar atmosphere along with detailed atomic and molecular data to reanalyze high $\mathrm{S} / \mathrm{N}$ solar spectra and so obtain revised solar abundances for $\mathrm{C}, \mathrm{N}$, $\mathrm{O}, \mathrm{Si}$, and $\mathrm{Fe}$. These abundances are generally lower than the previous estimates by Anders \& Grevesse (1989) and are quite similar to those found in the local ISM and surrounding B stars (Russell \& Bessell 1989; Russell \& Dopita 1990, 1992).

We have adopted the $\mathrm{C}, \mathrm{N}, \mathrm{O}, \mathrm{Si}$, and $\mathrm{Fe}$ abundances from Asplund et al., and the abundances of the remaining elements in our solar abundance set, excluding the noble gases, are obtained from the meteoritic abundances in the standard solar abundance set of Grevesse \& Sauval (1998). Ne and Ar are obtained from the photospheric measurements of Grevesse \& Sauval (1998).

Because of its absence from the solar photospheric spectrum, the He represents a special case. We have used the primordial measurements of Pagel et al. (1992) along with their linear fits of $Y$ with $[\mathrm{O} / \mathrm{H}]$ to estimate the solar helium abundance.

The final solar abundance set used within our models closely resembles that observed in the local ISM (Russell \& Bessell 1989; Russell \& Dopita 1990, 1992) and is given in Table 2.
TABLE 2

Solar Abundance Set

\begin{tabular}{|c|c|}
\hline Element & Abundance \\
\hline Н........................ & 0.000 \\
\hline Не & -0.987 \\
\hline С & -3.61 \\
\hline 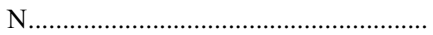 & -4.20 \\
\hline О & -3.31 \\
\hline $\mathrm{Ne}$ & -3.92 \\
\hline $\mathrm{Na}$ & -5.68 \\
\hline $\operatorname{Mg} . \ldots \ldots \ldots \ldots \ldots . . . . .$. & -4.42 \\
\hline (2) & -5.51 \\
\hline 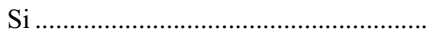 & -4.49 \\
\hline ..................... & -4.80 \\
\hline $\mathrm{Cl}$ & -6.72 \\
\hline $\operatorname{Ar}$ & -5.60 \\
\hline $\mathrm{Ca}$ & -5.65 \\
\hline $\mathrm{Fe}$ & -4.54 \\
\hline 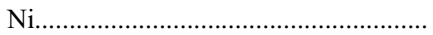 & -5.75 \\
\hline
\end{tabular}

${ }^{\text {a }}$ All abundances are logarithmic with respect to hydrogen.

\subsection{Scaling the Solar Abundance Set}

For primary nucleosynthetic elements, their abundance with respect to hydrogen scales with overall metallicity. The two exceptions to this relationship are $\mathrm{He}$ and $\mathrm{N}$.

For helium, the chemical yield from stars simply adds to the primordial abundance estimated by Pagel et al. (1992) to be $\mathrm{He} / \mathrm{H}=0.0737\left(Y_{P}=0.228\right)$. This gives

$$
\mathrm{He} / \mathrm{H}=0.0737+0.0293 Z / Z_{\odot} .
$$

Nitrogen is well known to possess both a primary and secondary nucleosynthetic component. For low-metallicity galaxies $(\log (\mathrm{O} / \mathrm{H}) \leq-4.0)$ the $\mathrm{N} / \mathrm{O}$ ratio is approximately constant, as expected for a "primary" element whose production is independent of metallicity. However, for highmetallicity galaxies $(\log (\mathrm{O} / \mathrm{H}) \geq-3.5)$ the $\mathrm{N} / \mathrm{O}$ ratio is found to rise steeply with metallicity, suggesting that nitrogen becomes a "secondary" element whose production is proportional to the abundance of other heavy elements, mainly $\mathrm{C}$ and $\mathrm{O}$.

To take account of this effect, we have created a linear combination of the primary and secondary components of nitrogen, fitting the resulting this curve to several abundance data sets, with the only condition being that it must pass through the solar abundance values.

Three data sets were used, with two of these coming from Mouhcine \& Contini (2002): the H II Galaxy sample and the starburst nuclear galaxy sample (SBNG), both of which are compilations of several surveys. The third data set comes from Kennicutt et al. (2003), which are measurements of H II regions within the giant spiral galaxy M101.

The analytic function obtained for the nitrogen abundance is

$$
[\mathrm{N} / \mathrm{H}]=[\mathrm{O} / \mathrm{H}]\left(10^{-1.6}+10^{\left(2.33+\log _{10}[\mathrm{O} / \mathrm{H}]\right)}\right) .
$$

The fit of this curve with data can be seen in Figure 2. The fit is generally good, but as the curve must pass through 


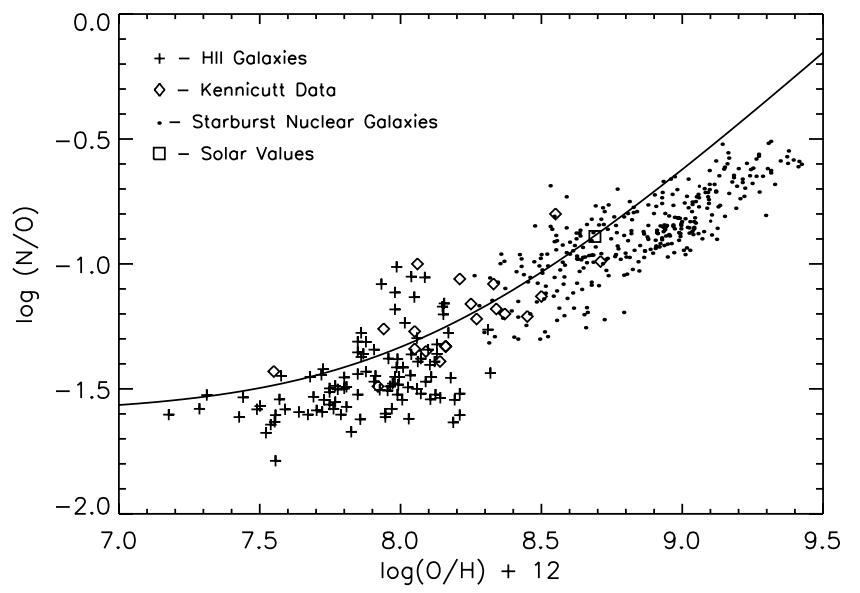

FIG. 2.- $[\mathrm{N} / \mathrm{O}]$ ratio against oxygen abundance for a sample of galaxies and $\mathrm{H}$ II regions, and our analytic fit to the $[\mathrm{N} / \mathrm{O}]$ variation with metallicity. The solar value is marked by the square and constrains our analytic fit. The data sets are as marked in the key, with the references given in the text.

the solar values, it tends to give a higher $\mathrm{N} / \mathrm{O}$ ratio than the observations at large metallicities.

\section{PHOTOIONIZATION MODELS}

In Dopita et al. (2002) we demonstrated the important effects of both radiation pressure and dust on the output line ratios in photoionization models. In this and a following paper (Groves et al. 2004, hereafter Paper II) we will systematically explore the parameter space of the ionization parameter, abundance, slope of power-law photoionizing spectrum, and density for both dusty, radiation pressure-dominated photoionization models and for the standard (dust and radiation-pressure free) photoionization models. The chosen physical parameters are common to both types of model and are chosen to cover the typical values inferred for the NLR of active galaxies.

The photoionization models are all one-dimensional, equilibrium models and have a plane-parallel geometry, representing the NLR clouds far from the AGN. Both sets of models are truncated at the point at which the fraction of $\mathrm{H} \mathrm{II}$ drops below $1 \%$.

\subsection{Abundance Variation}

As stated in the previous section $(\S 3)$ we have run five abundance sets with metallicities of $0.25 Z_{\odot}, 0.5 Z_{\odot}, 1 Z_{\odot}, 2 Z_{\odot}$, and $4 Z_{\odot}$ with relative abundances as discussed in that section.

The gas abundances in both the dusty and dust-free models are depleted by dust (by the amounts given in Table 1). In the dusty, radiation pressure models the depleted heavy elements reside in the dust, but in the standard photoionization models the depleted heavy elements are effectively lost. The reason for this is that the cooling and, to some extent, the ionization are determined by the gas phase abundance of heavy elements. It is therefore important for comparison purposes that the gas phase abundances in both sets of models is the same. To demonstrate this effect we have run a third set of standard, dust-free photoionization models that are not depleted. This third set reproduces the standard models seen in previous papers (e.g., Allen et al. 1998).

\subsection{Density Structure}

For each set of models we examine three densities: $n_{\mathrm{H}}=$ $10^{2}, 10^{3}$, and $10^{4} \mathrm{~cm}^{-3}$.
For the standard constant density (isochoric) dust-free models the concept of a hydrogen density is straightforward. However, in radiation pressure-dominated models that have constant gas plus photon pressure (isobaric models) the concept is not so straightforward, since the local density varies continually throughout the models. Since the density sensitive lines like [S II] $\lambda \lambda 6717,6730$ are most strongly emitted close to the ionization front, the local $U$ is low. It is in the same region that effectively all the radiation pressure in the ionizing radiation field $P_{*}$ has been accumulated, such that the local pressure $P_{\text {loc }}=P_{*}+P_{0}$. We therefore have chosen to set the hydrogen density in the models to be the density in the [S II] emission zone, physically near to the ionization front, but where there are still sufficient ionizing photons to ensure that $n_{\mathrm{H} \text { II }} / n_{\mathrm{H}} \sim 1.0$.

\subsection{Form of the Ionizing Spectrum}

Following the convention for photoionization models of AGNs we use a simple power law to represent the spectrum of the ionizing source, with

$$
F_{\nu} \propto \nu^{\alpha}, \quad \nu_{\min }<\nu<\nu_{\max } .
$$

In our models we set $\nu_{\min }=5 \mathrm{eV}$ and $\nu_{\max }=1000 \mathrm{eV}$.

We investigate four values of the power-law index $\alpha,-1.2$, $-1.4,-1.7$, and -2.0 . These encompass the "standard" values usually adopted. The factor of proportionality, which determines the total radiative flux entering the photoionized cloud, is set by the ionization parameter at the front of the cloud, $U_{0}=S_{\star} /\left(n_{0} c\right)$, where $S_{\star}$ is the flux of ionizing photons, and the initial value of the density $n_{0}$ is set by the initial pressure in the ionized gas, $P_{0}$, defined in the previous section.

As the final parameter of the models, we set the value of $U$ defined at the front of the cloud and hence the total flux entering the cloud. In the case of the standard isochoric photoionization models the density at the front of the cloud is fixed. However, for the dusty, radiation pressure-dominated, isobaric models, the front end density $n_{0}$ is an unknown quantity, because we do not a priori know the temperature of the plasma at photoionization equilibrium. We have estimated this by assuming a frontend temperature of $T_{0}=20,000 \mathrm{~K}$ and obtaining the density from $P_{0}$. This may be an underestimate for high ionization parameters (which give a high $T_{0}$ ) and an overestimate for the low ionization parameters. However, these errors will have very little effect on the observed density in the [S II] emission zone.

A better ionization parameter to use in this instance would be the ratio of gas pressure to radiation pressure $(\Xi=$ $\left.P_{\text {rad }} / P_{\text {gas }}\right)$, but we have chosen to adopt $U$ as the ionization parameter to facilitate comparison of our models with those published in the literature and to limit confusion.

\section{THE DENSITY, IONIZATION, AND TEMPERATURE STRUCTURE}

In this section, we investigate the temperature and ionization structure of the dusty, radiation pressure-dominated models. We explore the dependence of the dusty model structures upon the parameters and reveal how these differ from the corresponding structure of the dust-free models.

Figure 3 demonstrates the one-dimensional structure of the ionized region of a dusty NLR cloud from the front of the cloud to the point at which hydrogen is $99 \%$ recombined. The model has an initial ionization parameter of $U_{0}=10^{-2}$, a metallicity of $1 Z_{\odot}$, a power-law ionizing SED with an index of $\alpha=-1.4$, and a hydrogen density of $n_{\mathrm{H}}=1000 \mathrm{~cm}^{-3}$. The distance has been normalized to allow for easy comparison 

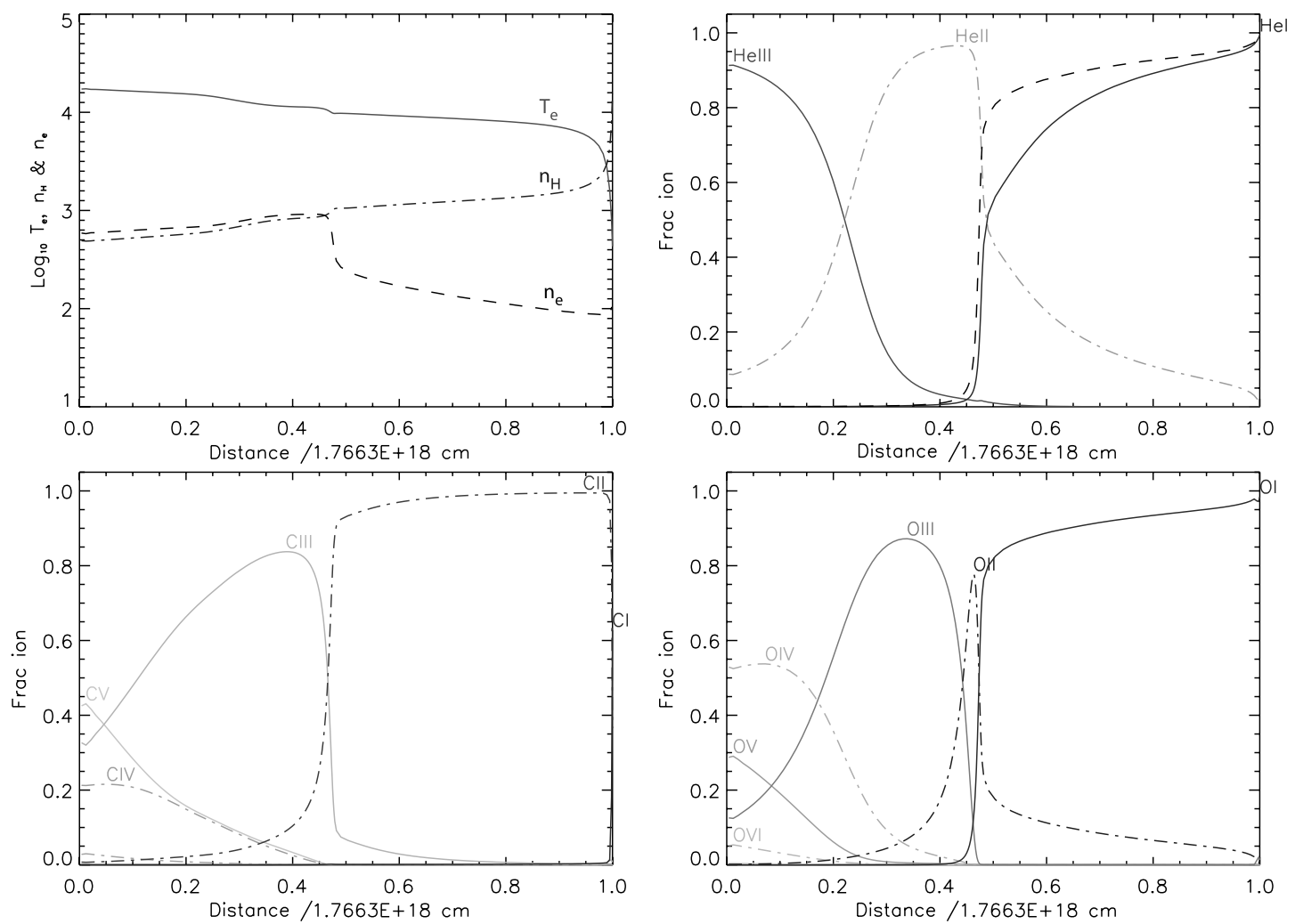

FIg. 3.- Structure diagram showing the variation of electron temperature, electron and hydrogen density, and the ionization states of hydrogen, helium, carbon, and oxygen with distance in a dusty model. The distance is normalized to 1 , with the distance in $\mathrm{cm}$ given within the figure. The model parameters are $1 Z_{\odot}$ metallicity, $\alpha=-2.0, n_{\mathrm{H}}=1000 \mathrm{~cm}^{-3}$, and $\log U_{0}=-2.0$.

between the different models. Figure $3 a$ (upper left corner) shows the temperature and density structure of the nebulae. The inverse correspondence between the temperature $\left(T_{e}\right)$ and hydrogen density $\left(n_{\mathrm{H}}\right)$ is due to the isobaric nature of the models. This inverse correspondence is not perfect owing to the addition of the radiation pressure term. The electron density $\left(n_{e}\right)$ follows the hydrogen density up until hydrogen begins to recombine, at which point $n_{e}$ drops sharply. This point is better seen in Figure $3 b$, which displays the ionization structure of helium and the fraction of neutral hydrogen.

The classical ionization front is marked by the point at which the fraction of neutral hydrogen sharply increases and the electron density drops. Beyond this is a partially ionized region dominated by X-ray heating and photoionization, including Auger processes. This region forms for all power-law ionizing spectra. The fully ionized region is marked by a high temperature $\left(\sim 2 \times 10^{4} \mathrm{~K}\right)$ and low density and is the region in which the higher ionization species are found. The X-ray ionized region has a lower temperature $\left(\sim 1 \times 10^{4} \mathrm{~K}\right)$ and higher density and contains mainly neutral or singly ionized species that are ionized and excited by secondary electrons. These electrons are produced as "knock on" electrons in slowing down the energetic primary electrons produced by inner-shell photoionization.

The transition from fully ionized to partially ionized is clearly demarcated in the ionization structure of helium and the heavier elements. Figures $3 c$ and $3 d$ show the fractional ionization structure for two other dominant species: carbon and oxygen. Each ionization species is labeled within the figure. The change from fully to partially ionized is seen in the transitions of $\mathrm{He}$ II to $\mathrm{He}_{\mathrm{I}}, \mathrm{C}_{\text {III }}$ to $\mathrm{C}$ II, and $\mathrm{O}$ III to $\mathrm{O}$ I. The ionization structure of oxygen is particularly interesting as the narrow peak of $\mathrm{O}$ II is a good marker of the transition point. The fraction of the $\mathrm{O}$ II ion rises once the local ionization parameter reaches low enough values but then quickly falls as hydrogen becomes neutral. This is due to the nearly resonant charge-exchange reaction between $\mathrm{O}$ II and $\mathrm{H} \mathrm{I}$, which locks the oxygen ionization balance to that of hydrogen.

\subsection{Determining Factors of the Model Structure}

Of all the parameters we use to define the models, the major determinant of the NLR cloud structure is the ionization parameter. At high ionization parameters (Fig. 4), the relative number of ionizing photons is increased, which increases the average temperature and ionization state of the nebulae. With a low ionization parameter (Fig. 5), there are not enough photons to maintain a high ionization state and the majority of the $\mathrm{H}$ II region consists of low ionization species. Both the ionized column and the distance from the front of the cloud to the ionization front are much smaller in the low ionization parameter case, owing to the smaller number of ionizing photons impinging on the ionized layer.

The same is seen for the dust-free models. At an ionization parameter of $U_{0}=10^{-1}$ the dust-free model with depleted abundance (Fig. 6) shows a similar high ionization state to the equivalent dusty model. Likewise, the low ionization parameter dust-free model (Fig. 7) shows the same low ionization state as the dusty model with $U_{0}=10^{-3}$.

There are several differences between the dusty models and the dust-free models. Most of these differences are more obvious at the higher ionization parameter, when dust dominates the opacity and are discussed in the next section. At the low ionization parameter of $U_{0}=10^{-3}$ there are few major differences, but one that is conspicuous is the larger distance covered 

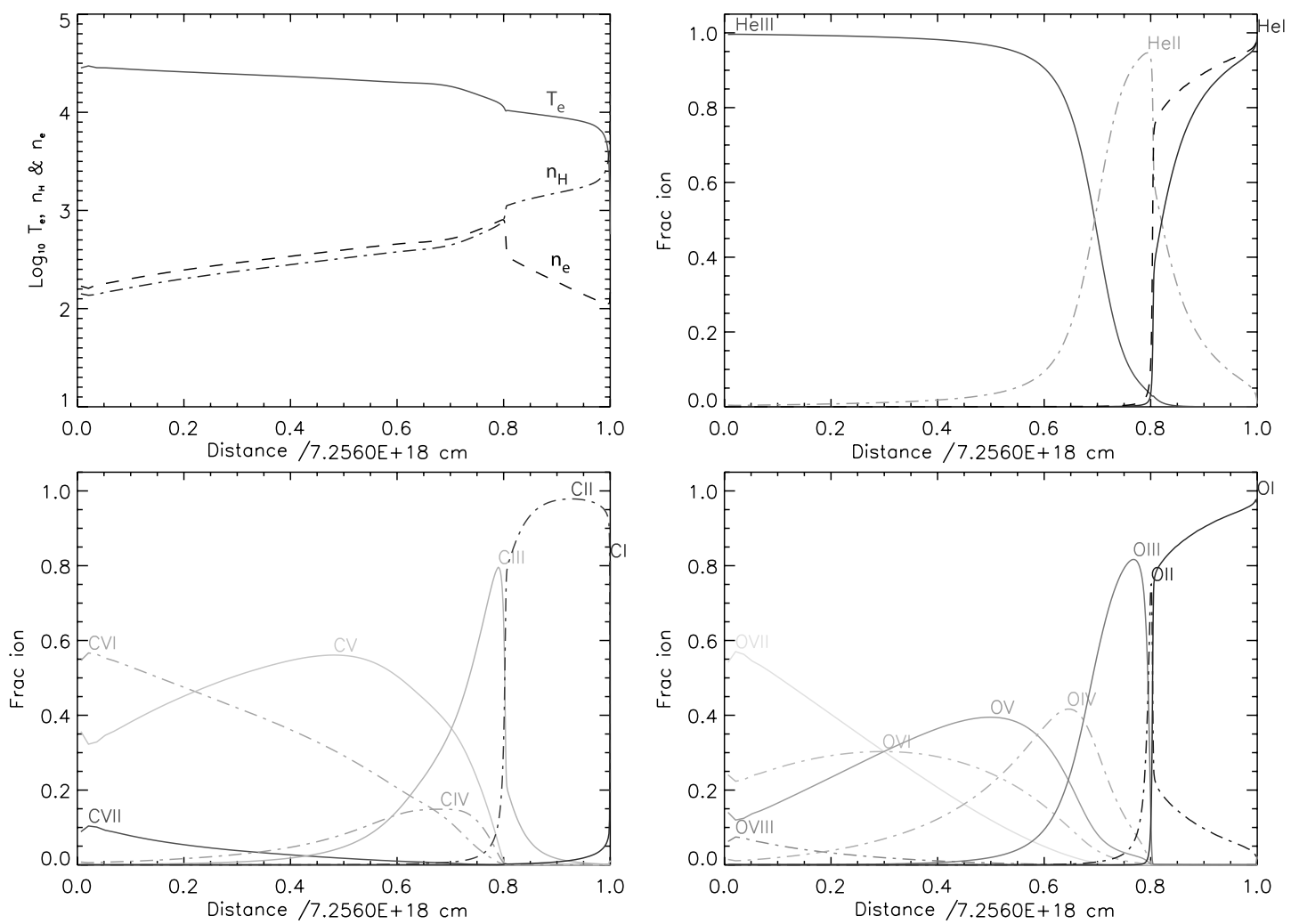

FIG. 4.-Structure diagram: $1 Z_{\odot}$ metallicity, $\alpha=-1.4, n_{\mathrm{H}}=1000 \mathrm{~cm}^{-3}, \log U_{0}=-1.0$.
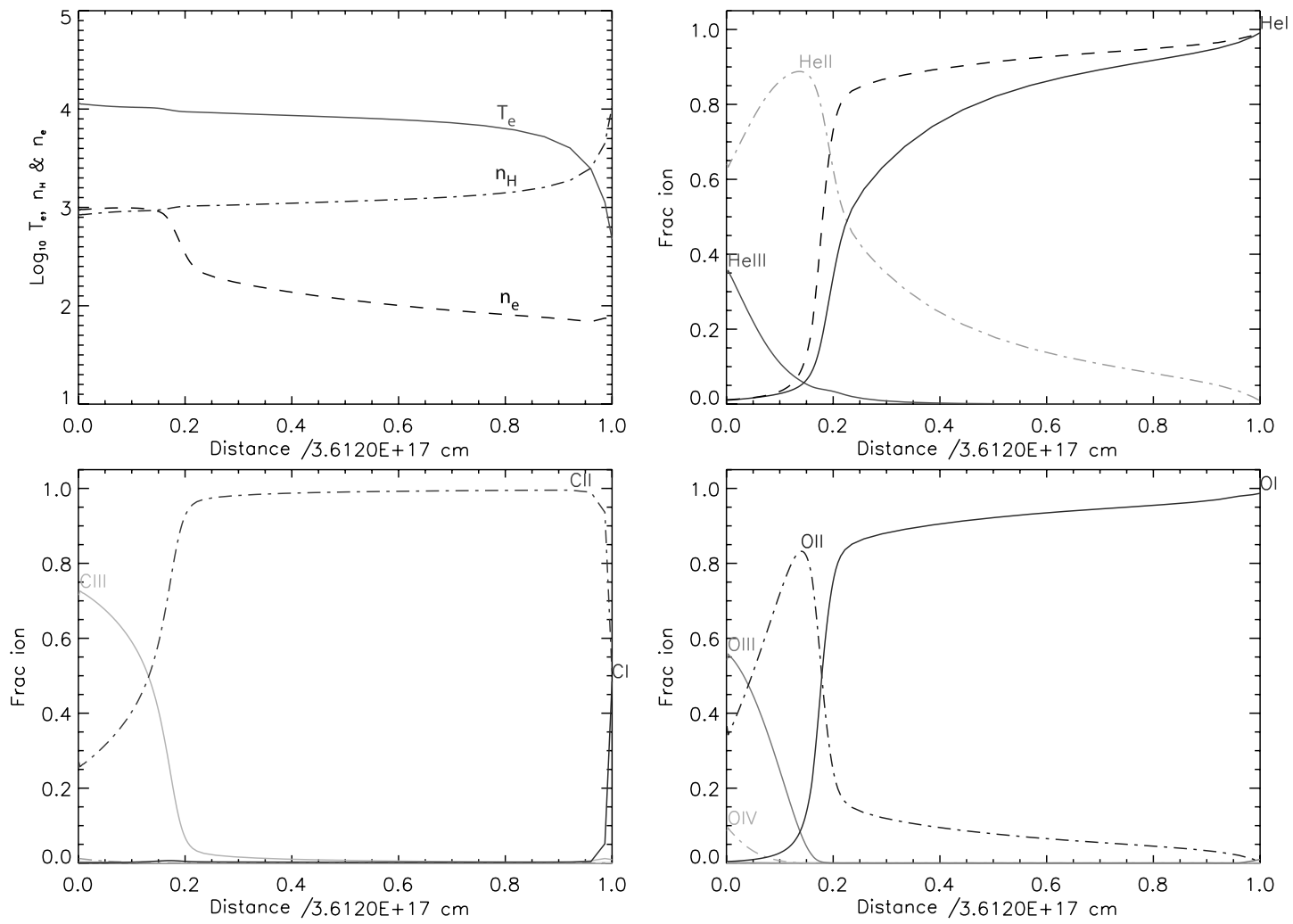

FIG. 5.-Structure diagram: $1 Z_{\odot}$ metallicity, $\alpha=-1.4, n_{\mathrm{H}}=1000 \mathrm{~cm}^{-3}, \log U_{0}=-3.0$. 

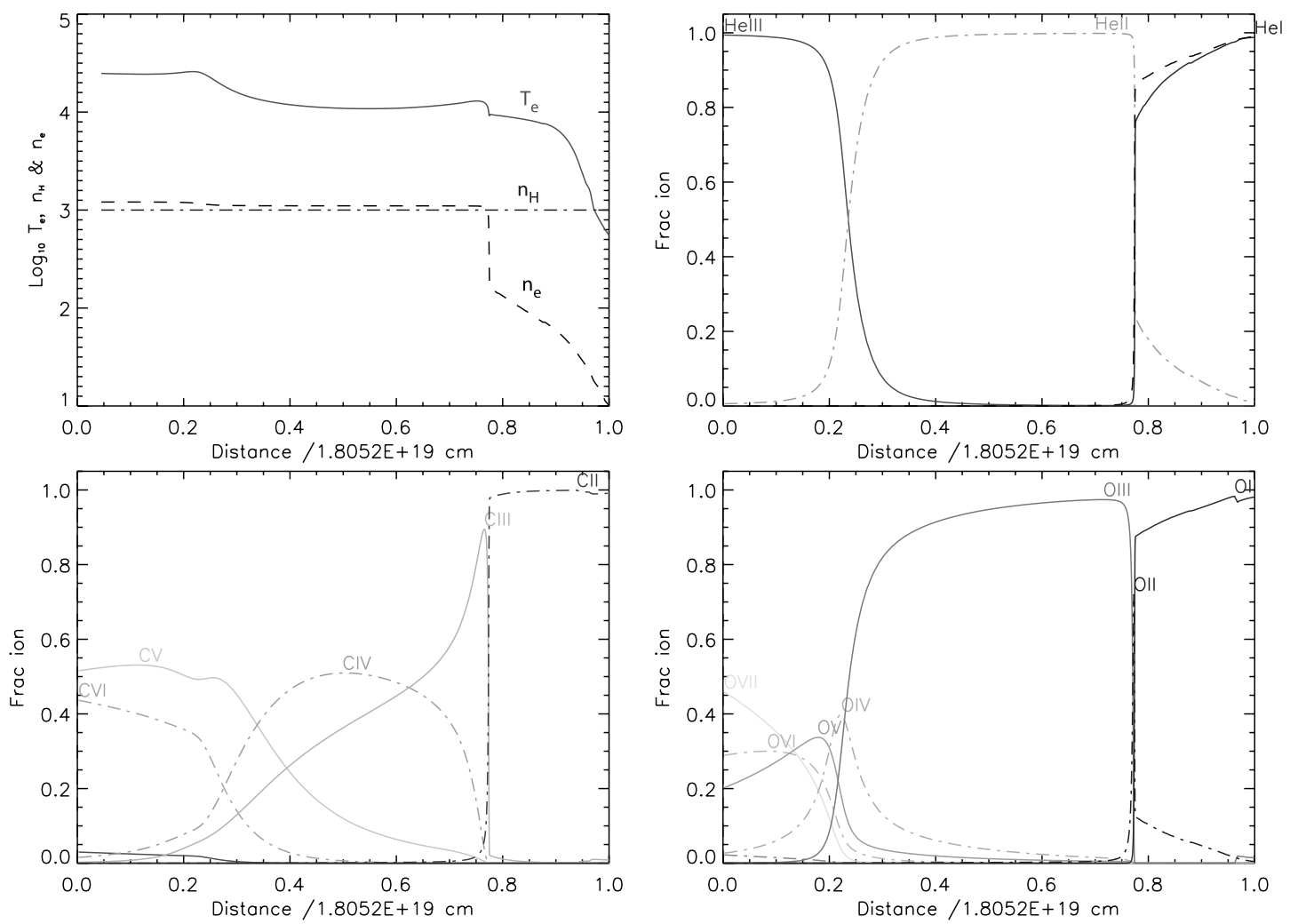

FIG. 6. - Structure diagram for dust-free model: $1 Z_{\odot}$ metallicity, $\alpha=-1.4, n_{\mathrm{H}}=1000 \mathrm{~cm}^{-3}, \log U_{0}=-1.0$.
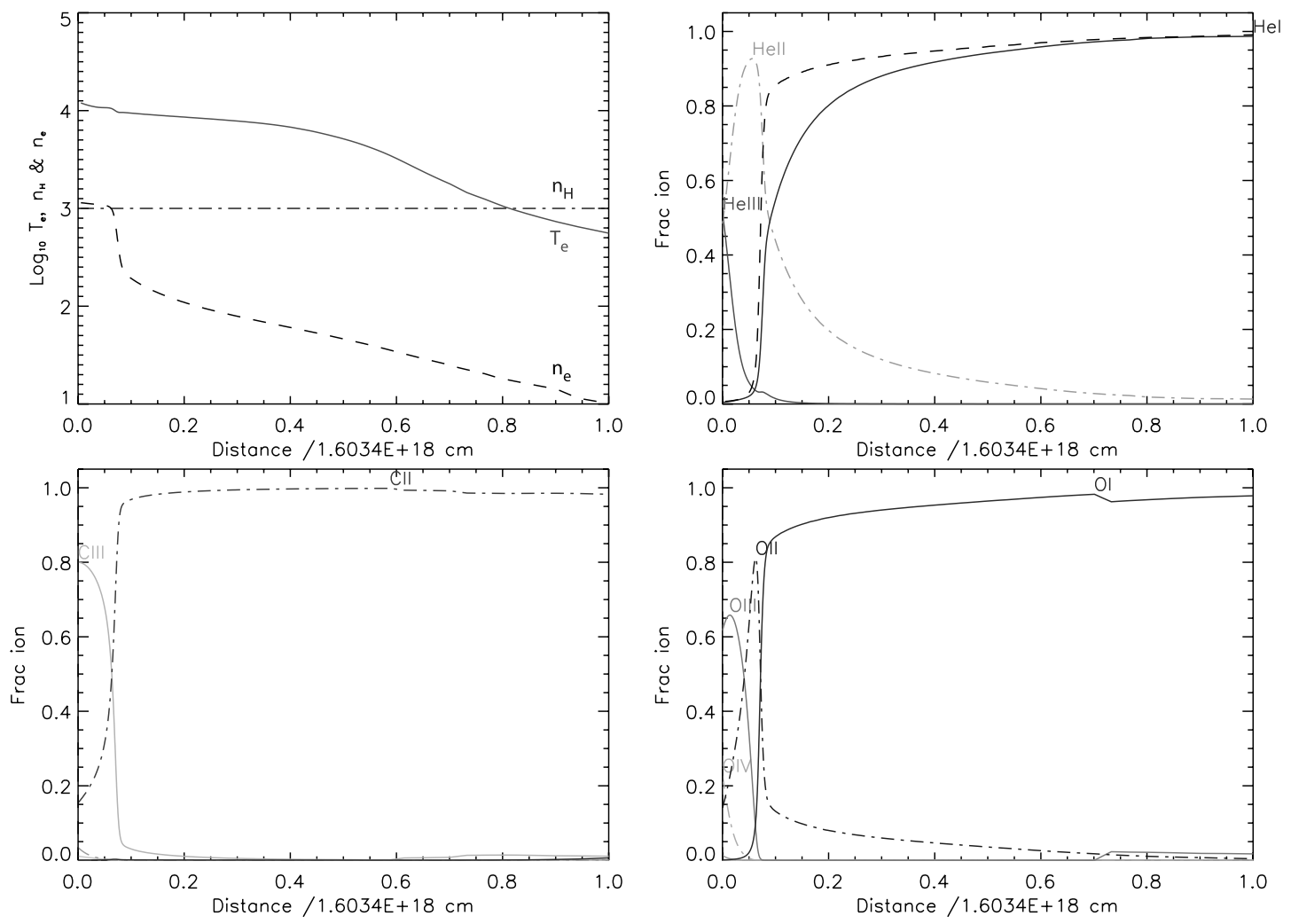

FIG. 7.-Structure diagram for dust-free model: $1 Z_{\odot}$ metallicity, $\alpha=-1.4, n_{\mathrm{H}}=1000 \mathrm{~cm}^{-3}, \log U_{0}=-3.0$. 

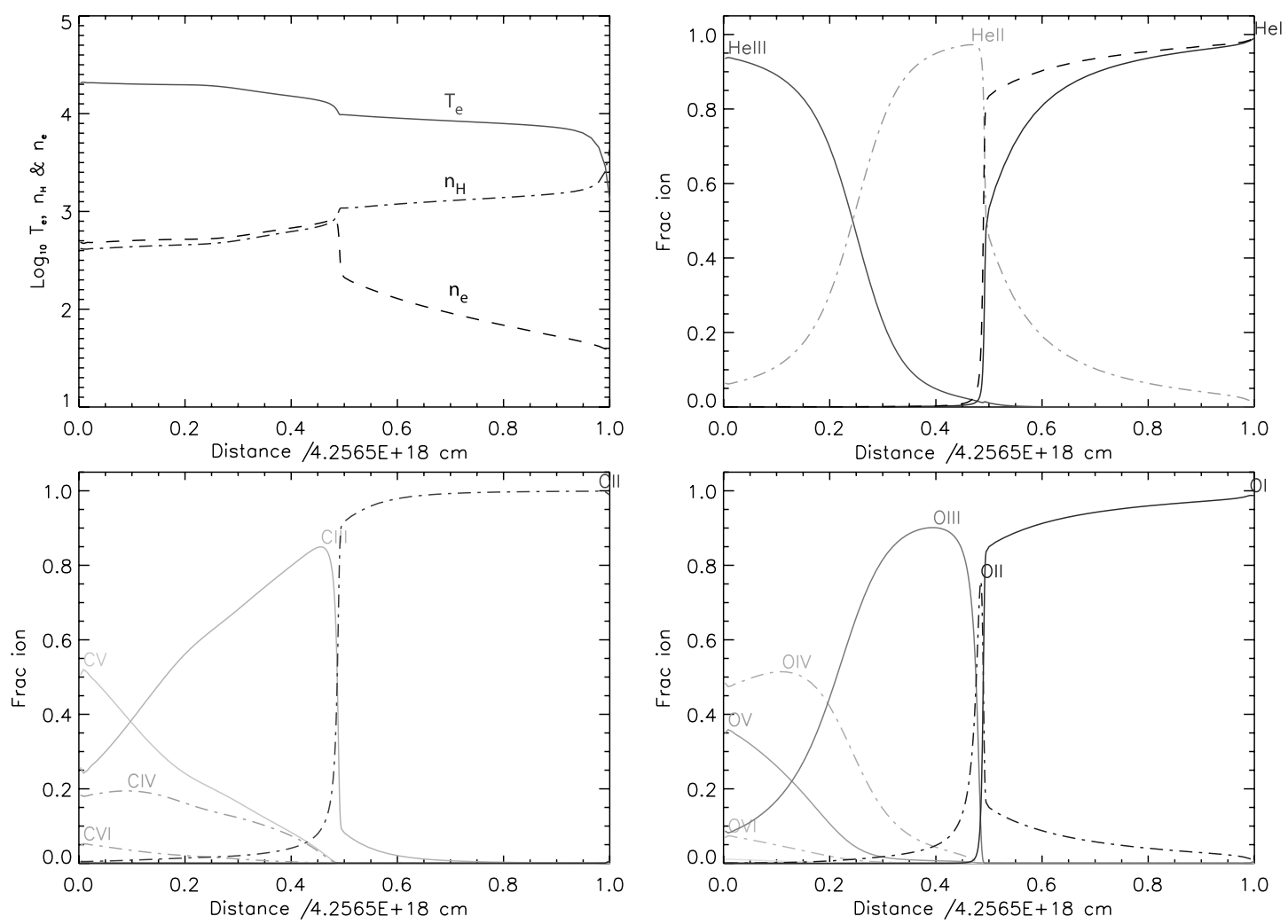

FIG. 8.-Structure diagram: $0.25 Z_{\odot}$ metallicity, $\alpha=-1.4, n_{\mathrm{H}}=1000 \mathrm{~cm}^{-3}, \log U_{0}=-2.0$.

by the dust-free models. This difference predominantly arises because of the dust opacity, which competes with hydrogen for the ionizing photons, and thus reduces the total ionized column (Netzer \& Laor 1993; Kraemer et al. 1994). Even with its low density, dust is such a dominant opacity source because of two reasons: it has a broad opacity (see Fig. 1), and it is able to multiply absorb ionizing photons. This is compared with the atoms of the gas, which have an ionizing opacity strongly peaked at their ionization potential and can only absorb one ionizing photon before their opacity is altered.

The other factor that causes the disparity in distance is the different density structure of the two models. For the dust-free models, their isochoric nature means that the total column depth of hydrogen is determined only by the distance. In the isobaric dusty models the increasing density means that the total column of hydrogen can be reached within a smaller distance.

Even with this effect, the density has little influence upon the overall temperature and ionization structure of the nebula in either model. The density does determine the distance to the recombination edge from the front of the cloud, but simply because the column depth is approximately constant for a given $U_{0}$, ionizing SED, and metallicity. As the distance is normalized within the diagrams, no effect is seen in the ionization structure. As the ionization structure does not change, the electron density just scales with the change in density parameter. The temperature structure varies little with density, as density only affects collisional de-excitation, which is a second-order effect in the cooling function. Cooling and heating by dust is a first-order effect with density, but this dependence is through the ionization parameter, which is kept constant when varying density.

In terms of the ionization and temperature structure, more interesting are the effects of the variation of either the met- allicity or the ionizing power-law spectrum. Of course, the inclusion of dust into the models also has a large effect upon both of these. The photoelectric process on dust is a strong heating mechanism within a photoionized nebula. The dust can also heat the nebula through the sticking of preferentially low-energy electrons to the grains, thus increasing the average energy of the gas locally. These processes explain the hotter temperatures seen in the dusty models in comparison with the dust-free ones (cf. Figs. 4-6). The slope in $T_{e}$ seen with distance (and $U_{\text {local }}$ in $\S 5.2$ ) in the dusty models can also be explained by these processes. Both these heating processes are dependent upon the local ionization parameter, being stronger at higher values, and thus increase closer toward the front of the cloud. Dust also has a secondary heating effect through the depletion of metals from the gas.

Metallicity strongly affects the temperature structure of a nebula as a result of the prominent part that heavy elements play in the cooling processes. The temperature of a nebula is set by the balance of the heating and cooling processes, which are functions of the temperature and metallicity of the gas. The heating is dominated by photoionization, with some contribution from recombination. The cooling is mainly through emission lines and collisional losses. Increasing the abundance of metals does increase the heating by providing greater absorption, with this being especially important at high $U_{0}$. However, the cooling is much more strongly affected by an increase in metals, with a higher abundance meaning stronger emission lines and greater cross section for collisions.

Figures 8 and 9 show two dusty models with metallicities of $0.25 Z_{\odot}$ and $4 Z_{\odot}$, respectively. The other parameters are set to the fiducial values of $\log U_{0}=-2.0, \alpha=-1.4$ and $n_{\mathrm{H}}=$ $1000 \mathrm{~cm}^{-3}$. The effect of varying the metal abundance upon the temperature structure is obvious in both diagrams, with the 

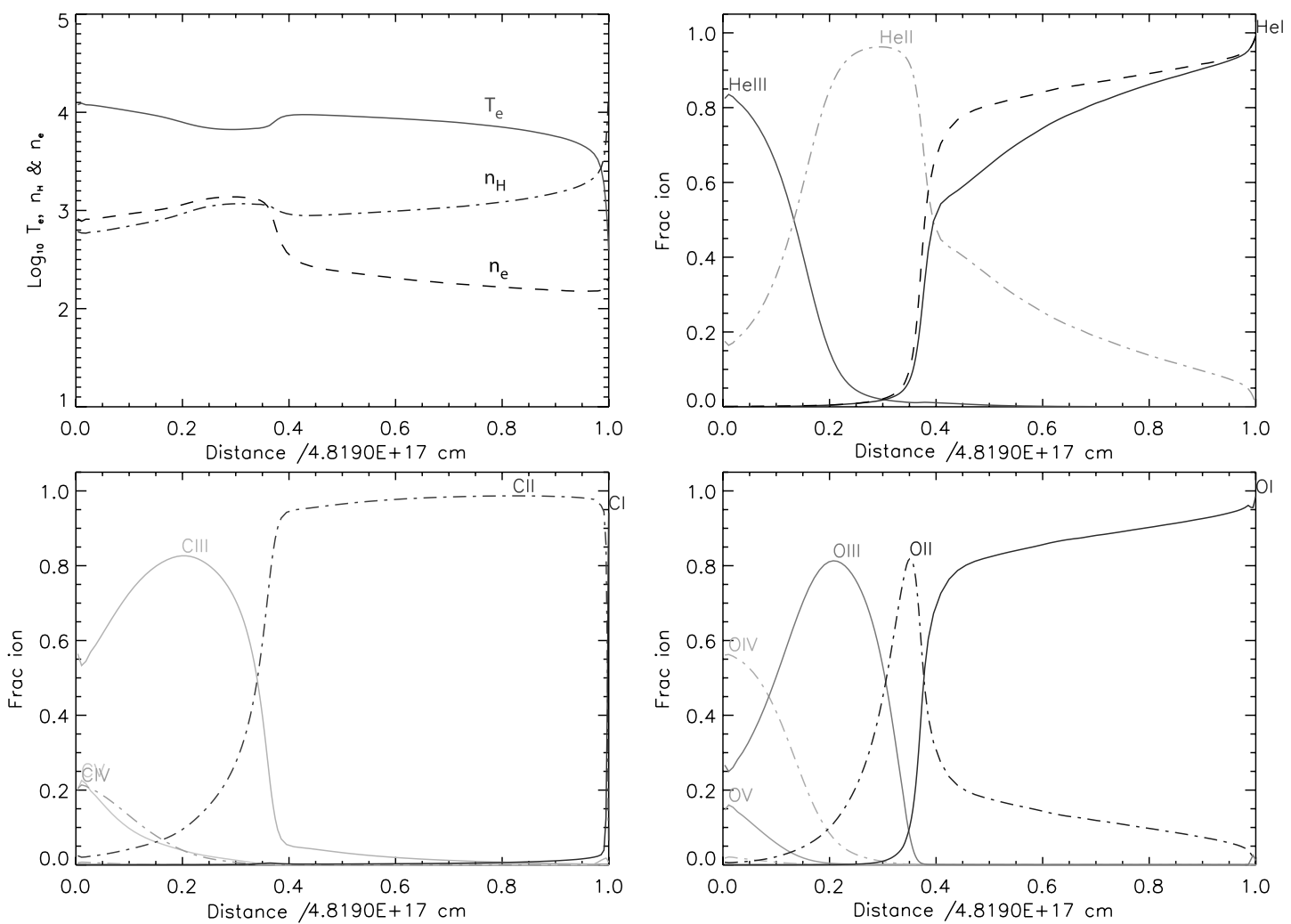

FIG. 9.-Structure diagram: $4 Z_{\odot}$ metallicity, $\alpha=-1.4, n_{\mathrm{H}}=1000 \mathrm{~cm}^{-3}, \log U_{0}=-2.0$.

low-metallicity model having a much higher temperature overall than the high-metallicity case. The higher temperature in the low- $Z$ model leads to a lower average density in these isobaric models. This means that the distance to the ionization front and the end of the model is increased in the low- $Z$ models to obtain the same column depth.

The change in the overall temperature resulting from the metallicity variation also affects the ionization state somewhat. This is because the recombination rate is temperature dependent, becoming slower at high temperatures, and therefore the low- $Z$ model displays a larger fraction of high ionization species in comparison with the model with $Z=4 Z_{\odot}$.

This region at the inner edge of the cloud that displays these high ionization species is dominated by the photoionization and line emission of the metals. As the photon field and temperature decrease, a second region occurs defined by He II. This region can be seen in the temperature structure of both the low- $Z$ model (Fig. 8) and the high- $Z$ model (Fig. 9). In the $Z=0.25 Z_{\odot}$ model the region appears as a change in slope in the temperature structure, but in the $Z=4 Z_{\odot}$ model the region can be seen as an obvious dip in the temperature. This larger dip is due to the increased abundance, and hence collisional cooling, of the metal ions in this region such as $\mathrm{O}$ III, $\mathrm{S}$ III, and Ne III. These are strong coolants in the visible, as can be seen by the strength of the [O III] $\lambda 5007$ line, and counteract the heating due to photoionization. The effects of this temperature drop can be seen in the density as well as in the ionization structure of carbon and oxygen due to the increase in the recombination rate. The $\mathrm{O}$ II peak is broad at high $Z$ compared with the low $-Z$ model as the low temperature leads to the early recombination of $\mathrm{O}$ III.

After the helium-dominated region there is a sharp transition zone in which hydrogen recombines and the He II fraction falls. This region is dominated by the $\mathrm{H}$ photoionization and line cooling. The equilibrium temperature in this region is $\sim 10^{4} \mathrm{~K}$, and hence there is a sharp drop seen in the temperature structure of the hot low- $Z$ model and a rise seen in the cool high- $Z$ model structure. The region is very narrow as a result of the rapid absorption of the $\mathrm{H}$ ionizing photons once the neutral ion fraction becomes significant. The final region is the partially ionized zone discussed before, dominated by $\mathrm{X}$-ray photoionization and secondary electron ionization. The temperature equilibrium in this region is reasonably insensitive to metallicity; thus, the similarity in appearance between the two models.

The change in structure due to metallicity variation has observational consequences. Apart from temperature sensitive diagnostic ratios like [O III] $\lambda 4363 /[\mathrm{O}$ III] $\lambda 5007$, there are diagnostics sensitive to the increased ionization state such as C III] $\lambda 1909 / \mathrm{C}$ II] $\lambda 2326$. These, along with abundance sensitive diagnostics like [ $\left.\mathrm{N}_{\text {II }}\right] \lambda 6583 / \mathrm{H} \alpha$, can be used to estimate the metallicity of NLR regions. These diagnostics are discussed in detail for both the dusty and the dust-free models in the following paper of this series (Paper II).

The index of the ionizing power law directly affects the temperature. As the power law becomes flatter, the average energy of the ionizing photons increases. This leads to an increase of the average energy per photoionization and thus an overall temperature increase in the nebula.

Figures 10 and 11 show two dusty models with a flatter ( $\alpha=-1.2)$ and steeper $(\alpha=-2.0)$ ionizing power-law spectrum, respectively. The other parameters are at the fiducial values of $U_{0}=10^{-2}, Z=1 Z_{\odot}$, and $n_{\mathrm{H}}=1000 \mathrm{~cm}^{-3}$. The temperature is clearly hotter in the $\alpha=-1.2$ model than the $\alpha=-2.0$ one. The ionization state within the $\alpha=-1.2$ model is also clearly higher than the steeper model. The reason for this is twofold. The flatter model has a hotter 

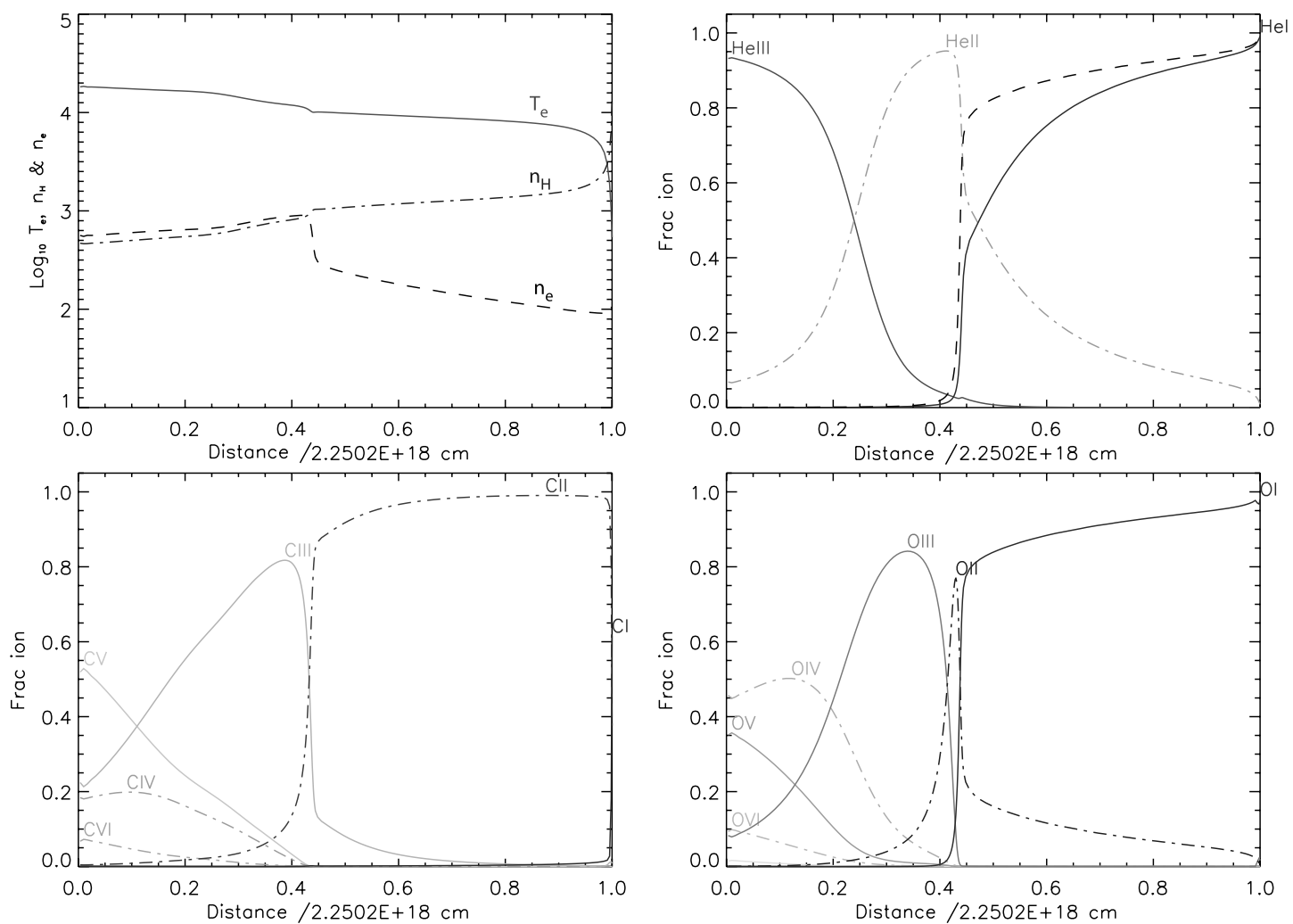

FIG. 10.-Structure diagram: $1 Z_{\odot}$ metallicity, $\alpha=-1.2, n_{\mathrm{H}}=1000 \mathrm{~cm}^{-3}, \log U_{0}=-2.0$.
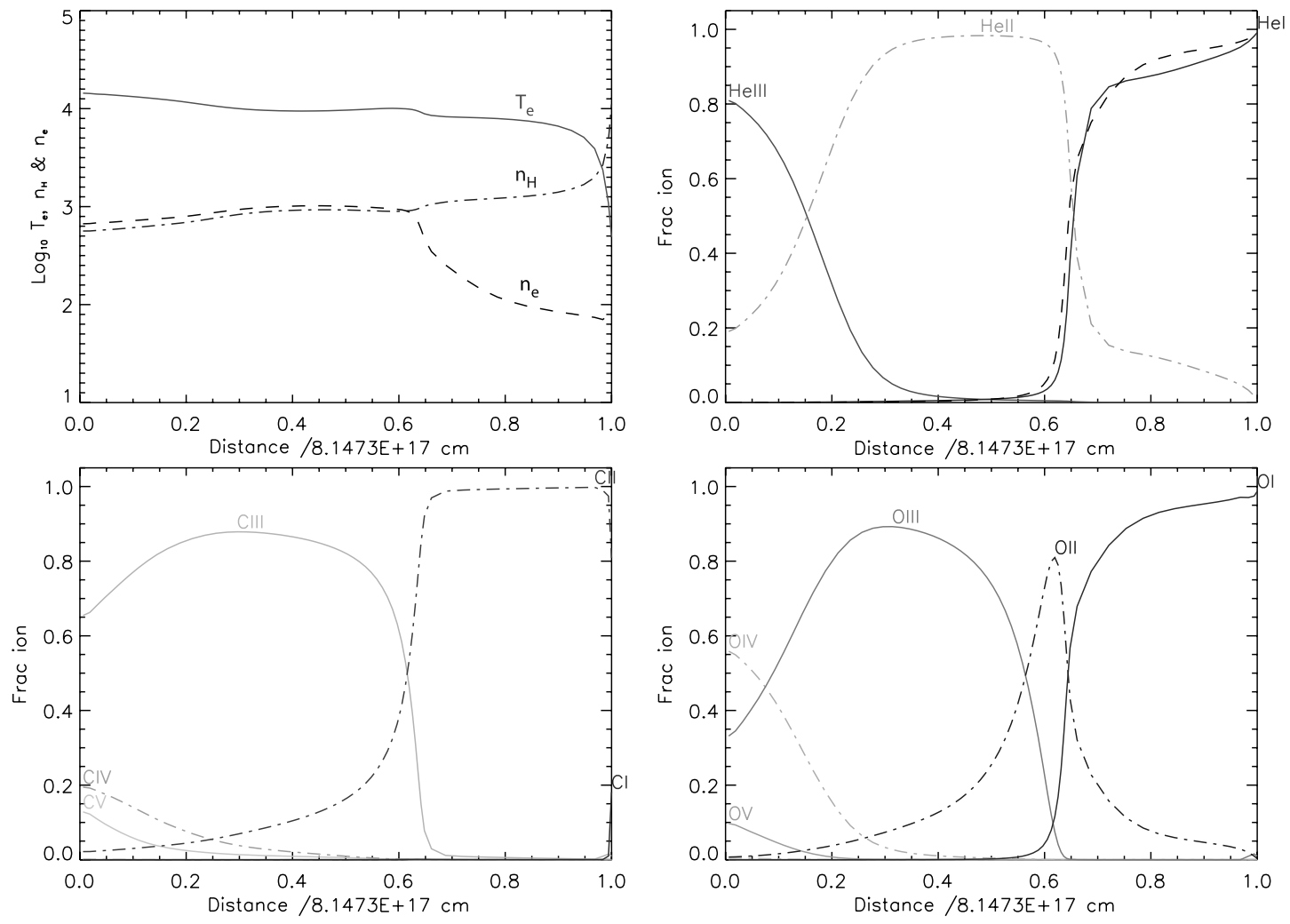

FIG. 11.-Structure diagram: $1 Z_{\odot}$ metallicity, $\alpha=-2.0, n_{\mathrm{H}}=1000 \mathrm{~cm}^{-3}, \log U_{0}=-2.0$. 

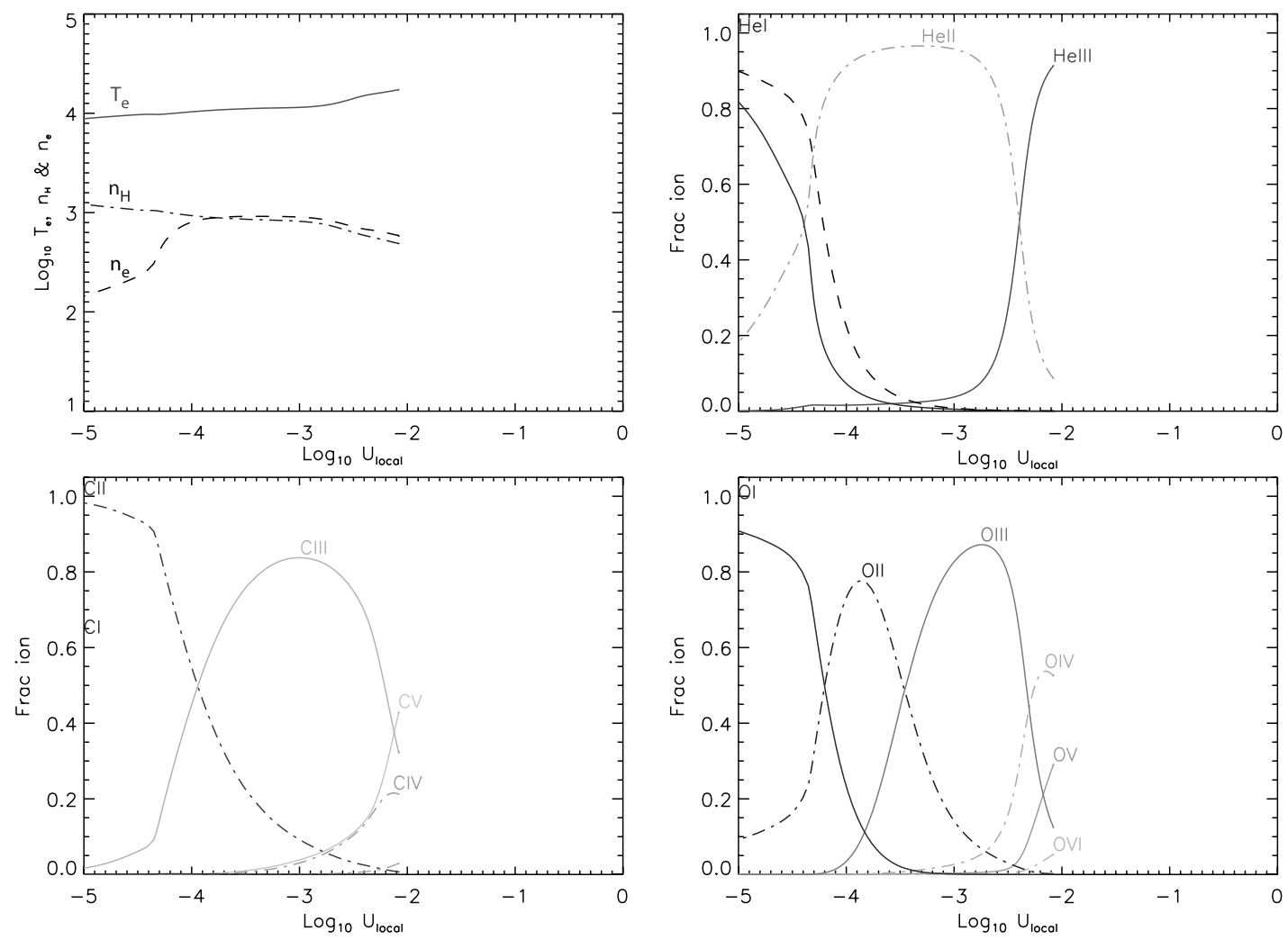

FIG. 12.- $U_{\text {local }}$ structure diagram showing the variation of electron temperature, electron and hydrogen density, and the ionization states of hydrogen, helium, carbon, and oxygen with local ionization parameter in a dusty model. The model parameters are $1 Z_{\odot}$ metallicity, $\alpha=-1.4, n_{\mathrm{H}}=1000 \mathrm{~cm}{ }^{-3}$, and log $U_{0}=-2.0$.

temperature than the $\alpha=-2.0$ model and thus a slower recombination rate. It also has more high-energy photons available for the ionization of the high ionization species. The relative increase of these high-energy photons to the lower energy ionizing photons is also why the He III zone appears much larger than the He II zone in the flatter spectrum model. The reverse is true for the steeper power-law model $(\alpha=$ -2.0 ) in which the $\mathrm{He}$ II zone becomes dominant as a result of the increase in $\mathrm{H}$ I and $\mathrm{He}$ i ionizing photons relative to the He II ionizing photons. This alters the temperature structure due to the He II cooling, and the cooling of the ions that coexist with He II in this zone.

Beyond the classical ionization front, the extended partially ionized region arises because of the additional X-rays from the AGN ionizing source. Thus, a change in the slope of the power-law ionizing spectrum leads to a change in the structure of this region. A flatter power law $(\alpha=-1.2)$ has relatively more X-rays and hence leads to a wider partially ionized zone relative to the model with a steeper power law $(\alpha=-2.0)$.

The effects of a change in the power-law index can also be seen observationally, such as the line diagnostic diagram Ar III] $\left.8.98 \mu \mathrm{m} / \mathrm{Ar}_{\mathrm{II}}\right] 6.98 \mu \mathrm{m}$ and $\mathrm{Ne}$ III] $15.5 \mu \mathrm{m} / \mathrm{Ne}$ II] $12.8 \mu \mathrm{m}$ discussed in Paper II.

\subsection{Ionization Structure with Local Ionization Parameter}

In order to understand where the emission of bright lines in NLRs is likely to originate from, we need to look at the cloud structure as a function of the local ionization parameter. The local ionization parameter is a better guide to the ionization of the nebula because the ionization state is largely determined the ratio of photon density to gas particle density. Through inspection of the diagrams of the structure against $U_{\text {local }}$ it becomes obvious as to why the dusty, radiation pressuredominated models provide an improvement over the standard isochoric photoionization models, and how the dusty models achieve the tight clustering on line diagnostic diagrams that is characteristic of NLRs.

Figure 12 displays the local ionization parameter structure diagram for a dusty model with solar metallicity $\left(1 Z_{\odot}\right)$, a power-law index of $\alpha=-1.4$, hydrogen density of $1000 \mathrm{~cm}^{-3}$, and an initial ionization parameter of $U_{0}=10^{-2}$. As in the previous structure diagrams this figure is divided into four parts, showing the temperature $\left(T_{e}\right)$ and density $\left(n_{\mathrm{H}}, n_{e}\right)$ structure in the upper left quadrant and the ionization structure of helium, carbon, and oxygen in the other three quadrants. The $x$-axis on all quadrants is $\log \left(U_{\text {local }}\right)$ and therefore is reversed with respect to the spatial coordinate used in the previous structure diagrams. A comparison with Figure 3 reveals the correspondence of $U_{\text {local }}$ with distance. Note that $U_{\text {local }}$ has been truncated at $10^{-5}$, which does not precisely correspond with the end of the photoionized model nebula. The transition to the partially ionized zone occurs at $\log U_{\text {local }} \sim-4.3$, but it does not appear as sharp in terms of $\log U_{\text {local }}$ as it does in terms of distance.

Figure 13 shows the dust-free model, which has the same model parameters as the dusty model in Figure 12. The density structure is clearly different, with the dust-free model having a constant hydrogen density, and an electron density determined entirely by the local state of ionization within the plasma. The differences between the ionization structure of the dusty and dust-free models is less obvious, as expected for the low initial ionization parameter. The high ionization zone is smaller in terms of $U_{\text {local }}$, and the ionization zone occupied by He II, C III, and $\mathrm{O}$ III appears to be broader. The transition to the partially 

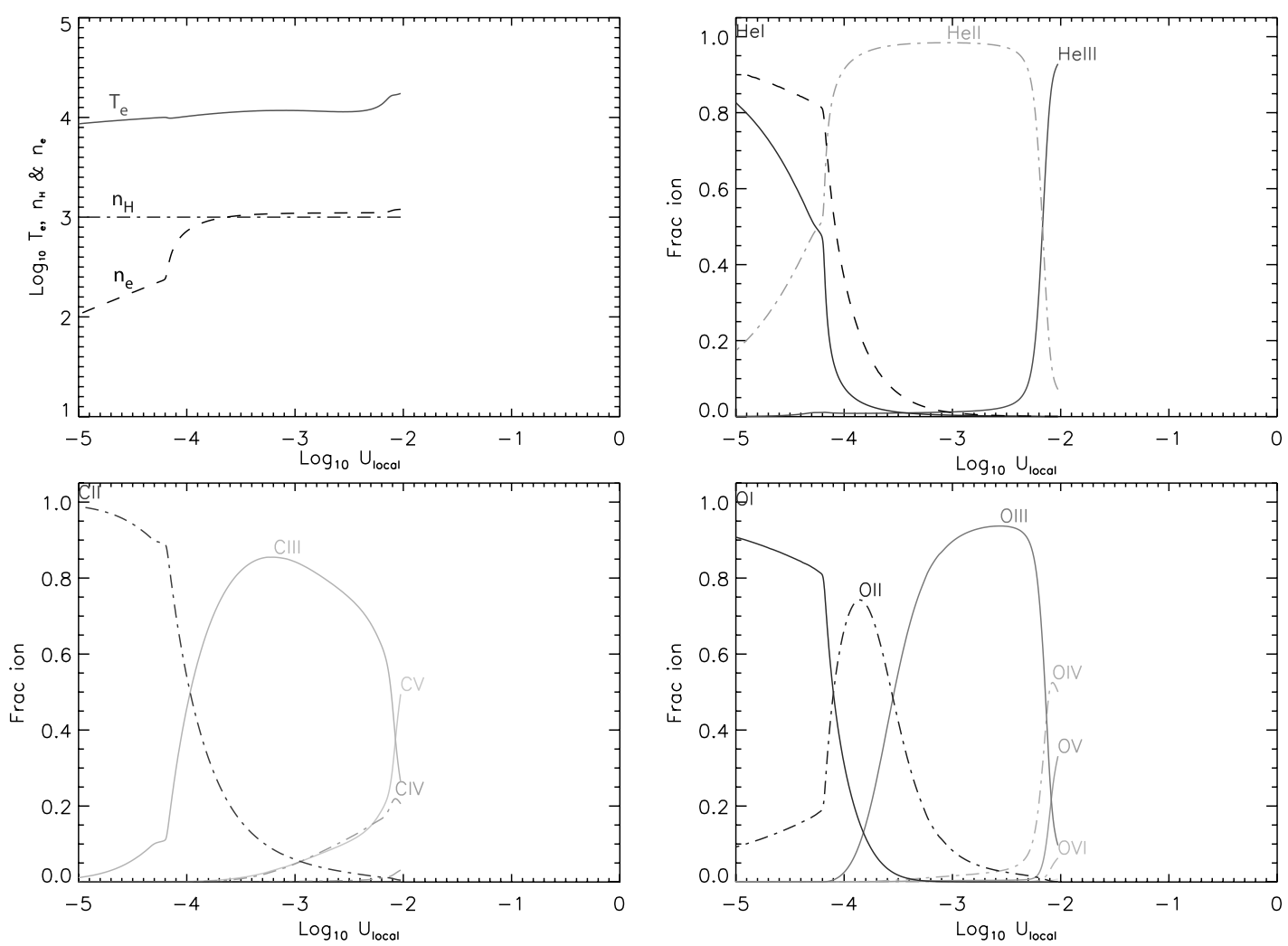

FIG. 13. $-U_{\text {local }}$ structure diagram showing the dust-free equivalent of Fig. 12 , with the same model parameters: $1 Z_{\odot}$ metallicity, $\alpha=-1.4, n_{\mathrm{H}}=1000 \mathrm{~cm}^{-3}$, $\log U_{0}=-2.0$.
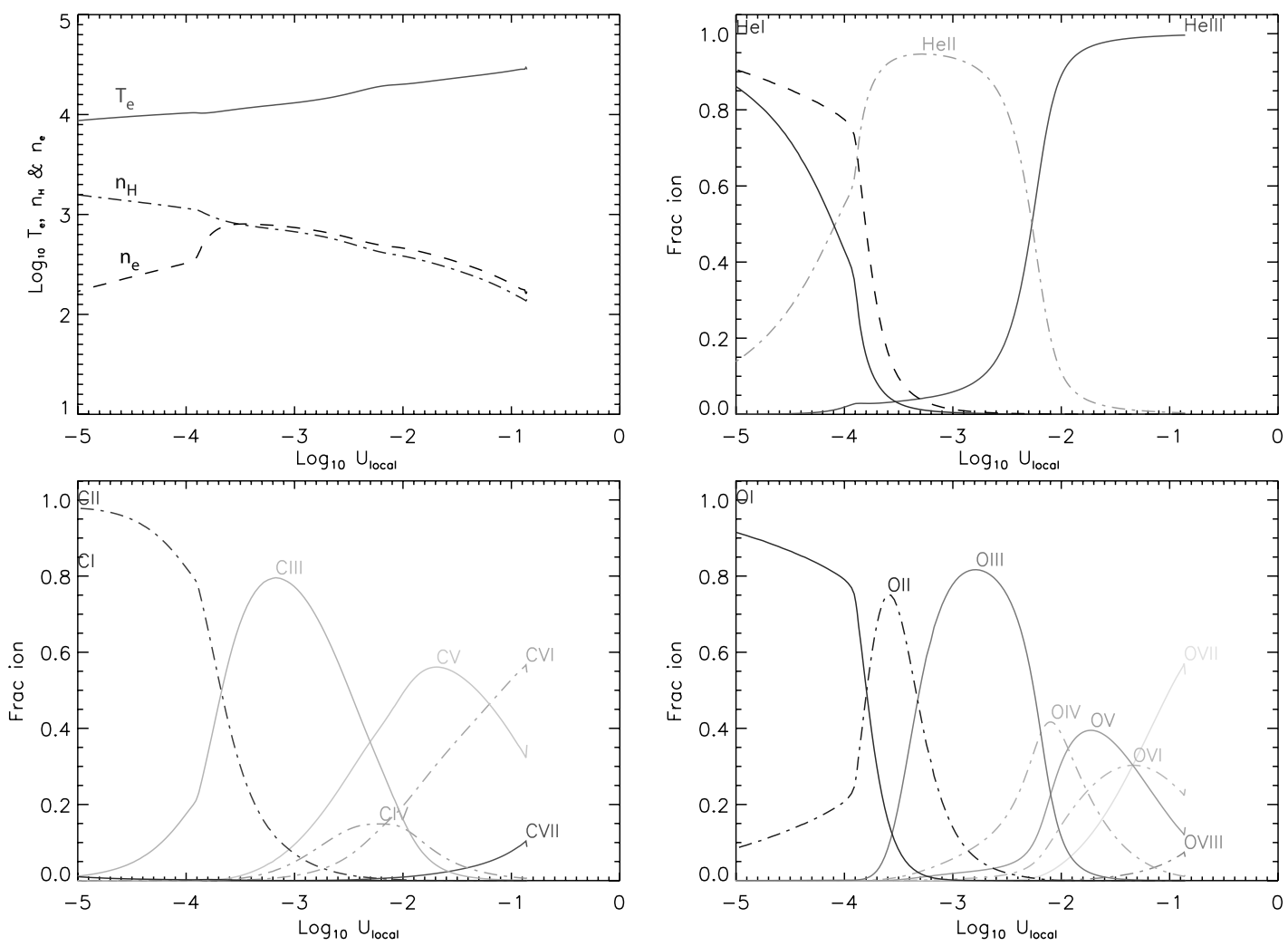

FIG. 14. $-U_{\text {local }}$ structure diagram showing a dusty model with initial ionization parameter $\log U_{0}=-1.0$, with $1 Z_{\odot}$ metallicity, $\alpha=-1.4$, and $n_{\mathrm{H}}=1000 \mathrm{~cm}{ }^{-3}$. 

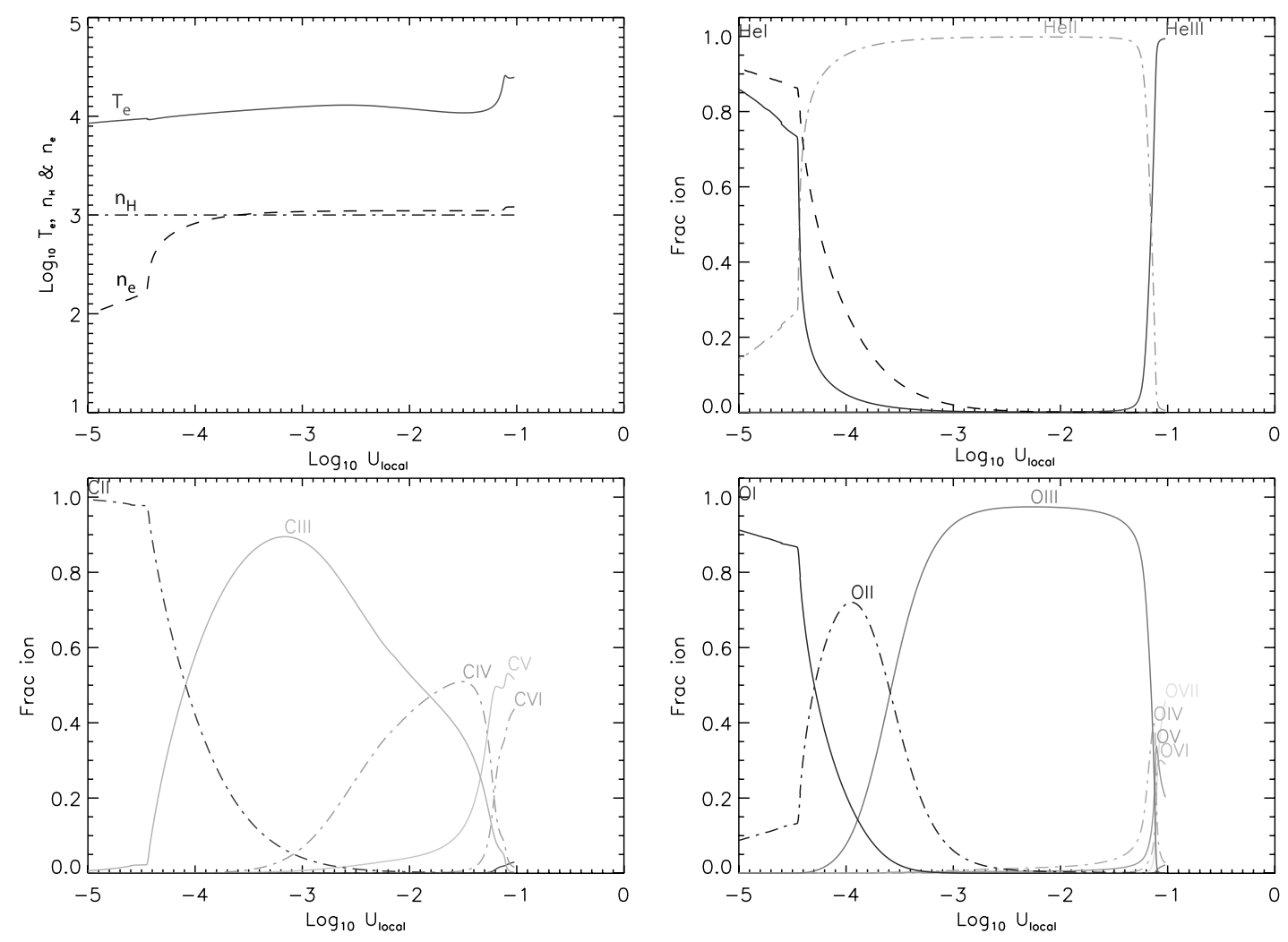

FIG. 15. $-U_{\text {local }}$ structure diagram showing a dust-free model with initial ionization parameter $\log U_{0}=-1.0$, with $1 Z_{\odot}$ metallicity, $\alpha=-1.4$, and $n_{\mathrm{H}}=$ $1000 \mathrm{~cm}^{-3}$

ionized zone in the dust-free model shows a sharp transition that is due to the nonvarying density of the isochoric model.

The distinctions between the models becomes more apparent at higher ionization parameters, when dust dominates the local opacity. Figure 14 shows the dusty model with an initial ionization parameter of $U_{0}=10^{-1}$. The other parameters are the same as Figure 12. Above $\log U_{\text {local }} \sim-2.5$. Several new ionization species appear because of the higher local ionization parameter reached in the early parts of the model, and the electron temperature in these regions is appreciably higher. Below $\log U_{\text {local }} \sim-2.5$ there is little to distinguish the $U_{0}=$ $10^{-1}$ dusty model from the model with $U_{0}=10^{-2}$ (Fig. 12) in either the density or ionization structure. The two models appear almost interchangeable below this value of $U_{\text {local }}$.

The dust-free model shown in Figure 15 has a distinctly different structure to the dusty model (Fig. 14) and to the low ionization dust-free model (Fig. 13). The partially ionized zone of the $U_{0}=-1.0$ model is similar in most respects to that seen in the $U_{0}=-2.0$ model, although the transition occurs at a lower $U_{\text {local }}$. The differences become more obvious in the zone where $\mathrm{O}$ III and $\mathrm{He}$ II are the dominant ionization species. These species occupy a large range of the $\log U_{\text {local }}$ space, with the higher ionization species occurring only at the highest values of $U_{\text {local }}$. For example, in oxygen all the species above $\mathrm{O}$ III occur only between $-1.2 \lesssim \log U_{\text {local }} \lesssim-1.0$.

In the dust-free models, the atomic species provide the only opacity source. At high ionization parameter, and with a ionizing spectrum that extends beyond the He II limit (such as an AGN or power-law spectrum), the gas is able to maintain a high ionization state. Thus, the high ionization species are the main opacity source and the harder ionizing photons are preferentially absorbed to the softer photons that ionize He I,
$\mathrm{O}$ II, and $\mathrm{H}$ I. Therefore, the high ionization regions in the dust-free models occupy only a small region of $U_{\text {local }}$ space, as the high-energy photons represent only a fraction of the total number of ionizing photons.

In the dusty models, dust, rather than the gas, provides the predominant opacity source at high ionization parameter. As its opacity peaks near $13.6 \mathrm{eV}$ (see Fig. 2), dust removes the $\mathrm{H}$ I ionizing photons preferentially before the harder, $\mathrm{He}$ II ionizing photons. Because of this preferential absorption and because dust opacity is proportional to the number of ionizing photons, the ionizing spectrum hardens with decreasing $U_{\text {local }}$. This results in a much larger extent of the $U_{\text {local }}$ space being occupied by the high ionization species. However, the only differences in the total column depths of the high ionization species in the two models arise as a result of the difference in recombination rates because of the addition of photoelectric heating to the electron temperature and the small addition of dust opacity to the high-energy photons.

The differences between the dusty and dust-free models become even more prominent at the very high ionization parameter of $\log U_{0}=0.0$. The dusty model in Figure 16 simply extends the structure seen in the lower $U_{0}$ dusty models to higher values. For values of $U_{\text {local }}$ below -1.0 , the $U_{0}=10^{0}$ model structure is almost identical to the dusty model at $U_{0}=10^{-1}$ in Figure 14. Above this value of $U_{\text {local }}$, coronal species start to appear as a result of the high ionization parameter. By comparison, the ionization structure of the dustfree model at $\log U_{0}=0.0$ (Fig. 17) is clearly distinguishable from both the dusty model (Fig. 16) and the lower ionization parameter dust-free model (Fig. 15). Again, the structure diagram is dominated by the $\mathrm{He}$ II and $\mathrm{O}$ III ionization zone, but unlike the $U_{0}=10^{-1}$ model, in the $U_{0}=10^{0}$ dust-free 

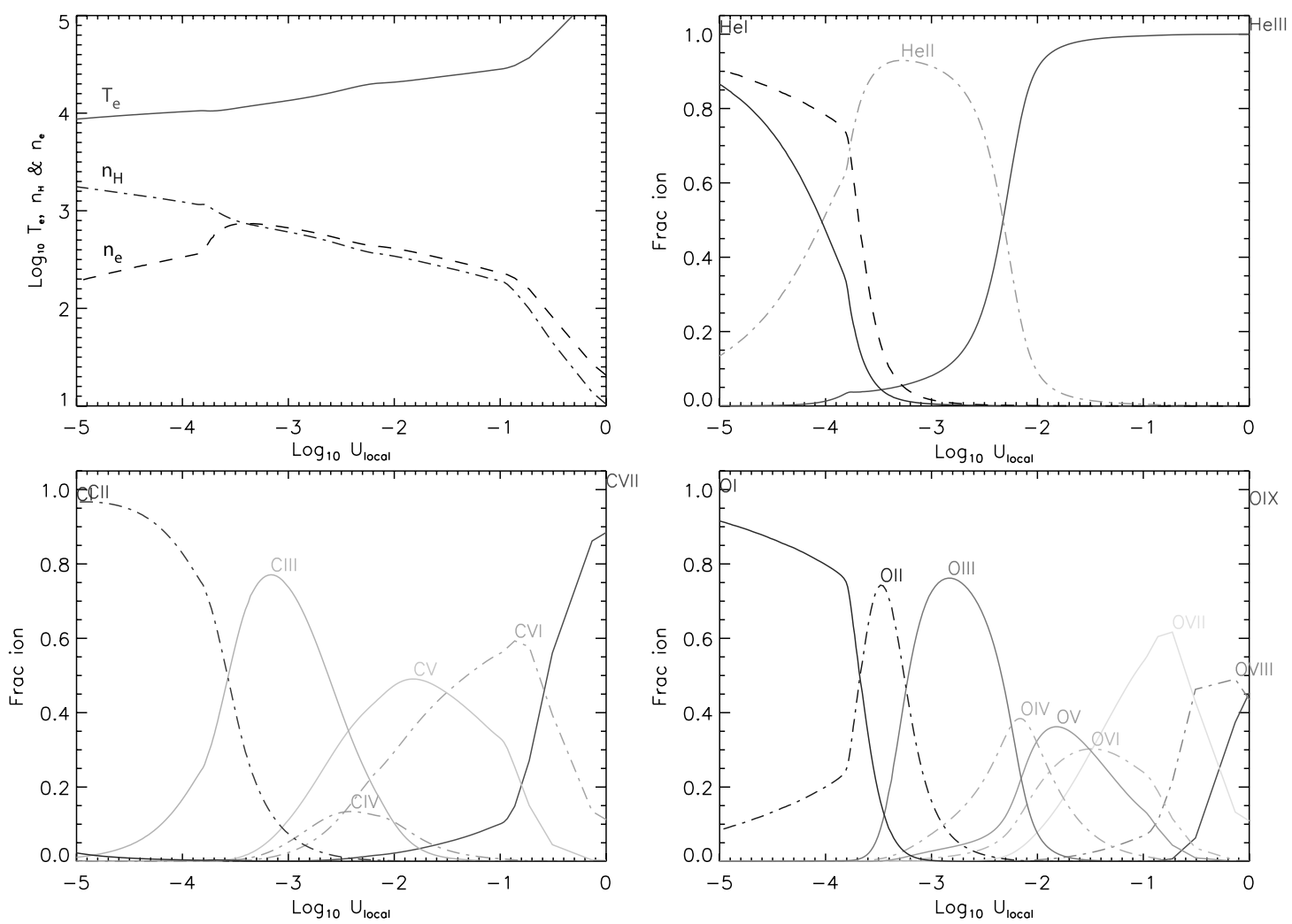

FIG. 16. $-U_{\text {local }}$ structure diagram showing a dusty model with initial ionization parameter $\log U_{0}=0.0$, with $1 Z_{\odot}$ metallicity, $\alpha=-1.4$, and $n_{\mathrm{H}}=1000 \mathrm{~cm}{ }^{-3}$.
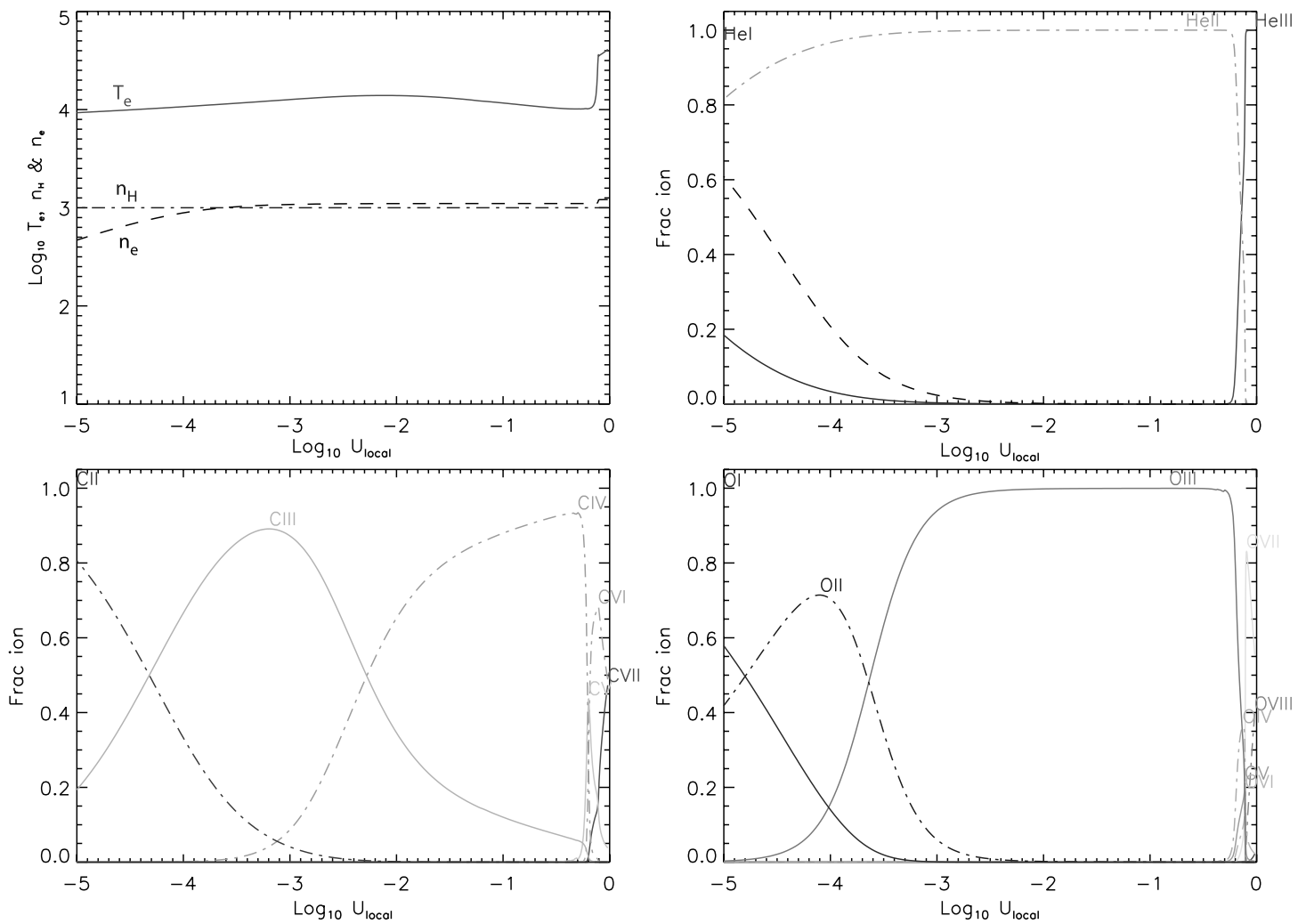

FIG. 17. $-U_{\text {local }}$ structure diagram showing a dust-free model with initial ionization parameter $\log U_{0}=0.0,1 Z_{\odot}$ metallicity, $\alpha=-1.4$, and $n_{\mathrm{H}}=1000 \mathrm{~cm}{ }^{-3}$. 

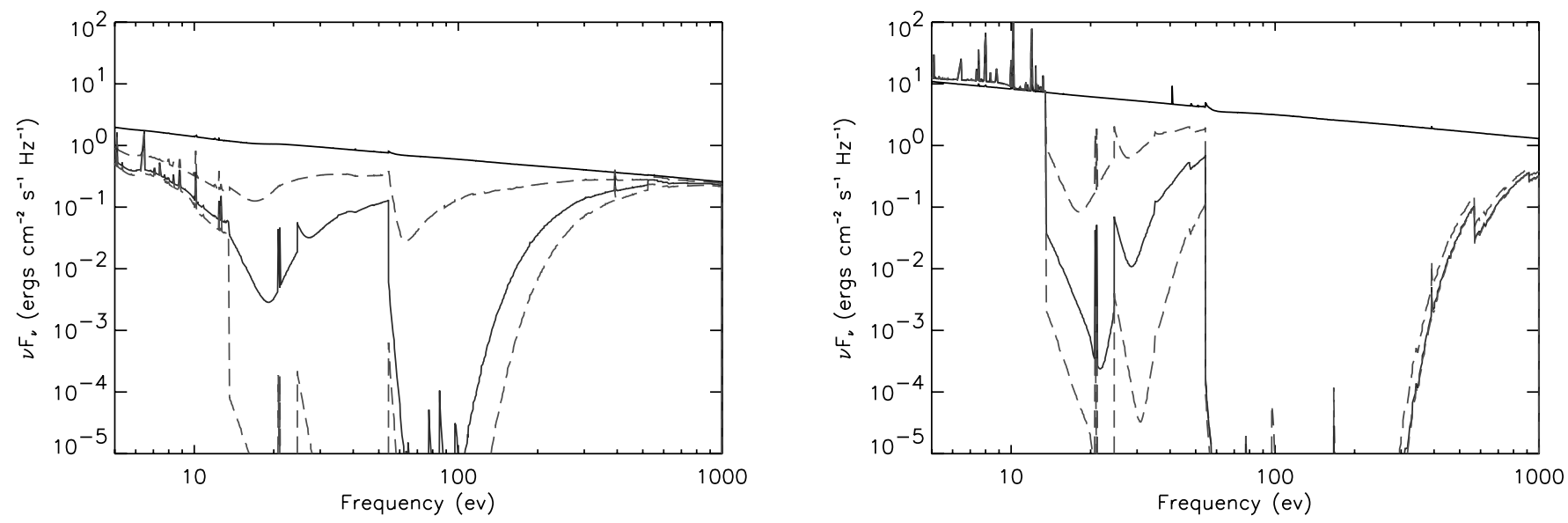

FIG. 18. - Variation of the ionizing spectrum $\left(\nu F_{\nu}\right)$ with $U_{\text {local }}$ for both the dusty (left $)$ and dust-free $(r i g h t)$ models. Curves are at $\log U_{\text {local }}=-1.0,-2.0,-3.0$, and -4.0 , with decreasing flux in the $10-100 \mathrm{eV}$ range for each curve, respectively. Both models have $\log U_{0}=-1.0,1 Z_{\odot}$ metallicity, $\alpha=-1.4$, and $n_{\mathrm{H}}=1000 \mathrm{~cm}{ }^{-3}$.

model this zone extends almost through the entire model. He III and the high ionization oxygen species occur only between $-0.2 \lesssim \log U_{\text {local }} \lesssim 0.0$. As in the other models, the partially ionized zone appears only when $\log U_{\text {local }} \lesssim-5.0$.

A large part of the distinction between the structure of the two models arises because of the dust opacity. As the dust extinction is broad (see Fig. 2) and dominates the opacity at high $U_{0}$, the ionizing spectrum in the dusty models alters in a different manner with $U_{\text {local }}$ to the dust-free case. Figure 18 shows the variation in ionizing spectrum with $U_{\text {local }}$ in both the dusty and dust-free models with $\log U_{0}=-1.0, Z=1 Z_{\odot}$, $\alpha=-1.4$, and $n_{\mathrm{H}}=1000 \mathrm{~cm}^{-3}$. The curves are at $\log U_{\text {local }}=$ $-1.0,-2.0,-3.0$, and -4.0 .

At $\log U_{\text {local }}=-1.0$ (top solid curve), the dusty model has less overall flux owing to the difference in density structure between the two models. Below $U_{\text {local }}=10^{-1}$ the most obvious difference is the way in which the dust removes photons across the spectrum, especially visible below the Lyman limit and between the $\mathrm{H}$ absorption and $\mathrm{He}$ absorption ( 30 and $54 \mathrm{eV}$ ). This demonstrates clearly the "hardening" of the spectrum with $U_{\text {local }}$ by dust discussed above, while in the dustfree case the heavier ions (especially $\mathrm{He}$ ) removed the majority of the high-energy photons above $50 \mathrm{eV}$. In the dust case the $\mathrm{H}$ edge $(13.6 \mathrm{eV})$ does not become obvious until $U_{\text {local }} \sim 10^{-3}$, demonstrating the competition between dust and $\mathrm{H}$ at high $U$.

As discussed in DG02, the dust not only dominates the local opacity at high ionization parameter $\left(U_{0} \gtrsim 10^{-2}\right)$, but also couples the radiation pressure to the gas pressure. At these values of $U_{0}$ the radiation pressure is comparable to or greater than the gas pressure, and hence the nebula structure is determined by the radiation pressure on dust. An increase in ionization parameter leads to an increase in dust opacity and radiation pressure, which in turn leads to a corresponding change in the density. Thus, the $U_{\text {local }}$ structure becomes independent of the initial ionization parameter. Because the $U_{\text {local }}$ parameter determines the local emission spectrum in a nebula, this coupling leads to an emission spectrum that is almost independent of the initial ionization parameter. This self-regulatory mechanism lies at the core of the dusty photoionization paradigm.

\section{SPECTRAL LINE INTENSITIES}

In Tables 3-17 we present the intensities of a wide range of emission lines with respect to $\mathrm{H} \beta$ from our dusty models. In
Tables 18-32 we present the same lines for the classical, dustfree isochoric models, which have been artificially depleted to the dusty models gas abundances. In Tables 33-47 we present the undepleted versions of the classical models. These lines cover a range from the far-UV to the mid-IR and encompass both temperature and density sensitive diagnostics. Also included are lines that are prime diagnostics to distinguish shock excitation from photoionization, as well as lines that can distinguish $\mathrm{H}$ II regions from AGNs. Most lines are fairly insensitive to density effects, and hence we have limited ourselves to models with a hydrogen density of $n_{\mathrm{H}} \sim 1000 \mathrm{~cm}^{-3}$. The effects of density variations will be explored further in the following paper. To minimize the size of the tables themselves we have only given the lines in steps of -1 dex over $\log U_{0}$. A full line list from all dusty and dust-free models are available on-line. ${ }^{2}$

For both the dusty and dust-free models we present the line ratios in three wavelength sets: UV, visible, and IR. Each set is comprised of five tables, one for each metallicity examined in the models. The tables themselves are separated into four sections, for the different power-law indices examined, with the index labeled above the quadrant. Note that the $\log U=0.0$ dust-free models are not included within the tables as these models become unphysical at such a high ionization parameter and with no corresponding change in the density structure.

One point to note is that in the UV tables we include several resonance lines like $\mathrm{C}$ IV $\lambda 1550$ and $\mathrm{Mg}$ II $\lambda 2800$. These lines are strong and observed in the NLR of AGNs and need to be evaluated for a reasonable comparison. However, MAPPINGS III does not evaluate the multiple scatterings, and hence the exact transfer, of these lines, but rather estimates the escape probability (including dust extinction) of these lines. Thus, these lines have a larger uncertainty associated with them compared with the forbidden lines, like [Ne v] $\lambda 3426$.

Though the full line sets will be explored in depth using line diagnostic diagrams in the following papers, there are some general patterns that are evident from the tables.

The most obvious is that as the ionization parameter is increased we see a corresponding increase in the ionization state of the nebula. This is as expected, although there is also a "stagnation" in ionization structure around an ionization parameter above $\log U_{0} \sim-2$ in the dusty models that is not

\footnotetext{
${ }^{2}$ See http://www.mso.anu.edu.au/ bgroves/linedata.
} 
TABLE 3

Dusty UV Line Ratios with Respect to $\mathrm{H} \beta, 0.25 Z_{\odot}$

\begin{tabular}{|c|c|c|c|c|c|}
\hline \multirow[b]{2}{*}{ LiNE } & \multicolumn{5}{|c|}{$\log U_{0}$} \\
\hline & 0.0 & -1.0 & -2.0 & -3.0 & -4.0 \\
\hline \multicolumn{6}{|c|}{$\alpha=-1.2$} \\
\hline C III $\lambda 977$............................ & $9.426 \mathrm{E}-02$ & $1.372 \mathrm{E}-01$ & $2.257 \mathrm{E}-01$ & $8.691 \mathrm{E}-03$ & $9.601 \mathrm{E}-05$ \\
\hline N III $\lambda 991$....................... & $1.022 \mathrm{E}-02$ & $1.462 \mathrm{E}-02$ & $1.975 \mathrm{E}-02$ & $6.670 \mathrm{E}-04$ & $0.000 \mathrm{E}+00$ \\
\hline O VI $\lambda \lambda 1032,1037 \ldots \ldots . .$. & $5.940 \mathrm{E}+00$ & $5.315 \mathrm{E}+00$ & $1.930 \mathrm{E}-01$ & $2.344 \mathrm{E}-05$ & $0.000 \mathrm{E}+00$ \\
\hline $\mathrm{N}$ v $\lambda 1240 \ldots \ldots \ldots \ldots \ldots \ldots . .$. & $1.880 \mathrm{E}-01$ & $2.083 \mathrm{E}-01$ & $5.492 \mathrm{E}-02$ & $5.414 \mathrm{E}-05$ & $0.000 \mathrm{E}+00$ \\
\hline Si IV+O IV] $\lambda 1400 \ldots \ldots \ldots$. & $2.034 \mathrm{E}-01$ & $2.392 \mathrm{E}-01$ & $1.752 \mathrm{E}-01$ & $9.293 \mathrm{E}-03$ & $8.287 \mathrm{E}-06$ \\
\hline $\mathrm{N}$ Iv] $\lambda 1486 \ldots \ldots \ldots \ldots \ldots \ldots$ & $3.326 \mathrm{E}-02$ & $4.521 \mathrm{E}-02$ & $4.235 \mathrm{E}-02$ & $2.845 \mathrm{E}-04$ & $0.000 \mathrm{E}+00$ \\
\hline 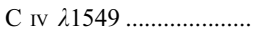 & $1.720 \mathrm{E}+00$ & $2.213 \mathrm{E}+00$ & $2.291 \mathrm{E}+00$ & $1.438 \mathrm{E}-02$ & $8.292 \mathrm{E}-06$ \\
\hline 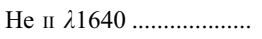 & $3.462 \mathrm{E}+00$ & $3.295 \mathrm{E}+00$ & $2.279 \mathrm{E}+00$ & $1.395 \mathrm{E}+00$ & $4.655 \mathrm{E}-01$ \\
\hline $\mathrm{O}$ iII] $\lambda 1664 \ldots \ldots \ldots \ldots \ldots . .$. & $2.089 \mathrm{E}-01$ & $2.698 \mathrm{E}-01$ & $3.051 \mathrm{E}-01$ & $3.515 \mathrm{E}-02$ & $1.303 \mathrm{E}-04$ \\
\hline $\mathrm{N}$ iII] $\lambda 1750 \ldots \ldots \ldots \ldots \ldots \ldots$ & $6.533 \mathrm{E}-03$ & $9.345 \mathrm{E}-03$ & $1.976 \mathrm{E}-02$ & $2.736 \mathrm{E}-03$ & $1.019 \mathrm{E}-05$ \\
\hline $\mathrm{C}$ III] $\lambda 1909 \ldots \ldots \ldots \ldots \ldots . .$. & $4.682 \mathrm{E}-01$ & $6.586 \mathrm{E}-01$ & $1.673 \mathrm{E}+00$ & $3.533 \mathrm{E}-01$ & $1.970 \mathrm{E}-02$ \\
\hline 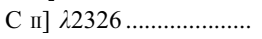 & $9.247 \mathrm{E}-02$ & $8.630 \mathrm{E}-02$ & $1.012 \mathrm{E}-01$ & $2.116 \mathrm{E}-01$ & $2.100 \mathrm{E}-01$ \\
\hline Mg II $\lambda 2800 \ldots \ldots \ldots \ldots \ldots . .$. & $1.245 \mathrm{E}-01$ & $1.245 \mathrm{E}-01$ & $1.706 \mathrm{E}-01$ & $3.256 \mathrm{E}-01$ & $4.497 \mathrm{E}-01$ \\
\hline$[\mathrm{Ne} v] \lambda 3426 \ldots \ldots \ldots \ldots . .$. & $8.408 \mathrm{E}-01$ & $8.656 \mathrm{E}-01$ & $3.742 \mathrm{E}-01$ & $3.582 \mathrm{E}-04$ & $0.000 \mathrm{E}+00$ \\
\hline$[\mathrm{Ne}$ III] $\lambda 3869 \ldots \ldots \ldots \ldots \ldots$ & $6.836 \mathrm{E}-01$ & $7.641 \mathrm{E}-01$ & $1.058 \mathrm{E}+00$ & $7.254 \mathrm{E}-01$ & $3.174 \mathrm{E}-01$ \\
\hline \multicolumn{6}{|c|}{$\alpha=-1.4$} \\
\hline 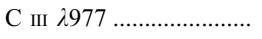 & $1.489 \mathrm{E}-01$ & $2.335 \mathrm{E}-01$ & $2.228 \mathrm{E}-01$ & $7.943 \mathrm{E}-03$ & $9.024 \mathrm{E}-05$ \\
\hline 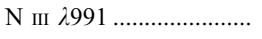 & $1.547 \mathrm{E}-02$ & $2.268 \mathrm{E}-02$ & $1.862 \mathrm{E}-02$ & $6.142 \mathrm{E}-04$ & $0.000 \mathrm{E}+00$ \\
\hline O vI $\lambda \lambda 1032,1037 \ldots \ldots . .$. & $6.788 \mathrm{E}+00$ & $4.929 \mathrm{E}+00$ & $8.263 \mathrm{E}-02$ & $9.041 \mathrm{E}-06$ & $0.000 \mathrm{E}+00$ \\
\hline 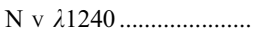 & $2.464 \mathrm{E}-01$ & $2.568 \mathrm{E}-01$ & $3.384 \mathrm{E}-02$ & $2.923 \mathrm{E}-05$ & $0.000 \mathrm{E}+00$ \\
\hline Si IV+O Iv] $\lambda 1400 \ldots \ldots \ldots$. & $2.648 \mathrm{E}-01$ & $3.049 \mathrm{E}-01$ & $1.575 \mathrm{E}-01$ & $7.861 \mathrm{E}-03$ & $6.417 \mathrm{E}-06$ \\
\hline 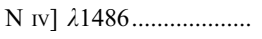 & $5.204 \mathrm{E}-02$ & $7.263 \mathrm{E}-02$ & $3.734 \mathrm{E}-02$ & $2.319 \mathrm{E}-04$ & $0.000 \mathrm{E}+00$ \\
\hline C IV $\lambda 1549 \ldots \ldots \ldots \ldots \ldots \ldots . .$. & $2.416 \mathrm{E}+00$ & $3.247 \mathrm{E}+00$ & $1.977 \mathrm{E}+00$ & $1.122 \mathrm{E}-02$ & $6.295 \mathrm{E}-06$ \\
\hline 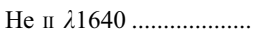 & $3.370 \mathrm{E}+00$ & $2.994 \mathrm{E}+00$ & $1.903 \mathrm{E}+00$ & $1.188 \mathrm{E}+00$ & $3.877 \mathrm{E}-01$ \\
\hline 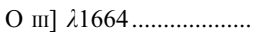 & $3.048 \mathrm{E}-01$ & $3.948 \mathrm{E}-01$ & $3.133 \mathrm{E}-01$ & $3.416 \mathrm{E}-02$ & $1.302 \mathrm{E}-04$ \\
\hline $\mathrm{N}$ iII] $\lambda 1750 \ldots \ldots \ldots \ldots \ldots \ldots . . . . . . . .$. & $9.217 \mathrm{E}-03$ & $1.385 \mathrm{E}-02$ & $2.030 \mathrm{E}-02$ & $2.663 \mathrm{E}-03$ & $1.041 \mathrm{E}-05$ \\
\hline C III] $\lambda 1909 \ldots \ldots \ldots \ldots \ldots . .$. & $6.692 \mathrm{E}-01$ & $1.046 \mathrm{E}+00$ & $1.808 \mathrm{E}+00$ & $3.431 \mathrm{E}-01$ & $1.878 \mathrm{E}-02$ \\
\hline $\mathrm{C}$ II] $\lambda 2326 \ldots \ldots \ldots \ldots \ldots . .$. & $7.210 \mathrm{E}-02$ & $6.449 \mathrm{E}-02$ & $8.567 \mathrm{E}-02$ & $1.879 \mathrm{E}-01$ & $1.850 \mathrm{E}-01$ \\
\hline $\mathrm{Mg}$ II $\lambda 2800 \ldots \ldots \ldots \ldots \ldots . . . . . . . .$. & $1.157 \mathrm{E}-01$ & $1.180 \mathrm{E}-01$ & $1.651 \mathrm{E}-01$ & $2.902 \mathrm{E}-01$ & $3.964 \mathrm{E}-01$ \\
\hline$[\mathrm{Ne} v] \lambda 3426 \ldots \ldots \ldots \ldots . .$. & $9.184 \mathrm{E}-01$ & $9.174 \mathrm{E}-01$ & $2.613 \mathrm{E}-01$ & $2.229 \mathrm{E}-04$ & $0.000 \mathrm{E}+00$ \\
\hline$[\mathrm{Ne}$ III] $\lambda 3869 \ldots \ldots \ldots \ldots . .$. & $7.698 \mathrm{E}-01$ & $9.192 \mathrm{E}-01$ & $1.128 \mathrm{E}+00$ & $6.778 \mathrm{E}-01$ & $2.640 \mathrm{E}-01$ \\
\hline \multicolumn{6}{|c|}{$\alpha=-1.7$} \\
\hline C III $\lambda 977$...................... & $2.526 \mathrm{E}-01$ & $3.306 \mathrm{E}-01$ & $1.692 \mathrm{E}-01$ & $6.273 \mathrm{E}-03$ & $7.647 \mathrm{E}-05$ \\
\hline 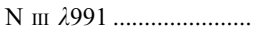 & $2.265 \mathrm{E}-02$ & $2.645 \mathrm{E}-02$ & $1.367 \mathrm{E}-02$ & $4.820 \mathrm{E}-04$ & $0.000 \mathrm{E}+00$ \\
\hline O vi $\lambda \lambda 1032,1037 \ldots \ldots .$. & $6.759 \mathrm{E}+00$ & $3.021 \mathrm{E}+00$ & $1.787 \mathrm{E}-02$ & $1.159 \mathrm{E}-06$ & $0.000 \mathrm{E}+00$ \\
\hline 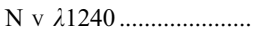 & $3.090 \mathrm{E}-01$ & $2.431 \mathrm{E}-01$ & $1.273 \mathrm{E}-02$ & $9.913 \mathrm{E}-06$ & $0.000 \mathrm{E}+00$ \\
\hline $\mathrm{Si}$ IV+O IV] $\lambda 1400 \ldots \ldots \ldots$. & $3.274 \mathrm{E}-01$ & $3.161 \mathrm{E}-01$ & $1.607 \mathrm{E}-01$ & $5.493 \mathrm{E}-03$ & $4.142 \mathrm{E}-06$ \\
\hline 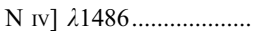 & $8.465 \mathrm{E}-02$ & $9.548 \mathrm{E}-02$ & $2.432 \mathrm{E}-02$ & $1.476 \mathrm{E}-04$ & $0.000 \mathrm{E}+00$ \\
\hline C IV $\lambda 1549 \ldots \ldots \ldots \ldots \ldots \ldots . .$. & $3.686 \mathrm{E}+00$ & $4.234 \mathrm{E}+00$ & $1.270 \mathrm{E}+00$ & $6.888 \mathrm{E}-03$ & $3.901 \mathrm{E}-06$ \\
\hline 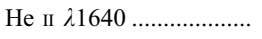 & $3.063 \mathrm{E}+00$ & $2.357 \mathrm{E}+00$ & $1.378 \mathrm{E}+00$ & $8.827 \mathrm{E}-01$ & $2.784 \mathrm{E}-01$ \\
\hline 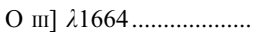 & $4.404 \mathrm{E}-01$ & $4.751 \mathrm{E}-01$ & $2.749 \mathrm{E}-01$ & $2.956 \mathrm{E}-02$ & $1.175 \mathrm{E}-04$ \\
\hline $\mathrm{N}$ III] $\lambda 1750 \ldots \ldots \ldots \ldots \ldots \ldots . .$. & $1.311 \mathrm{E}-02$ & $1.753 \mathrm{E}-02$ & $1.799 \mathrm{E}-02$ & $2.323 \mathrm{E}-03$ & $9.747 \mathrm{E}-06$ \\
\hline 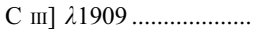 & $1.080 \mathrm{E}+00$ & $1.583 \mathrm{E}+00$ & $1.684 \mathrm{E}+00$ & $3.053 \mathrm{E}-01$ & $1.649 \mathrm{E}-02$ \\
\hline $\mathrm{C}$ II] $\lambda 2326 \ldots \ldots \ldots \ldots \ldots \ldots$ & $4.806 \mathrm{E}-02$ & 4.299E-02 & $7.125 \mathrm{E}-02$ & $1.667 \mathrm{E}-01$ & $1.605 \mathrm{E}-01$ \\
\hline 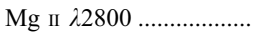 & $1.038 \mathrm{E}-01$ & $1.065 \mathrm{E}-01$ & $1.564 \mathrm{E}-01$ & $2.704 \mathrm{E}-01$ & $3.658 \mathrm{E}-01$ \\
\hline$[\mathrm{Ne} v] \lambda 3426 \ldots \ldots \ldots \ldots \ldots$ & $9.670 \mathrm{E}-01$ & $8.246 \mathrm{E}-01$ & $1.209 \mathrm{E}-01$ & $9.114 \mathrm{E}-05$ & $0.000 \mathrm{E}+00$ \\
\hline$[\mathrm{Ne}$ III] $\lambda 3869 \ldots \ldots \ldots \ldots \ldots . .$. & $9.543 \mathrm{E}-01$ & $1.138 \mathrm{E}+00$ & $1.153 \mathrm{E}+00$ & $6.020 \mathrm{E}-01$ & $1.973 \mathrm{E}-01$ \\
\hline \multicolumn{6}{|c|}{$\alpha=-2.0$} \\
\hline 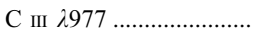 & $3.151 \mathrm{E}-01$ & $2.859 \mathrm{E}-01$ & $1.063 \mathrm{E}-01$ & $4.595 \mathrm{E}-03$ & $6.106 \mathrm{E}-05$ \\
\hline 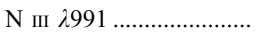 & $2.349 \mathrm{E}-02$ & $1.993 \mathrm{E}-02$ & $8.677 \mathrm{E}-03$ & $3.448 \mathrm{E}-04$ & $0.000 \mathrm{E}+00$ \\
\hline O vi $\lambda \lambda 1032,1037 \ldots \ldots .$. & $5.213 \mathrm{E}+00$ & $1.255 \mathrm{E}+00$ & $3.107 \mathrm{E}-03$ & $0.000 \mathrm{E}+00$ & $0.000 \mathrm{E}+00$ \\
\hline $\mathrm{N}$ v $\lambda 1240 \ldots \ldots \ldots \ldots \ldots \ldots \ldots$ & $3.016 \mathrm{E}-01$ & $1.631 \mathrm{E}-01$ & $3.958 \mathrm{E}-03$ & $2.963 \mathrm{E}-06$ & $0.000 \mathrm{E}+00$ \\
\hline $\mathrm{Si}$ Iv+O IV] $\lambda 1400 \ldots \ldots \ldots$. & $3.235 \mathrm{E}-01$ & $3.802 \mathrm{E}-01$ & $8.172 \mathrm{E}-02$ & $3.334 \mathrm{E}-03$ & $1.692 \mathrm{E}-06$ \\
\hline 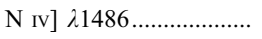 & $1.030 \mathrm{E}-01$ & $8.338 \mathrm{E}-02$ & $1.328 \mathrm{E}-02$ & $8.369 \mathrm{E}-05$ & $0.000 \mathrm{E}+00$ \\
\hline 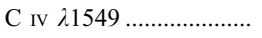 & $4.641 \mathrm{E}+00$ & $3.848 \mathrm{E}+00$ & $6.813 \mathrm{E}-01$ & $3.874 \mathrm{E}-03$ & $1.518 \mathrm{E}-06$ \\
\hline He II $\lambda 1640 \ldots \ldots \ldots \ldots \ldots \ldots . .$. & $2.570 \mathrm{E}+00$ & $1.691 \mathrm{E}+00$ & $9.571 \mathrm{E}-01$ & $6.261 \mathrm{E}-01$ & $1.907 \mathrm{E}-01$ \\
\hline $\mathrm{O}$ III] $\lambda 1664 \ldots \ldots \ldots \ldots \ldots \ldots . .$. & $4.929 \mathrm{E}-01$ & $4.139 \mathrm{E}-01$ & $2.152 \mathrm{E}-01$ & $2.347 \mathrm{E}-02$ & $9.752 \mathrm{E}-05$ \\
\hline $\mathrm{N}$ III] $\lambda 1750 \ldots \ldots \ldots \ldots \ldots \ldots$ & $1.495 \mathrm{E}-02$ & $1.651 \mathrm{E}-02$ & $1.433 \mathrm{E}-02$ & $1.876 \mathrm{E}-03$ & $8.414 \mathrm{E}-06$ \\
\hline C III] $\lambda 1909 \ldots \ldots \ldots \ldots \ldots . .$. & $1.461 \mathrm{E}+00$ & $1.713 \mathrm{E}+00$ & $1.363 \mathrm{E}+00$ & $2.564 \mathrm{E}-01$ & $1.378 \mathrm{E}-02$ \\
\hline 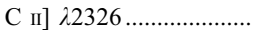 & $3.276 \mathrm{E}-02$ & $3.188 \mathrm{E}-02$ & $6.405 \mathrm{E}-02$ & $1.558 \mathrm{E}-01$ & $1.444 \mathrm{E}-01$ \\
\hline Mg II $\lambda 2800 \ldots \ldots \ldots \ldots \ldots . .$. & $9.170 \mathrm{E}-02$ & $9.406 \mathrm{E}-02$ & $1.497 \mathrm{E}-01$ & $2.766 \mathrm{E}-01$ & $3.612 \mathrm{E}-01$ \\
\hline$[\mathrm{Ne} v] \lambda 3426 \ldots \ldots \ldots \ldots \ldots . . . .$. & $8.971 \mathrm{E}-01$ & $5.963 \mathrm{E}-01$ & $4.444 \mathrm{E}-02$ & $3.205 \mathrm{E}-05$ & $0.000 \mathrm{E}+00$ \\
\hline$[\mathrm{Ne}$ III] $\lambda 3869 \ldots \ldots \ldots \ldots \ldots$ & $1.138 \mathrm{E}+00$ & $1.232 \mathrm{E}+00$ & $1.093 \mathrm{E}+00$ & $5.213 \mathrm{E}-01$ & $1.440 \mathrm{E}-01$ \\
\hline
\end{tabular}


TABLE 4

Dusty UV Line Ratios with Respect to $\mathrm{H} \beta, 0.5 Z$.

\begin{tabular}{|c|c|c|c|c|c|}
\hline \multirow[b]{2}{*}{ Line } & \multicolumn{5}{|c|}{$\log U_{0}$} \\
\hline & 0.0 & -1.0 & -2.0 & -3.0 & -4.0 \\
\hline \multicolumn{6}{|c|}{$\alpha=-1.2$} \\
\hline C III $\lambda 977$...................... & $1.514 \mathrm{E}-01$ & $1.975 \mathrm{E}-01$ & $2.590 \mathrm{E}-01$ & $9.684 \mathrm{E}-03$ & $1.336 \mathrm{E}-04$ \\
\hline N III $\lambda 991$...................... & $2.290 \mathrm{E}-02$ & $2.974 \mathrm{E}-02$ & $3.413 \mathrm{E}-02$ & $1.081 \mathrm{E}-03$ & $1.279 \mathrm{E}-06$ \\
\hline O vi $\lambda \lambda 1032,1037 \ldots \ldots .$. & $7.991 \mathrm{E}+00$ & $7.108 \mathrm{E}+00$ & $2.076 \mathrm{E}-01$ & $2.143 \mathrm{E}-05$ & $0.000 \mathrm{E}+00$ \\
\hline $\mathrm{N}$ v $\lambda 1240 \ldots \ldots \ldots \ldots \ldots \ldots . .$. & $4.478 \mathrm{E}-01$ & $4.710 \mathrm{E}-01$ & $1.020 \mathrm{E}-01$ & $8.759 \mathrm{E}-05$ & $0.000 \mathrm{E}+00$ \\
\hline $\mathrm{Si}$ IV+O IV] $\lambda 1400 \ldots \ldots \ldots$ & $2.911 \mathrm{E}-01$ & $3.296 \mathrm{E}-01$ & $2.354 \mathrm{E}-01$ & $1.030 \mathrm{E}-02$ & $1.234 \mathrm{E}-05$ \\
\hline $\mathrm{N}$ IV] $\lambda 1486 \ldots \ldots \ldots \ldots \ldots \ldots$ & $7.855 \mathrm{E}-02$ & $9.862 \mathrm{E}-02$ & $8.346 \mathrm{E}-02$ & $5.043 \mathrm{E}-04$ & $0.000 \mathrm{E}+00$ \\
\hline 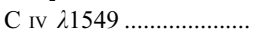 & $3.052 \mathrm{E}+00$ & $3.646 \mathrm{E}+00$ & $3.148 \mathrm{E}+00$ & $1.732 \mathrm{E}-02$ & $1.258 \mathrm{E}-05$ \\
\hline 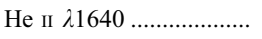 & $3.778 \mathrm{E}+00$ & $3.657 \mathrm{E}+00$ & $2.449 \mathrm{E}+00$ & $1.434 \mathrm{E}+00$ & $4.908 \mathrm{E}-01$ \\
\hline $\mathrm{O}$ iII] $\lambda 1664 \ldots \ldots \ldots \ldots \ldots \ldots . .$. & $2.895 \mathrm{E}-01$ & $3.551 \mathrm{E}-01$ & $4.142 \mathrm{E}-01$ & $4.823 \mathrm{E}-02$ & $2.039 \mathrm{E}-04$ \\
\hline $\mathrm{N}$ III] $\lambda 1750 \ldots \ldots \ldots \ldots \ldots \ldots$ & $1.836 \mathrm{E}-02$ & $2.427 \mathrm{E}-02$ & $4.372 \mathrm{E}-02$ & $5.708 \mathrm{E}-03$ & $2.416 \mathrm{E}-05$ \\
\hline C III] $\lambda 1909 \ldots \ldots \ldots \ldots \ldots \ldots \ldots$ & $9.461 \mathrm{E}-01$ & $1.221 \mathrm{E}+00$ & $2.535 \mathrm{E}+00$ & $5.261 \mathrm{E}-01$ & $3.264 \mathrm{E}-02$ \\
\hline $\mathrm{C}$ II] $\lambda 2326 \ldots \ldots \ldots \ldots \ldots . .$. & $2.222 \mathrm{E}-01$ & $2.064 \mathrm{E}-01$ & $1.943 \mathrm{E}-01$ & $3.606 \mathrm{E}-01$ & $3.450 \mathrm{E}-01$ \\
\hline 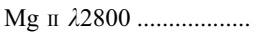 & $2.692 \mathrm{E}-01$ & $2.627 \mathrm{E}-01$ & $3.168 \mathrm{E}-01$ & $5.229 \mathrm{E}-01$ & $6.697 \mathrm{E}-01$ \\
\hline$[\mathrm{Ne} v] \lambda 3426 \ldots \ldots \ldots \ldots \ldots . . . .$. & $1.521 \mathrm{E}+00$ & $1.531 \mathrm{E}+00$ & $5.609 \mathrm{E}-01$ & $4.722 \mathrm{E}-04$ & $0.000 \mathrm{E}+00$ \\
\hline$[\mathrm{Ne}$ III] $\lambda 3869 \ldots \ldots \ldots \ldots \ldots$ & $1.304 \mathrm{E}+00$ & $1.399 \mathrm{E}+00$ & $1.863 \mathrm{E}+00$ & $1.275 \mathrm{E}+00$ & $5.638 \mathrm{E}-01$ \\
\hline \multicolumn{6}{|c|}{$\alpha=-1.4$} \\
\hline 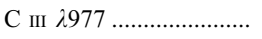 & $2.209 \mathrm{E}-01$ & $2.938 \mathrm{E}-01$ & $2.132 \mathrm{E}-01$ & $7.796 \mathrm{E}-03$ & $1.186 \mathrm{E}-04$ \\
\hline N III $\lambda 991$..................... & $3.242 \mathrm{E}-02$ & $4.136 \mathrm{E}-02$ & $2.701 \mathrm{E}-02$ & $8.688 \mathrm{E}-04$ & $1.205 \mathrm{E}-06$ \\
\hline O vi $\lambda \lambda 1032,1037 \ldots \ldots .$. & $8.562 \mathrm{E}+00$ & $6.085 \mathrm{E}+00$ & $7.496 \mathrm{E}-02$ & $6.956 \mathrm{E}-06$ & $0.000 \mathrm{E}+00$ \\
\hline $\mathrm{N}$ v $\lambda 1240 \ldots \ldots \ldots \ldots \ldots \ldots \ldots$ & $5.697 \mathrm{E}-01$ & $5.504 \mathrm{E}-01$ & $5.443 \mathrm{E}-02$ & $4.101 \mathrm{E}-05$ & $0.000 \mathrm{E}+00$ \\
\hline $\mathrm{Si}$ IV+O Iv] $\lambda 1400 \ldots \ldots \ldots$. & $3.674 \mathrm{E}-01$ & $4.037 \mathrm{E}-01$ & $1.899 \mathrm{E}-01$ & $8.672 \mathrm{E}-03$ & $9.025 \mathrm{E}-06$ \\
\hline $\mathrm{N}$ Iv] $\lambda 1486 \ldots \ldots \ldots \ldots \ldots \ldots$ & $1.180 \mathrm{E}-01$ & $1.482 \mathrm{E}-01$ & $6.466 \mathrm{E}-02$ & $3.644 \mathrm{E}-04$ & $0.000 \mathrm{E}+00$ \\
\hline 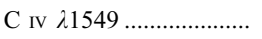 & $4.125 \mathrm{E}+00$ & $4.952 \mathrm{E}+00$ & $2.412 \mathrm{E}+00$ & $1.205 \mathrm{E}-02$ & $9.080 \mathrm{E}-06$ \\
\hline 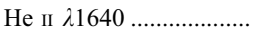 & $3.741 \mathrm{E}+00$ & $3.402 \mathrm{E}+00$ & $2.036 \mathrm{E}+00$ & $1.212 \mathrm{E}+00$ & $4.047 \mathrm{E}-01$ \\
\hline O III] $\lambda 1664 \ldots \ldots \ldots \ldots \ldots \ldots . .$. & $4.020 \mathrm{E}-01$ & $4.892 \mathrm{E}-01$ & $3.777 \mathrm{E}-01$ & $4.288 \mathrm{E}-02$ & $1.955 \mathrm{E}-04$ \\
\hline $\mathrm{N}$ III] $\lambda 1750 \ldots \ldots \ldots \ldots \ldots \ldots . .$. & $2.497 \mathrm{E}-02$ & $3.352 \mathrm{E}-02$ & $4.006 \mathrm{E}-02$ & $5.103 \mathrm{E}-03$ & $2.368 \mathrm{E}-05$ \\
\hline 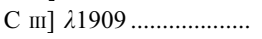 & $1.281 \mathrm{E}+00$ & $1.760 \mathrm{E}+00$ & $2.447 \mathrm{E}+00$ & $4.768 \mathrm{E}-01$ & $3.008 \mathrm{E}-02$ \\
\hline $\mathrm{C}$ II] $\lambda 2326 \ldots \ldots \ldots \ldots \ldots . .$. & $1.723 \mathrm{E}-01$ & $1.507 \mathrm{E}-01$ & $1.542 \mathrm{E}-01$ & $3.118 \mathrm{E}-01$ & $3.052 \mathrm{E}-01$ \\
\hline 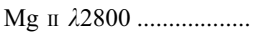 & $2.486 \mathrm{E}-01$ & $2.445 \mathrm{E}-01$ & $2.959 \mathrm{E}-01$ & $4.646 \mathrm{E}-01$ & $6.146 \mathrm{E}-01$ \\
\hline$[\mathrm{Ne} v] \lambda 3426 \ldots \ldots \ldots \ldots \ldots$ & $1.677 \mathrm{E}+00$ & $1.609 \mathrm{E}+00$ & $3.588 \mathrm{E}-01$ & $2.662 \mathrm{E}-04$ & $0.000 \mathrm{E}+00$ \\
\hline$[\mathrm{Ne}$ III] $\lambda 3869 \ldots \ldots \ldots \ldots \ldots$ & $1.380 \mathrm{E}+00$ & $1.556 \mathrm{E}+00$ & $1.861 \mathrm{E}+00$ & $1.153 \mathrm{E}+00$ & $4.633 \mathrm{E}-01$ \\
\hline \multicolumn{6}{|c|}{$\alpha=-1.7$} \\
\hline C III $\lambda 977$......................... & $3.110 \mathrm{E}-01$ & $3.258 \mathrm{E}-01$ & $1.217 \mathrm{E}-01$ & $5.078 \mathrm{E}-03$ & $9.098 \mathrm{E}-05$ \\
\hline N III $\lambda 991 \ldots \ldots \ldots \ldots \ldots \ldots \ldots . .$. & $4.054 \mathrm{E}-02$ & $3.945 \mathrm{E}-02$ & $1.499 \mathrm{E}-02$ & $5.544 \mathrm{E}-04$ & $0.000 \mathrm{E}+00$ \\
\hline O vi $\lambda \lambda 1032,1037 \ldots \ldots .$. & $7.696 \mathrm{E}+00$ & $3.225 \mathrm{E}+00$ & $1.242 \mathrm{E}-02$ & $0.000 \mathrm{E}+00$ & $0.000 \mathrm{E}+00$ \\
\hline $\mathrm{N}$ v $\lambda 1240 \ldots \ldots \ldots \ldots \ldots \ldots . . . . . . . . .$. & $6.631 \mathrm{E}-01$ & $4.664 \mathrm{E}-01$ & $1.636 \mathrm{E}-02$ & $1.134 \mathrm{E}-05$ & $0.000 \mathrm{E}+00$ \\
\hline $\mathrm{Si}$ Iv+O Iv] $\lambda 1400 \ldots \ldots \ldots$. & $4.280 \mathrm{E}-01$ & $3.870 \mathrm{E}-01$ & $1.598 \mathrm{E}-01$ & $5.131 \mathrm{E}-03$ & $5.264 \mathrm{E}-06$ \\
\hline $\mathrm{N}$ Iv] $\lambda 1486 \ldots \ldots \ldots \ldots \ldots \ldots$ & $1.729 \mathrm{E}-01$ & $1.720 \mathrm{E}-01$ & $3.433 \mathrm{E}-02$ & $1.967 \mathrm{E}-04$ & $0.000 \mathrm{E}+00$ \\
\hline C Iv $\lambda 1549$ & $5.591 \mathrm{E}+00$ & $5.536 \mathrm{E}+00$ & $1.274 \mathrm{E}+00$ & $6.302 \mathrm{E}-03$ & $5.152 \mathrm{E}-06$ \\
\hline 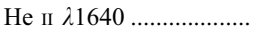 & $3.455 \mathrm{E}+00$ & $2.707 \mathrm{E}+00$ & $1.452 \mathrm{E}+00$ & $8.914 \mathrm{E}-01$ & $2.832 \mathrm{E}-01$ \\
\hline O III] $\lambda 1664 \ldots \ldots \ldots \ldots \ldots \ldots . . .$. & $5.264 \mathrm{E}-01$ & $5.231 \mathrm{E}-01$ & $2.721 \mathrm{E}-01$ & $3.238 \mathrm{E}-02$ & $1.639 \mathrm{E}-04$ \\
\hline 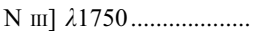 & $3.195 \mathrm{E}-02$ & $3.676 \mathrm{E}-02$ & $2.931 \mathrm{E}-02$ & $3.915 \mathrm{E}-03$ & $2.058 \mathrm{E}-05$ \\
\hline C III] $\lambda 1909 \ldots \ldots \ldots \ldots \ldots . .$. & $1.810 \mathrm{E}+00$ & $2.239 \mathrm{E}+00$ & $1.895 \mathrm{E}+00$ & $3.820 \mathrm{E}-01$ & $2.478 \mathrm{E}-02$ \\
\hline $\mathrm{C}$ II] $\lambda 2326 \ldots \ldots \ldots \ldots \ldots \ldots . .$. & $1.106 \mathrm{E}-01$ & $9.104 \mathrm{E}-02$ & $1.142 \mathrm{E}-01$ & $2.633 \mathrm{E}-01$ & $2.597 \mathrm{E}-01$ \\
\hline Mg II $\lambda 2800 \ldots \ldots \ldots \ldots \ldots . . . . . . . .$. & $2.173 \mathrm{E}-01$ & $2.118 \mathrm{E}-01$ & $2.629 \mathrm{E}-01$ & $4.291 \mathrm{E}-01$ & $5.776 \mathrm{E}-01$ \\
\hline$[\mathrm{Ne} v] \lambda 3426 \ldots \ldots \ldots \ldots \ldots . .$. & $1.743 \mathrm{E}+00$ & $1.369 \mathrm{E}+00$ & $1.430 \mathrm{E}-01$ & $9.655 \mathrm{E}-05$ & $0.000 \mathrm{E}+00$ \\
\hline$[\mathrm{Ne}$ III] $\lambda 3869 \ldots \ldots \ldots \ldots . . .$. & $1.558 \mathrm{E}+00$ & $1.749 \mathrm{E}+00$ & $1.707 \mathrm{E}+00$ & $9.686 \mathrm{E}-01$ & $3.342 \mathrm{E}-01$ \\
\hline \multicolumn{6}{|c|}{$\alpha=-2.0$} \\
\hline C III $\lambda 977 \ldots \ldots \ldots \ldots \ldots \ldots . .$. & $3.110 \mathrm{E}-01$ & $2.156 \mathrm{E}-01$ & $5.641 \mathrm{E}-02$ & $3.064 \mathrm{E}-03$ & $6.644 \mathrm{E}-05$ \\
\hline N III 2991 ..................... & $3.556 \mathrm{E}-02$ & $2.357 \mathrm{E}-02$ & $7.036 \mathrm{E}-03$ & $3.211 \mathrm{E}-04$ & $0.000 \mathrm{E}+00$ \\
\hline O vi $\lambda \lambda 1032,1037 \ldots \ldots .$. & $6.096 \mathrm{E}+00$ & $1.124 \mathrm{E}+00$ & $1.609 \mathrm{E}-03$ & $0.000 \mathrm{E}+00$ & $0.000 \mathrm{E}+00$ \\
\hline $\mathrm{N}$ v $\lambda 1240 \ldots \ldots \ldots \ldots \ldots \ldots \ldots$ & $6.145 \mathrm{E}-01$ & $2.709 \mathrm{E}-01$ & $3.976 \mathrm{E}-03$ & $1.812 \mathrm{E}-06$ & $0.000 \mathrm{E}+00$ \\
\hline Si IV+O IV] $\lambda 1400 \ldots \ldots \ldots$. & $3.998 \mathrm{E}-01$ & $2.744 \mathrm{E}-01$ & $6.565 \mathrm{E}-02$ & $2.783 \mathrm{E}-03$ & $2.991 \mathrm{E}-06$ \\
\hline $\mathrm{N}$ Iv] $\lambda 1486 \ldots \ldots \ldots \ldots \ldots \ldots$ & $1.855 \mathrm{E}-01$ & $1.295 \mathrm{E}-01$ & $1.498 \mathrm{E}-02$ & $9.422 \mathrm{E}-05$ & $0.000 \mathrm{E}+00$ \\
\hline C Iv $\lambda 1549 \ldots \ldots \ldots \ldots \ldots . .$. & $6.011 \mathrm{E}+00$ & $4.207 \mathrm{E}+00$ & $5.486 \mathrm{E}-01$ & $3.018 \mathrm{E}-03$ & $1.856 \mathrm{E}-06$ \\
\hline 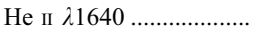 & $3.013 \mathrm{E}+00$ & $1.909 \mathrm{E}+00$ & $9.951 \mathrm{E}-01$ & $6.265 \mathrm{E}-01$ & $1.911 \mathrm{E}-01$ \\
\hline $\mathrm{O}$ III] $\lambda 1664 \ldots \ldots \ldots \ldots \ldots \ldots . .$. & $5.256 \mathrm{E}-01$ & $3.833 \mathrm{E}-01$ & $1.723 \mathrm{E}-01$ & $2.237 \mathrm{E}-02$ & $1.280 \mathrm{E}-04$ \\
\hline $\mathrm{N}$ III] $\lambda 1750 \ldots \ldots \ldots \ldots \ldots . .$. & $3.180 \mathrm{E}-02$ & $2.863 \mathrm{E}-02$ & $1.893 \mathrm{E}-02$ & $2.771 \mathrm{E}-03$ & $1.670 \mathrm{E}-05$ \\
\hline 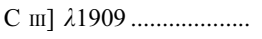 & $2.046 \mathrm{E}+00$ & $1.976 \mathrm{E}+00$ & $1.264 \mathrm{E}+00$ & $2.884 \mathrm{E}-01$ & $1.962 \mathrm{E}-02$ \\
\hline 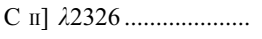 & $7.311 \mathrm{E}-02$ & $5.923 \mathrm{E}-02$ & $9.156 \mathrm{E}-02$ & $2.325 \mathrm{E}-01$ & $2.292 \mathrm{E}-01$ \\
\hline Mg II $\lambda 2800 \ldots \ldots \ldots \ldots \ldots . . . . . . . .$. & $1.890 \mathrm{E}-01$ & $1.799 \mathrm{E}-01$ & $2.377 \mathrm{E}-01$ & $4.281 \mathrm{E}-01$ & $5.711 \mathrm{E}-01$ \\
\hline$[\mathrm{Ne} v] \lambda 3426 \ldots \ldots \ldots \ldots \ldots . . . .$. & $1.583 \mathrm{E}+00$ & $8.929 \mathrm{E}-01$ & $4.449 \mathrm{E}-02$ & $3.019 \mathrm{E}-05$ & $0.000 \mathrm{E}+00$ \\
\hline$[\mathrm{Ne}$ III] $\lambda 3869 \ldots \ldots \ldots \ldots . .$. & $1.685 \mathrm{E}+00$ & $1.710 \mathrm{E}+00$ & $1.445 \mathrm{E}+00$ & $7.901 \mathrm{E}-01$ & $2.369 \mathrm{E}-01$ \\
\hline
\end{tabular}


TABLE 5

Dusty UV Line Ratios with Respect to $\mathrm{H} \beta, 1 Z_{\odot}$

\begin{tabular}{|c|c|c|c|c|c|}
\hline \multirow[b]{2}{*}{ LiNE } & \multicolumn{5}{|c|}{$\log U_{0}$} \\
\hline & 0.0 & -1.0 & -2.0 & -3.0 & -4.0 \\
\hline \multicolumn{6}{|c|}{$\alpha=-1.2$} \\
\hline C & $2.303 \mathrm{E}-01$ & $2.740 \mathrm{E}-01$ & $2.066 \mathrm{E}-01$ & $5.113 \mathrm{E}-03$ & $1.063 \mathrm{E}-04$ \\
\hline N III 2991 ..................... & $5.510 \mathrm{E}-02$ & $6.562 \mathrm{E}-02$ & $4.504 \mathrm{E}-02$ & $8.584 \mathrm{E}-04$ & $1.517 \mathrm{E}-06$ \\
\hline $\mathrm{O}$ vi $\lambda \lambda 1032,1037 \ldots .$. & $1.041 \mathrm{E}+01$ & $8.983 \mathrm{E}+00$ & $1.502 \mathrm{E}-01$ & $7.381 \mathrm{E}-06$ & $0.000 \mathrm{E}+00$ \\
\hline $\mathrm{N}$ v $\lambda 1240 \ldots \ldots \ldots \ldots \ldots . . . . . . .$. & $1.116 \mathrm{E}+00$ & $1.123 \mathrm{E}+00$ & $1.504 \mathrm{E}-01$ & $7.051 \mathrm{E}-05$ & $0.000 \mathrm{E}+00$ \\
\hline $\mathrm{Si} \mathrm{IV+O} \mathrm{IV]} \lambda 1400 \ldots \ldots$ & $4.276 \mathrm{E}-01$ & $4.638 \mathrm{E}-01$ & $2.430 \mathrm{E}-01$ & $7.366 \mathrm{E}-03$ & $1.173 \mathrm{E}-05$ \\
\hline $\mathrm{N}$ Iv] $\lambda 1486 \ldots \ldots \ldots \ldots \ldots$ & $1.999 \mathrm{E}-01$ & $2.374 \mathrm{E}-01$ & $1.394 \mathrm{E}-01$ & $5.017 \mathrm{E}-04$ & $0.000 \mathrm{E}+00$ \\
\hline 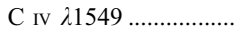 & $5.059 \mathrm{E}+00$ & $5.728 \mathrm{E}+00$ & $3.352 \mathrm{E}+00$ & $1.098 \mathrm{E}-02$ & $1.246 \mathrm{E}-05$ \\
\hline Не пा $\lambda 1640 \ldots \ldots \ldots \ldots \ldots . . . .$. & $4.232 \mathrm{E}+00$ & $4.140 \mathrm{E}+00$ & $2.659 \mathrm{E}+00$ & $1.480 \mathrm{E}+00$ & $5.153 \mathrm{E}-01$ \\
\hline $\mathrm{O}$ III] $\lambda 1664 \ldots \ldots \ldots \ldots \ldots . . . .$. & $4.218 \mathrm{E}-01$ & $4.911 \mathrm{E}-01$ & $4.360 \mathrm{E}-01$ & $3.998 \mathrm{E}-02$ & $2.142 \mathrm{E}-04$ \\
\hline $\mathrm{N}$ III] $\lambda 1750 \ldots \ldots \ldots \ldots \ldots . . . .$. & $5.465 \mathrm{E}-02$ & $6.803 \mathrm{E}-02$ & $8.384 \mathrm{E}-02$ & $8.003 \mathrm{E}-03$ & $4.254 \mathrm{E}-05$ \\
\hline 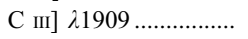 & $1.812 \mathrm{E}+00$ & $2.196 \mathrm{E}+00$ & $3.084 \mathrm{E}+00$ & $5.295 \mathrm{E}-01$ & $3.953 \mathrm{E}-02$ \\
\hline $\mathrm{C}$ II] $\lambda 2326 \ldots \ldots \ldots \ldots \ldots . . .$. & $5.040 \mathrm{E}-01$ & $4.648 \mathrm{E}-01$ & $3.418 \mathrm{E}-01$ & $5.215 \mathrm{E}-01$ & $4.742 \mathrm{E}-01$ \\
\hline $\mathrm{Mg}$ II $\lambda 2800 \ldots \ldots \ldots \ldots . . . .$. & $5.467 \mathrm{E}-01$ & $5.271 \mathrm{E}-01$ & $5.416 \mathrm{E}-01$ & $7.406 \mathrm{E}-01$ & $8.770 \mathrm{E}-01$ \\
\hline$[\mathrm{Ne} v] \lambda 3426 \ldots \ldots \ldots \ldots$ & $2.591 \mathrm{E}+00$ & $2.554 \mathrm{E}+00$ & $6.855 \mathrm{E}-01$ & $3.781 \mathrm{E}-04$ & $0.000 \mathrm{E}+00$ \\
\hline$[\mathrm{Ne}$ III] $\lambda 3869 \ldots \ldots \ldots \ldots$ & $2.568 \mathrm{E}+00$ & $2.671 \mathrm{E}+00$ & $2.961 \mathrm{E}+00$ & $1.892 \mathrm{E}+00$ & $8.511 \mathrm{E}-01$ \\
\hline \multicolumn{6}{|c|}{$\alpha=-1.4$} \\
\hline 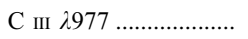 & $2.912 \mathrm{E}-01$ & $3.249 \mathrm{E}-01$ & $1.258 \mathrm{E}-01$ & $3.176 \mathrm{E}-03$ & $8.150 \mathrm{E}-05$ \\
\hline N III $\lambda 991$.................. & $6.818 \mathrm{E}-02$ & $7.394 \mathrm{E}-02$ & $2.645 \mathrm{E}-02$ & $5.219 \mathrm{E}-04$ & $1.222 \mathrm{E}-06$ \\
\hline $\mathrm{O}$ vi $\lambda \lambda 1032,1037 \ldots$. & $1.023 \mathrm{E}+01$ & $6.786 \mathrm{E}+00$ & $4.104 \mathrm{E}-02$ & $1.155 \mathrm{E}-06$ & $0.000 \mathrm{E}+00$ \\
\hline $\mathrm{N} v 21240 \ldots \ldots \ldots \ldots \ldots \ldots$ & $1.335 \mathrm{E}+00$ & $1.181 \mathrm{E}+00$ & $6.309 \mathrm{E}-02$ & $2.546 \mathrm{E}-05$ & $0.000 \mathrm{E}+00$ \\
\hline $\mathrm{Si} \mathrm{IV}+\mathrm{O}$ IV] $\lambda 1400 \ldots \ldots$ & $5.035 \mathrm{E}-01$ & $5.104 \mathrm{E}-01$ & $1.607 \mathrm{E}-01$ & $4.072 \mathrm{E}-03$ & $7.485 \mathrm{E}-06$ \\
\hline $\mathrm{N}$ Iv] $\lambda 1486 \ldots \ldots \ldots \ldots \ldots . . .$. & $2.789 \mathrm{E}-01$ & $3.152 \mathrm{E}-01$ & $8.731 \mathrm{E}-02$ & $2.946 \mathrm{E}-04$ & $0.000 \mathrm{E}+00$ \\
\hline $\mathrm{C}$ IV $\lambda 1549 \ldots \ldots \ldots \ldots \ldots$ & $6.387 \mathrm{E}+00$ & $6.906 \mathrm{E}+00$ & $2.097 \mathrm{E}+00$ & $6.216 \mathrm{E}-03$ & $7.913 \mathrm{E}-06$ \\
\hline 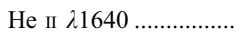 & $4.268 \mathrm{E}+00$ & $3.912 \mathrm{E}+00$ & $2.186 \mathrm{E}+00$ & $1.237 \mathrm{E}+00$ & $4.148 \mathrm{E}-01$ \\
\hline 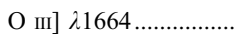 & $5.268 \mathrm{E}-01$ & $5.811 \mathrm{E}-01$ & $3.175 \mathrm{E}-01$ & $2.965 \mathrm{E}-02$ & $1.850 \mathrm{E}-04$ \\
\hline $\mathrm{N}$ III] $\lambda 1750 \ldots \ldots \ldots \ldots \ldots . . .$. & $6.859 \mathrm{E}-02$ & $8.183 \mathrm{E}-02$ & $6.215 \mathrm{E}-02$ & $6.023 \mathrm{E}-03$ & $3.753 \mathrm{E}-05$ \\
\hline 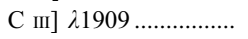 & $2.248 \mathrm{E}+00$ & $2.726 \mathrm{E}+00$ & $2.435 \mathrm{E}+00$ & $4.164 \mathrm{E}-01$ & $3.338 \mathrm{E}-02$ \\
\hline $\mathrm{C}$ II] $\lambda 2326 \ldots \ldots \ldots \ldots \ldots$ & $3.877 \mathrm{E}-01$ & $3.272 \mathrm{E}-01$ & $2.424 \mathrm{E}-01$ & $4.188 \mathrm{E}-01$ & $4.051 \mathrm{E}-01$ \\
\hline $\mathrm{Mg}$ II $\lambda 2800 \ldots \ldots \ldots \ldots . . . .$. & $5.018 \mathrm{E}-01$ & $4.769 \mathrm{E}-01$ & $4.633 \mathrm{E}-01$ & $6.335 \mathrm{E}-01$ & $8.051 \mathrm{E}-01$ \\
\hline$[\mathrm{Ne} v] \lambda 3426 \ldots \ldots \ldots \ldots$ & $2.822 \mathrm{E}+00$ & $2.575 \mathrm{E}+00$ & $3.794 \mathrm{E}-01$ & $1.861 \mathrm{E}-04$ & $0.000 \mathrm{E}+00$ \\
\hline$[\mathrm{Ne}$ III] $\lambda 3869 \ldots \ldots \ldots \ldots$ & $2.545 \mathrm{E}+00$ & $2.685 \mathrm{E}+00$ & $2.614 \mathrm{E}+00$ & $1.591 \mathrm{E}+00$ & $6.704 \mathrm{E}-01$ \\
\hline \multicolumn{6}{|c|}{$\alpha=-1.7$} \\
\hline C III 2977 ...................... & $3.107 \mathrm{E}-01$ & $2.449 \mathrm{E}-01$ & $4.523 \mathrm{E}-02$ & $1.408 \mathrm{E}-03$ & $5.112 \mathrm{E}-05$ \\
\hline $\mathrm{N}$ III $\lambda 991 \ldots \ldots \ldots \ldots \ldots \ldots . .$. & $6.612 \mathrm{E}-02$ & $4.946 \mathrm{E}-02$ & $9.292 \mathrm{E}-03$ & $2.178 \mathrm{E}-04$ & $0.000 \mathrm{E}+00$ \\
\hline $\mathrm{O}$ vi $\lambda \lambda 1032,1037 \ldots .$. & $7.960 \mathrm{E}+00$ & $2.894 \mathrm{E}+00$ & $4.260 \mathrm{E}-03$ & $0.000 \mathrm{E}+00$ & $0.000 \mathrm{E}+00$ \\
\hline $\mathrm{N} v \lambda 1240 \ldots \ldots \ldots \ldots \ldots \ldots$ & $1.378 \mathrm{E}+00$ & $8.293 \mathrm{E}-01$ & $1.274 \mathrm{E}-02$ & $4.683 \mathrm{E}-06$ & $0.000 \mathrm{E}+00$ \\
\hline $\mathrm{Si} \mathrm{IV+O} \mathrm{IV]} \lambda 1400 \ldots \ldots$. & $5.190 \mathrm{E}-01$ & $4.068 \mathrm{E}-01$ & $7.188 \mathrm{E}-02$ & $1.679 \mathrm{E}-03$ & $3.605 \mathrm{E}-06$ \\
\hline $\mathrm{N}$ Iv] $\lambda 1486 \ldots \ldots \ldots \ldots \ldots$ & $3.515 \mathrm{E}-01$ & $2.958 \mathrm{E}-01$ & $3.296 \mathrm{E}-02$ & $1.142 \mathrm{E}-04$ & $0.000 \mathrm{E}+00$ \\
\hline 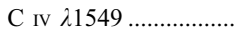 & $7.446 \mathrm{E}+00$ & $6.189 \mathrm{E}+00$ & $7.905 \mathrm{E}-01$ & $2.362 \mathrm{E}-03$ & $3.793 \mathrm{E}-06$ \\
\hline 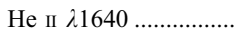 & $3.960 \mathrm{E}+00$ & $3.092 \mathrm{E}+00$ & $1.539 \mathrm{E}+00$ & $8.949 \mathrm{E}-01$ & $2.864 \mathrm{E}-01$ \\
\hline 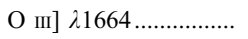 & $5.689 \mathrm{E}-01$ & $4.707 \mathrm{E}-01$ & $1.628 \mathrm{E}-01$ & $1.697 \mathrm{E}-02$ & $1.359 \mathrm{E}-04$ \\
\hline $\mathrm{N}$ III] $\lambda 1750 \ldots \ldots \ldots \ldots \ldots . . .$. & $7.385 \mathrm{E}-02$ & $6.834 \mathrm{E}-02$ & $3.271 \mathrm{E}-02$ & $3.546 \mathrm{E}-03$ & $2.853 \mathrm{E}-05$ \\
\hline 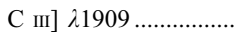 & $2.627 \mathrm{E}+00$ & $2.606 \mathrm{E}+00$ & $1.387 \mathrm{E}+00$ & $2.693 \mathrm{E}-01$ & $2.460 \mathrm{E}-02$ \\
\hline $\mathrm{C}$ II] $\lambda 2326 \ldots \ldots \ldots \ldots \ldots . . .$. & $2.333 \mathrm{E}-01$ & $1.710 \mathrm{E}-01$ & $1.477 \mathrm{E}-01$ & $3.123 \mathrm{E}-01$ & $3.292 \mathrm{E}-01$ \\
\hline $\mathrm{Mg}$ II $\lambda 2800 \ldots \ldots \ldots \ldots \ldots$ & $4.191 \mathrm{E}-01$ & $3.737 \mathrm{E}-01$ & $3.595 \mathrm{E}-01$ & $5.433 \mathrm{E}-01$ & $7.441 \mathrm{E}-01$ \\
\hline$[\mathrm{Ne} v] \lambda 3426 \ldots \ldots \ldots \ldots$ & $2.787 \mathrm{E}+00$ & $1.967 \mathrm{E}+00$ & $1.177 \mathrm{E}-01$ & $5.413 \mathrm{E}-05$ & $0.000 \mathrm{E}+00$ \\
\hline$[\mathrm{Ne}$ III] $\lambda 3869 \ldots \ldots \ldots . .$. & $2.520 \mathrm{E}+00$ & $2.508 \mathrm{E}+00$ & $1.988 \mathrm{E}+00$ & $1.189 \mathrm{E}+00$ & $4.598 \mathrm{E}-01$ \\
\hline \multicolumn{6}{|c|}{$\alpha=-2.0$} \\
\hline $\mathrm{C}$ III $\lambda 977$..................... & $2.448 \mathrm{E}-01$ & $1.163 \mathrm{E}-01$ & $1.261 \mathrm{E}-02$ & $6.047 \mathrm{E}-04$ & $3.047 \mathrm{E}-05$ \\
\hline 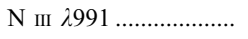 & $4.732 \mathrm{E}-02$ & $2.177 \mathrm{E}-02$ & $2.649 \mathrm{E}-03$ & $8.628 \mathrm{E}-05$ & $0.000 \mathrm{E}+00$ \\
\hline $\mathrm{O}$ vi $\lambda \lambda 1032,1037 \ldots .$. & $6.340 \mathrm{E}+00$ & $7.906 \mathrm{E}-01$ & $3.108 \mathrm{E}-04$ & $0.000 \mathrm{E}+00$ & $0.000 \mathrm{E}+00$ \\
\hline 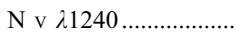 & $1.199 \mathrm{E}+00$ & $3.944 \mathrm{E}-01$ & $1.918 \mathrm{E}-03$ & $0.000 \mathrm{E}+00$ & $0.000 \mathrm{E}+00$ \\
\hline $\mathrm{Si}$ IV $+\mathrm{O}$ Iv] $\lambda 1400 \ldots \ldots$. & $4.421 \mathrm{E}-01$ & $2.377 \mathrm{E}-01$ & $3.343 \mathrm{E}-02$ & $6.790 \mathrm{E}-04$ & $1.092 \mathrm{E}-06$ \\
\hline $\mathrm{N}$ IV] $\lambda 1486 \ldots \ldots \ldots \ldots \ldots . . . .$. & $3.317 \mathrm{E}-01$ & $1.833 \mathrm{E}-01$ & $9.745 \mathrm{E}-03$ & $4.087 \mathrm{E}-05$ & $0.000 \mathrm{E}+00$ \\
\hline 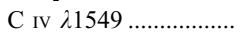 & $6.935 \mathrm{E}+00$ & $3.802 \mathrm{E}+00$ & $2.283 \mathrm{E}-01$ & $8.555 \mathrm{E}-04$ & $1.158 \mathrm{E}-06$ \\
\hline Не пा $\lambda 1640 \ldots \ldots \ldots \ldots \ldots . . . . . .$. & $3.500 \mathrm{E}+00$ & $2.141 \mathrm{E}+00$ & $1.042 \mathrm{E}+00$ & $6.205 \mathrm{E}-01$ & $1.909 \mathrm{E}-01$ \\
\hline $\mathrm{O}$ III] $\lambda 1664 \ldots \ldots \ldots \ldots . . . . .$. & $4.756 \mathrm{E}-01$ & $2.566 \mathrm{E}-01$ & $7.365 \mathrm{E}-02$ & $9.135 \mathrm{E}-03$ & $9.332 \mathrm{E}-05$ \\
\hline $\mathrm{N}$ III] $\lambda 1750 \ldots \ldots \ldots \ldots \ldots$ & $6.172 \mathrm{E}-02$ & $3.954 \mathrm{E}-02$ & $1.507 \mathrm{E}-02$ & $1.975 \mathrm{E}-03$ & $2.032 \mathrm{E}-05$ \\
\hline $\mathrm{C}$ III] $\lambda 1909 \ldots \ldots \ldots \ldots . . . .$. & $2.440 \mathrm{E}+00$ & $1.714 \mathrm{E}+00$ & $6.777 \mathrm{E}-01$ & $1.672 \mathrm{E}-01$ & $1.741 \mathrm{E}-02$ \\
\hline $\mathrm{C}_{\text {II] }} \lambda 2326 \ldots \ldots \ldots \ldots \ldots$ & $1.444 \mathrm{E}-01$ & $8.948 \mathrm{E}-02$ & $9.677 \mathrm{E}-02$ & $2.430 \mathrm{E}-01$ & $2.742 \mathrm{E}-01$ \\
\hline $\mathrm{Mg}$ II $\lambda 2800 \ldots \ldots \ldots \ldots \ldots$ & $3.453 \mathrm{E}-01$ & $2.785 \mathrm{E}-01$ & $2.906 \mathrm{E}-01$ & $4.987 \mathrm{E}-01$ & $7.028 \mathrm{E}-01$ \\
\hline$[\mathrm{Ne} v] \lambda 3426 \ldots \ldots \ldots \ldots . .$. & $2.440 \mathrm{E}+00$ & $1.122 \mathrm{E}+00$ & $2.711 \mathrm{E}-02$ & $1.408 \mathrm{E}-05$ & $0.000 \mathrm{E}+00$ \\
\hline$[\mathrm{Ne}$ III] $\lambda 3869 \ldots \ldots \ldots \ldots$ & $2.396 \mathrm{E}+00$ & $2.025 \mathrm{E}+00$ & $1.408 \mathrm{E}+00$ & $8.663 \mathrm{E}-01$ & $3.080 \mathrm{E}-01$ \\
\hline
\end{tabular}


TABLE 6

Dusty UV Line Ratios with Respect to $\mathrm{H} \beta, 2 Z_{\odot}$

\begin{tabular}{|c|c|c|c|c|c|}
\hline \multirow[b]{2}{*}{ LiNE } & \multicolumn{5}{|c|}{$\log U_{0}$} \\
\hline & 0.0 & -1.0 & -2.0 & -3.0 & -4.0 \\
\hline \multicolumn{6}{|c|}{$\alpha=-1.2$} \\
\hline 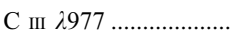 & $3.264 \mathrm{E}-01$ & $3.415 \mathrm{E}-01$ & $9.512 \mathrm{E}-02$ & $5.800 \mathrm{E}-04$ & $1.733 \mathrm{E}-05$ \\
\hline 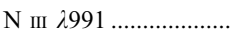 & $1.392 \mathrm{E}-01$ & $1.455 \mathrm{E}-01$ & $3.708 \mathrm{E}-02$ & $1.363 \mathrm{E}-04$ & $0.000 \mathrm{E}+00$ \\
\hline O VI $\lambda \lambda 1032,1037 \ldots .$. & $1.308 \mathrm{E}+01$ & $1.054 \mathrm{E}+01$ & $6.218 \mathrm{E}-02$ & $0.000 \mathrm{E}+00$ & $0.000 \mathrm{E}+00$ \\
\hline $\mathrm{N}$ v $\lambda 1240 \ldots \ldots \ldots \ldots \ldots \ldots$ & $2.904 \mathrm{E}+00$ & $2.735 \mathrm{E}+00$ & $1.500 \mathrm{E}-01$ & $1.279 \mathrm{E}-05$ & $0.000 \mathrm{E}+00$ \\
\hline $\mathrm{Si} \mathrm{IV+O} \mathrm{Iv]} \lambda 1400 \ldots \ldots$ & $6.241 \mathrm{E}-01$ & $6.304 \mathrm{E}-01$ & $1.650 \mathrm{E}-01$ & $1.086 \mathrm{E}-03$ & $3.382 \mathrm{E}-06$ \\
\hline $\mathrm{N}$ IV] $\lambda 1486 \ldots \ldots \ldots \ldots \ldots$ & $5.446 \mathrm{E}-01$ & $6.052 \mathrm{E}-01$ & $1.725 \mathrm{E}-01$ & $1.493 \mathrm{E}-04$ & $0.000 \mathrm{E}+00$ \\
\hline $\mathrm{C}$ Iv $\lambda 1549 \ldots \ldots \ldots \ldots \ldots$ & $7.881 \mathrm{E}+00$ & $8.343 \mathrm{E}+00$ & $2.406 \mathrm{E}+00$ & $2.010 \mathrm{E}-03$ & $3.906 \mathrm{E}-06$ \\
\hline He II $\lambda 1640 \ldots \ldots \ldots \ldots . . .$. & $4.957 \mathrm{E}+00$ & $4.853 \mathrm{E}+00$ & $2.906 \mathrm{E}+00$ & $1.517 \mathrm{E}+00$ & $5.202 \mathrm{E}-01$ \\
\hline $\mathrm{O}$ III] $\lambda 1664 \ldots \ldots \ldots \ldots \ldots . . .$. & $6.281 \mathrm{E}-01$ & $6.667 \mathrm{E}-01$ & $2.895 \mathrm{E}-01$ & $1.133 \mathrm{E}-02$ & $7.433 \mathrm{E}-05$ \\
\hline 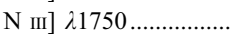 & $1.693 \mathrm{E}-01$ & $1.931 \mathrm{E}-01$ & $1.141 \mathrm{E}-01$ & $4.284 \mathrm{E}-03$ & $2.727 \mathrm{E}-05$ \\
\hline 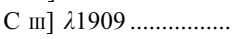 & $3.211 \mathrm{E}+00$ & $3.584 \mathrm{E}+00$ & $2.512 \mathrm{E}+00$ & $2.288 \mathrm{E}-01$ & $1.951 \mathrm{E}-02$ \\
\hline 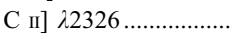 & $1.044 \mathrm{E}+00$ & $9.413 \mathrm{E}-01$ & $4.951 \mathrm{E}-01$ & $5.237 \mathrm{E}-01$ & $3.792 \mathrm{E}-01$ \\
\hline $\mathrm{Mg}$ II $\lambda 2800 \ldots \ldots \ldots \ldots \ldots . .$. & $1.039 \mathrm{E}+00$ & $9.840 \mathrm{E}-01$ & $7.682 \mathrm{E}-01$ & $8.036 \mathrm{E}-01$ & $7.806 \mathrm{E}-01$ \\
\hline$[\mathrm{Ne} v] \lambda 3426 \ldots \ldots \ldots \ldots . .$. & $4.176 \mathrm{E}+00$ & $3.973 \mathrm{E}+00$ & $6.224 \mathrm{E}-01$ & $1.301 \mathrm{E}-04$ & $0.000 \mathrm{E}+00$ \\
\hline$[\mathrm{Ne}$ III] $\lambda 3869 \ldots \ldots \ldots \ldots$ & $4.952 \mathrm{E}+00$ & $4.939 \mathrm{E}+00$ & $3.715 \mathrm{E}+00$ & $1.956 \mathrm{E}+00$ & $8.383 \mathrm{E}-01$ \\
\hline \multicolumn{6}{|c|}{$\alpha=-1.4$} \\
\hline 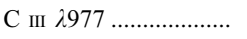 & $3.307 \mathrm{E}-01$ & $2.898 \mathrm{E}-01$ & $3.716 \mathrm{E}-02$ & $2.433 \mathrm{E}-04$ & $9.694 \mathrm{E}-06$ \\
\hline 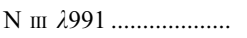 & $1.373 \mathrm{E}-01$ & $1.168 \mathrm{E}-01$ & $1.403 \mathrm{E}-02$ & $5.404 \mathrm{E}-05$ & $0.000 \mathrm{E}+00$ \\
\hline $\mathrm{O}$ VI $\lambda \lambda 1032,1037 \ldots .$. & $1.148 \mathrm{E}+01$ & $6.696 \mathrm{E}+00$ & $1.056 \mathrm{E}-02$ & $0.000 \mathrm{E}+00$ & $0.000 \mathrm{E}+00$ \\
\hline $\mathrm{N} v \lambda 1240 \ldots \ldots \ldots \ldots \ldots \ldots$ & $3.145 \mathrm{E}+00$ & $2.447 \mathrm{E}+00$ & $4.183 \mathrm{E}-02$ & $3.118 \mathrm{E}-06$ & $0.000 \mathrm{E}+00$ \\
\hline $\mathrm{Si} \mathrm{IV+O} \mathrm{IV]} \lambda 1400 \ldots \ldots$ & $6.524 \mathrm{E}-01$ & $5.772 \mathrm{E}-01$ & $7.846 \mathrm{E}-02$ & $4.405 \mathrm{E}-04$ & $1.081 \mathrm{E}-06$ \\
\hline $\mathrm{N}$ Iv] $\lambda 1486 \ldots \ldots \ldots \ldots \ldots$ & $6.781 \mathrm{E}-01$ & $6.660 \mathrm{E}-01$ & $7.682 \mathrm{E}-02$ & $6.396 \mathrm{E}-05$ & $0.000 \mathrm{E}+00$ \\
\hline 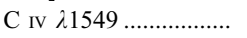 & $8.942 \mathrm{E}+00$ & $8.440 \mathrm{E}+00$ & $1.074 \mathrm{E}+00$ & $8.352 \mathrm{E}-04$ & $1.284 \mathrm{E}-06$ \\
\hline 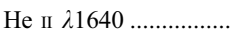 & $5.045 \mathrm{E}+00$ & $4.575 \mathrm{E}+00$ & $2.353 \mathrm{E}+00$ & $1.244 \mathrm{E}+00$ & $4.128 \mathrm{E}-01$ \\
\hline 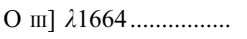 & $6.523 \mathrm{E}-01$ & $5.945 \mathrm{E}-01$ & $1.516 \mathrm{E}-01$ & $6.356 \mathrm{E}-03$ & $5.320 \mathrm{E}-05$ \\
\hline $\mathrm{N}$ III] $\lambda 1750 \ldots \ldots \ldots \ldots \ldots . . .$. & $1.821 \mathrm{E}-01$ & $1.797 \mathrm{E}-01$ & $6.155 \mathrm{E}-02$ & $2.465 \mathrm{E}-03$ & $1.991 \mathrm{E}-05$ \\
\hline C III] $\lambda 1909 \ldots \ldots \ldots \ldots . . .$. & $3.444 \mathrm{E}+00$ & $3.483 \mathrm{E}+00$ & $1.473 \mathrm{E}+00$ & $1.428 \mathrm{E}-01$ & $1.389 \mathrm{E}-02$ \\
\hline 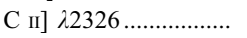 & $7.779 \mathrm{E}-01$ & $6.148 \mathrm{E}-01$ & $2.934 \mathrm{E}-01$ & $3.584 \mathrm{E}-01$ & $2.916 \mathrm{E}-01$ \\
\hline $\mathrm{Mg}$ II $\lambda 2800 \ldots \ldots \ldots \ldots \ldots$ & $9.280 \mathrm{E}-01$ & $8.320 \mathrm{E}-01$ & $5.671 \mathrm{E}-01$ & $6.184 \mathrm{E}-01$ & $6.705 \mathrm{E}-01$ \\
\hline$[\mathrm{Ne} v] \lambda 3426 \ldots \ldots \ldots \ldots$ & $4.371 \mathrm{E}+00$ & $3.687 \mathrm{E}+00$ & $2.693 \mathrm{E}-01$ & $5.270 \mathrm{E}-05$ & $0.000 \mathrm{E}+00$ \\
\hline$[\mathrm{Ne}$ III] $\lambda 3869 \ldots \ldots \ldots \ldots$ & $4.521 \mathrm{E}+00$ & $4.279 \mathrm{E}+00$ & $2.720 \mathrm{E}+00$ & $1.443 \mathrm{E}+00$ & $6.054 \mathrm{E}-01$ \\
\hline \multicolumn{6}{|c|}{$\alpha=-1.7$} \\
\hline C III 2977 ...................... & $2.420 \mathrm{E}-01$ & $1.378 \mathrm{E}-01$ & $6.032 \mathrm{E}-03$ & $6.541 \mathrm{E}-05$ & $3.756 \mathrm{E}-06$ \\
\hline 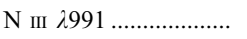 & $9.149 \mathrm{E}-02$ & $5.000 \mathrm{E}-02$ & $2.289 \mathrm{E}-03$ & $1.325 \mathrm{E}-05$ & $0.000 \mathrm{E}+00$ \\
\hline $\mathrm{O}$ VI $\lambda \lambda 1032,1037 \ldots .$. & $7.547 \mathrm{E}+00$ & $2.159 \mathrm{E}+00$ & $4.146 \mathrm{E}-04$ & $0.000 \mathrm{E}+00$ & $0.000 \mathrm{E}+00$ \\
\hline $\mathrm{N}$ v $\lambda 1240 \ldots \ldots \ldots \ldots \ldots \ldots$ & $2.755 \mathrm{E}+00$ & $1.340 \mathrm{E}+00$ & $3.754 \mathrm{E}-03$ & $0.000 \mathrm{E}+00$ & $0.000 \mathrm{E}+00$ \\
\hline $\mathrm{Si} \mathrm{IV+O} \mathrm{Iv]} \lambda 1400 \ldots \ldots$ & $5.536 \mathrm{E}-01$ & $3.512 \mathrm{E}-01$ & $2.524 \mathrm{E}-02$ & $1.082 \mathrm{E}-04$ & $0.000 \mathrm{E}+00$ \\
\hline 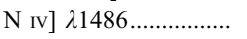 & $6.977 \mathrm{E}-01$ & $4.749 \mathrm{E}-01$ & $1.547 \mathrm{E}-02$ & $1.694 \mathrm{E}-05$ & $0.000 \mathrm{E}+00$ \\
\hline 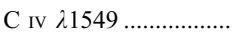 & $8.569 \mathrm{E}+00$ & $5.756 \mathrm{E}+00$ & $2.113 \mathrm{E}-01$ & $2.187 \mathrm{E}-04$ & $0.000 \mathrm{E}+00$ \\
\hline He II $\lambda 1640 \ldots \ldots \ldots \ldots \ldots . . . .$. & $4.642 \mathrm{E}+00$ & $3.529 \mathrm{E}+00$ & $1.627 \mathrm{E}+00$ & $8.776 \mathrm{E}-01$ & $2.700 \mathrm{E}-01$ \\
\hline 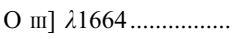 & $5.015 \mathrm{E}-01$ & $3.101 \mathrm{E}-01$ & $4.752 \mathrm{E}-02$ & $2.571 \mathrm{E}-03$ & $2.909 \mathrm{E}-05$ \\
\hline $\mathrm{N}$ III] $\lambda 1750 \ldots \ldots \ldots \ldots \ldots$ & $1.446 \mathrm{E}-01$ & $9.991 \mathrm{E}-02$ & $1.972 \mathrm{E}-02$ & $1.037 \mathrm{E}-03$ & $1.124 \mathrm{E}-05$ \\
\hline 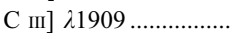 & $2.994 \mathrm{E}+00$ & $2.238 \mathrm{E}+00$ & $5.248 \mathrm{E}-01$ & $6.843 \mathrm{E}-02$ & $7.771 \mathrm{E}-03$ \\
\hline $\mathrm{C}$ II] $\lambda 2326 \ldots \ldots \ldots \ldots \ldots$ & $4.163 \mathrm{E}-01$ & $2.550 \mathrm{E}-01$ & $1.283 \mathrm{E}-01$ & $2.076 \mathrm{E}-01$ & $1.946 \mathrm{E}-01$ \\
\hline $\mathrm{Mg}$ II $\lambda 2800 \ldots \ldots \ldots \ldots \ldots$ & $7.006 \mathrm{E}-01$ & $5.340 \mathrm{E}-01$ & $3.483 \mathrm{E}-01$ & $4.498 \mathrm{E}-01$ & $5.289 \mathrm{E}-01$ \\
\hline$[\mathrm{Ne} \mathrm{v}] \lambda 3426 \ldots \ldots \ldots \ldots$ & $3.949 \mathrm{E}+00$ & $2.406 \mathrm{E}+00$ & $5.145 \mathrm{E}-02$ & $1.233 \mathrm{E}-05$ & $0.000 \mathrm{E}+00$ \\
\hline$[\mathrm{Ne}$ III] $\lambda 3869 \ldots \ldots \ldots \ldots$ & $3.654 \mathrm{E}+00$ & $2.969 \mathrm{E}+00$ & $1.582 \mathrm{E}+00$ & $8.932 \mathrm{E}-01$ & $3.506 \mathrm{E}-01$ \\
\hline \multicolumn{6}{|c|}{$\alpha=-2.0$} \\
\hline C III 2977 ...................... & $1.507 \mathrm{E}-01$ & $4.706 \mathrm{E}-02$ & $5.929 \mathrm{E}-04$ & $1.827 \mathrm{E}-05$ & $1.434 \mathrm{E}-06$ \\
\hline N III $\lambda 991 \ldots \ldots \ldots \ldots \ldots . . .$. & $5.273 \mathrm{E}-02$ & $1.637 \mathrm{E}-02$ & $2.402 \mathrm{E}-04$ & $3.437 \mathrm{E}-06$ & $0.000 \mathrm{E}+00$ \\
\hline $\mathrm{O}$ VI $\lambda \lambda 1032,1037 \ldots$. & $6.003 \mathrm{E}+00$ & $4.211 \mathrm{E}-01$ & $8.854 \mathrm{E}-06$ & $0.000 \mathrm{E}+00$ & $0.000 \mathrm{E}+00$ \\
\hline $\mathrm{N} v \mathrm{\lambda} 1240 \ldots \ldots \ldots \ldots \ldots \ldots$ & $2.238 \mathrm{E}+00$ & $4.856 \mathrm{E}-01$ & $2.057 \mathrm{E}-04$ & $0.000 \mathrm{E}+00$ & $0.000 \mathrm{E}+00$ \\
\hline $\mathrm{Si} \mathrm{IV+O} \mathrm{Iv]} \lambda 1400 \ldots \ldots$ & $6.648 \mathrm{E}-01$ & $1.610 \mathrm{E}-01$ & $3.412 \mathrm{E}-03$ & $2.684 \mathrm{E}-05$ & $0.000 \mathrm{E}+00$ \\
\hline $\mathrm{N}$ Iv] $\lambda 1486 \ldots \ldots \ldots \ldots \ldots$ & $5.792 \mathrm{E}-01$ & $2.329 \mathrm{E}-01$ & $2.126 \mathrm{E}-03$ & $4.339 \mathrm{E}-06$ & $0.000 \mathrm{E}+00$ \\
\hline $\mathrm{C}$ IV $\lambda 1549 \ldots \ldots \ldots \ldots \ldots . .$. & $6.973 \mathrm{E}+00$ & $2.777 \mathrm{E}+00$ & $2.687 \mathrm{E}-02$ & $5.820 \mathrm{E}-05$ & $0.000 \mathrm{E}+00$ \\
\hline 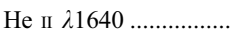 & $4.098 \mathrm{E}+00$ & $2.392 \mathrm{E}+00$ & $1.093 \mathrm{E}+00$ & $5.923 \mathrm{E}-01$ & $1.696 \mathrm{E}-01$ \\
\hline 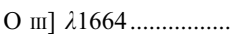 & $3.292 \mathrm{E}-01$ & $1.202 \mathrm{E}-01$ & $1.229 \mathrm{E}-02$ & $1.049 \mathrm{E}-03$ & $1.552 \mathrm{E}-05$ \\
\hline $\mathrm{N}$ III] $\lambda 1750 \ldots \ldots \ldots \ldots \ldots$ & $9.694 \mathrm{E}-02$ & $4.169 \mathrm{E}-02$ & $5.172 \mathrm{E}-03$ & $4.400 \mathrm{E}-04$ & $6.207 \mathrm{E}-06$ \\
\hline 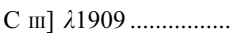 & $2.227 \mathrm{E}+00$ & $1.067 \mathrm{E}+00$ & $1.519 \mathrm{E}-01$ & $3.295 \mathrm{E}-02$ & $4.256 \mathrm{E}-03$ \\
\hline $\mathrm{C}$ II] $\lambda 2326 \ldots \ldots \ldots \ldots \ldots$ & $2.242 \mathrm{E}-01$ & $9.036 \mathrm{E}-02$ & $5.246 \mathrm{E}-02$ & $1.263 \mathrm{E}-01$ & $1.321 \mathrm{E}-01$ \\
\hline $\mathrm{Mg}$ II $\lambda 2800 \ldots \ldots \ldots \ldots \ldots$ & $5.122 \mathrm{E}-01$ & $2.997 \mathrm{E}-01$ & $2.039 \mathrm{E}-01$ & $3.524 \mathrm{E}-01$ & $4.198 \mathrm{E}-01$ \\
\hline$[\mathrm{Ne} \mathrm{v}] \lambda 3426 \ldots \ldots \ldots \ldots$ & $3.278 \mathrm{E}+00$ & $1.148 \mathrm{E}+00$ & $6.793 \mathrm{E}-03$ & $2.572 \mathrm{E}-06$ & $0.000 \mathrm{E}+00$ \\
\hline$[\mathrm{Ne}$ III] $\lambda 3869 \ldots \ldots \ldots \ldots$ & $2.915 \mathrm{E}+00$ & $1.830 \mathrm{E}+00$ & $8.569 \mathrm{E}-01$ & $5.468 \mathrm{E}-01$ & $1.967 \mathrm{E}-01$ \\
\hline
\end{tabular}


TABLE 7

Dusty UV Line Ratios with Respect to $\mathrm{H} \beta, 4 Z_{\odot}$

\begin{tabular}{|c|c|c|c|c|c|}
\hline \multirow[b]{2}{*}{ LiNE } & \multicolumn{5}{|c|}{$\log U_{0}$} \\
\hline & 0.0 & -1.0 & -2.0 & -3.0 & -4.0 \\
\hline \multicolumn{6}{|c|}{$\alpha=-1.2$} \\
\hline C III $\lambda 977$................... & $3.989 \mathrm{E}-01$ & $3.460 \mathrm{E}-01$ & $2.398 \mathrm{E}-02$ & $9.662 \mathrm{E}-06$ & $0.000 \mathrm{E}+00$ \\
\hline 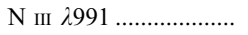 & $3.226 \mathrm{E}-01$ & $2.756 \mathrm{E}-01$ & $1.728 \mathrm{E}-02$ & $2.394 \mathrm{E}-06$ & $0.000 \mathrm{E}+00$ \\
\hline $\mathrm{O}$ vi $\lambda \lambda 1032,1037 \ldots$. & $1.563 \mathrm{E}+01$ & $1.110 \mathrm{E}+01$ & $1.224 \mathrm{E}-02$ & $0.000 \mathrm{E}+00$ & $0.000 \mathrm{E}+00$ \\
\hline 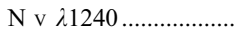 & $7.359 \mathrm{E}+00$ & $6.265 \mathrm{E}+00$ & $8.204 \mathrm{E}-02$ & $0.000 \mathrm{E}+00$ & $0.000 \mathrm{E}+00$ \\
\hline $\mathrm{Si} \mathrm{IV+O} \mathrm{Iv]} \lambda 1400 \ldots \ldots$ & $8.556 \mathrm{E}-01$ & $7.711 \mathrm{E}-01$ & $9.646 \mathrm{E}-02$ & $3.267 \mathrm{E}-05$ & $0.000 \mathrm{E}+00$ \\
\hline $\mathrm{N}$ Iv] $\lambda 1486 \ldots \ldots \ldots \ldots \ldots$ & $1.461 \mathrm{E}+00$ & $1.477 \mathrm{E}+00$ & $1.339 \mathrm{E}-01$ & $9.172 \mathrm{E}-06$ & $0.000 \mathrm{E}+00$ \\
\hline 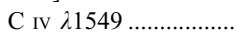 & $1.123 \mathrm{E}+01$ & $1.079 \mathrm{E}+01$ & $1.022 \mathrm{E}+00$ & $8.189 \mathrm{E}-05$ & $0.000 \mathrm{E}+00$ \\
\hline 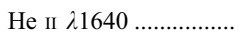 & $6.114 \mathrm{E}+00$ & $5.932 \mathrm{E}+00$ & $3.188 \mathrm{E}+00$ & $1.495 \mathrm{E}+00$ & $4.666 \mathrm{E}-01$ \\
\hline 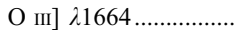 & $8.450 \mathrm{E}-01$ & $7.555 \mathrm{E}-01$ & $1.165 \mathrm{E}-01$ & $7.634 \mathrm{E}-04$ & $3.849 \mathrm{E}-06$ \\
\hline $\mathrm{N}$ III] $\lambda 1750 \ldots \ldots \ldots \ldots \ldots . . .$. & $4.758 \mathrm{E}-01$ & $4.640 \mathrm{E}-01$ & $9.893 \mathrm{E}-02$ & $5.766 \mathrm{E}-04$ & $2.765 \mathrm{E}-06$ \\
\hline 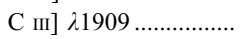 & $4.774 \mathrm{E}+00$ & $4.634 \mathrm{E}+00$ & $1.278 \mathrm{E}+00$ & $2.797 \mathrm{E}-02$ & $1.535 \mathrm{E}-03$ \\
\hline $\left.\mathrm{C}_{\text {II }}\right] \lambda 2326 \ldots \ldots \ldots \ldots \ldots$ & $1.881 \mathrm{E}+00$ & $1.585 \mathrm{E}+00$ & $5.280 \mathrm{E}-01$ & $2.722 \mathrm{E}-01$ & $7.540 \mathrm{E}-02$ \\
\hline Mg II $\lambda 2800 \ldots \ldots \ldots \ldots . . .$. & $1.805 \mathrm{E}+00$ & $1.618 \mathrm{E}+00$ & $8.259 \mathrm{E}-01$ & $5.442 \mathrm{E}-01$ & $2.968 \mathrm{E}-01$ \\
\hline$[\mathrm{Ne} v] \lambda 3426 \ldots \ldots \ldots \ldots$ & $6.331 \mathrm{E}+00$ & $5.686 \mathrm{E}+00$ & $3.750 \mathrm{E}-01$ & $1.705 \mathrm{E}-05$ & $0.000 \mathrm{E}+00$ \\
\hline$[\mathrm{Ne}$ III] $\lambda 3869 \ldots \ldots \ldots \ldots$ & $8.505 \mathrm{E}+00$ & $7.763 \mathrm{E}+00$ & $3.310 \mathrm{E}+00$ & $1.148 \mathrm{E}+00$ & $3.053 \mathrm{E}-01$ \\
\hline \multicolumn{6}{|c|}{$\alpha=-1.4$} \\
\hline C III $\lambda 977$................... & $3.090 \mathrm{E}-01$ & $2.064 \mathrm{E}-01$ & $4.112 \mathrm{E}-03$ & $2.099 \mathrm{E}-06$ & $0.000 \mathrm{E}+00$ \\
\hline N III $\lambda 991$.................. & $2.379 \mathrm{E}-01$ & $1.523 \mathrm{E}-01$ & $2.941 \mathrm{E}-03$ & $0.000 \mathrm{E}+00$ & $0.000 \mathrm{E}+00$ \\
\hline $\mathrm{O}$ vi $\lambda \lambda 1032,1037 \ldots$. & $1.202 \mathrm{E}+01$ & $5.691 \mathrm{E}+00$ & $7.588 \mathrm{E}-04$ & $0.000 \mathrm{E}+00$ & $0.000 \mathrm{E}+00$ \\
\hline $\mathrm{N}$ v $\lambda 1240 \ldots \ldots \ldots \ldots \ldots . . . . . .$. & $7.016 \mathrm{E}+00$ & $4.591 \mathrm{E}+00$ & $9.824 \mathrm{E}-03$ & $0.000 \mathrm{E}+00$ & $0.000 \mathrm{E}+00$ \\
\hline $\mathrm{Si} \mathrm{IV+O} \mathrm{IV]} \lambda 1400 \ldots \ldots$ & $7.630 \mathrm{E}-01$ & $5.638 \mathrm{E}-01$ & $1.728 \mathrm{E}-02$ & $6.872 \mathrm{E}-06$ & $0.000 \mathrm{E}+00$ \\
\hline $\mathrm{N}$ IV] $\lambda 1486 \ldots \ldots \ldots \ldots \ldots$ & $1.571 \mathrm{E}+00$ & $1.298 \mathrm{E}+00$ & $3.096 \mathrm{E}-02$ & $2.811 \mathrm{E}-06$ & $0.000 \mathrm{E}+00$ \\
\hline $\mathrm{C}$ IV $\lambda 1549 \ldots \ldots \ldots \ldots \ldots$ & $1.111 \mathrm{E}+01$ & $8.884 \mathrm{E}+00$ & $2.327 \mathrm{E}-01$ & $2.430 \mathrm{E}-05$ & $0.000 \mathrm{E}+00$ \\
\hline He II $\lambda 1640 \ldots \ldots \ldots \ldots \ldots . . . .$. & $6.194 \mathrm{E}+00$ & $5.463 \mathrm{E}+00$ & $2.534 \mathrm{E}+00$ & $1.196 \mathrm{E}+00$ & $3.519 \mathrm{E}-01$ \\
\hline 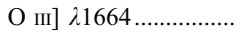 & $6.692 \mathrm{E}-01$ & $4.685 \mathrm{E}-01$ & $3.768 \mathrm{E}-02$ & $3.220 \mathrm{E}-04$ & $1.632 \mathrm{E}-06$ \\
\hline $\mathrm{N}$ III] $\lambda 1750 \ldots \ldots \ldots \ldots \ldots$ & $4.022 \mathrm{E}-01$ & $3.114 \mathrm{E}-01$ & $3.270 \mathrm{E}-02$ & $2.511 \mathrm{E}-04$ & $1.690 \mathrm{E}-06$ \\
\hline $\mathrm{C}$ III] $\lambda 1909 \ldots \ldots \ldots \ldots . . . .$. & $4.127 \mathrm{E}+00$ & $3.350 \mathrm{E}+00$ & $4.736 \mathrm{E}-01$ & $1.292 \mathrm{E}-02$ & $9.024 \mathrm{E}-04$ \\
\hline $\mathrm{C}$ II] $\lambda 2326 \ldots \ldots \ldots \ldots \ldots$ & $1.276 \mathrm{E}+00$ & $8.959 \mathrm{E}-01$ & $2.416 \mathrm{E}-01$ & $1.314 \mathrm{E}-01$ & $4.883 \mathrm{E}-02$ \\
\hline $\mathrm{Mg}$ II $\lambda 2800 \ldots \ldots \ldots \ldots \ldots$ & $1.475 \mathrm{E}+00$ & $1.172 \mathrm{E}+00$ & $4.870 \mathrm{E}-01$ & $3.348 \mathrm{E}-01$ & $2.235 \mathrm{E}-01$ \\
\hline$[\mathrm{Ne} v] \lambda 3426 \ldots \ldots \ldots \ldots$ & $6.204 \mathrm{E}+00$ & $4.673 \mathrm{E}+00$ & $1.002 \mathrm{E}-01$ & $5.675 \mathrm{E}-06$ & $0.000 \mathrm{E}+00$ \\
\hline$[\mathrm{Ne}$ III] $\lambda 3869 \ldots \ldots \ldots \ldots$ & $6.749 \mathrm{E}+00$ & $5.395 \mathrm{E}+00$ & $1.877 \mathrm{E}+00$ & $6.839 \mathrm{E}-01$ & $1.898 \mathrm{E}-01$ \\
\hline \multicolumn{6}{|c|}{$\alpha=-1.7$} \\
\hline $\mathrm{C}$ III $\lambda 977$................... & $1.579 \mathrm{E}-01$ & $6.472 \mathrm{E}-02$ & $1.041 \mathrm{E}-04$ & $0.000 \mathrm{E}+00$ & $0.000 \mathrm{E}+00$ \\
\hline 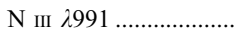 & $1.095 \mathrm{E}-01$ & $4.366 \mathrm{E}-02$ & $8.004 \mathrm{E}-05$ & $0.000 \mathrm{E}+00$ & $0.000 \mathrm{E}+00$ \\
\hline $\mathrm{O}$ vi $\lambda \lambda 1032,1037 \ldots .$. & $6.646 \mathrm{E}+00$ & $1.290 \mathrm{E}+00$ & $3.969 \mathrm{E}-06$ & $0.000 \mathrm{E}+00$ & $0.000 \mathrm{E}+00$ \\
\hline $\mathrm{N} v \lambda 1240 \ldots \ldots \ldots \ldots \ldots \ldots$ & $5.128 \mathrm{E}+00$ & $1.858 \mathrm{E}+00$ & $1.644 \mathrm{E}-04$ & $0.000 \mathrm{E}+00$ & $0.000 \mathrm{E}+00$ \\
\hline $\mathrm{Si} \mathrm{IV}+\mathrm{O}$ Iv] $\lambda 1400 \ldots \ldots$. & $5.199 \mathrm{E}-01$ & $2.547 \mathrm{E}-01$ & $1.174 \mathrm{E}-03$ & $0.000 \mathrm{E}+00$ & $0.000 \mathrm{E}+00$ \\
\hline $\mathrm{N}$ IV] $\lambda 1486 \ldots \ldots \ldots \ldots \ldots . . . .$. & $1.296 \mathrm{E}+00$ & $6.830 \mathrm{E}-01$ & $1.667 \mathrm{E}-03$ & $0.000 \mathrm{E}+00$ & $0.000 \mathrm{E}+00$ \\
\hline $\mathrm{C}$ IV $\lambda 1549 \ldots \ldots \ldots \ldots \ldots$ & $8.642 \mathrm{E}+00$ & $4.496 \mathrm{E}+00$ & $1.130 \mathrm{E}-02$ & $4.145 \mathrm{E}-06$ & $0.000 \mathrm{E}+00$ \\
\hline Не II $\lambda 1640 \ldots \ldots \ldots \ldots . . . . . .$. & $5.563 \mathrm{E}+00$ & $4.024 \mathrm{E}+00$ & $1.730 \mathrm{E}+00$ & $8.131 \mathrm{E}-01$ & $2.239 \mathrm{E}-01$ \\
\hline $\mathrm{O}$ III] $\lambda 1664 \ldots \ldots \ldots \ldots \ldots . . .$. & $3.499 \mathrm{E}-01$ & $1.589 \mathrm{E}-01$ & $4.331 \mathrm{E}-03$ & $9.045 \mathrm{E}-05$ & $0.000 \mathrm{E}+00$ \\
\hline $\mathrm{N}$ III] $\lambda 1750 \ldots \ldots \ldots \ldots \ldots . . .$. & $2.271 \mathrm{E}-01$ & $1.167 \mathrm{E}-01$ & $3.857 \mathrm{E}-03$ & $7.386 \mathrm{E}-05$ & $0.000 \mathrm{E}+00$ \\
\hline $\mathrm{C}$ III] $\lambda 1909 \ldots \ldots \ldots \ldots . . . .$. & $2.624 \mathrm{E}+00$ & $1.485 \mathrm{E}+00$ & $6.592 \mathrm{E}-02$ & $4.280 \mathrm{E}-03$ & $4.267 \mathrm{E}-04$ \\
\hline $\left.\mathrm{C}_{\text {II }}\right] \lambda 2326 \ldots \ldots \ldots \ldots \ldots$ & $5.616 \mathrm{E}-01$ & $2.629 \mathrm{E}-01$ & $5.507 \mathrm{E}-02$ & $4.353 \mathrm{E}-02$ & $2.805 \mathrm{E}-02$ \\
\hline $\mathrm{Mg}$ II $\lambda 2800 \ldots \ldots \ldots \ldots . . . . .$. & $9.194 \mathrm{E}-01$ & $5.435 \mathrm{E}-01$ & $1.798 \mathrm{E}-01$ & $1.688 \mathrm{E}-01$ & $1.535 \mathrm{E}-01$ \\
\hline$[\mathrm{Ne} \mathrm{v}] \lambda 3426 \ldots \ldots \ldots \ldots$ & $4.972 \mathrm{E}+00$ & $2.468 \mathrm{E}+00$ & $7.924 \mathrm{E}-03$ & $1.022 \mathrm{E}-06$ & $0.000 \mathrm{E}+00$ \\
\hline$[\mathrm{Ne}$ III] $\lambda 3869 \ldots \ldots \ldots . .$. & $4.152 \mathrm{E}+00$ & $2.590 \mathrm{E}+00$ & $6.791 \mathrm{E}-01$ & $3.131 \mathrm{E}-01$ & $9.578 \mathrm{E}-02$ \\
\hline \multicolumn{6}{|c|}{$\alpha=-2.0$} \\
\hline 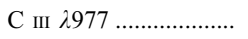 & $7.950 \mathrm{E}-02$ & $1.538 \mathrm{E}-02$ & $1.400 \mathrm{E}-06$ & $0.000 \mathrm{E}+00$ & $0.000 \mathrm{E}+00$ \\
\hline 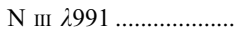 & $5.174 \mathrm{E}-02$ & $1.031 \mathrm{E}-02$ & $1.236 \mathrm{E}-06$ & $0.000 \mathrm{E}+00$ & $0.000 \mathrm{E}+00$ \\
\hline $\mathrm{O}$ vi $\lambda \lambda 1032,1037 \ldots$. & $5.212 \mathrm{E}+00$ & $1.569 \mathrm{E}-01$ & $0.000 \mathrm{E}+00$ & $0.000 \mathrm{E}+00$ & $0.000 \mathrm{E}+00$ \\
\hline 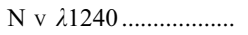 & $3.837 \mathrm{E}+00$ & $4.594 \mathrm{E}-01$ & $1.042 \mathrm{E}-06$ & $0.000 \mathrm{E}+00$ & $0.000 \mathrm{E}+00$ \\
\hline $\mathrm{Si} \mathrm{IV+O} \mathrm{Iv]} \lambda 1400 \ldots \ldots$ & $3.454 \mathrm{E}-01$ & $8.415 \mathrm{E}-02$ & $5.817 \mathrm{E}-05$ & $0.000 \mathrm{E}+00$ & $0.000 \mathrm{E}+00$ \\
\hline $\mathrm{N}$ Iv] $\lambda 1486 \ldots \ldots \ldots \ldots \ldots . . . .$. & $9.392 \mathrm{E}-01$ & $2.430 \mathrm{E}-01$ & $5.911 \mathrm{E}-05$ & $0.000 \mathrm{E}+00$ & $0.000 \mathrm{E}+00$ \\
\hline $\mathrm{C}$ IV $\lambda 1549 \ldots \ldots \ldots \ldots \ldots \ldots$ & $6.110 \mathrm{E}+00$ & $1.571 \mathrm{E}+00$ & $3.427 \mathrm{E}-04$ & $0.000 \mathrm{E}+00$ & $0.000 \mathrm{E}+00$ \\
\hline 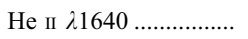 & $4.803 \mathrm{E}+00$ & $2.618 \mathrm{E}+00$ & $1.126 \mathrm{E}+00$ & $5.320 \mathrm{E}-01$ & $1.362 \mathrm{E}-01$ \\
\hline 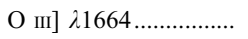 & $1.804 \mathrm{E}-01$ & $4.242 \mathrm{E}-02$ & $3.737 \mathrm{E}-04$ & $2.771 \mathrm{E}-05$ & $0.000 \mathrm{E}+00$ \\
\hline $\mathrm{N}$ III] $\lambda 1750 \ldots \ldots \ldots \ldots \ldots . .$. & $1.227 \mathrm{E}-01$ & $3.392 \mathrm{E}-02$ & $3.490 \mathrm{E}-04$ & $2.360 \mathrm{E}-05$ & $0.000 \mathrm{E}+00$ \\
\hline $\mathrm{C}$ III] $\lambda 1909 \ldots \ldots \ldots \ldots . . . .$. & $1.588 \mathrm{E}+00$ & $4.990 \mathrm{E}-01$ & $7.094 \mathrm{E}-03$ & $1.572 \mathrm{E}-03$ & $2.058 \mathrm{E}-04$ \\
\hline $\mathrm{C}_{\text {II] }} \lambda 2326 \ldots \ldots \ldots \ldots \ldots$ & $2.347 \mathrm{E}-01$ & $4.552 \mathrm{E}-02$ & $7.132 \mathrm{E}-03$ & $1.867 \mathrm{E}-02$ & $1.718 \mathrm{E}-02$ \\
\hline $\mathrm{Mg}$ II $\lambda 2800 \ldots \ldots \ldots \ldots \ldots$ & $5.351 \mathrm{E}-01$ & $1.617 \mathrm{E}-01$ & $4.406 \mathrm{E}-02$ & $9.959 \mathrm{E}-02$ & $1.080 \mathrm{E}-01$ \\
\hline$[\mathrm{Ne} v] \lambda 3426 \ldots \ldots \ldots \ldots$ & $3.805 \mathrm{E}+00$ & $9.069 \mathrm{E}-01$ & $4.404 \mathrm{E}-04$ & $0.000 \mathrm{E}+00$ & $0.000 \mathrm{E}+00$ \\
\hline$[\mathrm{Ne}$ III] $\lambda 3869$............ & $2.657 \mathrm{E}+00$ & $1.059 \mathrm{E}+00$ & $2.201 \mathrm{E}-01$ & $1.544 \mathrm{E}-01$ & $4.865 \mathrm{E}-02$ \\
\hline
\end{tabular}


TABLE 8

Dusty Visible Line Ratios with Respect to $\mathrm{H} \beta, 0.25 Z_{\odot}$

\begin{tabular}{|c|c|c|c|c|c|}
\hline \multirow[b]{2}{*}{ LiNE } & \multicolumn{5}{|c|}{$\log U_{0}$} \\
\hline & 0.0 & -1.0 & -2.0 & -3.0 & -4.0 \\
\hline \multicolumn{6}{|c|}{$\alpha=-1.2$} \\
\hline$[\mathrm{O}$ пा] $\lambda \lambda 3727,3729 \ldots \ldots$. & $3.694 \mathrm{E}-1$ & $3.645 \mathrm{E}-1$ & $5.551 \mathrm{E}-1$ & $1.873 \mathrm{E}+0$ & $1.359 \mathrm{E}+0$ \\
\hline$[\mathrm{S}$ II] $\lambda \lambda 4067,4076 \ldots \ldots$. & $3.056 \mathrm{E}-2$ & $2.853 \mathrm{E}-2$ & $2.840 \mathrm{E}-2$ & $7.306 \mathrm{E}-2$ & $1.538 \mathrm{E}-1$ \\
\hline 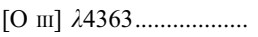 & $6.074 \mathrm{E}-2$ & $7.862 \mathrm{E}-2$ & $1.035 \mathrm{E}-1$ & $1.847 \mathrm{E}-2$ & $1.116 \mathrm{E}-4$ \\
\hline 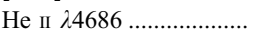 & $4.464 \mathrm{E}-1$ & $4.258 \mathrm{E}-1$ & $3.104 \mathrm{E}-1$ & $2.060 \mathrm{E}-1$ & $7.211 \mathrm{E}-2$ \\
\hline 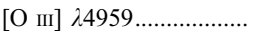 & $6.474 \mathrm{E}-1$ & $8.425 \mathrm{E}-1$ & $1.382 \mathrm{E}+0$ & $4.610 \mathrm{E}-1$ & $5.690 \mathrm{E}-3$ \\
\hline$[\mathrm{O}$ III] $\lambda 5007 \ldots \ldots \ldots \ldots \ldots \ldots$ & $1.865 \mathrm{E}+0$ & $2.427 \mathrm{E}+0$ & $3.981 \mathrm{E}+0$ & $1.328 \mathrm{E}+0$ & $1.639 \mathrm{E}-2$ \\
\hline 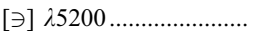 & $2.099 \mathrm{E}-2$ & $1.955 \mathrm{E}-2$ & $1.874 \mathrm{E}-2$ & $3.848 \mathrm{E}-2$ & $6.172 \mathrm{E}-2$ \\
\hline$[\mathrm{Fe}$ VII $] \lambda 6085 \ldots \ldots \ldots \ldots \ldots$ & $2.924 \mathrm{E}-4$ & $3.864 \mathrm{E}-4$ & $2.162 \mathrm{E}-4$ & $0.000 \mathrm{E}+0$ & $0.000 \mathrm{E}+0$ \\
\hline [O I] $\lambda 6300 \ldots \ldots \ldots \ldots \ldots \ldots . .$. & $2.567 \mathrm{E}-1$ & $2.294 \mathrm{E}-1$ & $1.909 \mathrm{E}-1$ & $3.748 \mathrm{E}-1$ & $6.137 \mathrm{E}-1$ \\
\hline $\mathrm{H} \alpha \quad \lambda 6563 \ldots \ldots \ldots \ldots \ldots \ldots \ldots$ & $3.435 \mathrm{E}+0$ & $3.346 \mathrm{E}+0$ & $3.097 \mathrm{E}+0$ & $3.053 \mathrm{E}+0$ & $3.043 \mathrm{E}+0$ \\
\hline$[\mathrm{N}$ II] $\lambda 6583 \ldots \ldots \ldots \ldots \ldots . .$. & $6.589 \mathrm{E}-2$ & $5.821 \mathrm{E}-2$ & $5.773 \mathrm{E}-2$ & $1.856 \mathrm{E}-1$ & $1.820 \mathrm{E}-1$ \\
\hline$[\mathrm{S}$ II] $\lambda \lambda 6717,6730 \ldots \ldots$. & $6.454 \mathrm{E}-1$ & $6.028 \mathrm{E}-1$ & $6.069 \mathrm{E}-1$ & $1.475 \mathrm{E}+0$ & $3.172 \mathrm{E}+0$ \\
\hline [O II] $\lambda \lambda 7318,7324 \ldots \ldots$. & $6.532 \mathrm{E}-3$ & $6.837 \mathrm{E}-3$ & $1.228 \mathrm{E}-2$ & $4.059 \mathrm{E}-2$ & $2.063 \mathrm{E}-2$ \\
\hline [S III] $\lambda \lambda 9069,9532 \ldots \ldots$. & $6.968 \mathrm{E}-1$ & $6.741 \mathrm{E}-1$ & $8.738 \mathrm{E}-1$ & $1.736 \mathrm{E}+0$ & $1.195 \mathrm{E}+0$ \\
\hline \multicolumn{6}{|c|}{$\alpha=-1.4$} \\
\hline [O пा] $\lambda \lambda 3727,3729 \ldots \ldots$. & $3.100 \mathrm{E}-1$ & $3.130 \mathrm{E}-1$ & $5.405 \mathrm{E}-1$ & $1.918 \mathrm{E}+0$ & $1.390 \mathrm{E}+0$ \\
\hline$[\mathrm{S}$ II] $\lambda \lambda 4067,4076 \ldots \ldots$. & $2.552 \mathrm{E}-2$ & $2.269 \mathrm{E}-2$ & $2.272 \mathrm{E}-2$ & $6.259 \mathrm{E}-2$ & $1.390 \mathrm{E}-1$ \\
\hline$[\mathrm{O}$ III] $\lambda 4363 \ldots \ldots \ldots \ldots \ldots . .$. & $8.713 \mathrm{E}-2$ & $1.148 \mathrm{E}-1$ & $1.100 \mathrm{E}-1$ & $1.830 \mathrm{E}-2$ & $1.124 \mathrm{E}-4$ \\
\hline 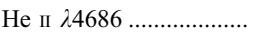 & $4.314 \mathrm{E}-1$ & $3.860 \mathrm{E}-1$ & $2.599 \mathrm{E}-1$ & $1.755 \mathrm{E}-1$ & $6.008 \mathrm{E}-2$ \\
\hline [O & $9.104 \mathrm{E}-1$ & $1.238 \mathrm{E}+0$ & $1.556 \mathrm{E}+0$ & $4.701 \mathrm{E}-1$ & $5.790 \mathrm{E}-3$ \\
\hline$[\mathrm{O}$ III] $\lambda 5007 \ldots \ldots \ldots \ldots \ldots . . .$. & $2.622 \mathrm{E}+0$ & $3.564 \mathrm{E}+0$ & $4.481 \mathrm{E}+0$ & $1.354 \mathrm{E}+0$ & $1.667 \mathrm{E}-2$ \\
\hline$[\ni] \lambda 5200 \ldots \ldots \ldots \ldots \ldots \ldots$ & $1.712 \mathrm{E}-2$ & $1.475 \mathrm{E}-2$ & $1.320 \mathrm{E}-2$ & $2.664 \mathrm{E}-2$ & $4.525 \mathrm{E}-2$ \\
\hline$[\mathrm{Fe}$ VII $] \lambda 6085 \ldots \ldots \ldots \ldots . .$. & $3.840 \mathrm{E}-4$ & $5.238 \mathrm{E}-4$ & $1.013 \mathrm{E}-4$ & $0.000 \mathrm{E}+0$ & $0.000 \mathrm{E}+0$ \\
\hline$[\mathrm{O}$ I] $\lambda 6300 \ldots \ldots \ldots \ldots \ldots \ldots . .$. & $1.928 \mathrm{E}-1$ & $1.567 \mathrm{E}-1$ & $1.288 \mathrm{E}-1$ & $2.630 \mathrm{E}-1$ & $4.650 \mathrm{E}-1$ \\
\hline $\mathrm{H} \alpha \quad \lambda 6563 \ldots \ldots \ldots \ldots \ldots \ldots$ & $3.280 \mathrm{E}+0$ & $3.157 \mathrm{E}+0$ & $2.995 \mathrm{E}+0$ & $2.999 \mathrm{E}+0$ & $3.024 \mathrm{E}+0$ \\
\hline$[\mathrm{N}$ II] $\lambda 6583 \ldots \ldots \ldots \ldots \ldots . .$. & $4.593 \mathrm{E}-2$ & $3.822 \mathrm{E}-2$ & $4.841 \mathrm{E}-2$ & $1.832 \mathrm{E}-1$ & $1.842 \mathrm{E}-1$ \\
\hline$[\mathrm{S}$ II] $\lambda \lambda 6717,6730 \ldots \ldots$. & $5.447 \mathrm{E}-1$ & $4.842 \mathrm{E}-1$ & $4.711 \mathrm{E}-1$ & $1.162 \mathrm{E}+0$ & $2.647 \mathrm{E}+0$ \\
\hline [O II] $\lambda \lambda 7318,7324 \ldots \ldots$. & $5.794 \mathrm{E}-3$ & $6.335 \mathrm{E}-3$ & $1.239 \mathrm{E}-2$ & $4.187 \mathrm{E}-2$ & $2.147 \mathrm{E}-2$ \\
\hline$[\mathrm{S}$ шII] $\lambda \lambda 9069,9532 \ldots \ldots$. & $5.854 \mathrm{E}-1$ & $5.762 \mathrm{E}-1$ & $8.514 \mathrm{E}-1$ & $1.573 \mathrm{E}+0$ & $9.359 \mathrm{E}-1$ \\
\hline \multicolumn{6}{|c|}{$\alpha=-1.7$} \\
\hline [O пा] $\lambda \lambda 3727,3729 \ldots \ldots$. & $2.511 \mathrm{E}-1$ & $2.710 \mathrm{E}-1$ & $5.436 \mathrm{E}-1$ & $1.972 \mathrm{E}+0$ & $1.410 \mathrm{E}+0$ \\
\hline$[\mathrm{S}$ II] $\lambda \lambda 4067,4076 \ldots \ldots$. & $1.847 \mathrm{E}-2$ & $1.508 \mathrm{E}-2$ & $1.652 \mathrm{E}-2$ & $5.296 \mathrm{E}-2$ & $1.253 \mathrm{E}-1$ \\
\hline$[\mathrm{O}$ III] $\lambda 4363 \ldots \ldots \ldots \ldots \ldots . .$. & $1.266 \mathrm{E}-1$ & $1.451 \mathrm{E}-1$ & $1.036 \mathrm{E}-1$ & $1.643 \mathrm{E}-2$ & $1.029 \mathrm{E}-4$ \\
\hline 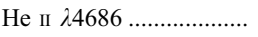 & $3.901 \mathrm{E}-1$ & $3.048 \mathrm{E}-1$ & $1.895 \mathrm{E}-1$ & $1.307 \mathrm{E}-1$ & $4.320 \mathrm{E}-2$ \\
\hline [O III] $\lambda 4959 \ldots \ldots \ldots \ldots \ldots . .$. & $1.348 \mathrm{E}+0$ & $1.704 \mathrm{E}+0$ & $1.639 \mathrm{E}+0$ & $4.454 \mathrm{E}-1$ & $5.416 \mathrm{E}-3$ \\
\hline [O III] $\lambda 5007 \ldots \ldots \ldots \ldots \ldots . .$. & $3.883 \mathrm{E}+0$ & $4.909 \mathrm{E}+0$ & $4.719 \mathrm{E}+0$ & $1.283 \mathrm{E}+0$ & $1.560 \mathrm{E}-2$ \\
\hline$[\ni] \lambda 5200 \ldots \ldots \ldots \ldots \ldots$ & $1.129 \mathrm{E}-2$ & $8.185 \mathrm{E}-3$ & $7.075 \mathrm{E}-3$ & $1.510 \mathrm{E}-2$ & $3.051 \mathrm{E}-2$ \\
\hline$[\mathrm{Fe}$ VII $] \lambda 6085 \ldots \ldots \ldots \ldots . . . .$. & $5.454 \mathrm{E}-4$ & $5.828 \mathrm{E}-4$ & $2.572 \mathrm{E}-5$ & $0.000 \mathrm{E}+0$ & $0.000 \mathrm{E}+0$ \\
\hline$\left[\mathrm{O}_{\mathrm{I}}\right] \lambda 6300 \ldots \ldots \ldots \ldots \ldots . .$. & $1.149 \mathrm{E}-1$ & $7.964 \mathrm{E}-2$ & $6.815 \mathrm{E}-2$ & $1.558 \mathrm{E}-1$ & $3.273 \mathrm{E}-1$ \\
\hline 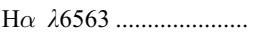 & $3.075 \mathrm{E}+0$ & $2.959 \mathrm{E}+0$ & $2.906 \mathrm{E}+0$ & $2.946 \mathrm{E}+0$ & $2.985 \mathrm{E}+0$ \\
\hline$[\mathrm{N}$ II] $\lambda 6583 \ldots \ldots \ldots \ldots \ldots . . .$. & $2.699 \mathrm{E}-2$ & $2.390 \mathrm{E}-2$ & $4.395 \mathrm{E}-2$ & $1.838 \mathrm{E}-1$ & $1.843 \mathrm{E}-1$ \\
\hline$[\mathrm{S}$ II] $\lambda \lambda 6717,6730 \ldots \ldots$. & $3.962 \mathrm{E}-1$ & $3.168 \mathrm{E}-1$ & $3.131 \mathrm{E}-1$ & $8.574 \mathrm{E}-1$ & $2.175 \mathrm{E}+0$ \\
\hline [O II] $\lambda \lambda 7318,7324 \ldots \ldots$. & $5.107 \mathrm{E}-3$ & $5.941 \mathrm{E}-3$ & $1.271 \mathrm{E}-2$ & $4.292 \mathrm{E}-2$ & $2.202 \mathrm{E}-2$ \\
\hline [S III] $\lambda \lambda 9069,9532 \ldots \ldots$. & $4.766 \mathrm{E}-1$ & $5.068 \mathrm{E}-1$ & $8.767 \mathrm{E}-1$ & $1.395 \mathrm{E}+0$ & $6.932 \mathrm{E}-1$ \\
\hline \multicolumn{6}{|c|}{$\alpha=-2.0$} \\
\hline [O пा] $\lambda \lambda 3727,3729 \ldots \ldots$. & $2.188 \mathrm{E}-1$ & $2.528 \mathrm{E}-1$ & $5.662 \mathrm{E}-1$ & $2.006 \mathrm{E}+0$ & $1.407 \mathrm{E}+0$ \\
\hline [S II] $\lambda \lambda 4067,4076 \ldots \ldots .$. & $1.252 \mathrm{E}-2$ & $9.708 \mathrm{E}-3$ & $1.302 \mathrm{E}-2$ & $4.800 \mathrm{E}-2$ & $1.161 \mathrm{E}-1$ \\
\hline$[\mathrm{O}$ III] $\lambda 4363 \ldots \ldots \ldots \ldots \ldots . .$. & $1.485 \mathrm{E}-1$ & $1.390 \mathrm{E}-1$ & $8.804 \mathrm{E}-2$ & $1.360 \mathrm{E}-2$ & $8.687 \mathrm{E}-5$ \\
\hline 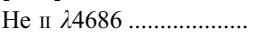 & $3.278 \mathrm{E}-1$ & $2.207 \mathrm{E}-1$ & $1.327 \mathrm{E}-1$ & $9.296 \mathrm{E}-2$ & $2.961 \mathrm{E}-2$ \\
\hline [O III] $\lambda 4959 \ldots \ldots \ldots \ldots \ldots . .$. & $1.710 \mathrm{E}+0$ & $1.885 \mathrm{E}+0$ & $1.575 \mathrm{E}+0$ & $3.920 \mathrm{E}-1$ & $4.688 \mathrm{E}-3$ \\
\hline 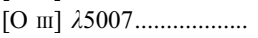 & $4.925 \mathrm{E}+0$ & $5.430 \mathrm{E}+0$ & $4.537 \mathrm{E}+0$ & $1.129 \mathrm{E}+0$ & $1.350 \mathrm{E}-2$ \\
\hline 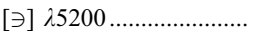 & $6.323 \mathrm{E}-3$ & $3.802 \mathrm{E}-3$ & $3.600 \mathrm{E}-3$ & $8.908 \mathrm{E}-3$ & $2.208 \mathrm{E}-2$ \\
\hline$[\mathrm{Fe}$ VII $] \lambda 6085 \ldots \ldots \ldots \ldots \ldots$ & $6.288 \mathrm{E}-4$ & $4.146 \mathrm{E}-4$ & $5.437 \mathrm{E}-6$ & $0.000 \mathrm{E}+0$ & $0.000 \mathrm{E}+0$ \\
\hline [O I] $\lambda 6300 \ldots \ldots \ldots \ldots \ldots . .$. & $6.097 \mathrm{E}-2$ & $3.655 \mathrm{E}-2$ & $3.607 \mathrm{E}-2$ & $9.779 \mathrm{E}-2$ & $2.448 \mathrm{E}-1$ \\
\hline $\mathrm{H} \alpha \quad \lambda 6563 \ldots \ldots \ldots \ldots \ldots \ldots \ldots$ & $2.931 \mathrm{E}+0$ & $2.862 \mathrm{E}+0$ & $2.863 \mathrm{E}+0$ & $2.917 \mathrm{E}+0$ & $2.961 \mathrm{E}+0$ \\
\hline$[\mathrm{N}$ п] $\lambda 26583 \ldots \ldots \ldots \ldots \ldots . . . . . . .$. & $1.849 \mathrm{E}-2$ & $1.952 \mathrm{E}-2$ & $4.472 \mathrm{E}-2$ & $1.858 \mathrm{E}-1$ & $1.817 \mathrm{E}-1$ \\
\hline$[\mathrm{S}$ II] $\lambda \lambda 6717,6730 \ldots \ldots$. & $2.633 \mathrm{E}-1$ & $1.910 \mathrm{E}-1$ & $2.173 \mathrm{E}-1$ & $6.975 \mathrm{E}-1$ & $1.889 \mathrm{E}+0$ \\
\hline [O II] $\lambda \lambda 7318,7324 \ldots \ldots$. & $4.718 \mathrm{E}-3$ & $5.701 \mathrm{E}-3$ & $1.318 \mathrm{E}-2$ & $4.307 \mathrm{E}-2$ & $2.196 \mathrm{E}-2$ \\
\hline [S III] $\lambda \lambda 9069,9532 \ldots \ldots$. & $4.181 \mathrm{E}-1$ & $4.869 \mathrm{E}-1$ & $9.317 \mathrm{E}-1$ & $1.273 \mathrm{E}+0$ & $5.553 \mathrm{E}-1$ \\
\hline
\end{tabular}


TABLE 9

Dusty Visible Line Ratios with Respect to $\mathrm{H} \beta, 0.5 Z_{\odot}$

\begin{tabular}{|c|c|c|c|c|c|}
\hline \multirow[b]{2}{*}{ LiNE } & \multicolumn{5}{|c|}{$\log U_{0}$} \\
\hline & 0.0 & -1.0 & -2.0 & -3.0 & -4.0 \\
\hline \multicolumn{6}{|c|}{$\alpha=-1.2$} \\
\hline [O п $] \lambda \lambda 3727,3729 \ldots \ldots$. & $9.318 \mathrm{E}-1$ & $9.107 \mathrm{E}-1$ & $1.166 \mathrm{E}+0$ & $3.477 \mathrm{E}+0$ & $2.529 \mathrm{E}+0$ \\
\hline [S II] $\lambda \lambda 4067,4076 \ldots \ldots .$. & $6.896 \mathrm{E}-2$ & $6.323 \mathrm{E}-2$ & $5.391 \mathrm{E}-2$ & $1.220 \mathrm{E}-1$ & $2.567 \mathrm{E}-1$ \\
\hline 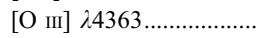 & $9.127 \mathrm{E}-2$ & $1.127 \mathrm{E}-1$ & $1.536 \mathrm{E}-1$ & $2.766 \mathrm{E}-2$ & $1.852 \mathrm{E}-4$ \\
\hline 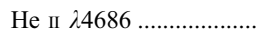 & $4.965 \mathrm{E}-1$ & $4.816 \mathrm{E}-1$ & $3.383 \mathrm{E}-1$ & $2.135 \mathrm{E}-1$ & $7.625 \mathrm{E}-2$ \\
\hline 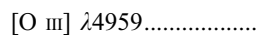 & $1.097 \mathrm{E}+0$ & $1.371 \mathrm{E}+0$ & $2.358 \mathrm{E}+0$ & $7.849 \mathrm{E}-1$ & $1.028 \mathrm{E}-2$ \\
\hline 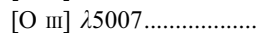 & $3.159 \mathrm{E}+0$ & $3.949 \mathrm{E}+0$ & $6.793 \mathrm{E}+0$ & $2.261 \mathrm{E}+0$ & $2.960 \mathrm{E}-2$ \\
\hline$[\ni] \lambda 5200 \ldots \ldots \ldots \ldots \ldots \ldots$ & $7.072 \mathrm{E}-2$ & $6.539 \mathrm{E}-2$ & $5.324 \mathrm{E}-2$ & $9.087 \mathrm{E}-2$ & $1.346 \mathrm{E}-1$ \\
\hline$[\mathrm{Fe}$ VII $] \lambda 6085 \ldots \ldots \ldots \ldots . . . . .$. & $6.681 \mathrm{E}-4$ & $8.217 \mathrm{E}-4$ & $3.178 \mathrm{E}-4$ & $0.000 \mathrm{E}+0$ & $0.000 \mathrm{E}+0$ \\
\hline 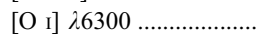 & $5.952 \mathrm{E}-1$ & $5.326 \mathrm{E}-1$ & $3.675 \mathrm{E}-1$ & $6.050 \mathrm{E}-1$ & $9.328 \mathrm{E}-1$ \\
\hline $\mathrm{H} \alpha \lambda 6563 \ldots \ldots \ldots \ldots \ldots \ldots$ & $3.428 \mathrm{E}+0$ & $3.340 \mathrm{E}+0$ & $3.032 \mathrm{E}+0$ & $3.006 \mathrm{E}+0$ & $3.015 \mathrm{E}+0$ \\
\hline$[\mathrm{N}$ II] $\lambda 6583 \ldots \ldots \ldots \ldots \ldots . . . . . . . .$. & $2.479 \mathrm{E}-1$ & $2.237 \mathrm{E}-1$ & $1.892 \mathrm{E}-1$ & $5.461 \mathrm{E}-1$ & $5.346 \mathrm{E}-1$ \\
\hline [S II] $\lambda \lambda 6717,6730 \ldots \ldots$. & $1.409 \mathrm{E}+0$ & $1.296 \mathrm{E}+0$ & $1.113 \mathrm{E}+0$ & $2.291 \mathrm{E}+0$ & $4.891 \mathrm{E}+0$ \\
\hline$[\mathrm{O}$ II] $\lambda \lambda 7318,7324 \ldots \ldots$. & $1.651 \mathrm{E}-2$ & $1.690 \mathrm{E}-2$ & $2.501 \mathrm{E}-2$ & $7.257 \mathrm{E}-2$ & $3.729 \mathrm{E}-2$ \\
\hline$[\mathrm{S}$ III] $\lambda \lambda 9069,9532 \ldots \ldots$. & $1.639 \mathrm{E}+0$ & $1.571 \mathrm{E}+0$ & $1.802 \mathrm{E}+0$ & $3.235 \mathrm{E}+0$ & $2.064 \mathrm{E}+0$ \\
\hline \multicolumn{6}{|c|}{$\alpha=-1.4$} \\
\hline [O пा] $\lambda \lambda 3727,3729 \ldots \ldots$. & $7.757 \mathrm{E}-1$ & $7.618 \mathrm{E}-1$ & $1.098 \mathrm{E}+0$ & $3.473 \mathrm{E}+0$ & $2.549 \mathrm{E}+0$ \\
\hline [S II] $\lambda \lambda 4067,4076 \ldots \ldots .$. & $5.682 \mathrm{E}-2$ & $4.932 \mathrm{E}-2$ & $4.194 \mathrm{E}-2$ & $1.058 \mathrm{E}-1$ & $2.386 \mathrm{E}-1$ \\
\hline 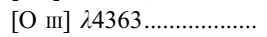 & $1.260 \mathrm{E}-1$ & $1.571 \mathrm{E}-1$ & $1.487 \mathrm{E}-1$ & $2.553 \mathrm{E}-2$ & $1.802 \mathrm{E}-4$ \\
\hline 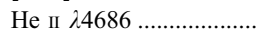 & $4.895 \mathrm{E}-1$ & $4.480 \mathrm{E}-1$ & $2.829 \mathrm{E}-1$ & $1.807 \mathrm{E}-1$ & $6.293 \mathrm{E}-2$ \\
\hline [O & $1.513 \mathrm{E}+0$ & $1.966 \mathrm{E}+0$ & $2.519 \mathrm{E}+0$ & $7.653 \mathrm{E}-1$ & $1.021 \mathrm{E}-2$ \\
\hline 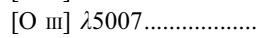 & $4.357 \mathrm{E}+0$ & $5.663 \mathrm{E}+0$ & $7.255 \mathrm{E}+0$ & $2.204 \mathrm{E}+0$ & $2.940 \mathrm{E}-2$ \\
\hline$[\ni] \lambda 5200 \ldots \ldots \ldots \ldots \ldots \ldots$ & $5.780 \mathrm{E}-2$ & $4.943 \mathrm{E}-2$ & $3.622 \mathrm{E}-2$ & $6.134 \mathrm{E}-2$ & $1.006 \mathrm{E}-1$ \\
\hline$[\mathrm{Fe}$ VII $] \lambda 6085 \ldots \ldots \ldots \ldots . . . . .$. & $8.724 \mathrm{E}-4$ & $1.045 \mathrm{E}-3$ & $1.341 \mathrm{E}-4$ & $0.000 \mathrm{E}+0$ & $0.000 \mathrm{E}+0$ \\
\hline 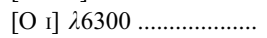 & $4.476 \mathrm{E}-1$ & $3.632 \mathrm{E}-1$ & $2.396 \mathrm{E}-1$ & $4.167 \mathrm{E}-1$ & $7.221 \mathrm{E}-1$ \\
\hline $\mathrm{H} \alpha \lambda 6563 \ldots \ldots \ldots \ldots \ldots \ldots$ & $3.249 \mathrm{E}+0$ & $3.127 \mathrm{E}+0$ & $2.937 \mathrm{E}+0$ & $2.958 \mathrm{E}+0$ & $2.994 \mathrm{E}+0$ \\
\hline 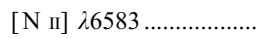 & $1.758 \mathrm{E}-1$ & $1.489 \mathrm{E}-1$ & $1.581 \mathrm{E}-1$ & $5.344 \mathrm{E}-1$ & $5.349 \mathrm{E}-1$ \\
\hline$\left[\mathrm{S}_{\text {II }}\right] \lambda \lambda 6717,6730 \ldots \ldots$. & $1.179 \mathrm{E}+0$ & $1.025 \mathrm{E}+0$ & $8.342 \mathrm{E}-1$ & $1.811 \mathrm{E}+0$ & $4.269 \mathrm{E}+0$ \\
\hline [O II] $\lambda \lambda 7318,7324 \ldots \ldots$. & $1.432 \mathrm{E}-2$ & $1.496 \mathrm{E}-2$ & $2.396 \mathrm{E}-2$ & $7.225 \mathrm{E}-2$ & $3.795 \mathrm{E}-2$ \\
\hline$\left[\mathrm{S}_{\mathrm{III}}\right] \lambda \lambda 9069,9532 \ldots \ldots$ & $1.371 \mathrm{E}+0$ & $1.318 \mathrm{E}+0$ & $1.725 \mathrm{E}+0$ & $2.897 \mathrm{E}+0$ & $1.628 \mathrm{E}+0$ \\
\hline \multicolumn{6}{|c|}{$\alpha=-1.7$} \\
\hline [O пा] $\lambda \lambda 3727,3729 \ldots \ldots$. & $6.150 \mathrm{E}-1$ & $6.269 \mathrm{E}-1$ & $1.038 \mathrm{E}+0$ & $3.414 \mathrm{E}+0$ & $2.494 \mathrm{E}+0$ \\
\hline$[\mathrm{S}$ II] $\lambda \lambda 4067,4076 \ldots \ldots$. & $3.992 \mathrm{E}-2$ & $3.158 \mathrm{E}-2$ & $2.979 \mathrm{E}-2$ & $9.148 \mathrm{E}-2$ & $2.163 \mathrm{E}-1$ \\
\hline 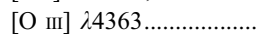 & $1.692 \mathrm{E}-1$ & $1.811 \mathrm{E}-1$ & $1.197 \mathrm{E}-1$ & $2.057 \mathrm{E}-2$ & $1.550 \mathrm{E}-4$ \\
\hline 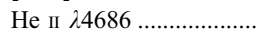 & $4.515 \mathrm{E}-1$ & $3.589 \mathrm{E}-1$ & $2.041 \mathrm{E}-1$ & $1.335 \mathrm{E}-1$ & $4.412 \mathrm{E}-2$ \\
\hline 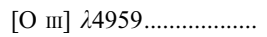 & $2.141 \mathrm{E}+0$ & $2.591 \mathrm{E}+0$ & $2.425 \mathrm{E}+0$ & $6.776 \mathrm{E}-1$ & $9.110 \mathrm{E}-3$ \\
\hline 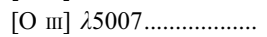 & $6.167 \mathrm{E}+0$ & $7.462 \mathrm{E}+0$ & $6.984 \mathrm{E}+0$ & $1.952 \mathrm{E}+0$ & $2.624 \mathrm{E}-2$ \\
\hline 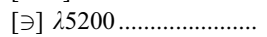 & $3.761 \mathrm{E}-2$ & $2.664 \mathrm{E}-2$ & $1.824 \mathrm{E}-2$ & $3.391 \mathrm{E}-2$ & $6.873 \mathrm{E}-2$ \\
\hline$[\mathrm{Fe}$ VII $] \lambda 6085 \ldots \ldots \ldots \ldots . . . . .$. & $1.155 \mathrm{E}-3$ & $1.026 \mathrm{E}-3$ & $2.907 \mathrm{E}-5$ & $0.000 \mathrm{E}+0$ & $0.000 \mathrm{E}+0$ \\
\hline 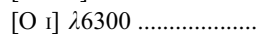 & $2.628 \mathrm{E}-1$ & $1.775 \mathrm{E}-1$ & $1.194 \mathrm{E}-1$ & $2.435 \mathrm{E}-1$ & $5.150 \mathrm{E}-1$ \\
\hline 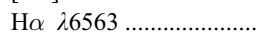 & $3.030 \mathrm{E}+0$ & $2.922 \mathrm{E}+0$ & $2.869 \mathrm{E}+0$ & $2.914 \mathrm{E}+0$ & $2.975 \mathrm{E}+0$ \\
\hline$\left[\mathrm{N}_{\mathrm{II}}\right] \lambda 6583 \ldots \ldots \ldots \ldots \ldots . . . . . .$. & $1.055 \mathrm{E}-1$ & $9.150 \mathrm{E}-2$ & $1.404 \mathrm{E}-1$ & $5.247 \mathrm{E}-1$ & $5.190 \mathrm{E}-1$ \\
\hline$[\mathrm{S}$ II] $\lambda \lambda 6717,6730 \ldots \ldots$. & $8.328 \mathrm{E}-1$ & $6.421 \mathrm{E}-1$ & $5.334 \mathrm{E}-1$ & $1.384 \mathrm{E}+0$ & $3.624 \mathrm{E}+0$ \\
\hline [O II] $\lambda \lambda 7318,7324 \ldots \ldots$. & $1.208 \mathrm{E}-2$ & $1.305 \mathrm{E}-2$ & $2.248 \mathrm{E}-2$ & $6.963 \mathrm{E}-2$ & $3.715 \mathrm{E}-2$ \\
\hline$[\mathrm{S}$ III] $\lambda \lambda 9069,9532 \ldots \ldots$ & $1.094 \mathrm{E}+0$ & $1.117 \mathrm{E}+0$ & $1.701 \mathrm{E}+0$ & $2.512 \mathrm{E}+0$ & $1.210 \mathrm{E}+0$ \\
\hline \multicolumn{6}{|c|}{$\alpha=-2.0$} \\
\hline [O пा] $\lambda \lambda 3727,3729 \ldots \ldots$. & $5.293 \mathrm{E}-1$ & $5.598 \mathrm{E}-1$ & $1.015 \mathrm{E}+0$ & $3.307 \mathrm{E}+0$ & $2.424 \mathrm{E}+0$ \\
\hline$[\mathrm{S}$ II] $\lambda \lambda 4067,4076 \ldots \ldots$. & $2.787 \mathrm{E}-2$ & $2.070 \mathrm{E}-2$ & $2.389 \mathrm{E}-2$ & $8.367 \mathrm{E}-2$ & $2.007 \mathrm{E}-1$ \\
\hline 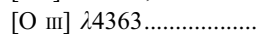 & $1.796 \mathrm{E}-1$ & $1.509 \mathrm{E}-1$ & $8.582 \mathrm{E}-2$ & $1.524 \mathrm{E}-2$ & $1.244 \mathrm{E}-4$ \\
\hline 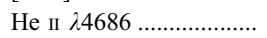 & $3.946 \mathrm{E}-1$ & $2.562 \mathrm{E}-1$ & $1.417 \mathrm{E}-1$ & $9.434 \mathrm{E}-2$ & $2.983 \mathrm{E}-2$ \\
\hline [O & $2.520 \mathrm{E}+0$ & $2.661 \mathrm{E}+0$ & $2.114 \mathrm{E}+0$ & $5.562 \mathrm{E}-1$ & $7.622 \mathrm{E}-3$ \\
\hline 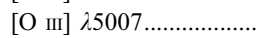 & $7.258 \mathrm{E}+0$ & $7.664 \mathrm{E}+0$ & $6.090 \mathrm{E}+0$ & $1.602 \mathrm{E}+0$ & $2.195 \mathrm{E}-2$ \\
\hline$[\ni] \lambda 5200 \ldots \ldots \ldots \ldots \ldots \ldots$ & $2.199 \mathrm{E}-2$ & $1.182 \mathrm{E}-2$ & $8.898 \mathrm{E}-3$ & $1.980 \mathrm{E}-2$ & $5.067 \mathrm{E}-2$ \\
\hline$[\mathrm{Fe}$ VII $] \lambda 6085 \ldots \ldots \ldots \ldots . . . . .$. & $1.193 \mathrm{E}-3$ & $6.191 \mathrm{E}-4$ & $5.209 \mathrm{E}-6$ & $0.000 \mathrm{E}+0$ & $0.000 \mathrm{E}+0$ \\
\hline$[\mathrm{O}$ I] $\lambda 6300 \ldots \ldots \ldots \ldots \ldots . . . . . . . . .$. & $1.456 \mathrm{E}-1$ & $7.682 \mathrm{E}-2$ & $6.073 \mathrm{E}-2$ & $1.526 \mathrm{E}-1$ & $3.906 \mathrm{E}-1$ \\
\hline 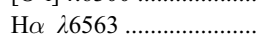 & $2.906 \mathrm{E}+0$ & $2.843 \mathrm{E}+0$ & $2.849 \mathrm{E}+0$ & $2.894 \mathrm{E}+0$ & $2.955 \mathrm{E}+0$ \\
\hline$[\mathrm{N}$ II] $\lambda 6583 \ldots \ldots \ldots \ldots \ldots . . . . . . . .$. & $7.516 \mathrm{E}-2$ & $7.333 \mathrm{E}-2$ & $1.391 \mathrm{E}-1$ & $5.169 \mathrm{E}-1$ & $5.019 \mathrm{E}-1$ \\
\hline$[\mathrm{S}$ II] $\lambda \lambda 6717,6730 \ldots \ldots$. & $5.645 \mathrm{E}-1$ & $3.864 \mathrm{E}-1$ & $3.796 \mathrm{E}-1$ & $1.168 \mathrm{E}+0$ & $3.209 \mathrm{E}+0$ \\
\hline [O II] $\lambda \lambda 7318,7324 \ldots \ldots$. & $1.078 \mathrm{E}-2$ & $1.158 \mathrm{E}-2$ & $2.122 \mathrm{E}-2$ & $6.547 \mathrm{E}-2$ & $3.577 \mathrm{E}-2$ \\
\hline [S III] $\lambda \lambda 9069,9532 \ldots \ldots$. & $9.509 \mathrm{E}-1$ & $1.037 \mathrm{E}+0$ & $1.715 \mathrm{E}+0$ & $2.234 \mathrm{E}+0$ & $9.747 \mathrm{E}-1$ \\
\hline
\end{tabular}


TABLE 10

Dusty Visible Line Ratios with Respect to $\mathrm{H} \beta, 1 Z_{\odot}$

\begin{tabular}{|c|c|c|c|c|c|}
\hline \multirow[b]{2}{*}{ LiNE } & \multicolumn{5}{|c|}{$\log U_{0}$} \\
\hline & 0.0 & -1.0 & -2.0 & -3.0 & -4.0 \\
\hline \multicolumn{6}{|c|}{$\alpha=-1.2$} \\
\hline [O II] $\lambda \lambda 3727,3729 \ldots \ldots$. & $2.301 \mathrm{E}+0$ & $2.221 \mathrm{E}+0$ & $2.296 \mathrm{E}+0$ & $5.651 \mathrm{E}+0$ & $4.086 \mathrm{E}+0$ \\
\hline$[\mathrm{S}$ II] $\lambda \lambda 4067,4076 \ldots \ldots .$. & $1.492 \mathrm{E}-1$ & $1.348 \mathrm{E}-1$ & $9.742 \mathrm{E}-2$ & $1.917 \mathrm{E}-1$ & $3.997 \mathrm{E}-1$ \\
\hline 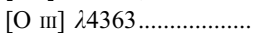 & $1.434 \mathrm{E}-1$ & $1.697 \mathrm{E}-1$ & $1.868 \mathrm{E}-1$ & $2.799 \mathrm{E}-2$ & $2.247 \mathrm{E}-4$ \\
\hline 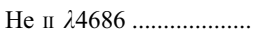 & $5.649 \mathrm{E}-1$ & $5.543 \mathrm{E}-1$ & $3.746 \mathrm{E}-1$ & $2.237 \mathrm{E}-1$ & $8.064 \mathrm{E}-2$ \\
\hline 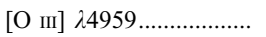 & $1.931 \mathrm{E}+0$ & $2.351 \mathrm{E}+0$ & $3.629 \mathrm{E}+0$ & $1.063 \mathrm{E}+0$ & $1.532 \mathrm{E}-2$ \\
\hline 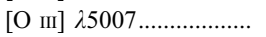 & $5.561 \mathrm{E}+0$ & $6.772 \mathrm{E}+0$ & $1.045 \mathrm{E}+1$ & $3.062 \mathrm{E}+0$ & $4.412 \mathrm{E}-2$ \\
\hline$[\ni] ~ \lambda 5200 \ldots \ldots \ldots \ldots \ldots \ldots$ & $2.421 \mathrm{E}-1$ & $2.224 \mathrm{E}-1$ & $1.542 \mathrm{E}-1$ & $2.123 \mathrm{E}-1$ & $2.866 \mathrm{E}-1$ \\
\hline$[\mathrm{Fe}$ VII $] \lambda 6085 \ldots \ldots \ldots \ldots \ldots$ & $1.394 \mathrm{E}-3$ & $1.624 \mathrm{E}-3$ & $3.738 \mathrm{E}-4$ & $0.000 \mathrm{E}+0$ & $0.000 \mathrm{E}+0$ \\
\hline 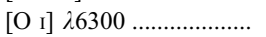 & $1.282 \mathrm{E}+0$ & $1.141 \mathrm{E}+0$ & $6.601 \mathrm{E}-1$ & $8.896 \mathrm{E}-1$ & $1.285 \mathrm{E}+0$ \\
\hline $\mathrm{H} \alpha \quad \lambda 6563 \ldots \ldots \ldots \ldots \ldots \ldots$ & $3.412 \mathrm{E}+0$ & $3.311 \mathrm{E}+0$ & $2.959 \mathrm{E}+0$ & $2.954 \mathrm{E}+0$ & $2.987 \mathrm{E}+0$ \\
\hline 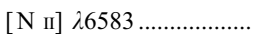 & $9.776 \mathrm{E}-1$ & $8.884 \mathrm{E}-1$ & $6.641 \mathrm{E}-1$ & $1.659 \mathrm{E}+0$ & $1.585 \mathrm{E}+0$ \\
\hline$[\mathrm{S}$ II] $\lambda \lambda 6717,6730 \ldots \ldots .$. & $2.913 \mathrm{E}+0$ & $2.650 \mathrm{E}+0$ & $1.933 \mathrm{E}+0$ & $3.352 \mathrm{E}+0$ & $7.294 \mathrm{E}+0$ \\
\hline$[\mathrm{O}$ II $] \lambda \lambda 7318,7324 \ldots \ldots$. & $4.108 \mathrm{E}-2$ & $4.104 \mathrm{E}-2$ & $4.589 \mathrm{E}-2$ & $1.074 \mathrm{E}-1$ & $5.535 \mathrm{E}-2$ \\
\hline [S III] $\lambda \lambda 9069,9532 \ldots \ldots$. & $3.673 \mathrm{E}+0$ & $3.505 \mathrm{E}+0$ & $3.588 \mathrm{E}+0$ & $5.529 \mathrm{E}+0$ & $3.195 \mathrm{E}+0$ \\
\hline \multicolumn{6}{|c|}{$\alpha=-1.4$} \\
\hline$[\mathrm{O}$ II $] \lambda \lambda 3727,3729 \ldots \ldots$. & $1.897 \mathrm{E}+0$ & $1.792 \mathrm{E}+0$ & $1.998 \mathrm{E}+0$ & $5.296 \mathrm{E}+0$ & $3.938 \mathrm{E}+0$ \\
\hline$[\mathrm{S}$ II] $\lambda \lambda 4067,4076 \ldots \ldots .$. & $1.214 \mathrm{E}-1$ & $1.021 \mathrm{E}-1$ & $7.229 \mathrm{E}-2$ & $1.669 \mathrm{E}-1$ & $3.701 \mathrm{E}-1$ \\
\hline 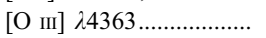 & $1.820 \mathrm{E}-1$ & $2.094 \mathrm{E}-1$ & $1.505 \mathrm{E}-1$ & $2.241 \mathrm{E}-2$ & $2.005 \mathrm{E}-4$ \\
\hline He II $\lambda 4686 \ldots \ldots \ldots \ldots \ldots . .$. & $5.694 \mathrm{E}-1$ & $5.259 \mathrm{E}-1$ & $3.112 \mathrm{E}-1$ & $1.880 \mathrm{E}-1$ & $6.505 \mathrm{E}-2$ \\
\hline 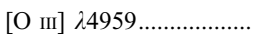 & $2.541 \mathrm{E}+0$ & $3.146 \mathrm{E}+0$ & $3.473 \mathrm{E}+0$ & $9.510 \mathrm{E}-1$ & $1.434 \mathrm{E}-2$ \\
\hline$[\mathrm{O}$ III] $\lambda 5007 \ldots \ldots \ldots \ldots \ldots \ldots$ & $7.320 \mathrm{E}+0$ & $9.060 \mathrm{E}+0$ & $1.000 \mathrm{E}+1$ & $2.739 \mathrm{E}+0$ & $4.131 \mathrm{E}-2$ \\
\hline 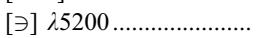 & $1.980 \mathrm{E}-1$ & $1.657 \mathrm{E}-1$ & $9.982 \mathrm{E}-2$ & $1.388 \mathrm{E}-1$ & $2.151 \mathrm{E}-1$ \\
\hline$[\mathrm{Fe}$ VII $] \lambda 6085 \ldots \ldots \ldots \ldots . .$. & $1.759 \mathrm{E}-3$ & $1.885 \mathrm{E}-3$ & $1.344 \mathrm{E}-4$ & $0.000 \mathrm{E}+0$ & $0.000 \mathrm{E}+0$ \\
\hline 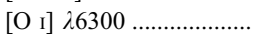 & $9.641 \mathrm{E}-1$ & $7.662 \mathrm{E}-1$ & $4.103 \mathrm{E}-1$ & $5.985 \mathrm{E}-1$ & $9.996 \mathrm{E}-1$ \\
\hline $\mathrm{H} \alpha \quad \lambda 6563 \ldots \ldots \ldots \ldots \ldots \ldots$ & $3.203 \mathrm{E}+0$ & $3.073 \mathrm{E}+0$ & $2.891 \mathrm{E}+0$ & $2.919 \mathrm{E}+0$ & $2.980 \mathrm{E}+0$ \\
\hline$\left[\mathrm{N}_{\mathrm{II}}\right] \lambda 6583 \ldots \ldots \ldots \ldots \ldots . . . . . . . .$. & $7.091 \mathrm{E}-1$ & $6.005 \mathrm{E}-1$ & $5.434 \mathrm{E}-1$ & $1.577 \mathrm{E}+0$ & $1.534 \mathrm{E}+0$ \\
\hline$[\mathrm{S}$ II] $\lambda \lambda 6717,6730 \ldots \ldots$. & $2.418 \mathrm{E}+0$ & $2.041 \mathrm{E}+0$ & $1.381 \mathrm{E}+0$ & $2.690 \mathrm{E}+0$ & $6.503 \mathrm{E}+0$ \\
\hline [O II $] \lambda \lambda 7318,7324 \ldots \ldots$. & $3.459 \mathrm{E}-2$ & $3.407 \mathrm{E}-2$ & $3.925 \mathrm{E}-2$ & $9.793 \mathrm{E}-2$ & $5.301 \mathrm{E}-2$ \\
\hline [S III] $\lambda \lambda 9069,9532 \ldots . .$. & $3.089 \mathrm{E}+0$ & $2.914 \mathrm{E}+0$ & $3.299 \mathrm{E}+0$ & $4.805 \mathrm{E}+0$ & $2.519 \mathrm{E}+0$ \\
\hline \multicolumn{6}{|c|}{$\alpha=-1.7$} \\
\hline$[\mathrm{O}$ II $] \lambda \lambda 3727,3729 \ldots \ldots$. & $1.427 \mathrm{E}+0$ & $1.311 \mathrm{E}+0$ & $1.667 \mathrm{E}+0$ & $4.708 \mathrm{E}+0$ & $3.678 \mathrm{E}+0$ \\
\hline$[\mathrm{S}$ II] $\lambda \lambda 4067,4076 \ldots \ldots .$. & $8.123 \mathrm{E}-2$ & $6.044 \mathrm{E}-2$ & $4.888 \mathrm{E}-2$ & $1.435 \mathrm{E}-1$ & $3.341 \mathrm{E}-1$ \\
\hline [O III] $\lambda 4363 \ldots \ldots \ldots \ldots \ldots . .$. & $2.095 \mathrm{E}-1$ & $1.925 \mathrm{E}-1$ & $9.179 \mathrm{E}-2$ & $1.451 \mathrm{E}-2$ & $1.554 \mathrm{E}-4$ \\
\hline 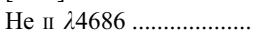 & $5.301 \mathrm{E}-1$ & $4.209 \mathrm{E}-1$ & $2.233 \mathrm{E}-1$ & $1.371 \mathrm{E}-1$ & $4.509 \mathrm{E}-2$ \\
\hline [O III] $\lambda 4959 \ldots \ldots \ldots \ldots \ldots . .$. & $3.303 \mathrm{E}+0$ & $3.643 \mathrm{E}+0$ & $2.838 \mathrm{E}+0$ & $7.362 \mathrm{E}-1$ & $1.201 \mathrm{E}-2$ \\
\hline 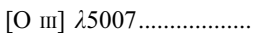 & $9.513 \mathrm{E}+0$ & $1.049 \mathrm{E}+1$ & $8.173 \mathrm{E}+0$ & $2.120 \mathrm{E}+0$ & $3.460 \mathrm{E}-2$ \\
\hline$[\ni] ~ \lambda 5200 \ldots \ldots \ldots \ldots \ldots \ldots$ & $1.242 \mathrm{E}-1$ & $8.392 \mathrm{E}-2$ & $4.658 \mathrm{E}-2$ & $7.438 \mathrm{E}-2$ & $1.503 \mathrm{E}-1$ \\
\hline$[\mathrm{Fe}$ VII $] \lambda 6085 \ldots \ldots \ldots \ldots \ldots$ & $2.119 \mathrm{E}-3$ & $1.566 \mathrm{E}-3$ & $2.267 \mathrm{E}-5$ & $0.000 \mathrm{E}+0$ & $0.000 \mathrm{E}+0$ \\
\hline 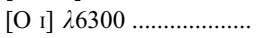 & $5.450 \mathrm{E}-1$ & $3.497 \mathrm{E}-1$ & $1.897 \mathrm{E}-1$ & $3.430 \mathrm{E}-1$ & $7.256 \mathrm{E}-1$ \\
\hline $\mathrm{H} \alpha \quad \lambda 6563 \ldots \ldots \ldots \ldots \ldots \ldots$ & $2.972 \mathrm{E}+0$ & $2.883 \mathrm{E}+0$ & $2.861 \mathrm{E}+0$ & $2.895 \mathrm{E}+0$ & $2.960 \mathrm{E}+0$ \\
\hline 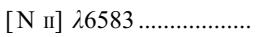 & $4.301 \mathrm{E}-1$ & $3.580 \mathrm{E}-1$ & $4.579 \mathrm{E}-1$ & $1.469 \mathrm{E}+0$ & $1.447 \mathrm{E}+0$ \\
\hline [S II] $\lambda \lambda 6717,6730 \ldots \ldots .$. & $1.633 \mathrm{E}+0$ & $1.188 \mathrm{E}+0$ & $8.433 \mathrm{E}-1$ & $2.132 \mathrm{E}+0$ & $5.667 \mathrm{E}+0$ \\
\hline$[\mathrm{O}$ II $] \lambda \lambda 7318,7324 \ldots \ldots$. & $2.665 \mathrm{E}-2$ & $2.492 \mathrm{E}-2$ & $3.091 \mathrm{E}-2$ & $8.242 \mathrm{E}-2$ & $4.839 \mathrm{E}-2$ \\
\hline$[\mathrm{S}$ III] $\lambda \lambda 9069,9532 \ldots \ldots$. & $2.428 \mathrm{E}+0$ & $2.347 \mathrm{E}+0$ & $3.004 \mathrm{E}+0$ & $3.959 \mathrm{E}+0$ & $1.897 \mathrm{E}+0$ \\
\hline \multicolumn{6}{|c|}{$\alpha=-2.0$} \\
\hline$[\mathrm{O}$ II $] \lambda \lambda 3727,3729 \ldots \ldots$. & $1.158 \mathrm{E}+0$ & $1.031 \mathrm{E}+0$ & $1.446 \mathrm{E}+0$ & $4.138 \mathrm{E}+0$ & $3.413 \mathrm{E}+0$ \\
\hline$[\mathrm{S}$ II] $\lambda \lambda 4067,4076 \ldots \ldots$. & $5.571 \mathrm{E}-2$ & $3.741 \mathrm{E}-2$ & $3.892 \mathrm{E}-2$ & $1.280 \mathrm{E}-1$ & $3.055 \mathrm{E}-1$ \\
\hline [O III] $\lambda 4363 \ldots \ldots \ldots \ldots \ldots . .$. & $1.910 \mathrm{E}-1$ & $1.246 \mathrm{E}-1$ & $5.042 \mathrm{E}-2$ & $8.831 \mathrm{E}-3$ & $1.130 \mathrm{E}-4$ \\
\hline 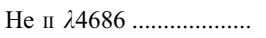 & $4.705 \mathrm{E}-1$ & $2.964 \mathrm{E}-1$ & $1.542 \mathrm{E}-1$ & $9.590 \mathrm{E}-2$ & $3.018 \mathrm{E}-2$ \\
\hline 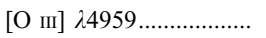 & $3.516 \mathrm{E}+0$ & $3.218 \mathrm{E}+0$ & $2.124 \mathrm{E}+0$ & $5.349 \mathrm{E}-1$ & $9.483 \mathrm{E}-3$ \\
\hline 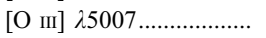 & $1.013 \mathrm{E}+1$ & $9.269 \mathrm{E}+0$ & $6.116 \mathrm{E}+0$ & $1.541 \mathrm{E}+0$ & $2.731 \mathrm{E}-2$ \\
\hline$[\ni] ~ \lambda 5200 \ldots \ldots \ldots \ldots \ldots \ldots$ & $7.255 \mathrm{E}-2$ & $3.413 \mathrm{E}-2$ & $2.118 \mathrm{E}-2$ & $4.253 \mathrm{E}-2$ & $1.133 \mathrm{E}-1$ \\
\hline$[\mathrm{Fe}$ VII $] \lambda 6085 \ldots \ldots \ldots \ldots \ldots$ & $1.985 \mathrm{E}-3$ & $7.811 \mathrm{E}-4$ & $3.049 \mathrm{E}-6$ & $0.000 \mathrm{E}+0$ & $0.000 \mathrm{E}+0$ \\
\hline 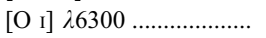 & $3.010 \mathrm{E}-1$ & $1.375 \mathrm{E}-1$ & $8.987 \mathrm{E}-2$ & $2.120 \mathrm{E}-1$ & $5.576 \mathrm{E}-1$ \\
\hline $\mathrm{H} \alpha \quad \lambda 6563 \ldots \ldots \ldots \ldots \ldots \ldots \ldots$ & $2.873 \mathrm{E}+0$ & $2.840 \mathrm{E}+0$ & $2.865 \mathrm{E}+0$ & $2.891 \mathrm{E}+0$ & $2.935 \mathrm{E}+0$ \\
\hline$[\mathrm{N}$ II] $\lambda 6583 \ldots \ldots \ldots \ldots \ldots . .$. & $3.090 \mathrm{E}-1$ & $2.690 \mathrm{E}-1$ & $4.257 \mathrm{E}-1$ & $1.368 \mathrm{E}+0$ & $1.362 \mathrm{E}+0$ \\
\hline [S II] $\lambda \lambda 6717,6730 \ldots \ldots .$. & $1.092 \mathrm{E}+0$ & $6.791 \mathrm{E}-1$ & $6.232 \mathrm{E}-1$ & $1.833 \mathrm{E}+0$ & $5.096 \mathrm{E}+0$ \\
\hline$[\mathrm{O}$ II $] \lambda \lambda 7318,7324 \ldots \ldots$. & $2.143 \mathrm{E}-2$ & $1.835 \mathrm{E}-2$ & $2.447 \mathrm{E}-2$ & $6.800 \mathrm{E}-2$ & $4.347 \mathrm{E}-2$ \\
\hline [S III] $\lambda \lambda 9069,9532 \ldots . .$. & $2.061 \mathrm{E}+0$ & $2.020 \mathrm{E}+0$ & $2.763 \mathrm{E}+0$ & $3.350 \mathrm{E}+0$ & $1.536 \mathrm{E}+0$ \\
\hline
\end{tabular}


TABLE 11

Dusty Visible Line Ratios with Respect to $\mathrm{H} \beta, 2 Z_{\odot}$

\begin{tabular}{|c|c|c|c|c|c|}
\hline \multirow[b]{2}{*}{ LiNe } & \multicolumn{5}{|c|}{$\log U_{0}$} \\
\hline & 0.0 & -1.0 & -2.0 & -3.0 & -4.0 \\
\hline \multicolumn{6}{|c|}{$\alpha=-1.2$} \\
\hline [O II] $\lambda \lambda 3727,3729 \ldots \ldots \ldots$ & $5.449 \mathrm{E}+0$ & $5.101 \mathrm{E}+0$ & $3.623 \mathrm{E}+0$ & $6.444 \mathrm{E}+0$ & $4.366 \mathrm{E}+0$ \\
\hline$[\mathrm{S}$ II] $\lambda \lambda 4067,4076 \ldots \ldots \ldots . .$. & $3.061 \mathrm{E}-1$ & $2.703 \mathrm{E}-1$ & $1.582 \mathrm{E}-1$ & $2.617 \mathrm{E}-1$ & $4.832 \mathrm{E}-1$ \\
\hline 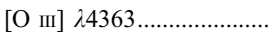 & $2.301 \mathrm{E}-1$ & $2.532 \mathrm{E}-1$ & $1.541 \mathrm{E}-1$ & $1.231 \mathrm{E}-2$ & $1.151 \mathrm{E}-4$ \\
\hline 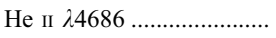 & $6.703 \mathrm{E}-1$ & $6.598 \mathrm{E}-1$ & $4.210 \mathrm{E}-1$ & $2.362 \mathrm{E}-1$ & $8.308 \mathrm{E}-2$ \\
\hline 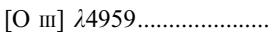 & $3.499 \mathrm{E}+0$ & $4.106 \mathrm{E}+0$ & $4.419 \mathrm{E}+0$ & $8.860 \mathrm{E}-1$ & $1.375 \mathrm{E}-2$ \\
\hline 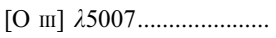 & $1.008 \mathrm{E}+1$ & $1.183 \mathrm{E}+1$ & $1.273 \mathrm{E}+1$ & $2.552 \mathrm{E}+0$ & $3.960 \mathrm{E}-2$ \\
\hline$[\ni] \lambda 5200 \ldots \ldots \ldots \ldots \ldots \ldots \ldots$ & $8.114 \mathrm{E}-1$ & $7.330 \mathrm{E}-1$ & $4.290 \mathrm{E}-1$ & $4.631 \mathrm{E}-1$ & $5.167 \mathrm{E}-1$ \\
\hline$[\mathrm{Fe}$ VII $] \lambda 6085 \ldots \ldots \ldots \ldots \ldots . . . . .$. & $2.641 \mathrm{E}-3$ & $2.898 \mathrm{E}-3$ & $3.186 \mathrm{E}-4$ & $0.000 \mathrm{E}+0$ & $0.000 \mathrm{E}+0$ \\
\hline$[\mathrm{O}$ I] $\lambda 6300 \ldots \ldots \ldots \ldots \ldots \ldots$ & $2.494 \mathrm{E}+0$ & $2.176 \mathrm{E}+0$ & $1.049 \mathrm{E}+0$ & $1.127 \mathrm{E}+0$ & $1.400 \mathrm{E}+0$ \\
\hline $\mathrm{H} \alpha \quad \lambda 6563 \ldots \ldots \ldots \ldots \ldots \ldots \ldots \ldots$ & $3.363 \mathrm{E}+0$ & $3.240 \mathrm{E}+0$ & $2.917 \mathrm{E}+0$ & $2.926 \mathrm{E}+0$ & $2.978 \mathrm{E}+0$ \\
\hline 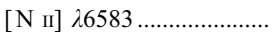 & $3.958 \mathrm{E}+0$ & $3.569 \mathrm{E}+0$ & $2.283 \mathrm{E}+0$ & $4.550 \mathrm{E}+0$ & $4.000 \mathrm{E}+0$ \\
\hline$[\mathrm{S}$ II] $\lambda \lambda 6717,6730 \ldots \ldots \ldots .$. & $5.649 \mathrm{E}+0$ & $5.036 \mathrm{E}+0$ & $3.067 \mathrm{E}+0$ & $4.554 \mathrm{E}+0$ & $9.607 \mathrm{E}+0$ \\
\hline$[\mathrm{O}$ II $] \lambda \lambda 7318,7324 \ldots \ldots \ldots$ & $9.821 \mathrm{E}-2$ & $9.329 \mathrm{E}-2$ & $6.122 \mathrm{E}-2$ & $9.646 \mathrm{E}-2$ & $4.534 \mathrm{E}-2$ \\
\hline$[\mathrm{S}$ III] $\lambda \lambda 29069,9532 \ldots \ldots \ldots$ & $7.679 \mathrm{E}+0$ & $7.277 \mathrm{E}+0$ & $6.407 \mathrm{E}+0$ & $7.885 \mathrm{E}+0$ & $3.985 \mathrm{E}+0$ \\
\hline \multicolumn{6}{|c|}{$\alpha=-1.4$} \\
\hline$[\mathrm{O}$ II $] \lambda \lambda 3727,3729 \ldots \ldots .$. & $4.295 \mathrm{E}+0$ & $3.699 \mathrm{E}+0$ & $2.678 \mathrm{E}+0$ & $5.345 \mathrm{E}+0$ & $3.902 \mathrm{E}+0$ \\
\hline$[\mathrm{S}$ II] $\lambda \lambda 4067,4076 \ldots \ldots \ldots . .$. & $2.424 \mathrm{E}-1$ & $1.944 \mathrm{E}-1$ & $1.076 \mathrm{E}-1$ & $2.190 \mathrm{E}-1$ & $4.297 \mathrm{E}-1$ \\
\hline 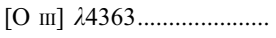 & $2.518 \mathrm{E}-1$ & $2.468 \mathrm{E}-1$ & $9.423 \mathrm{E}-2$ & $7.924 \mathrm{E}-3$ & $8.893 \mathrm{E}-5$ \\
\hline 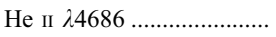 & $6.848 \mathrm{E}-1$ & $6.279 \mathrm{E}-1$ & $3.468 \mathrm{E}-1$ & $1.954 \mathrm{E}-1$ & $6.627 \mathrm{E}-2$ \\
\hline 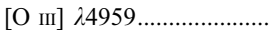 & $4.239 \mathrm{E}+0$ & $4.761 \mathrm{E}+0$ & $3.569 \mathrm{E}+0$ & $6.949 \mathrm{E}-1$ & $1.186 \mathrm{E}-2$ \\
\hline 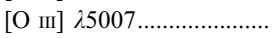 & $1.221 \mathrm{E}+1$ & $1.371 \mathrm{E}+1$ & $1.028 \mathrm{E}+1$ & $2.001 \mathrm{E}+0$ & $3.415 \mathrm{E}-2$ \\
\hline$[\ni] \lambda 5200 \ldots \ldots \ldots$. & $6.533 \mathrm{E}-1$ & $5.300 \mathrm{E}-1$ & $2.600 \mathrm{E}-1$ & $2.891 \mathrm{E}-1$ & $3.839 \mathrm{E}-1$ \\
\hline$[\mathrm{Fe}$ VII $] \lambda 6085 \ldots$ & $3.142 \mathrm{E}-3$ & $2.987 \mathrm{E}-3$ & $8.933 \mathrm{E}-5$ & $0.000 \mathrm{E}+0$ & $0.000 \mathrm{E}+0$ \\
\hline$[\mathrm{O}$ I] $\lambda 6300 \ldots \ldots \ldots \ldots \ldots \ldots . .$. & $1.845 \mathrm{E}+0$ & $1.413 \mathrm{E}+0$ & $6.091 \mathrm{E}-1$ & $7.276 \mathrm{E}-1$ & $1.075 \mathrm{E}+0$ \\
\hline $\mathrm{H} \alpha \quad \lambda 6563 \ldots \ldots \ldots \ldots \ldots \ldots \ldots \ldots$ & $3.128 \mathrm{E}+0$ & $3.002 \mathrm{E}+0$ & $2.888 \mathrm{E}+0$ & $2.916 \mathrm{E}+0$ & $2.959 \mathrm{E}+0$ \\
\hline$[\mathrm{N}$ II] $\lambda 6583 \ldots \ldots \ldots \ldots \ldots \ldots . .$. & $2.897 \mathrm{E}+0$ & $2.388 \mathrm{E}+0$ & $1.743 \mathrm{E}+0$ & $4.045 \mathrm{E}+0$ & $3.709 \mathrm{E}+0$ \\
\hline$[\mathrm{S}$ II] $\lambda \lambda 6717,6730 \ldots \ldots \ldots . .$. & $4.585 \mathrm{E}+0$ & $3.724 \mathrm{E}+0$ & $2.050 \mathrm{E}+0$ & $3.674 \mathrm{E}+0$ & $8.566 \mathrm{E}+0$ \\
\hline$[\mathrm{O}$ II $] \lambda \lambda 7318,7324 \ldots \ldots \ldots$ & $7.601 \mathrm{E}-2$ & $6.484 \mathrm{E}-2$ & $4.175 \mathrm{E}-2$ & $7.452 \mathrm{E}-2$ & $3.874 \mathrm{E}-2$ \\
\hline [S III] $\lambda \lambda 9069,9532 \ldots \ldots \ldots$. & $6.476 \mathrm{E}+0$ & $5.936 \mathrm{E}+0$ & $5.456 \mathrm{E}+0$ & $6.446 \mathrm{E}+0$ & $3.123 \mathrm{E}+0$ \\
\hline \multicolumn{6}{|c|}{$\alpha=-1.7$} \\
\hline [O II] $\lambda \lambda 3727,3729 \ldots \ldots \ldots$ & $2.772 \mathrm{E}+0$ & $2.116 \mathrm{E}+0$ & $1.767 \mathrm{E}+0$ & $4.024 \mathrm{E}+0$ & $3.173 \mathrm{E}+0$ \\
\hline [S II] $\lambda \lambda 4067,4076 \ldots \ldots \ldots . .$. & $1.505 \mathrm{E}-1$ & $1.008 \mathrm{E}-1$ & $6.436 \mathrm{E}-2$ & $1.732 \mathrm{E}-1$ & $3.497 \mathrm{E}-1$ \\
\hline 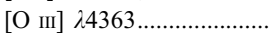 & $2.172 \mathrm{E}-1$ & $1.536 \mathrm{E}-1$ & $3.888 \mathrm{E}-2$ & $3.912 \mathrm{E}-3$ & $5.448 \mathrm{E}-5$ \\
\hline 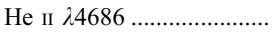 & $6.361 \mathrm{E}-1$ & $4.940 \mathrm{E}-1$ & $2.465 \mathrm{E}-1$ & $1.395 \mathrm{E}-1$ & $4.369 \mathrm{E}-2$ \\
\hline 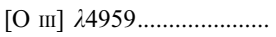 & $4.612 \mathrm{E}+0$ & $4.276 \mathrm{E}+0$ & $2.337 \mathrm{E}+0$ & $4.566 \mathrm{E}-1$ & $8.543 \mathrm{E}-3$ \\
\hline 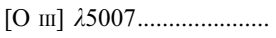 & $1.328 \mathrm{E}+1$ & $1.232 \mathrm{E}+1$ & $6.730 \mathrm{E}+0$ & $1.315 \mathrm{E}+0$ & $2.460 \mathrm{E}-2$ \\
\hline$[\ni] \quad \lambda 5200 \ldots \ldots \ldots \ldots \ldots \ldots \ldots$ & $3.932 \mathrm{E}-1$ & $2.465 \mathrm{E}-1$ & $1.087 \mathrm{E}-1$ & $1.431 \mathrm{E}-1$ & $2.545 \mathrm{E}-1$ \\
\hline$[\mathrm{Fe}$ VII $] \lambda 6085 \ldots \ldots \ldots \ldots \ldots . . . . .$. & $3.347 \mathrm{E}-3$ & $2.028 \mathrm{E}-3$ & $9.322 \mathrm{E}-6$ & $0.000 \mathrm{E}+0$ & $0.000 \mathrm{E}+0$ \\
\hline$[\mathrm{O}$ I] $\lambda 6300 \ldots \ldots \ldots \ldots \ldots \ldots . .$. & $9.945 \mathrm{E}-1$ & $5.886 \mathrm{E}-1$ & $2.492 \mathrm{E}-1$ & $3.877 \mathrm{E}-1$ & $7.360 \mathrm{E}-1$ \\
\hline $\mathrm{H} \alpha \quad \lambda 6563 \ldots \ldots \ldots \ldots \ldots \ldots \ldots \ldots$ & $2.919 \mathrm{E}+0$ & $2.870 \mathrm{E}+0$ & $2.893 \mathrm{E}+0$ & $2.918 \mathrm{E}+0$ & $2.975 \mathrm{E}+0$ \\
\hline$[\mathrm{N}$ II] $\lambda 6583 \ldots \ldots \ldots \ldots \ldots \ldots$ & $1.712 \mathrm{E}+0$ & $1.311 \mathrm{E}+0$ & $1.294 \mathrm{E}+0$ & $3.412 \mathrm{E}+0$ & $3.188 \mathrm{E}+0$ \\
\hline$[\mathrm{S}$ II] $\lambda \lambda 6717,6730 \ldots \ldots \ldots .$. & $2.930 \mathrm{E}+0$ & $1.962 \mathrm{E}+0$ & $1.176 \mathrm{E}+0$ & $2.890 \mathrm{E}+0$ & $7.125 \mathrm{E}+0$ \\
\hline$[\mathrm{O}$ II $] \lambda \lambda 7318,7324 \ldots \ldots \ldots$. & $4.588 \mathrm{E}-2$ & $3.313 \mathrm{E}-2$ & $2.363 \mathrm{E}-2$ & $4.993 \mathrm{E}-2$ & $2.915 \mathrm{E}-2$ \\
\hline [S III] $\lambda \lambda 9069,9532 \ldots \ldots \ldots$. & $4.906 \mathrm{E}+0$ & $4.349 \mathrm{E}+0$ & $4.370 \mathrm{E}+0$ & $4.873 \mathrm{E}+0$ & $2.243 \mathrm{E}+0$ \\
\hline \multicolumn{6}{|c|}{$\alpha=-2.0$} \\
\hline [O II] $\lambda \lambda 3727,3729 \ldots \ldots \ldots$ & $1.947 \mathrm{E}+0$ & $1.253 \mathrm{E}+0$ & $1.214 \mathrm{E}+0$ & $3.076 \mathrm{E}+0$ & $2.575 \mathrm{E}+0$ \\
\hline$[\mathrm{S}$ II] $\lambda \lambda 4067,4076 \ldots \ldots \ldots$. & $9.568 \mathrm{E}-2$ & $5.103 \mathrm{E}-2$ & $4.228 \mathrm{E}-2$ & $1.392 \mathrm{E}-1$ & $2.850 \mathrm{E}-1$ \\
\hline 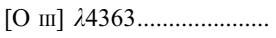 & $1.592 \mathrm{E}-1$ & $7.172 \mathrm{E}-2$ & $1.425 \mathrm{E}-2$ & $1.915 \mathrm{E}-3$ & $3.242 \mathrm{E}-5$ \\
\hline 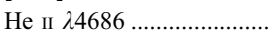 & $5.657 \mathrm{E}-1$ & $3.427 \mathrm{E}-1$ & $1.700 \mathrm{E}-1$ & $9.516 \mathrm{E}-2$ & $2.766 \mathrm{E}-2$ \\
\hline 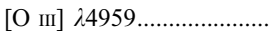 & $4.242 \mathrm{E}+0$ & $3.040 \mathrm{E}+0$ & $1.431 \mathrm{E}+0$ & $2.899 \mathrm{E}-1$ & $5.947 \mathrm{E}-3$ \\
\hline 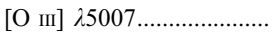 & $1.222 \mathrm{E}+1$ & $8.755 \mathrm{E}+0$ & $4.122 \mathrm{E}+0$ & $8.349 \mathrm{E}-1$ & $1.713 \mathrm{E}-2$ \\
\hline$[\ni] \quad \lambda 5200 \ldots \ldots \ldots \ldots \ldots \ldots$ & $2.224 \mathrm{E}-1$ & $8.611 \mathrm{E}-2$ & $3.829 \mathrm{E}-2$ & $7.329 \mathrm{E}-2$ & $1.783 \mathrm{E}-1$ \\
\hline$[\mathrm{Fe}$ VII $] \lambda 6085 \ldots \ldots \ldots \ldots \ldots$ & $2.870 \mathrm{E}-3$ & $8.065 \mathrm{E}-4$ & $0.000 \mathrm{E}+0$ & $0.000 \mathrm{E}+0$ & $0.000 \mathrm{E}+0$ \\
\hline$[\mathrm{O}$ I] $\lambda 6300 \ldots \ldots \ldots \ldots \ldots \ldots$ & $5.293 \mathrm{E}-1$ & $1.964 \mathrm{E}-1$ & $9.188 \mathrm{E}-2$ & $2.163 \mathrm{E}-1$ & $5.237 \mathrm{E}-1$ \\
\hline $\mathrm{H} \alpha \quad \lambda 6563 \ldots \ldots \ldots \ldots \ldots \ldots \ldots \ldots$ & $2.859 \mathrm{E}+0$ & $2.868 \mathrm{E}+0$ & $2.913 \mathrm{E}+0$ & $2.926 \mathrm{E}+0$ & $2.990 \mathrm{E}+0$ \\
\hline 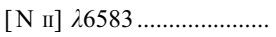 & $1.172 \mathrm{E}+0$ & $8.361 \mathrm{E}-1$ & $1.048 \mathrm{E}+0$ & $2.910 \mathrm{E}+0$ & $2.737 \mathrm{E}+0$ \\
\hline$[\mathrm{S}$ II] $\lambda \lambda 6717,6730 \ldots \ldots \ldots$. & $1.865 \mathrm{E}+0$ & $9.789 \mathrm{E}-1$ & $8.084 \mathrm{E}-1$ & $2.397 \mathrm{E}+0$ & $6.013 \mathrm{E}+0$ \\
\hline$[\mathrm{O}$ II $] \lambda \lambda 7318,7324 \ldots \ldots \ldots$ & $2.991 \mathrm{E}-2$ & $1.663 \mathrm{E}-2$ & $1.329 \mathrm{E}-2$ & $3.384 \mathrm{E}-2$ & $2.178 \mathrm{E}-2$ \\
\hline$[\mathrm{S}$ III $] \lambda \lambda 9069,9532 \ldots \ldots \ldots$ & $3.958 \mathrm{E}+0$ & $3.281 \mathrm{E}+0$ & $3.542 \mathrm{E}+0$ & $3.827 \mathrm{E}+0$ & $1.710 \mathrm{E}+0$ \\
\hline
\end{tabular}


TABLE 12

Dusty Visible Line Ratios with Respect to $\mathrm{H} \beta, 4 Z_{\odot}$

\begin{tabular}{|c|c|c|c|c|c|}
\hline \multirow[b]{2}{*}{ LiNE } & \multicolumn{5}{|c|}{$\log U_{0}$} \\
\hline & 0.0 & -1.0 & -2.0 & -3.0 & -4.0 \\
\hline \multicolumn{6}{|c|}{$\alpha=-1.2$} \\
\hline$[\mathrm{O}$ п] $] \lambda \lambda 3727,3729 \ldots \ldots \ldots$ & $1.141 \mathrm{E}+1$ & $9.677 \mathrm{E}+0$ & $3.704 \mathrm{E}+0$ & $3.946 \mathrm{E}+0$ & $1.941 \mathrm{E}+0$ \\
\hline$[\mathrm{S}$ II] $\lambda \lambda 4067,4076 \ldots \ldots \ldots$ & $5.893 \mathrm{E}-1$ & $4.950 \mathrm{E}-1$ & $2.081 \mathrm{E}-1$ & $2.481 \mathrm{E}-1$ & $2.867 \mathrm{E}-1$ \\
\hline 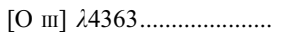 & $3.357 \mathrm{E}-1$ & $3.199 \mathrm{E}-1$ & $8.040 \mathrm{E}-2$ & $1.785 \mathrm{E}-3$ & $1.387 \mathrm{E}-5$ \\
\hline 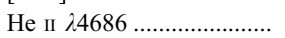 & $8.366 \mathrm{E}-1$ & $8.194 \mathrm{E}-1$ & $4.792 \mathrm{E}-1$ & $2.430 \mathrm{E}-1$ & $7.767 \mathrm{E}-2$ \\
\hline [O & $5.958 \mathrm{E}+0$ & $6.428 \mathrm{E}+0$ & $3.874 \mathrm{E}+0$ & $3.884 \mathrm{E}-1$ & $5.513 \mathrm{E}-3$ \\
\hline [O III] $\lambda 5007 \ldots \ldots$ & $1.716 \mathrm{E}+1$ & $1.851 \mathrm{E}+1$ & $1.116 \mathrm{E}+1$ & $1.119 \mathrm{E}+0$ & $1.588 \mathrm{E}-2$ \\
\hline 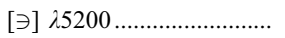 & $2.449 \mathrm{E}+0$ & $2.148 \mathrm{E}+0$ & $1.022 \mathrm{E}+0$ & $7.858 \mathrm{E}-1$ & $5.046 \mathrm{E}-1$ \\
\hline$[\mathrm{Fe}$ VII $] \lambda 6085 \ldots \ldots \ldots \ldots \ldots \ldots$ & $4.449 \mathrm{E}-3$ & $4.552 \mathrm{E}-3$ & $1.754 \mathrm{E}-4$ & $0.000 \mathrm{E}+0$ & $0.000 \mathrm{E}+0$ \\
\hline$\left[\mathrm{O}_{\mathrm{I}}\right] \mathrm{\lambda} 6300 \ldots \ldots \ldots \ldots \ldots \ldots \ldots$ & $4.244 \mathrm{E}+0$ & $3.566 \mathrm{E}+0$ & $1.365 \mathrm{E}+0$ & $1.049 \mathrm{E}+0$ & $8.316 \mathrm{E}-1$ \\
\hline 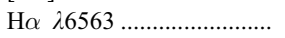 & $3.252 \mathrm{E}+0$ & $3.124 \mathrm{E}+0$ & $2.925 \mathrm{E}+0$ & $2.957 \mathrm{E}+0$ & $3.030 \mathrm{E}+0$ \\
\hline 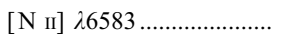 & $1.519 \mathrm{E}+1$ & $1.316 \mathrm{E}+1$ & $6.205 \mathrm{E}+0$ & $8.772 \mathrm{E}+0$ & $6.152 \mathrm{E}+0$ \\
\hline$[\mathrm{S}$ II] $\lambda \lambda 6717,6730 \ldots \ldots \ldots . .$. & $1.034 \mathrm{E}+1$ & $8.874 \mathrm{E}+0$ & $4.249 \mathrm{E}+0$ & $5.263 \mathrm{E}+0$ & $8.617 \mathrm{E}+0$ \\
\hline$[\mathrm{O}$ пI] $\lambda \lambda 7318,7324 \ldots \ldots \ldots$ & $2.019 \mathrm{E}-1$ & $1.648 \mathrm{E}-1$ & $4.559 \mathrm{E}-2$ & $3.547 \mathrm{E}-2$ & $1.036 \mathrm{E}-2$ \\
\hline$[\mathrm{S}$ III] $\lambda \lambda 9069,9532 \ldots \ldots \ldots$ & $1.461 \mathrm{E}+1$ & $1.350 \mathrm{E}+1$ & $9.262 \mathrm{E}+0$ & $8.327 \mathrm{E}+0$ & $3.209 \mathrm{E}+0$ \\
\hline \multicolumn{6}{|c|}{$\alpha=-1.4$} \\
\hline [O II] $\lambda \lambda 3727,3729 \ldots \ldots \ldots$. & $7.802 \mathrm{E}+0$ & $5.591 \mathrm{E}+0$ & $2.094 \mathrm{E}+0$ & $2.764 \mathrm{E}+0$ & $1.560 \mathrm{E}+0$ \\
\hline$[\mathrm{S}$ II] $\lambda \lambda 4067,4076 \ldots \ldots \ldots$. & $4.387 \mathrm{E}-1$ & $3.188 \mathrm{E}-1$ & $1.195 \mathrm{E}-1$ & $1.805 \mathrm{E}-1$ & $2.292 \mathrm{E}-1$ \\
\hline 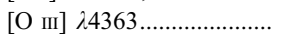 & $2.898 \mathrm{E}-1$ & $2.243 \mathrm{E}-1$ & $3.365 \mathrm{E}-2$ & $9.257 \mathrm{E}-4$ & $9.287 \mathrm{E}-6$ \\
\hline He II $\lambda 4686 \ldots \ldots \ldots \ldots \ldots \ldots \ldots$ & $8.552 \mathrm{E}-1$ & $7.668 \mathrm{E}-1$ & $3.908 \mathrm{E}-1$ & $1.963 \mathrm{E}-1$ & $5.895 \mathrm{E}-2$ \\
\hline 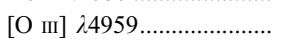 & $6.180 \mathrm{E}+0$ & $5.914 \mathrm{E}+0$ & $2.518 \mathrm{E}+0$ & $2.694 \mathrm{E}-1$ & $4.346 \mathrm{E}-3$ \\
\hline 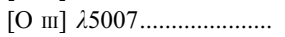 & $1.780 \mathrm{E}+1$ & $1.703 \mathrm{E}+1$ & $7.252 \mathrm{E}+0$ & $7.759 \mathrm{E}-1$ & $1.252 \mathrm{E}-2$ \\
\hline$[\ni] \quad \lambda 5200 \ldots \ldots \ldots \ldots \ldots \ldots \ldots \ldots$ & $1.915 \mathrm{E}+0$ & $1.464 \mathrm{E}+0$ & $5.653 \mathrm{E}-1$ & $4.250 \mathrm{E}-1$ & $3.475 \mathrm{E}-1$ \\
\hline$[\mathrm{Fe}$ VII $] \lambda 6085 \ldots \ldots \ldots \ldots \ldots . . . . . .$. & $4.938 \mathrm{E}-3$ & $4.109 \mathrm{E}-3$ & $3.061 \mathrm{E}-5$ & $0.000 \mathrm{E}+0$ & $0.000 \mathrm{E}+0$ \\
\hline 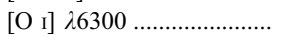 & $3.041 \mathrm{E}+0$ & $2.176 \mathrm{E}+0$ & $7.084 \mathrm{E}-1$ & $5.881 \mathrm{E}-1$ & $5.963 \mathrm{E}-1$ \\
\hline 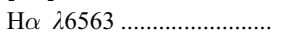 & $3.042 \mathrm{E}+0$ & $2.950 \mathrm{E}+0$ & $2.930 \mathrm{E}+0$ & $2.964 \mathrm{E}+0$ & $3.046 \mathrm{E}+0$ \\
\hline$[\mathrm{N}$ II] $\lambda 6583 \ldots \ldots \ldots \ldots \ldots \ldots \ldots$ & $1.076 \mathrm{E}+1$ & $8.152 \mathrm{E}+0$ & $4.060 \mathrm{E}+0$ & $7.084 \mathrm{E}+0$ & $5.316 \mathrm{E}+0$ \\
\hline$\left[\mathrm{S}_{\mathrm{II}}\right] \lambda \lambda 6717,6730 \ldots \ldots \ldots$. & $8.040 \mathrm{E}+0$ & $6.057 \mathrm{E}+0$ & $2.582 \mathrm{E}+0$ & $4.046 \mathrm{E}+0$ & $7.200 \mathrm{E}+0$ \\
\hline$[\mathrm{O}$ п] $] \lambda \lambda 7318,7324 \ldots \ldots \ldots$ & $1.245 \mathrm{E}-1$ & $8.171 \mathrm{E}-2$ & $2.124 \mathrm{E}-2$ & $2.133 \mathrm{E}-2$ & $7.648 \mathrm{E}-3$ \\
\hline$[\mathrm{S}$ III] $\lambda \lambda 29069,9532 \ldots \ldots \ldots$. & $1.202 \mathrm{E}+1$ & $1.032 \mathrm{E}+1$ & $7.011 \mathrm{E}+0$ & $6.222 \mathrm{E}+0$ & $2.413 \mathrm{E}+0$ \\
\hline \multicolumn{6}{|c|}{$\alpha=-1.7$} \\
\hline$[\mathrm{O}$ II $] \lambda \lambda 3727,3729 \ldots \ldots \ldots$ & $3.737 \mathrm{E}+0$ & $2.043 \mathrm{E}+0$ & $9.111 \mathrm{E}-1$ & $1.691 \mathrm{E}+0$ & $1.166 \mathrm{E}+0$ \\
\hline$\left[\mathrm{S}_{\mathrm{II}}\right] \lambda \lambda 4067,4076 \ldots \ldots \ldots$. & $2.352 \mathrm{E}-1$ & $1.282 \mathrm{E}-1$ & $5.208 \mathrm{E}-2$ & $1.138 \mathrm{E}-1$ & $1.708 \mathrm{E}-1$ \\
\hline$\left[\mathrm{O}\right.$ III] $\lambda_{14} 4363 \ldots \ldots \ldots \ldots \ldots \ldots \ldots$ & $1.748 \mathrm{E}-1$ & $9.158 \mathrm{E}-2$ & $6.706 \mathrm{E}-3$ & $3.469 \mathrm{E}-4$ & $5.083 \mathrm{E}-6$ \\
\hline He II $\lambda 4686 \ldots \ldots \ldots \ldots \ldots \ldots \ldots$ & $7.806 \mathrm{E}-1$ & $5.810 \mathrm{E}-1$ & $2.769 \mathrm{E}-1$ & $1.351 \mathrm{E}-1$ & $3.783 \mathrm{E}-2$ \\
\hline 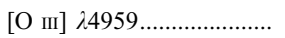 & $5.202 \mathrm{E}+0$ & $3.836 \mathrm{E}+0$ & $1.151 \mathrm{E}+0$ & $1.512 \mathrm{E}-1$ & $2.972 \mathrm{E}-3$ \\
\hline 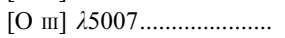 & $1.498 \mathrm{E}+1$ & $1.105 \mathrm{E}+1$ & $3.316 \mathrm{E}+0$ & $4.355 \mathrm{E}-1$ & $8.560 \mathrm{E}-3$ \\
\hline$[\ni] \quad \lambda 5200 \ldots \ldots \ldots \ldots \ldots \ldots \ldots \ldots$ & $1.076 \mathrm{E}+0$ & $5.989 \mathrm{E}-1$ & $1.872 \mathrm{E}-1$ & $1.538 \mathrm{E}-1$ & $2.195 \mathrm{E}-1$ \\
\hline [Fe vII] $\lambda 6085 \ldots \ldots \ldots \ldots \ldots \ldots$ & $4.583 \mathrm{E}-3$ & $2.176 \mathrm{E}-3$ & $1.496 \mathrm{E}-6$ & $0.000 \mathrm{E}+0$ & $0.000 \mathrm{E}+0$ \\
\hline 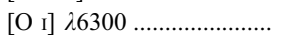 & $1.508 \mathrm{E}+0$ & $7.741 \mathrm{E}-1$ & $2.219 \mathrm{E}-1$ & $2.390 \mathrm{E}-1$ & $3.921 \mathrm{E}-1$ \\
\hline $\mathrm{H} \alpha \quad \lambda 6563 \ldots \ldots \ldots \ldots \ldots \ldots \ldots \ldots \ldots$ & $2.902 \mathrm{E}+0$ & $2.898 \mathrm{E}+0$ & $2.964 \mathrm{E}+0$ & $2.981 \mathrm{E}+0$ & $3.046 \mathrm{E}+0$ \\
\hline 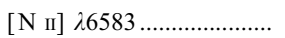 & $5.650 \mathrm{E}+0$ & $3.535 \mathrm{E}+0$ & $2.384 \mathrm{E}+0$ & $5.311 \mathrm{E}+0$ & $4.372 \mathrm{E}+0$ \\
\hline$\left[\mathrm{S}_{\mathrm{II}}\right] \lambda \lambda 6717,6730 \ldots \ldots \ldots$. & $4.673 \mathrm{E}+0$ & $2.731 \mathrm{E}+0$ & $1.280 \mathrm{E}+0$ & $2.861 \mathrm{E}+0$ & $5.732 \mathrm{E}+0$ \\
\hline$[\mathrm{O}$ пI] $\lambda \lambda 7318,7324 \ldots \ldots \ldots$ & $4.846 \mathrm{E}-2$ & $2.211 \mathrm{E}-2$ & $6.415 \mathrm{E}-3$ & $1.049 \mathrm{E}-2$ & $5.057 \mathrm{E}-3$ \\
\hline$[\mathrm{S}$ III] $] \lambda \lambda 9069,9532 \ldots \ldots \ldots$ & $8.307 \mathrm{E}+0$ & $6.336 \mathrm{E}+0$ & $4.615 \mathrm{E}+0$ & $4.195 \mathrm{E}+0$ & $1.718 \mathrm{E}+0$ \\
\hline \multicolumn{6}{|c|}{$\alpha=-2.0$} \\
\hline$[\mathrm{O}$ пा] $\lambda \lambda 3727,3729 \ldots \ldots \ldots$ & $1.954 \mathrm{E}+0$ & $6.904 \mathrm{E}-1$ & $4.238 \mathrm{E}-1$ & $1.117 \mathrm{E}+0$ & $8.816 \mathrm{E}-1$ \\
\hline$\left[\mathrm{S}_{\mathrm{II}}\right] \lambda \lambda 4067,4076 \ldots \ldots \ldots .$. & $1.250 \mathrm{E}-1$ & $4.184 \mathrm{E}-2$ & $2.107 \mathrm{E}-2$ & $7.646 \mathrm{E}-2$ & $1.300 \mathrm{E}-1$ \\
\hline$[\mathrm{O}$ III] $\lambda 4363 \ldots \ldots \ldots \ldots \ldots \ldots \ldots$ & $1.008 \mathrm{E}-1$ & $2.852 \mathrm{E}-2$ & $1.125 \mathrm{E}-3$ & $1.368 \mathrm{E}-4$ & $2.720 \mathrm{E}-6$ \\
\hline 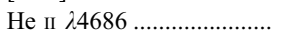 & $6.820 \mathrm{E}-1$ & $3.906 \mathrm{E}-1$ & $1.860 \mathrm{E}-1$ & $8.925 \mathrm{E}-2$ & $2.319 \mathrm{E}-2$ \\
\hline 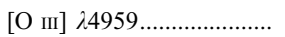 & $3.989 \mathrm{E}+0$ & $1.914 \mathrm{E}+0$ & $4.881 \mathrm{E}-1$ & $8.487 \mathrm{E}-2$ & $1.956 \mathrm{E}-3$ \\
\hline 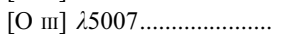 & $1.149 \mathrm{E}+1$ & $5.512 \mathrm{E}+0$ & $1.406 \mathrm{E}+0$ & $2.445 \mathrm{E}-1$ & $5.634 \mathrm{E}-3$ \\
\hline 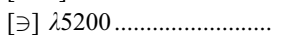 & $5.590 \mathrm{E}-1$ & $1.571 \mathrm{E}-1$ & $3.239 \mathrm{E}-2$ & $5.845 \mathrm{E}-2$ & $1.500 \mathrm{E}-1$ \\
\hline [Fe vII] $\lambda 6085 \ldots \ldots \ldots \ldots \ldots \ldots$ & $3.560 \mathrm{E}-3$ & $6.382 \mathrm{E}-4$ & $0.000 \mathrm{E}+0$ & $0.000 \mathrm{E}+0$ & $0.000 \mathrm{E}+0$ \\
\hline 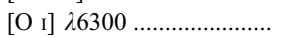 & $7.214 \mathrm{E}-1$ & $1.883 \mathrm{E}-1$ & $4.327 \mathrm{E}-2$ & $1.056 \mathrm{E}-1$ & $2.730 \mathrm{E}-1$ \\
\hline 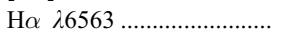 & $2.884 \mathrm{E}+0$ & $2.938 \mathrm{E}+0$ & $3.001 \mathrm{E}+0$ & $2.998 \mathrm{E}+0$ & $3.056 \mathrm{E}+0$ \\
\hline 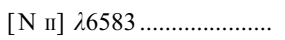 & $3.281 \mathrm{E}+0$ & $1.625 \mathrm{E}+0$ & $1.618 \mathrm{E}+0$ & $4.155 \mathrm{E}+0$ & $3.612 \mathrm{E}+0$ \\
\hline$[\mathrm{S}$ II $] \lambda \lambda 6717,6730 \ldots \ldots \ldots . .$. & $2.685 \mathrm{E}+0$ & $1.065 \mathrm{E}+0$ & $6.829 \mathrm{E}-1$ & $2.131 \mathrm{E}+0$ & $4.666 \mathrm{E}+0$ \\
\hline$[\mathrm{O}$ п] $] \lambda \lambda 7318,7324 \ldots \ldots \ldots$ & $2.078 \mathrm{E}-2$ & $4.964 \mathrm{E}-3$ & $1.966 \mathrm{E}-3$ & $5.705 \mathrm{E}-3$ & $3.396 \mathrm{E}-3$ \\
\hline$[\mathrm{S}$ III] $\lambda \lambda 9069,9532 \ldots \ldots \ldots$. & $6.016 \mathrm{E}+0$ & $3.766 \mathrm{E}+0$ & $3.065 \mathrm{E}+0$ & $3.044 \mathrm{E}+0$ & $1.286 \mathrm{E}+0$ \\
\hline
\end{tabular}


TABLE 13

Dusty IR Line Ratios with Respect to $\mathrm{H} \beta, 0.25 Z_{\odot}$

\begin{tabular}{|c|c|c|c|c|c|}
\hline \multirow[b]{2}{*}{ Line } & \multicolumn{5}{|c|}{$\log U_{0}$} \\
\hline & 0.0 & -1.0 & -2.0 & -3.0 & -4.0 \\
\hline \multicolumn{6}{|c|}{$\alpha=-1.2$} \\
\hline 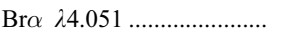 & $5.972 \mathrm{E}-2$ & $6.043 \mathrm{E}-2$ & $6.631 \mathrm{E}-2$ & $7.586 \mathrm{E}-2$ & $8.485 \mathrm{E}-2$ \\
\hline$[\mathrm{Ar}$ vI] $\lambda 4.530 \ldots \ldots \ldots \ldots \ldots \ldots$ & $9.960 \mathrm{E}-3$ & $9.314 \mathrm{E}-3$ & $9.535 \mathrm{E}-4$ & $0.000 \mathrm{E}+0$ & $0.000 \mathrm{E}+0$ \\
\hline 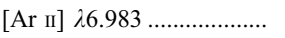 & $1.703 \mathrm{E}-2$ & $1.544 \mathrm{E}-2$ & $1.381 \mathrm{E}-2$ & $3.049 \mathrm{E}-2$ & $6.315 \mathrm{E}-2$ \\
\hline 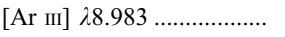 & $3.863 \mathrm{E}-1$ & $3.418 \mathrm{E}-1$ & $3.954 \mathrm{E}-2$ & $7.316 \mathrm{E}-6$ & $0.000 \mathrm{E}+0$ \\
\hline$[\mathrm{Ne} v \mathrm{vI}] \lambda 7.652 \ldots \ldots \ldots \ldots \ldots . .$. & $1.957 \mathrm{E}-2$ & $2.209 \mathrm{E}-2$ & $4.496 \mathrm{E}-2$ & $5.968 \mathrm{E}-2$ & $2.904 \mathrm{E}-2$ \\
\hline 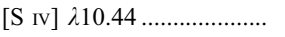 & $2.219 \mathrm{E}-1$ & $2.924 \mathrm{E}-1$ & $6.424 \mathrm{E}-1$ & $1.125 \mathrm{E}-1$ & $8.612 \mathrm{E}-4$ \\
\hline$[\mathrm{Ne}$ II] $\lambda 12.8 \ldots \ldots \ldots \ldots \ldots \ldots . . . . . . . .$. & $2.454 \mathrm{E}-1$ & $2.296 \mathrm{E}-1$ & $2.520 \mathrm{E}-1$ & $6.916 \mathrm{E}-1$ & $1.569 \mathrm{E}+0$ \\
\hline 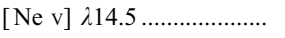 & $1.001 \mathrm{E}-1$ & $1.043 \mathrm{E}-1$ & $6.515 \mathrm{E}-2$ & $1.658 \mathrm{E}-4$ & $0.000 \mathrm{E}+0$ \\
\hline$[\mathrm{Ne}$ III] $\lambda 15.5 \ldots \ldots \ldots \ldots \ldots \ldots . .$. & $5.532 \mathrm{E}-1$ & $5.569 \mathrm{E}-1$ & $6.981 \mathrm{E}-1$ & $9.382 \mathrm{E}-1$ & $8.588 \mathrm{E}-1$ \\
\hline 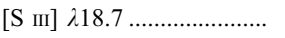 & $2.230 \mathrm{E}-1$ & $2.114 \mathrm{E}-1$ & $2.500 \mathrm{E}-1$ & $5.464 \mathrm{E}-1$ & $5.060 \mathrm{E}-1$ \\
\hline$[\mathrm{O}$ IV] $\lambda 25.9 \ldots \ldots \ldots \ldots \ldots \ldots \ldots \ldots \ldots \ldots \ldots$ & $7.046 \mathrm{E}-1$ & $7.988 \mathrm{E}-1$ & $1.115 \mathrm{E}+0$ & $1.676 \mathrm{E}-1$ & $1.463 \mathrm{E}-4$ \\
\hline \multicolumn{6}{|c|}{$\alpha=-1.4$} \\
\hline 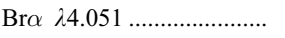 & $5.977 \mathrm{E}-2$ & $6.125 \mathrm{E}-2$ & $6.714 \mathrm{E}-2$ & $7.569 \mathrm{E}-2$ & $8.594 \mathrm{E}-2$ \\
\hline 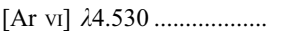 & $1.075 \mathrm{E}-2$ & $9.023 \mathrm{E}-3$ & $5.333 \mathrm{E}-4$ & $0.000 \mathrm{E}+0$ & $0.000 \mathrm{E}+0$ \\
\hline 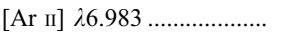 & $1.328 \mathrm{E}-2$ & $1.110 \mathrm{E}-2$ & $9.799 \mathrm{E}-3$ & $2.239 \mathrm{E}-2$ & $4.985 \mathrm{E}-2$ \\
\hline [Ar III] $\lambda 8.983 \ldots \ldots \ldots \ldots \ldots . . .$. & $3.825 \mathrm{E}-1$ & $2.920 \mathrm{E}-1$ & $1.988 \mathrm{E}-2$ & $3.195 \mathrm{E}-6$ & $0.000 \mathrm{E}+0$ \\
\hline$[\mathrm{Ne}$ vI $] 27.652 \ldots \ldots \ldots \ldots \ldots . .$. & $2.019 \mathrm{E}-2$ & $2.446 \mathrm{E}-2$ & $4.886 \mathrm{E}-2$ & $6.242 \mathrm{E}-2$ & $2.975 \mathrm{E}-2$ \\
\hline 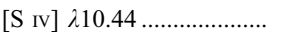 & $3.110 \mathrm{E}-1$ & $4.493 \mathrm{E}-1$ & $7.362 \mathrm{E}-1$ & $9.925 \mathrm{E}-2$ & $8.658 \mathrm{E}-4$ \\
\hline$[\mathrm{Ne}$ II] $\lambda 12.8 \ldots \ldots \ldots \ldots \ldots \ldots$ & $2.146 \mathrm{E}-1$ & $1.936 \mathrm{E}-1$ & $2.029 \mathrm{E}-1$ & $5.202 \mathrm{E}-1$ & $1.145 \mathrm{E}+0$ \\
\hline$[\mathrm{Ne} v] \lambda 14.5 \ldots \ldots \ldots \ldots \ldots \ldots$ & $1.050 \mathrm{E}-1$ & $1.079 \mathrm{E}-1$ & $4.699 \mathrm{E}-2$ & $1.066 \mathrm{E}-4$ & $0.000 \mathrm{E}+0$ \\
\hline$[\mathrm{Ne}$ III $] \lambda 15.5 \ldots \ldots \ldots \ldots \ldots \ldots . .$. & $5.099 \mathrm{E}-1$ & $5.383 \mathrm{E}-1$ & $6.919 \mathrm{E}-1$ & $8.261 \mathrm{E}-1$ & $6.939 \mathrm{E}-1$ \\
\hline 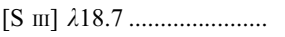 & $1.820 \mathrm{E}-1$ & $1.706 \mathrm{E}-1$ & $2.300 \mathrm{E}-1$ & $4.821 \mathrm{E}-1$ & $3.904 \mathrm{E}-1$ \\
\hline$[\mathrm{O}$ iv] $225.9 \ldots \ldots \ldots \ldots \ldots \ldots \ldots$ & $7.739 \mathrm{E}-1$ & $8.691 \mathrm{E}-1$ & $9.865 \mathrm{E}-1$ & $1.396 \mathrm{E}-1$ & $1.126 \mathrm{E}-4$ \\
\hline \multicolumn{6}{|c|}{$\alpha=-1.7$} \\
\hline 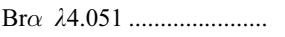 & $6.083 \mathrm{E}-2$ & $6.329 \mathrm{E}-2$ & $6.855 \mathrm{E}-2$ & $7.567 \mathrm{E}-2$ & $8.447 \mathrm{E}-2$ \\
\hline$[\mathrm{Ar} \mathrm{vI}] \lambda 4.530 \ldots \ldots \ldots \ldots \ldots . .$. & $1.069 \mathrm{E}-2$ & $7.015 \mathrm{E}-3$ & $1.821 \mathrm{E}-4$ & $0.000 \mathrm{E}+0$ & $0.000 \mathrm{E}+0$ \\
\hline 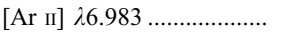 & $8.424 \mathrm{E}-3$ & $6.124 \mathrm{E}-3$ & $5.639 \mathrm{E}-3$ & $1.432 \mathrm{E}-2$ & $3.796 \mathrm{E}-2$ \\
\hline 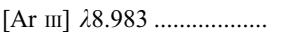 & $3.387 \mathrm{E}-1$ & $1.846 \mathrm{E}-1$ & $5.479 \mathrm{E}-3$ & $0.000 \mathrm{E}+0$ & $0.000 \mathrm{E}+0$ \\
\hline$[\mathrm{Ne}$ vI $] \lambda 7.652 \ldots \ldots \ldots \ldots \ldots . .$. & $2.189 \mathrm{E}-2$ & $2.830 \mathrm{E}-2$ & $5.401 \mathrm{E}-2$ & $6.428 \mathrm{E}-2$ & $2.909 \mathrm{E}-2$ \\
\hline [S IV] $\lambda 10.44 \ldots \ldots \ldots \ldots \ldots \ldots$ & $4.998 \mathrm{E}-1$ & $7.176 \mathrm{E}-1$ & $7.917 \mathrm{E}-1$ & $7.577 \mathrm{E}-2$ & $1.047 \mathrm{E}-3$ \\
\hline$[\mathrm{Ne}$ II] $\lambda 12.8 \ldots \ldots \ldots \ldots \ldots \ldots . .$. & $1.643 \mathrm{E}-1$ & $1.342 \mathrm{E}-1$ & $1.361 \mathrm{E}-1$ & $3.312 \mathrm{E}-1$ & $7.957 \mathrm{E}-1$ \\
\hline$[\mathrm{Ne} v] \lambda 14.5 \ldots \ldots \ldots \ldots \ldots \ldots \ldots$ & $1.069 \mathrm{E}-1$ & $9.803 \mathrm{E}-2$ & $2.329 \mathrm{E}-2$ & $4.651 \mathrm{E}-5$ & $0.000 \mathrm{E}+0$ \\
\hline$[\mathrm{Ne}$ III] $\lambda 15.5 \ldots \ldots \ldots \ldots \ldots \ldots . .$. & $5.005 \mathrm{E}-1$ & $5.749 \mathrm{E}-1$ & $7.039 \mathrm{E}-1$ & $7.025 \mathrm{E}-1$ & $5.064 \mathrm{E}-1$ \\
\hline$[\mathrm{S}$ III] $\lambda 18.7 \ldots \ldots \ldots \ldots \ldots \ldots . .$. & $1.369 \mathrm{E}-1$ & $1.347 \mathrm{E}-1$ & $2.231 \mathrm{E}-1$ & $4.152 \mathrm{E}-1$ & $2.813 \mathrm{E}-1$ \\
\hline$[\mathrm{O}$ IV $] \lambda 25.9 \ldots \ldots \ldots \ldots \ldots \ldots \ldots \ldots$ & $7.928 \mathrm{E}-1$ & $8.224 \mathrm{E}-1$ & $7.283 \mathrm{E}-1$ & $9.492 \mathrm{E}-2$ & $7.150 \mathrm{E}-5$ \\
\hline \multicolumn{6}{|c|}{$\alpha=-2.0$} \\
\hline 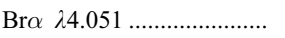 & $6.265 \mathrm{E}-2$ & $6.556 \mathrm{E}-2$ & $6.996 \mathrm{E}-2$ & $7.623 \mathrm{E}-2$ & $8.390 \mathrm{E}-2$ \\
\hline$[\mathrm{Ar}$ vI] $\lambda 4.530 \ldots \ldots \ldots \ldots \ldots \ldots$ & $9.110 \mathrm{E}-3$ & $4.421 \mathrm{E}-3$ & $5.263 \mathrm{E}-5$ & $0.000 \mathrm{E}+0$ & $0.000 \mathrm{E}+0$ \\
\hline 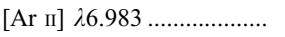 & $4.820 \mathrm{E}-3$ & $3.098 \mathrm{E}-3$ & $3.305 \mathrm{E}-3$ & $1.005 \mathrm{E}-2$ & $3.139 \mathrm{E}-2$ \\
\hline 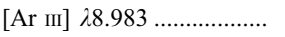 & $2.565 \mathrm{E}-1$ & $9.011 \mathrm{E}-2$ & $1.173 \mathrm{E}-3$ & $0.000 \mathrm{E}+0$ & $0.000 \mathrm{E}+0$ \\
\hline$[\mathrm{Ne} v \mathrm{vI}] \lambda 7.652 \ldots \ldots \ldots \ldots \ldots . .$. & $2.378 \mathrm{E}-2$ & $3.165 \mathrm{E}-2$ & $5.807 \mathrm{E}-2$ & $6.402 \mathrm{E}-2$ & $2.702 \mathrm{E}-2$ \\
\hline 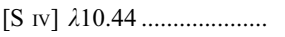 & $7.260 \mathrm{E}-1$ & $9.366 \mathrm{E}-1$ & $7.573 \mathrm{E}-1$ & $5.433 \mathrm{E}-2$ & $3.703 \mathrm{E}-4$ \\
\hline$[\mathrm{Ne}$ II] $\lambda 12.8 \ldots \ldots \ldots \ldots \ldots \ldots$ & $1.135 \mathrm{E}-1$ & $8.180 \mathrm{E}-2$ & $8.732 \mathrm{E}-2$ & $2.272 \mathrm{E}-1$ & $5.963 \mathrm{E}-1$ \\
\hline$[\mathrm{Ne} \mathrm{v}] \lambda 14.5 \ldots \ldots \ldots \ldots \ldots \ldots \ldots \ldots \ldots$ & $9.967 \mathrm{E}-2$ & $7.452 \mathrm{E}-2$ & $9.328 \mathrm{E}-3$ & $1.768 \mathrm{E}-5$ & $0.000 \mathrm{E}+0$ \\
\hline$[\mathrm{Ne}$ III] $\lambda 15.5 \ldots \ldots \ldots \ldots \ldots \ldots . .$. & $5.434 \mathrm{E}-1$ & $6.370 \mathrm{E}-1$ & $7.141 \mathrm{E}-1$ & $6.080 \mathrm{E}-1$ & $3.664 \mathrm{E}-1$ \\
\hline 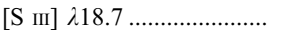 & $1.093 \mathrm{E}-1$ & $1.200 \mathrm{E}-1$ & $2.326 \mathrm{E}-1$ & $3.736 \mathrm{E}-1$ & $2.173 \mathrm{E}-1$ \\
\hline 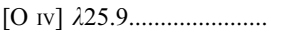 & $7.229 \mathrm{E}-1$ & $6.874 \mathrm{E}-1$ & $4.845 \mathrm{E}-1$ & $5.879 \mathrm{E}-2$ & $4.223 \mathrm{E}-5$ \\
\hline
\end{tabular}


TABLE 14

Dusty IR Line Ratios with Respect to $\mathrm{H} \beta, 0.5 Z_{\odot}$

\begin{tabular}{|c|c|c|c|c|c|}
\hline \multirow[b]{2}{*}{ LiNE } & \multicolumn{5}{|c|}{$\log U_{0}$} \\
\hline & 0.0 & -1.0 & -2.0 & -3.0 & -4.0 \\
\hline \multicolumn{6}{|c|}{$\alpha=-1.2$} \\
\hline 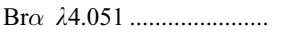 & $6.215 \mathrm{E}-2$ & $6.272 \mathrm{E}-2$ & $6.838 \mathrm{E}-2$ & $7.666 \mathrm{E}-2$ & $8.571 \mathrm{E}-2$ \\
\hline 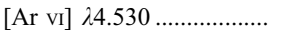 & $1.682 \mathrm{E}-2$ & $1.576 \mathrm{E}-2$ & $1.457 \mathrm{E}-3$ & $0.000 \mathrm{E}+0$ & $0.000 \mathrm{E}+0$ \\
\hline 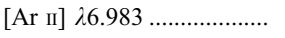 & $3.996 \mathrm{E}-2$ & $3.612 \mathrm{E}-2$ & $2.753 \mathrm{E}-2$ & $5.292 \mathrm{E}-2$ & $1.067 \mathrm{E}-1$ \\
\hline [Ar III] $\lambda 8.983 \ldots \ldots \ldots \ldots \ldots . .$. & $6.618 \mathrm{E}-1$ & $5.912 \mathrm{E}-1$ & $6.019 \mathrm{E}-2$ & $1.047 \mathrm{E}-5$ & $0.000 \mathrm{E}+0$ \\
\hline$[\mathrm{Ne} v \mathrm{vI}] \lambda 7.652 \ldots \ldots \ldots \ldots \ldots . .$. & $4.493 \mathrm{E}-2$ & $4.975 \mathrm{E}-2$ & $9.418 \mathrm{E}-2$ & $1.183 \mathrm{E}-1$ & $5.645 \mathrm{E}-2$ \\
\hline 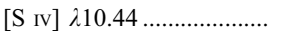 & $4.291 \mathrm{E}-1$ & $5.423 \mathrm{E}-1$ & $1.228 \mathrm{E}+0$ & $1.974 \mathrm{E}-1$ & $5.173 \mathrm{E}-3$ \\
\hline$[\mathrm{Ne}$ II] $\lambda 12.8 \ldots \ldots \ldots \ldots \ldots \ldots . . .$. & $5.111 \mathrm{E}-1$ & $4.687 \mathrm{E}-1$ & $4.429 \mathrm{E}-1$ & $1.004 \mathrm{E}+0$ & $2.056 \mathrm{E}+0$ \\
\hline 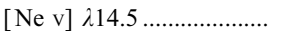 & $2.024 \mathrm{E}-1$ & $2.073 \mathrm{E}-1$ & $1.110 \mathrm{E}-1$ & $2.571 \mathrm{E}-4$ & $0.000 \mathrm{E}+0$ \\
\hline$[\mathrm{Ne}$ III] $\lambda 15.5 \ldots \ldots \ldots \ldots \ldots \ldots . .$. & $1.219 \mathrm{E}+0$ & $1.204 \mathrm{E}+0$ & $1.405 \mathrm{E}+0$ & $1.790 \mathrm{E}+0$ & $1.599 \mathrm{E}+0$ \\
\hline$[\mathrm{S}$ III] $\lambda 18.7 \ldots \ldots \ldots \ldots \ldots \ldots . .$. & $5.292 \mathrm{E}-1$ & $4.995 \mathrm{E}-1$ & $5.276 \mathrm{E}-1$ & $1.045 \mathrm{E}+0$ & $9.041 \mathrm{E}-1$ \\
\hline 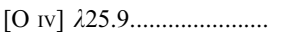 & $1.417 \mathrm{E}+0$ & $1.590 \mathrm{E}+0$ & $2.117 \mathrm{E}+0$ & $2.854 \mathrm{E}-1$ & $2.858 \mathrm{E}-4$ \\
\hline \multicolumn{6}{|c|}{$\alpha=-1.4$} \\
\hline $\operatorname{Br} \alpha \lambda 4.051 \ldots \ldots$. & $6.236 \mathrm{E}-2$ & $6.360 \mathrm{E}-2$ & $6.946 \mathrm{E}-2$ & $7.670 \mathrm{E}-2$ & $8.594 \mathrm{E}-2$ \\
\hline$[\mathrm{Ar} \mathrm{vI}] \lambda 4.530 \ldots \ldots$. & $1.804 \mathrm{E}-2$ & $1.529 \mathrm{E}-2$ & $7.634 \mathrm{E}-4$ & $0.000 \mathrm{E}+0$ & $0.000 \mathrm{E}+0$ \\
\hline$[$ Ar II $] \lambda 6.983 \ldots \ldots .$. & $3.133 \mathrm{E}-2$ & $2.610 \mathrm{E}-2$ & $1.915 \mathrm{E}-2$ & $3.863 \mathrm{E}-2$ & $8.663 \mathrm{E}-2$ \\
\hline$[\mathrm{Ar}$ III $] \quad \lambda 8.983 \ldots \ldots \ldots$. & $6.502 \mathrm{E}-1$ & $5.000 \mathrm{E}-1$ & $2.809 \mathrm{E}-2$ & $4.219 \mathrm{E}-6$ & $0.000 \mathrm{E}+0$ \\
\hline$[\mathrm{Ne} v \mathrm{vI}] \lambda 7.652 \ldots \ldots . .$. & $4.660 \mathrm{E}-2$ & $5.484 \mathrm{E}-2$ & $1.017 \mathrm{E}-1$ & $1.226 \mathrm{E}-1$ & $5.707 \mathrm{E}-2$ \\
\hline [S IV] $\lambda 10.44 \ldots \ldots \ldots \ldots \ldots \ldots$ & $5.852 \mathrm{E}-1$ & $8.061 \mathrm{E}-1$ & $1.363 \mathrm{E}+0$ & $1.675 \mathrm{E}-1$ & $1.669 \mathrm{E}-3$ \\
\hline$[\mathrm{Ne}$ II $] \lambda 12.8 \ldots \ldots \ldots \ldots \ldots \ldots$ & $4.454 \mathrm{E}-1$ & $3.905 \mathrm{E}-1$ & $3.433 \mathrm{E}-1$ & $7.462 \mathrm{E}-1$ & $1.568 \mathrm{E}+0$ \\
\hline$[\mathrm{Ne} v] \lambda 14.5 \ldots \ldots \ldots \ldots \ldots \ldots \ldots$ & $2.180 \mathrm{E}-1$ & $2.170 \mathrm{E}-1$ & $7.592 \mathrm{E}-2$ & $1.551 \mathrm{E}-4$ & $0.000 \mathrm{E}+0$ \\
\hline$[\mathrm{Ne}$ III] $] \lambda 15.5 .$. & $1.095 \mathrm{E}+0$ & $1.114 \mathrm{E}+0$ & $1.372 \mathrm{E}+0$ & $1.579 \mathrm{E}+0$ & $1.296 \mathrm{E}+0$ \\
\hline$[\mathrm{S}$ III] $\lambda 18.7 \ldots \ldots \ldots \ldots$. & $4.345 \mathrm{E}-1$ & $4.031 \mathrm{E}-1$ & $4.866 \mathrm{E}-1$ & $9.225 \mathrm{E}-1$ & $7.010 \mathrm{E}-1$ \\
\hline$[\mathrm{O}$ IV $] \lambda 25.9 \ldots \ldots \ldots \ldots \ldots \ldots \ldots \ldots \ldots \ldots$ & $1.590 \mathrm{E}+0$ & $1.776 \mathrm{E}+0$ & $1.837 \mathrm{E}+0$ & $2.283 \mathrm{E}-1$ & $2.046 \mathrm{E}-4$ \\
\hline \multicolumn{6}{|c|}{$\alpha=-1.7$} \\
\hline 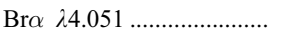 & $6.354 \mathrm{E}-2$ & $6.589 \mathrm{E}-2$ & $7.143 \mathrm{E}-2$ & $7.703 \mathrm{E}-2$ & $8.678 \mathrm{E}-2$ \\
\hline 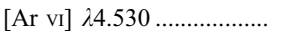 & $1.779 \mathrm{E}-2$ & $1.177 \mathrm{E}-2$ & $2.364 \mathrm{E}-4$ & $0.000 \mathrm{E}+0$ & $0.000 \mathrm{E}+0$ \\
\hline$[\mathrm{Ar}$ II] $\lambda 6.983 \ldots \ldots \ldots \ldots \ldots \ldots$ & $1.984 \mathrm{E}-2$ & $1.417 \mathrm{E}-2$ & $1.073 \mathrm{E}-2$ & $2.499 \mathrm{E}-2$ & $6.773 \mathrm{E}-2$ \\
\hline [Ar III] $\lambda 8.983 \ldots \ldots \ldots \ldots \ldots . . .$. & $5.606 \mathrm{E}-1$ & $3.002 \mathrm{E}-1$ & $6.844 \mathrm{E}-3$ & $0.000 \mathrm{E}+0$ & $0.000 \mathrm{E}+0$ \\
\hline$[\mathrm{Ne} v \mathrm{vI}] \lambda 7.652 \ldots \ldots \ldots \ldots \ldots \ldots$ & $5.057 \mathrm{E}-2$ & $6.346 \mathrm{E}-2$ & $1.102 \mathrm{E}-1$ & $1.245 \mathrm{E}-1$ & $5.407 \mathrm{E}-2$ \\
\hline 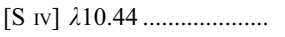 & $8.986 \mathrm{E}-1$ & $1.253 \mathrm{E}+0$ & $1.386 \mathrm{E}+0$ & $1.218 \mathrm{E}-1$ & $1.414 \mathrm{E}-3$ \\
\hline$[\mathrm{Ne}$ II] $\lambda 12.8 \ldots \ldots \ldots \ldots \ldots \ldots$ & $3.330 \mathrm{E}-1$ & $2.597 \mathrm{E}-1$ & $2.204 \mathrm{E}-1$ & $4.879 \mathrm{E}-1$ & $1.144 \mathrm{E}+0$ \\
\hline$[\mathrm{Ne} \mathrm{v}] \lambda 14.5 \ldots \ldots \ldots \ldots \ldots \ldots \ldots$ & $2.246 \mathrm{E}-1$ & $1.923 \mathrm{E}-1$ & $3.426 \mathrm{E}-2$ & $6.334 \mathrm{E}-5$ & $0.000 \mathrm{E}+0$ \\
\hline$[\mathrm{Ne}$ III] $] \lambda 15.5 \ldots \ldots \ldots \ldots \ldots \ldots . . . . .$. & $1.015 \mathrm{E}+0$ & $1.119 \mathrm{E}+0$ & $1.358 \mathrm{E}+0$ & $1.335 \mathrm{E}+0$ & $9.315 \mathrm{E}-1$ \\
\hline 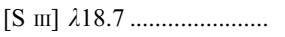 & $3.279 \mathrm{E}-1$ & $3.161 \mathrm{E}-1$ & $4.697 \mathrm{E}-1$ & $7.913 \mathrm{E}-1$ & $5.063 \mathrm{E}-1$ \\
\hline 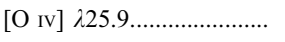 & $1.671 \mathrm{E}+0$ & $1.715 \mathrm{E}+0$ & $1.303 \mathrm{E}+0$ & $1.482 \mathrm{E}-1$ & $1.233 \mathrm{E}-4$ \\
\hline \multicolumn{6}{|c|}{$\alpha=-2.0$} \\
\hline 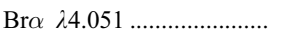 & $6.527 \mathrm{E}-2$ & $6.875 \mathrm{E}-2$ & $7.341 \mathrm{E}-2$ & $7.792 \mathrm{E}-2$ & $8.653 \mathrm{E}-2$ \\
\hline 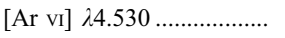 & $1.567 \mathrm{E}-2$ & $7.116 \mathrm{E}-3$ & $6.189 \mathrm{E}-5$ & $0.000 \mathrm{E}+0$ & $0.000 \mathrm{E}+0$ \\
\hline$[\mathrm{Ar}$ II] $\lambda 6.983 \ldots \ldots \ldots \ldots \ldots \ldots$ & $1.202 \mathrm{E}-2$ & $7.206 \mathrm{E}-3$ & $6.433 \mathrm{E}-3$ & $1.815 \mathrm{E}-2$ & $5.806 \mathrm{E}-2$ \\
\hline$[\mathrm{Ar}$ III] $\lambda 8.983 \ldots \ldots \ldots \ldots \ldots \ldots$ & $4.418 \mathrm{E}-1$ & $1.340 \mathrm{E}-1$ & $1.280 \mathrm{E}-3$ & $0.000 \mathrm{E}+0$ & $0.000 \mathrm{E}+0$ \\
\hline$[\mathrm{Ne} v \mathrm{vI}] \lambda 7.652 \ldots \ldots \ldots \ldots \ldots . . . .$. & $5.419 \mathrm{E}-2$ & $7.048 \mathrm{E}-2$ & $1.155 \mathrm{E}-1$ & $1.222 \mathrm{E}-1$ & $4.918 \mathrm{E}-2$ \\
\hline$[\mathrm{S}$ Iv] $\lambda 10.44 \ldots \ldots \ldots \ldots \ldots \ldots$ & $1.212 \mathrm{E}+0$ & $1.594 \mathrm{E}+0$ & $1.244 \mathrm{E}+0$ & $8.364 \mathrm{E}-2$ & $5.728 \mathrm{E}-4$ \\
\hline$[\mathrm{Ne}$ II $] \lambda 12.8 \ldots \ldots \ldots \ldots \ldots \ldots$ & $2.312 \mathrm{E}-1$ & $1.522 \mathrm{E}-1$ & $1.425 \mathrm{E}-1$ & $3.604 \mathrm{E}-1$ & $9.317 \mathrm{E}-1$ \\
\hline$[\mathrm{Ne} \mathrm{v}] \lambda 14.5 \ldots \ldots \ldots \ldots \ldots \ldots$ & $2.074 \mathrm{E}-1$ & $1.357 \mathrm{E}-1$ & $1.237 \mathrm{E}-2$ & $2.262 \mathrm{E}-5$ & $0.000 \mathrm{E}+0$ \\
\hline 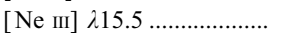 & $1.033 \mathrm{E}+0$ & $1.194 \mathrm{E}+0$ & $1.337 \mathrm{E}+0$ & $1.141 \mathrm{E}+0$ & $6.693 \mathrm{E}-1$ \\
\hline 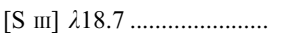 & $2.683 \mathrm{E}-1$ & $2.815 \mathrm{E}-1$ & $4.832 \mathrm{E}-1$ & $7.072 \mathrm{E}-1$ & $3.993 \mathrm{E}-1$ \\
\hline [O IV] $225.9 \ldots \ldots \ldots \ldots \ldots \ldots \ldots$ & $1.553 \mathrm{E}+0$ & $1.407 \mathrm{E}+0$ & $8.307 \mathrm{E}-1$ & $8.759 \mathrm{E}-2$ & $7.047 \mathrm{E}-5$ \\
\hline
\end{tabular}


TABLE 15

Dusty IR Line Ratios with Respect to $\mathrm{H} \beta, 1 Z_{\odot}$

\begin{tabular}{|c|c|c|c|c|c|}
\hline \multirow[b]{2}{*}{ LiNE } & \multicolumn{5}{|c|}{$\log U_{0}$} \\
\hline & 0.0 & -1.0 & -2.0 & -3.0 & -4.0 \\
\hline \multicolumn{6}{|c|}{$\alpha=-1.2$} \\
\hline 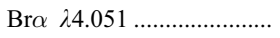 & $6.469 \mathrm{E}-2$ & $6.526 \mathrm{E}-2$ & $7.137 \mathrm{E}-2$ & $7.928 \mathrm{E}-2$ & $8.901 \mathrm{E}-2$ \\
\hline$[\mathrm{Ar}$ vI] $\lambda 4.530 \ldots \ldots \ldots \ldots \ldots \ldots . . .$. & $2.755 \mathrm{E}-2$ & $2.564 \mathrm{E}-2$ & $1.847 \mathrm{E}-3$ & $0.000 \mathrm{E}+0$ & $0.000 \mathrm{E}+0$ \\
\hline$[\mathrm{Ar}$ II] $\lambda 6.983 \ldots \ldots \ldots \ldots \ldots \ldots$ & $8.806 \mathrm{E}-2$ & $7.896 \mathrm{E}-2$ & $5.211 \mathrm{E}-2$ & $8.678 \mathrm{E}-2$ & $1.748 \mathrm{E}-1$ \\
\hline 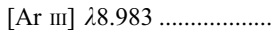 & $1.083 \mathrm{E}+0$ & $9.621 \mathrm{E}-1$ & $7.624 \mathrm{E}-2$ & $1.031 \mathrm{E}-5$ & $0.000 \mathrm{E}+0$ \\
\hline$[\mathrm{Ne} v \mathrm{vI}] \lambda 7.652 \ldots \ldots \ldots \ldots \ldots . . . .$. & $1.011 \mathrm{E}-1$ & $1.115 \mathrm{E}-1$ & $1.961 \mathrm{E}-1$ & $2.266 \mathrm{E}-1$ & $1.007 \mathrm{E}-1$ \\
\hline 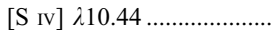 & $8.741 \mathrm{E}-1$ & $1.083 \mathrm{E}+0$ & $2.271 \mathrm{E}+0$ & $2.933 \mathrm{E}-1$ & $3.749 \mathrm{E}-3$ \\
\hline$[\mathrm{Ne}$ II] $\lambda 12.8 \ldots \ldots \ldots \ldots \ldots \ldots . . . . . . . .$. & $1.012 \mathrm{E}+0$ & $9.140 \mathrm{E}-1$ & $7.395 \mathrm{E}-1$ & $1.378 \mathrm{E}+0$ & $2.652 \mathrm{E}+0$ \\
\hline 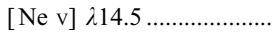 & $3.807 \mathrm{E}-1$ & $3.858 \mathrm{E}-1$ & $1.658 \mathrm{E}-1$ & $2.990 \mathrm{E}-4$ & $0.000 \mathrm{E}+0$ \\
\hline$[\mathrm{Ne}$ III] $\lambda 15.5 \ldots \ldots \ldots \ldots \ldots \ldots . .$. & $2.612 \mathrm{E}+0$ & $2.556 \mathrm{E}+0$ & $2.774 \mathrm{E}+0$ & $3.261 \mathrm{E}+0$ & $2.774 \mathrm{E}+0$ \\
\hline 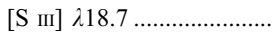 & $1.190 \mathrm{E}+0$ & $1.120 \mathrm{E}+0$ & $1.099 \mathrm{E}+0$ & $1.912 \mathrm{E}+0$ & $1.493 \mathrm{E}+0$ \\
\hline [O IV] $\lambda 25.9 \ldots \ldots \ldots \ldots \ldots \ldots \ldots \ldots \ldots \ldots \ldots . .$. & $2.805 \mathrm{E}+0$ & $3.134 \mathrm{E}+0$ & $3.737 \mathrm{E}+0$ & $3.974 \mathrm{E}-1$ & $4.481 \mathrm{E}-4$ \\
\hline \multicolumn{6}{|c|}{$\alpha=-1.4$} \\
\hline 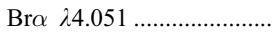 & $6.522 \mathrm{E}-2$ & $6.656 \mathrm{E}-2$ & $7.319 \mathrm{E}-2$ & $7.949 \mathrm{E}-2$ & $9.043 \mathrm{E}-2$ \\
\hline$[\mathrm{Ar}$ vI] $\lambda 4.530 \ldots \ldots \ldots \ldots \ldots . . .$. & $2.949 \mathrm{E}-2$ & $2.469 \mathrm{E}-2$ & $8.795 \mathrm{E}-4$ & $0.000 \mathrm{E}+0$ & $0.000 \mathrm{E}+0$ \\
\hline [Ar II] $26.983 \ldots \ldots \ldots \ldots \ldots . . . . . . . .$. & $6.945 \mathrm{E}-2$ & $5.674 \mathrm{E}-2$ & $3.536 \mathrm{E}-2$ & $6.360 \mathrm{E}-2$ & $1.456 \mathrm{E}-1$ \\
\hline 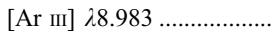 & $1.051 \mathrm{E}+0$ & $7.912 \mathrm{E}-1$ & $3.177 \mathrm{E}-2$ & $3.810 \mathrm{E}-6$ & $0.000 \mathrm{E}+0$ \\
\hline$[\mathrm{Ne}$ vI $] \lambda 7.652 \ldots \ldots \ldots \ldots \ldots . . .$. & $1.063 \mathrm{E}-1$ & $1.234 \mathrm{E}-1$ & $2.078 \mathrm{E}-1$ & $2.311 \mathrm{E}-1$ & $9.869 \mathrm{E}-2$ \\
\hline 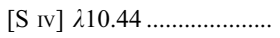 & $1.159 \mathrm{E}+0$ & $1.537 \mathrm{E}+0$ & $2.379 \mathrm{E}+0$ & $2.361 \mathrm{E}-1$ & $8.295 \mathrm{E}-3$ \\
\hline$[\mathrm{Ne}$ II] $\lambda 12.8 \ldots \ldots \ldots \ldots \ldots \ldots . .$. & $8.779 \mathrm{E}-1$ & $7.455 \mathrm{E}-1$ & $5.542 \mathrm{E}-1$ & $1.028 \mathrm{E}+0$ & $2.120 \mathrm{E}+0$ \\
\hline$[\mathrm{Ne} v] \lambda 14.5 \ldots \ldots \ldots \ldots \ldots \ldots \ldots$ & $4.155 \mathrm{E}-1$ & $4.008 \mathrm{E}-1$ & $1.043 \mathrm{E}-1$ & $1.692 \mathrm{E}-4$ & $0.000 \mathrm{E}+0$ \\
\hline$[\mathrm{Ne}$ III] $\lambda 15.5 \ldots \ldots \ldots \ldots \ldots \ldots . .$. & $2.328 \mathrm{E}+0$ & $2.311 \mathrm{E}+0$ & $2.654 \mathrm{E}+0$ & $2.867 \mathrm{E}+0$ & $2.224 \mathrm{E}+0$ \\
\hline 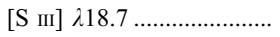 & $9.919 \mathrm{E}-1$ & $9.131 \mathrm{E}-1$ & $1.015 \mathrm{E}+0$ & $1.681 \mathrm{E}+0$ & $1.165 \mathrm{E}+0$ \\
\hline$[\mathrm{O}$ IV $] \lambda 25.9 \ldots \ldots \ldots \ldots \ldots \ldots \ldots$ & $3.184 \mathrm{E}+0$ & $3.509 \mathrm{E}+0$ & $3.118 \mathrm{E}+0$ & $3.027 \mathrm{E}-1$ & $3.238 \mathrm{E}-4$ \\
\hline \multicolumn{6}{|c|}{$\alpha=-1.7$} \\
\hline 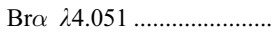 & $6.697 \mathrm{E}-2$ & $6.955 \mathrm{E}-2$ & $7.608 \mathrm{E}-2$ & $8.041 \mathrm{E}-2$ & $8.983 \mathrm{E}-2$ \\
\hline$[\mathrm{Ar}$ vI $] \lambda 4.530 \ldots \ldots \ldots \ldots \ldots . . . . . . . .$. & $2.861 \mathrm{E}-2$ & $1.832 \mathrm{E}-2$ & $2.349 \mathrm{E}-4$ & $0.000 \mathrm{E}+0$ & $0.000 \mathrm{E}+0$ \\
\hline [Ar II] $26.983 \ldots \ldots \ldots \ldots \ldots . . . . . . . .$. & $4.321 \mathrm{E}-2$ & $2.973 \mathrm{E}-2$ & $1.943 \mathrm{E}-2$ & $4.295 \mathrm{E}-2$ & $1.205 \mathrm{E}-1$ \\
\hline 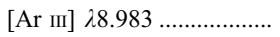 & $8.636 \mathrm{E}-1$ & $4.362 \mathrm{E}-1$ & $6.382 \mathrm{E}-3$ & $0.000 \mathrm{E}+0$ & $0.000 \mathrm{E}+0$ \\
\hline$[\mathrm{Ne}$ vI $] \lambda 7.652 \ldots \ldots \ldots \ldots \ldots . . . .$. & $1.165 \mathrm{E}-1$ & $1.412 \mathrm{E}-1$ & $2.179 \mathrm{E}-1$ & $2.284 \mathrm{E}-1$ & $9.102 \mathrm{E}-2$ \\
\hline 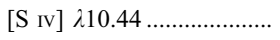 & $1.687 \mathrm{E}+0$ & $2.233 \mathrm{E}+0$ & $2.207 \mathrm{E}+0$ & $1.578 \mathrm{E}-1$ & $1.379 \mathrm{E}-3$ \\
\hline$[\mathrm{Ne}$ II $] \lambda 12.8 \ldots \ldots \ldots \ldots \ldots \ldots$ & $6.346 \mathrm{E}-1$ & $4.678 \mathrm{E}-1$ & $3.435 \mathrm{E}-1$ & $7.182 \mathrm{E}-1$ & $1.689 \mathrm{E}+0$ \\
\hline$[\mathrm{Ne} v] \lambda 14.5 \ldots \ldots \ldots \ldots \ldots \ldots$ & $4.227 \mathrm{E}-1$ & $3.354 \mathrm{E}-1$ & $4.076 \mathrm{E}-2$ & $6.193 \mathrm{E}-5$ & $0.000 \mathrm{E}+0$ \\
\hline$[\mathrm{Ne}$ III] $\lambda 15.5 \ldots \ldots \ldots \ldots \ldots \ldots$ & $2.081 \mathrm{E}+0$ & $2.203 \mathrm{E}+0$ & $2.531 \mathrm{E}+0$ & $2.389 \mathrm{E}+0$ & $1.599 \mathrm{E}+0$ \\
\hline 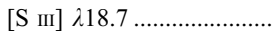 & $7.575 \mathrm{E}-1$ & $7.186 \mathrm{E}-1$ & $9.717 \mathrm{E}-1$ & $1.427 \mathrm{E}+0$ & $8.663 \mathrm{E}-1$ \\
\hline$[\mathrm{O}$ Iv $] 225.9 \ldots \ldots \ldots \ldots \ldots \ldots \ldots \ldots \ldots$ & $3.369 \mathrm{E}+0$ & $3.346 \mathrm{E}+0$ & $2.085 \mathrm{E}+0$ & $1.806 \mathrm{E}-1$ & $1.781 \mathrm{E}-4$ \\
\hline \multicolumn{6}{|c|}{$\alpha=-2.0$} \\
\hline 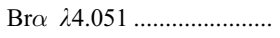 & $6.895 \mathrm{E}-2$ & $7.329 \mathrm{E}-2$ & $7.893 \mathrm{E}-2$ & $8.174 \mathrm{E}-2$ & $8.755 \mathrm{E}-2$ \\
\hline 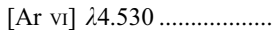 & $2.532 \mathrm{E}-2$ & $1.039 \mathrm{E}-2$ & $5.219 \mathrm{E}-5$ & $0.000 \mathrm{E}+0$ & $0.000 \mathrm{E}+0$ \\
\hline 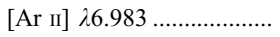 & $2.678 \mathrm{E}-2$ & $1.482 \mathrm{E}-2$ & $1.247 \mathrm{E}-2$ & $3.357 \mathrm{E}-2$ & $1.073 \mathrm{E}-1$ \\
\hline$[$ Ar III] $\lambda 8.983 \ldots \ldots \ldots \ldots \ldots . . . . . . .$. & $6.866 \mathrm{E}-1$ & $1.748 \mathrm{E}-1$ & $9.562 \mathrm{E}-4$ & $0.000 \mathrm{E}+0$ & $0.000 \mathrm{E}+0$ \\
\hline$[\mathrm{Ne} v \mathrm{vI}] \lambda 7.652 \ldots \ldots \ldots \ldots \ldots . . . .$. & $1.230 \mathrm{E}-1$ & $1.526 \mathrm{E}-1$ & $2.197 \mathrm{E}-1$ & $2.182 \mathrm{E}-1$ & $8.061 \mathrm{E}-2$ \\
\hline [S rv] $\lambda 10.44 \ldots \ldots \ldots \ldots \ldots \ldots \ldots$ & $2.117 \mathrm{E}+0$ & $2.662 \mathrm{E}+0$ & $1.809 \mathrm{E}+0$ & $1.010 \mathrm{E}-1$ & $1.350 \mathrm{E}-3$ \\
\hline$[\mathrm{Ne}$ II $] \lambda 12.8 \ldots \ldots \ldots \ldots \ldots . . . . . . . .$. & $4.355 \mathrm{E}-1$ & $2.614 \mathrm{E}-1$ & $2.384 \mathrm{E}-1$ & $5.886 \mathrm{E}-1$ & $1.441 \mathrm{E}+0$ \\
\hline$[\mathrm{Ne} v] \lambda 14.5 \ldots \ldots \ldots \ldots \ldots \ldots \ldots$ & $3.827 \mathrm{E}-1$ & $2.175 \mathrm{E}-1$ & $1.252 \mathrm{E}-2$ & $2.033 \mathrm{E}-5$ & $0.000 \mathrm{E}+0$ \\
\hline$[\mathrm{Ne}$ III] $\lambda 15.5 \ldots \ldots \ldots \ldots \ldots \ldots \ldots$ & $2.025 \mathrm{E}+0$ & $2.240 \mathrm{E}+0$ & $2.389 \mathrm{E}+0$ & $1.993 \mathrm{E}+0$ & $1.140 \mathrm{E}+0$ \\
\hline$[\mathrm{S}$ III] $\lambda 18.7 \ldots \ldots \ldots \ldots \ldots \ldots \ldots$ & $6.320 \mathrm{E}-1$ & $6.390 \mathrm{E}-1$ & $9.737 \mathrm{E}-1$ & $1.260 \mathrm{E}+0$ & $6.948 \mathrm{E}-1$ \\
\hline 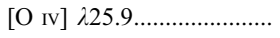 & $3.139 \mathrm{E}+0$ & $2.665 \mathrm{E}+0$ & $1.256 \mathrm{E}+0$ & $9.941 \mathrm{E}-2$ & $9.654 \mathrm{E}-5$ \\
\hline
\end{tabular}


TABLE 16

Dusty IR Line Ratios with Respect to $\mathrm{H} \beta, 2 Z_{\odot}$

\begin{tabular}{|c|c|c|c|c|c|}
\hline \multirow[b]{2}{*}{ LiNE } & \multicolumn{5}{|c|}{$\log U_{0}$} \\
\hline & 0.0 & -1.0 & -2.0 & -3.0 & -4.0 \\
\hline \multicolumn{6}{|c|}{$\alpha=-1.2$} \\
\hline 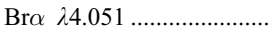 & $6.756 \mathrm{E}-2$ & $6.846 \mathrm{E}-2$ & $7.615 \mathrm{E}-2$ & $8.405 \mathrm{E}-2$ & $9.463 \mathrm{E}-2$ \\
\hline 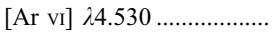 & $4.396 \mathrm{E}-2$ & $4.011 \mathrm{E}-2$ & $1.823 \mathrm{E}-3$ & $0.000 \mathrm{E}+0$ & $0.000 \mathrm{E}+0$ \\
\hline [Ar II] $26.983 \ldots \ldots \ldots \ldots \ldots \ldots . .$. & $1.818 \mathrm{E}-1$ & $1.598 \mathrm{E}-1$ & $9.183 \mathrm{E}-2$ & $1.390 \mathrm{E}-1$ & $2.878 \mathrm{E}-1$ \\
\hline [Ar III] $\lambda 8.983 \ldots \ldots \ldots \ldots \ldots . .$. & $1.695 \mathrm{E}+0$ & $1.467 \mathrm{E}+0$ & $7.517 \mathrm{E}-2$ & $5.999 \mathrm{E}-6$ & $0.000 \mathrm{E}+0$ \\
\hline$[\mathrm{Ne}$ vI $] \lambda 7.652 \ldots \ldots \ldots \ldots \ldots . .$. & $2.218 \mathrm{E}-1$ & $2.462 \mathrm{E}-1$ & $3.926 \mathrm{E}-1$ & $3.999 \mathrm{E}-1$ & $1.456 \mathrm{E}-1$ \\
\hline 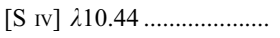 & $1.811 \mathrm{E}+0$ & $2.220 \mathrm{E}+0$ & $3.851 \mathrm{E}+0$ & $3.282 \mathrm{E}-1$ & $9.896 \mathrm{E}-3$ \\
\hline$[\mathrm{Ne}$ II $] \lambda 12.8 \ldots \ldots \ldots \ldots \ldots \ldots . .$. & $1.954 \mathrm{E}+0$ & $1.724 \mathrm{E}+0$ & $1.182 \mathrm{E}+0$ & $1.882 \mathrm{E}+0$ & $3.576 \mathrm{E}+0$ \\
\hline$[\mathrm{Ne} v] \lambda 14.5 \ldots \ldots \ldots \ldots \ldots \ldots \ldots \ldots$ & $6.717 \mathrm{E}-1$ & $6.693 \mathrm{E}-1$ & $2.054 \mathrm{E}-1$ & $2.311 \mathrm{E}-4$ & $0.000 \mathrm{E}+0$ \\
\hline$[\mathrm{Ne}$ III] $\lambda 15.5 \ldots \ldots \ldots \ldots \ldots \ldots . .$. & $5.318 \mathrm{E}+0$ & $5.181 \mathrm{E}+0$ & $5.224 \mathrm{E}+0$ & $5.519 \mathrm{E}+0$ & $4.329 \mathrm{E}+0$ \\
\hline 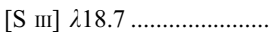 & $2.508 \mathrm{E}+0$ & $2.354 \mathrm{E}+0$ & $2.205 \mathrm{E}+0$ & $3.254 \mathrm{E}+0$ & $2.246 \mathrm{E}+0$ \\
\hline 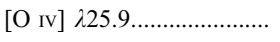 & $5.383 \mathrm{E}+0$ & $5.956 \mathrm{E}+0$ & $5.838 \mathrm{E}+0$ & $3.958 \mathrm{E}-1$ & $5.661 \mathrm{E}-4$ \\
\hline \multicolumn{6}{|c|}{$\alpha=-1.4$} \\
\hline $\operatorname{Br} \alpha \lambda 4.051 \ldots \ldots$. & $6.870 \mathrm{E}-2$ & $7.050 \mathrm{E}-2$ & $7.904 \mathrm{E}-2$ & $8.512 \mathrm{E}-2$ & $9.256 \mathrm{E}-2$ \\
\hline$[\mathrm{Ar}$ vI $] \lambda 4.530 \ldots \ldots \ldots \ldots \ldots . . . . . .$. & $4.671 \mathrm{E}-2$ & $3.764 \mathrm{E}-2$ & $7.493 \mathrm{E}-4$ & $0.000 \mathrm{E}+0$ & $0.000 \mathrm{E}+0$ \\
\hline$[\mathrm{Ar}$ II] $\lambda 6.983 \ldots \ldots \ldots \ldots \ldots \ldots$ & $1.424 \mathrm{E}-1$ & $1.122 \mathrm{E}-1$ & $6.092 \mathrm{E}-2$ & $1.052 \mathrm{E}-1$ & $2.536 \mathrm{E}-1$ \\
\hline 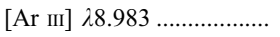 & $1.605 \mathrm{E}+0$ & $1.142 \mathrm{E}+0$ & $2.612 \mathrm{E}-2$ & $1.997 \mathrm{E}-6$ & $0.000 \mathrm{E}+0$ \\
\hline$[\mathrm{Ne} \mathrm{vI}] \lambda 7.652 \ldots \ldots \ldots \ldots \ldots . . . .$. & $2.381 \mathrm{E}-1$ & $2.725 \mathrm{E}-1$ & $4.044 \mathrm{E}-1$ & $3.962 \mathrm{E}-1$ & $1.385 \mathrm{E}-1$ \\
\hline [S IV] $\lambda 10.44 \ldots \ldots \ldots \ldots \ldots \ldots$ & $2.338 \mathrm{E}+0$ & $2.973 \mathrm{E}+0$ & $3.719 \mathrm{E}+0$ & $2.454 \mathrm{E}-1$ & $4.877 \mathrm{E}-3$ \\
\hline$[\mathrm{Ne}$ II $] \lambda 12.8 \ldots \ldots \ldots \ldots \ldots \ldots$ & $1.663 \mathrm{E}+0$ & $1.340 \mathrm{E}+0$ & $8.565 \mathrm{E}-1$ & $1.450 \mathrm{E}+0$ & $3.084 \mathrm{E}+0$ \\
\hline$[\mathrm{Ne} v] \lambda 14.5 \ldots \ldots \ldots \ldots \ldots \ldots \ldots$ & $7.286 \mathrm{E}-1$ & $6.710 \mathrm{E}-1$ & $1.130 \mathrm{E}-1$ & $1.203 \mathrm{E}-4$ & $0.000 \mathrm{E}+0$ \\
\hline$[\mathrm{Ne}$ III] $\lambda 15.5 \ldots \ldots \ldots \ldots \ldots \ldots$ & $4.760 \mathrm{E}+0$ & $4.648 \mathrm{E}+0$ & $4.879 \mathrm{E}+0$ & $4.792 \mathrm{E}+0$ & $3.471 \mathrm{E}+0$ \\
\hline 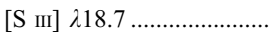 & $2.128 \mathrm{E}+0$ & $1.948 \mathrm{E}+0$ & $2.034 \mathrm{E}+0$ & $2.826 \mathrm{E}+0$ & $1.795 \mathrm{E}+0$ \\
\hline$[\mathrm{O}$ IV $] ~ \lambda 25.9 \ldots \ldots \ldots \ldots \ldots \ldots \ldots \ldots \ldots \ldots$ & $6.068 \mathrm{E}+0$ & $6.492 \mathrm{E}+0$ & $4.594 \mathrm{E}+0$ & $2.811 \mathrm{E}-1$ & $3.643 \mathrm{E}-4$ \\
\hline \multicolumn{6}{|c|}{$\alpha=-1.7$} \\
\hline 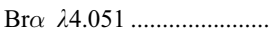 & $7.145 \mathrm{E}-2$ & $7.493 \mathrm{E}-2$ & $8.324 \mathrm{E}-2$ & $8.699 \mathrm{E}-2$ & $9.559 \mathrm{E}-2$ \\
\hline$[\mathrm{Ar} \mathrm{vI}] \lambda 4.530 \ldots \ldots \ldots \ldots \ldots . . .$. & $4.400 \mathrm{E}-2$ & $2.606 \mathrm{E}-2$ & $1.489 \mathrm{E}-4$ & $0.000 \mathrm{E}+0$ & $0.000 \mathrm{E}+0$ \\
\hline$[\mathrm{Ar}$ II] $\lambda 6.983 \ldots \ldots \ldots \ldots \ldots \ldots$ & $8.642 \mathrm{E}-2$ & $5.640 \mathrm{E}-2$ & $3.412 \mathrm{E}-2$ & $7.819 \mathrm{E}-2$ & $2.165 \mathrm{E}-1$ \\
\hline [Ar III] $\lambda 8.983 \ldots \ldots \ldots \ldots \ldots . . .$. & $1.234 \mathrm{E}+0$ & $5.601 \mathrm{E}-1$ & $3.694 \mathrm{E}-3$ & $0.000 \mathrm{E}+0$ & $0.000 \mathrm{E}+0$ \\
\hline 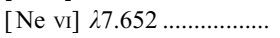 & $2.603 \mathrm{E}-1$ & $3.018 \mathrm{E}-1$ & $4.063 \mathrm{E}-1$ & $3.747 \mathrm{E}-1$ & $1.181 \mathrm{E}-1$ \\
\hline 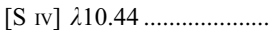 & $3.176 \mathrm{E}+0$ & $3.860 \mathrm{E}+0$ & $3.083 \mathrm{E}+0$ & $1.516 \mathrm{E}-1$ & $6.474 \mathrm{E}-3$ \\
\hline 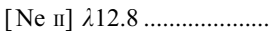 & $1.141 \mathrm{E}+0$ & $7.868 \mathrm{E}-1$ & $5.471 \mathrm{E}-1$ & $1.132 \mathrm{E}+0$ & $2.584 \mathrm{E}+0$ \\
\hline 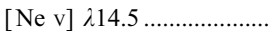 & $7.126 \mathrm{E}-1$ & $5.140 \mathrm{E}-1$ & $3.457 \mathrm{E}-2$ & $4.061 \mathrm{E}-5$ & $0.000 \mathrm{E}+0$ \\
\hline$[\mathrm{Ne}$ III] $\lambda 15.5 \ldots \ldots \ldots \ldots \ldots \ldots$ & $4.188 \mathrm{E}+0$ & $4.251 \mathrm{E}+0$ & $4.458 \mathrm{E}+0$ & $3.876 \mathrm{E}+0$ & $2.386 \mathrm{E}+0$ \\
\hline 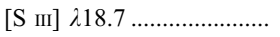 & $1.660 \mathrm{E}+0$ & $1.556 \mathrm{E}+0$ & $1.910 \mathrm{E}+0$ & $2.357 \mathrm{E}+0$ & $1.331 \mathrm{E}+0$ \\
\hline 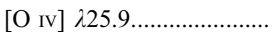 & $6.298 \mathrm{E}+0$ & $5.897 \mathrm{E}+0$ & $2.836 \mathrm{E}+0$ & $1.553 \mathrm{E}-1$ & $1.786 \mathrm{E}-4$ \\
\hline \multicolumn{6}{|c|}{$\alpha=-2.0$} \\
\hline 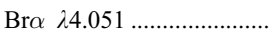 & $7.408 \mathrm{E}-2$ & $7.999 \mathrm{E}-2$ & $8.697 \mathrm{E}-2$ & $8.869 \mathrm{E}-2$ & $9.779 \mathrm{E}-2$ \\
\hline$[$ Ar vI] $\lambda 4.530 \ldots \ldots \ldots \ldots \ldots . . .$. & $3.839 \mathrm{E}-2$ & $1.330 \mathrm{E}-2$ & $2.590 \mathrm{E}-5$ & $0.000 \mathrm{E}+0$ & $0.000 \mathrm{E}+0$ \\
\hline$[\mathrm{Ar}$ II] $\lambda 6.983 \ldots \ldots \ldots \ldots \ldots \ldots$ & $5.323 \mathrm{E}-2$ & $2.697 \mathrm{E}-2$ & $2.421 \mathrm{E}-2$ & $6.833 \mathrm{E}-2$ & $1.948 \mathrm{E}-1$ \\
\hline$[$ Ar III $] \lambda 8.983 \ldots \ldots \ldots \ldots \ldots . . . . . .$. & $9.656 \mathrm{E}-1$ & $1.949 \mathrm{E}-1$ & $3.932 \mathrm{E}-4$ & $0.000 \mathrm{E}+0$ & $0.000 \mathrm{E}+0$ \\
\hline$[\mathrm{Ne}$ vI $] \lambda 7.652 \ldots \ldots \ldots \ldots \ldots . . . .$. & $2.701 \mathrm{E}-1$ & $3.155 \mathrm{E}-1$ & $3.930 \mathrm{E}-1$ & $3.422 \mathrm{E}-1$ & $9.681 \mathrm{E}-2$ \\
\hline [S rv] $\lambda 10.44 \ldots \ldots \ldots \ldots \ldots \ldots$ & $3.710 \mathrm{E}+0$ & $4.237 \mathrm{E}+0$ & $2.282 \mathrm{E}+0$ & $9.161 \mathrm{E}-2$ & $2.114 \mathrm{E}-3$ \\
\hline$[\mathrm{Ne}$ II $] \lambda 12.8 \ldots \ldots \ldots \ldots \ldots \ldots$ & $7.630 \mathrm{E}-1$ & $4.268 \mathrm{E}-1$ & $4.373 \mathrm{E}-1$ & $1.038 \mathrm{E}+0$ & $2.274 \mathrm{E}+0$ \\
\hline$[\mathrm{Ne} v] \lambda 14.5 \ldots \ldots \ldots \ldots \ldots \ldots \ldots \ldots \ldots \ldots \ldots \ldots$ & $6.257 \mathrm{E}-1$ & $2.997 \mathrm{E}-1$ & $8.443 \mathrm{E}-3$ & $1.194 \mathrm{E}-5$ & $0.000 \mathrm{E}+0$ \\
\hline$[\mathrm{Ne}$ III] $\lambda 15.5 \ldots \ldots \ldots \ldots \ldots \ldots$ & $3.967 \mathrm{E}+0$ & $4.135 \mathrm{E}+0$ & $4.014 \mathrm{E}+0$ & $3.101 \mathrm{E}+0$ & $1.604 \mathrm{E}+0$ \\
\hline 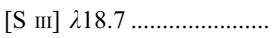 & $1.412 \mathrm{E}+0$ & $1.379 \mathrm{E}+0$ & $1.857 \mathrm{E}+0$ & $2.043 \mathrm{E}+0$ & $1.054 \mathrm{E}+0$ \\
\hline$[\mathrm{O}$ IV $] \lambda 25.9 \ldots \ldots \ldots \ldots \ldots \ldots \ldots$ & $5.814 \mathrm{E}+0$ & $4.513 \mathrm{E}+0$ & $1.622 \mathrm{E}+0$ & $7.825 \mathrm{E}-2$ & $8.506 \mathrm{E}-5$ \\
\hline
\end{tabular}


TABLE 17

Dusty IR Line Ratios with Respect to $\mathrm{H} \beta, 4 Z_{\odot}$

\begin{tabular}{|c|c|c|c|c|c|}
\hline \multirow[b]{2}{*}{ LiNE } & \multicolumn{5}{|c|}{$\log U_{0}$} \\
\hline & 0.0 & -1.0 & -2.0 & -3.0 & -4.0 \\
\hline \multicolumn{6}{|c|}{$\alpha=-1.2$} \\
\hline 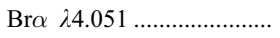 & $7.169 \mathrm{E}-2$ & $7.301 \mathrm{E}-2$ & $8.374 \mathrm{E}-2$ & $9.239 \mathrm{E}-2$ & $1.027 \mathrm{E}-1$ \\
\hline$[\mathrm{Ar}$ vI $] \lambda 4.530 \ldots \ldots \ldots \ldots \ldots . . . . . .$. & $6.792 \mathrm{E}-2$ & $5.985 \mathrm{E}-2$ & $1.301 \mathrm{E}-3$ & $0.000 \mathrm{E}+0$ & $0.000 \mathrm{E}+0$ \\
\hline$[\mathrm{Ar}$ II] $\lambda 6.983 \ldots \ldots \ldots \ldots \ldots \ldots$ & $3.553 \mathrm{E}-1$ & $3.036 \mathrm{E}-1$ & $1.549 \mathrm{E}-1$ & $2.373 \mathrm{E}-1$ & $4.844 \mathrm{E}-1$ \\
\hline 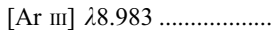 & $2.525 \mathrm{E}+0$ & $2.072 \mathrm{E}+0$ & $5.298 \mathrm{E}-2$ & $2.211 \mathrm{E}-6$ & $0.000 \mathrm{E}+0$ \\
\hline$[\mathrm{Ne} v \mathrm{vI}] \lambda 7.652 \ldots \ldots \ldots \ldots \ldots . . . .$. & $4.641 \mathrm{E}-1$ & $5.159 \mathrm{E}-1$ & $7.255 \mathrm{E}-1$ & $5.953 \mathrm{E}-1$ & $1.427 \mathrm{E}-1$ \\
\hline 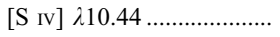 & $3.613 \mathrm{E}+0$ & $4.360 \mathrm{E}+0$ & $5.736 \mathrm{E}+0$ & $2.738 \mathrm{E}-1$ & $5.102 \mathrm{E}-3$ \\
\hline$[\mathrm{Ne}$ II $] \lambda 12.8 \ldots \ldots \ldots \ldots \ldots \ldots$ & $3.706 \mathrm{E}+0$ & $3.179 \mathrm{E}+0$ & $1.888 \mathrm{E}+0$ & $2.776 \mathrm{E}+0$ & $5.318 \mathrm{E}+0$ \\
\hline$[\mathrm{Ne} v] \lambda 14.5 \ldots \ldots \ldots \ldots \ldots \ldots \ldots$ & $1.112 \mathrm{E}+0$ & $1.080 \mathrm{E}+0$ & $1.983 \mathrm{E}-1$ & $1.209 \mathrm{E}-4$ & $0.000 \mathrm{E}+0$ \\
\hline$[\mathrm{Ne}$ III] $\lambda 15.5 \ldots \ldots \ldots \ldots \ldots \ldots . .$. & $1.015 \mathrm{E}+1$ & $9.834 \mathrm{E}+0$ & $9.115 \mathrm{E}+0$ & $8.434 \mathrm{E}+0$ & $5.735 \mathrm{E}+0$ \\
\hline$[\mathrm{S}$ III] $\lambda 18.7 \ldots \ldots \ldots \ldots \ldots \ldots . .$. & $4.957 \mathrm{E}+0$ & $4.635 \mathrm{E}+0$ & $4.146 \mathrm{E}+0$ & $5.031 \mathrm{E}+0$ & $3.002 \mathrm{E}+0$ \\
\hline 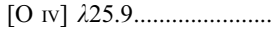 & $9.703 \mathrm{E}+0$ & $1.054 \mathrm{E}+1$ & $7.818 \mathrm{E}+0$ & $2.709 \mathrm{E}-1$ & $4.999 \mathrm{E}-4$ \\
\hline \multicolumn{6}{|c|}{$\alpha=-1.4$} \\
\hline 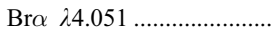 & $7.391 \mathrm{E}-2$ & $7.631 \mathrm{E}-2$ & $8.776 \mathrm{E}-2$ & $9.360 \mathrm{E}-2$ & $1.051 \mathrm{E}-1$ \\
\hline 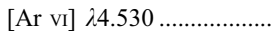 & $7.072 \mathrm{E}-2$ & $5.320 \mathrm{E}-2$ & $4.009 \mathrm{E}-4$ & $0.000 \mathrm{E}+0$ & $0.000 \mathrm{E}+0$ \\
\hline$[\mathrm{Ar}$ II] $\lambda 6.983 \ldots \ldots \ldots \ldots \ldots \ldots$ & $2.748 \mathrm{E}-1$ & $2.065 \mathrm{E}-1$ & $1.039 \mathrm{E}-1$ & $1.939 \mathrm{E}-1$ & $4.311 \mathrm{E}-1$ \\
\hline$[$ Ar III] $\lambda 8.983 \ldots \ldots \ldots \ldots \ldots . .$. & $2.292 \mathrm{E}+0$ & $1.476 \mathrm{E}+0$ & $1.322 \mathrm{E}-2$ & $0.000 \mathrm{E}+0$ & $0.000 \mathrm{E}+0$ \\
\hline$[\mathrm{Ne}$ VI] $\lambda 7.652 \ldots \ldots \ldots \ldots \ldots . .$. & $5.009 \mathrm{E}-1$ & $5.641 \mathrm{E}-1$ & $7.220 \mathrm{E}-1$ & $5.679 \mathrm{E}-1$ & $1.292 \mathrm{E}-1$ \\
\hline$[\mathrm{S}$ IV] $\lambda 10.44 \ldots \ldots \ldots \ldots \ldots \ldots$ & $4.453 \mathrm{E}+0$ & $5.367 \mathrm{E}+0$ & $5.055 \mathrm{E}+0$ & $1.945 \mathrm{E}-1$ & $3.563 \mathrm{E}-3$ \\
\hline$[\mathrm{Ne}$ II] $\lambda 12.8 \ldots \ldots \ldots \ldots \ldots \ldots$ & $3.062 \mathrm{E}+0$ & $2.345 \mathrm{E}+0$ & $1.382 \mathrm{E}+0$ & $2.311 \mathrm{E}+0$ & $4.686 \mathrm{E}+0$ \\
\hline$[\mathrm{Ne} v] \lambda 14.5 \ldots \ldots \ldots \ldots \ldots \ldots \ldots \ldots \ldots \ldots$ & $1.175 \mathrm{E}+0$ & $1.012 \mathrm{E}+0$ & $8.708 \mathrm{E}-2$ & $5.842 \mathrm{E}-5$ & $0.000 \mathrm{E}+0$ \\
\hline$[\mathrm{Ne}$ III] $\lambda 15.5 \ldots \ldots \ldots \ldots \ldots \ldots . .$. & $9.137 \mathrm{E}+0$ & $8.777 \mathrm{E}+0$ & $8.282 \mathrm{E}+0$ & $7.158 \mathrm{E}+0$ & $4.376 \mathrm{E}+0$ \\
\hline 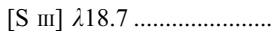 & $4.286 \mathrm{E}+0$ & $3.899 \mathrm{E}+0$ & $3.799 \mathrm{E}+0$ & $4.311 \mathrm{E}+0$ & $2.378 \mathrm{E}+0$ \\
\hline$[\mathrm{O}$ Iv $] \lambda 25.9 \ldots \ldots \ldots \ldots \ldots \ldots \ldots$ & $1.064 \mathrm{E}+1$ & $1.088 \mathrm{E}+1$ & $5.748 \mathrm{E}+0$ & $1.818 \mathrm{E}-1$ & $2.985 \mathrm{E}-4$ \\
\hline \multicolumn{6}{|c|}{$\alpha=-1.7$} \\
\hline 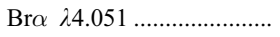 & $7.793 \mathrm{E}-2$ & $8.298 \mathrm{E}-2$ & $9.340 \mathrm{E}-2$ & $9.585 \mathrm{E}-2$ & $1.049 \mathrm{E}-1$ \\
\hline 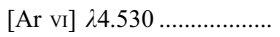 & $6.326 \mathrm{E}-2$ & $3.251 \mathrm{E}-2$ & $5.834 \mathrm{E}-5$ & $0.000 \mathrm{E}+0$ & $0.000 \mathrm{E}+0$ \\
\hline$[\mathrm{Ar}$ II] $\lambda 6.983 \ldots \ldots \ldots \ldots \ldots \ldots . .$. & $1.617 \mathrm{E}-1$ & $9.941 \mathrm{E}-2$ & $6.492 \mathrm{E}-2$ & $1.626 \mathrm{E}-1$ & $3.830 \mathrm{E}-1$ \\
\hline 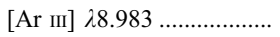 & $1.621 \mathrm{E}+0$ & $6.143 \mathrm{E}-1$ & $1.162 \mathrm{E}-3$ & $0.000 \mathrm{E}+0$ & $0.000 \mathrm{E}+0$ \\
\hline$[\mathrm{Ne}$ vI $] \lambda 7.652 \ldots \ldots \ldots \ldots \ldots . . .$. & $5.429 \mathrm{E}-1$ & $6.006 \mathrm{E}-1$ & $6.842 \mathrm{E}-1$ & $5.060 \mathrm{E}-1$ & $1.079 \mathrm{E}-1$ \\
\hline [S IV] $\lambda 10.44 \ldots \ldots \ldots \ldots \ldots \ldots$ & $5.540 \mathrm{E}+0$ & $6.185 \mathrm{E}+0$ & $3.613 \mathrm{E}+0$ & $1.126 \mathrm{E}-1$ & $5.342 \mathrm{E}-3$ \\
\hline$[\mathrm{Ne}$ II $] \lambda 12.8 \ldots \ldots \ldots \ldots \ldots \ldots$ & $1.987 \mathrm{E}+0$ & $1.300 \mathrm{E}+0$ & $1.003 \mathrm{E}+0$ & $2.038 \mathrm{E}+0$ & $4.100 \mathrm{E}+0$ \\
\hline$[\mathrm{Ne} v] \lambda 14.5 \ldots \ldots \ldots \ldots \ldots \ldots$ & $1.075 \mathrm{E}+0$ & $6.800 \mathrm{E}-1$ & $1.928 \mathrm{E}-2$ & $1.776 \mathrm{E}-5$ & $0.000 \mathrm{E}+0$ \\
\hline$[\mathrm{Ne}$ III] $\lambda 15.5 \ldots \ldots \ldots \ldots \ldots \ldots$ & $7.983 \mathrm{E}+0$ & $7.767 \mathrm{E}+0$ & $7.119 \mathrm{E}+0$ & $5.505 \mathrm{E}+0$ & $2.900 \mathrm{E}+0$ \\
\hline$[\mathrm{S}$ III] $\lambda 18.7 \ldots \ldots \ldots \ldots \ldots \ldots . .$. & $3.425 \mathrm{E}+0$ & $3.135 \mathrm{E}+0$ & $3.489 \mathrm{E}+0$ & $3.532 \mathrm{E}+0$ & $1.819 \mathrm{E}+0$ \\
\hline$[\mathrm{O}$ Iv $] 225.9 \ldots \ldots \ldots \ldots \ldots \ldots \ldots \ldots \ldots$ & $1.061 \mathrm{E}+1$ & $9.254 \mathrm{E}+0$ & $3.296 \mathrm{E}+0$ & $9.251 \mathrm{E}-2$ & $1.399 \mathrm{E}-4$ \\
\hline \multicolumn{6}{|c|}{$\alpha=-2.0$} \\
\hline 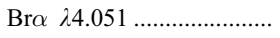 & $8.181 \mathrm{E}-2$ & $9.029 \mathrm{E}-2$ & $9.827 \mathrm{E}-2$ & $9.801 \mathrm{E}-2$ & $1.062 \mathrm{E}-1$ \\
\hline$[$ Ar vI] $\lambda 4.530 \ldots \ldots \ldots \ldots \ldots . . . . . . .$. & $5.271 \mathrm{E}-2$ & $1.383 \mathrm{E}-2$ & $7.540 \mathrm{E}-6$ & $0.000 \mathrm{E}+0$ & $0.000 \mathrm{E}+0$ \\
\hline 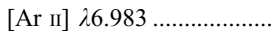 & $9.750 \mathrm{E}-2$ & $4.764 \mathrm{E}-2$ & $5.458 \mathrm{E}-2$ & $1.522 \mathrm{E}-1$ & $3.493 \mathrm{E}-1$ \\
\hline 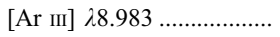 & $1.205 \mathrm{E}+0$ & $1.738 \mathrm{E}-1$ & $8.399 \mathrm{E}-5$ & $0.000 \mathrm{E}+0$ & $0.000 \mathrm{E}+0$ \\
\hline$[\mathrm{Ne}$ vI $] \lambda 7.652 \ldots \ldots \ldots \ldots \ldots . . . . .$. & $5.562 \mathrm{E}-1$ & $5.980 \mathrm{E}-1$ & $6.205 \mathrm{E}-1$ & $4.337 \mathrm{E}-1$ & $8.626 \mathrm{E}-2$ \\
\hline 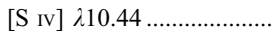 & $6.095 \mathrm{E}+0$ & $6.075 \mathrm{E}+0$ & $2.268 \mathrm{E}+0$ & $6.611 \mathrm{E}-2$ & $2.378 \mathrm{E}-3$ \\
\hline$[\mathrm{Ne}$ II $] \lambda 12.8 \ldots \ldots \ldots \ldots \ldots \ldots$ & $1.295 \mathrm{E}+0$ & $7.365 \mathrm{E}-1$ & $9.417 \mathrm{E}-1$ & $1.962 \mathrm{E}+0$ & $3.637 \mathrm{E}+0$ \\
\hline$[\mathrm{Ne} v] \lambda 14.5 \ldots \ldots \ldots \ldots \ldots \ldots \ldots$ & $8.925 \mathrm{E}-1$ & $3.374 \mathrm{E}-1$ & $3.622 \mathrm{E}-3$ & $5.010 \mathrm{E}-6$ & $0.000 \mathrm{E}+0$ \\
\hline$[\mathrm{Ne}$ III] $\lambda 15.5 \ldots \ldots \ldots \ldots \ldots \ldots . .$. & $7.440 \mathrm{E}+0$ & $7.150 \mathrm{E}+0$ & $5.929 \mathrm{E}+0$ & $4.125 \mathrm{E}+0$ & $1.863 \mathrm{E}+0$ \\
\hline$[\mathrm{S}$ III] $\lambda 18.7 \ldots \ldots \ldots \ldots \ldots \ldots \ldots$ & $2.946 \mathrm{E}+0$ & $2.756 \mathrm{E}+0$ & $3.276 \mathrm{E}+0$ & $3.000 \mathrm{E}+0$ & $1.463 \mathrm{E}+0$ \\
\hline 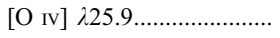 & $9.593 \mathrm{E}+0$ & $6.635 \mathrm{E}+0$ & $1.785 \mathrm{E}+0$ & $4.456 \mathrm{E}-2$ & $6.206 \mathrm{E}-5$ \\
\hline
\end{tabular}


TABLE 18

Dust-free UV Line Ratios with Respect to $\mathrm{H} \beta, 0.25 Z_{\odot}$

\begin{tabular}{|c|c|c|c|c|}
\hline \multirow[b]{2}{*}{ LiNE } & \multicolumn{4}{|c|}{$\log U_{0}$} \\
\hline & -1.0 & -2.0 & -3.0 & -4.0 \\
\hline \multicolumn{5}{|c|}{$\alpha=-1.2$} \\
\hline C III $\lambda 977$......................... & $2.436 \mathrm{E}-01$ & $2.981 \mathrm{E}-01$ & $1.816 \mathrm{E}-02$ & $3.224 \mathrm{E}-04$ \\
\hline $\mathrm{N}$ III 2991 ......................... & $3.760 \mathrm{E}-02$ & $4.178 \mathrm{E}-02$ & $2.485 \mathrm{E}-03$ & $6.870 \mathrm{E}-06$ \\
\hline $\mathrm{O}$ vi $\lambda \lambda 1032,1037 \ldots \ldots \ldots \ldots$ & $8.149 \mathrm{E}+00$ & $1.692 \mathrm{E}-01$ & $8.534 \mathrm{E}-05$ & $0.000 \mathrm{E}+00$ \\
\hline 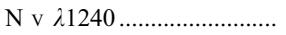 & $5.498 \mathrm{E}-01$ & $9.207 \mathrm{E}-02$ & $2.891 \mathrm{E}-04$ & $0.000 \mathrm{E}+00$ \\
\hline $\mathrm{Si}$ IV+O Iv] $\lambda 1400 \ldots \ldots \ldots \ldots . .$. & $6.388 \mathrm{E}-01$ & $2.989 \mathrm{E}-01$ & $2.189 \mathrm{E}-02$ & $4.685 \mathrm{E}-05$ \\
\hline 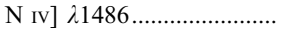 & $2.150 \mathrm{E}-01$ & $8.577 \mathrm{E}-02$ & $1.409 \mathrm{E}-03$ & $0.000 \mathrm{E}+00$ \\
\hline 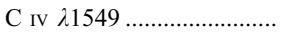 & $6.358 \mathrm{E}+00$ & $2.859 \mathrm{E}+00$ & $4.334 \mathrm{E}-02$ & $5.360 \mathrm{E}-05$ \\
\hline 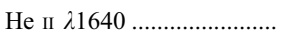 & $2.195 \mathrm{E}+00$ & $2.115 \mathrm{E}+00$ & $1.607 \mathrm{E}+00$ & $6.769 \mathrm{E}-01$ \\
\hline $\mathrm{O}$ III] $\lambda 1664 \ldots \ldots \ldots \ldots \ldots \ldots \ldots$ & $1.156 \mathrm{E}+00$ & $4.410 \mathrm{E}-01$ & $6.507 \mathrm{E}-02$ & $6.663 \mathrm{E}-04$ \\
\hline $\mathrm{N}$ III] $\lambda 1750 \ldots \ldots \ldots \ldots \ldots \ldots \ldots$ & $1.583 \mathrm{E}-02$ & 4.204E-02 & $8.505 \mathrm{E}-03$ & $8.103 \mathrm{E}-05$ \\
\hline C III] $\lambda 1909 \ldots \ldots \ldots \ldots \ldots \ldots \ldots . .$. & $9.547 \mathrm{E}-01$ & $2.033 \mathrm{E}+00$ & $5.586 \mathrm{E}-01$ & $4.515 \mathrm{E}-02$ \\
\hline $\mathrm{C}$ II] $\lambda 2326 \ldots \ldots \ldots \ldots \ldots \ldots \ldots \ldots$ & $9.787 \mathrm{E}-03$ & $7.305 \mathrm{E}-02$ & $1.971 \mathrm{E}-01$ & $2.284 \mathrm{E}-01$ \\
\hline 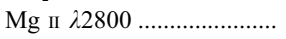 & $3.323 \mathrm{E}-02$ & $1.435 \mathrm{E}-01$ & $3.143 \mathrm{E}-01$ & $4.489 \mathrm{E}-01$ \\
\hline$[\mathrm{Ne} \mathrm{v}] \lambda 3426 \ldots \ldots \ldots \ldots \ldots \ldots$ & $7.645 \mathrm{E}-01$ & $3.813 \mathrm{E}-01$ & $1.574 \mathrm{E}-03$ & $0.000 \mathrm{E}+00$ \\
\hline$[\mathrm{Ne}$ III] $\lambda 3869$.................... & $1.776 \mathrm{E}+00$ & $1.259 \mathrm{E}+00$ & $8.633 \mathrm{E}-01$ & 4.149E-01 \\
\hline \multicolumn{5}{|c|}{$\alpha=-1.4$} \\
\hline 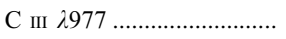 & $2.514 \mathrm{E}-01$ & $2.743 \mathrm{E}-01$ & $1.660 \mathrm{E}-02$ & $3.110 \mathrm{E}-04$ \\
\hline 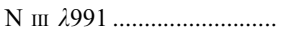 & $3.516 \mathrm{E}-02$ & $3.634 \mathrm{E}-02$ & $2.301 \mathrm{E}-03$ & $6.998 \mathrm{E}-06$ \\
\hline O vi $\lambda \lambda 1032,1037 \ldots \ldots \ldots \ldots . .$. & $5.099 \mathrm{E}+00$ & $7.249 \mathrm{E}-02$ & $3.394 \mathrm{E}-05$ & $0.000 \mathrm{E}+00$ \\
\hline $\mathrm{N}$ v $\lambda 1240 \ldots \ldots \ldots \ldots \ldots \ldots \ldots \ldots \ldots \ldots \ldots \ldots \ldots \ldots \ldots$ & $4.627 \mathrm{E}-01$ & $5.464 \mathrm{E}-02$ & $1.625 \mathrm{E}-04$ & $0.000 \mathrm{E}+00$ \\
\hline $\mathrm{Si}$ IV+O Iv] $\lambda 1400 \ldots \ldots \ldots \ldots . .$. & $4.384 \mathrm{E}-01$ & $2.459 \mathrm{E}-01$ & $1.891 \mathrm{E}-02$ & $3.882 \mathrm{E}-05$ \\
\hline $\mathrm{N}$ rv] $\lambda 1486 \ldots \ldots \ldots \ldots \ldots \ldots \ldots$ & $2.170 \mathrm{E}-01$ & $7.185 \mathrm{E}-02$ & $1.158 \mathrm{E}-03$ & $0.000 \mathrm{E}+00$ \\
\hline 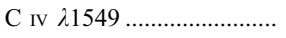 & $6.704 \mathrm{E}+00$ & $2.383 \mathrm{E}+00$ & $3.420 \mathrm{E}-02$ & $4.416 \mathrm{E}-05$ \\
\hline He II $\lambda 1640 \ldots \ldots \ldots \ldots \ldots \ldots \ldots . .$. & $1.776 \mathrm{E}+00$ & $1.720 \mathrm{E}+00$ & $1.354 \mathrm{E}+00$ & $5.776 \mathrm{E}-01$ \\
\hline 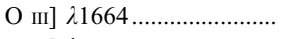 & $9.362 \mathrm{E}-01$ & 4.213E-01 & $6.433 \mathrm{E}-02$ & $6.773 \mathrm{E}-04$ \\
\hline $\mathrm{N}$ III] $\lambda 1750 \ldots \ldots \ldots \ldots \ldots \ldots \ldots$ & $1.715 \mathrm{E}-02$ & $4.079 \mathrm{E}-02$ & $8.319 \mathrm{E}-03$ & $8.449 \mathrm{E}-05$ \\
\hline 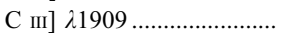 & $1.085 \mathrm{E}+00$ & $2.108 \mathrm{E}+00$ & $5.430 \mathrm{E}-01$ & $4.428 \mathrm{E}-02$ \\
\hline 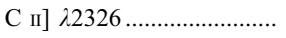 & $7.930 \mathrm{E}-03$ & $6.298 \mathrm{E}-02$ & $1.742 \mathrm{E}-01$ & $2.032 \mathrm{E}-01$ \\
\hline 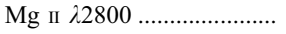 & $3.083 \mathrm{E}-02$ & $1.326 \mathrm{E}-01$ & $2.778 \mathrm{E}-01$ & $3.847 \mathrm{E}-01$ \\
\hline$[\mathrm{Ne} v] \quad \lambda 3426 \ldots \ldots \ldots \ldots \ldots \ldots \ldots$ & $6.549 \mathrm{E}-01$ & $2.598 \mathrm{E}-01$ & $9.903 \mathrm{E}-04$ & $0.000 \mathrm{E}+00$ \\
\hline$[\mathrm{Ne}$ III] $\lambda 3869 \ldots \ldots \ldots \ldots \ldots . .$. & $1.737 \mathrm{E}+00$ & $1.323 \mathrm{E}+00$ & $8.197 \mathrm{E}-01$ & $3.609 \mathrm{E}-01$ \\
\hline \multicolumn{5}{|c|}{$\alpha=-1.7$} \\
\hline C III $\lambda 977$.......................... & $2.166 \mathrm{E}-01$ & $1.976 \mathrm{E}-01$ & $1.311 \mathrm{E}-02$ & $2.708 \mathrm{E}-04$ \\
\hline $\mathrm{N}$ III $2991 \ldots \ldots \ldots \ldots \ldots \ldots \ldots . .$. & $2.342 \mathrm{E}-02$ & $2.514 \mathrm{E}-02$ & $1.834 \mathrm{E}-03$ & $6.379 \mathrm{E}-06$ \\
\hline O vi $\lambda \lambda 1032,1037 \ldots \ldots \ldots \ldots . . .$. & $2.088 \mathrm{E}+00$ & $1.681 \mathrm{E}-02$ & $7.076 \mathrm{E}-06$ & $0.000 \mathrm{E}+00$ \\
\hline 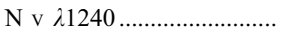 & $2.896 \mathrm{E}-01$ & $2.092 \mathrm{E}-02$ & $5.941 \mathrm{E}-05$ & $0.000 \mathrm{E}+00$ \\
\hline $\mathrm{Si} \mathrm{IV+O} \mathrm{Iv]} \lambda 1400 \ldots \ldots \ldots \ldots . .$. & $2.481 \mathrm{E}-01$ & $1.687 \mathrm{E}-01$ & $1.377 \mathrm{E}-02$ & $2.748 \mathrm{E}-05$ \\
\hline $\mathrm{N}$ IV] $\lambda 1486 \ldots \ldots \ldots \ldots \ldots \ldots \ldots$ & $1.676 \mathrm{E}-01$ & $4.617 \mathrm{E}-02$ & $7.603 \mathrm{E}-04$ & $0.000 \mathrm{E}+00$ \\
\hline $\mathrm{C}$ IV $\lambda 1549 \ldots \ldots \ldots \ldots \ldots \ldots \ldots$ & $5.857 \mathrm{E}+00$ & $1.530 \mathrm{E}+00$ & $2.154 \mathrm{E}-02$ & $3.005 \mathrm{E}-05$ \\
\hline 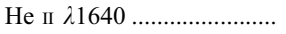 & $1.247 \mathrm{E}+00$ & $1.219 \mathrm{E}+00$ & $9.962 \mathrm{E}-01$ & 4.273E-01 \\
\hline 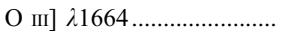 & $5.989 \mathrm{E}-01$ & $3.464 \mathrm{E}-01$ & $5.755 \mathrm{E}-02$ & $6.206 \mathrm{E}-04$ \\
\hline $\mathrm{N}$ III] $\lambda 1750 \ldots \ldots \ldots \ldots \ldots \ldots \ldots \ldots$ & $1.590 \mathrm{E}-02$ & $3.453 \mathrm{E}-02$ & $7.383 \mathrm{E}-03$ & $8.056 \mathrm{E}-05$ \\
\hline 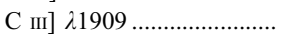 & $1.148 \mathrm{E}+00$ & $1.903 \mathrm{E}+00$ & $4.857 \mathrm{E}-01$ & $4.014 \mathrm{E}-02$ \\
\hline $\mathrm{C}$ II] $\lambda 2326 \ldots \ldots \ldots \ldots \ldots \ldots \ldots$ & $6.330 \mathrm{E}-03$ & $5.246 \mathrm{E}-02$ & $1.527 \mathrm{E}-01$ & $1.781 \mathrm{E}-01$ \\
\hline 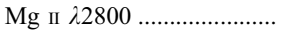 & $2.678 \mathrm{E}-02$ & $1.179 \mathrm{E}-01$ & $2.574 \mathrm{E}-01$ & $3.453 \mathrm{E}-01$ \\
\hline 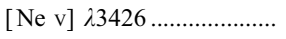 & $4.909 \mathrm{E}-01$ & $1.221 \mathrm{E}-01$ & $4.194 \mathrm{E}-04$ & $0.000 \mathrm{E}+00$ \\
\hline$[\mathrm{Ne}$ III] $\lambda 3869 \ldots \ldots \ldots \ldots \ldots \ldots . .$. & $1.567 \mathrm{E}+00$ & $1.317 \mathrm{E}+00$ & $7.458 \mathrm{E}-01$ & $2.876 \mathrm{E}-01$ \\
\hline \multicolumn{5}{|c|}{$\alpha=-2.0$} \\
\hline C III $\lambda 977$.......................... & $1.507 \mathrm{E}-01$ & $1.233 \mathrm{E}-01$ & $9.581 \mathrm{E}-03$ & $2.201 \mathrm{E}-04$ \\
\hline 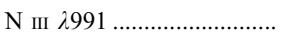 & $1.410 \mathrm{E}-02$ & $1.578 \mathrm{E}-02$ & $1.339 \mathrm{E}-03$ & $5.280 \mathrm{E}-06$ \\
\hline $\mathrm{O}$ VI $\lambda \lambda 1032,1037 \ldots \ldots \ldots \ldots$ & $6.805 \mathrm{E}-01$ & $3.284 \mathrm{E}-03$ & $0.000 \mathrm{E}+00$ & $0.000 \mathrm{E}+00$ \\
\hline 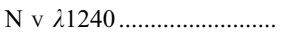 & $1.558 \mathrm{E}-01$ & $6.977 \mathrm{E}-03$ & $1.934 \mathrm{E}-05$ & $0.000 \mathrm{E}+00$ \\
\hline $\mathrm{Si}$ IV+O IV] $\lambda 1400 \ldots \ldots \ldots \ldots . .$. & $2.193 \mathrm{E}-01$ & $9.005 \mathrm{E}-02$ & $9.341 \mathrm{E}-03$ & $1.899 \mathrm{E}-05$ \\
\hline 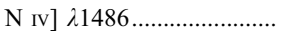 & $1.117 \mathrm{E}-01$ & $2.612 \mathrm{E}-02$ & $4.505 \mathrm{E}-04$ & $0.000 \mathrm{E}+00$ \\
\hline 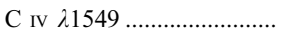 & $4.251 \mathrm{E}+00$ & $8.598 \mathrm{E}-01$ & $1.251 \mathrm{E}-02$ & $1.891 \mathrm{E}-05$ \\
\hline 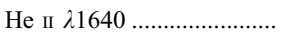 & $8.644 \mathrm{E}-01$ & $8.434 \mathrm{E}-01$ & $7.051 \mathrm{E}-01$ & $3.000 \mathrm{E}-01$ \\
\hline $\mathrm{O}$ III] $\lambda 1664 \ldots \ldots \ldots \ldots \ldots \ldots \ldots$ & $3.885 \mathrm{E}-01$ & $2.641 \mathrm{E}-01$ & $4.757 \mathrm{E}-02$ & $5.192 \mathrm{E}-04$ \\
\hline 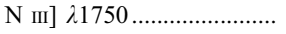 & $1.380 \mathrm{E}-02$ & $2.718 \mathrm{E}-02$ & $6.121 \mathrm{E}-03$ & 7.023E-05 \\
\hline 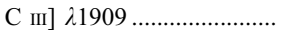 & $1.083 \mathrm{E}+00$ & $1.538 \mathrm{E}+00$ & $4.118 \mathrm{E}-01$ & $3.433 \mathrm{E}-02$ \\
\hline 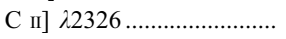 & $5.643 \mathrm{E}-03$ & $4.627 \mathrm{E}-02$ & $1.419 \mathrm{E}-01$ & $1.619 \mathrm{E}-01$ \\
\hline 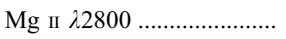 & $2.345 \mathrm{E}-02$ & $1.068 \mathrm{E}-01$ & $2.605 \mathrm{E}-01$ & $3.427 \mathrm{E}-01$ \\
\hline$[\mathrm{Ne} \mathrm{v}] \lambda 3426 \ldots \ldots \ldots \ldots \ldots \ldots$ & $3.324 \mathrm{E}-01$ & $4.780 \mathrm{E}-02$ & $1.548 \mathrm{E}-04$ & $0.000 \mathrm{E}+00$ \\
\hline$[\mathrm{Ne}$ III] $\lambda 33869 \ldots \ldots \ldots \ldots \ldots \ldots$ & $1.392 \mathrm{E}+00$ & $1.231 \mathrm{E}+00$ & $6.635 \mathrm{E}-01$ & $2.235 \mathrm{E}-01$ \\
\hline
\end{tabular}


TABLE 19

Dust-free UV Line Ratios with Respect to $\mathrm{H} \beta, 0.5 Z_{\odot}$

\begin{tabular}{|c|c|c|c|c|}
\hline \multirow[b]{2}{*}{ LiNE } & \multicolumn{4}{|c|}{$\log U_{0}$} \\
\hline & -1.0 & -2.0 & -3.0 & -4.0 \\
\hline \multicolumn{5}{|c|}{$\alpha=-1.2$} \\
\hline 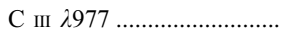 & $2.054 \mathrm{E}-01$ & $3.388 \mathrm{E}-01$ & $2.189 \mathrm{E}-02$ & $4.521 \mathrm{E}-04$ \\
\hline 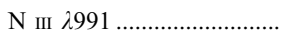 & $3.209 \mathrm{E}-02$ & $4.553 \mathrm{E}-02$ & $2.845 \mathrm{E}-03$ & $8.965 \mathrm{E}-06$ \\
\hline O VI $\lambda \lambda 1032,1037 \ldots \ldots \ldots \ldots . .$. & $1.035 \mathrm{E}+01$ & $2.083 \mathrm{E}-01$ & $9.726 \mathrm{E}-05$ & $0.000 \mathrm{E}+00$ \\
\hline $\mathrm{N}$ v $\lambda 1240 \ldots \ldots \ldots \ldots \ldots \ldots \ldots \ldots \ldots \ldots \ldots \ldots \ldots \ldots \ldots$ & $6.987 \mathrm{E}-01$ & $1.168 \mathrm{E}-01$ & $3.521 \mathrm{E}-04$ & $0.000 \mathrm{E}+00$ \\
\hline $\mathrm{Si}$ Iv+O Iv] $\lambda 1400 \ldots \ldots \ldots \ldots . . .$. & $6.964 \mathrm{E}-01$ & $2.798 \mathrm{E}-01$ & $3.033 \mathrm{E}-02$ & $7.002 \mathrm{E}-05$ \\
\hline $\mathrm{N}$ Iv] $\lambda 1486 \ldots \ldots \ldots \ldots \ldots \ldots \ldots$ & $2.359 \mathrm{E}-01$ & $1.116 \mathrm{E}-01$ & $1.837 \mathrm{E}-03$ & $0.000 \mathrm{E}+00$ \\
\hline C IV $\lambda 1549 \ldots \ldots \ldots \ldots \ldots \ldots \ldots$ & $7.151 \mathrm{E}+00$ & $4.094 \mathrm{E}+00$ & $6.017 \mathrm{E}-02$ & $8.333 \mathrm{E}-05$ \\
\hline 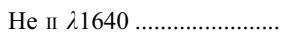 & $2.242 \mathrm{E}+00$ & $2.147 \mathrm{E}+00$ & $1.642 \mathrm{E}+00$ & $7.035 \mathrm{E}-01$ \\
\hline O III] $\lambda 1664 \ldots \ldots \ldots \ldots \ldots \ldots \ldots$ & $1.224 \mathrm{E}+00$ & $6.200 \mathrm{E}-01$ & $9.636 \mathrm{E}-02$ & $1.065 \mathrm{E}-03$ \\
\hline $\mathrm{N}$ III] $\lambda 1750 \ldots \ldots \ldots \ldots \ldots \ldots \ldots$ & $1.750 \mathrm{E}-02$ & $5.727 \mathrm{E}-02$ & $1.205 \mathrm{E}-02$ & $1.237 \mathrm{E}-04$ \\
\hline 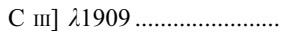 & $1.093 \mathrm{E}+00$ & $2.992 \mathrm{E}+00$ & $8.643 \mathrm{E}-01$ & $7.502 \mathrm{E}-02$ \\
\hline $\mathrm{C}$ II] $\lambda 2326 \ldots \ldots \ldots \ldots \ldots \ldots$ & $1.462 \mathrm{E}-02$ & $1.219 \mathrm{E}-01$ & $3.361 \mathrm{E}-01$ & $3.795 \mathrm{E}-01$ \\
\hline Mg II $\lambda 2800 \ldots \ldots \ldots \ldots \ldots \ldots \ldots$ & $5.594 \mathrm{E}-02$ & $2.554 \mathrm{E}-01$ & $5.256 \mathrm{E}-01$ & $6.699 \mathrm{E}-01$ \\
\hline$[\mathrm{Ne} v] \lambda 3426 \ldots \ldots \ldots \ldots \ldots \ldots \ldots$ & $1.249 \mathrm{E}+00$ & $6.018 \mathrm{E}-01$ & $2.489 \mathrm{E}-03$ & $0.000 \mathrm{E}+00$ \\
\hline$[\mathrm{Ne}$ III] $\lambda 3869 \ldots \ldots \ldots \ldots \ldots \ldots$ & $2.588 \mathrm{E}+00$ & $2.208 \mathrm{E}+00$ & $1.527 \mathrm{E}+00$ & $7.404 \mathrm{E}-01$ \\
\hline \multicolumn{5}{|c|}{$\alpha=-1.4$} \\
\hline 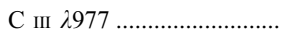 & $1.904 \mathrm{E}-01$ & $2.559 \mathrm{E}-01$ & $1.765 \mathrm{E}-02$ & $4.125 \mathrm{E}-04$ \\
\hline 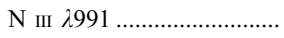 & $2.532 \mathrm{E}-02$ & $3.244 \mathrm{E}-02$ & $2.321 \mathrm{E}-03$ & $8.622 \mathrm{E}-06$ \\
\hline O vi $\lambda \lambda 1032,1037 \ldots \ldots \ldots \ldots . .$. & $5.918 \mathrm{E}+00$ & $7.879 \mathrm{E}-02$ & $3.431 \mathrm{E}-05$ & $0.000 \mathrm{E}+00$ \\
\hline 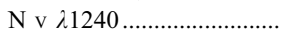 & $5.384 \mathrm{E}-01$ & $6.165 \mathrm{E}-02$ & $1.783 \mathrm{E}-04$ & $0.000 \mathrm{E}+00$ \\
\hline $\mathrm{Si}$ IV $+\mathrm{O}$ IV] $\lambda 1400 \ldots \ldots \ldots \ldots . . .$. & $4.399 \mathrm{E}-01$ & $2.124 \mathrm{E}-01$ & $2.406 \mathrm{E}-02$ & $5.662 \mathrm{E}-05$ \\
\hline $\mathrm{N}$ Iv] $\lambda 1486 \ldots \ldots \ldots \ldots \ldots \ldots \ldots$ & $2.067 \mathrm{E}-01$ & $8.176 \mathrm{E}-02$ & $1.386 \mathrm{E}-03$ & $0.000 \mathrm{E}+00$ \\
\hline 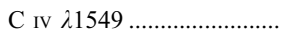 & $6.654 \mathrm{E}+00$ & $3.031 \mathrm{E}+00$ & $4.359 \mathrm{E}-02$ & $6.582 \mathrm{E}-05$ \\
\hline 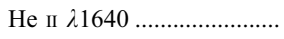 & $1.777 \mathrm{E}+00$ & $1.735 \mathrm{E}+00$ & $1.379 \mathrm{E}+00$ & $5.985 \mathrm{E}-01$ \\
\hline 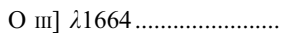 & $8.504 \mathrm{E}-01$ & $5.063 \mathrm{E}-01$ & $8.791 \mathrm{E}-02$ & $1.045 \mathrm{E}-03$ \\
\hline 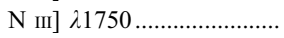 & $1.659 \mathrm{E}-02$ & $4.841 \mathrm{E}-02$ & $1.092 \mathrm{E}-02$ & $1.245 \mathrm{E}-04$ \\
\hline $\mathrm{C}$ III] $\lambda 1909 \ldots \ldots \ldots \ldots \ldots \ldots \ldots$ & $1.109 \mathrm{E}+00$ & $2.734 \mathrm{E}+00$ & $7.858 \mathrm{E}-01$ & $7.142 \mathrm{E}-02$ \\
\hline $\mathrm{C}$ II] $22326 \ldots \ldots \ldots \ldots \ldots \ldots \ldots$ & $1.115 \mathrm{E}-02$ & $9.719 \mathrm{E}-02$ & $2.859 \mathrm{E}-01$ & $3.375 \mathrm{E}-01$ \\
\hline $\mathrm{Mg}$ II $\lambda 2800 \ldots \ldots \ldots \ldots \ldots \ldots$ & $4.880 \mathrm{E}-02$ & $2.237 \mathrm{E}-01$ & $4.585 \mathrm{E}-01$ & $5.924 \mathrm{E}-01$ \\
\hline$[\mathrm{Ne} v] \lambda 3426 \ldots \ldots \ldots \ldots \ldots \ldots$ & $1.027 \mathrm{E}+00$ & $3.847 \mathrm{E}-01$ & $1.489 \mathrm{E}-03$ & $0.000 \mathrm{E}+00$ \\
\hline$[\mathrm{Ne}$ III] $\lambda 3869 \ldots \ldots \ldots \ldots \ldots . .$. & $2.321 \mathrm{E}+00$ & $2.135 \mathrm{E}+00$ & $1.403 \mathrm{E}+00$ & $6.382 \mathrm{E}-01$ \\
\hline \multicolumn{5}{|c|}{$\alpha=-1.7$} \\
\hline 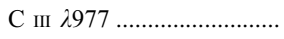 & $1.363 \mathrm{E}-01$ & $1.393 \mathrm{E}-01$ & $1.127 \mathrm{E}-02$ & $3.294 \mathrm{E}-04$ \\
\hline $\mathrm{N}$ III $\lambda 991 \ldots \ldots \ldots \ldots \ldots \ldots . .$. & $1.391 \mathrm{E}-02$ & $1.689 \mathrm{E}-02$ & $1.492 \mathrm{E}-03$ & $7.194 \mathrm{E}-06$ \\
\hline O vi $\lambda \lambda 1032,1037 \ldots \ldots \ldots \ldots . . .$. & $2.143 \mathrm{E}+00$ & $1.511 \mathrm{E}-02$ & $5.835 \mathrm{E}-06$ & $0.000 \mathrm{E}+00$ \\
\hline $\mathrm{N}$ v $\lambda 1240 \ldots \ldots \ldots \ldots \ldots \ldots \ldots \ldots$ & $3.056 \mathrm{E}-01$ & $1.993 \mathrm{E}-02$ & $5.463 \mathrm{E}-05$ & $0.000 \mathrm{E}+00$ \\
\hline Si IV+O IV] $\lambda 1400 \ldots \ldots \ldots \ldots$ & $2.293 \mathrm{E}-01$ & $1.288 \mathrm{E}-01$ & $1.515 \mathrm{E}-02$ & $3.592 \mathrm{E}-05$ \\
\hline $\mathrm{N}$ iv] $\lambda 1486 \ldots \ldots \ldots \ldots \ldots \ldots \ldots$ & $1.360 \mathrm{E}-01$ & $4.318 \mathrm{E}-02$ & $7.875 \mathrm{E}-04$ & $0.000 \mathrm{E}+00$ \\
\hline 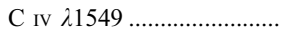 & $5.020 \mathrm{E}+00$ & $1.623 \mathrm{E}+00$ & $2.375 \mathrm{E}-02$ & $4.199 \mathrm{E}-05$ \\
\hline 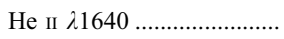 & $1.227 \mathrm{E}+00$ & $1.220 \mathrm{E}+00$ & $1.011 \mathrm{E}+00$ & $4.408 \mathrm{E}-01$ \\
\hline 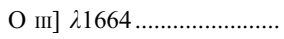 & $4.519 \mathrm{E}-01$ & $3.323 \mathrm{E}-01$ & $6.871 \mathrm{E}-02$ & $9.075 \mathrm{E}-04$ \\
\hline $\mathrm{N}$ III] $\lambda 1750 \ldots \ldots \ldots \ldots \ldots \ldots \ldots$ & $1.332 \mathrm{E}-02$ & $3.343 \mathrm{E}-02$ & $8.519 \mathrm{E}-03$ & $1.124 \mathrm{E}-04$ \\
\hline 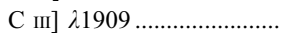 & $1.027 \mathrm{E}+00$ & $2.045 \mathrm{E}+00$ & $6.284 \mathrm{E}-01$ & $6.177 \mathrm{E}-02$ \\
\hline $\mathrm{C}$ II] $22326 \ldots \ldots \ldots \ldots \ldots$ & $8.143 \mathrm{E}-03$ & $7.132 \mathrm{E}-02$ & $2.343 \mathrm{E}-01$ & $2.927 \mathrm{E}-01$ \\
\hline 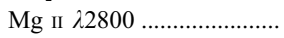 & $3.942 \mathrm{E}-02$ & $1.823 \mathrm{E}-01$ & $4.117 \mathrm{E}-01$ & $5.506 \mathrm{E}-01$ \\
\hline$[\mathrm{Ne} v] \lambda 3426 \ldots \ldots \ldots \ldots \ldots \ldots \ldots$ & $7.212 \mathrm{E}-01$ & $1.643 \mathrm{E}-01$ & $5.812 \mathrm{E}-04$ & $0.000 \mathrm{E}+00$ \\
\hline$[\mathrm{Ne}$ III] $\lambda 3869 \ldots \ldots \ldots \ldots \ldots \ldots$ & $1.902 \mathrm{E}+00$ & $1.884 \mathrm{E}+00$ & $1.205 \mathrm{E}+00$ & $4.993 \mathrm{E}-01$ \\
\hline \multicolumn{5}{|c|}{$\alpha=-2.0$} \\
\hline $\mathrm{C}_{\text {III }} 2977 \ldots \ldots \ldots \ldots \ldots \ldots \ldots$ & $7.969 \mathrm{E}-02$ & $6.656 \mathrm{E}-02$ & $6.543 \mathrm{E}-03$ & $2.450 \mathrm{E}-04$ \\
\hline 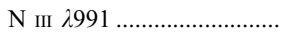 & $7.434 \mathrm{E}-03$ & $8.074 \mathrm{E}-03$ & $8.603 \mathrm{E}-04$ & $5.443 \mathrm{E}-06$ \\
\hline $\mathrm{O}$ vi $\lambda \lambda 1032,1037 \ldots \ldots \ldots \ldots$ & $6.217 \mathrm{E}-01$ & $2.416 \mathrm{E}-03$ & $0.000 \mathrm{E}+00$ & $0.000 \mathrm{E}+00$ \\
\hline 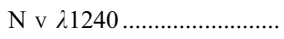 & $1.508 \mathrm{E}-01$ & $5.600 \mathrm{E}-03$ & $1.461 \mathrm{E}-05$ & $0.000 \mathrm{E}+00$ \\
\hline $\mathrm{Si}$ IV+O Iv] $\lambda 1400 \ldots \ldots \ldots \ldots . . .$. & $1.324 \mathrm{E}-01$ & $7.312 \mathrm{E}-02$ & $8.724 \mathrm{E}-03$ & $2.270 \mathrm{E}-05$ \\
\hline $\mathrm{N}$ Iv] $\lambda 1486 \ldots \ldots \ldots \ldots \ldots \ldots \ldots$ & $8.117 \mathrm{E}-02$ & $2.012 \mathrm{E}-02$ & $3.971 \mathrm{E}-04$ & $0.000 \mathrm{E}+00$ \\
\hline 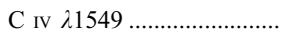 & $3.191 \mathrm{E}+00$ & $7.568 \mathrm{E}-01$ & $1.173 \mathrm{E}-02$ & $2.477 \mathrm{E}-05$ \\
\hline He II $\lambda 1640 \ldots \ldots \ldots \ldots \ldots \ldots \ldots$ & $8.447 \mathrm{E}-01$ & $8.422 \mathrm{E}-01$ & $7.140 \mathrm{E}-01$ & $3.081 \mathrm{E}-01$ \\
\hline 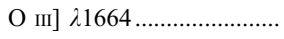 & $2.528 \mathrm{E}-01$ & $2.056 \mathrm{E}-01$ & $4.906 \mathrm{E}-02$ & $7.190 \mathrm{E}-04$ \\
\hline $\mathrm{N}$ III] $\lambda 1750 \ldots \ldots \ldots \ldots \ldots \ldots \ldots$ & $1.035 \mathrm{E}-02$ & $2.161 \mathrm{E}-02$ & $6.138 \mathrm{E}-03$ & $9.273 \mathrm{E}-05$ \\
\hline 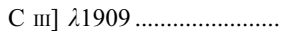 & $8.575 \mathrm{E}-01$ & $1.382 \mathrm{E}+00$ & $4.723 \mathrm{E}-01$ & $5.032 \mathrm{E}-02$ \\
\hline 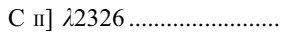 & $6.733 \mathrm{E}-03$ & $5.570 \mathrm{E}-02$ & $2.018 \mathrm{E}-01$ & $2.607 \mathrm{E}-01$ \\
\hline $\mathrm{Mg}$ II $\lambda 2800 \ldots \ldots \ldots \ldots \ldots \ldots \ldots$ & $3.264 \mathrm{E}-02$ & $1.524 \mathrm{E}-01$ & $3.981 \mathrm{E}-01$ & $5.503 \mathrm{E}-01$ \\
\hline$[\mathrm{Ne} \mathrm{v}] \lambda 3426 \ldots \ldots \ldots \ldots \ldots \ldots$ & 4.562E-01 & $5.841 \mathrm{E}-02$ & $1.963 \mathrm{E}-04$ & $0.000 \mathrm{E}+00$ \\
\hline$[\mathrm{Ne}$ III] $\lambda 3869 \ldots \ldots \ldots \ldots \ldots \ldots$ & $1.567 \mathrm{E}+00$ & $1.580 \mathrm{E}+00$ & $1.006 \mathrm{E}+00$ & $3.792 \mathrm{E}-01$ \\
\hline
\end{tabular}


TABLE 20

Dust-free UV Line Ratios with Respect to $\mathrm{H} \beta, 1 Z_{\odot}$

\begin{tabular}{|c|c|c|c|c|}
\hline \multirow[b]{2}{*}{ LiNE } & \multicolumn{4}{|c|}{$\log U_{0}$} \\
\hline & -1.0 & -2.0 & -3.0 & -4.0 \\
\hline \multicolumn{5}{|c|}{$\alpha=-1.2$} \\
\hline C III 2977 ........................... & $1.429 \mathrm{E}-01$ & $2.427 \mathrm{E}-01$ & $1.339 \mathrm{E}-02$ & $3.721 \mathrm{E}-04$ \\
\hline 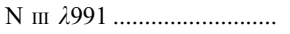 & $3.781 \mathrm{E}-02$ & $5.423 \mathrm{E}-02$ & $2.872 \mathrm{E}-03$ & $1.145 \mathrm{E}-05$ \\
\hline O vi $\lambda \lambda 1032,1037 \ldots \ldots \ldots \ldots . .$. & $1.082 \mathrm{E}+01$ & $1.803 \mathrm{E}-01$ & $5.535 \mathrm{E}-05$ & $0.000 \mathrm{E}+00$ \\
\hline 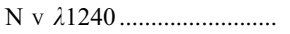 & $1.313 \mathrm{E}+00$ & $1.873 \mathrm{E}-01$ & $4.188 \mathrm{E}-04$ & $0.000 \mathrm{E}+00$ \\
\hline $\mathrm{Si}$ rv+O Iv] $\lambda 1400 \ldots \ldots \ldots \ldots . .$. & $6.131 \mathrm{E}-01$ & $2.754 \mathrm{E}-01$ & $2.282 \mathrm{E}-02$ & $6.873 \mathrm{E}-05$ \\
\hline 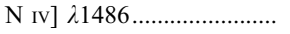 & $3.621 \mathrm{E}-01$ & $1.840 \mathrm{E}-01$ & $2.591 \mathrm{E}-03$ & $1.016 \mathrm{E}-06$ \\
\hline $\mathrm{C}$ IV $\lambda 1549$ & $6.193 \mathrm{E}+00$ & $4.341 \mathrm{E}+00$ & $5.194 \mathrm{E}-02$ & $8.841 \mathrm{E}-05$ \\
\hline He II $\lambda 1640 \ldots \ldots \ldots \ldots \ldots \ldots \ldots$ & $2.237 \mathrm{E}+00$ & $2.172 \mathrm{E}+00$ & $1.701 \mathrm{E}+00$ & $7.462 \mathrm{E}-01$ \\
\hline 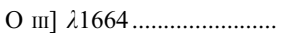 & $9.687 \mathrm{E}-01$ & $5.969 \mathrm{E}-01$ & $9.402 \mathrm{E}-02$ & $1.205 \mathrm{E}-03$ \\
\hline $\mathrm{N}$ III] $\lambda 1750 \ldots \ldots \ldots \ldots \ldots \ldots \ldots . .$. & $2.596 \mathrm{E}-02$ & $9.718 \mathrm{E}-02$ & $1.987 \mathrm{E}-02$ & $2.339 \mathrm{E}-04$ \\
\hline 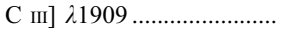 & $9.427 \mathrm{E}-01$ & $3.226 \mathrm{E}+00$ & $9.375 \mathrm{E}-01$ & $9.402 \mathrm{E}-02$ \\
\hline $\mathrm{C}$ II] $\lambda 2326 \ldots \ldots \ldots \ldots \ldots \ldots \ldots \ldots$ & $1.708 \mathrm{E}-02$ & $1.758 \mathrm{E}-01$ & $4.827 \mathrm{E}-01$ & $5.376 \mathrm{E}-01$ \\
\hline $\mathrm{Mg}$ II $\lambda 2800 \ldots \ldots \ldots \ldots \ldots \ldots \ldots$ & $7.565 \mathrm{E}-02$ & $3.984 \mathrm{E}-01$ & $7.772 \mathrm{E}-01$ & $8.807 \mathrm{E}-01$ \\
\hline$[\mathrm{Ne} v] \lambda 3426 \ldots \ldots \ldots \ldots \ldots \ldots$ & $1.820 \mathrm{E}+00$ & $7.900 \mathrm{E}-01$ & $2.973 \mathrm{E}-03$ & $0.000 \mathrm{E}+00$ \\
\hline$[\mathrm{Ne}$ III] $\lambda 3869 \ldots \ldots \ldots \ldots \ldots \ldots$ & $3.045 \mathrm{E}+00$ & $3.217 \mathrm{E}+00$ & $2.291 \mathrm{E}+00$ & $1.156 \mathrm{E}+00$ \\
\hline \multicolumn{5}{|c|}{$\alpha=-1.4$} \\
\hline 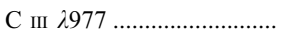 & $1.187 \mathrm{E}-01$ & $1.423 \mathrm{E}-01$ & $8.011 \mathrm{E}-03$ & $2.935 \mathrm{E}-04$ \\
\hline 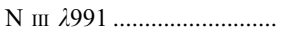 & $2.530 \mathrm{E}-02$ & $2.988 \mathrm{E}-02$ & $1.730 \mathrm{E}-03$ & $9.447 \mathrm{E}-06$ \\
\hline O vi $\lambda \lambda 1032,1037 \ldots \ldots \ldots \ldots . .$. & $5.601 \mathrm{E}+00$ & $5.652 \mathrm{E}-02$ & $1.476 \mathrm{E}-05$ & $0.000 \mathrm{E}+00$ \\
\hline $\mathrm{N}$ v $\lambda 1240 \ldots \ldots \ldots \ldots \ldots \ldots \ldots \ldots \ldots$ & $9.240 \mathrm{E}-01$ & $8.362 \mathrm{E}-02$ & $1.656 \mathrm{E}-04$ & $0.000 \mathrm{E}+00$ \\
\hline $\mathrm{Si}$ Iv+O Iv] $\lambda 1400 \ldots \ldots \ldots \ldots . .$. & $3.614 \mathrm{E}-01$ & $2.465 \mathrm{E}-01$ & $1.479 \mathrm{E}-02$ & $4.834 \mathrm{E}-05$ \\
\hline $\mathrm{N}$ Iv] $\lambda 1486 \ldots \ldots \ldots \ldots \ldots \ldots \ldots \ldots$ & $2.792 \mathrm{E}-01$ & $1.122 \mathrm{E}-01$ & $1.599 \mathrm{E}-03$ & $0.000 \mathrm{E}+00$ \\
\hline $\mathrm{C}$ IV $\lambda 1549 \ldots \ldots \ldots \ldots \ldots \ldots \ldots$ & $5.326 \mathrm{E}+00$ & $2.702 \mathrm{E}+00$ & $3.065 \mathrm{E}-02$ & $6.253 \mathrm{E}-05$ \\
\hline 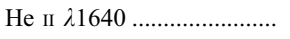 & $1.746 \mathrm{E}+00$ & $1.739 \mathrm{E}+00$ & $1.420 \mathrm{E}+00$ & $6.312 \mathrm{E}-01$ \\
\hline $\mathrm{O}$ III] $\lambda 1664 \ldots \ldots \ldots \ldots \ldots \ldots \ldots \ldots \ldots$ & $5.710 \mathrm{E}-01$ & $3.863 \mathrm{E}-01$ & $7.074 \mathrm{E}-02$ & $1.078 \mathrm{E}-03$ \\
\hline $\mathrm{N}$ III] $\lambda 1750 \ldots \ldots \ldots \ldots \ldots \ldots \ldots$ & $2.160 \mathrm{E}-02$ & $6.686 \mathrm{E}-02$ & $1.496 \mathrm{E}-02$ & $2.144 \mathrm{E}-04$ \\
\hline 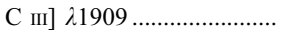 & $8.663 \mathrm{E}-01$ & $2.436 \mathrm{E}+00$ & $7.256 \mathrm{E}-01$ & $8.277 \mathrm{E}-02$ \\
\hline 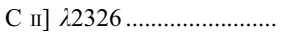 & $1.238 \mathrm{E}-02$ & $1.244 \mathrm{E}-01$ & $3.731 \mathrm{E}-01$ & $4.623 \mathrm{E}-01$ \\
\hline 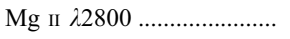 & $6.152 \mathrm{E}-02$ & $3.166 \mathrm{E}-01$ & $6.395 \mathrm{E}-01$ & $7.857 \mathrm{E}-01$ \\
\hline$[\mathrm{Ne} v] \lambda 3426 \ldots \ldots \ldots \ldots \ldots \ldots$ & $1.432 \mathrm{E}+00$ & $4.598 \mathrm{E}-01$ & $1.588 \mathrm{E}-03$ & $0.000 \mathrm{E}+00$ \\
\hline$[\mathrm{Ne}$ III $] \lambda 3869 \ldots \ldots \ldots \ldots \ldots \ldots$ & $2.476 \mathrm{E}+00$ & $2.716 \mathrm{E}+00$ & $1.939 \mathrm{E}+00$ & $9.650 \mathrm{E}-01$ \\
\hline \multicolumn{5}{|c|}{$\alpha=-1.7$} \\
\hline C III $\lambda 977 \ldots \ldots \ldots \ldots \ldots \ldots \ldots . .$. & $6.956 \mathrm{E}-02$ & $5.543 \mathrm{E}-02$ & $3.204 \mathrm{E}-03$ & $1.870 \mathrm{E}-04$ \\
\hline 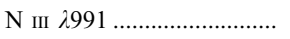 & $1.192 \mathrm{E}-02$ & $1.109 \mathrm{E}-02$ & $6.858 \mathrm{E}-04$ & $6.237 \mathrm{E}-06$ \\
\hline $\mathrm{O}$ vi $\lambda \lambda 1032,1037 \ldots \ldots \ldots \ldots$ & $1.759 \mathrm{E}+00$ & $8.074 \mathrm{E}-03$ & $1.040 \mathrm{E}-06$ & $0.000 \mathrm{E}+00$ \\
\hline 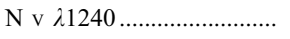 & $4.711 \mathrm{E}-01$ & $2.111 \mathrm{E}-02$ & $3.411 \mathrm{E}-05$ & $0.000 \mathrm{E}+00$ \\
\hline $\mathrm{Si}$ IV+O IV] $\lambda 1400 \ldots \ldots \ldots \ldots . .$. & $1.798 \mathrm{E}-01$ & $8.115 \mathrm{E}-02$ & $7.189 \mathrm{E}-03$ & $2.477 \mathrm{E}-05$ \\
\hline $\mathrm{N}$ IV] $\lambda 1486 \ldots \ldots \ldots \ldots \ldots \ldots \ldots$ & $1.619 \mathrm{E}-01$ & $4.617 \mathrm{E}-02$ & $6.562 \mathrm{E}-04$ & $0.000 \mathrm{E}+00$ \\
\hline C Iv $\lambda 1549$ & $3.590 \mathrm{E}+00$ & $1.136 \mathrm{E}+00$ & $1.199 \mathrm{E}-02$ & $3.373 \mathrm{E}-05$ \\
\hline He II $\lambda 1640 \ldots \ldots \ldots \ldots \ldots \ldots \ldots . .$. & $1.192 \mathrm{E}+00$ & $1.217 \mathrm{E}+00$ & $1.036 \mathrm{E}+00$ & $4.612 \mathrm{E}-01$ \\
\hline 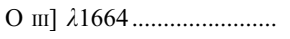 & $2.463 \mathrm{E}-01$ & $1.885 \mathrm{E}-01$ & $4.079 \mathrm{E}-02$ & $8.123 \mathrm{E}-04$ \\
\hline 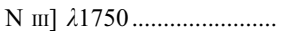 & $1.491 \mathrm{E}-02$ & $3.507 \mathrm{E}-02$ & $8.721 \mathrm{E}-03$ & $1.680 \mathrm{E}-04$ \\
\hline 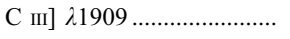 & $6.895 \mathrm{E}-01$ & $1.415 \mathrm{E}+00$ & $4.510 \mathrm{E}-01$ & $6.335 \mathrm{E}-02$ \\
\hline $\mathrm{C}$ II] $\lambda 2326 \ldots \ldots \ldots \ldots \ldots \ldots \ldots \ldots$ & $8.328 \mathrm{E}-03$ & $7.591 \mathrm{E}-02$ & $2.598 \mathrm{E}-01$ & $3.754 \mathrm{E}-01$ \\
\hline 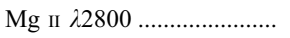 & $4.546 \mathrm{E}-02$ & $2.247 \mathrm{E}-01$ & $5.130 \mathrm{E}-01$ & $7.158 \mathrm{E}-01$ \\
\hline$[\mathrm{Ne} v] \lambda 3426 \ldots \ldots \ldots \ldots \ldots \ldots . .$. & $9.349 \mathrm{E}-01$ & $1.709 \mathrm{E}-01$ & $5.180 \mathrm{E}-04$ & $0.000 \mathrm{E}+00$ \\
\hline$[\mathrm{Ne}$ III] $\lambda 3869$.................... & $1.792 \mathrm{E}+00$ & $2.013 \mathrm{E}+00$ & $1.458 \mathrm{E}+00$ & $7.139 \mathrm{E}-01$ \\
\hline \multicolumn{5}{|c|}{$\alpha=-2.0$} \\
\hline C III 2977 .......................... & $3.484 \mathrm{E}-02$ & $1.888 \mathrm{E}-02$ & $1.198 \mathrm{E}-03$ & $1.103 \mathrm{E}-04$ \\
\hline N III $\lambda 991$......................... & $5.775 \mathrm{E}-03$ & $3.765 \mathrm{E}-03$ & $2.483 \mathrm{E}-04$ & $3.731 \mathrm{E}-06$ \\
\hline O vi $\lambda \lambda 1032,1037 \ldots \ldots \ldots \ldots$ & $4.371 \mathrm{E}-01$ & $9.257 \mathrm{E}-04$ & $0.000 \mathrm{E}+00$ & $0.000 \mathrm{E}+00$ \\
\hline 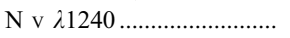 & $2.074 \mathrm{E}-01$ & $4.499 \mathrm{E}-03$ & $6.175 \mathrm{E}-06$ & $0.000 \mathrm{E}+00$ \\
\hline $\mathrm{Si}$ Iv+O Iv] $\lambda 1400 \ldots \ldots \ldots \ldots . .$. & $9.800 \mathrm{E}-02$ & $3.469 \mathrm{E}-02$ & $2.871 \mathrm{E}-03$ & $1.246 \mathrm{E}-05$ \\
\hline $\mathrm{N}$ Iv] $\lambda 1486 \ldots \ldots \ldots \ldots \ldots \ldots \ldots \ldots \ldots$ & $8.907 \mathrm{E}-02$ & $1.668 \mathrm{E}-02$ & $2.402 \mathrm{E}-04$ & $0.000 \mathrm{E}+00$ \\
\hline $\mathrm{C}$ IV $\lambda 1549 \ldots \ldots \ldots \ldots \ldots \ldots \ldots \ldots \ldots$ & $2.051 \mathrm{E}+00$ & $4.120 \mathrm{E}-01$ & $4.276 \mathrm{E}-03$ & $1.682 \mathrm{E}-05$ \\
\hline He II $\lambda 1640 \ldots \ldots \ldots \ldots \ldots \ldots \ldots \ldots . .$. & $8.148 \mathrm{E}-01$ & $8.410 \mathrm{E}-01$ & $7.300 \mathrm{E}-01$ & $3.193 \mathrm{E}-01$ \\
\hline 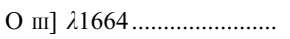 & $1.137 \mathrm{E}-01$ & $8.936 \mathrm{E}-02$ & $2.183 \mathrm{E}-02$ & $5.588 \mathrm{E}-04$ \\
\hline $\mathrm{N}$ III] $\lambda 1750 \ldots \ldots \ldots \ldots \ldots \ldots \ldots \ldots$ & $1.002 \mathrm{E}-02$ & $1.745 \mathrm{E}-02$ & $4.764 \mathrm{E}-03$ & $1.202 \mathrm{E}-04$ \\
\hline 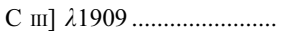 & $4.917 \mathrm{E}-01$ & $7.538 \mathrm{E}-01$ & $2.672 \mathrm{E}-01$ & $4.546 \mathrm{E}-02$ \\
\hline $\mathrm{C}$ II] $\lambda 2326 \ldots \ldots \ldots \ldots \ldots \ldots \ldots \ldots$ & $6.350 \mathrm{E}-03$ & $4.949 \mathrm{E}-02$ & $1.902 \mathrm{E}-01$ & $3.088 \mathrm{E}-01$ \\
\hline $\mathrm{Mg}$ II $\lambda 2800 \ldots \ldots \ldots \ldots \ldots \ldots \ldots \ldots \ldots$ & $3.493 \mathrm{E}-02$ & $1.654 \mathrm{E}-01$ & $4.431 \mathrm{E}-01$ & $6.778 \mathrm{E}-01$ \\
\hline 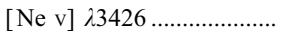 & $5.448 \mathrm{E}-01$ & $5.236 \mathrm{E}-02$ & $1.472 \mathrm{E}-04$ & $0.000 \mathrm{E}+00$ \\
\hline 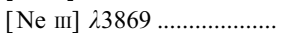 & $1.315 \mathrm{E}+00$ & $1.458 \mathrm{E}+00$ & $1.070 \mathrm{E}+00$ & $5.081 \mathrm{E}-01$ \\
\hline
\end{tabular}


TABLE 21

Dust-free UV Line Ratios with Respect to $\mathrm{H} \beta, 2 Z_{\odot}$

\begin{tabular}{|c|c|c|c|c|}
\hline \multirow[b]{2}{*}{ LiNE } & \multicolumn{4}{|c|}{$\log U_{0}$} \\
\hline & -1.0 & -2.0 & -3.0 & -4.0 \\
\hline \multicolumn{5}{|c|}{$\alpha=-1.2$} \\
\hline 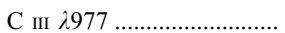 & $9.419 \mathrm{E}-02$ & $1.131 \mathrm{E}-01$ & $1.941 \mathrm{E}-03$ & $7.456 \mathrm{E}-05$ \\
\hline 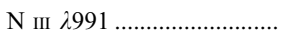 & $4.323 \mathrm{E}-02$ & $4.557 \mathrm{E}-02$ & $7.473 \mathrm{E}-04$ & $3.311 \mathrm{E}-06$ \\
\hline $\mathrm{O}$ vI $\lambda \lambda 1032,1037 \ldots \ldots \ldots \ldots$ & $9.454 \mathrm{E}+00$ & $1.031 \mathrm{E}-01$ & $7.990 \mathrm{E}-06$ & $0.000 \mathrm{E}+00$ \\
\hline $\mathrm{N}$ v $\lambda 1240 \ldots \ldots \ldots \ldots \ldots \ldots \ldots \ldots$ & $2.340 \mathrm{E}+00$ & $2.278 \mathrm{E}-01$ & $1.640 \mathrm{E}-04$ & $0.000 \mathrm{E}+00$ \\
\hline $\mathrm{Si}$ IV+O IV] $\lambda 1400 \ldots \ldots \ldots \ldots . .$. & $4.654 \mathrm{E}-01$ & $1.892 \mathrm{E}-01$ & $6.598 \mathrm{E}-03$ & $2.146 \mathrm{E}-05$ \\
\hline $\left.\mathrm{N}_{\text {IV }}\right] \lambda 1486 \ldots \ldots \ldots \ldots \ldots \ldots \ldots \ldots$ & $5.415 \mathrm{E}-01$ & $2.357 \mathrm{E}-01$ & $1.523 \mathrm{E}-03$ & $0.000 \mathrm{E}+00$ \\
\hline $\mathrm{C}$ IV $\lambda 1549$ & $5.060 \mathrm{E}+00$ & $3.271 \mathrm{E}+00$ & $1.675 \mathrm{E}-02$ & $3.377 \mathrm{E}-05$ \\
\hline 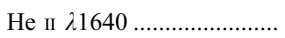 & $2.200 \mathrm{E}+00$ & $2.188 \mathrm{E}+00$ & $1.786 \mathrm{E}+00$ & $7.968 \mathrm{E}-01$ \\
\hline 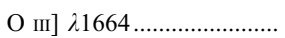 & $6.148 \mathrm{E}-01$ & $3.579 \mathrm{E}-01$ & $3.669 \mathrm{E}-02$ & $5.255 \mathrm{E}-04$ \\
\hline $\mathrm{N}$ III] $\lambda 1750 \ldots \ldots \ldots \ldots \ldots \ldots \ldots \ldots$ & $3.288 \mathrm{E}-02$ & $1.190 \mathrm{E}-01$ & $1.476 \mathrm{E}-02$ & $1.882 \mathrm{E}-04$ \\
\hline 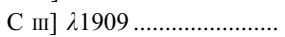 & $5.786 \mathrm{E}-01$ & $2.333 \mathrm{E}+00$ & $4.589 \mathrm{E}-01$ & $5.390 \mathrm{E}-02$ \\
\hline $\mathrm{C}$ II] $\lambda 2326 \ldots \ldots \ldots \ldots \ldots \ldots \ldots \ldots$ & $1.170 \mathrm{E}-02$ & $2.003 \mathrm{E}-01$ & $4.886 \mathrm{E}-01$ & $4.885 \mathrm{E}-01$ \\
\hline 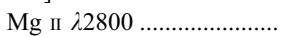 & $6.261 \mathrm{E}-02$ & $4.850 \mathrm{E}-01$ & $8.885 \mathrm{E}-01$ & $8.455 \mathrm{E}-01$ \\
\hline 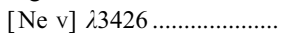 & $2.362 \mathrm{E}+00$ & $8.264 \mathrm{E}-01$ & $2.053 \mathrm{E}-03$ & $0.000 \mathrm{E}+00$ \\
\hline 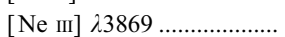 & $2.560 \mathrm{E}+00$ & $3.399 \mathrm{E}+00$ & $2.379 \mathrm{E}+00$ & $1.272 \mathrm{E}+00$ \\
\hline
\end{tabular}

\begin{tabular}{|c|c|c|c|c|}
\hline \multicolumn{5}{|c|}{$\alpha=-1.4$} \\
\hline 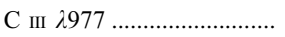 & $6.807 \mathrm{E}-02$ & $5.207 \mathrm{E}-02$ & $7.218 \mathrm{E}-04$ & $4.241 \mathrm{E}-05$ \\
\hline 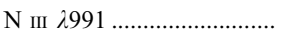 & $2.538 \mathrm{E}-02$ & $1.976 \mathrm{E}-02$ & $2.767 \mathrm{E}-04$ & $1.961 \mathrm{E}-06$ \\
\hline $\mathrm{O}$ VI $\lambda \lambda 1032,1037 \ldots \ldots \ldots \ldots$ & $4.437 \mathrm{E}+00$ & $2.523 \mathrm{E}-02$ & $0.000 \mathrm{E}+00$ & $0.000 \mathrm{E}+00$ \\
\hline $\mathrm{N}$ v $\lambda 1240 \ldots \ldots \ldots \ldots \ldots \ldots \ldots \ldots \ldots$ & $1.510 \mathrm{E}+00$ & $8.282 \mathrm{E}-02$ & $4.359 \mathrm{E}-05$ & $0.000 \mathrm{E}+00$ \\
\hline $\mathrm{Si} \mathrm{Iv+O} \mathrm{Iv]} \lambda 1400 \ldots \ldots \ldots \ldots . .$. & $2.671 \mathrm{E}-01$ & $9.902 \mathrm{E}-02$ & $3.214 \mathrm{E}-03$ & $1.136 \mathrm{E}-05$ \\
\hline $\mathrm{N}$ IV] $\lambda 1486 \ldots \ldots \ldots \ldots \ldots \ldots \ldots \ldots$ & $3.806 \mathrm{E}-01$ & $1.195 \mathrm{E}-01$ & $6.791 \mathrm{E}-04$ & $0.000 \mathrm{E}+00$ \\
\hline $\mathrm{C}$ IV $\lambda 1549 \ldots \ldots \ldots \ldots \ldots \ldots \ldots$ & $4.196 \mathrm{E}+00$ & $1.700 \mathrm{E}+00$ & $7.073 \mathrm{E}-03$ & $1.869 \mathrm{E}-05$ \\
\hline 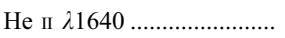 & $1.697 \mathrm{E}+00$ & $1.743 \mathrm{E}+00$ & $1.481 \mathrm{E}+00$ & $6.659 \mathrm{E}-01$ \\
\hline $\left.\mathrm{O}_{\mathrm{III}}\right] \lambda_{16}$ & $3.150 \mathrm{E}-01$ & $1.868 \mathrm{E}-01$ & $2.020 \mathrm{E}-02$ & $3.836 \mathrm{E}-04$ \\
\hline $\mathrm{N}$ III] $\lambda 1750 \ldots \ldots \ldots \ldots \ldots \ldots \ldots$ & $2.457 \mathrm{E}-02$ & $6.709 \mathrm{E}-02$ & $8.241 \mathrm{E}-03$ & $1.410 \mathrm{E}-04$ \\
\hline $\mathrm{C}$ III] $\lambda 1909 \ldots \ldots \ldots \ldots \ldots \ldots \ldots$ & $4.964 \mathrm{E}-01$ & $1.458 \mathrm{E}+00$ & $2.715 \mathrm{E}-01$ & $3.952 \mathrm{E}-02$ \\
\hline $\mathrm{C}$ II] $22326 \ldots \ldots \ldots \ldots \ldots \ldots \ldots$ & $7.646 \mathrm{E}-03$ & $1.225 \mathrm{E}-01$ & $3.150 \mathrm{E}-01$ & $3.697 \mathrm{E}-01$ \\
\hline $\mathrm{Mg}$ II $\lambda 2800 \ldots \ldots \ldots \ldots \ldots \ldots \ldots$ & $4.516 \mathrm{E}-02$ & $3.367 \mathrm{E}-01$ & $6.402 \mathrm{E}-01$ & $7.022 \mathrm{E}-01$ \\
\hline$[\mathrm{Ne} \mathrm{v}] \lambda 3426 \ldots \ldots \ldots \ldots \ldots \ldots$ & $1.774 \mathrm{E}+00$ & $4.284 \mathrm{E}-01$ & $9.153 \mathrm{E}-04$ & $0.000 \mathrm{E}+00$ \\
\hline$[\mathrm{Ne}$ III] $\lambda 3869 \ldots \ldots \ldots \ldots \ldots \ldots$ & $1.832 \mathrm{E}+00$ & $2.448 \mathrm{E}+00$ & $1.738 \mathrm{E}+00$ & $9.668 \mathrm{E}-01$ \\
\hline
\end{tabular}

\begin{tabular}{|c|c|c|c|c|}
\hline \multicolumn{5}{|c|}{$\alpha=-1.7$} \\
\hline C III $\lambda 977$. & $3.377 \mathrm{E}-02$ & $1.340 \mathrm{E}-02$ & $1.551 \mathrm{E}-04$ & $1.700 \mathrm{E}-05$ \\
\hline 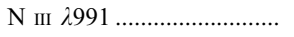 & $1.084 \mathrm{E}-02$ & $4.868 \mathrm{E}-03$ & $5.739 \mathrm{E}-05$ & $0.000 \mathrm{E}+00$ \\
\hline O vi $\lambda \lambda 1032,1037 \ldots \ldots \ldots \ldots . .$. & $1.188 \mathrm{E}+00$ & $2.320 \mathrm{E}-03$ & $0.000 \mathrm{E}+00$ & $0.000 \mathrm{E}+00$ \\
\hline 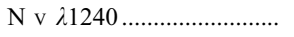 & $6.845 \mathrm{E}-01$ & $1.449 \mathrm{E}-02$ & $5.290 \mathrm{E}-06$ & $0.000 \mathrm{E}+00$ \\
\hline $\mathrm{Si}$ IV+O Iv] $\lambda 1400 \ldots \ldots \ldots \ldots . .$. & $1.303 \mathrm{E}-01$ & $4.609 \mathrm{E}-02$ & $7.720 \mathrm{E}-04$ & $4.083 \mathrm{E}-06$ \\
\hline 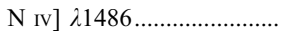 & $2.064 \mathrm{E}-01$ & $3.622 \mathrm{E}-02$ & $1.803 \mathrm{E}-04$ & $0.000 \mathrm{E}+00$ \\
\hline 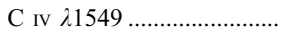 & $2.622 \mathrm{E}+00$ & $5.277 \mathrm{E}-01$ & $1.772 \mathrm{E}-03$ & $7.275 \mathrm{E}-06$ \\
\hline 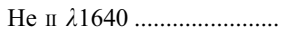 & $1.148 \mathrm{E}+00$ & $1.220 \mathrm{E}+00$ & $1.072 \mathrm{E}+00$ & $4.792 \mathrm{E}-01$ \\
\hline 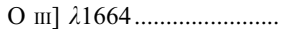 & $1.133 \mathrm{E}-01$ & $6.677 \mathrm{E}-02$ & $7.747 \mathrm{E}-03$ & $2.208 \mathrm{E}-04$ \\
\hline 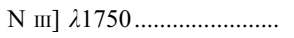 & $1.490 \mathrm{E}-02$ & $2.598 \mathrm{E}-02$ & $3.250 \mathrm{E}-03$ & $8.439 \mathrm{E}-05$ \\
\hline 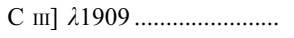 & $3.503 \mathrm{E}-01$ & $6.374 \mathrm{E}-01$ & $1.185 \mathrm{E}-01$ & $2.340 \mathrm{E}-02$ \\
\hline $\mathrm{C}$ II] $\lambda 2326 \ldots \ldots \ldots \ldots \ldots \ldots$ & $4.491 \mathrm{E}-03$ & $5.693 \mathrm{E}-02$ & $1.652 \mathrm{E}-01$ & $2.467 \mathrm{E}-01$ \\
\hline 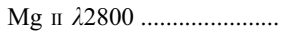 & $2.842 \mathrm{E}-02$ & $1.887 \mathrm{E}-01$ & $4.183 \mathrm{E}-01$ & $5.624 \mathrm{E}-01$ \\
\hline$[\mathrm{Ne} \mathrm{v}] \lambda 3426 \ldots \ldots \ldots \ldots \ldots \ldots$ & $1.068 \mathrm{E}+00$ & $1.312 \mathrm{E}-01$ & $2.354 \mathrm{E}-04$ & $0.000 \mathrm{E}+00$ \\
\hline$[\mathrm{Ne}$ III] $\lambda 3869 \ldots \ldots \ldots \ldots \ldots \ldots . .$. & $1.080 \mathrm{E}+00$ & $1.457 \mathrm{E}+00$ & $1.066 \mathrm{E}+00$ & $6.199 \mathrm{E}-01$ \\
\hline \multicolumn{5}{|c|}{$\alpha=-2.0$} \\
\hline 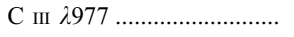 & $1.473 \mathrm{E}-02$ & $2.657 \mathrm{E}-03$ & $3.400 \mathrm{E}-05$ & $6.534 \mathrm{E}-06$ \\
\hline 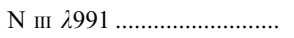 & $4.785 \mathrm{E}-03$ & $9.645 \mathrm{E}-04$ & $1.194 \mathrm{E}-05$ & $0.000 \mathrm{E}+00$ \\
\hline O vi $\lambda \lambda 1032,1037 \ldots \ldots \ldots \ldots . .$. & $2.425 \mathrm{E}-01$ & $1.503 \mathrm{E}-04$ & $0.000 \mathrm{E}+00$ & $0.000 \mathrm{E}+00$ \\
\hline 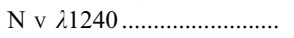 & $2.581 \mathrm{E}-01$ & $1.916 \mathrm{E}-03$ & $0.000 \mathrm{E}+00$ & $0.000 \mathrm{E}+00$ \\
\hline $\mathrm{Si}$ IV+O Iv] $\lambda 1400 \ldots \ldots \ldots \ldots . .$. & $6.552 \mathrm{E}-02$ & $1.237 \mathrm{E}-02$ & $2.100 \mathrm{E}-04$ & $0.000 \mathrm{E}+00$ \\
\hline 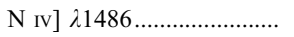 & $1.059 \mathrm{E}-01$ & $8.893 \mathrm{E}-03$ & $4.556 \mathrm{E}-05$ & $0.000 \mathrm{E}+00$ \\
\hline 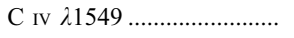 & $1.370 \mathrm{E}+00$ & $1.290 \mathrm{E}-01$ & $4.329 \mathrm{E}-04$ & $1.828 \mathrm{E}-06$ \\
\hline 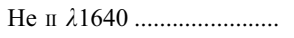 & $7.815 \mathrm{E}-01$ & $8.488 \mathrm{E}-01$ & $7.494 \mathrm{E}-01$ & $3.261 \mathrm{E}-01$ \\
\hline 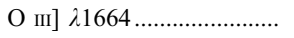 & $4.488 \mathrm{E}-02$ & $2.200 \mathrm{E}-02$ & $2.951 \mathrm{E}-03$ & $1.211 \mathrm{E}-04$ \\
\hline 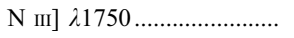 & $8.871 \mathrm{E}-03$ & $8.956 \mathrm{E}-03$ & $1.282 \mathrm{E}-03$ & $4.813 \mathrm{E}-05$ \\
\hline 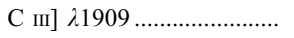 & $2.194 \mathrm{E}-01$ & $2.418 \mathrm{E}-01$ & $5.187 \mathrm{E}-02$ & $1.330 \mathrm{E}-02$ \\
\hline 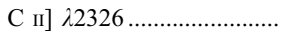 & $3.021 \mathrm{E}-03$ & $2.636 \mathrm{E}-02$ & $9.113 \mathrm{E}-02$ & $1.675 \mathrm{E}-01$ \\
\hline 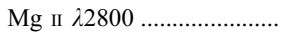 & $1.847 \mathrm{E}-02$ & $1.035 \mathrm{E}-01$ & $2.942 \mathrm{E}-01$ & $4.669 \mathrm{E}-01$ \\
\hline 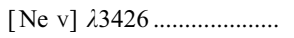 & $5.653 \mathrm{E}-01$ & $3.172 \mathrm{E}-02$ & $5.482 \mathrm{E}-05$ & $0.000 \mathrm{E}+00$ \\
\hline$[\mathrm{Ne}$ III] $\lambda 3869 \ldots \ldots \ldots \ldots \ldots \ldots$ & $6.328 \mathrm{E}-01$ & $8.447 \mathrm{E}-01$ & $6.513 \mathrm{E}-01$ & $3.835 \mathrm{E}-01$ \\
\hline
\end{tabular}


TABLE 22

Dust-free UV Line Ratios with Respect to $\mathrm{H} \beta, 4 Z$.

\begin{tabular}{lcccc}
\hline \hline & \multicolumn{4}{c}{$\log U_{0}$} \\
\cline { 2 - 5 } Line & \multicolumn{5}{c}{$\alpha=-1.0$} & -2.0 \\
& \multicolumn{5}{c}{-3.0} \\
\hline \\
\hline
\end{tabular}


TABLE 22-Continued

\begin{tabular}{|c|c|c|c|c|}
\hline \multirow[b]{2}{*}{ Line } & \multicolumn{4}{|c|}{$\log U_{0}$} \\
\hline & -1.0 & -2.0 & -3.0 & -4.0 \\
\hline \multicolumn{5}{|c|}{$\alpha=-1.7$} \\
\hline C III $\lambda 977 .$. & $1.791 \mathrm{E}-02$ & $2.034 \mathrm{E}-03$ & $1.020 \mathrm{E}-06$ & $0.000 \mathrm{E}+00$ \\
\hline 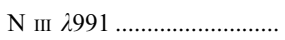 & $1.126 \mathrm{E}-02$ & $1.371 \mathrm{E}-03$ & $0.000 \mathrm{E}+00$ & $0.000 \mathrm{E}+00$ \\
\hline $\mathrm{O}$ vI $\lambda \lambda 1032,1037 \ldots \ldots \ldots \ldots$ & $6.734 \mathrm{E}-01$ & $3.350 \mathrm{E}-04$ & $0.000 \mathrm{E}+00$ & $0.000 \mathrm{E}+00$ \\
\hline $\mathrm{N} \vee \lambda 1240 \ldots \ldots \ldots \ldots \ldots \ldots \ldots \ldots$ & $9.120 \mathrm{E}-01$ & $5.815 \mathrm{E}-03$ & $0.000 \mathrm{E}+00$ & $0.000 \mathrm{E}+00$ \\
\hline $\mathrm{Si}$ IV+O Iv] $\lambda 1400 \ldots \ldots \ldots \ldots . . .$. & $9.403 \mathrm{E}-02$ & $1.280 \mathrm{E}-02$ & $2.315 \mathrm{E}-05$ & $0.000 \mathrm{E}+00$ \\
\hline $\left.\mathrm{N}_{\text {rv] }}\right] \lambda 1486 \ldots \ldots \ldots \ldots \ldots \ldots \ldots$ & $2.840 \mathrm{E}-01$ & $1.976 \mathrm{E}-02$ & $1.518 \mathrm{E}-05$ & $0.000 \mathrm{E}+00$ \\
\hline $\mathrm{C}$ IV $\lambda 1549 \ldots \ldots \ldots . . . .$. & $2.032 \mathrm{E}+00$ & $1.619 \mathrm{E}-01$ & $7.620 \mathrm{E}-05$ & $0.000 \mathrm{E}+00$ \\
\hline He II $\lambda 1640 \ldots \ldots \ldots \ldots \ldots \ldots \ldots$ & $1.112 \mathrm{E}+00$ & $1.245 \mathrm{E}+00$ & $1.103 \mathrm{E}+00$ & $4.804 \mathrm{E}-01$ \\
\hline 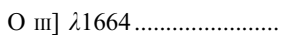 & $6.504 \mathrm{E}-02$ & $1.886 \mathrm{E}-02$ & $4.258 \mathrm{E}-04$ & $1.354 \mathrm{E}-05$ \\
\hline 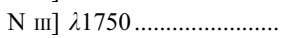 & $2.038 \mathrm{E}-02$ & $1.581 \mathrm{E}-02$ & $3.829 \mathrm{E}-04$ & $1.027 \mathrm{E}-05$ \\
\hline $\left.\mathrm{C}_{\mathrm{III}}\right] \lambda 1909 \ldots \ldots \ldots \ldots \ldots \ldots \ldots$ & $2.426 \mathrm{E}-01$ & $2.303 \mathrm{E}-01$ & $8.905 E-03$ & $1.940 \mathrm{E}-03$ \\
\hline $\mathrm{C}$ II] $\lambda 2326 \ldots \ldots \ldots \ldots \ldots \ldots \ldots \ldots$ & $5.489 \mathrm{E}-04$ & $2.533 \mathrm{E}-02$ & $4.444 \mathrm{E}-02$ & $4.749 \mathrm{E}-02$ \\
\hline $\mathrm{Mg}$ II $\lambda 2800 \ldots \ldots \ldots \ldots \ldots \ldots \ldots$ & $1.010 \mathrm{E}-03$ & $7.571 \mathrm{E}-02$ & $1.608 \mathrm{E}-01$ & $1.985 \mathrm{E}-01$ \\
\hline 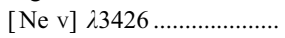 & $1.083 \mathrm{E}+00$ & $7.240 \mathrm{E}-02$ & $5.240 \mathrm{E}-05$ & $0.000 \mathrm{E}+00$ \\
\hline$[\mathrm{Ne}$ III $] \lambda 3869 \ldots \ldots \ldots \ldots \ldots . .$. & $4.292 \mathrm{E}-01$ & $6.521 \mathrm{E}-01$ & $3.847 \mathrm{E}-01$ & $2.313 \mathrm{E}-01$ \\
\hline \multicolumn{5}{|c|}{$\alpha=-2.0$} \\
\hline 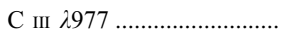 & $6.514 \mathrm{E}-03$ & $1.448 \mathrm{E}-04$ & $0.000 \mathrm{E}+00$ & $0.000 \mathrm{E}+00$ \\
\hline 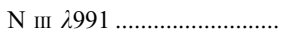 & $4.231 \mathrm{E}-03$ & $9.862 \mathrm{E}-05$ & $0.000 \mathrm{E}+00$ & $0.000 \mathrm{E}+00$ \\
\hline O VI $\lambda \lambda 1032,1037 \ldots \ldots \ldots \ldots$ & $1.082 \mathrm{E}-01$ & $7.599 \mathrm{E}-06$ & $0.000 \mathrm{E}+00$ & $0.000 \mathrm{E}+00$ \\
\hline $\mathrm{N}$ v $\lambda 1240$ & $2.807 \mathrm{E}-01$ & $3.214 \mathrm{E}-04$ & $0.000 \mathrm{E}+00$ & $0.000 \mathrm{E}+00$ \\
\hline $\mathrm{Si}$ IV+O IV] $\lambda 1400 \ldots \ldots \ldots \ldots . .$. & $6.406 \mathrm{E}-02$ & $1.269 \mathrm{E}-03$ & $1.353 \mathrm{E}-06$ & $0.000 \mathrm{E}+00$ \\
\hline $\mathrm{N}$ IV] $\lambda 1486 \ldots \ldots \ldots \ldots \ldots \ldots \ldots$ & $1.278 \mathrm{E}-01$ & $2.397 \mathrm{E}-03$ & $2.130 \mathrm{E}-06$ & $0.000 \mathrm{E}+00$ \\
\hline $\mathrm{C}$ IV $\lambda 1549$ & $9.139 \mathrm{E}-01$ & $1.903 \mathrm{E}-02$ & $1.032 \mathrm{E}-05$ & $0.000 \mathrm{E}+00$ \\
\hline 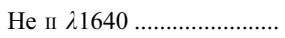 & $7.475 \mathrm{E}-01$ & $8.697 \mathrm{E}-01$ & $7.551 \mathrm{E}-01$ & $3.191 \mathrm{E}-01$ \\
\hline 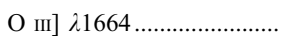 & $2.387 \mathrm{E}-02$ & $3.395 \mathrm{E}-03$ & $9.386 \mathrm{E}-05$ & $6.199 \mathrm{E}-06$ \\
\hline $\mathrm{N}$ III] $\lambda 1750 \ldots \ldots \ldots \ldots \ldots \ldots \ldots$ & $1.126 \mathrm{E}-02$ & $2.991 \mathrm{E}-03$ & $8.922 \mathrm{E}-05$ & $4.904 \mathrm{E}-06$ \\
\hline $\mathrm{C}$ III] $\lambda 1909 \ldots \ldots \ldots \ldots \ldots \ldots \ldots$ & $1.429 \mathrm{E}-01$ & $5.041 \mathrm{E}-02$ & $2.365 \mathrm{E}-03$ & $9.159 \mathrm{E}-04$ \\
\hline $\mathrm{C}$ II] $\lambda 2326 \ldots \ldots \ldots \ldots \ldots \ldots \ldots$ & $5.116 \mathrm{E}-04$ & $4.961 \mathrm{E}-03$ & $1.238 \mathrm{E}-02$ & $2.658 \mathrm{E}-02$ \\
\hline 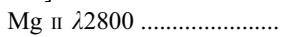 & $6.538 \mathrm{E}-04$ & $1.643 \mathrm{E}-02$ & $6.681 \mathrm{E}-02$ & $1.408 \mathrm{E}-01$ \\
\hline$[\mathrm{Ne} v] \lambda 3426 \ldots \ldots \ldots \ldots \ldots \ldots \ldots$ & $5.079 \mathrm{E}-01$ & $1.159 \mathrm{E}-02$ & $8.902 \mathrm{E}-06$ & $0.000 \mathrm{E}+00$ \\
\hline [Ne III] $\lambda 3869 \ldots \ldots \ldots \ldots \ldots \ldots$ & $2.157 \mathrm{E}-01$ & $2.535 \mathrm{E}-01$ & $1.693 \mathrm{E}-01$ & $1.244 \mathrm{E}-01$ \\
\hline
\end{tabular}


TABLE 23

Dust-free Visible Line Ratios with Respect to $\mathrm{H} \beta, 0.25 Z_{\odot}$

\begin{tabular}{|c|c|c|c|c|}
\hline \multirow[b]{2}{*}{ LiNE } & \multicolumn{4}{|c|}{$\log U_{0}$} \\
\hline & -1.0 & -2.0 & -3.0 & -4.0 \\
\hline \multicolumn{5}{|c|}{$\alpha=-1.2$} \\
\hline$[\mathrm{O}$ II] $\lambda \lambda 3727,3729 \ldots \ldots \ldots$. & $5.011 \mathrm{E}-2$ & $3.585 \mathrm{E}-1$ & $1.614 \mathrm{E}+0$ & $1.669 \mathrm{E}+0$ \\
\hline [S II] $\lambda \lambda 4067,4076 \ldots \ldots \ldots . .$. & $2.804 \mathrm{E}-3$ & $1.457 \mathrm{E}-2$ & $5.497 \mathrm{E}-2$ & $1.326 \mathrm{E}-1$ \\
\hline$[\mathrm{O}$ III] $\lambda 4363 \ldots \ldots \ldots \ldots \ldots \ldots \ldots$ & $2.874 \mathrm{E}-1$ & $1.446 \mathrm{E}-1$ & $3.176 \mathrm{E}-2$ & $5.066 \mathrm{E}-4$ \\
\hline Не пा $\lambda 4686 \ldots \ldots \ldots \ldots \ldots \ldots \ldots$ & $2.713 \mathrm{E}-1$ & $2.864 \mathrm{E}-1$ & $2.346 \mathrm{E}-1$ & $1.038 \mathrm{E}-1$ \\
\hline [O & $2.455 \mathrm{E}+0$ & $1.815 \mathrm{E}+0$ & $7.100 \mathrm{E}-1$ & $2.168 \mathrm{E}-2$ \\
\hline 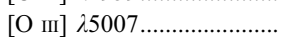 & $7.071 \mathrm{E}+0$ & $5.228 \mathrm{E}+0$ & $2.045 \mathrm{E}+0$ & $6.244 \mathrm{E}-2$ \\
\hline$\left[\mathrm{N}_{1}\right] \lambda 5200 \ldots \ldots \ldots \ldots \ldots \ldots \ldots \ldots \ldots \ldots \ldots \ldots$ & $2.663 \mathrm{E}-3$ & $1.586 \mathrm{E}-2$ & $4.953 \mathrm{E}-2$ & $9.055 \mathrm{E}-2$ \\
\hline$[\mathrm{Fe}$ vII $] \lambda 6085 \ldots \ldots \ldots \ldots \ldots \ldots$ & $5.552 \mathrm{E}-4$ & $1.219 \mathrm{E}-4$ & $0.000 \mathrm{E}+0$ & $0.000 \mathrm{E}+0$ \\
\hline [O I] $\lambda 6300 \ldots \ldots \ldots \ldots \ldots \ldots . .$. & $1.676 \mathrm{E}-2$ & $1.069 \mathrm{E}-1$ & $3.048 \mathrm{E}-1$ & $5.651 \mathrm{E}-1$ \\
\hline 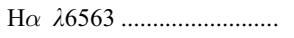 & $2.944 \mathrm{E}+0$ & $3.072 \mathrm{E}+0$ & $3.064 \mathrm{E}+0$ & $3.042 \mathrm{E}+0$ \\
\hline 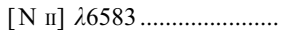 & $6.792 \mathrm{E}-3$ & $5.944 \mathrm{E}-2$ & $2.508 \mathrm{E}-1$ & $3.286 \mathrm{E}-1$ \\
\hline$[\mathrm{S}$ II] $\lambda \lambda 6717,6730 \ldots \ldots \ldots . .$. & $5.138 \mathrm{E}-2$ & $2.847 \mathrm{E}-1$ & $1.057 \mathrm{E}+0$ & $2.633 \mathrm{E}+0$ \\
\hline$[\mathrm{O}$ пI] $\lambda \lambda 7318,7324 \ldots \ldots \ldots .$. & $1.446 \mathrm{E}-3$ & $9.534 \mathrm{E}-3$ & $3.986 \mathrm{E}-2$ & $2.946 \mathrm{E}-2$ \\
\hline [S III] $\lambda \lambda 9069.9532 \ldots \ldots \ldots . .$. & $1.340 \mathrm{E}-1$ & $6.546 \mathrm{E}-1$ & $1.726 \mathrm{E}+0$ & $1.450 \mathrm{E}+0$ \\
\hline \multicolumn{5}{|c|}{$\alpha=-1.4$} \\
\hline$[\mathrm{O}$ пा] $\lambda \lambda 3727,3729 \ldots \ldots \ldots$. & $4.626 \mathrm{E}-2$ & $3.373 \mathrm{E}-1$ & $1.632 \mathrm{E}+0$ & $1.718 \mathrm{E}+0$ \\
\hline [S II] $\lambda \lambda 4067,4076 \ldots \ldots \ldots . .$. & $2.348 \mathrm{E}-3$ & $1.226 \mathrm{E}-2$ & $4.736 \mathrm{E}-2$ & $1.202 \mathrm{E}-1$ \\
\hline 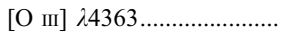 & $2.552 \mathrm{E}-1$ & $1.445 \mathrm{E}-1$ & $3.202 \mathrm{E}-2$ & $5.194 \mathrm{E}-4$ \\
\hline 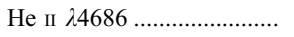 & $2.222 \mathrm{E}-1$ & $2.337 \mathrm{E}-1$ & $1.976 \mathrm{E}-1$ & $8.861 \mathrm{E}-2$ \\
\hline [O & $2.545 \mathrm{E}+0$ & $1.947 \mathrm{E}+0$ & $7.370 \mathrm{E}-1$ & $2.250 \mathrm{E}-2$ \\
\hline$[\mathrm{O}$ III] $\lambda 5007 \ldots \ldots \ldots \ldots \ldots \ldots \ldots$ & $7.330 \mathrm{E}+0$ & $5.608 \mathrm{E}+0$ & $2.123 \mathrm{E}+0$ & $6.479 \mathrm{E}-2$ \\
\hline 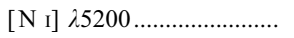 & $1.926 \mathrm{E}-3$ & $1.153 \mathrm{E}-2$ & $3.451 \mathrm{E}-2$ & $6.504 \mathrm{E}-2$ \\
\hline$[\mathrm{Fe}$ VII $] \lambda 6085 \ldots \ldots \ldots \ldots \ldots \ldots \ldots \ldots \ldots \ldots \ldots$ & $5.517 \mathrm{E}-4$ & $6.278 \mathrm{E}-5$ & $0.000 \mathrm{E}+0$ & $0.000 \mathrm{E}+0$ \\
\hline$\left[\mathrm{O}_{\mathrm{I}}\right] \mathrm{\lambda}, 6300 \ldots \ldots \ldots \ldots \ldots \ldots \ldots$ & $1.143 \mathrm{E}-2$ & $7.281 \mathrm{E}-2$ & $2.130 \mathrm{E}-1$ & $4.204 \mathrm{E}-1$ \\
\hline $\mathrm{H} \alpha \quad \lambda 6563 \ldots \ldots \ldots \ldots \ldots \ldots \ldots \ldots$ & $2.863 \mathrm{E}+0$ & $2.971 \mathrm{E}+0$ & $3.000 \mathrm{E}+0$ & $3.004 \mathrm{E}+0$ \\
\hline$[\mathrm{N}$ II] $\lambda 6583 \ldots \ldots \ldots \ldots \ldots \ldots \ldots$ & $5.141 \mathrm{E}-3$ & $4.760 \mathrm{E}-2$ & $2.413 \mathrm{E}-1$ & $3.341 \mathrm{E}-1$ \\
\hline$[\mathrm{S}$ II $] \lambda \lambda 6717,6730 \ldots \ldots \ldots . .$. & $4.287 \mathrm{E}-2$ & $2.319 \mathrm{E}-1$ & $8.352 \mathrm{E}-1$ & $2.169 \mathrm{E}+0$ \\
\hline$[\mathrm{O}$ II $] \lambda \lambda 7318,7324 \ldots \ldots \ldots .$. & $1.374 \mathrm{E}-3$ & $9.330 \mathrm{E}-3$ & $4.078 \mathrm{E}-2$ & $3.089 \mathrm{E}-2$ \\
\hline$[\mathrm{S}$ III] $\lambda \lambda 9069.9532 \ldots \ldots \ldots . .$. & $1.226 \mathrm{E}-1$ & $6.571 \mathrm{E}-1$ & $1.607 \mathrm{E}+0$ & $1.200 \mathrm{E}+0$ \\
\hline \multicolumn{5}{|c|}{$\alpha=-1.7$} \\
\hline$[\mathrm{O}$ пा] $\lambda \lambda 3727,3729 \ldots \ldots \ldots$. & $4.334 \mathrm{E}-2$ & $3.266 \mathrm{E}-1$ & $1.667 \mathrm{E}+0$ & $1.745 \mathrm{E}+0$ \\
\hline$[\mathrm{S}$ II] $\lambda \lambda 4067,4076 \ldots \ldots \ldots . .$. & $1.703 \mathrm{E}-3$ & $9.361 \mathrm{E}-3$ & $3.965 \mathrm{E}-2$ & $1.082 \mathrm{E}-1$ \\
\hline$[\mathrm{O}$ III] $\lambda 4363 \ldots \ldots \ldots \ldots \ldots \ldots \ldots$ & $1.904 \mathrm{E}-1$ & $1.285 \mathrm{E}-1$ & $2.975 \mathrm{E}-2$ & $4.837 \mathrm{E}-4$ \\
\hline 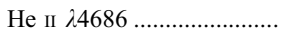 & $1.588 \mathrm{E}-1$ & $1.667 \mathrm{E}-1$ & $1.457 \mathrm{E}-1$ & $6.559 \mathrm{E}-2$ \\
\hline 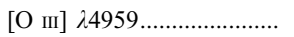 & $2.399 \mathrm{E}+0$ & $1.959 \mathrm{E}+0$ & $7.246 \mathrm{E}-1$ & $2.145 \mathrm{E}-2$ \\
\hline 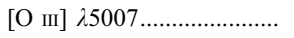 & $6.909 \mathrm{E}+0$ & $5.644 \mathrm{E}+0$ & $2.087 \mathrm{E}+0$ & $6.179 \mathrm{E}-2$ \\
\hline$\left[\mathrm{N}_{1}\right] \lambda 5200 \ldots \ldots \ldots \ldots \ldots \ldots \ldots \ldots \ldots \ldots \ldots$ & $1.041 \mathrm{E}-3$ & $6.468 \mathrm{E}-3$ & $1.908 \mathrm{E}-2$ & $4.081 \mathrm{E}-2$ \\
\hline 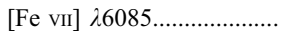 & $4.240 \mathrm{E}-4$ & $1.928 \mathrm{E}-5$ & $0.000 \mathrm{E}+0$ & $0.000 \mathrm{E}+0$ \\
\hline [O I] $\lambda 6300 \ldots \ldots \ldots \ldots \ldots \ldots \ldots$ & $6.071 \mathrm{E}-3$ & $3.947 \mathrm{E}-2$ & $1.221 \mathrm{E}-1$ & $2.789 \mathrm{E}-1$ \\
\hline 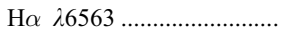 & $2.813 \mathrm{E}+0$ & $2.886 \mathrm{E}+0$ & $2.938 \mathrm{E}+0$ & $2.963 \mathrm{E}+0$ \\
\hline 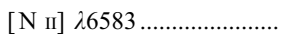 & $4.212 \mathrm{E}-3$ & $4.083 \mathrm{E}-2$ & $2.379 \mathrm{E}-1$ & $3.351 \mathrm{E}-1$ \\
\hline$[\mathrm{S}$ II $] \lambda \lambda 6717,6730 \ldots \ldots \ldots . .$. & $2.996 \mathrm{E}-2$ & $1.626 \mathrm{E}-1$ & $5.974 \mathrm{E}-1$ & $1.730 \mathrm{E}+0$ \\
\hline$[\mathrm{O}$ II] $\lambda \lambda 27318,7324 \ldots \ldots \ldots$. & $1.294 \mathrm{E}-3$ & $9.217 \mathrm{E}-3$ & $4.160 \mathrm{E}-2$ & $3.173 \mathrm{E}-2$ \\
\hline [S III] $\lambda \lambda 9069.9532 \ldots \ldots \ldots . .$. & $1.160 \mathrm{E}-1$ & $6.899 \mathrm{E}-1$ & $1.481 \mathrm{E}+0$ & $9.394 \mathrm{E}-1$ \\
\hline \multicolumn{5}{|c|}{$\alpha=-2.0$} \\
\hline$[\mathrm{O}$ II] $\lambda \lambda 3727,3729 \ldots \ldots \ldots$. & $4.310 \mathrm{E}-2$ & $3.314 \mathrm{E}-1$ & $1.706 \mathrm{E}+0$ & $1.732 \mathrm{E}+0$ \\
\hline$[\mathrm{S}$ II $] \lambda \lambda 4067,4076 \ldots \ldots \ldots . .$. & $1.214 \mathrm{E}-3$ & $7.308 \mathrm{E}-3$ & $3.544 \mathrm{E}-2$ & $1.007 \mathrm{E}-1$ \\
\hline$[\mathrm{O}$ III] $\lambda 4363 \ldots \ldots \ldots \ldots \ldots \ldots \ldots$ & $1.420 \mathrm{E}-1$ & $1.066 \mathrm{E}-1$ & $2.570 \mathrm{E}-2$ & $4.124 \mathrm{E}-4$ \\
\hline 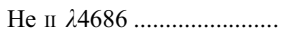 & $1.120 \mathrm{E}-1$ & $1.163 \mathrm{E}-1$ & $1.035 \mathrm{E}-1$ & $4.609 \mathrm{E}-2$ \\
\hline [O & $2.176 \mathrm{E}+0$ & $1.848 \mathrm{E}+0$ & $6.684 \mathrm{E}-1$ & $1.880 \mathrm{E}-2$ \\
\hline$[\mathrm{O}$ III $] \lambda 5007 \ldots \ldots \ldots \ldots \ldots \ldots \ldots$ & $6.267 \mathrm{E}+0$ & $5.321 \mathrm{E}+0$ & $1.925 \mathrm{E}+0$ & $5.416 \mathrm{E}-2$ \\
\hline$[\mathrm{N}$ I] $\lambda 5200 \ldots \ldots \ldots \ldots \ldots \ldots \ldots \ldots \ldots \ldots \ldots \ldots \ldots$ & $4.899 \mathrm{E}-4$ & $3.289 \mathrm{E}-3$ & $1.043 \mathrm{E}-2$ & $2.732 \mathrm{E}-2$ \\
\hline$[\mathrm{Fe}$ vII $] \lambda 6085 \ldots \ldots \ldots \ldots \ldots \ldots$ & $2.369 \mathrm{E}-4$ & $4.969 \mathrm{E}-6$ & $0.000 \mathrm{E}+0$ & $0.000 \mathrm{E}+0$ \\
\hline [O I] $\lambda 6300 \ldots \ldots \ldots \ldots \ldots \ldots . .$. & $3.056 \mathrm{E}-3$ & $2.086 \mathrm{E}-2$ & $7.158 \mathrm{E}-2$ & $1.963 \mathrm{E}-1$ \\
\hline $\mathrm{H} \alpha \quad \lambda 6563 \ldots \ldots \ldots \ldots \ldots \ldots \ldots \ldots . .$. & $2.800 \mathrm{E}+0$ & $2.848 \mathrm{E}+0$ & $2.902 \mathrm{E}+0$ & $2.936 \mathrm{E}+0$ \\
\hline$[\mathrm{N}$ II] $\lambda 6583 \ldots \ldots \ldots \ldots \ldots \ldots \ldots . .$. & $4.055 \mathrm{E}-3$ & $4.022 \mathrm{E}-2$ & $2.410 \mathrm{E}-1$ & $3.302 \mathrm{E}-1$ \\
\hline [S II] $\lambda \lambda 6717,6730 \ldots \ldots \ldots . .$. & $1.941 \mathrm{E}-2$ & $1.117 \mathrm{E}-1$ & $4.609 \mathrm{E}-1$ & $1.476 \mathrm{E}+0$ \\
\hline$[\mathrm{O}$ п] $] \lambda \lambda 7318,7324 \ldots \ldots \ldots .$. & $1.272 \mathrm{E}-3$ & $9.281 \mathrm{E}-3$ & $4.200 \mathrm{E}-2$ & $3.150 \mathrm{E}-2$ \\
\hline [S III] $\lambda \lambda 9069.9532 \ldots \ldots \ldots . .$. & $1.208 \mathrm{E}-1$ & $7.404 \mathrm{E}-1$ & $1.396 \mathrm{E}+0$ & $7.791 \mathrm{E}-1$ \\
\hline
\end{tabular}


TABLE 24

Dust-free Visible Line Ratios with Respect to $\mathrm{H} \beta, 0.5 Z$

\begin{tabular}{|c|c|c|c|c|}
\hline \multirow[b]{2}{*}{ LiNE } & \multicolumn{4}{|c|}{$\log U_{0}$} \\
\hline & -1.0 & -2.0 & -3.0 & -4.0 \\
\hline \multicolumn{5}{|c|}{$\alpha=-1.2$} \\
\hline [O II] $\lambda \lambda 3727,3729 \ldots \ldots \ldots$. & $8.501 \mathrm{E}-2$ & $6.627 \mathrm{E}-1$ & $2.952 \mathrm{E}+0$ & $3.064 \mathrm{E}+0$ \\
\hline$[\mathrm{S}$ II $] \lambda \lambda 4067,4076 \ldots \ldots \ldots . .$. & $4.873 \mathrm{E}-3$ & $2.599 \mathrm{E}-2$ & $9.310 \mathrm{E}-2$ & $2.191 \mathrm{E}-1$ \\
\hline 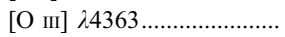 & $3.444 \mathrm{E}-1$ & $2.223 \mathrm{E}-1$ & $5.074 \mathrm{E}-2$ & $8.570 \mathrm{E}-4$ \\
\hline He II $\lambda 4686 \ldots . .$. & $2.825 \mathrm{E}-1$ & $2.940 \mathrm{E}-1$ & $2.414 \mathrm{E}-1$ & $1.082 \mathrm{E}-1$ \\
\hline$[\mathrm{O}$ III $] \lambda 4959 \ldots$. & $3.781 \mathrm{E}+0$ & $3.226 \mathrm{E}+0$ & $1.269 \mathrm{E}+0$ & $3.977 \mathrm{E}-2$ \\
\hline$[\mathrm{O}$ III $] \lambda 5007 \ldots$. & $1.089 \mathrm{E}+1$ & $9.290 \mathrm{E}+0$ & $3.654 \mathrm{E}+0$ & $1.146 \mathrm{E}-1$ \\
\hline$\left[\mathrm{N}_{\mathrm{I}}\right] \quad \lambda 5200 \ldots \ldots \ldots \ldots \ldots \ldots \ldots \ldots \ldots$ & $4.073 \mathrm{E}-3$ & $2.612 \mathrm{E}-2$ & $7.705 \mathrm{E}-2$ & $1.276 \mathrm{E}-1$ \\
\hline$[\mathrm{Fe}$ VII $] \lambda 6085 \ldots \ldots \ldots \ldots \ldots \ldots \ldots \ldots$ & $9.721 \mathrm{E}-4$ & $1.913 \mathrm{E}-4$ & $0.000 \mathrm{E}+0$ & $0.000 \mathrm{E}+0$ \\
\hline$\left[\mathrm{O}_{\mathrm{I}}\right], \lambda 6300 \ldots \ldots \ldots \ldots \ldots \ldots \ldots$ & $2.639 \mathrm{E}-2$ & $1.868 \mathrm{E}-1$ & $5.092 \mathrm{E}-1$ & $8.734 \mathrm{E}-1$ \\
\hline 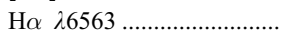 & $2.865 \mathrm{E}+0$ & $2.979 \mathrm{E}+0$ & $3.014 \mathrm{E}+0$ & $3.018 \mathrm{E}+0$ \\
\hline 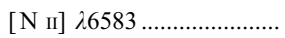 & $1.100 \mathrm{E}-2$ & $1.065 \mathrm{E}-1$ & $4.539 \mathrm{E}-1$ & $5.969 \mathrm{E}-1$ \\
\hline$[\mathrm{S}$ II] $\lambda \lambda 6717,6730 \ldots \ldots \ldots \ldots$ & $8.832 \mathrm{E}-2$ & $4.951 \mathrm{E}-1$ & $1.708 \mathrm{E}+0$ & $4.040 \mathrm{E}+0$ \\
\hline$[\mathrm{O}$ II] $\lambda \lambda 7318,7324 \ldots \ldots \ldots .$. & $2.241 \mathrm{E}-3$ & $1.674 \mathrm{E}-2$ & $6.977 \mathrm{E}-2$ & $5.247 \mathrm{E}-2$ \\
\hline [S III] $\lambda \lambda 9069.9532 \ldots \ldots \ldots \ldots$ & $2.171 \mathrm{E}-1$ & $1.242 \mathrm{E}+0$ & $3.229 \mathrm{E}+0$ & $2.591 \mathrm{E}+0$ \\
\hline \multicolumn{5}{|c|}{$\alpha=-1.4$} \\
\hline$[\mathrm{O}$ II] $\lambda \lambda 3727,3729 \ldots \ldots \ldots$. & $7.674 \mathrm{E}-2$ & $5.984 \mathrm{E}-1$ & $2.895 \mathrm{E}+0$ & $3.115 \mathrm{E}+0$ \\
\hline$[\mathrm{S}$ пा] $\lambda \lambda 4067,4076 \ldots \ldots \ldots \ldots$ & $3.938 \mathrm{E}-3$ & $2.131 \mathrm{E}-2$ & $7.977 \mathrm{E}-2$ & $2.039 \mathrm{E}-1$ \\
\hline 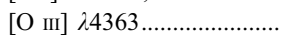 & $2.688 \mathrm{E}-1$ & $1.956 \mathrm{E}-1$ & $4.807 \mathrm{E}-2$ & $8.548 \mathrm{E}-4$ \\
\hline He II $\lambda 4686 \ldots \ldots \ldots \ldots \ldots \ldots$ & $2.272 \mathrm{E}-1$ & $2.391 \mathrm{E}-1$ & $2.031 \mathrm{E}-1$ & $9.212 \mathrm{E}-2$ \\
\hline 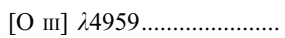 & $3.604 \mathrm{E}+0$ & $3.205 \mathrm{E}+0$ & $1.272 \mathrm{E}+0$ & $4.062 \mathrm{E}-2$ \\
\hline 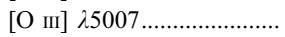 & $1.038 \mathrm{E}+1$ & $9.231 \mathrm{E}+0$ & $3.664 \mathrm{E}+0$ & $1.170 \mathrm{E}-1$ \\
\hline$\left[\mathrm{N}_{\mathrm{I}}\right] \lambda 5200 \ldots \ldots \ldots \ldots \ldots \ldots \ldots$ & $2.800 \mathrm{E}-3$ & $1.852 \mathrm{E}-2$ & $5.235 \mathrm{E}-2$ & $9.192 \mathrm{E}-2$ \\
\hline$[\mathrm{Fe}$ VII $] \lambda 6085 \ldots \ldots \ldots \ldots \ldots \ldots$ & $9.311 \mathrm{E}-4$ & $9.327 \mathrm{E}-5$ & $0.000 \mathrm{E}+0$ & $0.000 \mathrm{E}+0$ \\
\hline$\left[\mathrm{O}_{\mathrm{I}}\right], \lambda 6300 \ldots \ldots \ldots \ldots \ldots \ldots$ & $1.744 \mathrm{E}-2$ & $1.247 \mathrm{E}-1$ & $3.482 \mathrm{E}-1$ & $6.547 \mathrm{E}-1$ \\
\hline $\mathrm{H} \alpha \quad \lambda 6563 \ldots \ldots \ldots \ldots \ldots \ldots \ldots \ldots$ & $2.824 \mathrm{E}+0$ & $2.901 \mathrm{E}+0$ & $2.956 \mathrm{E}+0$ & $2.981 \mathrm{E}+0$ \\
\hline 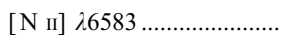 & $8.700 \mathrm{E}-3$ & $8.499 \mathrm{E}-2$ & $4.312 \mathrm{E}-1$ & $6.023 \mathrm{E}-1$ \\
\hline$\left[\mathrm{S}_{\mathrm{II}}\right] \lambda \lambda 6717,6730 \ldots \ldots \ldots \ldots$ & $7.064 \mathrm{E}-2$ & $3.932 \mathrm{E}-1$ & $1.336 \mathrm{E}+0$ & $3.449 \mathrm{E}+0$ \\
\hline$[\mathrm{O}$ II] $\lambda \lambda 7318,7324 \ldots \ldots \ldots .$. & $2.051 \mathrm{E}-3$ & $1.535 \mathrm{E}-2$ & $6.829 \mathrm{E}-2$ & $5.389 \mathrm{E}-2$ \\
\hline [S III] $\lambda \lambda 9069.9532 \ldots \ldots \ldots \ldots$. & $2.009 \mathrm{E}-1$ & $1.217 \mathrm{E}+0$ & $2.972 \mathrm{E}+0$ & $2.148 \mathrm{E}+0$ \\
\hline \multicolumn{5}{|c|}{$\alpha=-1.7$} \\
\hline$[\mathrm{O}$ II] $\lambda \lambda 3727,3729 \ldots \ldots \ldots$. & $6.985 \mathrm{E}-2$ & $5.413 \mathrm{E}-1$ & $2.811 \mathrm{E}+0$ & $3.098 \mathrm{E}+0$ \\
\hline$[\mathrm{S}$ II] $\lambda \lambda 4067,4076 \ldots \ldots \ldots . .$. & $2.752 \mathrm{E}-3$ & $1.565 \mathrm{E}-2$ & $6.653 \mathrm{E}-2$ & $1.878 \mathrm{E}-1$ \\
\hline 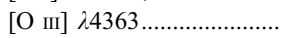 & $1.732 \mathrm{E}-1$ & $1.451 \mathrm{E}-1$ & $4.027 \mathrm{E}-2$ & $7.637 \mathrm{E}-4$ \\
\hline 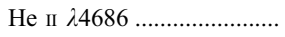 & $1.601 \mathrm{E}-1$ & $1.701 \mathrm{E}-1$ & $1.497 \mathrm{E}-1$ & $6.794 \mathrm{E}-2$ \\
\hline 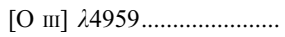 & $3.114 \mathrm{E}+0$ & $2.897 \mathrm{E}+0$ & $1.181 \mathrm{E}+0$ & $3.783 \mathrm{E}-2$ \\
\hline 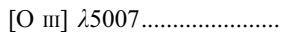 & $8.970 \mathrm{E}+0$ & $8.344 \mathrm{E}+0$ & $3.402 \mathrm{E}+0$ & $1.090 \mathrm{E}-1$ \\
\hline$\left[\mathrm{N}_{\mathrm{I}}\right] \lambda 5200 \ldots \ldots \ldots \ldots \ldots \ldots \ldots \ldots \ldots \ldots \ldots$ & $1.394 \mathrm{E}-3$ & $9.925 \mathrm{E}-3$ & $2.798 \mathrm{E}-2$ & $5.857 \mathrm{E}-2$ \\
\hline$[\mathrm{Fe}$ VII $] \lambda 6085 \ldots \ldots \ldots \ldots \ldots \ldots \ldots \ldots$ & $6.595 \mathrm{E}-4$ & $2.637 \mathrm{E}-5$ & $0.000 \mathrm{E}+0$ & $0.000 \mathrm{E}+0$ \\
\hline$\left[\mathrm{O}_{\mathrm{I}}\right] \mathrm{\lambda}, 6300 \ldots \ldots \ldots \ldots \ldots \ldots \ldots$ & $8.778 \mathrm{E}-3$ & $6.505 \mathrm{E}-2$ & $1.945 \mathrm{E}-1$ & $4.425 \mathrm{E}-1$ \\
\hline 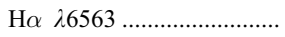 & $2.813 \mathrm{E}+0$ & $2.852 \mathrm{E}+0$ & $2.905 \mathrm{E}+0$ & $2.943 \mathrm{E}+0$ \\
\hline 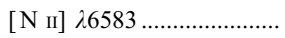 & $7.382 \mathrm{E}-3$ & $7.121 \mathrm{E}-2$ & $4.146 \mathrm{E}-1$ & $5.962 \mathrm{E}-1$ \\
\hline$[\mathrm{S}$ II] $\lambda \lambda 6717,6730 \ldots \ldots \ldots \ldots$ & $4.672 \mathrm{E}-2$ & $2.657 \mathrm{E}-1$ & $9.617 \mathrm{E}-1$ & $2.889 \mathrm{E}+0$ \\
\hline$[\mathrm{O}$ II $] \lambda \lambda 7318,7324 \ldots \ldots \ldots$. & $1.856 \mathrm{E}-3$ & $1.372 \mathrm{E}-2$ & $6.476 \mathrm{E}-2$ & $5.362 \mathrm{E}-2$ \\
\hline [S III] $\lambda \lambda 9069.9532 \ldots \ldots \ldots \ldots$ & $1.937 \mathrm{E}-1$ & $1.222 \mathrm{E}+0$ & $2.679 \mathrm{E}+0$ & $1.691 \mathrm{E}+0$ \\
\hline \multicolumn{5}{|c|}{$\alpha=-2.0$} \\
\hline$[\mathrm{O}$ II] $\lambda \lambda 3727,3729 \ldots \ldots \ldots$ & $6.704 \mathrm{E}-2$ & $5.122 \mathrm{E}-1$ & $2.723 \mathrm{E}+0$ & $3.007 \mathrm{E}+0$ \\
\hline$[\mathrm{S}$ II] $\lambda \lambda 4067,4076 \ldots \ldots \ldots \ldots$ & $1.920 \mathrm{E}-3$ & $1.182 \mathrm{E}-2$ & $5.889 \mathrm{E}-2$ & $1.756 \mathrm{E}-1$ \\
\hline 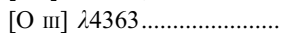 & $1.151 \mathrm{E}-1$ & $1.018 \mathrm{E}-1$ & $3.112 \mathrm{E}-2$ & $6.245 \mathrm{E}-4$ \\
\hline He II $\lambda 4686 \ldots \ldots \ldots \ldots \ldots \ldots \ldots$ & $1.124 \mathrm{E}-1$ & $1.188 \mathrm{E}-1$ & $1.064 \mathrm{E}-1$ & $4.757 \mathrm{E}-2$ \\
\hline 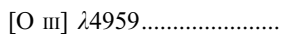 & $2.647 \mathrm{E}+0$ & $2.483 \mathrm{E}+0$ & $1.025 \mathrm{E}+0$ & $3.240 \mathrm{E}-2$ \\
\hline$[\mathrm{O}$ III $] \lambda 5007 \ldots \ldots \ldots \ldots \ldots \ldots \ldots$ & $7.623 \mathrm{E}+0$ & $7.153 \mathrm{E}+0$ & $2.953 \mathrm{E}+0$ & $9.330 \mathrm{E}-2$ \\
\hline$\left[\mathrm{N}_{\mathrm{I}}\right] \lambda 5200 \ldots \ldots \ldots \ldots \ldots \ldots \ldots \ldots \ldots \ldots$ & $5.807 \mathrm{E}-4$ & $4.767 \mathrm{E}-3$ & $1.493 \mathrm{E}-2$ & $3.990 \mathrm{E}-2$ \\
\hline$[\mathrm{Fe}$ vII $] \lambda 6085 \ldots \ldots \ldots \ldots \ldots \ldots$ & $3.388 \mathrm{E}-4$ & $6.264 \mathrm{E}-6$ & $0.000 \mathrm{E}+0$ & $0.000 \mathrm{E}+0$ \\
\hline$\left[\mathrm{O}_{\mathrm{I}}\right] \mathrm{\lambda} 6300 \ldots \ldots \ldots \ldots \ldots \ldots \ldots$ & $4.111 \mathrm{E}-3$ & $3.295 \mathrm{E}-2$ & $1.126 \mathrm{E}-1$ & $3.163 \mathrm{E}-1$ \\
\hline 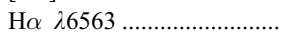 & $2.820 \mathrm{E}+0$ & $2.840 \mathrm{E}+0$ & $2.880 \mathrm{E}+0$ & $2.920 \mathrm{E}+0$ \\
\hline$\left[\mathrm{N}_{\text {II }}\right] \lambda 6583 \ldots \ldots \ldots \ldots \ldots \ldots \ldots$ & $7.109 \mathrm{E}-3$ & $6.770 \mathrm{E}-2$ & $4.077 \mathrm{E}-1$ & $5.793 \mathrm{E}-1$ \\
\hline$\left[\mathrm{S}_{\text {II }}\right] \lambda \lambda 6717,6730 \ldots \ldots \ldots \ldots$ & $2.878 \mathrm{E}-2$ & $1.776 \mathrm{E}-1$ & $7.576 \mathrm{E}-1$ & $2.538 \mathrm{E}+0$ \\
\hline$[\mathrm{O}$ II] $\lambda \lambda 7318,7324 \ldots \ldots \ldots .$. & $1.741 \mathrm{E}-3$ & $1.253 \mathrm{E}-2$ & $6.044 \mathrm{E}-2$ & $5.152 \mathrm{E}-2$ \\
\hline [S III] $\lambda \lambda 9069.9532 \ldots \ldots \ldots \ldots$ & $2.019 \mathrm{E}-1$ & $1.252 \mathrm{E}+0$ & $2.456 \mathrm{E}+0$ & $1.410 \mathrm{E}+0$ \\
\hline
\end{tabular}


TABLE 25

Dust-free Visible Line Ratios with Respect to $\mathrm{H} \beta, 1 Z_{\odot}$

\begin{tabular}{|c|c|c|c|c|}
\hline \multirow[b]{2}{*}{ Line } & \multicolumn{4}{|c|}{$\log U_{0}$} \\
\hline & -1.0 & -2.0 & -3.0 & -4.0 \\
\hline \multicolumn{5}{|c|}{$\alpha=-1.2$} \\
\hline [O II] $\lambda \lambda 3727,3729 \ldots . .$. & $1.245 \mathrm{E}-1$ & $1.083 \mathrm{E}+0$ & $4.689 \mathrm{E}+0$ & $4.952 \mathrm{E}+0$ \\
\hline$[\mathrm{S}$ II] $\lambda \lambda 4067,4076 \ldots \ldots$. & $7.141 \mathrm{E}-3$ & $4.279 \mathrm{E}-2$ & $1.448 \mathrm{E}-1$ & $3.392 \mathrm{E}-1$ \\
\hline 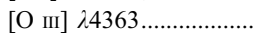 & $3.137 \mathrm{E}-1$ & $2.521 \mathrm{E}-1$ & $5.913 \mathrm{E}-2$ & $1.118 \mathrm{E}-3$ \\
\hline 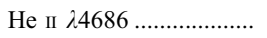 & $2.882 \mathrm{E}-1$ & $3.027 \mathrm{E}-1$ & $2.539 \mathrm{E}-1$ & $1.156 \mathrm{E}-1$ \\
\hline 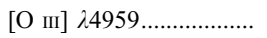 & $4.903 \mathrm{E}+0$ & $4.857 \mathrm{E}+0$ & $1.924 \mathrm{E}+0$ & $6.366 \mathrm{E}-2$ \\
\hline 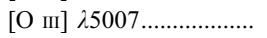 & $1.412 \mathrm{E}+1$ & $1.399 \mathrm{E}+1$ & $5.542 \mathrm{E}+0$ & $1.833 \mathrm{E}-1$ \\
\hline$\left[\mathrm{N}_{\mathrm{I}}\right] \lambda 5200 \ldots \ldots \ldots \ldots \ldots \ldots$ & $8.432 \mathrm{E}-3$ & $6.787 \mathrm{E}-2$ & $1.893 \mathrm{E}-1$ & $2.810 \mathrm{E}-1$ \\
\hline$[\mathrm{Fe}$ VII $] \lambda 6085 \ldots \ldots \ldots \ldots \ldots$ & $1.594 \mathrm{E}-3$ & $2.505 \mathrm{E}-4$ & $0.000 \mathrm{E}+0$ & $0.000 \mathrm{E}+0$ \\
\hline$\left[\mathrm{O}_{\mathrm{I}}\right] \lambda 6300 \ldots \ldots \ldots$ & $3.213 \mathrm{E}-2$ & $2.955 \mathrm{E}-1$ & $7.752 \mathrm{E}-1$ & $1.229 \mathrm{E}+0$ \\
\hline $\mathrm{H} \alpha \quad \lambda 6563 \ldots \ldots \ldots \ldots \ldots \ldots \ldots$ & $2.833 \mathrm{E}+0$ & $2.900 \mathrm{E}+0$ & $2.954 \mathrm{E}+0$ & $2.984 \mathrm{E}+0$ \\
\hline 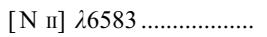 & $2.732 \mathrm{E}-2$ & $3.085 \mathrm{E}-1$ & $1.326 \mathrm{E}+0$ & $1.755 \mathrm{E}+0$ \\
\hline$[\mathrm{S}$ II] $\lambda \lambda 6717,6730 \ldots \ldots$. & $1.281 \mathrm{E}-1$ & $7.960 \mathrm{E}-1$ & $2.562 \mathrm{E}+0$ & $5.953 \mathrm{E}+0$ \\
\hline$[\mathrm{O}$ II $] \lambda \lambda 7318,7324 \ldots \ldots$. & $2.932 \mathrm{E}-3$ & $2.438 \mathrm{E}-2$ & $9.909 \mathrm{E}-2$ & $7.777 \mathrm{E}-2$ \\
\hline [S III] $\lambda \lambda 9069.9532 \ldots \ldots .$. & $3.395 \mathrm{E}-1$ & $2.200 \mathrm{E}+0$ & $5.583 \mathrm{E}+0$ & $4.247 \mathrm{E}+0$ \\
\hline \multicolumn{5}{|c|}{$\alpha=-1.4$} \\
\hline$[\mathrm{O}$ пा] $\lambda \lambda 3727,3729 \ldots \ldots$. & $1.104 \mathrm{E}-1$ & $9.044 \mathrm{E}-1$ & $4.268 \mathrm{E}+0$ & $4.853 \mathrm{E}+0$ \\
\hline$[\mathrm{S}$ II] $\lambda \lambda 4067,4076 \ldots \ldots$. & $5.489 \mathrm{E}-3$ & $3.339 \mathrm{E}-2$ & $1.208 \mathrm{E}-1$ & $3.175 \mathrm{E}-1$ \\
\hline 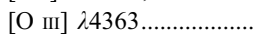 & $2.121 \mathrm{E}-1$ & $1.821 \mathrm{E}-1$ & $4.833 \mathrm{E}-2$ & $1.038 \mathrm{E}-3$ \\
\hline 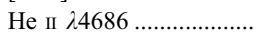 & $2.286 \mathrm{E}-1$ & $2.450 \mathrm{E}-1$ & $2.133 \mathrm{E}-1$ & $9.798 \mathrm{E}-2$ \\
\hline 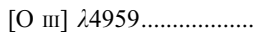 & $4.244 \mathrm{E}+0$ & $4.263 \mathrm{E}+0$ & $1.779 \mathrm{E}+0$ & $6.250 \mathrm{E}-2$ \\
\hline 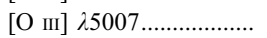 & $1.222 \mathrm{E}+1$ & $1.228 \mathrm{E}+1$ & $5.124 \mathrm{E}+0$ & $1.800 \mathrm{E}-1$ \\
\hline$\left[\mathrm{N}_{\mathrm{I}}\right] \lambda 5200 \ldots \ldots \ldots \ldots \ldots$ & $5.331 \mathrm{E}-3$ & $4.626 \mathrm{E}-2$ & $1.244 \mathrm{E}-1$ & $2.021 \mathrm{E}-1$ \\
\hline$[\mathrm{Fe}$ VII] $\lambda 6085 \ldots \ldots \ldots \ldots \ldots . . . .$. & $1.434 \mathrm{E}-3$ & $1.128 \mathrm{E}-4$ & $0.000 \mathrm{E}+0$ & $0.000 \mathrm{E}+0$ \\
\hline 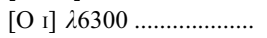 & $2.022 \mathrm{E}-2$ & $1.910 \mathrm{E}-1$ & $5.145 \mathrm{E}-1$ & $9.236 \mathrm{E}-1$ \\
\hline $\mathrm{H} \alpha \quad \lambda 6563 \ldots \ldots \ldots \ldots \ldots \ldots \ldots$ & $2.827 \mathrm{E}+0$ & $2.863 \mathrm{E}+0$ & $2.913 \mathrm{E}+0$ & $2.953 \mathrm{E}+0$ \\
\hline 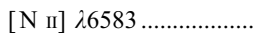 & $2.330 \mathrm{E}-2$ & $2.418 \mathrm{E}-1$ & $1.215 \mathrm{E}+0$ & $1.733 \mathrm{E}+0$ \\
\hline$[\mathrm{S}$ II] $\lambda \lambda 6717,6730 \ldots \ldots .$. & $9.647 \mathrm{E}-2$ & $6.087 \mathrm{E}-1$ & $1.980 \mathrm{E}+0$ & $5.246 \mathrm{E}+0$ \\
\hline$[\mathrm{O}$ Іा $] \lambda \lambda 7318,7324 \ldots \ldots$. & $2.586 \mathrm{E}-3$ & $1.994 \mathrm{E}-2$ & $8.717 \mathrm{E}-2$ & $7.561 \mathrm{E}-2$ \\
\hline [S III] $\lambda \lambda 9069.9532 \ldots \ldots .$. & $3.193 \mathrm{E}-1$ & $2.062 \mathrm{E}+0$ & $4.978 \mathrm{E}+0$ & $3.511 \mathrm{E}+0$ \\
\hline \multicolumn{5}{|c|}{$\alpha=-1.7$} \\
\hline$[\mathrm{O}$ II] $\lambda \lambda 3727,3729 \ldots \ldots$. & $9.701 \mathrm{E}-2$ & $7.299 \mathrm{E}-1$ & $3.681 \mathrm{E}+0$ & $4.557 \mathrm{E}+0$ \\
\hline$[\mathrm{S}$ II] $\lambda \lambda 4067,4076 \ldots \ldots$. & $3.618 \mathrm{E}-3$ & $2.287 \mathrm{E}-2$ & $9.628 \mathrm{E}-2$ & $2.888 \mathrm{E}-1$ \\
\hline 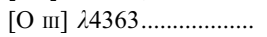 & $1.139 \mathrm{E}-1$ & $1.049 \mathrm{E}-1$ & $3.210 \mathrm{E}-2$ & $8.333 \mathrm{E}-4$ \\
\hline 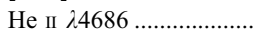 & $1.595 \mathrm{E}-1$ & $1.744 \mathrm{E}-1$ & $1.573 \mathrm{E}-1$ & $7.185 \mathrm{E}-2$ \\
\hline 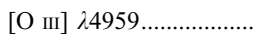 & $3.275 \mathrm{E}+0$ & $3.297 \mathrm{E}+0$ & $1.456 \mathrm{E}+0$ & $5.492 \mathrm{E}-2$ \\
\hline 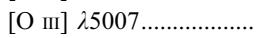 & $9.434 \mathrm{E}+0$ & $9.496 \mathrm{E}+0$ & $4.194 \mathrm{E}+0$ & $1.582 \mathrm{E}-1$ \\
\hline$\left[\mathrm{N}_{\mathrm{N}}\right] \lambda 5200 \ldots \ldots \ldots \ldots \ldots . .$. & $2.236 \mathrm{E}-3$ & $2.329 \mathrm{E}-2$ & $6.332 \mathrm{E}-2$ & $1.290 \mathrm{E}-1$ \\
\hline$[\mathrm{Fe}$ VII $] \lambda 6085 \ldots \ldots \ldots \ldots \ldots . . . . .$. & $9.089 \mathrm{E}-4$ & $2.836 \mathrm{E}-5$ & $0.000 \mathrm{E}+0$ & $0.000 \mathrm{E}+0$ \\
\hline$\left[\mathrm{O}_{\mathrm{I}}\right] \lambda 6300 \ldots \ldots \ldots \ldots \ldots \ldots$ & $9.162 \mathrm{E}-3$ & $9.462 \mathrm{E}-2$ & $2.759 \mathrm{E}-1$ & $6.258 \mathrm{E}-1$ \\
\hline $\mathrm{H} \alpha \quad \lambda 6563 \ldots \ldots \ldots \ldots \ldots \ldots \ldots$ & $2.839 \mathrm{E}+0$ & $2.853 \mathrm{E}+0$ & $2.887 \mathrm{E}+0$ & $2.922 \mathrm{E}+0$ \\
\hline 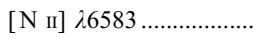 & $2.077 \mathrm{E}-2$ & $1.933 \mathrm{E}-1$ & $1.096 \mathrm{E}+0$ & $1.658 \mathrm{E}+0$ \\
\hline$[\mathrm{S}$ II] $\lambda \lambda 6717,6730 \ldots \ldots$. & $5.829 \mathrm{E}-2$ & $3.909 \mathrm{E}-1$ & $1.419 \mathrm{E}+0$ & $4.508 \mathrm{E}+0$ \\
\hline$[\mathrm{O}$ Іा $] \lambda \lambda 7318,7324 \ldots \ldots$. & $2.217 \mathrm{E}-3$ & $1.528 \mathrm{E}-2$ & $6.994 \mathrm{E}-2$ & $6.916 \mathrm{E}-2$ \\
\hline [S III] $\lambda \lambda 9069.9532 \ldots \ldots .$. & $3.092 \mathrm{E}-1$ & $1.924 \mathrm{E}+0$ & $4.232 \mathrm{E}+0$ & $2.758 \mathrm{E}+0$ \\
\hline \multicolumn{5}{|c|}{$\alpha=-2.0$} \\
\hline [O пा] $\lambda \lambda 3727,3729 \ldots . .$. & $8.841 \mathrm{E}-2$ & $6.200 \mathrm{E}-1$ & $3.184 \mathrm{E}+0$ & $4.166 \mathrm{E}+0$ \\
\hline$[\mathrm{S}$ II] $\lambda \lambda 4067,4076 \ldots \ldots$. & $2.452 \mathrm{E}-3$ & $1.613 \mathrm{E}-2$ & $8.071 \mathrm{E}-2$ & $2.621 \mathrm{E}-1$ \\
\hline$[\mathrm{O}$ III] $\lambda 4363 \ldots \ldots \ldots \ldots \ldots . .$. & $6.379 \mathrm{E}-2$ & $5.903 \mathrm{E}-2$ & $1.995 \mathrm{E}-2$ & $6.122 \mathrm{E}-4$ \\
\hline 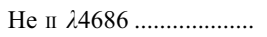 & $1.114 \mathrm{E}-1$ & $1.226 \mathrm{E}-1$ & $1.121 \mathrm{E}-1$ & $4.995 \mathrm{E}-2$ \\
\hline 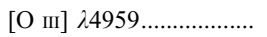 & $2.518 \mathrm{E}+0$ & $2.487 \mathrm{E}+0$ & $1.125 \mathrm{E}+0$ & $4.437 \mathrm{E}-2$ \\
\hline 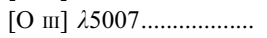 & $7.252 \mathrm{E}+0$ & $7.164 \mathrm{E}+0$ & $3.240 \mathrm{E}+0$ & $1.278 \mathrm{E}-1$ \\
\hline$\left[\mathrm{N}_{\mathrm{I}}\right] \lambda 5200 \ldots \ldots \ldots \ldots \ldots$ & $6.865 \mathrm{E}-4$ & $1.028 \mathrm{E}-2$ & $3.235 \mathrm{E}-2$ & $8.764 \mathrm{E}-2$ \\
\hline$[\mathrm{Fe}$ VII $] \lambda 6085 \ldots \ldots \ldots \ldots \ldots$ & $4.216 \mathrm{E}-4$ & $5.966 \mathrm{E}-6$ & $0.000 \mathrm{E}+0$ & $0.000 \mathrm{E}+0$ \\
\hline 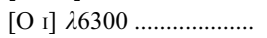 & $3.755 \mathrm{E}-3$ & $4.494 \mathrm{E}-2$ & $1.539 \mathrm{E}-1$ & $4.439 \mathrm{E}-1$ \\
\hline $\mathrm{H} \alpha \quad \lambda 6563 \ldots \ldots \ldots \ldots \ldots \ldots \ldots$ & $2.857 \mathrm{E}+0$ & $2.864 \mathrm{E}+0$ & $2.881 \mathrm{E}+0$ & $2.908 \mathrm{E}+0$ \\
\hline 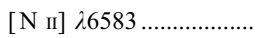 & $1.993 \mathrm{E}-2$ & $1.735 \mathrm{E}-1$ & $1.012 \mathrm{E}+0$ & $1.553 \mathrm{E}+0$ \\
\hline$[\mathrm{S}$ п] $\lambda \lambda 6717,6730 \ldots \ldots$. & $3.317 \mathrm{E}-2$ & $2.504 \mathrm{E}-1$ & $1.116 \mathrm{E}+0$ & $3.969 \mathrm{E}+0$ \\
\hline$[\mathrm{O}$ II] $\lambda \lambda 7318,7324 \ldots \ldots$. & $1.936 \mathrm{E}-3$ & $1.209 \mathrm{E}-2$ & $5.574 \mathrm{E}-2$ & $6.098 \mathrm{E}-2$ \\
\hline [S III] $\lambda \lambda 9069.9532 \ldots \ldots .$. & $3.158 \mathrm{E}-1$ & $1.838 \mathrm{E}+0$ & $3.658 \mathrm{E}+0$ & $2.272 \mathrm{E}+0$ \\
\hline
\end{tabular}


TABLE 26

Dust-free Visible Line Ratios with Respect to $\mathrm{H} \beta, 2 Z_{\odot}$

\begin{tabular}{|c|c|c|c|c|}
\hline \multirow[b]{2}{*}{ LiNE } & \multicolumn{4}{|c|}{$\log U_{0}$} \\
\hline & -1.0 & -2.0 & -3.0 & -4.0 \\
\hline \multicolumn{5}{|c|}{$\alpha=-1.2$} \\
\hline$[\mathrm{O}$ II] $\lambda \lambda 3727,3729 \ldots \ldots \ldots .$. & $1.278 \mathrm{E}-1$ & $1.328 \mathrm{E}+0$ & $5.194 \mathrm{E}+0$ & $5.537 \mathrm{E}+0$ \\
\hline$\left[\mathrm{S}_{\mathrm{II}}\right] \lambda \lambda 4067,4076 \ldots \ldots \ldots \ldots$ & $7.140 \mathrm{E}-3$ & $6.006 \mathrm{E}-2$ & $1.878 \mathrm{E}-1$ & $4.214 \mathrm{E}-1$ \\
\hline 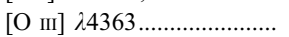 & $2.141 \mathrm{E}-1$ & $1.850 \mathrm{E}-1$ & $3.430 \mathrm{E}-2$ & $7.116 \mathrm{E}-4$ \\
\hline 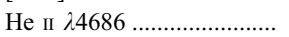 & $2.897 \mathrm{E}-1$ & $3.126 \mathrm{E}-1$ & $2.749 \mathrm{E}-1$ & $1.259 \mathrm{E}-1$ \\
\hline 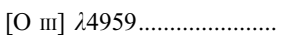 & $4.760 \mathrm{E}+0$ & $5.492 \mathrm{E}+0$ & $2.018 \mathrm{E}+0$ & $7.007 \mathrm{E}-2$ \\
\hline 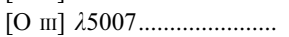 & $1.371 \mathrm{E}+1$ & $1.582 \mathrm{E}+1$ & $5.813 \mathrm{E}+0$ & $2.018 \mathrm{E}-1$ \\
\hline$\left[\mathrm{N}_{\mathrm{I}}\right] \lambda 5200 \ldots \ldots \ldots \ldots \ldots \ldots \ldots$ & $1.190 \mathrm{E}-2$ & $1.649 \mathrm{E}-1$ & $4.399 \mathrm{E}-1$ & $5.484 \mathrm{E}-1$ \\
\hline$[\mathrm{Fe}$ VII] $\lambda 6085 \ldots \ldots \ldots \ldots \ldots \ldots \ldots \ldots \ldots \ldots$ & $2.369 \mathrm{E}-3$ & $2.611 \mathrm{E}-4$ & $0.000 \mathrm{E}+0$ & $0.000 \mathrm{E}+0$ \\
\hline [O I] $\lambda 6300 \ldots \ldots \ldots \ldots \ldots \ldots \ldots$ & $2.535 \mathrm{E}-2$ & $3.995 \mathrm{E}-1$ & $1.021 \mathrm{E}+0$ & $1.416 \mathrm{E}+0$ \\
\hline 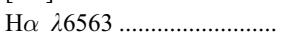 & $2.855 \mathrm{E}+0$ & $2.879 \mathrm{E}+0$ & $2.928 \mathrm{E}+0$ & $2.970 \mathrm{E}+0$ \\
\hline 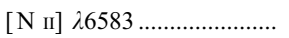 & $6.297 \mathrm{E}-2$ & $8.238 \mathrm{E}-1$ & $3.447 \mathrm{E}+0$ & $4.521 \mathrm{E}+0$ \\
\hline$[\mathrm{S}$ II] $\lambda \lambda 6717,6730 \ldots \ldots \ldots \ldots$ & $1.342 \mathrm{E}-1$ & $1.137 \mathrm{E}+0$ & $3.450 \mathrm{E}+0$ & $7.832 \mathrm{E}+0$ \\
\hline$[\mathrm{O}$ II] $\lambda \lambda 7318,7324 \ldots \ldots \ldots .$. & $2.432 \mathrm{E}-3$ & $2.362 \mathrm{E}-2$ & $8.328 \mathrm{E}-2$ & $6.775 \mathrm{E}-2$ \\
\hline$[\mathrm{S}$ III] $\lambda \lambda 9069.9532 \ldots \ldots \ldots \ldots$ & $4.646 \mathrm{E}-1$ & $3.351 \mathrm{E}+0$ & $8.126 \mathrm{E}+0$ & $5.820 \mathrm{E}+0$ \\
\hline \multicolumn{5}{|c|}{$\alpha=-1.4$} \\
\hline$[\mathrm{O}$ II] $\lambda \lambda 3727,3729 \ldots \ldots \ldots .$. & $1.087 \mathrm{E}-1$ & $9.700 \mathrm{E}-1$ & $4.101 \mathrm{E}+0$ & $4.940 \mathrm{E}+0$ \\
\hline$\left[\mathrm{S}_{\text {II }}\right] \lambda \lambda 4067,4076 \ldots \ldots \ldots . .$. & $4.975 \mathrm{E}-3$ & $4.305 \mathrm{E}-2$ & $1.448 \mathrm{E}-1$ & $3.738 \mathrm{E}-1$ \\
\hline$[\mathrm{O}$ III] $\lambda 4363 \ldots \ldots \ldots \ldots \ldots \ldots \ldots$ & $1.248 \mathrm{E}-1$ & $1.089 \mathrm{E}-1$ & $2.208 \mathrm{E}-2$ & $5.670 \mathrm{E}-4$ \\
\hline He II $\lambda 4686 \ldots \ldots \ldots \ldots \ldots \ldots \ldots$ & $2.274 \mathrm{E}-1$ & $2.526 \mathrm{E}-1$ & $2.303 \mathrm{E}-1$ & $1.058 \mathrm{E}-1$ \\
\hline 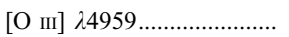 & $3.650 \mathrm{E}+0$ & $4.160 \mathrm{E}+0$ & $1.639 \mathrm{E}+0$ & $6.333 \mathrm{E}-2$ \\
\hline$[\mathrm{O}$ III] $25007 \ldots \ldots \ldots \ldots \ldots \ldots \ldots$ & $1.051 \mathrm{E}+1$ & $1.198 \mathrm{E}+1$ & $4.721 \mathrm{E}+0$ & $1.824 \mathrm{E}-1$ \\
\hline$\left[\mathrm{N}_{\mathrm{I}}\right] \lambda 5200 \ldots \ldots \ldots \ldots \ldots \ldots \ldots \ldots \ldots \ldots \ldots \ldots \ldots$ & $6.204 \mathrm{E}-3$ & $1.073 \mathrm{E}-1$ & $2.740 \mathrm{E}-1$ & $3.794 \mathrm{E}-1$ \\
\hline$[\mathrm{Fe}$ VII $] \lambda 6085 \ldots \ldots \ldots \ldots \ldots \ldots \ldots \ldots \ldots \ldots$ & $1.947 \mathrm{E}-3$ & $1.066 \mathrm{E}-4$ & $0.000 \mathrm{E}+0$ & $0.000 \mathrm{E}+0$ \\
\hline$\left[\mathrm{O}_{\mathrm{I}}\right] \mathrm{\lambda} 6300 \ldots \ldots \ldots \ldots \ldots \ldots \ldots$ & $1.405 \mathrm{E}-2$ & $2.481 \mathrm{E}-1$ & $6.414 \mathrm{E}-1$ & $1.026 \mathrm{E}+0$ \\
\hline 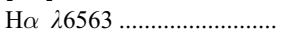 & $2.870 \mathrm{E}+0$ & $2.878 \mathrm{E}+0$ & $2.916 \mathrm{E}+0$ & $2.953 \mathrm{E}+0$ \\
\hline 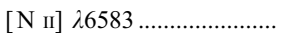 & $5.649 \mathrm{E}-2$ & $6.129 \mathrm{E}-1$ & $2.921 \mathrm{E}+0$ & $4.223 \mathrm{E}+0$ \\
\hline$\left[\mathrm{S}_{\mathrm{II}}\right] \lambda \lambda 6717,6730 \ldots \ldots \ldots . .$. & $9.044 \mathrm{E}-2$ & $8.249 \mathrm{E}-1$ & $2.576 \mathrm{E}+0$ & $6.830 \mathrm{E}+0$ \\
\hline$[\mathrm{O}$ II $] \lambda \lambda 7318,7324 \ldots \ldots \ldots$. & $2.002 \mathrm{E}-3$ & $1.611 \mathrm{E}-2$ & $6.023 \mathrm{E}-2$ & $5.768 \mathrm{E}-2$ \\
\hline$[\mathrm{S}$ III] $\lambda \lambda 9069.9532 \ldots \ldots \ldots \ldots$ & $4.349 \mathrm{E}-1$ & $2.933 \mathrm{E}+0$ & $6.789 \mathrm{E}+0$ & $4.681 \mathrm{E}+0$ \\
\hline \multicolumn{5}{|c|}{$\alpha=-1.7$} \\
\hline$[\mathrm{O}$ II] $\lambda \lambda 3727,3729 \ldots \ldots \ldots$ & $8.736 \mathrm{E}-2$ & $6.361 \mathrm{E}-1$ & $2.926 \mathrm{E}+0$ & $4.076 \mathrm{E}+0$ \\
\hline$[\mathrm{S}$ II $] \lambda \lambda 4067,4076 \ldots \ldots \ldots . .$. & $2.916 \mathrm{E}-3$ & $2.534 \mathrm{E}-2$ & $1.022 \mathrm{E}-1$ & $3.099 \mathrm{E}-1$ \\
\hline 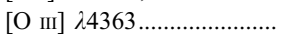 & $5.382 \mathrm{E}-2$ & $4.703 \mathrm{E}-2$ & $1.078 \mathrm{E}-2$ & $3.715 \mathrm{E}-4$ \\
\hline He II $\lambda 4686 \ldots \ldots \ldots \ldots \ldots \ldots \ldots$ & $1.575 \mathrm{E}-1$ & $1.807 \mathrm{E}-1$ & $1.693 \mathrm{E}-1$ & $7.668 \mathrm{E}-2$ \\
\hline 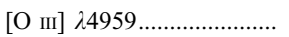 & $2.344 \mathrm{E}+0$ & $2.640 \mathrm{E}+0$ & $1.140 \mathrm{E}+0$ & $4.999 \mathrm{E}-2$ \\
\hline$[\mathrm{O}$ III] $\lambda 5007 \ldots \ldots \ldots \ldots \ldots \ldots \ldots$ & $6.752 \mathrm{E}+0$ & $7.604 \mathrm{E}+0$ & $3.283 \mathrm{E}+0$ & $1.440 \mathrm{E}-1$ \\
\hline$\left[\mathrm{N}_{\mathrm{I}}\right] \quad \lambda 5200 \ldots \ldots \ldots \ldots \ldots \ldots \ldots \ldots \ldots \ldots \ldots$ & $1.615 \mathrm{E}-3$ & $4.901 \mathrm{E}-2$ & $1.273 \mathrm{E}-1$ & $2.270 \mathrm{E}-1$ \\
\hline$[\mathrm{Fe}$ VII $] \lambda 6085 \ldots \ldots \ldots \ldots \ldots \ldots$ & $1.088 \mathrm{E}-3$ & $2.291 \mathrm{E}-5$ & $0.000 \mathrm{E}+0$ & $0.000 \mathrm{E}+0$ \\
\hline$\left[\mathrm{O}_{\mathrm{I}}\right] \mathrm{\lambda} 6300 \ldots \ldots \ldots \ldots \ldots \ldots \ldots$ & $4.981 \mathrm{E}-3$ & $1.120 \mathrm{E}-1$ & $3.127 \mathrm{E}-1$ & $6.520 \mathrm{E}-1$ \\
\hline 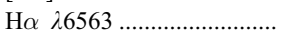 & $2.900 \mathrm{E}+0$ & $2.898 \mathrm{E}+0$ & $2.916 \mathrm{E}+0$ & $2.936 \mathrm{E}+0$ \\
\hline$[\mathrm{N}$ II] $\lambda 6583 \ldots \ldots \ldots \ldots \ldots \ldots \ldots$ & $5.038 \mathrm{E}-2$ & $4.417 \mathrm{E}-1$ & $2.369 \mathrm{E}+0$ & $3.740 \mathrm{E}+0$ \\
\hline$[\mathrm{S}$ II $] \lambda \lambda 6717,6730 \ldots \ldots \ldots . .$. & $4.629 \mathrm{E}-2$ & $4.841 \mathrm{E}-1$ & $1.763 \mathrm{E}+0$ & $5.664 \mathrm{E}+0$ \\
\hline$[\mathrm{O}$ II $] \lambda \lambda 7318,7324 \ldots \ldots \ldots .$. & $1.504 \mathrm{E}-3$ & $9.411 \mathrm{E}-3$ & $3.730 \mathrm{E}-2$ & $4.392 \mathrm{E}-2$ \\
\hline$[\mathrm{S}$ III] $\lambda \lambda 9069.9532 \ldots \ldots \ldots \ldots$ & $4.058 \mathrm{E}-1$ & $2.456 \mathrm{E}+0$ & $5.263 \mathrm{E}+0$ & $3.530 \mathrm{E}+0$ \\
\hline \multicolumn{5}{|c|}{$\alpha=-2.0$} \\
\hline$[\mathrm{O}$ II] $\lambda \lambda 3727,3729 \ldots \ldots \ldots$. & $6.762 \mathrm{E}-2$ & $4.364 \mathrm{E}-1$ & $2.156 \mathrm{E}+0$ & $3.319 \mathrm{E}+0$ \\
\hline$[\mathrm{S}$ II] $\lambda \lambda 4067,4076 \ldots \ldots \ldots \ldots$ & $1.877 \mathrm{E}-3$ & $1.477 \mathrm{E}-2$ & $7.533 \mathrm{E}-2$ & $2.554 \mathrm{E}-1$ \\
\hline 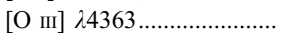 & $2.420 \mathrm{E}-2$ & $1.934 \mathrm{E}-2$ & $5.202 \mathrm{E}-3$ & $2.303 \mathrm{E}-4$ \\
\hline 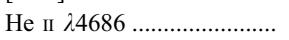 & $1.097 \mathrm{E}-1$ & $1.286 \mathrm{E}-1$ & $1.200 \mathrm{E}-1$ & $5.257 \mathrm{E}-2$ \\
\hline 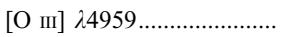 & $1.471 \mathrm{E}+0$ & $1.643 \mathrm{E}+0$ & $7.710 \mathrm{E}-1$ & $3.692 \mathrm{E}-2$ \\
\hline$[\mathrm{O}$ III] $\lambda 5007 \ldots \ldots \ldots \ldots \ldots \ldots \ldots$ & $4.236 \mathrm{E}+0$ & $4.732 \mathrm{E}+0$ & $2.221 \mathrm{E}+0$ & $1.063 \mathrm{E}-1$ \\
\hline$\left[\mathrm{N}_{\mathrm{I}}\right] \lambda 5200 \ldots \ldots \ldots \ldots \ldots \ldots \ldots$ & $3.534 \mathrm{E}-4$ & $1.838 \mathrm{E}-2$ & $5.700 \mathrm{E}-2$ & $1.438 \mathrm{E}-1$ \\
\hline$[\mathrm{Fe}$ VII $] \lambda 6085 \ldots \ldots \ldots \ldots \ldots \ldots$ & $4.500 \mathrm{E}-4$ & $4.000 \mathrm{E}-6$ & $0.000 \mathrm{E}+0$ & $0.000 \mathrm{E}+0$ \\
\hline$\left[\mathrm{O}_{\text {I }}\right] \lambda 6300 \ldots \ldots \ldots \ldots \ldots \ldots \ldots$ & $2.129 \mathrm{E}-3$ & $4.521 \mathrm{E}-2$ & $1.524 \mathrm{E}-1$ & $4.301 \mathrm{E}-1$ \\
\hline 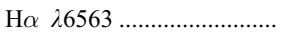 & $2.932 \mathrm{E}+0$ & $2.925 \mathrm{E}+0$ & $2.924 \mathrm{E}+0$ & $2.933 \mathrm{E}+0$ \\
\hline$[\mathrm{N}$ II] $\lambda 6583 \ldots \ldots \ldots \ldots \ldots \ldots \ldots$ & $4.485 \mathrm{E}-2$ & $3.546 \mathrm{E}-1$ & $1.991 \mathrm{E}+0$ & $3.265 \mathrm{E}+0$ \\
\hline$\left[\mathrm{S}_{\mathrm{II}}\right] \lambda \lambda 6717,6730 \ldots \ldots \ldots . .$. & $2.508 \mathrm{E}-2$ & $2.766 \mathrm{E}-1$ & $1.306 \mathrm{E}+0$ & $4.753 \mathrm{E}+0$ \\
\hline$[\mathrm{O}$ II $] \lambda \lambda 7318,7324 \ldots \ldots \ldots .$. & $1.040 \mathrm{E}-3$ & $5.566 \mathrm{E}-3$ & $2.382 \mathrm{E}-2$ & $3.287 \mathrm{E}-2$ \\
\hline$[\mathrm{S}$ III] $\lambda \lambda 9069.9532 \ldots \ldots \ldots \ldots$ & $3.868 \mathrm{E}-1$ & $2.107 \mathrm{E}+0$ & $4.201 \mathrm{E}+0$ & $2.786 \mathrm{E}+0$ \\
\hline
\end{tabular}


TABLE 27

Dust-free Visible Line Ratios with Respect to $\mathrm{H} \beta, 4 Z_{\odot}$

\begin{tabular}{|c|c|c|c|c|}
\hline \multirow[b]{2}{*}{ LiNE } & \multicolumn{4}{|c|}{$\log U_{0}$} \\
\hline & -1.0 & -2.0 & -3.0 & -4.0 \\
\hline \multicolumn{5}{|c|}{$\alpha=-1.2$} \\
\hline$[\mathrm{O}$ II] $\lambda \lambda 3727,3729 \ldots \ldots \ldots$ & $4.478 \mathrm{E}-2$ & $9.577 \mathrm{E}-1$ & $3.242 \mathrm{E}+0$ & $2.998 \mathrm{E}+0$ \\
\hline$[\mathrm{S}$ II $] \lambda \lambda 4067,4076 \ldots \ldots \ldots . .$. & $2.603 \mathrm{E}-3$ & $6.414 \mathrm{E}-2$ & $1.776 \mathrm{E}-1$ & $3.002 \mathrm{E}-1$ \\
\hline 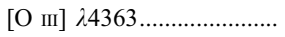 & $1.431 \mathrm{E}-1$ & $1.064 \mathrm{E}-1$ & $8.556 \mathrm{E}-3$ & $1.447 \mathrm{E}-4$ \\
\hline 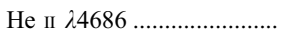 & $2.920 \mathrm{E}-1$ & $3.274 \mathrm{E}-1$ & $3.043 \mathrm{E}-1$ & $1.364 \mathrm{E}-1$ \\
\hline 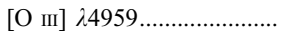 & $3.065 \mathrm{E}+0$ & $4.525 \mathrm{E}+0$ & $1.334 \mathrm{E}+0$ & $4.306 \mathrm{E}-2$ \\
\hline 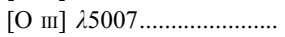 & $8.826 \mathrm{E}+0$ & $1.303 \mathrm{E}+1$ & $3.841 \mathrm{E}+0$ & $1.240 \mathrm{E}-1$ \\
\hline$\left[\mathrm{N}_{\mathrm{I}}\right] \lambda 5200 \ldots \ldots \ldots \ldots \ldots \ldots$ & $7.177 \mathrm{E}-3$ & $3.372 \mathrm{E}-1$ & $8.396 \mathrm{E}-1$ & $6.737 \mathrm{E}-1$ \\
\hline 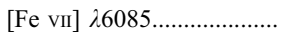 & $3.096 \mathrm{E}-3$ & $2.159 \mathrm{E}-4$ & $0.000 \mathrm{E}+0$ & $0.000 \mathrm{E}+0$ \\
\hline$[\mathrm{O}$ I] $\lambda 6300 \ldots \ldots \ldots \ldots \ldots \ldots$ & $8.991 \mathrm{E}-3$ & $4.302 \mathrm{E}-1$ & $1.045 \mathrm{E}+0$ & $1.001 \mathrm{E}+0$ \\
\hline 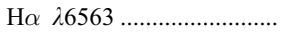 & $2.956 \mathrm{E}+0$ & $2.919 \mathrm{E}+0$ & $2.957 \mathrm{E}+0$ & $3.020 \mathrm{E}+0$ \\
\hline 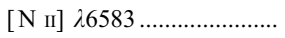 & $8.131 \mathrm{E}-2$ & $1.593 \mathrm{E}+0$ & $6.487 \mathrm{E}+0$ & $7.771 \mathrm{E}+0$ \\
\hline$[\mathrm{S}$ II $] \lambda \lambda 6717,6730 \ldots \ldots \ldots . .$. & $7.132 \mathrm{E}-2$ & $1.364 \mathrm{E}+0$ & $3.910 \mathrm{E}+0$ & $7.509 \mathrm{E}+0$ \\
\hline$[\mathrm{O}$ II] $\lambda \lambda 7318,7324 \ldots \ldots \ldots . .$. & $6.146 \mathrm{E}-4$ & $1.189 \mathrm{E}-2$ & $3.152 \mathrm{E}-2$ & $2.113 \mathrm{E}-2$ \\
\hline [S III] $\lambda \lambda 9069.9532 \ldots \ldots \ldots . .$. & $3.787 \mathrm{E}-1$ & $3.902 \mathrm{E}+0$ & $9.142 \mathrm{E}+0$ & $5.709 \mathrm{E}+0$ \\
\hline \multicolumn{5}{|c|}{$\alpha=-1.4$} \\
\hline$[\mathrm{O}$ II] $\lambda \lambda 3727,3729 \ldots \ldots \ldots$ & $2.880 \mathrm{E}-2$ & $5.294 \mathrm{E}-1$ & $2.066 \mathrm{E}+0$ & $2.376 \mathrm{E}+0$ \\
\hline$[\mathrm{S}$ II $] \lambda \lambda 4067,4076 \ldots \ldots \ldots . .$. & $1.303 \mathrm{E}-3$ & $3.875 \mathrm{E}-2$ & $1.161 \mathrm{E}-1$ & $2.366 \mathrm{E}-1$ \\
\hline 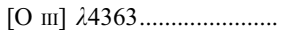 & $7.671 \mathrm{E}-2$ & $5.226 \mathrm{E}-2$ & $4.217 \mathrm{E}-3$ & $1.001 \mathrm{E}-4$ \\
\hline 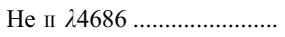 & $2.275 \mathrm{E}-1$ & $2.647 \mathrm{E}-1$ & $2.524 \mathrm{E}-1$ & $1.132 \mathrm{E}-1$ \\
\hline 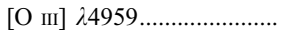 & $1.974 \mathrm{E}+0$ & $2.813 \mathrm{E}+0$ & $9.373 \mathrm{E}-1$ & $3.617 \mathrm{E}-2$ \\
\hline [O III] $25007 \ldots \ldots \ldots \ldots \ldots \ldots$ & $5.685 \mathrm{E}+0$ & $8.101 \mathrm{E}+0$ & $2.699 \mathrm{E}+0$ & $1.042 \mathrm{E}-1$ \\
\hline$\left[\mathrm{N}_{\mathrm{I}}\right] \lambda 5200 \ldots \ldots \ldots \ldots \ldots \ldots \ldots$ & $2.620 \mathrm{E}-3$ & $2.026 \mathrm{E}-1$ & $4.675 \mathrm{E}-1$ & $4.104 \mathrm{E}-1$ \\
\hline$[\mathrm{Fe}$ vII $] \lambda 6085 \ldots \ldots \ldots \ldots \ldots \ldots$ & $2.299 \mathrm{E}-3$ & $7.843 \mathrm{E}-5$ & $0.000 \mathrm{E}+0$ & $0.000 \mathrm{E}+0$ \\
\hline 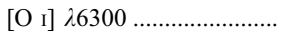 & $3.794 \mathrm{E}-3$ & $2.458 \mathrm{E}-1$ & $5.792 \mathrm{E}-1$ & $6.477 \mathrm{E}-1$ \\
\hline $\mathrm{H} \alpha \quad \lambda 6563 \ldots \ldots \ldots \ldots \ldots \ldots \ldots$ & $2.995 \mathrm{E}+0$ & $2.947 \mathrm{E}+0$ & $2.966 \mathrm{E}+0$ & $3.009 \mathrm{E}+0$ \\
\hline 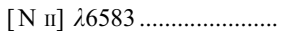 & $6.310 \mathrm{E}-2$ & $1.013 \mathrm{E}+0$ & $4.873 \mathrm{E}+0$ & $6.805 \mathrm{E}+0$ \\
\hline$[\mathrm{S}$ II $] \lambda \lambda 6717,6730 \ldots \ldots \ldots . .$. & $3.822 \mathrm{E}-2$ & $8.955 \mathrm{E}-1$ & $2.680 \mathrm{E}+0$ & $6.135 \mathrm{E}+0$ \\
\hline$[\mathrm{O}$ II] $\lambda \lambda 7318,7324 \ldots \ldots \ldots$. & $3.637 \mathrm{E}-4$ & $5.756 \mathrm{E}-3$ & $1.693 \mathrm{E}-2$ & $1.519 \mathrm{E}-2$ \\
\hline$[\mathrm{S}$ III] $\lambda \lambda 9069.9532 \ldots \ldots \ldots \ldots$. & $3.064 \mathrm{E}-1$ & $2.996 \mathrm{E}+0$ & $6.960 \mathrm{E}+0$ & $4.388 \mathrm{E}+0$ \\
\hline \multicolumn{5}{|c|}{$\alpha=-1.7$} \\
\hline$[\mathrm{O}$ II] $\lambda \lambda 3727,3729 \ldots \ldots \ldots$. & $1.354 \mathrm{E}-2$ & $2.195 \mathrm{E}-1$ & $1.101 \mathrm{E}+0$ & $1.713 \mathrm{E}+0$ \\
\hline$[\mathrm{S}$ II] $\lambda \lambda 4067,4076 \ldots \ldots \ldots . .$. & $2.774 \mathrm{E}-4$ & $1.578 \mathrm{E}-2$ & $6.131 \mathrm{E}-2$ & $1.695 \mathrm{E}-1$ \\
\hline 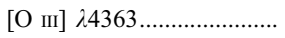 & $3.056 \mathrm{E}-2$ & $1.615 \mathrm{E}-2$ & $1.382 \mathrm{E}-3$ & $5.511 \mathrm{E}-5$ \\
\hline 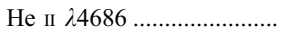 & $1.562 \mathrm{E}-1$ & $1.914 \mathrm{E}-1$ & $1.822 \mathrm{E}-1$ & $8.006 \mathrm{E}-2$ \\
\hline 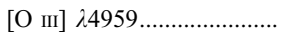 & $9.683 \mathrm{E}-1$ & $1.280 \mathrm{E}+0$ & $5.274 \mathrm{E}-1$ & $2.603 \mathrm{E}-2$ \\
\hline 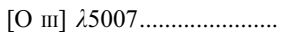 & $2.789 \mathrm{E}+0$ & $3.688 \mathrm{E}+0$ & $1.519 \mathrm{E}+0$ & $7.496 \mathrm{E}-2$ \\
\hline$\left[\mathrm{N}_{\mathrm{I}}\right] \lambda 5200 \ldots \ldots \ldots \ldots \ldots \ldots \ldots \ldots \ldots \ldots$ & $2.446 \mathrm{E}-4$ & $7.572 \mathrm{E}-2$ & $1.650 \mathrm{E}-1$ & $2.061 \mathrm{E}-1$ \\
\hline$[\mathrm{Fe}$ VII $] \lambda 6085 \ldots \ldots \ldots \ldots \ldots \ldots$ & $1.117 \mathrm{E}-3$ & $1.343 \mathrm{E}-5$ & $0.000 \mathrm{E}+0$ & $0.000 \mathrm{E}+0$ \\
\hline$\left[\mathrm{O}_{\mathrm{I}}\right]$ & $4.243 \mathrm{E}-4$ & $8.750 \mathrm{E}-2$ & $2.107 \mathrm{E}-1$ & $3.546 \mathrm{E}-1$ \\
\hline 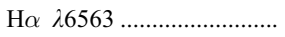 & $3.057 \mathrm{E}+0$ & $2.997 \mathrm{E}+0$ & $2.987 \mathrm{E}+0$ & $3.003 \mathrm{E}+0$ \\
\hline 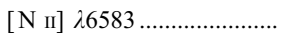 & $4.605 \mathrm{E}-2$ & $5.702 \mathrm{E}-1$ & $3.386 \mathrm{E}+0$ & $5.589 \mathrm{E}+0$ \\
\hline$[\mathrm{S}$ II] $\lambda \lambda 6717,6730 \ldots \ldots \ldots . .$. & $1.013 \mathrm{E}-2$ & $4.254 \mathrm{E}-1$ & $1.563 \mathrm{E}+0$ & $4.662 \mathrm{E}+0$ \\
\hline$[\mathrm{O}$ II] $\lambda \lambda 7318,7324 \ldots \ldots \ldots$. & $1.474 \mathrm{E}-4$ & $1.893 \mathrm{E}-3$ & $6.899 \mathrm{E}-3$ & $9.531 \mathrm{E}-3$ \\
\hline [S III] $\lambda \lambda 9069.9532 \ldots \ldots \ldots \ldots$ & $2.182 \mathrm{E}-1$ & $1.985 \mathrm{E}+0$ & $4.703 \mathrm{E}+0$ & $3.143 \mathrm{E}+0$ \\
\hline \multicolumn{5}{|c|}{$\alpha=-2.0$} \\
\hline$[\mathrm{O}$ пा] $\lambda \lambda 3727,3729 \ldots \ldots \ldots$ & $2.601 \mathrm{E}-2$ & $1.262 \mathrm{E}-1$ & $6.599 \mathrm{E}-1$ & $1.272 \mathrm{E}+0$ \\
\hline$[\mathrm{S}$ II $] \lambda \lambda 4067,4076 \ldots \ldots \ldots . .$. & $2.153 \mathrm{E}-4$ & $5.440 \mathrm{E}-3$ & $3.471 \mathrm{E}-2$ & $1.268 \mathrm{E}-1$ \\
\hline 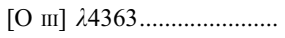 & $1.280 \mathrm{E}-2$ & $4.116 \mathrm{E}-3$ & $4.460 \mathrm{E}-4$ & $2.988 \mathrm{E}-5$ \\
\hline 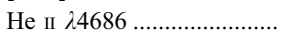 & $1.077 \mathrm{E}-1$ & $1.378 \mathrm{E}-1$ & $1.266 \mathrm{E}-1$ & $5.357 \mathrm{E}-2$ \\
\hline 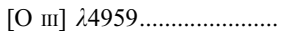 & $4.700 \mathrm{E}-1$ & $5.397 \mathrm{E}-1$ & $2.899 \mathrm{E}-1$ & $1.789 \mathrm{E}-2$ \\
\hline 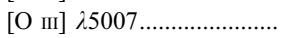 & $1.354 \mathrm{E}+0$ & $1.554 \mathrm{E}+0$ & $8.349 \mathrm{E}-1$ & $5.152 \mathrm{E}-2$ \\
\hline$\left[\mathrm{N}_{\mathrm{I}}\right] \lambda 5200 \ldots \ldots \ldots \ldots \ldots \ldots \ldots \ldots \ldots \ldots \ldots$ & $7.518 \mathrm{E}-5$ & $1.380 \mathrm{E}-2$ & $4.468 \mathrm{E}-2$ & $1.149 \mathrm{E}-1$ \\
\hline 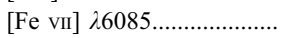 & $4.032 \mathrm{E}-4$ & $1.718 \mathrm{E}-6$ & $0.000 \mathrm{E}+0$ & $0.000 \mathrm{E}+0$ \\
\hline$\left[\mathrm{O}_{\mathrm{I}}\right]$ & $1.773 \mathrm{E}-4$ & $1.706 \mathrm{E}-2$ & $6.421 \mathrm{E}-2$ & $2.112 \mathrm{E}-1$ \\
\hline 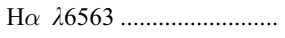 & $3.113 \mathrm{E}+0$ & $3.043 \mathrm{E}+0$ & $3.006 \mathrm{E}+0$ & $3.005 \mathrm{E}+0$ \\
\hline 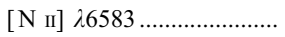 & $1.300 \mathrm{E}-1$ & $4.759 \mathrm{E}-1$ & $2.574 \mathrm{E}+0$ & $4.644 \mathrm{E}+0$ \\
\hline$[\mathrm{S}$ II $] \lambda \lambda 6717,6730 \ldots \ldots \ldots . .$. & $7.599 \mathrm{E}-3$ & $1.770 \mathrm{E}-1$ & $9.880 \mathrm{E}-1$ & $3.698 \mathrm{E}+0$ \\
\hline$[\mathrm{O}$ II] $\lambda \lambda 7318,7324 \ldots \ldots \ldots .$. & $1.361 \mathrm{E}-4$ & $7.695 \mathrm{E}-4$ & $3.235 \mathrm{E}-3$ & $6.226 \mathrm{E}-3$ \\
\hline [S III] $\lambda \lambda 9069.9532 \ldots \ldots \ldots . .$. & $2.518 \mathrm{E}-1$ & $1.370 \mathrm{E}+0$ & $3.340 \mathrm{E}+0$ & $2.399 \mathrm{E}+0$ \\
\hline
\end{tabular}


TABLE 28

Dust-free IR Line Ratios with Respect to $\mathrm{H} \beta, 0.25 Z_{\odot}$

\begin{tabular}{|c|c|c|c|c|}
\hline \multirow[b]{2}{*}{ LiNE } & \multicolumn{4}{|c|}{$\log U_{0}$} \\
\hline & -1.0 & -2.0 & -3.0 & -4.0 \\
\hline \multicolumn{5}{|c|}{$\alpha=-1.2$} \\
\hline 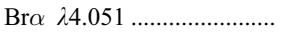 & $5.819 \mathrm{E}-2$ & $6.462 \mathrm{E}-2$ & $7.327 \mathrm{E}-2$ & $8.064 \mathrm{E}-2$ \\
\hline 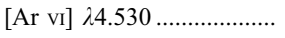 & $8.949 \mathrm{E}-3$ & $9.005 \mathrm{E}-4$ & $0.000 \mathrm{E}+0$ & $0.000 \mathrm{E}+0$ \\
\hline 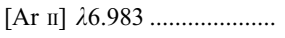 & $1.132 \mathrm{E}-3$ & $7.294 \mathrm{E}-3$ & $2.367 \mathrm{E}-2$ & $5.422 \mathrm{E}-2$ \\
\hline 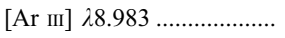 & $3.142 \mathrm{E}-1$ & $3.925 \mathrm{E}-2$ & $3.902 \mathrm{E}-5$ & $0.000 \mathrm{E}+0$ \\
\hline$[\mathrm{Ne}$ vI $] \lambda 7.652 \ldots \ldots \ldots \ldots \ldots . . . .$. & $7.458 \mathrm{E}-3$ & $4.097 \mathrm{E}-2$ & $6.394 \mathrm{E}-2$ & $4.018 \mathrm{E}-2$ \\
\hline 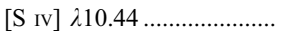 & $8.766 \mathrm{E}-1$ & $7.646 \mathrm{E}-1$ & $2.230 \mathrm{E}-1$ & $3.026 \mathrm{E}-3$ \\
\hline$[\mathrm{Ne}$ II] $\lambda 12.8 \ldots \ldots \ldots \ldots \ldots \ldots \ldots$ & $1.453 \mathrm{E}-2$ & $1.051 \mathrm{E}-1$ & $4.903 \mathrm{E}-1$ & $1.338 \mathrm{E}+0$ \\
\hline 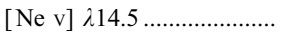 & $7.141 \mathrm{E}-2$ & $6.510 \mathrm{E}-2$ & $6.260 \mathrm{E}-4$ & $0.000 \mathrm{E}+0$ \\
\hline$[\mathrm{Ne}$ III] $\lambda 15.5 \ldots \ldots \ldots \ldots \ldots \ldots . .$. & $5.677 \mathrm{E}-1$ & $6.650 \mathrm{E}-1$ & $9.389 \mathrm{E}-1$ & $9.469 \mathrm{E}-1$ \\
\hline$[\mathrm{S}$ III] $\lambda 18.7 \ldots \ldots \ldots \ldots \ldots \ldots \ldots$ & $3.098 \mathrm{E}-2$ & $1.723 \mathrm{E}-1$ & $5.087 \mathrm{E}-1$ & $5.622 \mathrm{E}-1$ \\
\hline 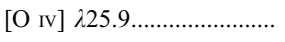 & $6.972 \mathrm{E}-1$ & $9.790 \mathrm{E}-1$ & $2.999 \mathrm{E}-1$ & $9.314 \mathrm{E}-4$ \\
\hline \multicolumn{5}{|c|}{$\alpha=-1.4$} \\
\hline 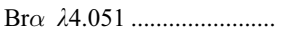 & $6.087 \mathrm{E}-2$ & $6.571 \mathrm{E}-2$ & $7.326 \mathrm{E}-2$ & $8.003 \mathrm{E}-2$ \\
\hline$[\mathrm{Ar} \mathrm{vI}] \lambda 4.530 \ldots \ldots \ldots \ldots \ldots \ldots . . . . . . .$. & $7.078 \mathrm{E}-3$ & $5.064 \mathrm{E}-4$ & $0.000 \mathrm{E}+0$ & $0.000 \mathrm{E}+0$ \\
\hline$[\mathrm{Ar}$ II $] \lambda 6.983 \ldots \ldots \ldots \ldots \ldots \ldots . . . . . . .$. & $8.185 \mathrm{E}-4$ & $5.249 \mathrm{E}-3$ & $1.734 \mathrm{E}-2$ & $4.191 \mathrm{E}-2$ \\
\hline [Ar III] $\lambda 8.983 \ldots \ldots \ldots \ldots \ldots . . . . . . .$. & $2.294 \mathrm{E}-1$ & $1.956 \mathrm{E}-2$ & $1.740 \mathrm{E}-5$ & $0.000 \mathrm{E}+0$ \\
\hline$[\mathrm{Ne}$ vI $] \lambda 7.652 \ldots \ldots \ldots \ldots \ldots . . . . .$. & $7.966 \mathrm{E}-3$ & $4.360 \mathrm{E}-2$ & $6.661 \mathrm{E}-2$ & $4.208 \mathrm{E}-2$ \\
\hline [S IV] $\lambda 10.44 \ldots \ldots \ldots \ldots \ldots \ldots \ldots$ & $1.027 \mathrm{E}+0$ & $8.820 \mathrm{E}-1$ & $2.021 \mathrm{E}-1$ & $2.595 \mathrm{E}-3$ \\
\hline$[\mathrm{Ne}$ II] $\lambda 12.8 \ldots \ldots \ldots \ldots \ldots \ldots$ & $1.318 \mathrm{E}-2$ & $9.064 \mathrm{E}-2$ & $3.865 \mathrm{E}-1$ & $9.732 \mathrm{E}-1$ \\
\hline 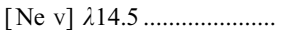 & $6.495 \mathrm{E}-2$ & $4.591 \mathrm{E}-2$ & $4.057 \mathrm{E}-4$ & $0.000 \mathrm{E}+0$ \\
\hline$[\mathrm{Ne}$ III] $\lambda 15.5 \ldots \ldots \ldots \ldots \ldots \ldots \ldots$ & $6.357 \mathrm{E}-1$ & $6.888 \mathrm{E}-1$ & $8.432 \mathrm{E}-1$ & $7.894 \mathrm{E}-1$ \\
\hline [S III] $\lambda 18.7 \ldots \ldots \ldots \ldots \ldots \ldots \ldots . .$. & $2.782 \mathrm{E}-2$ & $1.647 \mathrm{E}-1$ & $4.613 \mathrm{E}-1$ & $4.580 \mathrm{E}-1$ \\
\hline$[\mathrm{O}$ Iv $] \lambda 25.9 \ldots \ldots \ldots \ldots \ldots \ldots \ldots \ldots \ldots \ldots \ldots \ldots \ldots \ldots$ & $5.537 \mathrm{E}-1$ & $8.300 \mathrm{E}-1$ & $2.499 \mathrm{E}-1$ & $8.138 \mathrm{E}-4$ \\
\hline \multicolumn{5}{|c|}{$\alpha=-1.7$} \\
\hline 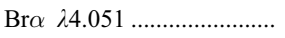 & $6.414 \mathrm{E}-2$ & $6.733 \mathrm{E}-2$ & $7.347 \mathrm{E}-2$ & $7.944 \mathrm{E}-2$ \\
\hline$[\mathrm{Ar}$ vI] $\lambda 4.530 \ldots \ldots \ldots \ldots \ldots . . . . . . . .$. & $4.509 \mathrm{E}-3$ & $1.840 \mathrm{E}-4$ & $0.000 \mathrm{E}+0$ & $0.000 \mathrm{E}+0$ \\
\hline$[\mathrm{Ar}$ II] $\lambda 6.983 \ldots \ldots \ldots \ldots \ldots . . .$. & $4.747 \mathrm{E}-4$ & $3.096 \mathrm{E}-3$ & $1.073 \mathrm{E}-2$ & $2.977 \mathrm{E}-2$ \\
\hline 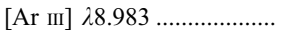 & $1.213 \mathrm{E}-1$ & $5.631 \mathrm{E}-3$ & $4.355 \mathrm{E}-6$ & $0.000 \mathrm{E}+0$ \\
\hline$[\mathrm{Ne}$ vI] $\lambda 7.652 \ldots \ldots \ldots \ldots \ldots \ldots$ & $9.023 \mathrm{E}-3$ & $4.734 \mathrm{E}-2$ & $6.868 \mathrm{E}-2$ & $4.250 \mathrm{E}-2$ \\
\hline 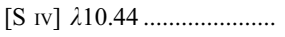 & $1.198 \mathrm{E}+0$ & $9.702 \mathrm{E}-1$ & $1.624 \mathrm{E}-1$ & $1.946 \mathrm{E}-3$ \\
\hline$[\mathrm{Ne}$ II] $\lambda 12.8 \ldots \ldots \ldots \ldots \ldots \ldots$ & $1.044 \mathrm{E}-2$ & $6.760 \mathrm{E}-2$ & $2.576 \mathrm{E}-1$ & $6.166 \mathrm{E}-1$ \\
\hline$[\mathrm{Ne} \mathrm{v}] \lambda 14.5 \ldots \ldots \ldots \ldots \ldots \ldots \ldots \ldots \ldots \ldots \ldots$ & $5.389 \mathrm{E}-2$ & $2.304 \mathrm{E}-2$ & $1.827 \mathrm{E}-4$ & $0.000 \mathrm{E}+0$ \\
\hline$[\mathrm{Ne}$ III] $\lambda 15.5 \ldots \ldots \ldots \ldots \ldots \ldots \ldots$ & $6.987 \mathrm{E}-1$ & $7.212 \mathrm{E}-1$ & $7.406 \mathrm{E}-1$ & $6.021 \mathrm{E}-1$ \\
\hline 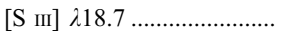 & $2.591 \mathrm{E}-2$ & $1.657 \mathrm{E}-1$ & $4.141 \mathrm{E}-1$ & $3.470 \mathrm{E}-1$ \\
\hline 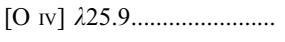 & $3.959 \mathrm{E}-1$ & $5.961 \mathrm{E}-1$ & $1.736 \mathrm{E}-1$ & $5.866 \mathrm{E}-4$ \\
\hline \multicolumn{5}{|c|}{$\alpha=-2.0$} \\
\hline 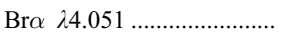 & $6.660 \mathrm{E}-2$ & $6.883 \mathrm{E}-2$ & $7.384 \mathrm{E}-2$ & $7.923 \mathrm{E}-2$ \\
\hline$[\mathrm{Ar}$ vI] $\lambda 4.530 \ldots \ldots \ldots \ldots \ldots . . . . . . . . .$. & $2.553 \mathrm{E}-3$ & $5.887 \mathrm{E}-5$ & $0.000 \mathrm{E}+0$ & $0.000 \mathrm{E}+0$ \\
\hline 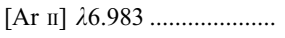 & $2.618 \mathrm{E}-4$ & $1.793 \mathrm{E}-3$ & $6.920 \mathrm{E}-3$ & $2.313 \mathrm{E}-2$ \\
\hline 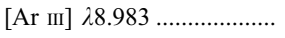 & $5.350 \mathrm{E}-2$ & $1.323 \mathrm{E}-3$ & $0.000 \mathrm{E}+0$ & $0.000 \mathrm{E}+0$ \\
\hline$[\mathrm{Ne}$ vI $] \lambda 7.652 \ldots \ldots \ldots \ldots \ldots . . . . .$. & $1.052 \mathrm{E}-2$ & $5.089 \mathrm{E}-2$ & $6.908 \mathrm{E}-2$ & $4.078 \mathrm{E}-2$ \\
\hline 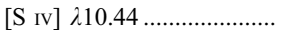 & $1.312 \mathrm{E}+0$ & $9.671 \mathrm{E}-1$ & $1.237 \mathrm{E}-1$ & $1.397 \mathrm{E}-3$ \\
\hline$[\mathrm{Ne}$ II] $\lambda 12.8 \ldots \ldots \ldots \ldots \ldots \ldots \ldots$ & $7.533 \mathrm{E}-3$ & $4.743 \mathrm{E}-2$ & $1.742 \mathrm{E}-1$ & $4.306 \mathrm{E}-1$ \\
\hline$[\mathrm{Ne} v] \lambda 14.5 \ldots \ldots \ldots \ldots \ldots \ldots \ldots$ & $4.053 \mathrm{E}-2$ & $9.756 \mathrm{E}-3$ & $7.282 \mathrm{E}-5$ & $0.000 \mathrm{E}+0$ \\
\hline$[\mathrm{Ne}$ III] $\lambda 15.5 \ldots \ldots \ldots \ldots \ldots \ldots . . . . . .$. & $7.326 \mathrm{E}-1$ & $7.399 \mathrm{E}-1$ & $6.651 \mathrm{E}-1$ & $4.576 \mathrm{E}-1$ \\
\hline 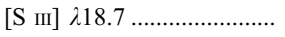 & $2.701 \mathrm{E}-2$ & $1.765 \mathrm{E}-1$ & $3.863 \mathrm{E}-1$ & $2.787 \mathrm{E}-1$ \\
\hline 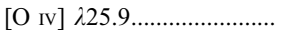 & $3.041 \mathrm{E}-1$ & $3.990 \mathrm{E}-1$ & $1.120 \mathrm{E}-1$ & $3.789 \mathrm{E}-4$ \\
\hline
\end{tabular}


TABLE 29

Dust-free IR Line Ratios with Respect to $\mathrm{H} \beta, 0.5 Z_{\odot}$

\begin{tabular}{|c|c|c|c|c|}
\hline \multirow[b]{2}{*}{ LiNE } & \multicolumn{4}{|c|}{$\log U_{0}$} \\
\hline & -1.0 & -2.0 & -3.0 & -4.0 \\
\hline \multicolumn{5}{|c|}{$\alpha=-1.2$} \\
\hline 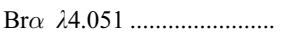 & $6.189 \mathrm{E}-2$ & $6.664 \mathrm{E}-2$ & $7.467 \mathrm{E}-2$ & $8.267 \mathrm{E}-2$ \\
\hline 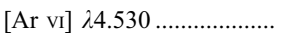 & $1.462 \mathrm{E}-2$ & $1.562 \mathrm{E}-3$ & $0.000 \mathrm{E}+0$ & $0.000 \mathrm{E}+0$ \\
\hline 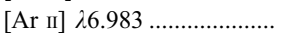 & $1.881 \mathrm{E}-3$ & $1.322 \mathrm{E}-2$ & $4.247 \mathrm{E}-2$ & $9.450 \mathrm{E}-2$ \\
\hline$[\mathrm{Ar}$ III] $\lambda 8.983 \ldots \ldots \ldots \ldots \ldots \ldots . . . . . . . .$. & $5.531 \mathrm{E}-1$ & $6.565 \mathrm{E}-2$ & $6.782 \mathrm{E}-5$ & $0.000 \mathrm{E}+0$ \\
\hline$[\mathrm{Ne} v \mathrm{vI}] \lambda 7.652 \ldots \ldots \ldots \ldots \ldots \ldots$ & $1.468 \mathrm{E}-2$ & $8.242 \mathrm{E}-2$ & $1.262 \mathrm{E}-1$ & $7.822 \mathrm{E}-2$ \\
\hline 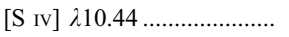 & $1.707 \mathrm{E}+0$ & $1.526 \mathrm{E}+0$ & $4.246 \mathrm{E}-1$ & $5.873 \mathrm{E}-3$ \\
\hline$[\mathrm{Ne}$ II] $\lambda 12.8 \ldots \ldots \ldots \ldots \ldots \ldots \ldots$ & $2.491 \mathrm{E}-2$ & $1.793 \mathrm{E}-1$ & $7.837 \mathrm{E}-1$ & $1.955 \mathrm{E}+0$ \\
\hline 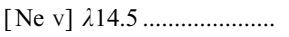 & $1.297 \mathrm{E}-1$ & $1.140 \mathrm{E}-1$ & $1.133 \mathrm{E}-3$ & $0.000 \mathrm{E}+0$ \\
\hline$[\mathrm{Ne}$ III $] \quad \lambda 15.5 \ldots \ldots \ldots \ldots \ldots \ldots \ldots$ & $1.119 \mathrm{E}+0$ & $1.323 \mathrm{E}+0$ & $1.795 \mathrm{E}+0$ & $1.774 \mathrm{E}+0$ \\
\hline 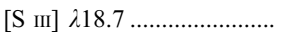 & $5.389 \mathrm{E}-2$ & $3.343 \mathrm{E}-1$ & $9.768 \mathrm{E}-1$ & $1.047 \mathrm{E}+0$ \\
\hline [O Iv] $\lambda 25.9 \ldots \ldots \ldots \ldots \ldots \ldots \ldots . .$. & $1.155 \mathrm{E}+0$ & $1.791 \mathrm{E}+0$ & $5.534 \mathrm{E}-1$ & $1.789 \mathrm{E}-3$ \\
\hline \multicolumn{5}{|c|}{$\alpha=-1.4$} \\
\hline 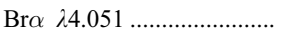 & $6.488 \mathrm{E}-2$ & $6.817 \mathrm{E}-2$ & $7.483 \mathrm{E}-2$ & $8.173 \mathrm{E}-2$ \\
\hline$[\mathrm{Ar} \mathrm{vI}] \quad \lambda 4.530 \ldots \ldots \ldots \ldots \ldots \ldots \ldots \ldots$ & $1.125 \mathrm{E}-2$ & $8.569 \mathrm{E}-4$ & $0.000 \mathrm{E}+0$ & $0.000 \mathrm{E}+0$ \\
\hline 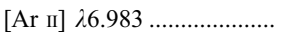 & $1.336 \mathrm{E}-3$ & $9.445 \mathrm{E}-3$ & $3.088 \mathrm{E}-2$ & $7.360 \mathrm{E}-2$ \\
\hline$[\mathrm{Ar}$ III] $\lambda 8.983 \ldots \ldots \ldots \ldots \ldots \ldots . . . . . . . .$. & $3.858 \mathrm{E}-1$ & $3.154 \mathrm{E}-2$ & $2.954 \mathrm{E}-5$ & $0.000 \mathrm{E}+0$ \\
\hline$[\mathrm{Ne} v \mathrm{vI}] \lambda 7.652 \ldots \ldots \ldots \ldots \ldots \ldots$ & $1.576 \mathrm{E}-2$ & $8.661 \mathrm{E}-2$ & $1.306 \mathrm{E}-1$ & $8.147 \mathrm{E}-2$ \\
\hline 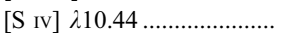 & $1.953 \mathrm{E}+0$ & $1.713 \mathrm{E}+0$ & $3.793 \mathrm{E}-1$ & $4.999 \mathrm{E}-3$ \\
\hline$[\mathrm{Ne}$ II] $\lambda 12.8 \ldots \ldots \ldots \ldots \ldots \ldots \ldots$ & $2.176 \mathrm{E}-2$ & $1.517 \mathrm{E}-1$ & $6.093 \mathrm{E}-1$ & $1.430 \mathrm{E}+0$ \\
\hline$[\mathrm{Ne} v]$ & $1.155 \mathrm{E}-1$ & $7.789 \mathrm{E}-2$ & $7.206 \mathrm{E}-4$ & $0.000 \mathrm{E}+0$ \\
\hline 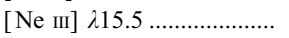 & $1.217 \mathrm{E}+0$ & $1.351 \mathrm{E}+0$ & $1.614 \mathrm{E}+0$ & $1.484 \mathrm{E}+0$ \\
\hline$[\mathrm{S}$ III] $\lambda 18.7 \ldots \ldots \ldots \ldots \ldots \ldots \ldots . .$. & $4.954 \mathrm{E}-2$ & $3.199 \mathrm{E}-1$ & $8.866 \mathrm{E}-1$ & $8.541 \mathrm{E}-1$ \\
\hline$[\mathrm{O}$ IV] $\lambda 25.9 \ldots \ldots \ldots \ldots \ldots \ldots \ldots$ & $9.013 \mathrm{E}-1$ & $1.488 \mathrm{E}+0$ & $4.555 \mathrm{E}-1$ & $1.549 \mathrm{E}-3$ \\
\hline \multicolumn{5}{|c|}{$\alpha=-1.7$} \\
\hline 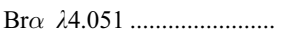 & $6.856 \mathrm{E}-2$ & $7.046 \mathrm{E}-2$ & $7.531 \mathrm{E}-2$ & $8.080 \mathrm{E}-2$ \\
\hline 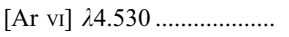 & $7.121 \mathrm{E}-3$ & $3.010 \mathrm{E}-4$ & $0.000 \mathrm{E}+0$ & $0.000 \mathrm{E}+0$ \\
\hline 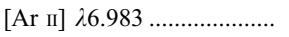 & $7.584 \mathrm{E}-4$ & $5.500 \mathrm{E}-3$ & $1.904 \mathrm{E}-2$ & $5.361 \mathrm{E}-2$ \\
\hline$[\mathrm{Ar}$ III] $28.983 \ldots \ldots \ldots \ldots \ldots \ldots . . . . . . . .$. & $1.929 \mathrm{E}-1$ & $8.601 \mathrm{E}-3$ & $7.135 \mathrm{E}-6$ & $0.000 \mathrm{E}+0$ \\
\hline$[\mathrm{Ne}$ vI $] \lambda 7.652 \ldots \ldots \ldots \ldots \ldots . . . . . .$. & $1.797 \mathrm{E}-2$ & $9.230 \mathrm{E}-2$ & $1.332 \mathrm{E}-1$ & $8.153 \mathrm{E}-2$ \\
\hline [S IV] $\lambda 10.44 \ldots \ldots \ldots \ldots \ldots \ldots . .$. & $2.229 \mathrm{E}+0$ & $1.817 \mathrm{E}+0$ & $2.982 \mathrm{E}-1$ & $3.706 \mathrm{E}-3$ \\
\hline$[\mathrm{Ne}$ II] $\lambda 12.8 \ldots \ldots \ldots \ldots \ldots \ldots \ldots$ & $1.638 \mathrm{E}-2$ & $1.102 \mathrm{E}-1$ & $4.045 \mathrm{E}-1$ & $9.441 \mathrm{E}-1$ \\
\hline$[\mathrm{Ne} v] \lambda 14.5 \ldots \ldots \ldots \ldots \ldots \ldots \ldots \ldots \ldots \ldots \ldots \ldots \ldots$ & $9.247 \mathrm{E}-2$ & $3.734 \mathrm{E}-2$ & $3.161 \mathrm{E}-4$ & $0.000 \mathrm{E}+0$ \\
\hline 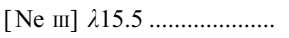 & $1.304 \mathrm{E}+0$ & $1.381 \mathrm{E}+0$ & $1.414 \mathrm{E}+0$ & $1.134 \mathrm{E}+0$ \\
\hline 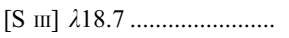 & $4.804 \mathrm{E}-2$ & $3.204 \mathrm{E}-1$ & $7.940 \mathrm{E}-1$ & $6.511 \mathrm{E}-1$ \\
\hline$[\mathrm{O}$ IV $] \lambda 25.9 \ldots \ldots \ldots \ldots \ldots \ldots \ldots \ldots \ldots \ldots$ & $6.478 \mathrm{E}-1$ & $1.044 \mathrm{E}+0$ & $3.114 \mathrm{E}-1$ & $1.102 \mathrm{E}-3$ \\
\hline \multicolumn{5}{|c|}{$\alpha=-2.0$} \\
\hline 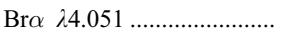 & $7.142 \mathrm{E}-2$ & $7.260 \mathrm{E}-2$ & $7.599 \mathrm{E}-2$ & $8.047 \mathrm{E}-2$ \\
\hline$[\mathrm{Ar} \mathrm{vI}] \quad \lambda 4.530 \ldots \ldots \ldots \ldots \ldots \ldots \ldots$ & $4.046 \mathrm{E}-3$ & $9.363 \mathrm{E}-5$ & $0.000 \mathrm{E}+0$ & $0.000 \mathrm{E}+0$ \\
\hline 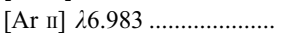 & $4.100 \mathrm{E}-4$ & $3.160 \mathrm{E}-3$ & $1.244 \mathrm{E}-2$ & $4.291 \mathrm{E}-2$ \\
\hline 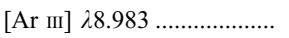 & $8.120 \mathrm{E}-2$ & $1.918 \mathrm{E}-3$ & $1.489 \mathrm{E}-6$ & $0.000 \mathrm{E}+0$ \\
\hline$[\mathrm{Ne}$ vI] $\lambda 7.652 \ldots \ldots \ldots \ldots \ldots \ldots . . .$. & $2.103 \mathrm{E}-2$ & $9.757 \mathrm{E}-2$ & $1.325 \mathrm{E}-1$ & $7.744 \mathrm{E}-2$ \\
\hline 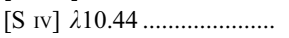 & $2.404 \mathrm{E}+0$ & $1.758 \mathrm{E}+0$ & $2.224 \mathrm{E}-1$ & $2.631 \mathrm{E}-3$ \\
\hline$[\mathrm{Ne}$ II] $\lambda 12.8 \ldots \ldots \ldots \ldots \ldots \ldots \ldots . .$. & $1.132 \mathrm{E}-2$ & $7.640 \mathrm{E}-2$ & $2.823 \mathrm{E}-1$ & $6.998 \mathrm{E}-1$ \\
\hline$[\mathrm{Ne} v] \lambda 14.5 \ldots \ldots \ldots \ldots \ldots \ldots \ldots \ldots \ldots \ldots \ldots \ldots \ldots$ & $6.681 \mathrm{E}-2$ & $1.515 \mathrm{E}-2$ & $1.229 \mathrm{E}-4$ & $0.000 \mathrm{E}+0$ \\
\hline$[\mathrm{Ne}$ III] $\lambda 15.5 \ldots \ldots \ldots \ldots \ldots \ldots . .$. & $1.347 \mathrm{E}+0$ & $1.386 \mathrm{E}+0$ & $1.260 \mathrm{E}+0$ & $8.609 \mathrm{E}-1$ \\
\hline 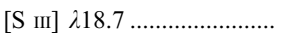 & $5.143 \mathrm{E}-2$ & $3.385 \mathrm{E}-1$ & $7.363 \mathrm{E}-1$ & $5.273 \mathrm{E}-1$ \\
\hline [O Iv] $\lambda 25.9 \ldots \ldots \ldots \ldots \ldots \ldots \ldots$ & $5.040 \mathrm{E}-1$ & $6.864 \mathrm{E}-1$ & $1.982 \mathrm{E}-1$ & $7.034 \mathrm{E}-4$ \\
\hline
\end{tabular}


TABLE 30

Dust-free IR Line Ratios with Respect to $\mathrm{H} \beta, 1 Z_{\odot}$

\begin{tabular}{|c|c|c|c|c|}
\hline \multirow[b]{2}{*}{ LiNE } & \multicolumn{4}{|c|}{$\log U_{0}$} \\
\hline & -1.0 & -2.0 & -3.0 & -4.0 \\
\hline \multicolumn{5}{|c|}{$\alpha=-1.2$} \\
\hline 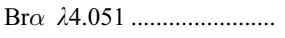 & $6.737 \mathrm{E}-2$ & $7.021 \mathrm{E}-2$ & $7.744 \mathrm{E}-2$ & $8.597 \mathrm{E}-2$ \\
\hline 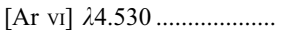 & $2.138 \mathrm{E}-2$ & $2.400 \mathrm{E}-3$ & $0.000 \mathrm{E}+0$ & $0.000 \mathrm{E}+0$ \\
\hline 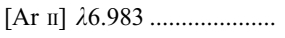 & $2.583 \mathrm{E}-3$ & $2.216 \mathrm{E}-2$ & $7.179 \mathrm{E}-2$ & $1.573 \mathrm{E}-1$ \\
\hline 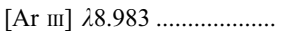 & $8.689 \mathrm{E}-1$ & $9.545 \mathrm{E}-2$ & $1.017 \mathrm{E}-4$ & $0.000 \mathrm{E}+0$ \\
\hline$[\mathrm{Ne}$ vI $] \lambda 7.652 \ldots \ldots \ldots \ldots \ldots . . . .$. & $2.971 \mathrm{E}-2$ & $1.634 \mathrm{E}-1$ & $2.428 \mathrm{E}-1$ & $1.460 \mathrm{E}-1$ \\
\hline 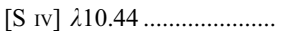 & $3.386 \mathrm{E}+0$ & $2.972 \mathrm{E}+0$ & $7.600 \mathrm{E}-1$ & $1.088 \mathrm{E}-2$ \\
\hline$[\mathrm{Ne}$ II] $\lambda 12.8 \ldots \ldots \ldots \ldots \ldots \ldots \ldots$ & $3.747 \mathrm{E}-2$ & $2.860 \mathrm{E}-1$ & $1.176 \mathrm{E}+0$ & $2.699 \mathrm{E}+0$ \\
\hline 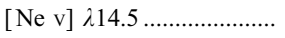 & $2.168 \mathrm{E}-1$ & $1.788 \mathrm{E}-1$ & $1.847 \mathrm{E}-3$ & $0.000 \mathrm{E}+0$ \\
\hline$[\mathrm{Ne}$ III] $\lambda 15.5 \ldots \ldots \ldots \ldots \ldots \ldots . .$. & $2.181 \mathrm{E}+0$ & $2.567 \mathrm{E}+0$ & $3.298 \mathrm{E}+0$ & $3.164 \mathrm{E}+0$ \\
\hline 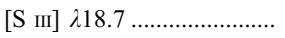 & $9.315 \mathrm{E}-2$ & $6.317 \mathrm{E}-1$ & $1.807 \mathrm{E}+0$ & $1.851 \mathrm{E}+0$ \\
\hline 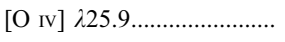 & $1.706 \mathrm{E}+0$ & $3.016 \mathrm{E}+0$ & $9.412 \mathrm{E}-1$ & $3.251 \mathrm{E}-3$ \\
\hline \multicolumn{5}{|c|}{$\alpha=-1.4$} \\
\hline 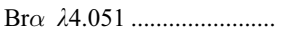 & $7.069 \mathrm{E}-2$ & $7.250 \mathrm{E}-2$ & $7.803 \mathrm{E}-2$ & $8.472 \mathrm{E}-2$ \\
\hline$[\mathrm{Ar} \mathrm{vI}] \lambda 4.530 \ldots \ldots \ldots \ldots \ldots \ldots . . . . . . .$. & $1.639 \mathrm{E}-2$ & $1.270 \mathrm{E}-3$ & $0.000 \mathrm{E}+0$ & $0.000 \mathrm{E}+0$ \\
\hline$[\mathrm{Ar}$ II $] \lambda 6.983 \ldots \ldots \ldots \ldots \ldots \ldots . . . . . . .$. & $1.799 \mathrm{E}-3$ & $1.567 \mathrm{E}-2$ & $5.189 \mathrm{E}-2$ & $1.246 \mathrm{E}-1$ \\
\hline [Ar III] $\lambda 8.983 \ldots \ldots \ldots \ldots \ldots \ldots . .$. & $5.785 \mathrm{E}-1$ & $4.355 \mathrm{E}-2$ & $4.222 \mathrm{E}-5$ & $0.000 \mathrm{E}+0$ \\
\hline$[\mathrm{Ne}$ vI $] \lambda 7.652 \ldots \ldots \ldots \ldots \ldots . . . . .$. & $3.204 \mathrm{E}-2$ & $1.682 \mathrm{E}-1$ & $2.475 \mathrm{E}-1$ & $1.503 \mathrm{E}-1$ \\
\hline [S IV] $\lambda 10.44 \ldots \ldots \ldots \ldots \ldots \ldots \ldots$ & $3.744 \mathrm{E}+0$ & $3.187 \mathrm{E}+0$ & $6.587 \mathrm{E}-1$ & $9.109 \mathrm{E}-3$ \\
\hline$[\mathrm{Ne}$ II] $\lambda 12.8 \ldots \ldots \ldots \ldots \ldots \ldots$ & $3.122 \mathrm{E}-2$ & $2.366 \mathrm{E}-1$ & $9.063 \mathrm{E}-1$ & $2.018 \mathrm{E}+0$ \\
\hline 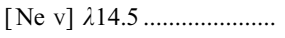 & $1.892 \mathrm{E}-1$ & $1.168 \mathrm{E}-1$ & $1.134 \mathrm{E}-3$ & $0.000 \mathrm{E}+0$ \\
\hline$[\mathrm{Ne}$ III] $\lambda 15.5 \ldots \ldots \ldots \ldots \ldots \ldots \ldots$ & $2.302 \mathrm{E}+0$ & $2.565 \mathrm{E}+0$ & $2.959 \mathrm{E}+0$ & $2.653 \mathrm{E}+0$ \\
\hline [S III] $\lambda 18.7 \ldots \ldots \ldots \ldots \ldots \ldots \ldots . .$. & $8.972 \mathrm{E}-2$ & $6.074 \mathrm{E}-1$ & $1.636 \mathrm{E}+0$ & $1.515 \mathrm{E}+0$ \\
\hline$[\mathrm{O}$ Iv $] \lambda 25.9 \ldots \ldots \ldots \ldots \ldots \ldots \ldots \ldots \ldots \ldots \ldots \ldots \ldots \ldots$ & $1.334 \mathrm{E}+0$ & $2.438 \mathrm{E}+0$ & $7.579 \mathrm{E}-1$ & $2.760 \mathrm{E}-3$ \\
\hline \multicolumn{5}{|c|}{$\alpha=-1.7$} \\
\hline 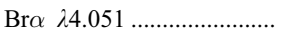 & $7.490 \mathrm{E}-2$ & $7.582 \mathrm{E}-2$ & $7.923 \mathrm{E}-2$ & $8.347 \mathrm{E}-2$ \\
\hline$[\mathrm{Ar}$ vI] $\lambda 4.530 \ldots \ldots \ldots \ldots \ldots . . . . . . . .$. & $1.049 \mathrm{E}-2$ & $4.275 \mathrm{E}-4$ & $0.000 \mathrm{E}+0$ & $0.000 \mathrm{E}+0$ \\
\hline$[\mathrm{Ar}$ II] $\lambda 6.983 \ldots \ldots \ldots \ldots \ldots . . .$. & $9.892 \mathrm{E}-4$ & $9.035 \mathrm{E}-3$ & $3.218 \mathrm{E}-2$ & $9.440 \mathrm{E}-2$ \\
\hline 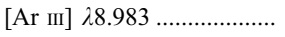 & $2.729 \mathrm{E}-1$ & $1.104 \mathrm{E}-2$ & $9.526 \mathrm{E}-6$ & $0.000 \mathrm{E}+0$ \\
\hline$[\mathrm{Ne}$ vI] $\lambda 7.652 \ldots \ldots \ldots \ldots \ldots \ldots$ & $3.675 \mathrm{E}-2$ & $1.750 \mathrm{E}-1$ & $2.470 \mathrm{E}-1$ & $1.476 \mathrm{E}-1$ \\
\hline 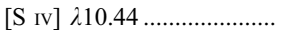 & $4.120 \mathrm{E}+0$ & $3.210 \mathrm{E}+0$ & $4.962 \mathrm{E}-1$ & $6.592 \mathrm{E}-3$ \\
\hline$[\mathrm{Ne}$ II] $\lambda 12.8 \ldots \ldots \ldots \ldots \ldots \ldots$ & $2.219 \mathrm{E}-2$ & $1.686 \mathrm{E}-1$ & $6.116 \mathrm{E}-1$ & $1.417 \mathrm{E}+0$ \\
\hline$[\mathrm{Ne} \mathrm{v}] \lambda 14.5 \ldots \ldots \ldots \ldots \ldots \ldots \ldots \ldots \ldots \ldots \ldots$ & $1.454 \mathrm{E}-1$ & $5.274 \mathrm{E}-2$ & $4.739 \mathrm{E}-4$ & $0.000 \mathrm{E}+0$ \\
\hline$[\mathrm{Ne}$ III $] \lambda 15.5 \ldots \ldots \ldots \ldots \ldots \ldots . . .$. & $2.395 \mathrm{E}+0$ & $2.543 \mathrm{E}+0$ & $2.563 \mathrm{E}+0$ & $2.026 \mathrm{E}+0$ \\
\hline 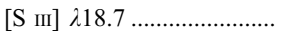 & $9.183 \mathrm{E}-2$ & $6.076 \mathrm{E}-1$ & $1.452 \mathrm{E}+0$ & $1.166 \mathrm{E}+0$ \\
\hline 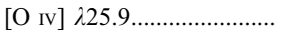 & $9.859 \mathrm{E}-1$ & $1.663 \mathrm{E}+0$ & $5.043 \mathrm{E}-1$ & $1.915 \mathrm{E}-3$ \\
\hline \multicolumn{5}{|c|}{$\alpha=-2.0$} \\
\hline 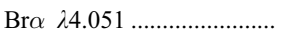 & $7.835 \mathrm{E}-2$ & $7.883 \mathrm{E}-2$ & $8.044 \mathrm{E}-2$ & $8.325 \mathrm{E}-2$ \\
\hline$[\mathrm{Ar}$ vI] $\lambda 4.530 \ldots \ldots \ldots \ldots \ldots . . . . . . . .$. & $5.922 \mathrm{E}-3$ & $1.284 \mathrm{E}-4$ & $0.000 \mathrm{E}+0$ & $0.000 \mathrm{E}+0$ \\
\hline 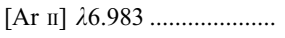 & $5.176 \mathrm{E}-4$ & $5.204 \mathrm{E}-3$ & $2.174 \mathrm{E}-2$ & $7.853 \mathrm{E}-2$ \\
\hline 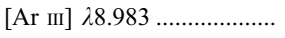 & $1.092 \mathrm{E}-1$ & $2.295 \mathrm{E}-3$ & $1.876 \mathrm{E}-6$ & $0.000 \mathrm{E}+0$ \\
\hline$[\mathrm{Ne}$ vI $] \lambda 7.652 \ldots \ldots \ldots \ldots \ldots . . . . .$. & $4.327 \mathrm{E}-2$ & $1.815 \mathrm{E}-1$ & $2.409 \mathrm{E}-1$ & $1.371 \mathrm{E}-1$ \\
\hline [S Iv] $\lambda 10.44 \ldots \ldots \ldots \ldots \ldots \ldots$ & $4.323 \mathrm{E}+0$ & $2.988 \mathrm{E}+0$ & $3.572 \mathrm{E}-1$ & $4.567 \mathrm{E}-3$ \\
\hline$[\mathrm{Ne}$ II] $\lambda 12.8 \ldots \ldots \ldots \ldots \ldots \ldots \ldots$ & $1.469 \mathrm{E}-2$ & $1.185 \mathrm{E}-1$ & $4.509 \mathrm{E}-1$ & $1.127 \mathrm{E}+0$ \\
\hline$[\mathrm{Ne} v] \lambda 14.5 \ldots \ldots \ldots \ldots \ldots \ldots \ldots$ & $1.003 \mathrm{E}-1$ & $2.027 \mathrm{E}-2$ & $1.771 \mathrm{E}-4$ & $0.000 \mathrm{E}+0$ \\
\hline$[\mathrm{Ne}$ III] $\lambda 15.5 \ldots \ldots \ldots \ldots \ldots \ldots . . .$. & $2.428 \mathrm{E}+0$ & $2.495 \mathrm{E}+0$ & $2.248 \mathrm{E}+0$ & $1.525 \mathrm{E}+0$ \\
\hline 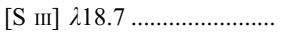 & $1.016 \mathrm{E}-1$ & $6.375 \mathrm{E}-1$ & $1.330 \mathrm{E}+0$ & $9.531 \mathrm{E}-1$ \\
\hline 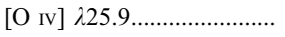 & $7.810 \mathrm{E}-1$ & $1.075 \mathrm{E}+0$ & $3.151 \mathrm{E}-1$ & $1.194 \mathrm{E}-3$ \\
\hline
\end{tabular}


TABLE 31

Dust-free IR Line Ratios with Respect to $\mathrm{H} \beta, 2 Z_{\odot}$

\begin{tabular}{|c|c|c|c|c|}
\hline \multirow[b]{2}{*}{ LiNE } & \multicolumn{4}{|c|}{$\log U_{0}$} \\
\hline & -1.0 & -2.0 & -3.0 & -4.0 \\
\hline \multicolumn{5}{|c|}{$\alpha=-1.2$} \\
\hline 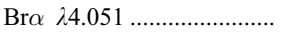 & $7.581 \mathrm{E}-2$ & $7.631 \mathrm{E}-2$ & $8.303 \mathrm{E}-2$ & $9.206 \mathrm{E}-2$ \\
\hline$[\mathrm{Ar} \mathrm{vI}] \lambda 4.530 \ldots \ldots \ldots \ldots \ldots \ldots . .$. & $2.896 \mathrm{E}-2$ & $3.119 \mathrm{E}-3$ & $0.000 \mathrm{E}+0$ & $0.000 \mathrm{E}+0$ \\
\hline 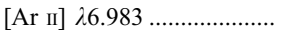 & $2.794 \mathrm{E}-3$ & $3.371 \mathrm{E}-2$ & $1.141 \mathrm{E}-1$ & $2.526 \mathrm{E}-1$ \\
\hline$[\mathrm{Ar}$ III] $\lambda 8.983 \ldots \ldots \ldots \ldots \ldots \ldots . .$. & $1.193 \mathrm{E}+0$ & $1.159 \mathrm{E}-1$ & $1.185 \mathrm{E}-4$ & $0.000 \mathrm{E}+0$ \\
\hline$[\mathrm{Ne}$ vI $] \lambda 7.652 \ldots \ldots \ldots \ldots \ldots \ldots$ & $6.266 \mathrm{E}-2$ & $3.139 \mathrm{E}-1$ & $4.385 \mathrm{E}-1$ & $2.441 \mathrm{E}-1$ \\
\hline [S IV] $\lambda 10.44 \ldots \ldots \ldots \ldots \ldots \ldots . .$. & $6.637 \mathrm{E}+0$ & $5.472 \mathrm{E}+0$ & $1.208 \mathrm{E}+0$ & $1.800 \mathrm{E}-2$ \\
\hline$[\mathrm{Ne}$ II] $\lambda 12.8 \ldots \ldots \ldots \ldots \ldots \ldots \ldots$ & $4.879 \mathrm{E}-2$ & $4.291 \mathrm{E}-1$ & $1.690 \mathrm{E}+0$ & $3.639 \mathrm{E}+0$ \\
\hline$[\mathrm{Ne} v] \quad \lambda 14.5 \ldots \ldots \ldots \ldots \ldots \ldots \ldots \ldots \ldots$ & $3.295 \mathrm{E}-1$ & $2.428 \mathrm{E}-1$ & $2.534 \mathrm{E}-3$ & $1.620 \mathrm{E}-6$ \\
\hline$[\mathrm{Ne}$ III] $\lambda 15.5 \ldots \ldots \ldots \ldots \ldots \ldots . .$. & $4.132 \mathrm{E}+0$ & $4.761 \mathrm{E}+0$ & $5.654 \mathrm{E}+0$ & $5.199 \mathrm{E}+0$ \\
\hline$[\mathrm{S}$ III] $\lambda 18.7 \ldots \ldots \ldots \ldots \ldots \ldots \ldots \ldots \ldots \ldots \ldots$ & $1.676 \mathrm{E}-1$ & $1.155 \mathrm{E}+0$ & $3.136 \mathrm{E}+0$ & $3.027 \mathrm{E}+0$ \\
\hline$[\mathrm{O}$ IV $] \lambda 25.9 \ldots \ldots \ldots \ldots \ldots \ldots \ldots \ldots \ldots \ldots \ldots \ldots \ldots$ & $2.265 \mathrm{E}+0$ & $4.556 \mathrm{E}+0$ & $1.406 \mathrm{E}+0$ & $5.215 \mathrm{E}-3$ \\
\hline \multicolumn{5}{|c|}{$\alpha=-1.4$} \\
\hline 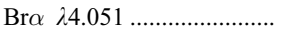 & $7.969 \mathrm{E}-2$ & $7.960 \mathrm{E}-2$ & $8.457 \mathrm{E}-2$ & $9.094 \mathrm{E}-2$ \\
\hline$[\mathrm{Ar} \mathrm{VI}] \mathrm{\lambda}, 24.530 \ldots \ldots \ldots \ldots \ldots \ldots . .$. & $2.247 \mathrm{E}-2$ & $1.589 \mathrm{E}-3$ & $0.000 \mathrm{E}+0$ & $0.000 \mathrm{E}+0$ \\
\hline$[$ Ar II] $\lambda 6.983 \ldots \ldots \ldots \ldots \ldots . . .$. & $1.892 \mathrm{E}-3$ & $2.373 \mathrm{E}-2$ & $8.279 \mathrm{E}-2$ & $2.056 \mathrm{E}-1$ \\
\hline$[$ Ar III] $28.983 \ldots \ldots \ldots \ldots \ldots . .$. & $7.592 \mathrm{E}-1$ & $4.985 \mathrm{E}-2$ & $4.616 \mathrm{E}-5$ & $0.000 \mathrm{E}+0$ \\
\hline$[\mathrm{Ne} \mathrm{vI}] \lambda 7.652 \ldots \ldots \ldots \ldots \ldots \ldots . .$. & $6.813 \mathrm{E}-2$ & $3.169 \mathrm{E}-1$ & $4.373 \mathrm{E}-1$ & $2.450 \mathrm{E}-1$ \\
\hline [S IV] $\lambda 10.44 \ldots \ldots \ldots \ldots \ldots \ldots . .$. & $7.019 \mathrm{E}+0$ & $5.565 \mathrm{E}+0$ & $1.004 \mathrm{E}+0$ & $1.459 \mathrm{E}-2$ \\
\hline$[\mathrm{Ne}$ II] $\lambda 12.8 \ldots \ldots \ldots \ldots \ldots \ldots . .$. & $4.027 \mathrm{E}-2$ & $3.510 \mathrm{E}-1$ & $1.312 \mathrm{E}+0$ & $2.827 \mathrm{E}+0$ \\
\hline$[\mathrm{Ne} v] \quad \lambda 14.5 \ldots \ldots \ldots \ldots \ldots \ldots \ldots \ldots$ & $2.805 \mathrm{E}-1$ & $1.510 \mathrm{E}-1$ & $1.484 \mathrm{E}-3$ & $0.000 \mathrm{E}+0$ \\
\hline$[\mathrm{Ne}$ III] $\lambda 15.5 \ldots \ldots \ldots \ldots \ldots \ldots . .$. & $4.226 \mathrm{E}+0$ & $4.655 \mathrm{E}+0$ & $5.035 \mathrm{E}+0$ & $4.336 \mathrm{E}+0$ \\
\hline$[\mathrm{S}$ III] $\lambda 18.7 \ldots \ldots \ldots \ldots \ldots \ldots \ldots . .$. & $1.704 \mathrm{E}-1$ & $1.123 \mathrm{E}+0$ & $2.823 \mathrm{E}+0$ & $2.481 \mathrm{E}+0$ \\
\hline [O Iv] $\lambda 25.9 \ldots \ldots \ldots \ldots \ldots \ldots$ & $1.815 \mathrm{E}+0$ & $3.586 \mathrm{E}+0$ & $1.105 \mathrm{E}+0$ & $4.281 \mathrm{E}-3$ \\
\hline \multicolumn{5}{|c|}{$\alpha=-1.7$} \\
\hline 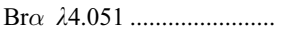 & $8.468 \mathrm{E}-2$ & $8.425 \mathrm{E}-2$ & $8.652 \mathrm{E}-2$ & $8.953 \mathrm{E}-2$ \\
\hline$[\mathrm{Ar} \mathrm{vI}] \quad \lambda 4.530 \ldots \ldots \ldots \ldots \ldots \ldots . .$. & $1.434 \mathrm{E}-2$ & $5.100 \mathrm{E}-4$ & $0.000 \mathrm{E}+0$ & $0.000 \mathrm{E}+0$ \\
\hline$[\mathrm{Ar}$ II] $\lambda 6.983 \ldots \ldots \ldots \ldots \ldots \ldots . .$. & $1.004 \mathrm{E}-3$ & $1.374 \mathrm{E}-2$ & $5.316 \mathrm{E}-2$ & $1.635 \mathrm{E}-1$ \\
\hline 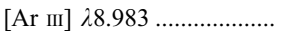 & $3.382 \mathrm{E}-1$ & $1.157 \mathrm{E}-2$ & $9.690 \mathrm{E}-6$ & $0.000 \mathrm{E}+0$ \\
\hline$[\mathrm{Ne}$ vI $] \lambda 7.652 \ldots \ldots \ldots \ldots \ldots \ldots$ & $7.926 \mathrm{E}-2$ & $3.215 \mathrm{E}-1$ & $4.249 \mathrm{E}-1$ & $2.319 \mathrm{E}-1$ \\
\hline [S IV] $\lambda 10.44 \ldots \ldots \ldots \ldots \ldots \ldots . .$. & $7.325 \mathrm{E}+0$ & $5.271 \mathrm{E}+0$ & $7.220 \mathrm{E}-1$ & $1.013 \mathrm{E}-2$ \\
\hline$[\mathrm{Ne}$ II] $\lambda 12.8 \ldots \ldots \ldots \ldots \ldots \ldots \ldots$ & $2.663 \mathrm{E}-2$ & $2.532 \mathrm{E}-1$ & $9.306 \mathrm{E}-1$ & $2.139 \mathrm{E}+0$ \\
\hline 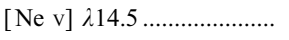 & $2.059 \mathrm{E}-1$ & $6.367 \mathrm{E}-2$ & $5.882 \mathrm{E}-4$ & $0.000 \mathrm{E}+0$ \\
\hline 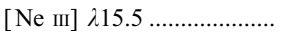 & $4.252 \mathrm{E}+0$ & $4.480 \mathrm{E}+0$ & $4.294 \mathrm{E}+0$ & $3.270 \mathrm{E}+0$ \\
\hline 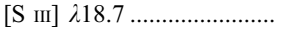 & $1.858 \mathrm{E}-1$ & $1.130 \mathrm{E}+0$ & $2.482 \mathrm{E}+0$ & $1.925 \mathrm{E}+0$ \\
\hline$[\mathrm{O}$ Iv $] \lambda 25.9 \ldots \ldots \ldots \ldots \ldots \ldots \ldots \ldots \ldots \ldots \ldots \ldots \ldots$ & $1.396 \mathrm{E}+0$ & $2.385 \mathrm{E}+0$ & $7.198 \mathrm{E}-1$ & $2.855 \mathrm{E}-3$ \\
\hline \multicolumn{5}{|c|}{$\alpha=-2.0$} \\
\hline 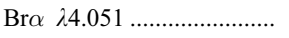 & $8.924 \mathrm{E}-2$ & $8.832 \mathrm{E}-2$ & $8.832 \mathrm{E}-2$ & $8.962 \mathrm{E}-2$ \\
\hline$[\mathrm{Ar} \mathrm{vI}] \lambda 4.530 \ldots \ldots \ldots \ldots \ldots \ldots . .$. & $7.826 \mathrm{E}-3$ & $1.469 \mathrm{E}-4$ & $0.000 \mathrm{E}+0$ & $0.000 \mathrm{E}+0$ \\
\hline$[\mathrm{Ar}$ II] $\lambda 6.983 \ldots \ldots \ldots \ldots \ldots \ldots \ldots \ldots$ & $5.387 \mathrm{E}-4$ & $8.277 \mathrm{E}-3$ & $3.881 \mathrm{E}-2$ & $1.413 \mathrm{E}-1$ \\
\hline$[\mathrm{Ar}$ III] $28.983 \ldots \ldots \ldots \ldots \ldots \ldots . .$. & $1.283 \mathrm{E}-1$ & $2.187 \mathrm{E}-3$ & $1.810 \mathrm{E}-6$ & $0.000 \mathrm{E}+0$ \\
\hline$[\mathrm{Ne}$ vI] $\lambda 7.652 \ldots \ldots \ldots \ldots \ldots \ldots$ & $9.451 \mathrm{E}-2$ & $3.261 \mathrm{E}-1$ & $4.035 \mathrm{E}-1$ & $2.073 \mathrm{E}-1$ \\
\hline [S IV] $\lambda 10.44 \ldots \ldots \ldots \ldots \ldots \ldots \ldots . .$. & $7.324 \mathrm{E}+0$ & $4.669 \mathrm{E}+0$ & $5.015 \mathrm{E}-1$ & $6.771 \mathrm{E}-3$ \\
\hline$[\mathrm{Ne}$ II] $\lambda 12.8 \ldots \ldots \ldots \ldots \ldots \ldots \ldots . .$. & $1.981 \mathrm{E}-2$ & $1.927 \mathrm{E}-1$ & $7.518 \mathrm{E}-1$ & $1.809 \mathrm{E}+0$ \\
\hline 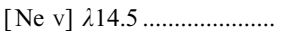 & $1.350 \mathrm{E}-1$ & $2.288 \mathrm{E}-2$ & $2.112 \mathrm{E}-4$ & $0.000 \mathrm{E}+0$ \\
\hline$[\mathrm{Ne}$ III] $\lambda 15.5 \ldots \ldots \ldots \ldots \ldots \ldots \ldots$ & $4.189 \mathrm{E}+0$ & $4.277 \mathrm{E}+0$ & $3.674 \mathrm{E}+0$ & $2.410 \mathrm{E}+0$ \\
\hline$[\mathrm{S}$ III] $\lambda 18.7 \ldots \ldots \ldots \ldots \ldots \ldots \ldots . .$. & $2.161 \mathrm{E}-1$ & $1.182 \mathrm{E}+0$ & $2.250 \mathrm{E}+0$ & $1.580 \mathrm{E}+0$ \\
\hline [O Iv] $\lambda 25.9 \ldots \ldots \ldots \ldots \ldots \ldots \ldots \ldots \ldots \ldots \ldots \ldots$ & $1.121 \mathrm{E}+0$ & $1.519 \mathrm{E}+0$ & $4.434 \mathrm{E}-1$ & $1.720 \mathrm{E}-3$ \\
\hline
\end{tabular}


TABLE 32

Dust-free IR Line Ratios with Respect to $\mathrm{H} \beta, 4 Z_{\odot}$

\begin{tabular}{|c|c|c|c|c|}
\hline \multirow[b]{2}{*}{ LiNE } & \multicolumn{4}{|c|}{$\log U_{0}$} \\
\hline & -1.0 & -2.0 & -3.0 & -4.0 \\
\hline \multicolumn{5}{|c|}{$\alpha=-1.2$} \\
\hline 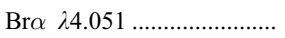 & $9.124 \mathrm{E}-2$ & $8.575 \mathrm{E}-2$ & $9.158 \mathrm{E}-2$ & $1.015 \mathrm{E}-1$ \\
\hline 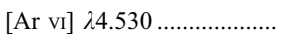 & $3.737 \mathrm{E}-2$ & $3.348 \mathrm{E}-3$ & $1.033 \mathrm{E}-6$ & $0.000 \mathrm{E}+0$ \\
\hline 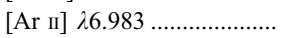 & $2.546 \mathrm{E}-3$ & $4.794 \mathrm{E}-2$ & $1.788 \mathrm{E}-1$ & $3.988 \mathrm{E}-1$ \\
\hline 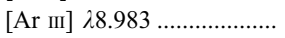 & $1.433 \mathrm{E}+0$ & $1.167 \mathrm{E}-1$ & $1.060 \mathrm{E}-4$ & $0.000 \mathrm{E}+0$ \\
\hline$[\mathrm{Ne} \mathrm{vI}] \lambda 7.652 \ldots \ldots \ldots \ldots \ldots \ldots$ & $1.422 \mathrm{E}-1$ & $5.838 \mathrm{E}-1$ & $7.211 \mathrm{E}-1$ & $3.329 \mathrm{E}-1$ \\
\hline [S Iv] $\lambda 10.44 \ldots \ldots \ldots \ldots \ldots \ldots$ & $1.147 \mathrm{E}+1$ & $9.238 \mathrm{E}+0$ & $1.688 \mathrm{E}+0$ & $2.554 \mathrm{E}-2$ \\
\hline$[\mathrm{Ne}$ II $] \lambda 12.8 \ldots \ldots \ldots \ldots \ldots \ldots \ldots$ & $6.070 \mathrm{E}-2$ & $6.445 \mathrm{E}-1$ & $2.447 \mathrm{E}+0$ & $4.965 \mathrm{E}+0$ \\
\hline 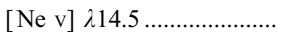 & $4.554 \mathrm{E}-1$ & $2.861 \mathrm{E}-1$ & $2.887 \mathrm{E}-3$ & $2.294 \mathrm{E}-6$ \\
\hline$[\mathrm{Ne}$ III] $\lambda 15.5 \ldots \ldots \ldots \ldots \ldots \ldots \ldots$ & $7.106 \mathrm{E}+0$ & $8.329 \mathrm{E}+0$ & $8.912 \mathrm{E}+0$ & $7.599 \mathrm{E}+0$ \\
\hline 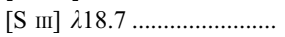 & $3.429 \mathrm{E}-1$ & $2.083 \mathrm{E}+0$ & $5.088 \mathrm{E}+0$ & $4.498 \mathrm{E}+0$ \\
\hline$[\mathrm{O}$ Iv $] \lambda 25.9 \ldots \ldots \ldots \ldots \ldots \ldots \ldots$ & $2.812 \mathrm{E}+0$ & $6.201 \mathrm{E}+0$ & $1.835 \mathrm{E}+0$ & $7.060 \mathrm{E}-3$ \\
\hline \multicolumn{5}{|c|}{$\alpha=-1.4$} \\
\hline 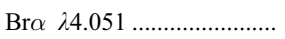 & $9.702 \mathrm{E}-2$ & $9.058 \mathrm{E}-2$ & $9.364 \mathrm{E}-2$ & $9.985 \mathrm{E}-2$ \\
\hline 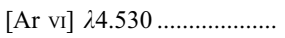 & $2.889 \mathrm{E}-2$ & $1.642 \mathrm{E}-3$ & $0.000 \mathrm{E}+0$ & $0.000 \mathrm{E}+0$ \\
\hline 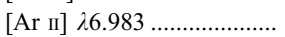 & $1.839 \mathrm{E}-3$ & $3.470 \mathrm{E}-2$ & $1.339 \mathrm{E}-1$ & $3.359 \mathrm{E}-1$ \\
\hline 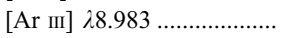 & $8.734 \mathrm{E}-1$ & $4.696 \mathrm{E}-2$ & $3.896 \mathrm{E}-5$ & $0.000 \mathrm{E}+0$ \\
\hline$[\mathrm{Ne}$ vI $] \lambda 7.652 \ldots \ldots \ldots \ldots \ldots \ldots$ & $1.539 \mathrm{E}-1$ & $5.713 \mathrm{E}-1$ & $7.007 \mathrm{E}-1$ & $3.266 \mathrm{E}-1$ \\
\hline$[\mathrm{S}$ IV $] \lambda 10.44 \ldots \ldots \ldots \ldots \ldots \ldots \ldots$ & $1.129 \mathrm{E}+1$ & $8.757 \mathrm{E}+0$ & $1.347 \mathrm{E}+0$ & $2.011 \mathrm{E}-2$ \\
\hline$[\mathrm{Ne}$ II $] \lambda 12.8 \ldots \ldots \ldots \ldots \ldots \ldots$ & $5.464 \mathrm{E}-2$ & $5.414 \mathrm{E}-1$ & $1.961 \mathrm{E}+0$ & $4.041 \mathrm{E}+0$ \\
\hline$[\mathrm{Ne} v] \lambda 14.5 \ldots \ldots \ldots \ldots \ldots \ldots \ldots \ldots \ldots$ & $3.763 \mathrm{E}-1$ & $1.687 \mathrm{E}-1$ & $1.612 \mathrm{E}-3$ & $1.249 \mathrm{E}-6$ \\
\hline 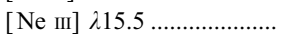 & $6.932 \mathrm{E}+0$ & $7.892 \mathrm{E}+0$ & $7.826 \mathrm{E}+0$ & $6.274 \mathrm{E}+0$ \\
\hline 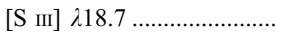 & $3.666 \mathrm{E}-1$ & $2.051 \mathrm{E}+0$ & $4.562 \mathrm{E}+0$ & $3.707 \mathrm{E}+0$ \\
\hline 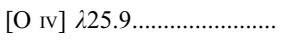 & $2.339 \mathrm{E}+0$ & $4.759 \mathrm{E}+0$ & $1.415 \mathrm{E}+0$ & $5.605 \mathrm{E}-3$ \\
\hline \multicolumn{5}{|c|}{$\alpha=-1.7$} \\
\hline 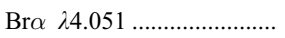 & $1.058 \mathrm{E}-1$ & $9.768 \mathrm{E}-2$ & $9.655 \mathrm{E}-2$ & $9.882 \mathrm{E}-2$ \\
\hline$[\mathrm{Ar}$ VI] $\lambda 4.530 \ldots \ldots \ldots \ldots \ldots . . . . . . .$. & $1.767 \mathrm{E}-2$ & $4.983 \mathrm{E}-4$ & $0.000 \mathrm{E}+0$ & $0.000 \mathrm{E}+0$ \\
\hline$[\mathrm{Ar}$ II] $\lambda 6.983 \ldots \ldots \ldots \ldots \ldots \ldots$ & $2.096 \mathrm{E}-3$ & $2.336 \mathrm{E}-2$ & $9.470 \mathrm{E}-2$ & $2.787 \mathrm{E}-1$ \\
\hline$[\mathrm{Ar}$ III] $28.983 \ldots \ldots \ldots \ldots \ldots \ldots$ & $3.658 \mathrm{E}-1$ & $9.703 \mathrm{E}-3$ & $7.574 \mathrm{E}-6$ & $0.000 \mathrm{E}+0$ \\
\hline$[\mathrm{Ne}$ vI] $\lambda 7.652 \ldots \ldots \ldots \ldots \ldots . . . . . .$. & $1.727 \mathrm{E}-1$ & $5.456 \mathrm{E}-1$ & $6.506 \mathrm{E}-1$ & $2.971 \mathrm{E}-1$ \\
\hline [S IV] $\lambda 10.44 \ldots \ldots \ldots \ldots \ldots \ldots$ & $1.050 \mathrm{E}+1$ & $7.489 \mathrm{E}+0$ & $9.147 \mathrm{E}-1$ & $1.339 \mathrm{E}-2$ \\
\hline$[\mathrm{Ne}$ II $] \lambda 12.8 \ldots \ldots \ldots \ldots \ldots \ldots \ldots$ & $6.304 \mathrm{E}-2$ & $4.439 \mathrm{E}-1$ & $1.522 \mathrm{E}+0$ & $3.265 \mathrm{E}+0$ \\
\hline 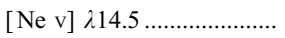 & $2.615 \mathrm{E}-1$ & $6.532 \mathrm{E}-2$ & $5.984 \mathrm{E}-4$ & $0.000 \mathrm{E}+0$ \\
\hline 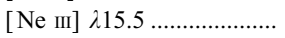 & $6.454 \mathrm{E}+0$ & $7.144 \mathrm{E}+0$ & $6.439 \mathrm{E}+0$ & $4.601 \mathrm{E}+0$ \\
\hline 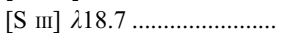 & $4.345 \mathrm{E}-1$ & $2.060 \mathrm{E}+0$ & $3.968 \mathrm{E}+0$ & $2.901 \mathrm{E}+0$ \\
\hline$[\mathrm{O}$ IV $]$ $\lambda 25.9 \ldots \ldots \ldots \ldots \ldots \ldots \ldots \ldots \ldots \ldots$ & $1.860 \mathrm{E}+0$ & $3.084 \mathrm{E}+0$ & $8.988 \mathrm{E}-1$ & $3.562 \mathrm{E}-3$ \\
\hline \multicolumn{5}{|c|}{$\alpha=-2.0$} \\
\hline $\operatorname{Br} \alpha \quad \lambda 4.051 \ldots \ldots \ldots \ldots \ldots \ldots \ldots \ldots$ & $1.138 \mathrm{E}-1$ & $1.039 \mathrm{E}-1$ & $9.900 \mathrm{E}-2$ & $9.899 \mathrm{E}-2$ \\
\hline 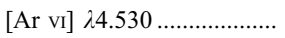 & $8.943 \mathrm{E}-3$ & $1.351 \mathrm{E}-4$ & $0.000 \mathrm{E}+0$ & $0.000 \mathrm{E}+0$ \\
\hline 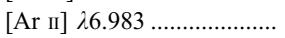 & $9.813 \mathrm{E}-3$ & $2.310 \mathrm{E}-2$ & $8.031 \mathrm{E}-2$ & $2.485 \mathrm{E}-1$ \\
\hline [Ar III] $\lambda 8.983 \ldots \ldots \ldots \ldots \ldots \ldots$ & $1.292 \mathrm{E}-1$ & $1.594 \mathrm{E}-3$ & $1.314 \mathrm{E}-6$ & $0.000 \mathrm{E}+0$ \\
\hline$[\mathrm{Ne}$ vI $] \lambda 7.652 \ldots \ldots \ldots \ldots \ldots . . . . .$. & $1.800 \mathrm{E}-1$ & $5.050 \mathrm{E}-1$ & $5.845 \mathrm{E}-1$ & $2.553 \mathrm{E}-1$ \\
\hline [S IV $] \lambda 10.44 \ldots \ldots \ldots \ldots \ldots \ldots \ldots$ & $9.161 \mathrm{E}+0$ & $5.971 \mathrm{E}+0$ & $6.004 \mathrm{E}-1$ & $9.046 \mathrm{E}-3$ \\
\hline$[\mathrm{Ne}$ II] $\lambda 12.8 \ldots \ldots \ldots \ldots \ldots \ldots . . . . . . . .$. & $1.620 \mathrm{E}-1$ & $4.525 \mathrm{E}-1$ & $1.362 \mathrm{E}+0$ & $2.878 \mathrm{E}+0$ \\
\hline$[\mathrm{Ne} v] \lambda 14.5 \ldots \ldots \ldots \ldots \ldots \ldots \ldots \ldots \ldots$ & $1.604 \mathrm{E}-1$ & $2.129 \mathrm{E}-2$ & $2.015 \mathrm{E}-4$ & $0.000 \mathrm{E}+0$ \\
\hline$[\mathrm{Ne}$ III] $\lambda 15.5 \ldots \ldots \ldots \ldots \ldots \ldots \ldots$ & $5.683 \mathrm{E}+0$ & $6.253 \mathrm{E}+0$ & $5.223 \mathrm{E}+0$ & $3.285 \mathrm{E}+0$ \\
\hline$\left[\mathrm{S}_{\mathrm{III}}\right] \lambda 18.7 \ldots \ldots \ldots \ldots \ldots \ldots \ldots \ldots \ldots$ & $6.079 \mathrm{E}-1$ & $2.127 \mathrm{E}+0$ & $3.545 \mathrm{E}+0$ & $2.395 \mathrm{E}+0$ \\
\hline 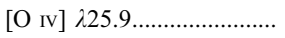 & $1.476 \mathrm{E}+0$ & $1.934 \mathrm{E}+0$ & $5.389 \mathrm{E}-1$ & $2.062 \mathrm{E}-3$ \\
\hline
\end{tabular}


TABLE 33

Undepleted Dust-Free UV Line Ratios with Respect to $\mathrm{H} \beta, 0.25 Z_{\odot}$

\begin{tabular}{|c|c|c|c|c|}
\hline \multirow[b]{2}{*}{ LiNE } & \multicolumn{4}{|c|}{$\log U_{0}$} \\
\hline & -1.0 & -2.0 & -3.0 & -4.0 \\
\hline \multicolumn{5}{|c|}{$\alpha=-1.2$} \\
\hline 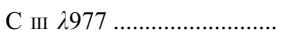 & $2.756 \mathrm{E}-01$ & $4.011 \mathrm{E}-01$ & $2.664 \mathrm{E}-02$ & $4.586 \mathrm{E}-04$ \\
\hline 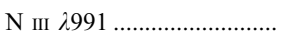 & $2.314 \mathrm{E}-02$ & $2.967 \mathrm{E}-02$ & $1.912 \mathrm{E}-03$ & $5.040 \mathrm{E}-06$ \\
\hline $\mathrm{O}$ VI $\lambda \lambda 1032,1037 \ldots \ldots \ldots \ldots . .$. & $1.016 \mathrm{E}+01$ & $2.059 \mathrm{E}-01$ & $1.038 \mathrm{E}-04$ & $0.000 \mathrm{E}+00$ \\
\hline 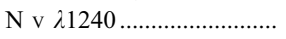 & $4.516 \mathrm{E}-01$ & $7.453 \mathrm{E}-02$ & $2.354 \mathrm{E}-04$ & $0.000 \mathrm{E}+00$ \\
\hline $\mathrm{Si}$ IV+O IV] $\lambda 1400 \ldots \ldots \ldots \ldots . .$. & $7.651 \mathrm{E}-01$ & $4.484 \mathrm{E}-01$ & $1.152 \mathrm{E}-01$ & $3.448 \mathrm{E}-04$ \\
\hline $\mathrm{N}$ Iv] $\lambda 1486 \ldots \ldots \ldots \ldots \ldots \ldots \ldots$ & $1.588 \mathrm{E}-01$ & $6.977 \mathrm{E}-02$ & $1.195 \mathrm{E}-03$ & $0.000 \mathrm{E}+00$ \\
\hline $\mathrm{C}$ iv $\lambda 1549 \ldots \ldots \ldots \ldots \ldots \ldots \ldots$ & $8.774 \mathrm{E}+00$ & $4.602 \mathrm{E}+00$ & $7.024 \mathrm{E}-02$ & $8.503 \mathrm{E}-05$ \\
\hline 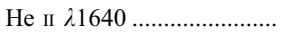 & $2.235 \mathrm{E}+00$ & $2.126 \mathrm{E}+00$ & $1.608 \mathrm{E}+00$ & $6.824 \mathrm{E}-01$ \\
\hline 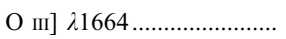 & $1.260 \mathrm{E}+00$ & $5.747 \mathrm{E}-01$ & $9.018 \mathrm{E}-02$ & $9.026 \mathrm{E}-04$ \\
\hline 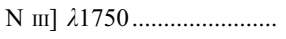 & $1.165 \mathrm{E}-02$ & $3.517 \mathrm{E}-02$ & $7.467 \mathrm{E}-03$ & $6.933 \mathrm{E}-05$ \\
\hline 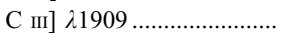 & $1.318 \mathrm{E}+00$ & $3.311 \mathrm{E}+00$ & $9.568 \mathrm{E}-01$ & $7.638 \mathrm{E}-02$ \\
\hline 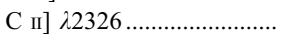 & $1.619 \mathrm{E}-02$ & $1.276 \mathrm{E}-01$ & $3.448 \mathrm{E}-01$ & $3.670 \mathrm{E}-01$ \\
\hline 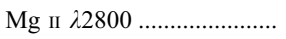 & $1.475 \mathrm{E}-01$ & $6.343 \mathrm{E}-01$ & $1.253 \mathrm{E}+00$ & $1.523 \mathrm{E}+00$ \\
\hline$[\mathrm{Ne} v] \lambda 3426 \ldots \ldots \ldots \ldots \ldots \ldots$ & $6.894 \mathrm{E}-01$ & $3.361 \mathrm{E}-01$ & $1.400 \mathrm{E}-03$ & $0.000 \mathrm{E}+00$ \\
\hline 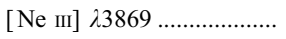 & $1.421 \mathrm{E}+00$ & $1.141 \mathrm{E}+00$ & $8.012 \mathrm{E}-01$ & $3.751 \mathrm{E}-01$ \\
\hline \multicolumn{5}{|c|}{$\alpha=-1.4$} \\
\hline 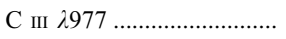 & $2.764 \mathrm{E}-01$ & $3.623 \mathrm{E}-01$ & $2.628 \mathrm{E}-02$ & $5.050 \mathrm{E}-04$ \\
\hline 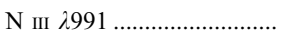 & $1.995 \mathrm{E}-02$ & $2.524 \mathrm{E}-02$ & $1.906 \mathrm{E}-03$ & $5.927 \mathrm{E}-06$ \\
\hline O vI $\lambda \lambda 1032,1037 \ldots \ldots \ldots \ldots$ & $6.054 \mathrm{E}+00$ & $8.940 \mathrm{E}-02$ & $4.459 \mathrm{E}-05$ & $0.000 \mathrm{E}+00$ \\
\hline 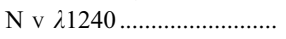 & $3.618 \mathrm{E}-01$ & $4.435 \mathrm{E}-02$ & $1.406 \mathrm{E}-04$ & $0.000 \mathrm{E}+00$ \\
\hline $\mathrm{Si}$ Iv $+\mathrm{O}$ Iv] $\lambda 1400 \ldots \ldots \ldots \ldots . .$. & $5.850 \mathrm{E}-01$ & $4.813 \mathrm{E}-01$ & $1.140 \mathrm{E}-01$ & $3.238 \mathrm{E}-04$ \\
\hline 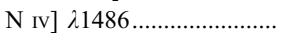 & $1.508 \mathrm{E}-01$ & $5.785 \mathrm{E}-02$ & $1.035 \mathrm{E}-03$ & $0.000 \mathrm{E}+00$ \\
\hline $\mathrm{C}$ IV $\lambda 1549$ & $8.955 \mathrm{E}+00$ & $3.793 \mathrm{E}+00$ & $5.831 \mathrm{E}-02$ & $7.664 \mathrm{E}-05$ \\
\hline 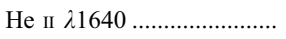 & $1.788 \mathrm{E}+00$ & $1.724 \mathrm{E}+00$ & $1.354 \mathrm{E}+00$ & $5.786 \mathrm{E}-01$ \\
\hline 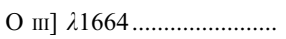 & $9.731 \mathrm{E}-01$ & $5.375 \mathrm{E}-01$ & $9.370 \mathrm{E}-02$ & $1.005 \mathrm{E}-03$ \\
\hline 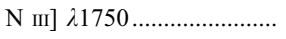 & $1.379 \mathrm{E}-02$ & $3.821 \mathrm{E}-02$ & $8.698 \mathrm{E}-03$ & $9.015 \mathrm{E}-05$ \\
\hline 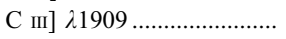 & $1.455 \mathrm{E}+00$ & $3.335 \mathrm{E}+00$ & $9.547 \mathrm{E}-01$ & $7.885 \mathrm{E}-02$ \\
\hline $\mathrm{C}$ II] $\lambda 2326 \ldots \ldots \ldots \ldots \ldots \ldots \ldots$ & $1.299 \mathrm{E}-02$ & $1.095 \mathrm{E}-01$ & $3.186 \mathrm{E}-01$ & $3.601 \mathrm{E}-01$ \\
\hline 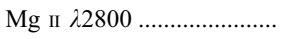 & $1.368 \mathrm{E}-01$ & $6.010 \mathrm{E}-01$ & $1.229 \mathrm{E}+00$ & $1.591 \mathrm{E}+00$ \\
\hline$[\mathrm{Ne} v] \lambda 3426 \ldots \ldots \ldots \ldots \ldots \ldots$ & $5.823 \mathrm{E}-01$ & $2.300 \mathrm{E}-01$ & $9.146 \mathrm{E}-04$ & $0.000 \mathrm{E}+00$ \\
\hline$[\mathrm{Ne}$ III] $\lambda 3869 \ldots \ldots \ldots \ldots \ldots \ldots$ & $1.365 \mathrm{E}+00$ & $1.180 \mathrm{E}+00$ & $7.766 \mathrm{E}-01$ & $3.408 \mathrm{E}-01$ \\
\hline \multicolumn{5}{|c|}{$\alpha=-1.7$} \\
\hline 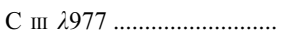 & $1.940 \mathrm{E}-01$ & $2.012 \mathrm{E}-01$ & $1.615 \mathrm{E}-02$ & $3.386 \mathrm{E}-04$ \\
\hline $\mathrm{N}$ III $\lambda 991 \ldots \ldots \ldots \ldots \ldots \ldots \ldots \ldots$ & $1.077 \mathrm{E}-02$ & $1.357 \mathrm{E}-02$ & $1.185 \mathrm{E}-03$ & $4.117 \mathrm{E}-06$ \\
\hline O vI $\lambda \lambda 1032,1037 \ldots \ldots \ldots \ldots$ & $2.231 \mathrm{E}+00$ & $1.657 \mathrm{E}-02$ & $7.287 \mathrm{E}-06$ & $0.000 \mathrm{E}+00$ \\
\hline 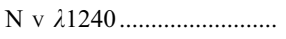 & $2.052 \mathrm{E}-01$ & $1.414 \mathrm{E}-02$ & $4.196 \mathrm{E}-05$ & $0.000 \mathrm{E}+00$ \\
\hline $\mathrm{Si}$ IV+O IV] $\lambda 1400 \ldots \ldots \ldots \ldots . .$. & $2.965 \mathrm{E}-01$ & $4.173 \mathrm{E}-01$ & $7.566 \mathrm{E}-02$ & $1.872 \mathrm{E}-04$ \\
\hline 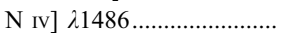 & $9.974 \mathrm{E}-02$ & $3.105 \mathrm{E}-02$ & $5.738 \mathrm{E}-04$ & $0.000 \mathrm{E}+00$ \\
\hline $\mathrm{C}$ IV $\lambda 1549 \ldots \ldots \ldots \ldots \ldots \ldots \ldots$ & $6.845 \mathrm{E}+00$ & $2.077 \mathrm{E}+00$ & $3.108 \mathrm{E}-02$ & $4.350 \mathrm{E}-05$ \\
\hline 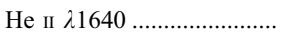 & $1.236 \mathrm{E}+00$ & $1.217 \mathrm{E}+00$ & $9.956 \mathrm{E}-01$ & $4.263 \mathrm{E}-01$ \\
\hline 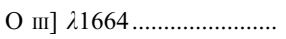 & $5.330 \mathrm{E}-01$ & $3.728 \mathrm{E}-01$ & $7.152 \mathrm{E}-02$ & $7.764 \mathrm{E}-04$ \\
\hline 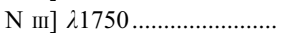 & $9.830 \mathrm{E}-03$ & $2.420 \mathrm{E}-02$ & $5.839 \mathrm{E}-03$ & $6.363 \mathrm{E}-05$ \\
\hline 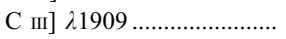 & $1.386 \mathrm{E}+00$ & $2.626 \mathrm{E}+00$ & $7.584 \mathrm{E}-01$ & $6.320 \mathrm{E}-02$ \\
\hline $\mathrm{C}$ II] $\lambda 2326 \ldots \ldots \ldots \ldots \ldots \ldots \ldots$ & $9.311 \mathrm{E}-03$ & $8.136 \mathrm{E}-02$ & $2.551 \mathrm{E}-01$ & $2.874 \mathrm{E}-01$ \\
\hline 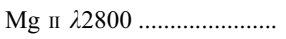 & $1.093 \mathrm{E}-01$ & $4.895 \mathrm{E}-01$ & $1.046 \mathrm{E}+00$ & $1.312 \mathrm{E}+00$ \\
\hline$[\mathrm{Ne} v] \lambda 3426 \ldots \ldots \ldots \ldots \ldots \ldots$ & $4.105 \mathrm{E}-01$ & $9.727 \mathrm{E}-02$ & $3.501 \mathrm{E}-04$ & $0.000 \mathrm{E}+00$ \\
\hline$[\mathrm{Ne}$ III] $\lambda 3869 \ldots \ldots \ldots \ldots \ldots \ldots . .$. & $1.151 \mathrm{E}+00$ & $1.085 \mathrm{E}+00$ & $6.607 \mathrm{E}-01$ & $2.537 \mathrm{E}-01$ \\
\hline \multicolumn{5}{|c|}{$\alpha=-2.0$} \\
\hline 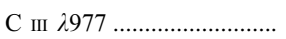 & $1.191 \mathrm{E}-01$ & $1.086 \mathrm{E}-01$ & $1.050 \mathrm{E}-02$ & $2.524 \mathrm{E}-04$ \\
\hline 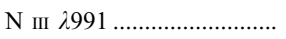 & $6.031 \mathrm{E}-03$ & $7.365 \mathrm{E}-03$ & $7.685 \mathrm{E}-04$ & $3.129 \mathrm{E}-06$ \\
\hline O vI $\lambda \lambda 1032,1037 \ldots \ldots \ldots \ldots . .$. & $6.728 \mathrm{E}-01$ & $2.848 \mathrm{E}-03$ & $0.000 \mathrm{E}+00$ & $0.000 \mathrm{E}+00$ \\
\hline 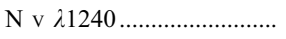 & $1.039 \mathrm{E}-01$ & $4.243 \mathrm{E}-03$ & $1.240 \mathrm{E}-05$ & $0.000 \mathrm{E}+00$ \\
\hline $\mathrm{Si}$ IV+O IV] $\lambda 1400 \ldots \ldots \ldots \ldots . .$. & $1.960 \mathrm{E}-01$ & $3.107 \mathrm{E}-01$ & $5.063 \mathrm{E}-02$ & $1.192 \mathrm{E}-04$ \\
\hline 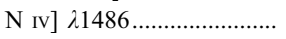 & $6.213 \mathrm{E}-02$ & $1.580 \mathrm{E}-02$ & $3.140 \mathrm{E}-04$ & $0.000 \mathrm{E}+00$ \\
\hline $\mathrm{C}$ IV $\lambda 1549$ & $4.592 \mathrm{E}+00$ & $1.055 \mathrm{E}+00$ & $1.667 \mathrm{E}-02$ & $2.578 \mathrm{E}-05$ \\
\hline 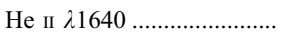 & $8.529 \mathrm{E}-01$ & $8.407 \mathrm{E}-01$ & $7.045 \mathrm{E}-01$ & $2.979 \mathrm{E}-01$ \\
\hline 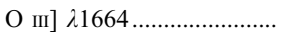 & $3.261 \mathrm{E}-01$ & $2.578 \mathrm{E}-01$ & $5.495 \mathrm{E}-02$ & $6.167 \mathrm{E}-04$ \\
\hline 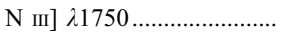 & $8.160 \mathrm{E}-03$ & $1.734 \mathrm{E}-02$ & $4.512 \mathrm{E}-03$ & $5.265 \mathrm{E}-05$ \\
\hline $\mathrm{C}$ III] $\lambda 1909 \ldots \ldots \ldots \ldots \ldots \ldots \ldots$ & $1.235 \mathrm{E}+00$ & $1.948 \mathrm{E}+00$ & $6.044 \mathrm{E}-01$ & $5.156 \mathrm{E}-02$ \\
\hline $\mathrm{C}$ II] $\lambda 2326 \ldots \ldots \ldots \ldots \ldots \ldots \ldots$ & $7.992 \mathrm{E}-03$ & $6.781 \mathrm{E}-02$ & $2.287 \mathrm{E}-01$ & $2.566 \mathrm{E}-01$ \\
\hline $\mathrm{Mg}$ II $\lambda 2800 \ldots \ldots \ldots \ldots \ldots \ldots . .$. & $9.329 \mathrm{E}-02$ & $4.315 \mathrm{E}-01$ & $1.061 \mathrm{E}+00$ & $1.328 \mathrm{E}+00$ \\
\hline$[\mathrm{Ne} v] \lambda 3426 \ldots \ldots \ldots \ldots \ldots \ldots$ & $2.651 \mathrm{E}-01$ & $3.588 \mathrm{E}-02$ & $1.239 \mathrm{E}-04$ & $0.000 \mathrm{E}+00$ \\
\hline$[\mathrm{Ne}$ III] $\lambda 3869 \ldots \ldots \ldots \ldots \ldots \ldots$ & $9.980 \mathrm{E}-01$ & $9.664 \mathrm{E}-01$ & $5.690 \mathrm{E}-01$ & $1.930 \mathrm{E}-01$ \\
\hline
\end{tabular}


TABLE 34

Undepleted Dust-Free UV Line Ratios with Respect to $\mathrm{H} \beta, 0.5 Z_{\odot}$

\begin{tabular}{|c|c|c|c|c|}
\hline \multirow[b]{2}{*}{ LiNE } & \multicolumn{4}{|c|}{$\log U_{0}$} \\
\hline & -1.0 & -2.0 & -3.0 & -4.0 \\
\hline \multicolumn{5}{|c|}{$\alpha=-1.2$} \\
\hline 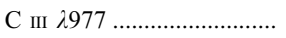 & $2.033 \mathrm{E}-01$ & $3.333 \mathrm{E}-01$ & $2.226 \mathrm{E}-02$ & $3.971 \mathrm{E}-04$ \\
\hline 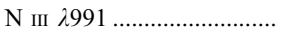 & $2.585 \mathrm{E}-02$ & $3.742 \mathrm{E}-02$ & $2.396 \mathrm{E}-03$ & $6.212 \mathrm{E}-06$ \\
\hline O vi $\lambda \lambda 1032,1037 \ldots \ldots \ldots \ldots . .$. & $1.130 \mathrm{E}+01$ & $1.997 \mathrm{E}-01$ & $8.221 \mathrm{E}-05$ & $0.000 \mathrm{E}+00$ \\
\hline 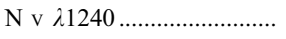 & $8.150 \mathrm{E}-01$ & $1.214 \mathrm{E}-01$ & $3.352 \mathrm{E}-04$ & $0.000 \mathrm{E}+00$ \\
\hline Si IV+O IV] $\lambda 1400 \ldots \ldots \ldots \ldots . .$. & $7.035 \mathrm{E}-01$ & $5.098 \mathrm{E}-01$ & $1.277 \mathrm{E}-01$ & $3.544 \mathrm{E}-04$ \\
\hline $\mathrm{N}$ IV] $\lambda 1486 \ldots \ldots \ldots \ldots \ldots \ldots \ldots \ldots$ & $2.398 \mathrm{E}-01$ & $1.172 \mathrm{E}-01$ & $1.923 \mathrm{E}-03$ & $0.000 \mathrm{E}+00$ \\
\hline $\mathrm{C}$ IV $\lambda 1549 \ldots \ldots \ldots \ldots \ldots \ldots \ldots$ & $8.641 \mathrm{E}+00$ & $5.476 \mathrm{E}+00$ & $7.592 \mathrm{E}-02$ & $9.431 \mathrm{E}-05$ \\
\hline 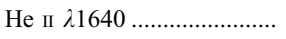 & $2.240 \mathrm{E}+00$ & $2.138 \mathrm{E}+00$ & $1.635 \mathrm{E}+00$ & $7.043 \mathrm{E}-01$ \\
\hline 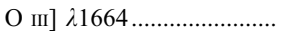 & $1.101 \mathrm{E}+00$ & $6.483 \mathrm{E}-01$ & $1.067 \mathrm{E}-01$ & $1.062 \mathrm{E}-03$ \\
\hline $\mathrm{N}$ III] $\lambda 1750 \ldots \ldots \ldots \ldots \ldots \ldots \ldots$ & $1.765 \mathrm{E}-02$ & $6.230 \mathrm{E}-02$ & $1.348 \mathrm{E}-02$ & $1.237 \mathrm{E}-04$ \\
\hline 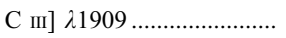 & $1.328 \mathrm{E}+00$ & $4.075 \mathrm{E}+00$ & $1.225 \mathrm{E}+00$ & $9.907 \mathrm{E}-02$ \\
\hline $\mathrm{C}$ II] $\lambda 2326 \ldots \ldots \ldots \ldots \ldots \ldots \ldots$ & $2.045 \mathrm{E}-02$ & $1.924 \mathrm{E}-01$ & $5.212 \mathrm{E}-01$ & $5.114 \mathrm{E}-01$ \\
\hline $\mathrm{Mg}$ II $\lambda 2800 \ldots \ldots \ldots \ldots \ldots \ldots \ldots$ & $2.161 \mathrm{E}-01$ & $1.018 \mathrm{E}+00$ & $1.832 \mathrm{E}+00$ & $1.917 \mathrm{E}+00$ \\
\hline$[\mathrm{Ne} v] \lambda 3426 \ldots \ldots \ldots \ldots \ldots \ldots$ & $1.064 \mathrm{E}+00$ & $4.797 \mathrm{E}-01$ & $1.935 \mathrm{E}-03$ & $0.000 \mathrm{E}+00$ \\
\hline$[\mathrm{Ne}$ III] $\lambda 3869 \ldots \ldots \ldots \ldots \ldots \ldots$ & $1.847 \mathrm{E}+00$ & $1.811 \mathrm{E}+00$ & $1.294 \mathrm{E}+00$ & $5.903 \mathrm{E}-01$ \\
\hline \multicolumn{5}{|c|}{$\alpha=-1.4$} \\
\hline 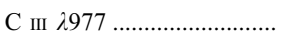 & $1.695 \mathrm{E}-01$ & $2.149 \mathrm{E}-01$ & $1.559 \mathrm{E}-02$ & $3.244 \mathrm{E}-04$ \\
\hline 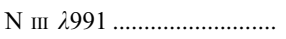 & $1.765 \mathrm{E}-02$ & $2.283 \mathrm{E}-02$ & $1.697 \mathrm{E}-03$ & $5.350 \mathrm{E}-06$ \\
\hline O vi $\lambda \lambda 1032,1037 \ldots \ldots \ldots \ldots . .$. & $5.996 \mathrm{E}+00$ & $6.646 \mathrm{E}-02$ & $2.539 \mathrm{E}-05$ & $0.000 \mathrm{E}+00$ \\
\hline 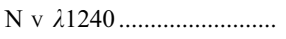 & $5.816 \mathrm{E}-01$ & $5.744 \mathrm{E}-02$ & $1.513 \mathrm{E}-04$ & $0.000 \mathrm{E}+00$ \\
\hline Si IV+O Iv] $\lambda 1400 \ldots \ldots \ldots \ldots . .$. & $4.356 \mathrm{E}-01$ & $4.077 \mathrm{E}-01$ & $9.928 \mathrm{E}-02$ & $2.585 \mathrm{E}-04$ \\
\hline 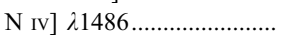 & $1.894 \mathrm{E}-01$ & $7.698 \mathrm{E}-02$ & $1.321 \mathrm{E}-03$ & $0.000 \mathrm{E}+00$ \\
\hline 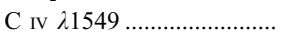 & $7.591 \mathrm{E}+00$ & $3.657 \mathrm{E}+00$ & $5.003 \mathrm{E}-02$ & $6.893 \mathrm{E}-05$ \\
\hline 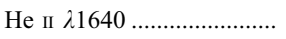 & $1.755 \mathrm{E}+00$ & $1.720 \mathrm{E}+00$ & $1.372 \mathrm{E}+00$ & $5.973 \mathrm{E}-01$ \\
\hline $\mathrm{O}$ III] $\lambda 1664 \ldots \ldots \ldots \ldots \ldots \ldots . .$. & $6.936 \mathrm{E}-01$ & $4.718 \mathrm{E}-01$ & $8.913 \mathrm{E}-02$ & $9.748 \mathrm{E}-04$ \\
\hline 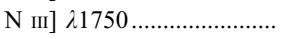 & $1.540 \mathrm{E}-02$ & $4.736 \mathrm{E}-02$ & $1.122 \mathrm{E}-02$ & $1.165 \mathrm{E}-04$ \\
\hline $\left.\mathrm{C}_{\mathrm{III}}\right] \lambda 1909 \ldots \ldots \ldots \ldots \ldots \ldots \ldots$ & $1.283 \mathrm{E}+00$ & $3.368 \mathrm{E}+00$ & $1.033 \mathrm{E}+00$ & $8.890 \mathrm{E}-02$ \\
\hline $\mathrm{C}_{\text {II] }} \lambda 2326 \ldots \ldots \ldots \ldots \ldots \ldots \ldots$ & $1.491 \mathrm{E}-02$ & $1.432 \mathrm{E}-01$ & $4.251 \mathrm{E}-01$ & $4.489 \mathrm{E}-01$ \\
\hline 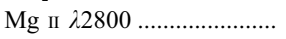 & $1.809 \mathrm{E}-01$ & $8.533 \mathrm{E}-01$ & $1.591 \mathrm{E}+00$ & $1.783 \mathrm{E}+00$ \\
\hline$[\mathrm{Ne} v] \lambda 3426 \ldots \ldots \ldots \ldots \ldots \ldots$ & $8.489 \mathrm{E}-01$ & $2.889 \mathrm{E}-01$ & $1.101 \mathrm{E}-03$ & $0.000 \mathrm{E}+00$ \\
\hline$[\mathrm{Ne}$ III] $\lambda 3869 \ldots \ldots \ldots \ldots \ldots \ldots$ & $1.598 \mathrm{E}+00$ & $1.650 \mathrm{E}+00$ & $1.145 \mathrm{E}+00$ & $4.989 \mathrm{E}-01$ \\
\hline \multicolumn{5}{|c|}{$\alpha=-1.7$} \\
\hline 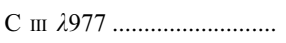 & $1.012 \mathrm{E}-01$ & $9.622 \mathrm{E}-02$ & $8.001 \mathrm{E}-03$ & $2.147 \mathrm{E}-04$ \\
\hline 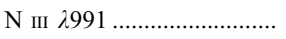 & $8.531 \mathrm{E}-03$ & $9.807 \mathrm{E}-03$ & $8.747 \mathrm{E}-04$ & $3.708 \mathrm{E}-06$ \\
\hline O vi $\lambda \lambda 1032,1037 \ldots \ldots \ldots \ldots$ & $1.955 \mathrm{E}+00$ & $1.053 \mathrm{E}-02$ & $3.508 \mathrm{E}-06$ & $0.000 \mathrm{E}+00$ \\
\hline $\mathrm{N}$ v $\lambda 1240 \ldots \ldots \ldots \ldots \ldots \ldots \ldots \ldots$ & $3.024 \mathrm{E}-01$ & $1.589 \mathrm{E}-02$ & $3.875 \mathrm{E}-05$ & $0.000 \mathrm{E}+00$ \\
\hline $\mathrm{Si}$ Iv+O Iv] $\lambda 1400 \ldots \ldots \ldots \ldots . .$. & $2.346 \mathrm{E}-01$ & $2.689 \mathrm{E}-01$ & $5.698 \mathrm{E}-02$ & $1.440 \mathrm{E}-04$ \\
\hline 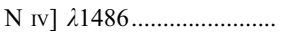 & $1.127 \mathrm{E}-01$ & $3.527 \mathrm{E}-02$ & $6.480 \mathrm{E}-04$ & $0.000 \mathrm{E}+00$ \\
\hline $\mathrm{C}$ IV $\lambda 1549 \ldots \ldots \ldots \ldots \ldots \ldots \ldots$ & $5.237 \mathrm{E}+00$ & $1.706 \mathrm{E}+00$ & $2.348 \mathrm{E}-02$ & $3.857 \mathrm{E}-05$ \\
\hline 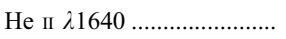 & $1.202 \mathrm{E}+00$ & $1.209 \mathrm{E}+00$ & $1.007 \mathrm{E}+00$ & $4.350 \mathrm{E}-01$ \\
\hline 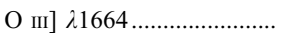 & $3.365 \mathrm{E}-01$ & $2.701 \mathrm{E}-01$ & $6.071 \mathrm{E}-02$ & $7.552 \mathrm{E}-04$ \\
\hline $\mathrm{N}$ III] $\lambda 1750 \ldots \ldots \ldots \ldots \ldots \ldots \ldots$ & $1.151 \mathrm{E}-02$ & $2.869 \mathrm{E}-02$ & $7.674 \mathrm{E}-03$ & $9.386 \mathrm{E}-05$ \\
\hline $\mathrm{C}$ III] $\lambda 1909 \ldots \ldots \ldots \ldots \ldots \ldots . .$. & $1.101 \mathrm{E}+00$ & $2.232 \mathrm{E}+00$ & $7.352 \mathrm{E}-01$ & $6.931 \mathrm{E}-02$ \\
\hline $\mathrm{C}$ II] $\lambda 2326 \ldots \ldots \ldots \ldots \ldots \ldots$ & $1.048 \mathrm{E}-02$ & $9.586 \mathrm{E}-02$ & $3.248 \mathrm{E}-01$ & $3.704 \mathrm{E}-01$ \\
\hline 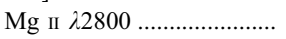 & $1.407 \mathrm{E}-01$ & $6.612 \mathrm{E}-01$ & $1.416 \mathrm{E}+00$ & $1.689 \mathrm{E}+00$ \\
\hline$[\mathrm{Ne} v] \lambda 3426 \ldots \ldots \ldots \ldots \ldots \ldots$ & $5.657 \mathrm{E}-01$ & $1.134 \mathrm{E}-01$ & $3.975 \mathrm{E}-04$ & $0.000 \mathrm{E}+00$ \\
\hline 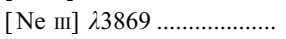 & $1.269 \mathrm{E}+00$ & $1.356 \mathrm{E}+00$ & $9.252 \mathrm{E}-01$ & $3.725 \mathrm{E}-01$ \\
\hline \multicolumn{5}{|c|}{$\alpha=-2.0$} \\
\hline 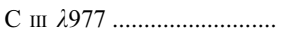 & $5.313 \mathrm{E}-02$ & $3.891 \mathrm{E}-02$ & $3.762 \mathrm{E}-03$ & $1.308 \mathrm{E}-04$ \\
\hline 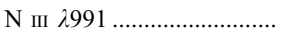 & $4.364 \mathrm{E}-03$ & $3.971 \mathrm{E}-03$ & $4.073 \mathrm{E}-04$ & $2.310 \mathrm{E}-06$ \\
\hline O vi $\lambda \lambda 1032,1037 \ldots \ldots \ldots \ldots . .$. & $5.138 \mathrm{E}-01$ & $1.390 \mathrm{E}-03$ & $0.000 \mathrm{E}+00$ & $0.000 \mathrm{E}+00$ \\
\hline 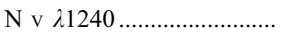 & $1.385 \mathrm{E}-01$ & $3.826 \mathrm{E}-03$ & $8.697 \mathrm{E}-06$ & $0.000 \mathrm{E}+00$ \\
\hline $\mathrm{Si} \mathrm{IV+O} \mathrm{Iv]} \lambda 1400 \ldots \ldots \ldots \ldots . .$. & $2.031 \mathrm{E}-01$ & $1.638 \mathrm{E}-01$ & $2.955 \mathrm{E}-02$ & $7.554 \mathrm{E}-05$ \\
\hline $\mathrm{N}$ iv] $\lambda 1486 \ldots \ldots \ldots \ldots \ldots \ldots \ldots$ & $6.429 \mathrm{E}-02$ & $1.448 \mathrm{E}-02$ & $2.830 \mathrm{E}-04$ & $0.000 \mathrm{E}+00$ \\
\hline $\mathrm{C}$ IV $\lambda 1549 \ldots \ldots \ldots \ldots \ldots \ldots . .$. & $3.091 \mathrm{E}+00$ & $7.003 \mathrm{E}-01$ & $1.001 \mathrm{E}-02$ & $1.988 \mathrm{E}-05$ \\
\hline 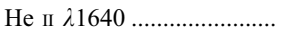 & $8.237 \mathrm{E}-01$ & $8.355 \mathrm{E}-01$ & $7.107 \mathrm{E}-01$ & $3.018 \mathrm{E}-01$ \\
\hline 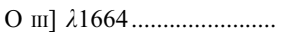 & $1.786 \mathrm{E}-01$ & $1.507 \mathrm{E}-01$ & $3.794 \mathrm{E}-02$ & $5.323 \mathrm{E}-04$ \\
\hline 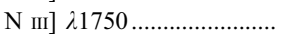 & $8.565 \mathrm{E}-03$ & $1.668 \mathrm{E}-02$ & $4.867 \mathrm{E}-03$ & $6.890 \mathrm{E}-05$ \\
\hline 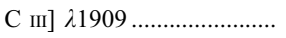 & $8.639 \mathrm{E}-01$ & $1.371 \mathrm{E}+00$ & $4.929 \mathrm{E}-01$ & $5.066 \mathrm{E}-02$ \\
\hline 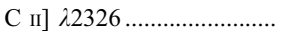 & $8.643 \mathrm{E}-03$ & $6.984 \mathrm{E}-02$ & $2.589 \mathrm{E}-01$ & $3.085 \mathrm{E}-01$ \\
\hline 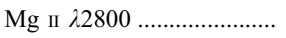 & $1.155 \mathrm{E}-01$ & $5.356 \mathrm{E}-01$ & $1.336 \mathrm{E}+00$ & $1.641 \mathrm{E}+00$ \\
\hline$[\mathrm{Ne} v] \lambda 3426 \ldots \ldots \ldots \ldots \ldots \ldots$ & $3.390 \mathrm{E}-01$ & $3.716 \mathrm{E}-02$ & $1.244 \mathrm{E}-04$ & $0.000 \mathrm{E}+00$ \\
\hline$[\mathrm{Ne}$ III] $\lambda 3869 \ldots \ldots \ldots \ldots \ldots \ldots$ & $1.020 \mathrm{E}+00$ & $1.080 \mathrm{E}+00$ & $7.261 \mathrm{E}-01$ & $2.683 \mathrm{E}-01$ \\
\hline
\end{tabular}


TABLE 35

Undepleted Dust-Free UV Line Ratios with Respect to $\mathrm{H} \beta, 1 Z_{\odot}$

\begin{tabular}{|c|c|c|c|c|}
\hline \multirow[b]{2}{*}{ LiNe } & \multicolumn{4}{|c|}{$\log U_{0}$} \\
\hline & -1.0 & -2.0 & -3.0 & -4.0 \\
\hline \multicolumn{5}{|c|}{$\alpha=-1.2$} \\
\hline 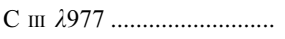 & $1.264 \mathrm{E}-01$ & $1.660 \mathrm{E}-01$ & $6.588 \mathrm{E}-03$ & $1.080 \mathrm{E}-04$ \\
\hline 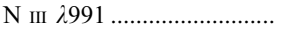 & $2.527 \mathrm{E}-02$ & $3.081 \mathrm{E}-02$ & $1.161 \mathrm{E}-03$ & $2.406 \mathrm{E}-06$ \\
\hline O vi $\lambda \lambda 1032,1037 \ldots \ldots \ldots \ldots$ & $1.040 \mathrm{E}+01$ & $1.281 \mathrm{E}-01$ & $2.320 \mathrm{E}-05$ & $0.000 \mathrm{E}+00$ \\
\hline 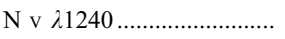 & $1.374 \mathrm{E}+00$ & $1.495 \mathrm{E}-01$ & $2.188 \mathrm{E}-04$ & $0.000 \mathrm{E}+00$ \\
\hline $\mathrm{Si}$ IV+O IV] $\lambda 1400 \ldots \ldots \ldots \ldots . .$. & $5.129 \mathrm{E}-01$ & $3.723 \mathrm{E}-01$ & $6.725 \mathrm{E}-02$ & $1.467 \mathrm{E}-04$ \\
\hline $\mathrm{N}$ Iv] $\lambda 1486 \ldots \ldots \ldots \ldots \ldots \ldots \ldots$ & $3.252 \mathrm{E}-01$ & $1.515 \mathrm{E}-01$ & $1.669 \mathrm{E}-03$ & $0.000 \mathrm{E}+00$ \\
\hline 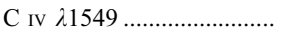 & $7.118 \mathrm{E}+00$ & $4.583 \mathrm{E}+00$ & $3.995 \mathrm{E}-02$ & $4.597 \mathrm{E}-05$ \\
\hline 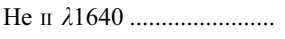 & $2.188 \mathrm{E}+00$ & $2.130 \mathrm{E}+00$ & $1.680 \mathrm{E}+00$ & $7.331 \mathrm{E}-01$ \\
\hline $\left.\mathrm{O}_{\mathrm{III}}\right] \lambda_{16}$ & $7.010 \mathrm{E}-01$ & $4.657 \mathrm{E}-01$ & $6.626 \mathrm{E}-02$ & $5.867 \mathrm{E}-04$ \\
\hline $\mathrm{N}$ III] $\lambda 1750 \ldots \ldots \ldots \ldots \ldots \ldots \ldots$ & $2.221 \mathrm{E}-02$ & $8.144 \mathrm{E}-02$ & $1.435 \mathrm{E}-02$ & $1.154 \mathrm{E}-04$ \\
\hline $\mathrm{C}$ III] $\lambda 1909 \ldots \ldots$. & $9.926 \mathrm{E}-01$ & $3.470 \mathrm{E}+00$ & $9.045 \mathrm{E}-01$ & $6.875 \mathrm{E}-02$ \\
\hline 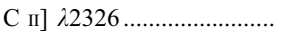 & $1.666 \mathrm{E}-02$ & $2.364 \mathrm{E}-01$ & $5.931 \mathrm{E}-01$ & $4.690 \mathrm{E}-01$ \\
\hline 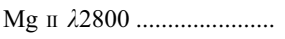 & $2.217 \mathrm{E}-01$ & $1.340 \mathrm{E}+00$ & $2.164 \mathrm{E}+00$ & $1.797 \mathrm{E}+00$ \\
\hline$[\mathrm{Ne} v] \lambda 3426 \ldots \ldots \ldots \ldots \ldots \ldots$ & $1.469 \mathrm{E}+00$ & $5.539 \mathrm{E}-01$ & $1.790 \mathrm{E}-03$ & $0.000 \mathrm{E}+00$ \\
\hline$[\mathrm{Ne}$ III] $\lambda 3869 \ldots \ldots \ldots \ldots \ldots \ldots . .$. & $1.873 \mathrm{E}+00$ & $2.274 \mathrm{E}+00$ & $1.613 \mathrm{E}+00$ & $6.834 \mathrm{E}-01$ \\
\hline \multicolumn{5}{|c|}{$\alpha=-1.4$} \\
\hline C III $\lambda 977$.......................... & $9.002 \mathrm{E}-02$ & $8.266 \mathrm{E}-02$ & $3.185 \mathrm{E}-03$ & $6.769 \mathrm{E}-05$ \\
\hline 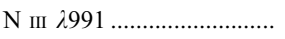 & $1.486 \mathrm{E}-02$ & $1.450 \mathrm{E}-02$ & $5.636 \mathrm{E}-04$ & $1.608 \mathrm{E}-06$ \\
\hline $\mathrm{O}$ vi $\lambda \lambda 1032,1037 \ldots \ldots \ldots \ldots$ & $5.010 \mathrm{E}+00$ & $3.361 \mathrm{E}-02$ & $5.043 \mathrm{E}-06$ & $0.000 \mathrm{E}+00$ \\
\hline 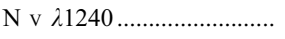 & $8.992 \mathrm{E}-01$ & $5.790 \mathrm{E}-02$ & $7.256 \mathrm{E}-05$ & $0.000 \mathrm{E}+00$ \\
\hline $\mathrm{Si}$ Iv+O Iv] $\lambda 1400 \ldots \ldots \ldots \ldots . .$. & $3.033 \mathrm{E}-01$ & $2.925 \mathrm{E}-01$ & $3.683 \mathrm{E}-02$ & $8.525 \mathrm{E}-05$ \\
\hline $\mathrm{N}$ IV] $\lambda 1486 \ldots \ldots \ldots \ldots \ldots \ldots \ldots$ & $2.293 \mathrm{E}-01$ & $8.204 \mathrm{E}-02$ & $8.921 \mathrm{E}-04$ & $0.000 \mathrm{E}+00$ \\
\hline $\mathrm{C}$ Iv $\lambda 1549 \ldots \ldots \ldots \ldots \ldots \ldots \ldots \ldots$ & $5.809 \mathrm{E}+00$ & $2.535 \mathrm{E}+00$ & $2.035 \mathrm{E}-02$ & $2.793 \mathrm{E}-05$ \\
\hline 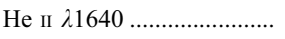 & $1.694 \mathrm{E}+00$ & $1.704 \mathrm{E}+00$ & $1.403 \mathrm{E}+00$ & $6.146 \mathrm{E}-01$ \\
\hline 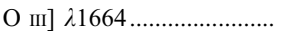 & $3.811 \mathrm{E}-01$ & $2.701 \mathrm{E}-01$ & $4.356 \mathrm{E}-02$ & $4.611 \mathrm{E}-04$ \\
\hline $\mathrm{N}$ III] $\lambda 1750 \ldots \ldots \ldots \ldots \ldots \ldots \ldots \ldots$ & $1.721 \mathrm{E}-02$ & $5.035 \mathrm{E}-02$ & $9.499 \mathrm{E}-03$ & $9.308 \mathrm{E}-05$ \\
\hline $\left.\mathrm{C}_{\mathrm{III}}\right] \lambda 1909 \ldots \ldots \ldots \ldots \ldots \ldots \ldots$ & $8.661 \mathrm{E}-01$ & $2.368 \mathrm{E}+00$ & $6.227 \mathrm{E}-01$ & $5.332 \mathrm{E}-02$ \\
\hline $\mathrm{C}$ II] $\lambda 2326 \ldots \ldots \ldots \ldots \ldots \ldots \ldots$ & $1.166 \mathrm{E}-02$ & $1.536 \mathrm{E}-01$ & $4.215 \mathrm{E}-01$ & $3.717 \mathrm{E}-01$ \\
\hline $\mathrm{Mg}$ II $22800 \ldots \ldots \ldots \ldots \ldots \ldots . .$. & $1.730 \mathrm{E}-01$ & $1.006 \mathrm{E}+00$ & $1.729 \mathrm{E}+00$ & $1.601 \mathrm{E}+00$ \\
\hline 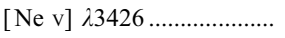 & $1.122 \mathrm{E}+00$ & $2.989 \mathrm{E}-01$ & $8.857 \mathrm{E}-04$ & $0.000 \mathrm{E}+00$ \\
\hline$[\mathrm{Ne}$ III] $\lambda 3869$.................... & $1.488 \mathrm{E}+00$ & $1.810 \mathrm{E}+00$ & $1.281 \mathrm{E}+00$ & $5.363 \mathrm{E}-01$ \\
\hline \multicolumn{5}{|c|}{$\alpha=-1.7$} \\
\hline C III $\lambda 977$.......................... & $4.480 \mathrm{E}-02$ & $2.542 \mathrm{E}-02$ & $9.849 \mathrm{E}-04$ & $3.008 \mathrm{E}-05$ \\
\hline 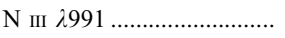 & $6.464 \mathrm{E}-03$ & $4.275 \mathrm{E}-03$ & $1.727 \mathrm{E}-04$ & $0.000 \mathrm{E}+00$ \\
\hline O vi $\lambda \lambda 1032,1037 \ldots \ldots \ldots \ldots . .$. & $1.406 \mathrm{E}+00$ & $3.615 \mathrm{E}-03$ & $0.000 \mathrm{E}+00$ & $0.000 \mathrm{E}+00$ \\
\hline 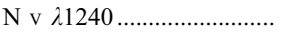 & $4.200 \mathrm{E}-01$ & $1.162 \mathrm{E}-02$ & $1.212 \mathrm{E}-05$ & $0.000 \mathrm{E}+00$ \\
\hline $\mathrm{Si}$ IV+O IV] $\lambda 1400 \ldots \ldots \ldots \ldots . .$. & $1.563 \mathrm{E}-01$ & $1.331 \mathrm{E}-01$ & $1.388 \mathrm{E}-02$ & $3.409 \mathrm{E}-05$ \\
\hline 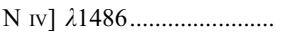 & $1.260 \mathrm{E}-01$ & $2.837 \mathrm{E}-02$ & $3.086 \mathrm{E}-04$ & $0.000 \mathrm{E}+00$ \\
\hline 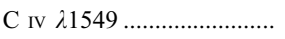 & $3.609 \mathrm{E}+00$ & $8.948 \mathrm{E}-01$ & $6.676 \mathrm{E}-03$ & $1.203 \mathrm{E}-05$ \\
\hline He II $\lambda 1640 \ldots \ldots \ldots \ldots \ldots \ldots \ldots$ & $1.149 \mathrm{E}+00$ & $1.198 \mathrm{E}+00$ & $1.025 \mathrm{E}+00$ & $4.421 \mathrm{E}-01$ \\
\hline 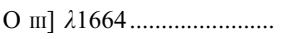 & $1.530 \mathrm{E}-01$ & $1.156 \mathrm{E}-01$ & $2.139 \mathrm{E}-02$ & $2.864 \mathrm{E}-04$ \\
\hline $\mathrm{N}$ III] $\lambda 1750 \ldots \ldots \ldots \ldots \ldots \ldots \ldots$ & $1.120 \mathrm{E}-02$ & $2.304 \mathrm{E}-02$ & $4.751 \mathrm{E}-03$ & $6.024 \mathrm{E}-05$ \\
\hline 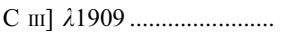 & $6.368 \mathrm{E}-01$ & $1.214 \mathrm{E}+00$ & $3.353 \mathrm{E}-01$ & $3.348 \mathrm{E}-02$ \\
\hline $\mathrm{C}$ II] $\lambda 2326 \ldots \ldots \ldots \ldots \ldots \ldots \ldots \ldots$ & $8.181 \mathrm{E}-03$ & $8.262 \mathrm{E}-02$ & $2.596 \mathrm{E}-01$ & $2.607 \mathrm{E}-01$ \\
\hline $\mathrm{Mg}$ II $\lambda 2800 \ldots \ldots \ldots \ldots \ldots \ldots \ldots \ldots$ & $1.283 \mathrm{E}-01$ & $6.663 \mathrm{E}-01$ & $1.345 \mathrm{E}+00$ & $1.378 \mathrm{E}+00$ \\
\hline$[\mathrm{Ne} v] \lambda 3426 \ldots \ldots \ldots \ldots \ldots \ldots \ldots$ & $6.920 \mathrm{E}-01$ & $9.916 \mathrm{E}-02$ & $2.642 \mathrm{E}-04$ & $0.000 \mathrm{E}+00$ \\
\hline$[\mathrm{Ne}$ III] $\lambda 3869 \ldots \ldots \ldots \ldots \ldots \ldots$ & $1.048 \mathrm{E}+00$ & $1.259 \mathrm{E}+00$ & $8.863 \mathrm{E}-01$ & $3.567 \mathrm{E}-01$ \\
\hline \multicolumn{5}{|c|}{$\alpha=-2.0$} \\
\hline 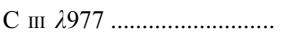 & $2.038 \mathrm{E}-02$ & $6.725 \mathrm{E}-03$ & $3.031 \mathrm{E}-04$ & $1.233 \mathrm{E}-05$ \\
\hline $\mathrm{N}$ III $2991 \ldots \ldots \ldots \ldots \ldots \ldots \ldots . .$. & $3.004 \mathrm{E}-03$ & $1.130 \mathrm{E}-03$ & $5.210 \mathrm{E}-05$ & $0.000 \mathrm{E}+00$ \\
\hline O vi $\lambda \lambda 1032,1037 \ldots \ldots \ldots \ldots . .$. & $3.076 \mathrm{E}-01$ & $3.044 \mathrm{E}-04$ & $0.000 \mathrm{E}+00$ & $0.000 \mathrm{E}+00$ \\
\hline 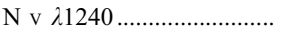 & $1.672 \mathrm{E}-01$ & $1.925 \mathrm{E}-03$ & $1.252 \mathrm{E}-06$ & $0.000 \mathrm{E}+00$ \\
\hline $\mathrm{Si}$ Iv+O Iv] $\lambda 1400 \ldots \ldots \ldots \ldots . .$. & $8.610 \mathrm{E}-02$ & $5.011 \mathrm{E}-02$ & $4.972 \mathrm{E}-03$ & $1.277 \mathrm{E}-05$ \\
\hline $\mathrm{N}$ IV] $\lambda 1486 \ldots \ldots \ldots \ldots \ldots \ldots \ldots$ & $6.709 \mathrm{E}-02$ & $8.539 \mathrm{E}-03$ & $1.006 \mathrm{E}-04$ & $0.000 \mathrm{E}+00$ \\
\hline $\mathrm{C}$ IV $\lambda 1549 \ldots \ldots \ldots \ldots \ldots \ldots \ldots$ & $1.922 \mathrm{E}+00$ & $2.677 \mathrm{E}-01$ & $2.110 \mathrm{E}-03$ & $4.865 \mathrm{E}-06$ \\
\hline 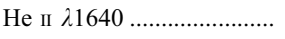 & $7.835 \mathrm{E}-01$ & $8.329 \mathrm{E}-01$ & $7.212 \mathrm{E}-01$ & $3.027 \mathrm{E}-01$ \\
\hline 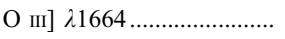 & $6.776 \mathrm{E}-02$ & $4.848 \mathrm{E}-02$ & $1.022 \mathrm{E}-02$ & $1.639 \mathrm{E}-04$ \\
\hline $\mathrm{N}$ III] $\lambda 1750 \ldots \ldots \ldots \ldots \ldots \ldots \ldots . .$. & $7.171 \mathrm{E}-03$ & $1.007 \mathrm{E}-02$ & $2.331 \mathrm{E}-03$ & $3.597 \mathrm{E}-05$ \\
\hline 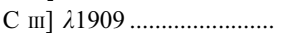 & $4.237 \mathrm{E}-01$ & $5.765 \mathrm{E}-01$ & $1.778 \mathrm{E}-01$ & $1.973 \mathrm{E}-02$ \\
\hline $\mathrm{C}$ II] $\lambda 2326 \ldots \ldots \ldots \ldots \ldots \ldots \ldots$ & $6.767 \mathrm{E}-03$ & $4.872 \mathrm{E}-02$ & $1.692 \mathrm{E}-01$ & $1.828 \mathrm{E}-01$ \\
\hline 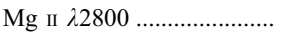 & $1.037 \mathrm{E}-01$ & $4.656 \mathrm{E}-01$ & $1.119 \mathrm{E}+00$ & $1.193 \mathrm{E}+00$ \\
\hline$[\mathrm{Ne} \mathrm{v}] \lambda 3426 \ldots \ldots \ldots \ldots \ldots \ldots$ & $3.781 \mathrm{E}-01$ & $2.699 \mathrm{E}-02$ & $7.073 \mathrm{E}-05$ & $0.000 \mathrm{E}+00$ \\
\hline$[\mathrm{Ne}$ III] $\lambda 3869 \ldots \ldots \ldots \ldots \ldots \ldots$ & $7.497 \mathrm{E}-01$ & $8.671 \mathrm{E}-01$ & $6.075 \mathrm{E}-01$ & $2.278 \mathrm{E}-01$ \\
\hline
\end{tabular}


TABLE 36

Undepleted Dust-Free UV Line Ratios with Respect to $\mathrm{H} \beta, 2 Z_{\odot}$

\begin{tabular}{|c|c|c|c|c|}
\hline \multirow[b]{2}{*}{ LINE } & \multicolumn{4}{|c|}{$\log U_{0}$} \\
\hline & -1.0 & -2.0 & -3.0 & -4.0 \\
\hline \multicolumn{5}{|c|}{$\alpha=-1.2$} \\
\hline C III $\lambda 977$........................... & $7.313 \mathrm{E}-02$ & $5.804 \mathrm{E}-02$ & $3.829 \mathrm{E}-04$ & $2.882 \mathrm{E}-06$ \\
\hline 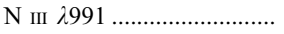 & $2.461 \mathrm{E}-02$ & $1.932 \mathrm{E}-02$ & $1.245 \mathrm{E}-04$ & $0.000 \mathrm{E}+00$ \\
\hline O vi $\lambda \lambda 1032,1037 \ldots \ldots \ldots \ldots$ & $8.290 \mathrm{E}+00$ & $5.389 \mathrm{E}-02$ & $0.000 \mathrm{E}+00$ & $0.000 \mathrm{E}+00$ \\
\hline 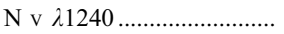 & $2.248 \mathrm{E}+00$ & $1.406 \mathrm{E}-01$ & $4.174 \mathrm{E}-05$ & $0.000 \mathrm{E}+00$ \\
\hline Si Iv+O Iv] $\lambda 1400 \ldots \ldots \ldots \ldots . .$. & $3.402 \mathrm{E}-01$ & $2.496 \mathrm{E}-01$ & $9.849 \mathrm{E}-03$ & $1.248 \mathrm{E}-05$ \\
\hline 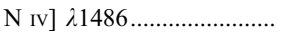 & $4.479 \mathrm{E}-01$ & $1.584 \mathrm{E}-01$ & $5.502 \mathrm{E}-04$ & $0.000 \mathrm{E}+00$ \\
\hline $\mathrm{C}$ IV $\lambda 1549 \ldots \ldots \ldots \ldots \ldots \ldots \ldots$ & $5.974 \mathrm{E}+00$ & $2.801 \mathrm{E}+00$ & $7.117 \mathrm{E}-03$ & $5.379 \mathrm{E}-06$ \\
\hline 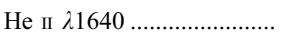 & $2.112 \mathrm{E}+00$ & $2.123 \mathrm{E}+00$ & $1.746 \mathrm{E}+00$ & $7.538 \mathrm{E}-01$ \\
\hline 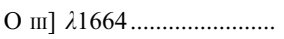 & $3.724 \mathrm{E}-01$ & $2.228 \mathrm{E}-01$ & $1.488 \mathrm{E}-02$ & $8.916 \mathrm{E}-05$ \\
\hline $\mathrm{N}$ III] $\lambda 1750 \ldots \ldots \ldots \ldots \ldots \ldots \ldots$ & $2.558 \mathrm{E}-02$ & $8.131 \mathrm{E}-02$ & $6.279 \mathrm{E}-03$ & $3.326 \mathrm{E}-05$ \\
\hline $\mathrm{C}$ III] $\lambda 1909 \ldots \ldots \ldots \ldots \ldots \ldots$ & $5.658 \mathrm{E}-01$ & $2.075 \mathrm{E}+00$ & $2.651 \mathrm{E}-01$ & $1.346 \mathrm{E}-02$ \\
\hline 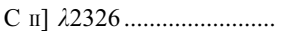 & $5.172 \mathrm{E}-03$ & $2.067 \mathrm{E}-01$ & $4.008 \mathrm{E}-01$ & $1.481 \mathrm{E}-01$ \\
\hline $\mathrm{Mg}$ II $\lambda 2800 \ldots \ldots \ldots \ldots \ldots \ldots \ldots$ & $9.799 \mathrm{E}-02$ & $1.248 \mathrm{E}+00$ & $1.732 \mathrm{E}+00$ & $8.732 \mathrm{E}-01$ \\
\hline$[\mathrm{Ne} \mathrm{v}] \lambda 3426 \ldots \ldots \ldots \ldots \ldots \ldots$ & $1.827 \mathrm{E}+00$ & $5.095 \mathrm{E}-01$ & $9.178 \mathrm{E}-04$ & $0.000 \mathrm{E}+00$ \\
\hline$[\mathrm{Ne}$ III] $\lambda 3869 \ldots \ldots \ldots \ldots \ldots . .$. & $1.313 \mathrm{E}+00$ & $2.075 \mathrm{E}+00$ & $1.277 \mathrm{E}+00$ & $3.901 \mathrm{E}-01$ \\
\hline \multicolumn{5}{|c|}{$\alpha=-1.4$} \\
\hline 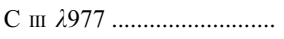 & $4.607 \mathrm{E}-02$ & $2.108 \mathrm{E}-02$ & $1.137 \mathrm{E}-04$ & $1.158 \mathrm{E}-06$ \\
\hline 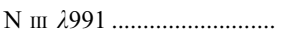 & $1.327 \mathrm{E}-02$ & $6.655 \mathrm{E}-03$ & $3.831 \mathrm{E}-05$ & $0.000 \mathrm{E}+00$ \\
\hline O vi $\lambda \lambda 1032,1037 \ldots \ldots \ldots \ldots . . .$. & $3.615 \mathrm{E}+00$ & $1.006 \mathrm{E}-02$ & $0.000 \mathrm{E}+00$ & $0.000 \mathrm{E}+00$ \\
\hline $\mathrm{N}$ v $\lambda 1240 \ldots \ldots \ldots \ldots \ldots \ldots \ldots \ldots$ & $1.359 \mathrm{E}+00$ & $4.098 \mathrm{E}-02$ & $9.587 \mathrm{E}-06$ & $0.000 \mathrm{E}+00$ \\
\hline Si IV+O Iv] $\lambda 1400 \ldots \ldots \ldots \ldots . .$. & $2.020 \mathrm{E}-01$ & $1.209 \mathrm{E}-01$ & $3.788 \mathrm{E}-03$ & $5.276 \mathrm{E}-06$ \\
\hline $\mathrm{N}$ Iv] $\lambda 1486 \ldots \ldots \ldots \ldots \ldots \ldots$ & $2.981 \mathrm{E}-01$ & $6.778 \mathrm{E}-02$ & $2.174 \mathrm{E}-04$ & $0.000 \mathrm{E}+00$ \\
\hline $\mathrm{C}$ IV $\lambda 1549$ & $4.598 \mathrm{E}+00$ & $1.227 \mathrm{E}+00$ & $2.655 \mathrm{E}-03$ & $1.671 \mathrm{E}-06$ \\
\hline 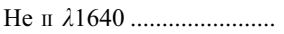 & $1.620 \mathrm{E}+00$ & $1.695 \mathrm{E}+00$ & $1.446 \mathrm{E}+00$ & $6.243 \mathrm{E}-01$ \\
\hline 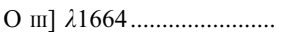 & $1.797 \mathrm{E}-01$ & $1.026 \mathrm{E}-01$ & $7.304 \mathrm{E}-03$ & $5.640 \mathrm{E}-05$ \\
\hline 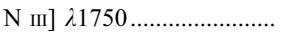 & $1.810 \mathrm{E}-02$ & $4.027 \mathrm{E}-02$ & $3.146 \mathrm{E}-03$ & $2.171 \mathrm{E}-05$ \\
\hline 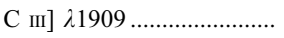 & $4.658 \mathrm{E}-01$ & $1.152 \mathrm{E}+00$ & $1.388 \mathrm{E}-01$ & $8.278 \mathrm{E}-03$ \\
\hline $\mathrm{C}$ II] $\lambda 2326 \ldots \ldots \ldots \ldots \ldots \ldots \ldots$ & $4.037 \mathrm{E}-03$ & $1.072 \mathrm{E}-01$ & $2.155 \mathrm{E}-01$ & $9.571 \mathrm{E}-02$ \\
\hline 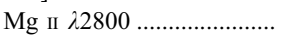 & $7.629 \mathrm{E}-02$ & $7.603 \mathrm{E}-01$ & $1.147 \mathrm{E}+00$ & $6.971 \mathrm{E}-01$ \\
\hline$[\mathrm{Ne} v] \lambda 3426 \ldots \ldots \ldots \ldots \ldots \ldots$ & $1.326 \mathrm{E}+00$ & $2.377 \mathrm{E}-01$ & $3.834 \mathrm{E}-04$ & $0.000 \mathrm{E}+00$ \\
\hline$[\mathrm{Ne}$ III] $\lambda 3869 \ldots \ldots \ldots \ldots \ldots \ldots$ & $9.169 \mathrm{E}-01$ & $1.406 \mathrm{E}+00$ & $8.574 \mathrm{E}-01$ & $2.679 \mathrm{E}-01$ \\
\hline \multicolumn{5}{|c|}{$\alpha=-1.7$} \\
\hline 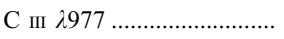 & $2.001 \mathrm{E}-02$ & $3.429 \mathrm{E}-03$ & $1.789 \mathrm{E}-05$ & $0.000 \mathrm{E}+00$ \\
\hline 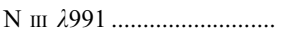 & $5.487 \mathrm{E}-03$ & $1.040 \mathrm{E}-03$ & $6.333 \mathrm{E}-06$ & $0.000 \mathrm{E}+00$ \\
\hline O vi $\lambda \lambda 1032,1037 \ldots \ldots \ldots \ldots . .$. & $8.529 \mathrm{E}-01$ & $5.478 \mathrm{E}-04$ & $0.000 \mathrm{E}+00$ & $0.000 \mathrm{E}+00$ \\
\hline 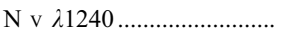 & $5.581 \mathrm{E}-01$ & $4.663 \mathrm{E}-03$ & $0.000 \mathrm{E}+00$ & $0.000 \mathrm{E}+00$ \\
\hline Si IV+O Iv] $\lambda 1400 \ldots \ldots \ldots \ldots . .$. & $1.021 \mathrm{E}-01$ & $3.500 \mathrm{E}-02$ & $8.298 \mathrm{E}-04$ & $0.000 \mathrm{E}+00$ \\
\hline $\mathrm{N}$ IV] $\lambda 1486 \ldots \ldots \ldots \ldots \ldots \ldots \ldots$ & $1.576 \mathrm{E}-01$ & $1.485 \mathrm{E}-02$ & $5.014 \mathrm{E}-05$ & $0.000 \mathrm{E}+00$ \\
\hline 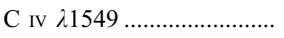 & $2.572 \mathrm{E}+00$ & $2.728 \mathrm{E}-01$ & $5.750 \mathrm{E}-04$ & $0.000 \mathrm{E}+00$ \\
\hline 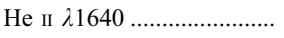 & $1.090 \mathrm{E}+00$ & $1.201 \mathrm{E}+00$ & $1.045 \mathrm{E}+00$ & $4.414 \mathrm{E}-01$ \\
\hline 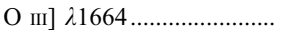 & $6.399 \mathrm{E}-02$ & $2.919 \mathrm{E}-02$ & $2.432 \mathrm{E}-03$ & $2.588 \mathrm{E}-05$ \\
\hline 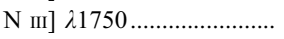 & $1.070 \mathrm{E}-02$ & $1.226 \mathrm{E}-02$ & $1.086 \mathrm{E}-03$ & $1.047 \mathrm{E}-05$ \\
\hline 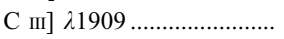 & $3.072 \mathrm{E}-01$ & $4.064 \mathrm{E}-01$ & $5.167 \mathrm{E}-02$ & $3.807 \mathrm{E}-03$ \\
\hline 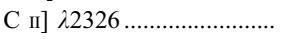 & $2.851 \mathrm{E}-03$ & $3.628 \mathrm{E}-02$ & $8.256 \mathrm{E}-02$ & $5.164 \mathrm{E}-02$ \\
\hline $\mathrm{Mg}$ II $\lambda 2800 \ldots \ldots \ldots \ldots \ldots \ldots$ & $5.203 \mathrm{E}-02$ & $3.369 \mathrm{E}-01$ & $6.481 \mathrm{E}-01$ & $5.044 \mathrm{E}-01$ \\
\hline 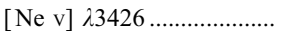 & $7.482 \mathrm{E}-01$ & $6.002 \mathrm{E}-02$ & $9.159 \mathrm{E}-05$ & $0.000 \mathrm{E}+00$ \\
\hline$[\mathrm{Ne}$ III] $\lambda 3869 \ldots \ldots \ldots \ldots \ldots \ldots$ & $5.234 \mathrm{E}-01$ & $7.615 \mathrm{E}-01$ & $4.684 \mathrm{E}-01$ & $1.495 \mathrm{E}-01$ \\
\hline \multicolumn{5}{|c|}{$\alpha=-2.0$} \\
\hline 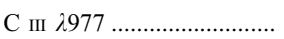 & $7.618 \mathrm{E}-03$ & $3.730 \mathrm{E}-04$ & $2.971 \mathrm{E}-06$ & $0.000 \mathrm{E}+00$ \\
\hline 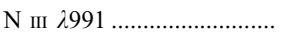 & $2.211 \mathrm{E}-03$ & $1.144 \mathrm{E}-04$ & $1.085 \mathrm{E}-06$ & $0.000 \mathrm{E}+00$ \\
\hline $\mathrm{O}$ VI $\lambda \lambda 1032,1037 \ldots \ldots \ldots \ldots . .$. & $1.472 \mathrm{E}-01$ & $1.821 \mathrm{E}-05$ & $0.000 \mathrm{E}+00$ & $0.000 \mathrm{E}+00$ \\
\hline $\mathrm{N} v \mathrm{\lambda} 1240 \ldots \ldots \ldots \ldots \ldots \ldots \ldots \ldots$ & $1.825 \mathrm{E}-01$ & $3.590 \mathrm{E}-04$ & $0.000 \mathrm{E}+00$ & $0.000 \mathrm{E}+00$ \\
\hline $\mathrm{Si}$ IV+O Iv] $\lambda 1400 \ldots \ldots \ldots \ldots . .$. & $7.596 \mathrm{E}-02$ & $7.014 \mathrm{E}-03$ & $1.881 \mathrm{E}-04$ & $0.000 \mathrm{E}+00$ \\
\hline $\mathrm{N}$ Iv] $\lambda 1486 \ldots \ldots \ldots \ldots \ldots \ldots$ & $7.499 \mathrm{E}-02$ & $2.429 \mathrm{E}-03$ & $1.131 \mathrm{E}-05$ & $0.000 \mathrm{E}+00$ \\
\hline 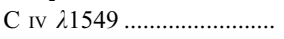 & $1.192 \mathrm{E}+00$ & $4.343 \mathrm{E}-02$ & $1.249 \mathrm{E}-04$ & $0.000 \mathrm{E}+00$ \\
\hline He & $7.443 \mathrm{E}-01$ & $8.442 \mathrm{E}-01$ & $7.275 \mathrm{E}-01$ & $2.960 \mathrm{E}-01$ \\
\hline $\mathrm{O}$ III] $\lambda 1664 \ldots \ldots \ldots \ldots \ldots \ldots \ldots$ & $2.457 \mathrm{E}-02$ & $7.209 \mathrm{E}-03$ & $8.201 \mathrm{E}-04$ & $1.136 \mathrm{E}-05$ \\
\hline 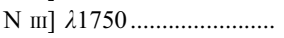 & $6.068 \mathrm{E}-03$ & $3.152 \mathrm{E}-03$ & $3.824 \mathrm{E}-04$ & $4.834 \mathrm{E}-06$ \\
\hline 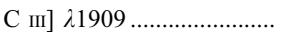 & $1.800 \mathrm{E}-01$ & $1.184 \mathrm{E}-01$ & $1.978 \mathrm{E}-02$ & $1.738 \mathrm{E}-03$ \\
\hline 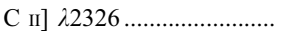 & $1.741 \mathrm{E}-03$ & $1.267 \mathrm{E}-02$ & $3.683 \mathrm{E}-02$ & $2.918 \mathrm{E}-02$ \\
\hline 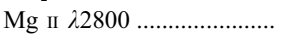 & $2.893 \mathrm{E}-02$ & $1.474 \mathrm{E}-01$ & $4.013 \mathrm{E}-01$ & $3.645 \mathrm{E}-01$ \\
\hline$[\mathrm{Ne} v] \lambda 3426 \ldots \ldots \ldots \ldots \ldots \ldots$ & $3.644 \mathrm{E}-01$ & $1.151 \mathrm{E}-02$ & $2.012 \mathrm{E}-05$ & $0.000 \mathrm{E}+00$ \\
\hline$[\mathrm{Ne}$ III] $\lambda 3869 \ldots \ldots \ldots \ldots \ldots \ldots$ & $2.931 \mathrm{E}-01$ & $3.981 \mathrm{E}-01$ & $2.632 \mathrm{E}-01$ & $8.219 \mathrm{E}-02$ \\
\hline
\end{tabular}


TABLE 37

Undepleted Dust-Free UV Line Ratios with Respect to $\mathrm{H} \beta, 4 Z_{\odot}$

\begin{tabular}{|c|c|c|c|c|}
\hline \multirow[b]{2}{*}{ Line } & \multicolumn{4}{|c|}{$\log U_{0}$} \\
\hline & -1.0 & -2.0 & -3.0 & -4.0 \\
\hline \multicolumn{5}{|c|}{$\alpha=-1.2$} \\
\hline 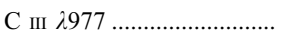 & $4.531 \mathrm{E}-02$ & $1.786 \mathrm{E}-02$ & $4.617 \mathrm{E}-06$ & $0.000 \mathrm{E}+00$ \\
\hline 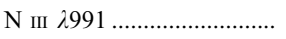 & $2.702 \mathrm{E}-02$ & $1.105 \mathrm{E}-02$ & $2.984 \mathrm{E}-06$ & $0.000 \mathrm{E}+00$ \\
\hline O vi $\lambda \lambda 1032,1037 \ldots \ldots \ldots \ldots . .$. & $5.945 \mathrm{E}+00$ & $1.623 \mathrm{E}-02$ & $0.000 \mathrm{E}+00$ & $0.000 \mathrm{E}+00$ \\
\hline $\mathrm{N}$ v $\lambda 1240 \ldots \ldots \ldots \ldots \ldots \ldots \ldots \ldots$ & $3.576 \mathrm{E}+00$ & $1.049 \mathrm{E}-01$ & $1.462 \mathrm{E}-06$ & $0.000 \mathrm{E}+00$ \\
\hline $\mathrm{Si}$ IV+O IV] $\lambda 1400 \ldots \ldots \ldots \ldots . .$. & $2.474 \mathrm{E}-01$ & $8.973 \mathrm{E}-02$ & $4.169 \mathrm{E}-04$ & $0.000 \mathrm{E}+00$ \\
\hline $\mathrm{N}$ Iv] $\lambda 1486 \ldots \ldots \ldots \ldots \ldots \ldots \ldots$ & $6.703 \mathrm{E}-01$ & $1.446 \mathrm{E}-01$ & $6.527 \mathrm{E}-05$ & $0.000 \mathrm{E}+00$ \\
\hline 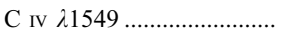 & $5.360 \mathrm{E}+00$ & $1.410 \mathrm{E}+00$ & $4.294 \mathrm{E}-04$ & $0.000 \mathrm{E}+00$ \\
\hline He II $\lambda 1640$ & $2.049 \mathrm{E}+00$ & $2.150 \mathrm{E}+00$ & $1.812 \mathrm{E}+00$ & $7.467 \mathrm{E}-01$ \\
\hline 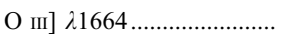 & $2.111 \mathrm{E}-01$ & $9.710 \mathrm{E}-02$ & $1.170 \mathrm{E}-03$ & $1.760 \mathrm{E}-06$ \\
\hline $\mathrm{N}$ III] $\lambda 1750 \ldots \ldots \ldots \ldots \ldots \ldots \ldots$ & $3.807 \mathrm{E}-02$ & $7.734 \mathrm{E}-02$ & $1.047 \mathrm{E}-03$ & $1.926 \mathrm{E}-06$ \\
\hline 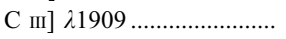 & $4.423 \mathrm{E}-01$ & $1.123 \mathrm{E}+00$ & $2.715 \mathrm{E}-02$ & $4.668 \mathrm{E}-04$ \\
\hline $\mathrm{C}$ II] $\lambda 2326 \ldots \ldots \ldots \ldots \ldots \ldots \ldots$ & $8.094 \mathrm{E}-04$ & $1.030 \mathrm{E}-01$ & $1.097 \mathrm{E}-01$ & $1.003 \mathrm{E}-02$ \\
\hline $\mathrm{Mg}$ II $\lambda 2800 \ldots \ldots \ldots \ldots \ldots \ldots \ldots$ & $5.722 \mathrm{E}-03$ & $6.007 \mathrm{E}-01$ & $6.755 \mathrm{E}-01$ & $1.368 \mathrm{E}-01$ \\
\hline$[\mathrm{Ne} v] \lambda 3426 \ldots \ldots \ldots \ldots \ldots \ldots$ & $2.063 \mathrm{E}+00$ & $3.862 \mathrm{E}-01$ & $2.535 \mathrm{E}-04$ & $0.000 \mathrm{E}+00$ \\
\hline$[\mathrm{Ne}$ III] $\lambda 3869 \ldots \ldots \ldots \ldots \ldots \ldots$ & $6.897 \mathrm{E}-01$ & $1.361 \mathrm{E}+00$ & $5.347 \mathrm{E}-01$ & $8.298 \mathrm{E}-02$ \\
\hline \multicolumn{5}{|c|}{$\alpha=-1.4$} \\
\hline 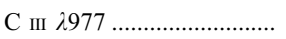 & $2.616 \mathrm{E}-02$ & $3.888 \mathrm{E}-03$ & $0.000 \mathrm{E}+00$ & $0.000 \mathrm{E}+00$ \\
\hline 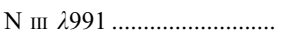 & $1.429 \mathrm{E}-02$ & $2.277 \mathrm{E}-03$ & $0.000 \mathrm{E}+00$ & $0.000 \mathrm{E}+00$ \\
\hline $\mathrm{O}$ vi $\lambda \lambda 1032,1037 \ldots \ldots \ldots \ldots$ & $2.326 \mathrm{E}+00$ & $1.780 \mathrm{E}-03$ & $0.000 \mathrm{E}+00$ & $0.000 \mathrm{E}+00$ \\
\hline $\mathrm{N}$ v $\lambda 1240 \ldots \ldots \ldots \ldots \ldots \ldots \ldots \ldots$ & $1.992 \mathrm{E}+00$ & $1.948 \mathrm{E}-02$ & $0.000 \mathrm{E}+00$ & $0.000 \mathrm{E}+00$ \\
\hline $\mathrm{Si}$ IV+O Iv] $\lambda 1400 \ldots \ldots \ldots \ldots . .$. & $1.514 \mathrm{E}-01$ & $4.036 \mathrm{E}-02$ & $9.277 \mathrm{E}-05$ & $0.000 \mathrm{E}+00$ \\
\hline $\mathrm{N}$ Iv] $\lambda 1486 \ldots \ldots \ldots \ldots \ldots \ldots \ldots$ & $4.347 \mathrm{E}-01$ & $4.282 \mathrm{E}-02$ & $1.637 \mathrm{E}-05$ & $0.000 \mathrm{E}+00$ \\
\hline $\mathrm{C}_{\text {IV }} \lambda 1549 \ldots \ldots \ldots \ldots \ldots \ldots \ldots$ & $3.778 \mathrm{E}+00$ & $4.265 \mathrm{E}-01$ & $1.009 \mathrm{E}-04$ & $0.000 \mathrm{E}+00$ \\
\hline He II $\lambda 1640 \ldots \ldots \ldots \ldots \ldots \ldots \ldots$ & $1.554 \mathrm{E}+00$ & $1.719 \mathrm{E}+00$ & $1.478 \mathrm{E}+00$ & $6.090 \mathrm{E}-01$ \\
\hline 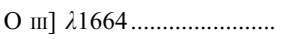 & $1.004 \mathrm{E}-01$ & $3.299 \mathrm{E}-02$ & $3.711 \mathrm{E}-04$ & $0.000 \mathrm{E}+00$ \\
\hline $\mathrm{N}$ III] $\lambda 1750 \ldots \ldots \ldots \ldots \ldots \ldots \ldots$ & $2.637 \mathrm{E}-02$ & $2.819 \mathrm{E}-02$ & $3.453 \mathrm{E}-04$ & $0.000 \mathrm{E}+00$ \\
\hline 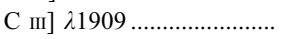 & $3.511 \mathrm{E}-01$ & $4.746 \mathrm{E}-01$ & $9.342 \mathrm{E}-03$ & $2.246 \mathrm{E}-04$ \\
\hline $\mathrm{C}$ II] $\lambda 2326 \ldots \ldots \ldots \ldots \ldots \ldots \ldots$ & $6.333 \mathrm{E}-04$ & $3.685 \mathrm{E}-02$ & $3.151 \mathrm{E}-02$ & $5.418 \mathrm{E}-03$ \\
\hline $\mathrm{Mg}$ II $\lambda 2800 \ldots \ldots \ldots \ldots \ldots \ldots \ldots$ & $2.227 \mathrm{E}-03$ & $2.290 \mathrm{E}-01$ & $2.755 \mathrm{E}-01$ & $9.124 \mathrm{E}-02$ \\
\hline$[\mathrm{Ne} \mathrm{v}] \lambda 3426 \ldots \ldots \ldots \ldots \ldots \ldots$ & $1.413 \mathrm{E}+00$ & $1.448 \mathrm{E}-01$ & $8.210 \mathrm{E}-05$ & $0.000 \mathrm{E}+00$ \\
\hline$[\mathrm{Ne}$ III] $\lambda 3869 \ldots \ldots \ldots \ldots \ldots \ldots$ & $4.345 \mathrm{E}-01$ & $7.338 \mathrm{E}-01$ & $2.683 \mathrm{E}-01$ & $4.996 \mathrm{E}-02$ \\
\hline \multicolumn{5}{|c|}{$\alpha=-1.7$} \\
\hline 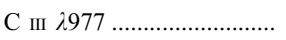 & $9.740 \mathrm{E}-03$ & $1.715 \mathrm{E}-04$ & $0.000 \mathrm{E}+00$ & $0.000 \mathrm{E}+00$ \\
\hline 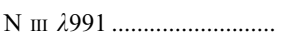 & $5.367 \mathrm{E}-03$ & $9.729 \mathrm{E}-05$ & $0.000 \mathrm{E}+00$ & $0.000 \mathrm{E}+00$ \\
\hline $\mathrm{O}$ vi $\lambda \lambda 1032,1037 \ldots \ldots \ldots \ldots$ & $4.494 \mathrm{E}-01$ & $2.512 \mathrm{E}-05$ & $0.000 \mathrm{E}+00$ & $0.000 \mathrm{E}+00$ \\
\hline 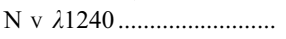 & $6.938 \mathrm{E}-01$ & $7.239 \mathrm{E}-04$ & $0.000 \mathrm{E}+00$ & $0.000 \mathrm{E}+00$ \\
\hline $\mathrm{Si}$ IV+O Iv] $\lambda 1400 \ldots \ldots \ldots \ldots . .$. & $7.139 \mathrm{E}-02$ & $4.743 \mathrm{E}-03$ & $7.985 \mathrm{E}-06$ & $0.000 \mathrm{E}+00$ \\
\hline $\mathrm{N}$ IV] $\lambda 1486 \ldots \ldots \ldots \ldots \ldots \ldots \ldots$ & $2.094 \mathrm{E}-01$ & $3.813 \mathrm{E}-03$ & $1.778 \mathrm{E}-06$ & $0.000 \mathrm{E}+00$ \\
\hline $\mathrm{C}$ IV $\lambda 1549 \ldots \ldots \ldots \ldots \ldots \ldots \ldots$ & $1.811 \mathrm{E}+00$ & $3.794 \mathrm{E}-02$ & $1.025 \mathrm{E}-05$ & $0.000 \mathrm{E}+00$ \\
\hline 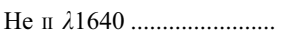 & $1.030 \mathrm{E}+00$ & $1.225 \mathrm{E}+00$ & $1.045 \mathrm{E}+00$ & $4.209 \mathrm{E}-01$ \\
\hline 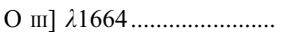 & $3.367 \mathrm{E}-02$ & $4.479 \mathrm{E}-03$ & $6.035 \mathrm{E}-05$ & $0.000 \mathrm{E}+00$ \\
\hline 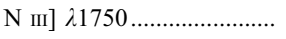 & $1.454 \mathrm{E}-02$ & $4.101 \mathrm{E}-03$ & $5.996 \mathrm{E}-05$ & $0.000 \mathrm{E}+00$ \\
\hline 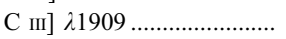 & $2.127 \mathrm{E}-01$ & $8.632 \mathrm{E}-02$ & $1.827 \mathrm{E}-03$ & $7.594 \mathrm{E}-05$ \\
\hline 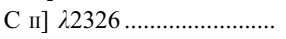 & $4.964 \mathrm{E}-04$ & $5.428 \mathrm{E}-03$ & $4.918 \mathrm{E}-03$ & $2.355 \mathrm{E}-03$ \\
\hline $\mathrm{Mg}$ II $22800 \ldots \ldots \ldots \ldots \ldots \ldots$ & $1.018 \mathrm{E}-03$ & $3.201 \mathrm{E}-02$ & $7.555 \mathrm{E}-02$ & $5.159 \mathrm{E}-02$ \\
\hline$[\mathrm{Ne} v] \lambda 3426 \ldots \ldots \ldots \ldots \ldots \ldots$ & $7.142 \mathrm{E}-01$ & $2.203 \mathrm{E}-02$ & $1.298 \mathrm{E}-05$ & $0.000 \mathrm{E}+00$ \\
\hline$[\mathrm{Ne}$ III] $\lambda 38869 \ldots \ldots \ldots \ldots \ldots \ldots$ & $2.162 \mathrm{E}-01$ & $2.586 \mathrm{E}-01$ & $9.813 \mathrm{E}-02$ & $2.381 \mathrm{E}-02$ \\
\hline \multicolumn{5}{|c|}{$\alpha=-2.0$} \\
\hline 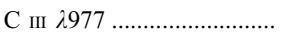 & $2.834 \mathrm{E}-03$ & $2.363 \mathrm{E}-06$ & $0.000 \mathrm{E}+00$ & $0.000 \mathrm{E}+00$ \\
\hline 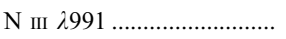 & $1.654 \mathrm{E}-03$ & $1.412 \mathrm{E}-06$ & $0.000 \mathrm{E}+00$ & $0.000 \mathrm{E}+00$ \\
\hline $\mathrm{O}$ vi $\lambda \lambda 1032,1037 \ldots \ldots \ldots \ldots$ & $5.703 \mathrm{E}-02$ & $0.000 \mathrm{E}+00$ & $0.000 \mathrm{E}+00$ & $0.000 \mathrm{E}+00$ \\
\hline $\mathrm{N} v 21240 \ldots \ldots \ldots \ldots \ldots \ldots \ldots \ldots \ldots$ & $1.723 \mathrm{E}-01$ & $1.024 \mathrm{E}-05$ & $0.000 \mathrm{E}+00$ & $0.000 \mathrm{E}+00$ \\
\hline $\mathrm{Si}$ IV+O IV] $\lambda 1400 \ldots \ldots \ldots \ldots$ & $2.786 \mathrm{E}-02$ & $2.330 \mathrm{E}-04$ & $0.000 \mathrm{E}+00$ & $0.000 \mathrm{E}+00$ \\
\hline $\left.\mathrm{N}_{\text {IV }}\right] \lambda 1486 \ldots \ldots \ldots \ldots \ldots \ldots \ldots$ & $7.982 \mathrm{E}-02$ & $1.591 \mathrm{E}-04$ & $0.000 \mathrm{E}+00$ & $0.000 \mathrm{E}+00$ \\
\hline $\mathrm{C}$ Iv $\lambda 1549 \ldots \ldots \ldots \ldots \ldots \ldots \ldots$ & $6.704 \mathrm{E}-01$ & $1.486 \mathrm{E}-03$ & $0.000 \mathrm{E}+00$ & $0.000 \mathrm{E}+00$ \\
\hline 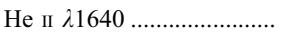 & $6.882 \mathrm{E}-01$ & $8.510 \mathrm{E}-01$ & $7.137 \mathrm{E}-01$ & $2.759 \mathrm{E}-01$ \\
\hline 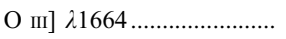 & $1.084 \mathrm{E}-02$ & $3.424 \mathrm{E}-04$ & $9.352 \mathrm{E}-06$ & $0.000 \mathrm{E}+00$ \\
\hline $\mathrm{N}$ III] $\lambda 1750 \ldots \ldots \ldots \ldots \ldots \ldots \ldots$ & $6.821 \mathrm{E}-03$ & $3.358 \mathrm{E}-04$ & $1.000 \mathrm{E}-05$ & $0.000 \mathrm{E}+00$ \\
\hline 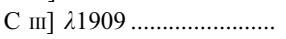 & $1.065 \mathrm{E}-01$ & $8.814 \mathrm{E}-03$ & $3.596 \mathrm{E}-04$ & $2.652 \mathrm{E}-05$ \\
\hline $\mathrm{C}_{\text {II] }} \lambda 2326 \ldots \ldots \ldots \ldots \ldots \ldots \ldots$ & $5.221 \mathrm{E}-04$ & $1.206 \mathrm{E}-03$ & $1.561 \mathrm{E}-03$ & $1.090 \mathrm{E}-03$ \\
\hline $\mathrm{Mg}$ II $22800 \ldots \ldots \ldots \ldots \ldots \ldots \ldots$ & $1.053 \mathrm{E}-03$ & $9.468 \mathrm{E}-03$ & $3.315 \mathrm{E}-02$ & $2.965 \mathrm{E}-02$ \\
\hline$[\mathrm{Ne} v] \lambda 3426 \ldots \ldots \ldots \ldots \ldots \ldots$ & $2.943 \mathrm{E}-01$ & $2.004 \mathrm{E}-03$ & $1.825 \mathrm{E}-06$ & $0.000 \mathrm{E}+00$ \\
\hline$[\mathrm{Ne}$ III] $\lambda 3869 \ldots \ldots \ldots \ldots \ldots \ldots$ & $1.088 \mathrm{E}-01$ & $7.500 \mathrm{E}-02$ & $4.017 \mathrm{E}-02$ & $1.143 \mathrm{E}-02$ \\
\hline
\end{tabular}


TABLE 38

Undepleted Dust-free Visible Line Ratios with Respect to $\mathrm{H} \beta, 0.25 Z_{\odot}$

\begin{tabular}{|c|c|c|c|c|}
\hline \multirow[b]{2}{*}{ LiNE } & \multicolumn{4}{|c|}{$\log U_{0}$} \\
\hline & -1.0 & -2.0 & -3.0 & -4.0 \\
\hline \multicolumn{5}{|c|}{$\alpha=-1.2$} \\
\hline$[\mathrm{O}$ II] $\lambda \lambda 3727,3729$. & $7.521 \mathrm{E}-02$ & $5.684 \mathrm{E}-01$ & $2.527 \mathrm{E}+00$ & $2.548 \mathrm{E}+00$ \\
\hline$[\mathrm{S}$ II $] \lambda \lambda 4067,4076 \ldots \ldots \ldots . .$. & $2.502 \mathrm{E}-03$ & $1.265 \mathrm{E}-02$ & $4.475 \mathrm{E}-02$ & $1.039 \mathrm{E}-01$ \\
\hline 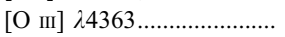 & $3.402 \mathrm{E}-01$ & $2.002 \mathrm{E}-01$ & $4.613 \mathrm{E}-02$ & $7.257 \mathrm{E}-04$ \\
\hline He II $\lambda 4686 \ldots \ldots$ & $2.804 \mathrm{E}-01$ & $2.905 \mathrm{E}-01$ & $2.358 \mathrm{E}-01$ & $1.050 \mathrm{E}-01$ \\
\hline [O III $] \lambda 4959 \ldots$ & $3.403 \mathrm{E}+00$ & $2.759 \mathrm{E}+00$ & $1.105 \mathrm{E}+00$ & $3.367 \mathrm{E}-02$ \\
\hline$[\mathrm{O} \mathrm{III}] \lambda 5007 \ldots \ldots \ldots$ & $9.802 \mathrm{E}+00$ & $7.946 \mathrm{E}+00$ & $3.181 \mathrm{E}+00$ & $9.698 \mathrm{E}-02$ \\
\hline$\left[\mathrm{N}_{\mathrm{I}}\right] \lambda 5200 \ldots \ldots \ldots \ldots \ldots \ldots$ & $2.424 \mathrm{E}-03$ & $1.459 \mathrm{E}-02$ & $4.141 \mathrm{E}-02$ & $6.508 \mathrm{E}-02$ \\
\hline$[\mathrm{Fe}$ VII $] \lambda 6085 \ldots \ldots \ldots \ldots \ldots \ldots$ & $5.254 \mathrm{E}-02$ & $1.086 \mathrm{E}-02$ & $2.495 \mathrm{E}-05$ & $0.000 \mathrm{E}+00$ \\
\hline$\left[\mathrm{O}_{\text {I] }}\right] \lambda 6300 \ldots \ldots \ldots \ldots \ldots \ldots \ldots$ & $2.422 \mathrm{E}-02$ & $1.600 \mathrm{E}-01$ & $4.189 \mathrm{E}-01$ & $6.859 \mathrm{E}-01$ \\
\hline $\mathrm{H} \alpha \quad \lambda 6563 \ldots \ldots \ldots \ldots \ldots \ldots \ldots \ldots \ldots \ldots \ldots$ & $2.888 \mathrm{E}+00$ & $3.008 \mathrm{E}+00$ & $3.029 \mathrm{E}+00$ & $3.017 \mathrm{E}+00$ \\
\hline 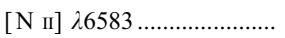 & $6.637 \mathrm{E}-03$ & $6.104 \mathrm{E}-02$ & $2.557 \mathrm{E}-01$ & $3.320 \mathrm{E}-01$ \\
\hline$[\mathrm{S}$ II] $\lambda \lambda 6717,6730 \ldots \ldots \ldots . .$. & $4.532 \mathrm{E}-02$ & $2.407 \mathrm{E}-01$ & $8.014 \mathrm{E}-01$ & $1.860 \mathrm{E}+00$ \\
\hline$[\mathrm{O}$ II] $\lambda \lambda 7318,7324 \ldots \ldots \ldots .$. & $2.030 \mathrm{E}-03$ & $1.461 \mathrm{E}-02$ & $6.070 \mathrm{E}-02$ & $4.353 \mathrm{E}-02$ \\
\hline [S III] $\lambda \lambda 9069.9532 \ldots \ldots \ldots . .$. & $1.158 \mathrm{E}-01$ & $6.302 \mathrm{E}-01$ & $1.653 \mathrm{E}+00$ & $1.296 \mathrm{E}+00$ \\
\hline \multicolumn{5}{|c|}{$\alpha=-1.4$} \\
\hline$[\mathrm{O}$ II] $\lambda \lambda 3727,3729 \ldots \ldots \ldots$. & $3.596 \mathrm{E}-02$ & $2.730 \mathrm{E}-01$ & $1.273 \mathrm{E}+00$ & $1.408 \mathrm{E}+00$ \\
\hline$[\mathrm{S}$ II $] \lambda \lambda 4067,4076 \ldots \ldots \ldots . .$. & $3.614 \mathrm{E}-03$ & $1.878 \mathrm{E}-02$ & $7.429 \mathrm{E}-02$ & $1.980 \mathrm{E}-01$ \\
\hline 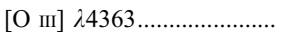 & $2.983 \mathrm{E}-01$ & $2.015 \mathrm{E}-01$ & $4.966 \mathrm{E}-02$ & $8.185 \mathrm{E}-04$ \\
\hline 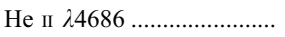 & $2.264 \mathrm{E}-01$ & $2.350 \mathrm{E}-01$ & $1.970 \mathrm{E}-01$ & $8.832 \mathrm{E}-02$ \\
\hline 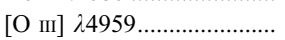 & $3.424 \mathrm{E}+00$ & $2.896 \mathrm{E}+00$ & $1.157 \mathrm{E}+00$ & $3.574 \mathrm{E}-02$ \\
\hline 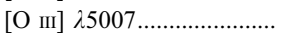 & $9.861 \mathrm{E}+00$ & $8.341 \mathrm{E}+00$ & $3.333 \mathrm{E}+00$ & $1.029 \mathrm{E}-01$ \\
\hline$\left[\mathrm{N}_{\mathrm{I}}\right] \lambda 5200 \ldots \ldots \ldots \ldots \ldots \ldots \ldots \ldots \ldots \ldots$ & $1.038 \mathrm{E}-03$ & $6.337 \mathrm{E}-03$ & $1.886 \mathrm{E}-02$ & $3.259 \mathrm{E}-02$ \\
\hline 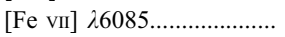 & $5.158 \mathrm{E}-02$ & $5.604 \mathrm{E}-03$ & $1.120 \mathrm{E}-05$ & $0.000 \mathrm{E}+00$ \\
\hline$\left[\mathrm{O}_{\mathrm{I}}\right], \lambda 6300 \ldots \ldots \ldots \ldots \ldots \ldots \ldots$ & $1.684 \mathrm{E}-02$ & $1.129 \mathrm{E}-01$ & $3.183 \mathrm{E}-01$ & $5.979 \mathrm{E}-01$ \\
\hline 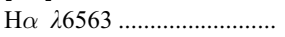 & $2.833 \mathrm{E}+00$ & $2.928 \mathrm{E}+00$ & $2.972 \mathrm{E}+00$ & $2.978 \mathrm{E}+00$ \\
\hline 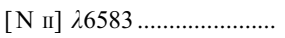 & $4.767 \mathrm{E}-03$ & $4.539 \mathrm{E}-02$ & $2.258 \mathrm{E}-01$ & $3.135 \mathrm{E}-01$ \\
\hline$[\mathrm{S}$ II $] \lambda \lambda 6717,6730 \ldots \ldots \ldots . .$. & $3.056 \mathrm{E}-02$ & $1.658 \mathrm{E}-01$ & $5.435 \mathrm{E}-01$ & $1.307 \mathrm{E}+00$ \\
\hline$[\mathrm{O}$ II] $\lambda \lambda 7318,7324 \ldots \ldots \ldots .$. & $3.747 \mathrm{E}-03$ & $2.782 \mathrm{E}-02$ & $1.272 \mathrm{E}-01$ & $9.714 \mathrm{E}-02$ \\
\hline [S III] $\lambda \lambda 9069.9532 \ldots \ldots \ldots \ldots$. & $1.088 \mathrm{E}-01$ & $6.371 \mathrm{E}-01$ & $1.584 \mathrm{E}+00$ & $1.160 \mathrm{E}+00$ \\
\hline \multicolumn{5}{|c|}{$\alpha=-1.7$} \\
\hline$[\mathrm{O}$ II] $\lambda \lambda 3727,3729 \ldots \ldots \ldots$ & $6.294 \mathrm{E}-02$ & $4.881 \mathrm{E}-01$ & $2.500 \mathrm{E}+00$ & $2.581 \mathrm{E}+00$ \\
\hline$[\mathrm{S}$ II $] \lambda \lambda 4067,4076 \ldots \ldots \ldots . .$. & $1.418 \mathrm{E}-03$ & $7.809 \mathrm{E}-03$ & $3.341 \mathrm{E}-02$ & $9.158 \mathrm{E}-02$ \\
\hline$[\mathrm{O}$ III $] \lambda 4363 \ldots \ldots \ldots \ldots \ldots \ldots \ldots$ & $1.934 \mathrm{E}-01$ & $1.532 \mathrm{E}-01$ & $3.974 \mathrm{E}-02$ & $6.513 \mathrm{E}-04$ \\
\hline 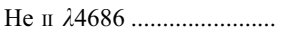 & $1.605 \mathrm{E}-01$ & $1.687 \mathrm{E}-01$ & $1.467 \mathrm{E}-01$ & $6.576 \mathrm{E}-02$ \\
\hline 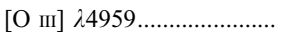 & $3.057 \mathrm{E}+00$ & $2.741 \mathrm{E}+00$ & $1.077 \mathrm{E}+00$ & $3.214 \mathrm{E}-02$ \\
\hline 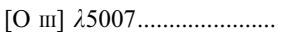 & $8.805 \mathrm{E}+00$ & $7.893 \mathrm{E}+00$ & $3.103 \mathrm{E}+00$ & $9.257 \mathrm{E}-02$ \\
\hline$\left[\mathrm{N}_{\mathrm{I}}\right] \lambda 5200 \ldots \ldots \ldots \ldots \ldots \ldots \ldots \ldots \ldots \ldots \ldots \ldots$ & $8.106 \mathrm{E}-04$ & $5.414 \mathrm{E}-03$ & $1.486 \mathrm{E}-02$ & $3.033 \mathrm{E}-02$ \\
\hline$[\mathrm{Fe}$ VII $] \lambda 6085 \ldots \ldots \ldots \ldots \ldots \ldots$ & $3.734 \mathrm{E}-02$ & $1.567 \mathrm{E}-03$ & $2.549 \mathrm{E}-06$ & $0.000 \mathrm{E}+00$ \\
\hline$\left[\mathrm{O}_{\mathrm{I}}\right] \mathrm{\lambda}, 6300 \ldots \ldots \ldots \ldots \ldots \ldots \ldots$ & $7.739 \mathrm{E}-03$ & $5.418 \mathrm{E}-02$ & $1.591 \mathrm{E}-01$ & $3.539 \mathrm{E}-01$ \\
\hline $\mathrm{H} \alpha \quad \lambda 6563 \ldots \ldots \ldots \ldots \ldots \ldots \ldots \ldots \ldots \ldots \ldots \ldots \ldots \ldots$ & $2.807 \mathrm{E}+00$ & $2.859 \mathrm{E}+00$ & $2.915 \mathrm{E}+00$ & $2.943 \mathrm{E}+00$ \\
\hline 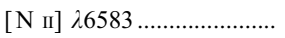 & $4.212 \mathrm{E}-03$ & $4.082 \mathrm{E}-02$ & $2.369 \mathrm{E}-01$ & $3.305 \mathrm{E}-01$ \\
\hline$[\mathrm{S}$ II] $\lambda \lambda 6717,6730 \ldots \ldots \ldots . .$. & $2.384 \mathrm{E}-02$ & $1.285 \mathrm{E}-01$ & $4.614 \mathrm{E}-01$ & $1.389 \mathrm{E}+00$ \\
\hline$[\mathrm{O}$ II] $\lambda \lambda 7318,7324 \ldots \ldots \ldots$. & $1.744 \mathrm{E}-03$ & $1.296 \mathrm{E}-02$ & $5.951 \mathrm{E}-02$ & $4.457 \mathrm{E}-02$ \\
\hline [S III] $\lambda \lambda 29069.9532 \ldots \ldots \ldots . .$. & $1.020 \mathrm{E}-01$ & $6.384 \mathrm{E}-01$ & $1.392 \mathrm{E}+00$ & $8.449 \mathrm{E}-01$ \\
\hline \multicolumn{5}{|c|}{$\alpha=-2.0$} \\
\hline$[\mathrm{O}$ II] $\lambda \lambda 3727,3729 \ldots \ldots \ldots$. & $6.167 \mathrm{E}-02$ & $4.784 \mathrm{E}-01$ & $2.485 \mathrm{E}+00$ & $2.503 \mathrm{E}+00$ \\
\hline$[\mathrm{S}$ II $] \lambda \lambda 4067,4076 \ldots \ldots \ldots . .$. & $9.867 \mathrm{E}-04$ & $6.061 \mathrm{E}-03$ & $3.045 \mathrm{E}-02$ & $8.611 \mathrm{E}-02$ \\
\hline 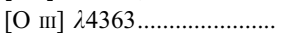 & $1.385 \mathrm{E}-01$ & $1.179 \mathrm{E}-01$ & $3.249 \mathrm{E}-02$ & $5.338 \mathrm{E}-04$ \\
\hline He II $\lambda 4686 \ldots \ldots \ldots \ldots \ldots \ldots \ldots$ & $1.128 \mathrm{E}-01$ & $1.178 \mathrm{E}-01$ & $1.044 \mathrm{E}-01$ & $4.603 \mathrm{E}-02$ \\
\hline 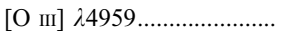 & $2.716 \mathrm{E}+00$ & $2.479 \mathrm{E}+00$ & $9.647 \mathrm{E}-01$ & $2.757 \mathrm{E}-02$ \\
\hline 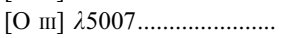 & $7.821 \mathrm{E}+00$ & $7.141 \mathrm{E}+00$ & $2.779 \mathrm{E}+00$ & $7.939 \mathrm{E}-02$ \\
\hline$\left[\mathrm{N}_{\mathrm{I}}\right] \lambda 5200 \ldots \ldots \ldots \ldots \ldots \ldots \ldots \ldots \ldots$ & $3.177 \mathrm{E}-04$ & $2.531 \mathrm{E}-03$ & $7.932 \mathrm{E}-03$ & $2.080 \mathrm{E}-02$ \\
\hline$[\mathrm{Fe}$ VII $] \lambda 6085 \ldots \ldots \ldots \ldots \ldots \ldots$ & $1.973 \mathrm{E}-02$ & $3.832 \mathrm{E}-04$ & $0.000 \mathrm{E}+00$ & $0.000 \mathrm{E}+00$ \\
\hline$\left[\mathrm{O}_{\mathrm{I}}\right], \lambda 6300 \ldots \ldots \ldots \ldots \ldots \ldots \ldots$ & $3.438 \mathrm{E}-03$ & $2.692 \mathrm{E}-02$ & $9.275 \mathrm{E}-02$ & $2.547 \mathrm{E}-01$ \\
\hline $\mathrm{H} \alpha \quad \lambda 6563 \ldots \ldots \ldots \ldots \ldots \ldots \ldots \ldots \ldots \ldots \ldots \ldots \ldots \ldots$ & $2.809 \mathrm{E}+00$ & $2.838 \mathrm{E}+00$ & $2.886 \mathrm{E}+00$ & $2.919 \mathrm{E}+00$ \\
\hline 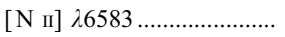 & $4.049 \mathrm{E}-03$ & $3.937 \mathrm{E}-02$ & $2.358 \mathrm{E}-01$ & $3.206 \mathrm{E}-01$ \\
\hline$\left[\mathrm{S}_{\mathrm{II}}\right] \lambda \lambda 6717,6730 \ldots \ldots \ldots \ldots$ & $1.439 \mathrm{E}-02$ & $8.648 \mathrm{E}-02$ & $3.746 \mathrm{E}-01$ & $1.235 \mathrm{E}+00$ \\
\hline$[\mathrm{O}$ II] $\lambda \lambda 7318,7324 \ldots \ldots \ldots .$. & $1.685 \mathrm{E}-03$ & $1.243 \mathrm{E}-02$ & $5.754 \mathrm{E}-02$ & $4.278 \mathrm{E}-02$ \\
\hline$[\mathrm{S}$ III] $\lambda \lambda 9069.9532 \ldots \ldots \ldots \ldots$ & $1.071 \mathrm{E}-01$ & $6.707 \mathrm{E}-01$ & $1.293 \mathrm{E}+00$ & $7.053 \mathrm{E}-01$ \\
\hline
\end{tabular}


TABLE 39

Undepleted Dust-free Visible Line Ratios with Respect to $\mathrm{H} \beta, 0.5 Z_{\odot}$

\begin{tabular}{|c|c|c|c|c|}
\hline \multirow[b]{2}{*}{ LiNe } & \multicolumn{4}{|c|}{$\log U_{0}$} \\
\hline & -1.0 & -2.0 & -3.0 & -4.0 \\
\hline \multicolumn{5}{|c|}{$\alpha=-1.2$} \\
\hline$[\mathrm{O}$ II $] \lambda \lambda 3727,3729 \ldots \ldots \ldots . .$. & $1.173 \mathrm{E}-01$ & $9.848 \mathrm{E}-01$ & $4.253 \mathrm{E}+00$ & $4.123 \mathrm{E}+00$ \\
\hline [S II] $\lambda \lambda 4067,4076 \ldots \ldots \ldots . .$. & $3.810 \mathrm{E}-03$ & $2.065 \mathrm{E}-02$ & $6.903 \mathrm{E}-02$ & $1.583 \mathrm{E}-01$ \\
\hline$[\mathrm{O}$ III] $\lambda 4363 \ldots \ldots \ldots \ldots \ldots \ldots$ & $3.470 \mathrm{E}-01$ & $2.595 \mathrm{E}-01$ & $6.220 \mathrm{E}-02$ & $9.762 \mathrm{E}-04$ \\
\hline He II $\lambda 4686 \ldots \ldots \ldots \ldots \ldots \ldots \ldots$ & $2.874 \mathrm{E}-01$ & $2.969 \mathrm{E}-01$ & $2.427 \mathrm{E}-01$ & $1.092 \mathrm{E}-01$ \\
\hline 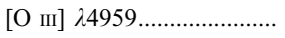 & $4.797 \mathrm{E}+00$ & $4.477 \mathrm{E}+00$ & $1.807 \mathrm{E}+00$ & $5.498 \mathrm{E}-02$ \\
\hline$[\mathrm{O}$ III $] \lambda 5007 \ldots \ldots \ldots \ldots \ldots \ldots \ldots$ & $1.382 \mathrm{E}+01$ & $1.289 \mathrm{E}+01$ & $5.204 \mathrm{E}+00$ & $1.583 \mathrm{E}-01$ \\
\hline$\left[\mathrm{N}_{\mathrm{I}}\right] \lambda 5200 \ldots \ldots \ldots \ldots \ldots \ldots$ & $4.823 \mathrm{E}-03$ & $3.456 \mathrm{E}-02$ & $9.062 \mathrm{E}-02$ & $1.242 \mathrm{E}-01$ \\
\hline$[\mathrm{Fe}$ VII $] \lambda 6085 \ldots \ldots \ldots \ldots \ldots \ldots$ & $9.113 \mathrm{E}-02$ & $1.562 \mathrm{E}-02$ & $3.295 \mathrm{E}-05$ & $0.000 \mathrm{E}+00$ \\
\hline 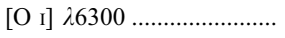 & $3.133 \mathrm{E}-02$ & $2.572 \mathrm{E}-01$ & $6.339 \mathrm{E}-01$ & $9.396 \mathrm{E}-01$ \\
\hline 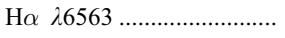 & $2.834 \mathrm{E}+00$ & $2.919 \mathrm{E}+00$ & $2.973 \mathrm{E}+00$ & $2.992 \mathrm{E}+00$ \\
\hline 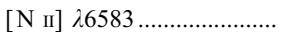 & $1.550 \mathrm{E}-02$ & $1.665 \mathrm{E}-01$ & $7.001 \mathrm{E}-01$ & $8.901 \mathrm{E}-01$ \\
\hline$[\mathrm{S}$ II] $\lambda \lambda 6717,6730 \ldots \ldots \ldots . .$. & $6.780 \mathrm{E}-02$ & $3.796 \mathrm{E}-01$ & $1.157 \mathrm{E}+00$ & $2.683 \mathrm{E}+00$ \\
\hline$[\mathrm{O}$ II $] \lambda \lambda 7318,7324 \ldots \ldots \ldots . .$. & $2.888 \mathrm{E}-03$ & $2.333 \mathrm{E}-02$ & $9.430 \mathrm{E}-02$ & $6.468 \mathrm{E}-02$ \\
\hline [S III] $\lambda \lambda 9069.9532 \ldots \ldots \ldots . .$. & $1.844 \mathrm{E}-01$ & $1.149 \mathrm{E}+00$ & $2.941 \mathrm{E}+00$ & $2.106 \mathrm{E}+00$ \\
\hline \multicolumn{5}{|c|}{$\alpha=-1.4$} \\
\hline [O II] $\lambda \lambda 3727,3729 \ldots \ldots \ldots .$. & $1.050 \mathrm{E}-01$ & $8.548 \mathrm{E}-01$ & $4.029 \mathrm{E}+00$ & $4.088 \mathrm{E}+00$ \\
\hline [S пा] $\lambda \lambda 4067,4076 \ldots \ldots \ldots . .$. & $2.935 \mathrm{E}-03$ & $1.643 \mathrm{E}-02$ & $5.992 \mathrm{E}-02$ & $1.519 \mathrm{E}-01$ \\
\hline 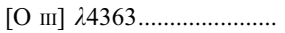 & $2.517 \mathrm{E}-01$ & $2.083 \mathrm{E}-01$ & $5.510 \mathrm{E}-02$ & $9.261 \mathrm{E}-04$ \\
\hline 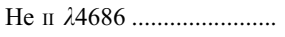 & $2.288 \mathrm{E}-01$ & $2.410 \mathrm{E}-01$ & $2.045 \mathrm{E}-01$ & $9.278 \mathrm{E}-02$ \\
\hline 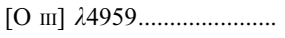 & $4.389 \mathrm{E}+00$ & $4.221 \mathrm{E}+00$ & $1.748 \mathrm{E}+00$ & $5.468 \mathrm{E}-02$ \\
\hline 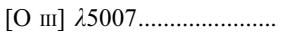 & $1.264 \mathrm{E}+01$ & $1.216 \mathrm{E}+01$ & $5.034 \mathrm{E}+00$ & $1.575 \mathrm{E}-01$ \\
\hline$\left[\mathrm{N}_{\mathrm{I}}\right] \lambda 5200 \ldots \ldots \ldots \ldots \ldots \ldots \ldots$ & $2.985 \mathrm{E}-03$ & $2.331 \mathrm{E}-02$ & $5.921 \mathrm{E}-02$ & $9.055 \mathrm{E}-02$ \\
\hline 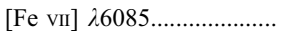 & $8.414 \mathrm{E}-02$ & $7.218 \mathrm{E}-03$ & $1.312 \mathrm{E}-05$ & $0.000 \mathrm{E}+00$ \\
\hline 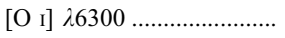 & $1.908 \mathrm{E}-02$ & $1.638 \mathrm{E}-01$ & $4.199 \mathrm{E}-01$ & $7.180 \mathrm{E}-01$ \\
\hline 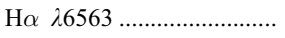 & $2.818 \mathrm{E}+00$ & $2.868 \mathrm{E}+00$ & $2.927 \mathrm{E}+00$ & $2.955 \mathrm{E}+00$ \\
\hline 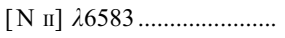 & $1.270 \mathrm{E}-02$ & $1.308 \mathrm{E}-01$ & $6.526 \mathrm{E}-01$ & $8.839 \mathrm{E}-01$ \\
\hline$[\mathrm{S}$ п] $] \lambda \lambda 6717,6730 \ldots \ldots \ldots . .$. & $5.074 \mathrm{E}-02$ & $2.897 \mathrm{E}-01$ & $9.120 \mathrm{E}-01$ & $2.444 \mathrm{E}+00$ \\
\hline$[\mathrm{O}$ II $] \lambda \lambda 7318,7324 \ldots \ldots \ldots .$. & $2.603 \mathrm{E}-03$ & $2.023 \mathrm{E}-02$ & $8.772 \mathrm{E}-02$ & $6.377 \mathrm{E}-02$ \\
\hline [S III] $\lambda \lambda 9069.9532 \ldots \ldots \ldots . .$. & $1.726 \mathrm{E}-01$ & $1.100 \mathrm{E}+00$ & $2.665 \mathrm{E}+00$ & $1.756 \mathrm{E}+00$ \\
\hline \multicolumn{5}{|c|}{$\alpha=-1.7$} \\
\hline$[\mathrm{O}$ II] $\lambda \lambda 3727,3729 \ldots \ldots \ldots$. & $9.480 \mathrm{E}-02$ & $7.346 \mathrm{E}-01$ & $3.697 \mathrm{E}+00$ & $3.859 \mathrm{E}+00$ \\
\hline$[\mathrm{S}$ II] $\lambda \lambda 4067,4076 \ldots \ldots \ldots . .$. & $1.940 \mathrm{E}-03$ & $1.174 \mathrm{E}-02$ & $5.107 \mathrm{E}-02$ & $1.404 \mathrm{E}-01$ \\
\hline 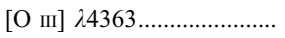 & $1.521 \mathrm{E}-01$ & $1.387 \mathrm{E}-01$ & $4.159 \mathrm{E}-02$ & $7.583 \mathrm{E}-04$ \\
\hline 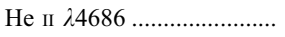 & $1.602 \mathrm{E}-01$ & $1.719 \mathrm{E}-01$ & $1.512 \mathrm{E}-01$ & $6.780 \mathrm{E}-02$ \\
\hline 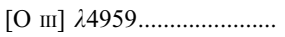 & $3.669 \mathrm{E}+00$ & $3.593 \mathrm{E}+00$ & $1.536 \mathrm{E}+00$ & $4.852 \mathrm{E}-02$ \\
\hline 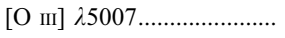 & $1.057 \mathrm{E}+01$ & $1.035 \mathrm{E}+01$ & $4.423 \mathrm{E}+00$ & $1.397 \mathrm{E}-01$ \\
\hline$\left[\mathrm{N}_{\mathrm{I}}\right] \lambda 5200 \ldots \ldots \ldots \ldots \ldots \ldots \ldots \ldots \ldots \ldots \ldots \ldots$ & $1.166 \mathrm{E}-03$ & $1.136 \mathrm{E}-02$ & $3.010 \mathrm{E}-02$ & $5.828 \mathrm{E}-02$ \\
\hline 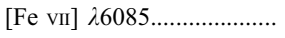 & $5.532 \mathrm{E}-02$ & $1.895 \mathrm{E}-03$ & $2.838 \mathrm{E}-06$ & $0.000 \mathrm{E}+00$ \\
\hline$\left[\mathrm{O}_{\mathrm{I}}\right]$ & $8.072 \mathrm{E}-03$ & $7.873 \mathrm{E}-02$ & $2.274 \mathrm{E}-01$ & $4.921 \mathrm{E}-01$ \\
\hline 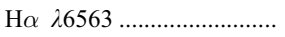 & $2.823 \mathrm{E}+00$ & $2.845 \mathrm{E}+00$ & $2.889 \mathrm{E}+00$ & $2.925 \mathrm{E}+00$ \\
\hline$[\mathrm{N}$ II $] \lambda 6583 \ldots \ldots \ldots \ldots \ldots \ldots$ & $1.114 \mathrm{E}-02$ & $1.066 \mathrm{E}-01$ & $6.079 \mathrm{E}-01$ & $8.449 \mathrm{E}-01$ \\
\hline$[\mathrm{S}$ пा] $\lambda \lambda 6717,6730 \ldots \ldots \ldots . .$. & $2.996 \mathrm{E}-02$ & $1.870 \mathrm{E}-01$ & $6.909 \mathrm{E}-01$ & $2.152 \mathrm{E}+00$ \\
\hline$[\mathrm{O}$ II $] \lambda \lambda 7318,7324 \ldots \ldots \ldots .$. & $2.323 \mathrm{E}-03$ & $1.688 \mathrm{E}-02$ & $7.671 \mathrm{E}-02$ & $5.887 \mathrm{E}-02$ \\
\hline$[\mathrm{S}$ III] $\lambda \lambda 9069.9532 \ldots \ldots \ldots \ldots$ & $1.689 \mathrm{E}-01$ & $1.070 \mathrm{E}+00$ & $2.340 \mathrm{E}+00$ & $1.386 \mathrm{E}+00$ \\
\hline \multicolumn{5}{|c|}{$\alpha=-2.0$} \\
\hline$[\mathrm{O}$ II] $\lambda \lambda 3727,3729 \ldots \ldots \ldots$ & $8.987 \mathrm{E}-02$ & $6.654 \mathrm{E}-01$ & $3.376 \mathrm{E}+00$ & $3.549 \mathrm{E}+00$ \\
\hline$[\mathrm{S}$ II $] \lambda \lambda 4067,4076 \ldots \ldots \ldots . .$. & $1.346 \mathrm{E}-03$ & $8.818 \mathrm{E}-03$ & $4.519 \mathrm{E}-02$ & $1.286 \mathrm{E}-01$ \\
\hline 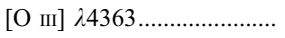 & $9.711 \mathrm{E}-02$ & $8.988 \mathrm{E}-02$ & $2.907 \mathrm{E}-02$ & $5.679 \mathrm{E}-04$ \\
\hline He II $\lambda 4686 \ldots \ldots \ldots \ldots \ldots \ldots . . . . . . . . . .$. & $1.121 \mathrm{E}-01$ & $1.205 \mathrm{E}-01$ & $1.077 \mathrm{E}-01$ & $4.722 \mathrm{E}-02$ \\
\hline 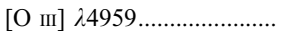 & $3.052 \mathrm{E}+00$ & $2.951 \mathrm{E}+00$ & $1.264 \mathrm{E}+00$ & $3.966 \mathrm{E}-02$ \\
\hline 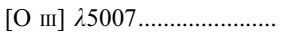 & $8.791 \mathrm{E}+00$ & $8.501 \mathrm{E}+00$ & $3.639 \mathrm{E}+00$ & $1.142 \mathrm{E}-01$ \\
\hline 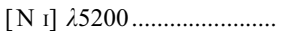 & $2.946 \mathrm{E}-04$ & $4.766 \mathrm{E}-03$ & $1.537 \mathrm{E}-02$ & $3.992 \mathrm{E}-02$ \\
\hline$[\mathrm{Fe}$ vII $] \lambda 6085 \ldots \ldots \ldots \ldots \ldots \ldots$ & $2.655 \mathrm{E}-02$ & $4.200 \mathrm{E}-04$ & $0.000 \mathrm{E}+00$ & $0.000 \mathrm{E}+00$ \\
\hline$[\mathrm{O}$ I] $\lambda 6300 \ldots \ldots \ldots \ldots \ldots \ldots \ldots$ & $2.966 \mathrm{E}-03$ & $3.642 \mathrm{E}-02$ & $1.294 \mathrm{E}-01$ & $3.526 \mathrm{E}-01$ \\
\hline 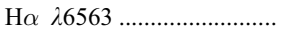 & $2.836 \mathrm{E}+00$ & $2.847 \mathrm{E}+00$ & $2.876 \mathrm{E}+00$ & $2.908 \mathrm{E}+00$ \\
\hline 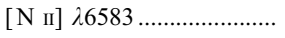 & $1.078 \mathrm{E}-02$ & $9.863 \mathrm{E}-02$ & $5.771 \mathrm{E}-01$ & $7.930 \mathrm{E}-01$ \\
\hline$[\mathrm{S}$ II] $\lambda \lambda 6717,6730 \ldots \ldots \ldots . .$. & $1.667 \mathrm{E}-02$ & $1.232 \mathrm{E}-01$ & $5.734 \mathrm{E}-01$ & $1.923 \mathrm{E}+00$ \\
\hline$[\mathrm{O}$ II] $\lambda \lambda 7318,7324 \ldots \ldots \ldots .$. & $2.136 \mathrm{E}-03$ & $1.453 \mathrm{E}-02$ & $6.588 \mathrm{E}-02$ & $5.233 \mathrm{E}-02$ \\
\hline [S III] $\lambda \lambda 9069.9532 \ldots \ldots \ldots . .$. & $1.764 \mathrm{E}-01$ & $1.069 \mathrm{E}+00$ & $2.088 \mathrm{E}+00$ & $1.152 \mathrm{E}+00$ \\
\hline
\end{tabular}


TABLE 40

Undepleted Dust-free Visible Line Ratios with Respect to $\mathrm{H} \beta, 1 Z_{\odot}$

\begin{tabular}{|c|c|c|c|c|}
\hline \multirow[b]{2}{*}{ LiNE } & \multicolumn{4}{|c|}{$\log U_{0}$} \\
\hline & -1.0 & -2.0 & -3.0 & -4.0 \\
\hline \multicolumn{5}{|c|}{$\alpha=-1.2$} \\
\hline [O пा $] \lambda \lambda 3727,3729 .$. & $1.449 \mathrm{E}-01$ & $1.411 \mathrm{E}+00$ & $5.630 \mathrm{E}+00$ & $4.889 \mathrm{E}+00$ \\
\hline$[\mathrm{S}$ II] $\lambda \lambda 4067,4076 \ldots \ldots \ldots \ldots$ & $4.197 \mathrm{E}-03$ & $2.954 \mathrm{E}-02$ & $9.301 \mathrm{E}-02$ & $1.972 \mathrm{E}-01$ \\
\hline 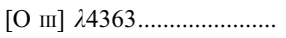 & $2.599 \mathrm{E}-01$ & $2.325 \mathrm{E}-01$ & $5.157 \mathrm{E}-02$ & $7.460 \mathrm{E}-04$ \\
\hline Не II $\lambda 4686 \ldots \ldots \ldots \ldots \ldots \ldots \ldots$ & $2.875 \mathrm{E}-01$ & $3.029 \mathrm{E}-01$ & $2.553 \mathrm{E}-01$ & $1.157 \mathrm{E}-01$ \\
\hline 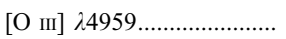 & $5.504 \mathrm{E}+00$ & $5.909 \mathrm{E}+00$ & $2.297 \mathrm{E}+00$ & $6.707 \mathrm{E}-02$ \\
\hline 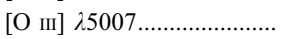 & $1.585 \mathrm{E}+01$ & $1.702 \mathrm{E}+01$ & $6.614 \mathrm{E}+00$ & $1.932 \mathrm{E}-01$ \\
\hline$\left[\mathrm{N}_{\mathrm{I}}\right] \mathrm{\lambda}, 25200 \ldots \ldots \ldots \ldots \ldots \ldots \ldots$ & $6.311 \mathrm{E}-03$ & $7.694 \mathrm{E}-02$ & $1.879 \mathrm{E}-01$ & $2.037 \mathrm{E}-01$ \\
\hline$[\mathrm{Fe}$ VII] $\lambda 6085 \ldots \ldots \ldots \ldots \ldots \ldots$ & $1.469 \mathrm{E}-01$ & $1.837 \mathrm{E}-02$ & $2.883 \mathrm{E}-05$ & $0.000 \mathrm{E}+00$ \\
\hline$[\mathrm{O}$ I] $\lambda 6300 \ldots \ldots \ldots \ldots \ldots \ldots \ldots$ & $2.509 \mathrm{E}-02$ & $3.544 \mathrm{E}-01$ & $8.348 \mathrm{E}-01$ & $1.042 \mathrm{E}+00$ \\
\hline $\mathrm{H} \alpha \quad \lambda 6563 \ldots \ldots \ldots \ldots \ldots \ldots \ldots \ldots$ & $2.832 \mathrm{E}+00$ & $2.872 \mathrm{E}+00$ & $2.932 \mathrm{E}+00$ & $2.987 \mathrm{E}+00$ \\
\hline$[\mathrm{N}$ II $] \lambda 6583 \ldots \ldots \ldots \ldots \ldots \ldots$ & $3.469 \mathrm{E}-02$ & $4.430 \mathrm{E}-01$ & $1.842 \mathrm{E}+00$ & $2.187 \mathrm{E}+00$ \\
\hline$\left[\mathrm{S}_{\mathrm{II}}\right] \lambda \lambda 6717,6730 \ldots \ldots \ldots \ldots$ & $7.343 \mathrm{E}-02$ & $5.321 \mathrm{E}-01$ & $1.526 \mathrm{E}+00$ & $3.493 \mathrm{E}+00$ \\
\hline$[\mathrm{O}$ II] $\lambda \lambda 7318,7324 \ldots \ldots \ldots$. & $3.104 \mathrm{E}-03$ & $2.841 \mathrm{E}-02$ & $1.032 \mathrm{E}-01$ & $6.154 \mathrm{E}-02$ \\
\hline [S III] $\lambda \lambda 9069.9532 \ldots \ldots \ldots . .$. & $2.726 \mathrm{E}-01$ & $1.896 \mathrm{E}+00$ & $4.619 \mathrm{E}+00$ & $2.910 \mathrm{E}+00$ \\
\hline \multicolumn{5}{|c|}{$\alpha=-1.4$} \\
\hline$[\mathrm{O}$ пा] $\lambda \lambda 3727,3729 \ldots \ldots \ldots$. & $1.287 \mathrm{E}-01$ & $1.117 \mathrm{E}+00$ & $4.816 \mathrm{E}+00$ & $4.493 \mathrm{E}+00$ \\
\hline$[\mathrm{S}$ II $] \lambda \lambda 4067,4076 \ldots \ldots \ldots \ldots$ & $2.996 \mathrm{E}-03$ & $2.206 \mathrm{E}-02$ & $7.780 \mathrm{E}-02$ & $1.814 \mathrm{E}-01$ \\
\hline [O & $1.656 \mathrm{E}-01$ & $1.537 \mathrm{E}-01$ & $3.806 \mathrm{E}-02$ & $6.288 \mathrm{E}-04$ \\
\hline 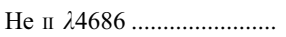 & $2.265 \mathrm{E}-01$ & $2.454 \mathrm{E}-01$ & $2.150 \mathrm{E}-01$ & $9.745 \mathrm{E}-02$ \\
\hline 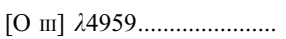 & $4.620 \mathrm{E}+00$ & $4.928 \mathrm{E}+00$ & $2.012 \mathrm{E}+00$ & $6.253 \mathrm{E}-02$ \\
\hline 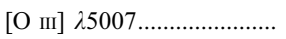 & $1.331 \mathrm{E}+01$ & $1.419 \mathrm{E}+01$ & $5.794 \mathrm{E}+00$ & $1.801 \mathrm{E}-01$ \\
\hline$\left[\mathrm{N}_{\mathrm{I}}\right] \mathrm{z} 25200 \ldots \ldots \ldots \ldots \ldots \ldots \ldots$ & $2.999 \mathrm{E}-03$ & $4.878 \mathrm{E}-02$ & $1.164 \mathrm{E}-01$ & $1.424 \mathrm{E}-01$ \\
\hline$[\mathrm{Fe}$ VII] $\lambda 6085 \ldots \ldots \ldots \ldots \ldots \ldots$ & $1.246 \mathrm{E}-01$ & $7.734 \mathrm{E}-03$ & $1.026 \mathrm{E}-05$ & $0.000 \mathrm{E}+00$ \\
\hline 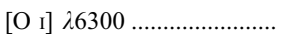 & $1.285 \mathrm{E}-02$ & $2.140 \mathrm{E}-01$ & $5.279 \mathrm{E}-01$ & $7.708 \mathrm{E}-01$ \\
\hline $\mathrm{H} \alpha \quad \lambda 6563 \ldots \ldots \ldots \ldots \ldots \ldots \ldots \ldots \ldots$ & $2.839 \mathrm{E}+00$ & $2.860 \mathrm{E}+00$ & $2.910 \mathrm{E}+00$ & $2.960 \mathrm{E}+00$ \\
\hline$[\mathrm{N}$ II] $\lambda 6583 \ldots \ldots \ldots \ldots \ldots \ldots \ldots$ & $3.101 \mathrm{E}-02$ & $3.385 \mathrm{E}-01$ & $1.628 \mathrm{E}+00$ & $2.074 \mathrm{E}+00$ \\
\hline$[\mathrm{S}$ II $] \lambda \lambda 6717,6730 \ldots \ldots \ldots . .$. & $4.852 \mathrm{E}-02$ & $3.871 \mathrm{E}-01$ & $1.202 \mathrm{E}+00$ & $3.169 \mathrm{E}+00$ \\
\hline$[\mathrm{O}$ II] $\lambda \lambda 7318,7324 \ldots \ldots \ldots .$. & $2.712 \mathrm{E}-03$ & $2.162 \mathrm{E}-02$ & $8.318 \mathrm{E}-02$ & $5.450 \mathrm{E}-02$ \\
\hline [S III] $\lambda \lambda 9069.9532 \ldots \ldots \ldots . .$. & $2.605 \mathrm{E}-01$ & $1.729 \mathrm{E}+00$ & $4.000 \mathrm{E}+00$ & $2.392 \mathrm{E}+00$ \\
\hline \multicolumn{5}{|c|}{$\alpha=-1.7$} \\
\hline$[\mathrm{O}$ II $] \lambda \lambda 3727,3729 \ldots \ldots \ldots$. & $1.131 \mathrm{E}-01$ & $8.437 \mathrm{E}-01$ & $3.817 \mathrm{E}+00$ & $3.798 \mathrm{E}+00$ \\
\hline$[\mathrm{S}$ II] $\lambda \lambda 4067,4076 \ldots \ldots \ldots . .$. & $1.924 \mathrm{E}-03$ & $1.440 \mathrm{E}-02$ & $6.170 \mathrm{E}-02$ & $1.549 \mathrm{E}-01$ \\
\hline$[\mathrm{O}$ III] $\lambda 4363 \ldots \ldots \ldots \ldots \ldots \ldots . .$. & $8.410 \mathrm{E}-02$ & $8.016 \mathrm{E}-02$ & $2.242 \mathrm{E}-02$ & $4.362 \mathrm{E}-04$ \\
\hline 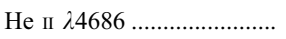 & $1.572 \mathrm{E}-01$ & $1.759 \mathrm{E}-01$ & $1.590 \mathrm{E}-01$ & $7.062 \mathrm{E}-02$ \\
\hline 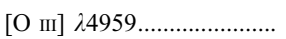 & $3.463 \mathrm{E}+00$ & $3.623 \mathrm{E}+00$ & $1.548 \mathrm{E}+00$ & $5.085 \mathrm{E}-02$ \\
\hline 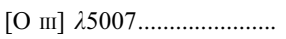 & $9.973 \mathrm{E}+00$ & $1.043 \mathrm{E}+01$ & $4.460 \mathrm{E}+00$ & $1.465 \mathrm{E}-01$ \\
\hline$\left[\mathrm{N}_{\mathrm{I}}\right] \mathrm{j}, 25200 \ldots \ldots \ldots \ldots \ldots \ldots \ldots$ & $5.304 \mathrm{E}-04$ & $2.089 \mathrm{E}-02$ & $5.385 \mathrm{E}-02$ & $8.619 \mathrm{E}-02$ \\
\hline 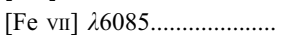 & $7.241 \mathrm{E}-02$ & $1.767 \mathrm{E}-03$ & $1.911 \mathrm{E}-06$ & $0.000 \mathrm{E}+00$ \\
\hline$[\mathrm{O}$ I] $\lambda 6300 \ldots \ldots \ldots \ldots \ldots \ldots \ldots$ & $3.829 \mathrm{E}-03$ & $9.255 \mathrm{E}-02$ & $2.652 \mathrm{E}-01$ & $4.999 \mathrm{E}-01$ \\
\hline $\mathrm{H} \alpha \quad \lambda 6563 \ldots \ldots \ldots \ldots \ldots \ldots \ldots \ldots$ & $2.861 \mathrm{E}+00$ & $2.869 \mathrm{E}+00$ & $2.899 \mathrm{E}+00$ & $2.941 \mathrm{E}+00$ \\
\hline$[\mathrm{N}$ II] $\lambda 6583 \ldots \ldots \ldots \ldots \ldots \ldots$ & $2.857 \mathrm{E}-02$ & $2.609 \mathrm{E}-01$ & $1.399 \mathrm{E}+00$ & $1.854 \mathrm{E}+00$ \\
\hline$[\mathrm{S}$ II $] \lambda \lambda 6717,6730 \ldots \ldots \ldots . .$. & $2.453 \mathrm{E}-02$ & $2.347 \mathrm{E}-01$ & $9.109 \mathrm{E}-01$ & $2.716 \mathrm{E}+00$ \\
\hline$[\mathrm{O}$ п] $] \lambda \lambda 7318,7324 \ldots \ldots \ldots$. & $2.285 \mathrm{E}-03$ & $1.518 \mathrm{E}-02$ & $5.931 \mathrm{E}-02$ & $4.298 \mathrm{E}-02$ \\
\hline [S III] $\lambda \lambda 9069.9532 \ldots \ldots \ldots . .$. & $2.562 \mathrm{E}-01$ & $1.563 \mathrm{E}+00$ & $3.282 \mathrm{E}+00$ & $1.851 \mathrm{E}+00$ \\
\hline \multicolumn{5}{|c|}{$\alpha=-2.0$} \\
\hline$[\mathrm{O}$ п] $] \lambda \lambda 3727,3729 \ldots \ldots \ldots .$. & $1.017 \mathrm{E}-01$ & $6.770 \mathrm{E}-01$ & $3.069 \mathrm{E}+00$ & $3.127 \mathrm{E}+00$ \\
\hline$[\mathrm{S}$ II] $\lambda \lambda 4067,4076 \ldots \ldots \ldots \ldots$ & $1.527 \mathrm{E}-03$ & $9.904 \mathrm{E}-03$ & $5.008 \mathrm{E}-02$ & $1.299 \mathrm{E}-01$ \\
\hline 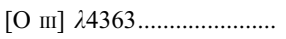 & $4.518 \mathrm{E}-02$ & $4.147 \mathrm{E}-02$ & $1.281 \mathrm{E}-02$ & $2.795 \mathrm{E}-04$ \\
\hline 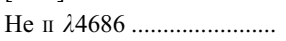 & $1.096 \mathrm{E}-01$ & $1.247 \mathrm{E}-01$ & $1.132 \mathrm{E}-01$ & $4.872 \mathrm{E}-02$ \\
\hline 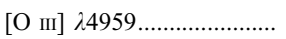 & $2.601 \mathrm{E}+00$ & $2.635 \mathrm{E}+00$ & $1.146 \mathrm{E}+00$ & $3.834 \mathrm{E}-02$ \\
\hline 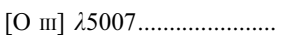 & $7.492 \mathrm{E}+00$ & $7.590 \mathrm{E}+00$ & $3.300 \mathrm{E}+00$ & $1.104 \mathrm{E}-01$ \\
\hline$\left[\mathrm{N}_{\mathrm{I}}\right] \lambda 5200 \ldots \ldots \ldots \ldots \ldots \ldots \ldots \ldots$ & $1.528 \mathrm{E}-04$ & $6.979 \mathrm{E}-03$ & $2.417 \mathrm{E}-02$ & $5.589 \mathrm{E}-02$ \\
\hline 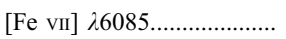 & $3.112 \mathrm{E}-02$ & $3.378 \mathrm{E}-04$ & $0.000 \mathrm{E}+00$ & $0.000 \mathrm{E}+00$ \\
\hline 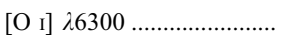 & $2.178 \mathrm{E}-03$ & $3.682 \mathrm{E}-02$ & $1.371 \mathrm{E}-01$ & $3.367 \mathrm{E}-01$ \\
\hline $\mathrm{H} \alpha \quad \lambda 6563 \ldots \ldots \ldots \ldots \ldots \ldots \ldots \ldots \ldots$ & $2.883 \mathrm{E}+00$ & $2.886 \mathrm{E}+00$ & $2.902 \mathrm{E}+00$ & $2.933 \mathrm{E}+00$ \\
\hline 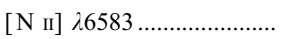 & $2.748 \mathrm{E}-02$ & $2.268 \mathrm{E}-01$ & $1.234 \mathrm{E}+00$ & $1.627 \mathrm{E}+00$ \\
\hline$[\mathrm{S}$ II $] \lambda \lambda 6717,6730 \ldots \ldots \ldots . .$. & $1.680 \mathrm{E}-02$ & $1.471 \mathrm{E}-01$ & $7.368 \mathrm{E}-01$ & $2.327 \mathrm{E}+00$ \\
\hline$[\mathrm{O}$ II] $\lambda \lambda 7318,7324 \ldots \ldots \ldots$. & $1.927 \mathrm{E}-03$ & $1.108 \mathrm{E}-02$ & $4.266 \mathrm{E}-02$ & $3.267 \mathrm{E}-02$ \\
\hline [S III] $\lambda \lambda 9069.9532 \ldots \ldots \ldots . .$. & $2.632 \mathrm{E}-01$ & $1.460 \mathrm{E}+00$ & $2.763 \mathrm{E}+00$ & $1.495 \mathrm{E}+00$ \\
\hline
\end{tabular}


TABLE 41

Undepleted Dust-free Visible Line Ratios with Respect to $\mathrm{H} \beta, 2 Z_{\odot}$

\begin{tabular}{|c|c|c|c|c|}
\hline \multirow[b]{2}{*}{ LiNe } & \multicolumn{4}{|c|}{$\log U_{0}$} \\
\hline & -1.0 & -2.0 & -3.0 & -4.0 \\
\hline \multicolumn{5}{|c|}{$\alpha=-1.2$} \\
\hline [O II] $\lambda \lambda 3727,3729 \ldots \ldots \ldots .$. & $1.025 \mathrm{E}-01$ & $1.353 \mathrm{E}+00$ & $4.569 \mathrm{E}+00$ & $2.974 \mathrm{E}+00$ \\
\hline$[\mathrm{S}$ II $] \lambda \lambda 4067,4076 \ldots \ldots \ldots . .$. & $2.071 \mathrm{E}-03$ & $3.280 \mathrm{E}-02$ & $9.314 \mathrm{E}-02$ & $1.397 \mathrm{E}-01$ \\
\hline 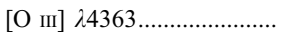 & $1.496 \mathrm{E}-01$ & $1.405 \mathrm{E}-01$ & $1.980 \mathrm{E}-02$ & $2.184 \mathrm{E}-04$ \\
\hline 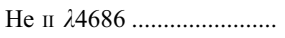 & $2.840 \mathrm{E}-01$ & $3.104 \mathrm{E}-01$ & $2.752 \mathrm{E}-01$ & $1.234 \mathrm{E}-01$ \\
\hline 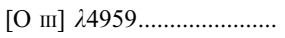 & $4.555 \mathrm{E}+00$ & $5.934 \mathrm{E}+00$ & $1.953 \mathrm{E}+00$ & $5.022 \mathrm{E}-02$ \\
\hline$[\mathrm{O}$ III $] \lambda 5007 \ldots .$. & $1.312 \mathrm{E}+01$ & $1.709 \mathrm{E}+01$ & $5.624 \mathrm{E}+00$ & $1.446 \mathrm{E}-01$ \\
\hline$\left[\mathrm{N}_{\mathrm{I}}\right] \lambda 5200 \ldots \ldots \ldots \ldots \ldots \ldots \ldots$ & $1.679 \mathrm{E}-03$ & $1.482 \mathrm{E}-01$ & $3.317 \mathrm{E}-01$ & $1.921 \mathrm{E}-01$ \\
\hline$[\mathrm{Fe}$ VII $] \lambda 6085 \ldots \ldots \ldots \ldots \ldots \ldots$ & $2.104 \mathrm{E}-01$ & $1.724 \mathrm{E}-02$ & $1.455 \mathrm{E}-05$ & $0.000 \mathrm{E}+00$ \\
\hline$\left[\mathrm{O}_{\mathrm{I}}\right] \lambda 6300 \ldots \ldots \ldots \ldots \ldots \ldots \ldots$ & $5.549 \mathrm{E}-03$ & $3.829 \mathrm{E}-01$ & $8.519 \mathrm{E}-01$ & $6.548 \mathrm{E}-01$ \\
\hline 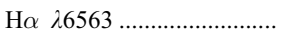 & $2.879 \mathrm{E}+00$ & $2.884 \mathrm{E}+00$ & $2.945 \mathrm{E}+00$ & $3.048 \mathrm{E}+00$ \\
\hline$[\mathrm{N}$ II] $\lambda 6583 \ldots \ldots \ldots \ldots \ldots \ldots$ & $6.672 \mathrm{E}-02$ & $1.001 \mathrm{E}+00$ & $3.972 \mathrm{E}+00$ & $3.926 \mathrm{E}+00$ \\
\hline$[\mathrm{S}$ II] $\lambda \lambda 6717,6730 \ldots \ldots \ldots . .$. & $3.580 \mathrm{E}-02$ & $6.297 \mathrm{E}-01$ & $1.721 \mathrm{E}+00$ & $3.233 \mathrm{E}+00$ \\
\hline$[\mathrm{O}$ II] $\lambda \lambda 7318,7324 \ldots \ldots \ldots$. & $1.603 \mathrm{E}-03$ & $1.995 \mathrm{E}-02$ & $5.627 \mathrm{E}-02$ & $2.302 \mathrm{E}-02$ \\
\hline [S III] $\lambda \lambda 9069.9532 \ldots \ldots \ldots . .$. & $3.376 \mathrm{E}-01$ & $2.605 \mathrm{E}+00$ & $5.813 \mathrm{E}+00$ & $2.907 \mathrm{E}+00$ \\
\hline $\log U_{0} \ldots \ldots \ldots \ldots \ldots \ldots$ & -1.0 & -2.0 & -3.0 & -4.0 \\
\hline \multicolumn{5}{|c|}{$\alpha=-1.4$} \\
\hline$[\mathrm{O}$ II $] \lambda \lambda 3727,3729 \ldots \ldots \ldots$. & $8.820 \mathrm{E}-02$ & $8.958 \mathrm{E}-01$ & $3.295 \mathrm{E}+00$ & $2.420 \mathrm{E}+00$ \\
\hline$[\mathrm{S}$ пा] $\lambda \lambda 4067,4076 \ldots \ldots \ldots . .$. & $1.555 \mathrm{E}-03$ & $2.114 \mathrm{E}-02$ & $6.867 \mathrm{E}-02$ & $1.150 \mathrm{E}-01$ \\
\hline 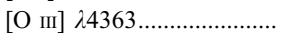 & $8.313 \mathrm{E}-02$ & $7.532 \mathrm{E}-02$ & $1.171 \mathrm{E}-02$ & $1.567 \mathrm{E}-04$ \\
\hline 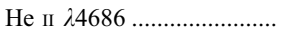 & $2.217 \mathrm{E}-01$ & $2.521 \mathrm{E}-01$ & $2.305 \mathrm{E}-01$ & $1.029 \mathrm{E}-01$ \\
\hline$[\mathrm{O}$ III] $\lambda 4959 \ldots \ldots \ldots \ldots \ldots \ldots \ldots$ & $3.381 \mathrm{E}+00$ & $4.278 \mathrm{E}+00$ & $1.520 \mathrm{E}+00$ & $4.310 \mathrm{E}-02$ \\
\hline 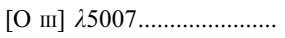 & $9.738 \mathrm{E}+00$ & $1.232 \mathrm{E}+01$ & $4.377 \mathrm{E}+00$ & $1.241 \mathrm{E}-01$ \\
\hline$\left[\mathrm{N}_{\mathrm{I}}\right] \lambda 5200 \ldots \ldots \ldots \ldots \ldots \ldots \ldots$ & $3.245 \mathrm{E}-04$ & $8.375 \mathrm{E}-02$ & $1.801 \mathrm{E}-01$ & $1.190 \mathrm{E}-01$ \\
\hline 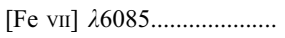 & $1.614 \mathrm{E}-01$ & $6.441 \mathrm{E}-03$ & $4.610 \mathrm{E}-06$ & $0.000 \mathrm{E}+00$ \\
\hline$\left[\mathrm{O}_{\mathrm{I}}\right] \mathrm{\lambda}, 26300 \ldots \ldots \ldots \ldots \ldots \ldots \ldots$ & $2.370 \mathrm{E}-03$ & $2.079 \mathrm{E}-01$ & $4.740 \mathrm{E}-01$ & $4.373 \mathrm{E}-01$ \\
\hline 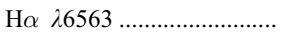 & $2.901 \mathrm{E}+00$ & $2.897 \mathrm{E}+00$ & $2.944 \mathrm{E}+00$ & $3.026 \mathrm{E}+00$ \\
\hline$[\mathrm{N}$ II] $\lambda 6583 \ldots \ldots \ldots \ldots \ldots \ldots$ & $6.188 \mathrm{E}-02$ & $7.082 \mathrm{E}-01$ & $3.199 \mathrm{E}+00$ & $3.465 \mathrm{E}+00$ \\
\hline$\left[\mathrm{S}_{\mathrm{II}}\right] \lambda \lambda 6717,6730 \ldots \ldots \ldots . .$. & $2.208 \mathrm{E}-02$ & $4.185 \mathrm{E}-01$ & $1.288 \mathrm{E}+00$ & $2.761 \mathrm{E}+00$ \\
\hline$[\mathrm{O}$ II] $\lambda \lambda 7318,7324 \ldots \ldots \ldots .$. & $1.285 \mathrm{E}-03$ & $1.187 \mathrm{E}-02$ & $3.579 \mathrm{E}-02$ & $1.713 \mathrm{E}-02$ \\
\hline [S III] $\lambda \lambda 9069.9532 \ldots \ldots \ldots . .$. & $3.225 \mathrm{E}-01$ & $2.217 \mathrm{E}+00$ & $4.687 \mathrm{E}+00$ & $2.323 \mathrm{E}+00$ \\
\hline \multicolumn{5}{|c|}{$\alpha=-1.7$} \\
\hline [O II] $\lambda \lambda 3727,3729 \ldots \ldots \ldots$. & $6.891 \mathrm{E}-02$ & $5.141 \mathrm{E}-01$ & $2.087 \mathrm{E}+00$ & $1.740 \mathrm{E}+00$ \\
\hline$[\mathrm{S}$ II $] \lambda \lambda 4067,4076 \ldots \ldots \ldots . .$. & $1.161 \mathrm{E}-03$ & $1.047 \mathrm{E}-02$ & $4.383 \mathrm{E}-02$ & $8.480 \mathrm{E}-02$ \\
\hline 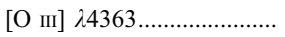 & $3.510 \mathrm{E}-02$ & $2.779 \mathrm{E}-02$ & $5.156 \mathrm{E}-03$ & $8.686 \mathrm{E}-05$ \\
\hline 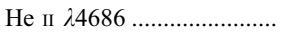 & $1.530 \mathrm{E}-01$ & $1.831 \mathrm{E}-01$ & $1.691 \mathrm{E}-01$ & $7.348 \mathrm{E}-02$ \\
\hline 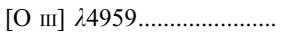 & $2.087 \mathrm{E}+00$ & $2.533 \mathrm{E}+00$ & $1.004 \mathrm{E}+00$ & $3.129 \mathrm{E}-02$ \\
\hline 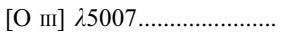 & $6.012 \mathrm{E}+00$ & $7.294 \mathrm{E}+00$ & $2.892 \mathrm{E}+00$ & $9.012 \mathrm{E}-02$ \\
\hline$\left[\mathrm{N}_{\mathrm{I}}\right] \lambda 5200 \ldots \ldots \ldots \ldots \ldots \ldots \ldots$ & $2.167 \mathrm{E}-04$ & $2.666 \mathrm{E}-02$ & $6.014 \mathrm{E}-02$ & $6.335 \mathrm{E}-02$ \\
\hline 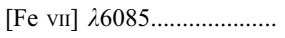 & $8.219 \mathrm{E}-02$ & $1.184 \mathrm{E}-03$ & $0.000 \mathrm{E}+00$ & $0.000 \mathrm{E}+00$ \\
\hline 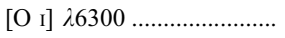 & $1.806 \mathrm{E}-03$ & $6.854 \mathrm{E}-02$ & $1.784 \mathrm{E}-01$ & $2.515 \mathrm{E}-01$ \\
\hline 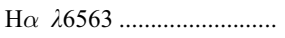 & $2.938 \mathrm{E}+00$ & $2.928 \mathrm{E}+00$ & $2.953 \mathrm{E}+00$ & $3.009 \mathrm{E}+00$ \\
\hline$[\mathrm{N}$ II] $\lambda 6583 \ldots \ldots \ldots \ldots \ldots \ldots . . . . . . . .$. & $5.529 \mathrm{E}-02$ & $4.793 \mathrm{E}-01$ & $2.428 \mathrm{E}+00$ & $2.818 \mathrm{E}+00$ \\
\hline$[\mathrm{S}$ II] $\lambda \lambda 6717,6730 \ldots \ldots \ldots . .$. & $1.681 \mathrm{E}-02$ & $2.143 \mathrm{E}-01$ & $8.737 \mathrm{E}-01$ & $2.182 \mathrm{E}+00$ \\
\hline$[\mathrm{O}$ II] $\lambda \lambda 7318,7324 \ldots \ldots \ldots . .$. & $8.857 \mathrm{E}-04$ & $5.684 \mathrm{E}-03$ & $1.861 \mathrm{E}-02$ & $1.068 \mathrm{E}-02$ \\
\hline$[\mathrm{S}$ III] $\lambda \lambda 9069.9532 \ldots \ldots \ldots \ldots$ & $3.046 \mathrm{E}-01$ & $1.793 \mathrm{E}+00$ & $3.495 \mathrm{E}+00$ & $1.740 \mathrm{E}+00$ \\
\hline \multicolumn{5}{|c|}{$\alpha=-2.0$} \\
\hline [O II] $\lambda \lambda 3727,3729 \ldots \ldots \ldots . .$. & $4.727 \mathrm{E}-02$ & $3.185 \mathrm{E}-01$ & $1.405 \mathrm{E}+00$ & $1.246 \mathrm{E}+00$ \\
\hline$[\mathrm{S}$ пा] $\lambda \lambda 4067,4076 \ldots \ldots \ldots . .$. & $7.275 \mathrm{E}-04$ & $5.219 \mathrm{E}-03$ & $2.923 \mathrm{E}-02$ & $6.282 \mathrm{E}-02$ \\
\hline 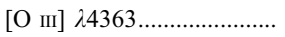 & $1.529 \mathrm{E}-02$ & $9.485 \mathrm{E}-03$ & $2.277 \mathrm{E}-03$ & $4.572 \mathrm{E}-05$ \\
\hline 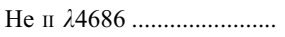 & $1.071 \mathrm{E}-01$ & $1.319 \mathrm{E}-01$ & $1.192 \mathrm{E}-01$ & $4.973 \mathrm{E}-02$ \\
\hline 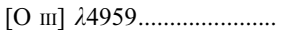 & $1.242 \mathrm{E}+00$ & $1.468 \mathrm{E}+00$ & $6.500 \mathrm{E}-01$ & $2.132 \mathrm{E}-02$ \\
\hline 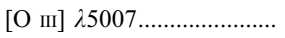 & $3.576 \mathrm{E}+00$ & $4.227 \mathrm{E}+00$ & $1.872 \mathrm{E}+00$ & $6.140 \mathrm{E}-02$ \\
\hline$\left[\mathrm{N}_{\mathrm{I}}\right] \lambda 5200 \ldots \ldots \ldots \ldots \ldots \ldots \ldots$ & $1.708 \mathrm{E}-04$ & $4.548 \mathrm{E}-03$ & $1.735 \mathrm{E}-02$ & $3.721 \mathrm{E}-02$ \\
\hline 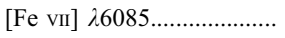 & $3.124 \mathrm{E}-02$ & $1.726 \mathrm{E}-04$ & $0.000 \mathrm{E}+00$ & $0.000 \mathrm{E}+00$ \\
\hline 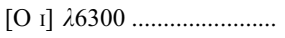 & $1.283 \mathrm{E}-03$ & $1.598 \mathrm{E}-02$ & $6.495 \mathrm{E}-02$ & $1.536 \mathrm{E}-01$ \\
\hline 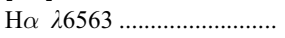 & $2.973 \mathrm{E}+00$ & $2.959 \mathrm{E}+00$ & $2.966 \mathrm{E}+00$ & $3.008 \mathrm{E}+00$ \\
\hline 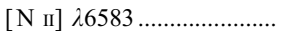 & $4.665 \mathrm{E}-02$ & $3.693 \mathrm{E}-01$ & $1.939 \mathrm{E}+00$ & $2.279 \mathrm{E}+00$ \\
\hline$[\mathrm{S}$ II] $\lambda \lambda 6717,6730 \ldots \ldots \ldots . .$. & $1.194 \mathrm{E}-02$ & $1.086 \mathrm{E}-01$ & $6.278 \mathrm{E}-01$ & $1.745 \mathrm{E}+00$ \\
\hline$[\mathrm{O}$ II] $\lambda \lambda 7318,7324 \ldots \ldots \ldots . .$. & $4.979 \mathrm{E}-04$ & $2.813 \mathrm{E}-03$ & $1.037 \mathrm{E}-02$ & $6.643 \mathrm{E}-03$ \\
\hline [S III] $\lambda \lambda 9069.9532 \ldots \ldots \ldots . .$. & $2.866 \mathrm{E}-01$ & $1.496 \mathrm{E}+00$ & $2.731 \mathrm{E}+00$ & $1.355 \mathrm{E}+00$ \\
\hline
\end{tabular}


TABLE 42

Undepleted Dust-free Visible Line Ratios with Respect to $\mathrm{H} \beta, 4 Z_{\odot}$

\begin{tabular}{|c|c|c|c|c|}
\hline \multirow[b]{2}{*}{ LiNE } & \multicolumn{4}{|c|}{$\log U_{0}$} \\
\hline & -1.0 & -2.0 & -3.0 & -4.0 \\
\hline \multicolumn{5}{|c|}{$\alpha=-1.2$} \\
\hline$[\mathrm{O}$ II] $\lambda \lambda 3727,3729 \ldots \ldots \ldots$ & $1.949 \mathrm{E}-02$ & $6.496 \mathrm{E}-01$ & $1.818 \mathrm{E}+00$ & $7.229 \mathrm{E}-01$ \\
\hline$[\mathrm{S}$ II] $\lambda \lambda 4067,4076 \ldots \ldots \ldots . .$. & $2.530 \mathrm{E}-04$ & $2.207 \mathrm{E}-02$ & $5.401 \mathrm{E}-02$ & $4.302 \mathrm{E}-02$ \\
\hline$[\mathrm{O}$ III] $\lambda 4363 \ldots \ldots \ldots \ldots \ldots \ldots \ldots$ & $9.184 \mathrm{E}-02$ & $7.188 \mathrm{E}-02$ & $3.350 \mathrm{E}-03$ & $1.830 \mathrm{E}-05$ \\
\hline He II $\lambda 4686 \ldots \ldots$. & $2.812 \mathrm{E}-01$ & $3.234 \mathrm{E}-01$ & $2.980 \mathrm{E}-01$ & $1.281 \mathrm{E}-01$ \\
\hline$[\mathrm{O}$ III] $\lambda 4959 \ldots$ & $2.539 \mathrm{E}+00$ & $4.409 \mathrm{E}+00$ & $1.055 \mathrm{E}+00$ & $2.018 \mathrm{E}-02$ \\
\hline$[\mathrm{O}$ III] $\lambda 5007 \ldots \ldots \ldots \ldots \ldots \ldots \ldots$ & $7.312 \mathrm{E}+00$ & $1.270 \mathrm{E}+01$ & $3.039 \mathrm{E}+00$ & $5.811 \mathrm{E}-02$ \\
\hline 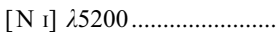 & $1.198 \mathrm{E}-04$ & $1.963 \mathrm{E}-01$ & $3.611 \mathrm{E}-01$ & $7.695 \mathrm{E}-02$ \\
\hline$[\mathrm{Fe}$ VII $] \lambda 6085 \ldots \ldots \ldots \ldots \ldots \ldots$ & $2.616 \mathrm{E}-01$ & $1.301 \mathrm{E}-02$ & $4.379 \mathrm{E}-06$ & $0.000 \mathrm{E}+00$ \\
\hline$\left[\mathrm{O}_{\mathrm{I}}\right] \lambda 6300 \ldots \ldots \ldots \ldots \ldots \ldots \ldots$ & $4.978 \mathrm{E}-04$ & $2.663 \mathrm{E}-01$ & $5.105 \mathrm{E}-01$ & $1.885 \mathrm{E}-01$ \\
\hline $\mathrm{H} \alpha \quad \lambda 6563 \ldots \ldots \ldots \ldots \ldots \ldots \ldots \ldots \ldots \ldots$ & $3.017 \mathrm{E}+00$ & $2.948 \mathrm{E}+00$ & $3.013 \mathrm{E}+00$ & $3.152 \mathrm{E}+00$ \\
\hline$[\mathrm{N}$ II $] \lambda 6583 \ldots \ldots \ldots \ldots \ldots \ldots \ldots$ & $5.335 \mathrm{E}-02$ & $1.434 \mathrm{E}+00$ & $5.710 \mathrm{E}+00$ & $4.234 \mathrm{E}+00$ \\
\hline$[\mathrm{S}$ II] $\lambda \lambda 6717,6730 \ldots \ldots \ldots . .$. & $7.600 \mathrm{E}-03$ & $5.413 \mathrm{E}-01$ & $1.423 \mathrm{E}+00$ & $1.763 \mathrm{E}+00$ \\
\hline$[\mathrm{O}$ II] $\lambda \lambda 7318,7324 \ldots \ldots \ldots .$. & $2.905 \mathrm{E}-04$ & $6.646 \mathrm{E}-03$ & $1.169 \mathrm{E}-02$ & $2.353 \mathrm{E}-03$ \\
\hline$[\mathrm{S}$ III $] \lambda \lambda 9069.9532 \ldots \ldots \ldots \ldots$ & $1.993 \mathrm{E}-01$ & $2.605 \mathrm{E}+00$ & $5.333 \mathrm{E}+00$ & $1.909 \mathrm{E}+00$ \\
\hline \multicolumn{5}{|c|}{$\alpha=-1.4$} \\
\hline$[\mathrm{O}$ пा $] \lambda \lambda 3727,3729 \ldots \ldots \ldots . .$. & $1.141 \mathrm{E}-02$ & $2.962 \mathrm{E}-01$ & $9.829 \mathrm{E}-01$ & $5.133 \mathrm{E}-01$ \\
\hline$[\mathrm{S}$ II] $\lambda \lambda 4067,4076 \ldots \ldots \ldots \ldots$ & $1.059 \mathrm{E}-04$ & $1.013 \mathrm{E}-02$ & $2.941 \mathrm{E}-02$ & $3.132 \mathrm{E}-02$ \\
\hline 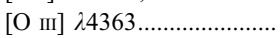 & $4.933 \mathrm{E}-02$ & $2.986 \mathrm{E}-02$ & $1.419 \mathrm{E}-03$ & $1.049 \mathrm{E}-05$ \\
\hline 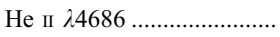 & $2.171 \mathrm{E}-01$ & $2.642 \mathrm{E}-01$ & $2.462 \mathrm{E}-01$ & $1.053 \mathrm{E}-01$ \\
\hline 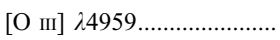 & $1.634 \mathrm{E}+00$ & $2.504 \mathrm{E}+00$ & $6.859 \mathrm{E}-01$ & $1.542 \mathrm{E}-02$ \\
\hline$[\mathrm{O}$ III] $\lambda 5007 \ldots \ldots \ldots \ldots \ldots \ldots$ & $4.706 \mathrm{E}+00$ & $7.212 \mathrm{E}+00$ & $1.976 \mathrm{E}+00$ & $4.442 \mathrm{E}-02$ \\
\hline$\left[\mathrm{N}_{\mathrm{I}}\right] \lambda 5200 \ldots \ldots \ldots \ldots \ldots \ldots \ldots \ldots \ldots$ & $4.943 \mathrm{E}-05$ & $8.729 \mathrm{E}-02$ & $1.376 \mathrm{E}-01$ & $4.227 \mathrm{E}-02$ \\
\hline$[\mathrm{Fe}$ vII $] \lambda 6085 \ldots \ldots \ldots \ldots \ldots \ldots$ & $1.806 \mathrm{E}-01$ & $4.088 \mathrm{E}-03$ & $1.181 \mathrm{E}-06$ & $0.000 \mathrm{E}+00$ \\
\hline$\left[\mathrm{O}_{\mathrm{I}}\right], \lambda 6300 \ldots \ldots \ldots \ldots \ldots \ldots$ & $1.735 \mathrm{E}-04$ & $1.140 \mathrm{E}-01$ & $2.030 \mathrm{E}-01$ & $1.145 \mathrm{E}-01$ \\
\hline $\mathrm{H} \alpha \quad \lambda 6563 \ldots \ldots \ldots \ldots \ldots \ldots \ldots \ldots$ & $3.061 \mathrm{E}+00$ & $2.986 \mathrm{E}+00$ & $3.026 \mathrm{E}+00$ & $3.127 \mathrm{E}+00$ \\
\hline$\left[\mathrm{N}_{\mathrm{II}}\right] \lambda 6583 \ldots \ldots \ldots \ldots \ldots \ldots \ldots \ldots \ldots \ldots$ & $3.734 \mathrm{E}-02$ & $8.325 \mathrm{E}-01$ & $3.971 \mathrm{E}+00$ & $3.457 \mathrm{E}+00$ \\
\hline$[\mathrm{S}$ II] $\lambda \lambda 6717,6730 \ldots \ldots \ldots . .$. & $4.132 \mathrm{E}-03$ & $2.931 \mathrm{E}-01$ & $8.965 \mathrm{E}-01$ & $1.393 \mathrm{E}+00$ \\
\hline$[\mathrm{O}$ II] $\lambda \lambda 7318,7324 \ldots \ldots \ldots .$. & $1.723 \mathrm{E}-04$ & $2.574 \mathrm{E}-03$ & $4.894 \mathrm{E}-03$ & $1.434 \mathrm{E}-03$ \\
\hline$[\mathrm{S}$ III $] \lambda \lambda 9069.9532 \ldots \ldots \ldots \ldots$ & $1.501 \mathrm{E}-01$ & $1.876 \mathrm{E}+00$ & $3.831 \mathrm{E}+00$ & $1.483 \mathrm{E}+00$ \\
\hline \multicolumn{5}{|c|}{$\alpha=-1.7$} \\
\hline$[\mathrm{O}$ II] $\lambda \lambda 33727,3729 \ldots \ldots \ldots$. & $1.275 \mathrm{E}-02$ & $1.043 \mathrm{E}-01$ & $4.355 \mathrm{E}-01$ & $3.099 \mathrm{E}-01$ \\
\hline$[\mathrm{S}$ II] $\lambda \lambda 4067,4076 \ldots \ldots \ldots . .$. & $6.730 \mathrm{E}-05$ & $2.466 \mathrm{E}-03$ & $1.229 \mathrm{E}-02$ & $1.997 \mathrm{E}-02$ \\
\hline$[\mathrm{O}$ III] $\lambda 4363 \ldots \ldots \ldots \ldots \ldots \ldots \ldots$ & $1.943 \mathrm{E}-02$ & $6.252 \mathrm{E}-03$ & $3.639 \mathrm{E}-04$ & $4.355 \mathrm{E}-06$ \\
\hline 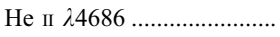 & $1.478 \mathrm{E}-01$ & $1.949 \mathrm{E}-01$ & $1.770 \mathrm{E}-01$ & $7.354 \mathrm{E}-02$ \\
\hline 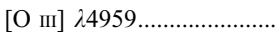 & $8.106 \mathrm{E}-01$ & $9.547 \mathrm{E}-01$ & $3.409 \mathrm{E}-01$ & $9.646 \mathrm{E}-03$ \\
\hline$[\mathrm{O}$ III $] \lambda 5007 \ldots \ldots \ldots \ldots \ldots \ldots \ldots$ & $2.335 \mathrm{E}+00$ & $2.750 \mathrm{E}+00$ & $9.819 \mathrm{E}-01$ & $2.778 \mathrm{E}-02$ \\
\hline$\left[\mathrm{N}_{\mathrm{I}}\right] \lambda 5200 \ldots \ldots \ldots \ldots \ldots \ldots \ldots \ldots \ldots \ldots \ldots \ldots$ & $2.714 \mathrm{E}-05$ & $1.166 \mathrm{E}-02$ & $1.953 \mathrm{E}-02$ & $2.047 \mathrm{E}-02$ \\
\hline$[\mathrm{Fe}$ vII $] \lambda 6085 \ldots \ldots \ldots \ldots \ldots \ldots$ & $7.951 \mathrm{E}-02$ & $5.079 \mathrm{E}-04$ & $0.000 \mathrm{E}+00$ & $0.000 \mathrm{E}+00$ \\
\hline$\left[\mathrm{O}_{\mathrm{I}}\right] \lambda 6300 \ldots \ldots \ldots \ldots \ldots \ldots \ldots$ & $8.299 \mathrm{E}-05$ & $1.632 \mathrm{E}-02$ & $3.711 \mathrm{E}-02$ & $6.052 \mathrm{E}-02$ \\
\hline 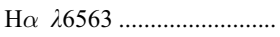 & $3.127 \mathrm{E}+00$ & $3.043 \mathrm{E}+00$ & $3.045 \mathrm{E}+00$ & $3.109 \mathrm{E}+00$ \\
\hline$[\mathrm{N}$ II $] \lambda 6583 \ldots \ldots \ldots \ldots \ldots \ldots \ldots$ & $7.372 \mathrm{E}-02$ & $4.762 \mathrm{E}-01$ & $2.514 \mathrm{E}+00$ & $2.548 \mathrm{E}+00$ \\
\hline$[\mathrm{S}$ II $] \lambda \lambda 6717,6730 \ldots \ldots \ldots . .$. & $3.301 \mathrm{E}-03$ & $9.814 \mathrm{E}-02$ & $4.658 \mathrm{E}-01$ & $1.009 \mathrm{E}+00$ \\
\hline$[\mathrm{O}$ пा] $\lambda \lambda 7318,7324 \ldots \ldots \ldots .$. & $1.022 \mathrm{E}-04$ & $6.394 \mathrm{E}-04$ & $1.506 \mathrm{E}-03$ & $6.930 \mathrm{E}-04$ \\
\hline [S III] $\lambda \lambda 9069.9532 \ldots \ldots \ldots \ldots$ & $1.292 \mathrm{E}-01$ & $1.131 \mathrm{E}+00$ & $2.440 \mathrm{E}+00$ & $1.065 \mathrm{E}+00$ \\
\hline \multicolumn{5}{|c|}{$\alpha=-2.0$} \\
\hline$[\mathrm{O}$ пा $] \lambda \lambda 3727,3729 \ldots \ldots \ldots$. & $3.983 \mathrm{E}-02$ & $7.153 \mathrm{E}-02$ & $2.293 \mathrm{E}-01$ & $1.899 \mathrm{E}-01$ \\
\hline$[\mathrm{S}$ II] $\lambda \lambda 4067,4076 \ldots \ldots \ldots . .$. & $1.149 \mathrm{E}-04$ & $1.157 \mathrm{E}-03$ & $6.912 \mathrm{E}-03$ & $1.306 \mathrm{E}-02$ \\
\hline 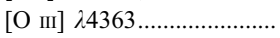 & $7.349 \mathrm{E}-03$ & $8.841 \mathrm{E}-04$ & $9.080 \mathrm{E}-05$ & $1.785 \mathrm{E}-06$ \\
\hline He II $\lambda 4686 \ldots \ldots \ldots \ldots \ldots \ldots \ldots$ & $1.017 \mathrm{E}-01$ & $1.401 \mathrm{E}-01$ & $1.228 \mathrm{E}-01$ & $4.859 \mathrm{E}-02$ \\
\hline$[\mathrm{O}$ III] $\lambda 49959 \ldots \ldots \ldots \ldots \ldots \ldots \ldots \ldots \ldots \ldots \ldots$ & $3.848 \mathrm{E}-01$ & $3.114 \mathrm{E}-01$ & $1.655 \mathrm{E}-01$ & $5.769 \mathrm{E}-03$ \\
\hline$[\mathrm{O}$ III] $\lambda 5007 \ldots \ldots \ldots \ldots \ldots \ldots \ldots$ & $1.108 \mathrm{E}+00$ & $8.970 \mathrm{E}-01$ & $4.768 \mathrm{E}-01$ & $1.662 \mathrm{E}-02$ \\
\hline$\left[\mathrm{N}_{\mathrm{I}}\right] \lambda 5200 \ldots \ldots \ldots \ldots \ldots \ldots \ldots \ldots \ldots \ldots \ldots$ & $3.295 \mathrm{E}-05$ & $3.884 \mathrm{E}-04$ & $3.226 \mathrm{E}-03$ & $1.157 \mathrm{E}-02$ \\
\hline$[\mathrm{Fe}$ VII $] \lambda 6085 \ldots \ldots \ldots \ldots \ldots \ldots$ & $2.567 \mathrm{E}-02$ & $4.265 \mathrm{E}-05$ & $0.000 \mathrm{E}+00$ & $0.000 \mathrm{E}+00$ \\
\hline$\left[\mathrm{O}_{\mathrm{I}}\right] \lambda 6300 \ldots \ldots \ldots \ldots \ldots \ldots \ldots$ & $8.866 \mathrm{E}-05$ & $1.092 \mathrm{E}-03$ & $9.255 \mathrm{E}-03$ & $3.542 \mathrm{E}-02$ \\
\hline 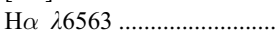 & $3.178 \mathrm{E}+00$ & $3.093 \mathrm{E}+00$ & $3.063 \mathrm{E}+00$ & $3.109 \mathrm{E}+00$ \\
\hline$[\mathrm{N}$ II $] \lambda 6583 \ldots \ldots \ldots \ldots \ldots \ldots \ldots$ & $2.721 \mathrm{E}-01$ & $4.636 \mathrm{E}-01$ & $1.771 \mathrm{E}+00$ & $1.887 \mathrm{E}+00$ \\
\hline$\left[\mathrm{S}_{\mathrm{II}}\right] \lambda \lambda 6717,6730 \ldots \ldots \ldots \ldots$ & $5.385 \mathrm{E}-03$ & $4.891 \mathrm{E}-02$ & $3.042 \mathrm{E}-01$ & $7.540 \mathrm{E}-01$ \\
\hline$[\mathrm{O}$ пा $] \lambda \lambda 7318,7324 \ldots \ldots \ldots .$. & $1.320 \mathrm{E}-04$ & $2.480 \mathrm{E}-04$ & $5.687 \mathrm{E}-04$ & $3.435 \mathrm{E}-04$ \\
\hline [S III] $\lambda \lambda 9069.9532 \ldots \ldots \ldots \ldots$ & $2.480 \mathrm{E}-01$ & $7.651 \mathrm{E}-01$ & $1.691 \mathrm{E}+00$ & $7.861 \mathrm{E}-01$ \\
\hline
\end{tabular}


TABLE 43

Undepleted Dust-free IR Line Ratios with Respect to $\mathrm{H} \beta, 0.25 Z_{\odot}$

\begin{tabular}{|c|c|c|c|c|}
\hline \multirow[b]{2}{*}{ LiNE } & \multicolumn{4}{|c|}{$\log U_{0}$} \\
\hline & -1.0 & -2.0 & -3.0 & -4.0 \\
\hline \multicolumn{5}{|c|}{$\alpha=-1.2$} \\
\hline 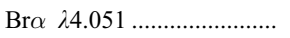 & $6.039 \mathrm{E}-02$ & $6.596 \mathrm{E}-02$ & $7.452 \mathrm{E}-02$ & $8.328 \mathrm{E}-02$ \\
\hline 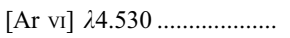 & $8.136 \mathrm{E}-03$ & $8.660 \mathrm{E}-04$ & $0.000 \mathrm{E}+00$ & $0.000 \mathrm{E}+00$ \\
\hline 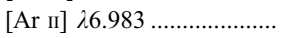 & $1.043 \mathrm{E}-03$ & $6.903 \mathrm{E}-03$ & $2.136 \mathrm{E}-02$ & $4.531 \mathrm{E}-02$ \\
\hline$[\mathrm{Ar}$ III] $\lambda 8.983 \ldots \ldots \ldots \ldots \ldots \ldots . . . . . . .$. & $3.066 \mathrm{E}-01$ & $3.678 \mathrm{E}-02$ & $3.714 \mathrm{E}-05$ & $0.000 \mathrm{E}+00$ \\
\hline$[\mathrm{Ne} v \mathrm{vI}] \lambda 7.652 \ldots \ldots \ldots \ldots \ldots \ldots$ & $7.238 \mathrm{E}-03$ & $4.091 \mathrm{E}-02$ & $6.353 \mathrm{E}-02$ & $4.014 \mathrm{E}-02$ \\
\hline 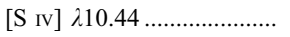 & $8.303 \mathrm{E}-01$ & $7.548 \mathrm{E}-01$ & $2.187 \mathrm{E}-01$ & $2.993 \mathrm{E}-03$ \\
\hline$[\mathrm{Ne}$ II $] \lambda 12.8 \ldots \ldots \ldots \ldots \ldots \ldots \ldots$ & $1.261 \mathrm{E}-02$ & $8.822 \mathrm{E}-02$ & $3.694 \mathrm{E}-01$ & $8.580 \mathrm{E}-01$ \\
\hline 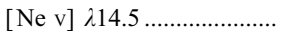 & $6.973 \mathrm{E}-02$ & $6.242 \mathrm{E}-02$ & $6.067 \mathrm{E}-04$ & $0.000 \mathrm{E}+00$ \\
\hline$[\mathrm{Ne}$ III $] \lambda 15.5 \ldots \ldots \ldots \ldots \ldots \ldots \ldots$ & $5.475 \mathrm{E}-01$ & $6.571 \mathrm{E}-01$ & $9.211 \mathrm{E}-01$ & $9.143 \mathrm{E}-01$ \\
\hline 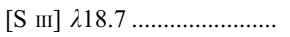 & $2.842 \mathrm{E}-02$ & $1.696 \mathrm{E}-01$ & $4.991 \mathrm{E}-01$ & $5.304 \mathrm{E}-01$ \\
\hline [O Iv] $\lambda 25.9 \ldots \ldots \ldots \ldots \ldots \ldots \ldots . .$. & $1.038 \mathrm{E}+00$ & $1.601 \mathrm{E}+00$ & $4.915 \mathrm{E}-01$ & $1.543 \mathrm{E}-03$ \\
\hline \multicolumn{5}{|c|}{$\alpha=-1.4$} \\
\hline 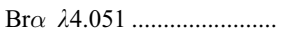 & $6.238 \mathrm{E}-02$ & $6.610 \mathrm{E}-02$ & $7.275 \mathrm{E}-02$ & $7.951 \mathrm{E}-02$ \\
\hline$[\mathrm{Ar} \mathrm{vI}] \lambda 4.530 \ldots \ldots \ldots \ldots \ldots \ldots \ldots$ & $6.341 \mathrm{E}-03$ & $4.854 \mathrm{E}-04$ & $0.000 \mathrm{E}+00$ & $0.000 \mathrm{E}+00$ \\
\hline$[\mathrm{Ar}$ II $] \lambda 6.983 \ldots \ldots \ldots \ldots \ldots \ldots$ & $7.575 \mathrm{E}-04$ & $5.059 \mathrm{E}-03$ & $1.641 \mathrm{E}-02$ & $3.876 \mathrm{E}-02$ \\
\hline$[\mathrm{Ar}$ III] $\lambda 8.983 \ldots \ldots \ldots \ldots \ldots \ldots$ & $2.169 \mathrm{E}-01$ & $1.813 \mathrm{E}-02$ & $1.662 \mathrm{E}-05$ & $0.000 \mathrm{E}+00$ \\
\hline$[\mathrm{Ne} v \mathrm{vI}] \lambda 7.652 \ldots \ldots \ldots \ldots \ldots \ldots$ & $7.261 \mathrm{E}-03$ & $4.029 \mathrm{E}-02$ & $6.146 \mathrm{E}-02$ & $3.920 \mathrm{E}-02$ \\
\hline [S IV] $\lambda 10.44 \ldots \ldots \ldots \ldots \ldots \ldots \ldots$ & $7.110 \mathrm{E}-01$ & $6.289 \mathrm{E}-01$ & $1.412 \mathrm{E}-01$ & $1.860 \mathrm{E}-03$ \\
\hline$[\mathrm{Ne}$ II] $\lambda 12.8 \ldots \ldots \ldots \ldots \ldots \ldots . .$. & $1.205 \mathrm{E}-02$ & $8.232 \mathrm{E}-02$ & $3.337 \mathrm{E}-01$ & $7.932 \mathrm{E}-01$ \\
\hline$[\mathrm{Ne} v] \lambda 14.5 \ldots \ldots \ldots \ldots \ldots \ldots \ldots \ldots \ldots \ldots \ldots$ & $6.477 \mathrm{E}-02$ & $4.548 \mathrm{E}-02$ & $4.154 \mathrm{E}-04$ & $0.000 \mathrm{E}+00$ \\
\hline$[\mathrm{Ne}$ III] $\lambda 15.5 \ldots \ldots \ldots \ldots \ldots \ldots \ldots . .$. & $5.413 \mathrm{E}-01$ & $6.075 \mathrm{E}-01$ & $7.546 \mathrm{E}-01$ & $7.240 \mathrm{E}-01$ \\
\hline 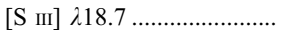 & $1.815 \mathrm{E}-02$ & $1.177 \mathrm{E}-01$ & $3.319 \mathrm{E}-01$ & $3.780 \mathrm{E}-01$ \\
\hline 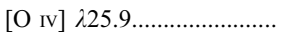 & $3.058 \mathrm{E}-01$ & $4.753 \mathrm{E}-01$ & $1.372 \mathrm{E}-01$ & $4.545 \mathrm{E}-04$ \\
\hline \multicolumn{5}{|c|}{$\alpha=-1.7$} \\
\hline 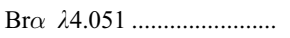 & $6.674 \mathrm{E}-02$ & $6.917 \mathrm{E}-02$ & $7.477 \mathrm{E}-02$ & $8.101 \mathrm{E}-02$ \\
\hline$[\mathrm{Ar}$ vI] $\lambda 4.530 \ldots \ldots \ldots \ldots \ldots . . . . . . . .$. & $4.017 \mathrm{E}-03$ & $1.709 \mathrm{E}-04$ & $0.000 \mathrm{E}+00$ & $0.000 \mathrm{E}+00$ \\
\hline 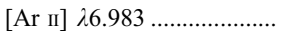 & $4.105 \mathrm{E}-04$ & $2.805 \mathrm{E}-03$ & $9.344 \mathrm{E}-03$ & $2.579 \mathrm{E}-02$ \\
\hline$[\mathrm{Ar}$ III] $\lambda 8.983 \ldots \ldots \ldots \ldots \ldots . . . . . . . . .$. & $1.105 \mathrm{E}-01$ & $4.978 \mathrm{E}-03$ & $4.039 \mathrm{E}-06$ & $0.000 \mathrm{E}+00$ \\
\hline$[\mathrm{Ne}$ vI $] \lambda 7.652 \ldots \ldots \ldots \ldots \ldots . . . . . . .$. & $8.884 \mathrm{E}-03$ & $4.655 \mathrm{E}-02$ & $6.765 \mathrm{E}-02$ & $4.191 \mathrm{E}-02$ \\
\hline [S IV] $\lambda 10.44 \ldots \ldots \ldots \ldots \ldots \ldots . .$. & $1.130 \mathrm{E}+00$ & $9.354 \mathrm{E}-01$ & $1.568 \mathrm{E}-01$ & $1.889 \mathrm{E}-03$ \\
\hline$[\mathrm{Ne}$ II] $\lambda 12.8 \ldots \ldots \ldots \ldots \ldots \ldots \ldots$ & $8.269 \mathrm{E}-03$ & $5.307 \mathrm{E}-02$ & $1.853 \mathrm{E}-01$ & $4.247 \mathrm{E}-01$ \\
\hline$[\mathrm{Ne} \mathrm{v}] \lambda 14.5 \ldots \ldots \ldots \ldots \ldots \ldots \ldots \ldots \ldots \ldots \ldots \ldots \ldots$ & $5.090 \mathrm{E}-02$ & $2.109 \mathrm{E}-02$ & $1.739 \mathrm{E}-04$ & $0.000 \mathrm{E}+00$ \\
\hline 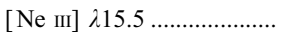 & $6.621 \mathrm{E}-01$ & $7.004 \mathrm{E}-01$ & $7.247 \mathrm{E}-01$ & $5.831 \mathrm{E}-01$ \\
\hline$[\mathrm{S}$ III] $\lambda 18.7 \ldots \ldots \ldots \ldots \ldots \ldots \ldots$ & $2.436 \mathrm{E}-02$ & $1.614 \mathrm{E}-01$ & $4.038 \mathrm{E}-01$ & $3.262 \mathrm{E}-01$ \\
\hline [O Iv ] $225.9 \ldots \ldots \ldots \ldots \ldots \ldots \ldots$ & $5.945 \mathrm{E}-01$ & $9.559 \mathrm{E}-01$ & $2.819 \mathrm{E}-01$ & $9.554 \mathrm{E}-04$ \\
\hline \multicolumn{5}{|c|}{$\alpha=-2.0$} \\
\hline 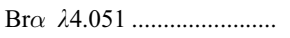 & $6.933 \mathrm{E}-02$ & $7.091 \mathrm{E}-02$ & $7.518 \mathrm{E}-02$ & $8.060 \mathrm{E}-02$ \\
\hline$[\mathrm{Ar} \mathrm{vI}] \lambda 4.530 \ldots \ldots \ldots \ldots \ldots \ldots \ldots$ & $2.292 \mathrm{E}-03$ & $5.374 \mathrm{E}-05$ & $0.000 \mathrm{E}+00$ & $0.000 \mathrm{E}+00$ \\
\hline 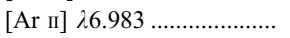 & $2.135 \mathrm{E}-04$ & $1.574 \mathrm{E}-03$ & $6.037 \mathrm{E}-03$ & $2.075 \mathrm{E}-02$ \\
\hline 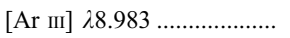 & $4.718 \mathrm{E}-02$ & $1.132 \mathrm{E}-03$ & $0.000 \mathrm{E}+00$ & $0.000 \mathrm{E}+00$ \\
\hline$[\mathrm{Ne}$ vI] $\lambda 7.652 \ldots \ldots \ldots \ldots \ldots \ldots . . .$. & $1.041 \mathrm{E}-02$ & $4.964 \mathrm{E}-02$ & $6.768 \mathrm{E}-02$ & $3.995 \mathrm{E}-02$ \\
\hline 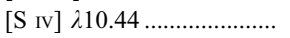 & $1.238 \mathrm{E}+00$ & $9.213 \mathrm{E}-01$ & $1.183 \mathrm{E}-01$ & $1.344 \mathrm{E}-03$ \\
\hline$[\mathrm{Ne}$ II] $\lambda 12.8 \ldots \ldots \ldots \ldots \ldots \ldots \ldots$ & $5.542 \mathrm{E}-03$ & $3.609 \mathrm{E}-02$ & $1.296 \mathrm{E}-01$ & $3.236 \mathrm{E}-01$ \\
\hline$[\mathrm{Ne} v] \quad \lambda 14.5 \ldots \ldots \ldots \ldots \ldots \ldots \ldots$ & $3.732 \mathrm{E}-02$ & $8.705 E-03$ & $6.862 \mathrm{E}-05$ & $0.000 \mathrm{E}+00$ \\
\hline$[\mathrm{Ne}$ III] $\lambda 15.5 \ldots \ldots \ldots \ldots \ldots \ldots . .$. & $6.927 \mathrm{E}-01$ & $7.122 \mathrm{E}-01$ & $6.485 \mathrm{E}-01$ & $4.429 \mathrm{E}-01$ \\
\hline [S III] $\lambda 18.7 \ldots \ldots \ldots \ldots \ldots \ldots . .$. & $2.580 \mathrm{E}-02$ & $1.711 \mathrm{E}-01$ & $3.752 \mathrm{E}-01$ & $2.641 \mathrm{E}-01$ \\
\hline [O Iv] $\lambda 25.9 \ldots \ldots \ldots \ldots \ldots \ldots \ldots \ldots \ldots \ldots \ldots \ldots$ & $4.647 \mathrm{E}-01$ & $6.341 \mathrm{E}-01$ & $1.810 \mathrm{E}-01$ & $6.123 \mathrm{E}-04$ \\
\hline
\end{tabular}


TABLE 44

Undepleted Dust-free IR Line Ratios with Respect to $\mathrm{H} \beta, 0.5 Z_{\odot}$

\begin{tabular}{|c|c|c|c|c|}
\hline \multirow[b]{2}{*}{ LiNE } & \multicolumn{4}{|c|}{$\log U_{0}$} \\
\hline & -1.0 & -2.0 & -3.0 & -4.0 \\
\hline \multicolumn{5}{|c|}{$\alpha=-1.2$} \\
\hline $\operatorname{Br} \alpha \lambda 4.051 \ldots \ldots \ldots \ldots \ldots \ldots \ldots$ & $6.483 \mathrm{E}-02$ & $6.901 \mathrm{E}-02$ & $7.752 \mathrm{E}-02$ & $8.786 \mathrm{E}-02$ \\
\hline 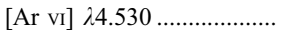 & $1.266 \mathrm{E}-02$ & $1.450 \mathrm{E}-03$ & $0.000 \mathrm{E}+00$ & $0.000 \mathrm{E}+00$ \\
\hline$[\mathrm{Ar}$ II] $\lambda 6.983 \ldots \ldots \ldots \ldots \ldots \ldots$ & $1.529 \mathrm{E}-03$ & $1.186 \mathrm{E}-02$ & $3.586 \mathrm{E}-02$ & $7.306 \mathrm{E}-02$ \\
\hline$[\mathrm{Ar}$ mI] $28.983 \ldots \ldots \ldots \ldots \ldots \ldots$ & $5.220 \mathrm{E}-01$ & $5.836 \mathrm{E}-02$ & $6.113 \mathrm{E}-05$ & $0.000 \mathrm{E}+00$ \\
\hline 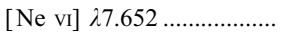 & $1.439 \mathrm{E}-02$ & $8.143 \mathrm{E}-02$ & $1.236 \mathrm{E}-01$ & $7.663 \mathrm{E}-02$ \\
\hline$[\mathrm{S}$ Iv] $\lambda 10.44 \ldots \ldots \ldots \ldots \ldots \ldots$ & $1.622 \mathrm{E}+00$ & $1.482 \mathrm{E}+00$ & $4.054 \mathrm{E}-01$ & $5.621 \mathrm{E}-03$ \\
\hline$[\mathrm{Ne}$ II] $\lambda 12.8 \ldots \ldots \ldots \ldots \ldots \ldots \ldots$ & $1.951 \mathrm{E}-02$ & $1.407 \mathrm{E}-01$ & $5.367 \mathrm{E}-01$ & $1.126 \mathrm{E}+00$ \\
\hline 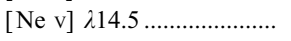 & $1.237 \mathrm{E}-01$ & $1.053 \mathrm{E}-01$ & $1.059 \mathrm{E}-03$ & $0.000 \mathrm{E}+00$ \\
\hline$[\mathrm{Ne}$ III] $\lambda 15.5 \ldots \ldots \ldots \ldots \ldots \ldots$ & $1.065 \mathrm{E}+00$ & $1.283 \mathrm{E}+00$ & $1.724 \mathrm{E}+00$ & $1.657 \mathrm{E}+00$ \\
\hline$[\mathrm{S}$ III] $\lambda 18.7 \ldots \ldots \ldots \ldots \ldots \ldots \ldots$ & $4.885 \mathrm{E}-02$ & $3.233 \mathrm{E}-01$ & $9.363 \mathrm{E}-01$ & $9.359 \mathrm{E}-01$ \\
\hline$[\mathrm{O}$ гv] $225.9 \ldots \ldots \ldots \ldots \ldots \ldots$ & $1.608 \mathrm{E}+00$ & $2.862 \mathrm{E}+00$ & $8.872 \mathrm{E}-01$ & $2.887 \mathrm{E}-03$ \\
\hline \multicolumn{5}{|c|}{$\alpha=-1.4$} \\
\hline $\operatorname{Br} \alpha \lambda .051 \ldots \ldots \ldots \ldots \ldots \ldots \ldots$ & $6.798 \mathrm{E}-02$ & $7.081 \mathrm{E}-02$ & $7.765 \mathrm{E}-02$ & $8.531 \mathrm{E}-02$ \\
\hline$[\mathrm{Ar} \mathrm{vI}] \lambda 4.530 \ldots \ldots \ldots \ldots \ldots \ldots$ & $9.752 \mathrm{E}-03$ & $7.787 \mathrm{E}-04$ & $0.000 \mathrm{E}+00$ & $0.000 \mathrm{E}+00$ \\
\hline 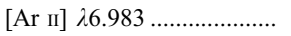 & $1.043 \mathrm{E}-03$ & $8.261 \mathrm{E}-03$ & $2.550 \mathrm{E}-02$ & $5.828 \mathrm{E}-02$ \\
\hline [Ar mI] $28.983 \ldots \ldots \ldots \ldots \ldots \ldots$ & $3.526 \mathrm{E}-01$ & $2.713 \mathrm{E}-02$ & $2.611 \mathrm{E}-05$ & $0.000 \mathrm{E}+00$ \\
\hline$[\mathrm{Ne} v \mathrm{vI}] \lambda 7.652 \ldots \ldots \ldots \ldots \ldots \ldots$ & $1.556 \mathrm{E}-02$ & $8.475 \mathrm{E}-02$ & $1.271 \mathrm{E}-01$ & $7.941 \mathrm{E}-02$ \\
\hline [S Iv] $\lambda 10.44 \ldots \ldots \ldots \ldots \ldots \ldots$ & $1.852 \mathrm{E}+00$ & $1.637 \mathrm{E}+00$ & $3.579 \mathrm{E}-01$ & $4.735 \mathrm{E}-03$ \\
\hline$[\mathrm{Ne}$ II] $\lambda 12.8 \ldots \ldots \ldots \ldots \ldots \ldots \ldots$ & $1.627 \mathrm{E}-02$ & $1.151 \mathrm{E}-01$ & $4.068 \mathrm{E}-01$ & $8.546 \mathrm{E}-01$ \\
\hline$[\mathrm{Ne} \mathrm{v}] \lambda 14.5 \ldots \ldots \ldots \ldots \ldots \ldots \ldots$ & $1.092 \mathrm{E}-01$ & $7.008 \mathrm{E}-02$ & $6.653 \mathrm{E}-04$ & $0.000 \mathrm{E}+00$ \\
\hline 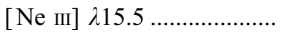 & $1.151 \mathrm{E}+00$ & $1.298 \mathrm{E}+00$ & $1.548 \mathrm{E}+00$ & $1.394 \mathrm{E}+00$ \\
\hline$[\mathrm{S}$ III] $\lambda 18.7 \ldots \ldots \ldots \ldots \ldots \ldots \ldots$ & $4.584 \mathrm{E}-02$ & $3.084 \mathrm{E}-01$ & $8.465 \mathrm{E}-01$ & $7.636 \mathrm{E}-01$ \\
\hline 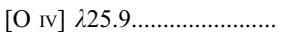 & $1.273 \mathrm{E}+00$ & $2.352 \mathrm{E}+00$ & $7.256 \mathrm{E}-01$ & $2.477 \mathrm{E}-03$ \\
\hline \multicolumn{5}{|c|}{$\alpha=-1.7$} \\
\hline 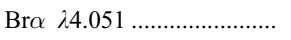 & $7.179 \mathrm{E}-02$ & $7.331 \mathrm{E}-02$ & $7.790 \mathrm{E}-02$ & $8.402 \mathrm{E}-02$ \\
\hline 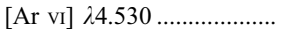 & $6.284 \mathrm{E}-03$ & $2.672 \mathrm{E}-04$ & $0.000 \mathrm{E}+00$ & $0.000 \mathrm{E}+00$ \\
\hline$[\mathrm{Ar}$ II] $\lambda 6.983 \ldots \ldots \ldots \ldots \ldots \ldots$ & $5.460 \mathrm{E}-04$ & $4.610 \mathrm{E}-03$ & $1.549 \mathrm{E}-02$ & $4.440 \mathrm{E}-02$ \\
\hline$[\mathrm{Ar}$ III] $28.983 \ldots \ldots \ldots \ldots \ldots \ldots$ & $1.693 \mathrm{E}-01$ & $7.083 \mathrm{E}-03$ & $6.140 \mathrm{E}-06$ & $0.000 \mathrm{E}+00$ \\
\hline$[\mathrm{Ne} v \mathrm{vI}] 27.652 \ldots \ldots \ldots \ldots \ldots . . . .$. & $1.789 \mathrm{E}-02$ & $8.944 \mathrm{E}-02$ & $1.286 \mathrm{E}-01$ & $7.829 \mathrm{E}-02$ \\
\hline$[\mathrm{S}$ Iv] $\lambda 10.44 \ldots \ldots \ldots \ldots \ldots \ldots \ldots$ & $2.109 \mathrm{E}+00$ & $1.708 \mathrm{E}+00$ & $2.768 \mathrm{E}-01$ & $3.436 \mathrm{E}-03$ \\
\hline 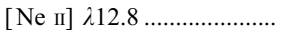 & $1.109 \mathrm{E}-02$ & $7.935 \mathrm{E}-02$ & $2.707 \mathrm{E}-01$ & $6.252 \mathrm{E}-01$ \\
\hline$[\mathrm{Ne} v] \lambda 14.5 \ldots \ldots \ldots \ldots \ldots \ldots \ldots$ & $8.541 \mathrm{E}-02$ & $3.250 \mathrm{E}-02$ & $2.868 \mathrm{E}-04$ & $0.000 \mathrm{E}+00$ \\
\hline$[\mathrm{Ne}$ III] $\lambda 15.5 \ldots \ldots \ldots \ldots \ldots \ldots \ldots$ & $1.229 \mathrm{E}+00$ & $1.314 \mathrm{E}+00$ & $1.351 \mathrm{E}+00$ & $1.064 \mathrm{E}+00$ \\
\hline$[\mathrm{S}$ III] $\lambda 18.7 \ldots \ldots \ldots \ldots \ldots \ldots \ldots$ & $4.579 \mathrm{E}-02$ & $3.078 \mathrm{E}-01$ & $7.536 \mathrm{E}-01$ & $5.860 \mathrm{E}-01$ \\
\hline 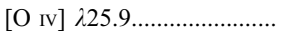 & $9.518 \mathrm{E}-01$ & $1.634 \mathrm{E}+00$ & $4.925 \mathrm{E}-01$ & $1.731 \mathrm{E}-03$ \\
\hline \multicolumn{5}{|c|}{$\alpha=-2.0$} \\
\hline 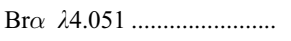 & $7.475 \mathrm{E}-02$ & $7.570 \mathrm{E}-02$ & $7.874 \mathrm{E}-02$ & $8.341 \mathrm{E}-02$ \\
\hline$[\mathrm{Ar} v \mathrm{vI}] \lambda .530 \ldots \ldots \ldots \ldots \ldots . . . . .$. & $3.590 \mathrm{E}-03$ & $8.164 \mathrm{E}-05$ & $0.000 \mathrm{E}+00$ & $0.000 \mathrm{E}+00$ \\
\hline 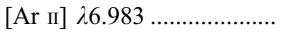 & $2.597 \mathrm{E}-04$ & $2.540 \mathrm{E}-03$ & $1.027 \mathrm{E}-02$ & $3.716 \mathrm{E}-02$ \\
\hline$[\mathrm{Ar}$ III] $28.983 \ldots \ldots \ldots \ldots \ldots \ldots$ & $6.880 \mathrm{E}-02$ & $1.520 \mathrm{E}-03$ & $1.249 \mathrm{E}-06$ & $0.000 \mathrm{E}+00$ \\
\hline$[\mathrm{Ne} v \mathrm{vI}] \lambda 7.652 \ldots \ldots \ldots \ldots \ldots . . .$. & $2.107 \mathrm{E}-02$ & $9.389 \mathrm{E}-02$ & $1.267 \mathrm{E}-01$ & $7.348 \mathrm{E}-02$ \\
\hline$[\mathrm{S}$ iv] $\lambda 10.44 \ldots \ldots \ldots \ldots \ldots \ldots$ & $2.267 \mathrm{E}+00$ & $1.633 \mathrm{E}+00$ & $2.032 \mathrm{E}-01$ & $2.400 \mathrm{E}-03$ \\
\hline$[\mathrm{Ne}$ II] $\lambda 12.8 \ldots \ldots \ldots \ldots \ldots \ldots \ldots$ & $6.759 \mathrm{E}-03$ & $5.411 \mathrm{E}-02$ & $2.035 \mathrm{E}-01$ & $5.154 \mathrm{E}-01$ \\
\hline$[\mathrm{Ne} v] \lambda 14.5 \ldots \ldots \ldots \ldots \ldots \ldots \ldots$ & $6.001 \mathrm{E}-02$ & $1.282 \mathrm{E}-02$ & $1.098 \mathrm{E}-04$ & $0.000 \mathrm{E}+00$ \\
\hline$[\mathrm{Ne}$ III] $\lambda 15.5 \ldots \ldots \ldots \ldots \ldots \ldots$ & $1.268 \mathrm{E}+00$ & $1.311 \mathrm{E}+00$ & $1.195 \mathrm{E}+00$ & $8.053 \mathrm{E}-01$ \\
\hline$[\mathrm{S}$ III] $\lambda 18.7 \ldots \ldots \ldots \ldots \ldots \ldots \ldots$ & $4.995 \mathrm{E}-02$ & $3.242 \mathrm{E}-01$ & $6.940 \mathrm{E}-01$ & $4.807 \mathrm{E}-01$ \\
\hline$[\mathrm{O}$ гv] $\lambda 25.9 \ldots \ldots \ldots \ldots \ldots \ldots$ & $7.628 \mathrm{E}-01$ & $1.068 \mathrm{E}+00$ & $3.114 \mathrm{E}-01$ & $1.091 \mathrm{E}-03$ \\
\hline
\end{tabular}


TABLE 45

Undepleted Dust-free IR Line Ratios with Respect to $\mathrm{H} \beta, 1 Z_{\odot}$

\begin{tabular}{|c|c|c|c|c|}
\hline \multirow[b]{2}{*}{ LiNE } & \multicolumn{4}{|c|}{$\log U_{0}$} \\
\hline & -1.0 & -2.0 & -3.0 & -4.0 \\
\hline \multicolumn{5}{|c|}{$\alpha=-1.2$} \\
\hline 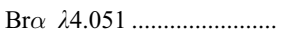 & $7.129 \mathrm{E}-02$ & $7.362 \mathrm{E}-02$ & $8.232 \mathrm{E}-02$ & $9.470 \mathrm{E}-02$ \\
\hline 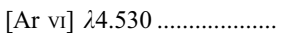 & $1.835 \mathrm{E}-02$ & $2.130 \mathrm{E}-03$ & $0.000 \mathrm{E}+00$ & $0.000 \mathrm{E}+00$ \\
\hline 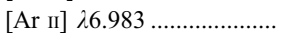 & $1.669 \mathrm{E}-03$ & $1.828 \mathrm{E}-02$ & $5.589 \mathrm{E}-02$ & $1.129 \mathrm{E}-01$ \\
\hline$[\mathrm{Ar}$ III] $\lambda 8.983 \ldots \ldots \ldots \ldots \ldots \ldots . . . . . . .$. & $7.890 \mathrm{E}-01$ & $7.953 \mathrm{E}-02$ & $8.375 \mathrm{E}-05$ & $0.000 \mathrm{E}+00$ \\
\hline$[\mathrm{Ne} v \mathrm{vI}] \lambda 7.652 \ldots \ldots \ldots \ldots \ldots \ldots$ & $2.949 \mathrm{E}-02$ & $1.587 \mathrm{E}-01$ & $2.312 \mathrm{E}-01$ & $1.363 \mathrm{E}-01$ \\
\hline 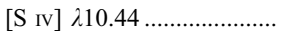 & $3.240 \mathrm{E}+00$ & $2.823 \mathrm{E}+00$ & $6.924 \mathrm{E}-01$ & $9.919 \mathrm{E}-03$ \\
\hline$[\mathrm{Ne}$ II $] \lambda 12.8 \ldots \ldots \ldots \ldots \ldots \ldots \ldots$ & $2.478 \mathrm{E}-02$ & $2.052 \mathrm{E}-01$ & $7.339 \mathrm{E}-01$ & $1.444 \mathrm{E}+00$ \\
\hline 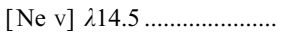 & $2.025 \mathrm{E}-01$ & $1.575 \mathrm{E}-01$ & $1.633 \mathrm{E}-03$ & $0.000 \mathrm{E}+00$ \\
\hline$[\mathrm{Ne}$ III $] \lambda 15.5 \ldots \ldots \ldots \ldots \ldots \ldots \ldots$ & $2.053 \mathrm{E}+00$ & $2.427 \mathrm{E}+00$ & $3.064 \mathrm{E}+00$ & $2.807 \mathrm{E}+00$ \\
\hline 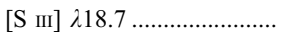 & $8.418 \mathrm{E}-02$ & $5.953 \mathrm{E}-01$ & $1.668 \mathrm{E}+00$ & $1.532 \mathrm{E}+00$ \\
\hline [O Iv] $\lambda 25.9 \ldots \ldots \ldots \ldots \ldots \ldots \ldots . .$. & $2.242 \mathrm{E}+00$ & $4.688 \mathrm{E}+00$ & $1.460 \mathrm{E}+00$ & $4.981 \mathrm{E}-03$ \\
\hline \multicolumn{5}{|c|}{$\alpha=-1.4$} \\
\hline 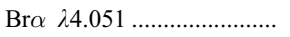 & $7.463 \mathrm{E}-02$ & $7.620 \mathrm{E}-02$ & $8.282 \mathrm{E}-02$ & $9.203 \mathrm{E}-02$ \\
\hline$[\mathrm{Ar} \mathrm{vI}] \lambda 4.530 \ldots \ldots \ldots \ldots \ldots \ldots \ldots$ & $1.439 \mathrm{E}-02$ & $1.103 \mathrm{E}-03$ & $0.000 \mathrm{E}+00$ & $0.000 \mathrm{E}+00$ \\
\hline$[\mathrm{Ar}$ II $] \lambda 6.983 \ldots \ldots \ldots \ldots \ldots \ldots$ & $1.061 \mathrm{E}-03$ & $1.251 \mathrm{E}-02$ & $3.950 \mathrm{E}-02$ & $9.284 \mathrm{E}-02$ \\
\hline$[\mathrm{Ar}$ III] $\lambda 8.983 \ldots \ldots \ldots \ldots \ldots \ldots$ & $5.091 \mathrm{E}-01$ & $3.497 \mathrm{E}-02$ & $3.394 \mathrm{E}-05$ & $0.000 \mathrm{E}+00$ \\
\hline$[\mathrm{Ne} v \mathrm{vI}] \lambda 7.652 \ldots \ldots \ldots \ldots \ldots \ldots$ & $3.211 \mathrm{E}-02$ & $1.622 \mathrm{E}-01$ & $2.338 \mathrm{E}-01$ & $1.386 \mathrm{E}-01$ \\
\hline [S IV] $\lambda 10.44 \ldots \ldots \ldots \ldots \ldots \ldots \ldots$ & $3.566 \mathrm{E}+00$ & $2.980 \mathrm{E}+00$ & $5.913 \mathrm{E}-01$ & $8.245 \mathrm{E}-03$ \\
\hline$[\mathrm{Ne}$ II] $\lambda 12.8 \ldots \ldots \ldots \ldots \ldots \ldots . .$. & $1.869 \mathrm{E}-02$ & $1.642 \mathrm{E}-01$ & $5.590 \mathrm{E}-01$ & $1.160 \mathrm{E}+00$ \\
\hline$[\mathrm{Ne} v] \lambda 14.5 \ldots \ldots \ldots \ldots \ldots \ldots \ldots \ldots \ldots \ldots \ldots$ & $1.753 \mathrm{E}-01$ & $1.001 \mathrm{E}-01$ & $9.872 \mathrm{E}-04$ & $0.000 \mathrm{E}+00$ \\
\hline$[\mathrm{Ne}$ III] $\lambda 15.5 \ldots \ldots \ldots \ldots \ldots \ldots \ldots . . . . . . .$. & $2.162 \mathrm{E}+00$ & $2.412 \mathrm{E}+00$ & $2.742 \mathrm{E}+00$ & $2.358 \mathrm{E}+00$ \\
\hline 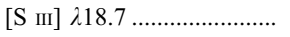 & $8.359 \mathrm{E}-02$ & $5.732 \mathrm{E}-01$ & $1.502 \mathrm{E}+00$ & $1.258 \mathrm{E}+00$ \\
\hline 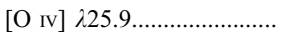 & $1.827 \mathrm{E}+00$ & $3.761 \mathrm{E}+00$ & $1.170 \mathrm{E}+00$ & $4.163 \mathrm{E}-03$ \\
\hline \multicolumn{5}{|c|}{$\alpha=-1.7$} \\
\hline 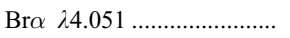 & $7.890 \mathrm{E}-02$ & $7.972 \mathrm{E}-02$ & $8.371 \mathrm{E}-02$ & $9.026 \mathrm{E}-02$ \\
\hline$[\mathrm{Ar}$ vI] $\lambda 4.530 \ldots \ldots \ldots \ldots \ldots \ldots . . . . . . . .$. & $9.337 \mathrm{E}-03$ & $3.629 \mathrm{E}-04$ & $0.000 \mathrm{E}+00$ & $0.000 \mathrm{E}+00$ \\
\hline 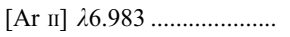 & $4.774 \mathrm{E}-04$ & $6.819 \mathrm{E}-03$ & $2.443 \mathrm{E}-02$ & $7.433 \mathrm{E}-02$ \\
\hline$[\mathrm{Ar}$ III] $\lambda 8.983 \ldots \ldots \ldots \ldots \ldots . . . . . . . . .$. & $2.307 \mathrm{E}-01$ & $8.451 \mathrm{E}-03$ & $7.488 \mathrm{E}-06$ & $0.000 \mathrm{E}+00$ \\
\hline$[\mathrm{Ne}$ vI $] \lambda 7.652 \ldots \ldots \ldots \ldots \ldots . . . . . .$. & $3.733 \mathrm{E}-02$ & $1.678 \mathrm{E}-01$ & $2.315 \mathrm{E}-01$ & $1.333 \mathrm{E}-01$ \\
\hline [S IV] $\lambda 10.44 \ldots \ldots \ldots \ldots \ldots \ldots . .$. & $3.903 \mathrm{E}+00$ & $2.961 \mathrm{E}+00$ & $4.393 \mathrm{E}-01$ & $5.863 \mathrm{E}-03$ \\
\hline$[\mathrm{Ne}$ II] $\lambda 12.8 \ldots \ldots \ldots \ldots \ldots \ldots \ldots$ & $1.133 \mathrm{E}-02$ & $1.133 \mathrm{E}-01$ & $3.959 \mathrm{E}-01$ & $9.281 \mathrm{E}-01$ \\
\hline$[\mathrm{Ne} \mathrm{v}] \lambda 14.5 \ldots \ldots \ldots \ldots \ldots \ldots \ldots \ldots \ldots \ldots \ldots \ldots$ & $1.313 \mathrm{E}-01$ & $4.374 \mathrm{E}-02$ & $4.068 \mathrm{E}-04$ & $0.000 \mathrm{E}+00$ \\
\hline 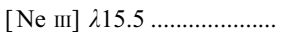 & $2.249 \mathrm{E}+00$ & $2.385 \mathrm{E}+00$ & $2.370 \mathrm{E}+00$ & $1.791 \mathrm{E}+00$ \\
\hline 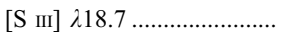 & $8.886 \mathrm{E}-02$ & $5.756 \mathrm{E}-01$ & $1.327 \mathrm{E}+00$ & $9.814 \mathrm{E}-01$ \\
\hline$[\mathrm{O}$ IV $]$ $225.9 \ldots \ldots \ldots \ldots \ldots \ldots \ldots \ldots \ldots \ldots$ & $1.436 \mathrm{E}+00$ & $2.557 \mathrm{E}+00$ & $7.779 \mathrm{E}-01$ & $2.824 \mathrm{E}-03$ \\
\hline \multicolumn{5}{|c|}{$\alpha=-2.0$} \\
\hline 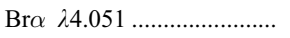 & $8.249 \mathrm{E}-02$ & $8.279 \mathrm{E}-02$ & $8.492 \mathrm{E}-02$ & $8.963 \mathrm{E}-02$ \\
\hline 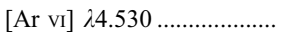 & $5.202 \mathrm{E}-03$ & $1.072 \mathrm{E}-04$ & $0.000 \mathrm{E}+00$ & $0.000 \mathrm{E}+00$ \\
\hline 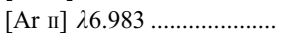 & $1.994 \mathrm{E}-04$ & $3.733 \mathrm{E}-03$ & $1.710 \mathrm{E}-02$ & $6.480 \mathrm{E}-02$ \\
\hline 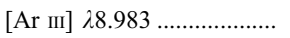 & $8.894 \mathrm{E}-02$ & $1.681 \mathrm{E}-03$ & $1.457 \mathrm{E}-06$ & $0.000 \mathrm{E}+00$ \\
\hline$[\mathrm{Ne}$ vI] $\lambda 7.652 \ldots \ldots \ldots \ldots \ldots \ldots . . .$. & $4.443 \mathrm{E}-02$ & $1.735 \mathrm{E}-01$ & $2.242 \mathrm{E}-01$ & $1.218 \mathrm{E}-01$ \\
\hline 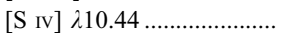 & $4.063 \mathrm{E}+00$ & $2.733 \mathrm{E}+00$ & $3.134 \mathrm{E}-01$ & $4.076 \mathrm{E}-03$ \\
\hline$[\mathrm{Ne}$ II] $\lambda 12.8 \ldots \ldots \ldots \ldots \ldots \ldots \ldots$ & $7.655 \mathrm{E}-03$ & $8.134 \mathrm{E}-02$ & $3.285 \mathrm{E}-01$ & $8.230 \mathrm{E}-01$ \\
\hline$[\mathrm{Ne} v] \lambda 14.5 \ldots \ldots \ldots \ldots \ldots \ldots \ldots$ & $8.785 \mathrm{E}-02$ & $1.634 \mathrm{E}-02$ & $1.508 \mathrm{E}-04$ & $0.000 \mathrm{E}+00$ \\
\hline$[\mathrm{Ne}$ III] $\lambda 15.5 \ldots \ldots \ldots \ldots \ldots \ldots . .$. & $2.279 \mathrm{E}+00$ & $2.335 \mathrm{E}+00$ & $2.066 \mathrm{E}+00$ & $1.338 \mathrm{E}+00$ \\
\hline$[\mathrm{S}$ III] $\lambda 18.7 \ldots \ldots \ldots \ldots \ldots \ldots \ldots$ & $1.010 \mathrm{E}-01$ & $6.057 \mathrm{E}-01$ & $1.214 \mathrm{E}+00$ & $8.146 \mathrm{E}-01$ \\
\hline [O Iv] $\lambda 25.9 \ldots \ldots \ldots \ldots \ldots \ldots \ldots \ldots \ldots \ldots \ldots$ & $1.177 \mathrm{E}+00$ & $1.653 \mathrm{E}+00$ & $4.867 \mathrm{E}-01$ & $1.736 \mathrm{E}-03$ \\
\hline
\end{tabular}


TABLE 46

Undepleted Dust-free IR Line Ratios with Respect to $\mathrm{H} \beta, 2 Z_{\odot}$

\begin{tabular}{|c|c|c|c|c|}
\hline \multirow[b]{2}{*}{ LINE } & \multicolumn{4}{|c|}{$\log U_{0}$} \\
\hline & -1.0 & -2.0 & -3.0 & -4.0 \\
\hline \multicolumn{5}{|c|}{$\alpha=-1.2$} \\
\hline $\operatorname{Br} \alpha \quad \lambda 4.051 \ldots \ldots \ldots \ldots \ldots \ldots \ldots$ & $8.081 \mathrm{E}-02$ & $8.062 \mathrm{E}-02$ & $9.000 \mathrm{E}-02$ & $1.058 \mathrm{E}-01$ \\
\hline 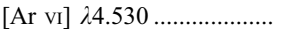 & $2.560 \mathrm{E}-02$ & $2.663 \mathrm{E}-03$ & $0.000 \mathrm{E}+00$ & $0.000 \mathrm{E}+00$ \\
\hline 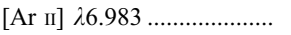 & $1.123 \mathrm{E}-03$ & $2.504 \mathrm{E}-02$ & $8.220 \mathrm{E}-02$ & $1.687 \mathrm{E}-01$ \\
\hline$[\mathrm{Ar} \mathrm{mI}] \quad 28.983 \ldots \ldots \ldots \ldots \ldots \ldots$ & $1.044 \mathrm{E}+00$ & $9.110 \mathrm{E}-02$ & $9.031 \mathrm{E}-05$ & $0.000 \mathrm{E}+00$ \\
\hline$[\mathrm{Ne} v \mathrm{vI}] \lambda 7.652 \ldots \ldots \ldots \ldots \ldots \ldots$ & $6.437 \mathrm{E}-02$ & $3.023 \mathrm{E}-01$ & $4.051 \mathrm{E}-01$ & $2.118 \mathrm{E}-01$ \\
\hline 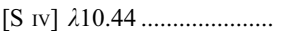 & $6.315 \mathrm{E}+00$ & $5.112 \mathrm{E}+00$ & $1.057 \mathrm{E}+00$ & $1.825 \mathrm{E}-02$ \\
\hline$[\mathrm{Ne}$ I] $\lambda 12.8 \ldots \ldots \ldots \ldots \ldots \ldots \ldots$ & $2.312 \mathrm{E}-02$ & $2.884 \mathrm{E}-01$ & $1.001 \mathrm{E}+00$ & $1.896 \mathrm{E}+00$ \\
\hline$[\mathrm{Ne} v], \lambda 14.5 \ldots \ldots \ldots \ldots \ldots \ldots \ldots$ & $3.036 \mathrm{E}-01$ & $2.057 \mathrm{E}-01$ & $2.140 \mathrm{E}-03$ & $1.380 \mathrm{E}-06$ \\
\hline$[\mathrm{Ne}$ III] $\lambda 15.5 \ldots \ldots \ldots \ldots \ldots \ldots \ldots$ & $3.850 \mathrm{E}+00$ & $4.425 \mathrm{E}+00$ & $5.074 \mathrm{E}+00$ & $4.290 \mathrm{E}+00$ \\
\hline 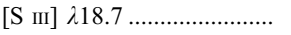 & $1.580 \mathrm{E}-01$ & $1.067 \mathrm{E}+00$ & $2.781 \mathrm{E}+00$ & $2.283 \mathrm{E}+00$ \\
\hline$[\mathrm{O}$ Iv] $225.9 \ldots \ldots \ldots \ldots \ldots \ldots \ldots . .$. & $2.909 \mathrm{E}+00$ & $6.951 \mathrm{E}+00$ & $2.141 \mathrm{E}+00$ & $7.791 \mathrm{E}-03$ \\
\hline \multicolumn{5}{|c|}{$\alpha=-1.4$} \\
\hline $\operatorname{Br} \alpha \lambda .051 \ldots \ldots \ldots \ldots \ldots \ldots \ldots$ & $8.461 \mathrm{E}-02$ & $8.405 \mathrm{E}-02$ & $9.097 \mathrm{E}-02$ & $1.025 \mathrm{E}-01$ \\
\hline 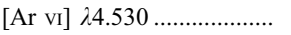 & $2.017 \mathrm{E}-02$ & $1.327 \mathrm{E}-03$ & $0.000 \mathrm{E}+00$ & $0.000 \mathrm{E}+00$ \\
\hline 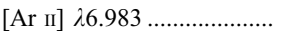 & $5.936 \mathrm{E}-04$ & $1.695 \mathrm{E}-02$ & $5.865 \mathrm{E}-02$ & $1.440 \mathrm{E}-01$ \\
\hline$[\mathrm{Ar}$ III] $28.983 \ldots \ldots \ldots \ldots \ldots \ldots$ & $6.447 \mathrm{E}-01$ & $3.758 \mathrm{E}-02$ & $3.471 \mathrm{E}-05$ & $0.000 \mathrm{E}+00$ \\
\hline$[\mathrm{Ne} v \mathrm{vI}] 77.652 \ldots \ldots \ldots \ldots \ldots . . . .$. & $7.100 \mathrm{E}-02$ & $3.038 \mathrm{E}-01$ & $4.021 \mathrm{E}-01$ & $2.113 \mathrm{E}-01$ \\
\hline [S Iv] $\lambda 10.44 \ldots \ldots \ldots \ldots \ldots \ldots \ldots$ & $6.638 \mathrm{E}+00$ & $5.125 \mathrm{E}+00$ & $8.719 \mathrm{E}-01$ & $1.581 \mathrm{E}-02$ \\
\hline$[\mathrm{Ne}$ п] $\lambda 12.8 \ldots \ldots \ldots \ldots \ldots \ldots$ & $1.660 \mathrm{E}-02$ & $2.316 \mathrm{E}-01$ & $7.898 \mathrm{E}-01$ & $1.625 \mathrm{E}+00$ \\
\hline$[\mathrm{Ne} v] \quad \lambda 14.5 \ldots \ldots \ldots \ldots \ldots \ldots \ldots$ & $2.554 \mathrm{E}-01$ & $1.244 \mathrm{E}-01$ & $1.241 \mathrm{E}-03$ & $0.000 \mathrm{E}+00$ \\
\hline$[\mathrm{Ne}$ III] $\lambda 15.5 \ldots \ldots \ldots \ldots \ldots \ldots . . .$. & $3.938 \mathrm{E}+00$ & $4.323 \mathrm{E}+00$ & $4.516 \mathrm{E}+00$ & $3.590 \mathrm{E}+00$ \\
\hline$[\mathrm{S}$ III] $\lambda 18.7 \ldots \ldots \ldots \ldots \ldots \ldots \ldots . .$. & $1.662 \mathrm{E}-01$ & $1.047 \mathrm{E}+00$ & $2.498 \mathrm{E}+00$ & $1.901 \mathrm{E}+00$ \\
\hline 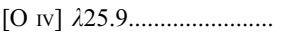 & $2.474 \mathrm{E}+00$ & $5.456 \mathrm{E}+00$ & $1.686 \mathrm{E}+00$ & $6.277 \mathrm{E}-03$ \\
\hline \multicolumn{5}{|c|}{$\alpha=-1.7$} \\
\hline $\operatorname{Br} \alpha \quad 24.051 \ldots \ldots \ldots \ldots \ldots \ldots \ldots$ & $8.982 \mathrm{E}-02$ & $8.877 \mathrm{E}-02$ & $9.235 \mathrm{E}-02$ & $9.983 \mathrm{E}-02$ \\
\hline 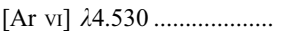 & $1.273 \mathrm{E}-02$ & $4.143 \mathrm{E}-04$ & $0.000 \mathrm{E}+00$ & $0.000 \mathrm{E}+00$ \\
\hline$[\mathrm{Ar}$ п] $\lambda 6.983 \ldots \ldots \ldots \ldots \ldots \ldots . . . . . . . . .$. & $2.896 \mathrm{E}-04$ & $9.192 \mathrm{E}-03$ & $3.830 \mathrm{E}-02$ & $1.215 \mathrm{E}-01$ \\
\hline 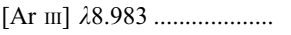 & $2.759 \mathrm{E}-01$ & $8.172 \mathrm{E}-03$ & $7.211 \mathrm{E}-06$ & $0.000 \mathrm{E}+00$ \\
\hline$[\mathrm{Ne} v \mathrm{vI}] \lambda 7.652 \ldots \ldots \ldots \ldots \ldots . . .$. & $8.385 \mathrm{E}-02$ & $3.071 \mathrm{E}-01$ & $3.887 \mathrm{E}-01$ & $1.977 \mathrm{E}-01$ \\
\hline [S Iv] $\lambda 10.44 \ldots \ldots \ldots \ldots \ldots \ldots \ldots$ & $6.834 \mathrm{E}+00$ & $4.783 \mathrm{E}+00$ & $6.220 \mathrm{E}-01$ & $1.006 \mathrm{E}-02$ \\
\hline 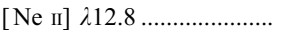 & $1.331 \mathrm{E}-02$ & $1.690 \mathrm{E}-01$ & $6.196 \mathrm{E}-01$ & $1.413 \mathrm{E}+00$ \\
\hline 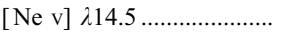 & $1.819 \mathrm{E}-01$ & $5.043 \mathrm{E}-02$ & $4.872 \mathrm{E}-04$ & $0.000 \mathrm{E}+00$ \\
\hline$[\mathrm{Ne}$ III] $\lambda 15.5 \ldots \ldots \ldots \ldots \ldots \ldots . .$. & $3.956 \mathrm{E}+00$ & $4.156 \mathrm{E}+00$ & $3.843 \mathrm{E}+00$ & $2.692 \mathrm{E}+00$ \\
\hline$[\mathrm{S}$ III] $\lambda 18.7 \ldots \ldots \ldots \ldots \ldots \ldots \ldots$ & $1.891 \mathrm{E}-01$ & $1.066 \mathrm{E}+00$ & $2.200 \mathrm{E}+00$ & $1.517 \mathrm{E}+00$ \\
\hline$[\mathrm{O}$ Iv] $225.9 \ldots \ldots \ldots \ldots \ldots \ldots$ & $2.041 \mathrm{E}+00$ & $3.635 \mathrm{E}+00$ & $1.104 \mathrm{E}+00$ & $4.073 \mathrm{E}-03$ \\
\hline \multicolumn{5}{|c|}{$\alpha=-2.0$} \\
\hline $\operatorname{Br} \alpha \lambda .051 \ldots \ldots \ldots \ldots \ldots \ldots \ldots$ & $9.451 \mathrm{E}-02$ & $9.290 \mathrm{E}-02$ & $9.401 \mathrm{E}-02$ & $9.949 \mathrm{E}-02$ \\
\hline 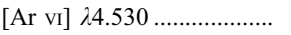 & $6.734 \mathrm{E}-03$ & $1.164 \mathrm{E}-04$ & $0.000 \mathrm{E}+00$ & $0.000 \mathrm{E}+00$ \\
\hline 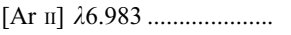 & $2.933 \mathrm{E}-04$ & $5.437 \mathrm{E}-03$ & $2.974 \mathrm{E}-02$ & $1.101 \mathrm{E}-01$ \\
\hline 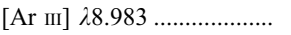 & $1.006 \mathrm{E}-01$ & $1.446 \mathrm{E}-03$ & $1.338 \mathrm{E}-06$ & $0.000 \mathrm{E}+00$ \\
\hline$[\mathrm{Ne} v \mathrm{vi}] \lambda 7.652 \ldots \ldots \ldots \ldots \ldots . . . .$. & $1.007 \mathrm{E}-01$ & $3.098 \mathrm{E}-01$ & $3.673 \mathrm{E}-01$ & $1.751 \mathrm{E}-01$ \\
\hline [S Iv] $\lambda 10.44 \ldots \ldots \ldots \ldots \ldots \ldots$ & $6.719 \mathrm{E}+00$ & $4.179 \mathrm{E}+00$ & $4.289 \mathrm{E}-01$ & $6.609 \mathrm{E}-03$ \\
\hline$[\mathrm{Ne}$ II] $\lambda 12.8 \ldots \ldots \ldots \ldots \ldots \ldots \ldots$ & $1.739 \mathrm{E}-02$ & $1.429 \mathrm{E}-01$ & $5.747 \mathrm{E}-01$ & $1.323 \mathrm{E}+00$ \\
\hline 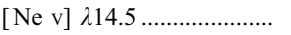 & $1.152 \mathrm{E}-01$ & $1.742 \mathrm{E}-02$ & $1.736 \mathrm{E}-04$ & $0.000 \mathrm{E}+00$ \\
\hline 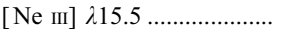 & $3.880 \mathrm{E}+00$ & $3.948 \mathrm{E}+00$ & $3.270 \mathrm{E}+00$ & $1.968 \mathrm{E}+00$ \\
\hline 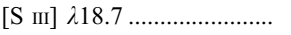 & $2.276 \mathrm{E}-01$ & $1.123 \mathrm{E}+00$ & $2.007 \mathrm{E}+00$ & $1.277 \mathrm{E}+00$ \\
\hline [O Iv] $\lambda 25.9 \ldots \ldots \ldots \ldots \ldots \ldots \ldots . .$. & $1.689 \mathrm{E}+00$ & $2.328 \mathrm{E}+00$ & $6.828 \mathrm{E}-01$ & $2.422 \mathrm{E}-03$ \\
\hline
\end{tabular}


TABLE 47

Undepleted Dust-free IR Line Ratios with Respect to $\mathrm{H} \beta, 4 Z_{\odot}$

\begin{tabular}{|c|c|c|c|c|}
\hline \multirow[b]{2}{*}{ LiNE } & \multicolumn{4}{|c|}{$\log U_{0}$} \\
\hline & -1.0 & -2.0 & -3.0 & -4.0 \\
\hline \multicolumn{5}{|c|}{$\alpha=-1.2$} \\
\hline 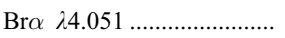 & $9.994 \mathrm{E}-02$ & $9.103 \mathrm{E}-02$ & $1.004 \mathrm{E}-01$ & $1.199 \mathrm{E}-01$ \\
\hline$[\mathrm{Ar} \mathrm{vI}] \lambda 4.530 \ldots \ldots \ldots \ldots \ldots \ldots$ & $3.375 \mathrm{E}-02$ & $2.776 \mathrm{E}-03$ & $0.000 \mathrm{E}+00$ & $0.000 \mathrm{E}+00$ \\
\hline 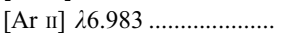 & $6.272 \mathrm{E}-04$ & $3.243 \mathrm{E}-02$ & $1.218 \mathrm{E}-01$ & $2.477 \mathrm{E}-01$ \\
\hline [Ar III] $\lambda 8.983 \ldots \ldots \ldots \ldots \ldots \ldots$ & $1.211 \mathrm{E}+00$ & $8.762 \mathrm{E}-02$ & $7.691 \mathrm{E}-05$ & $0.000 \mathrm{E}+00$ \\
\hline$[\mathrm{Ne}$ vI $] \lambda 7.652 \ldots \ldots \ldots \ldots \ldots \ldots$ & $1.472 \mathrm{E}-01$ & $5.580 \mathrm{E}-01$ & $6.466 \mathrm{E}-01$ & $2.759 \mathrm{E}-01$ \\
\hline 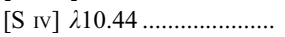 & $1.015 \mathrm{E}+01$ & $8.392 \mathrm{E}+00$ & $1.440 \mathrm{E}+00$ & $2.794 \mathrm{E}-02$ \\
\hline$[\mathrm{Ne}$ II $] \lambda 12.8 \ldots \ldots \ldots \ldots \ldots \ldots$ & $3.186 \mathrm{E}-02$ & $4.515 \mathrm{E}-01$ & $1.482 \mathrm{E}+00$ & $2.634 \mathrm{E}+00$ \\
\hline$[\mathrm{Ne} v] \lambda 14.5 \ldots \ldots \ldots \ldots \ldots \ldots \ldots \ldots$ & $4.132 \mathrm{E}-01$ & $2.354 \mathrm{E}-01$ & $2.357 \mathrm{E}-03$ & $1.806 \mathrm{E}-06$ \\
\hline$[\mathrm{Ne}$ III] $\lambda 15.5 \ldots \ldots \ldots \ldots \ldots \ldots \ldots$ & $6.314 \mathrm{E}+00$ & $7.574 \mathrm{E}+00$ & $7.716 \mathrm{E}+00$ & $5.810 \mathrm{E}+00$ \\
\hline [S III] $\lambda 18.7 \ldots \ldots \ldots \ldots \ldots \ldots \ldots$ & $3.463 \mathrm{E}-01$ & $1.930 \mathrm{E}+00$ & $4.365 \mathrm{E}+00$ & $3.142 \mathrm{E}+00$ \\
\hline [O IV] $\lambda 25.9 \ldots \ldots \ldots \ldots \ldots \ldots \ldots . .$. & $3.667 \mathrm{E}+00$ & $9.350 \mathrm{E}+00$ & $2.777 \mathrm{E}+00$ & $1.035 \mathrm{E}-02$ \\
\hline \multicolumn{5}{|c|}{$\alpha=-1.4$} \\
\hline 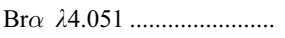 & $1.064 \mathrm{E}-01$ & $9.625 \mathrm{E}-02$ & $1.020 \mathrm{E}-01$ & $1.161 \mathrm{E}-01$ \\
\hline$[\mathrm{Ar}$ vI $] \lambda 4.530 \ldots \ldots \ldots \ldots \ldots \ldots$ & $2.586 \mathrm{E}-02$ & $1.322 \mathrm{E}-03$ & $0.000 \mathrm{E}+00$ & $0.000 \mathrm{E}+00$ \\
\hline 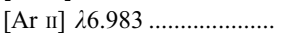 & $7.790 \mathrm{E}-04$ & $2.328 \mathrm{E}-02$ & $9.115 \mathrm{E}-02$ & $2.188 \mathrm{E}-01$ \\
\hline [Ar III] $\lambda 8.983 \ldots \ldots \ldots \ldots \ldots \ldots \ldots$ & $7.146 \mathrm{E}-01$ & $3.321 \mathrm{E}-02$ & $2.763 \mathrm{E}-05$ & $0.000 \mathrm{E}+00$ \\
\hline$[\mathrm{Ne}$ vI $] \lambda 7.652 \ldots \ldots \ldots \ldots \ldots \ldots$ & $1.577 \mathrm{E}-01$ & $5.384 \mathrm{E}-01$ & $6.237 \mathrm{E}-01$ & $2.691 \mathrm{E}-01$ \\
\hline [S IV] $\lambda 10.44 \ldots \ldots \ldots \ldots \ldots \ldots \ldots$ & $9.774 \mathrm{E}+00$ & $7.777 \mathrm{E}+00$ & $1.132 \mathrm{E}+00$ & $2.108 \mathrm{E}-02$ \\
\hline$[\mathrm{Ne}$ II] $\lambda 12.8 \ldots \ldots \ldots \ldots \ldots \ldots$ & $3.994 \mathrm{E}-02$ & $3.952 \mathrm{E}-01$ & $1.252 \mathrm{E}+00$ & $2.379 \mathrm{E}+00$ \\
\hline$[\mathrm{Ne} v] \lambda 14.5 \ldots \ldots \ldots \ldots \ldots \ldots \ldots \ldots \ldots \ldots \ldots$ & $3.342 \mathrm{E}-01$ & $1.339 \mathrm{E}-01$ & $1.289 \mathrm{E}-03$ & $0.000 \mathrm{E}+00$ \\
\hline 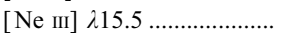 & $6.083 \mathrm{E}+00$ & $7.103 \mathrm{E}+00$ & $6.739 \mathrm{E}+00$ & $4.790 \mathrm{E}+00$ \\
\hline 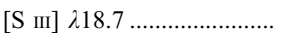 & $3.794 \mathrm{E}-01$ & $1.913 \mathrm{E}+00$ & $3.909 \mathrm{E}+00$ & $2.657 \mathrm{E}+00$ \\
\hline$[\mathrm{O}$ Iv $] \lambda 25.9 \ldots \ldots \ldots \ldots \ldots \ldots \ldots \ldots \ldots \ldots \ldots \ldots$ & $3.235 \mathrm{E}+00$ & $7.169 \mathrm{E}+00$ & $2.141 \mathrm{E}+00$ & $7.956 \mathrm{E}-03$ \\
\hline \multicolumn{5}{|c|}{$\alpha=-1.7$} \\
\hline 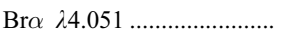 & $1.155 \mathrm{E}-01$ & $1.040 \mathrm{E}-01$ & $1.042 \mathrm{E}-01$ & $1.132 \mathrm{E}-01$ \\
\hline$[\mathrm{Ar}$ vI $] \lambda 4.530 \ldots \ldots \ldots \ldots \ldots \ldots$ & $1.518 \mathrm{E}-02$ & $3.809 \mathrm{E}-04$ & $0.000 \mathrm{E}+00$ & $0.000 \mathrm{E}+00$ \\
\hline 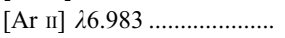 & $4.662 \mathrm{E}-03$ & $1.784 \mathrm{E}-02$ & $6.797 \mathrm{E}-02$ & $1.932 \mathrm{E}-01$ \\
\hline [Ar III] $\lambda 8.983 \ldots \ldots \ldots \ldots \ldots \ldots$ & $2.852 \mathrm{E}-01$ & $6.117 \mathrm{E}-03$ & $5.197 \mathrm{E}-06$ & $0.000 \mathrm{E}+00$ \\
\hline$[\mathrm{Ne}$ vI $] \lambda 7.652 \ldots \ldots \ldots \ldots \ldots \ldots$ & $1.685 \mathrm{E}-01$ & $5.002 \mathrm{E}-01$ & $5.722 \mathrm{E}-01$ & $2.426 \mathrm{E}-01$ \\
\hline [S IV] $\lambda 10.44 \ldots \ldots \ldots \ldots \ldots \ldots$ & $8.754 \mathrm{E}+00$ & $6.437 \mathrm{E}+00$ & $7.518 \mathrm{E}-01$ & $1.345 \mathrm{E}-02$ \\
\hline$[\mathrm{Ne}$ II $] \lambda 12.8 \ldots \ldots \ldots \ldots \ldots \ldots \ldots$ & $1.034 \mathrm{E}-01$ & $3.760 \mathrm{E}-01$ & $1.102 \mathrm{E}+00$ & $2.187 \mathrm{E}+00$ \\
\hline$[\mathrm{Ne} v] \lambda 14.5 \ldots \ldots \ldots \ldots \ldots \ldots \ldots$ & $2.222 \mathrm{E}-01$ & $4.848 \mathrm{E}-02$ & $4.648 \mathrm{E}-04$ & $0.000 \mathrm{E}+00$ \\
\hline$[\mathrm{Ne}$ III] $\lambda 15.5 \ldots \ldots \ldots \ldots \ldots \ldots \ldots$ & $5.489 \mathrm{E}+00$ & $6.285 \mathrm{E}+00$ & $5.482 \mathrm{E}+00$ & $3.493 \mathrm{E}+00$ \\
\hline$[\mathrm{S}$ III] $\lambda 18.7 \ldots \ldots \ldots \ldots \ldots \ldots \ldots$ & $4.867 \mathrm{E}-01$ & $1.934 \mathrm{E}+00$ & $3.409 \mathrm{E}+00$ & $2.169 \mathrm{E}+00$ \\
\hline [O Iv] $\lambda 25.9 \ldots \ldots \ldots \ldots \ldots \ldots \ldots$ & $2.698 \mathrm{E}+00$ & $4.662 \mathrm{E}+00$ & $1.358 \mathrm{E}+00$ & $4.917 \mathrm{E}-03$ \\
\hline \multicolumn{5}{|c|}{$\alpha=-2.0$} \\
\hline 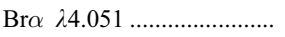 & $1.222 \mathrm{E}-01$ & $1.108 \mathrm{E}-01$ & $1.065 \mathrm{E}-01$ & $1.129 \mathrm{E}-01$ \\
\hline$[\mathrm{Ar} \mathrm{vI}] \lambda 4.530 \ldots \ldots \ldots \ldots \ldots \ldots$ & $7.272 \mathrm{E}-03$ & $9.629 \mathrm{E}-05$ & $0.000 \mathrm{E}+00$ & $0.000 \mathrm{E}+00$ \\
\hline$[$ Ar II $] \lambda 6.983 \ldots \ldots \ldots \ldots \ldots \ldots$ & $1.792 \mathrm{E}-02$ & $2.307 \mathrm{E}-02$ & $6.272 \mathrm{E}-02$ & $1.796 \mathrm{E}-01$ \\
\hline$[$ Ar III] $\lambda 8.983 \ldots \ldots \ldots \ldots \ldots \ldots$ & $9.490 \mathrm{E}-02$ & $8.755 \mathrm{E}-04$ & $0.000 \mathrm{E}+00$ & $0.000 \mathrm{E}+00$ \\
\hline$[\mathrm{Ne} v \mathrm{vI}] \lambda 7.652 \ldots \ldots \ldots \ldots \ldots \ldots$ & $1.654 \mathrm{E}-01$ & $4.448 \mathrm{E}-01$ & $5.092 \mathrm{E}-01$ & $2.065 \mathrm{E}-01$ \\
\hline 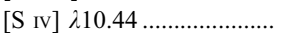 & $7.334 \mathrm{E}+00$ & $4.915 \mathrm{E}+00$ & $4.843 \mathrm{E}-01$ & $8.469 \mathrm{E}-03$ \\
\hline$[\mathrm{Ne}$ II $] \lambda 12.8 \ldots \ldots \ldots \ldots \ldots \ldots \ldots$ & $2.778 \mathrm{E}-01$ & $4.585 \mathrm{E}-01$ & $1.102 \mathrm{E}+00$ & $2.089 \mathrm{E}+00$ \\
\hline$[\mathrm{Ne} v] \lambda 14.5 \ldots \ldots \ldots \ldots \ldots \ldots \ldots$ & $1.293 \mathrm{E}-01$ & $1.443 \mathrm{E}-02$ & $1.524 \mathrm{E}-04$ & $0.000 \mathrm{E}+00$ \\
\hline$[\mathrm{Ne}$ III] $\lambda 15.5 \ldots \ldots \ldots \ldots \ldots \ldots \ldots$ & $4.655 \mathrm{E}+00$ & $5.305 \mathrm{E}+00$ & $4.405 \mathrm{E}+00$ & $2.471 \mathrm{E}+00$ \\
\hline 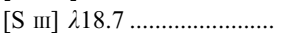 & $7.434 \mathrm{E}-01$ & $2.007 \mathrm{E}+00$ & $3.068 \mathrm{E}+00$ & $1.846 \mathrm{E}+00$ \\
\hline [O Iv] $225.9 \ldots \ldots \ldots \ldots \ldots \ldots \ldots$ & $2.151 \mathrm{E}+00$ & $2.911 \mathrm{E}+00$ & $8.123 \mathrm{E}-01$ & $2.792 \mathrm{E}-03$ \\
\hline
\end{tabular}


seen in the dust-free models. It is above this value of $U_{0}$ that dust begins to dominate the absorption and hence the radiation pressure. As discussed in the previous section, the radiation pressure on dust at this value of $U$ dominates the gas pressure and determines the density, and hence the local ionization parameter, toward the recombination zone.

The effects of metallicity variation is also apparent. As the amount of metals increases from $Z=0.25 Z_{\odot}$ there is a corresponding increase in the relative strength of the metal emission lines. However, at $Z \gtrsim 2 Z_{\odot}$ the effect of metal cooling begins to dominate the line emission, and the higher ionization lines becoming weaker above this value. This corresponds with the obvious dip seen in the temperature structure in Figure 9. A comparison between the depleted and undepleted models demonstrates the effect of dust depletion. In most ways it is similar to a change in overall metallicity caused by the effect upon temperature. However, there are variations that are different to metallicity, especially in lines from heavily depleted elements such as iron. This is due to the sudden abundance increase in these elements.

The change in the ionizing spectrum has a visible effect on the line emission. As the incident power law becomes steeper, with the index changing from -1.2 to -2.0 , not only do the high ionization lines become weaker, but the metal line ratios tend to become weaker relative to $\mathrm{H} \beta$ and other hydrogen lines. With a steeper power law, a larger fraction of the ionizing photons lies in the hydrogen ionizing band ( $1-1.8$ ryd), and there are correspondingly fewer high-energy photons available to ionize the high ionization species. Because hydrogen sees more ionizing photons at the same ionization parameter, there is a relative increase in the hydrogen line strength.

\section{CONCLUSIONS}

We have described here the implementation of dust and radiation pressure in the shock and photoionization code MAPPINGS III. To demonstrate the applicability of this model and to gain a deeper understanding of the physics, we have run a set of models covering a wide range in density, metallicity, power-law index of the ionizing spectra, and photoionization parameter. We have presented an examination of the physical and ionization structure of these models and the effects that variation of these parameters has upon the structure and emission lines. In addition, we have presented a grid of commonly used UV, visible, and IR diagnostic spectral lines from the models covering the full parameter space. These data are intended for use in the comparison with the narrowline regions of active galaxies and the understanding of the physical properties of such regions. In the next paper we will discuss further the resulting emission lines from the models and compare the grids of both the dusty models and of the dust-free models with the observational material for the commonly diagnostic ratios as well as presenting new FUV and IR diagnostics.

M. D. acknowledges the support of the Australian National University and of the Australian Research Council through his ARC Australian Federation Fellowship, and M. D. and R. S. acknowledge support through the ARC Discovery project DP0208445. The authors would like to thank the referee for incisive and detailed comments, which have much improved these papers.
Alexander, R. D., Casali, M. M., André, P., Persi, P., \& Eiroa, C. 2003, A\&A, 401,613

Alexander, T., Lutz, D., Sturm, E., Genzel, R., Sternberg, A., \& Netzer, H. 2000, ApJ, 536, 710

Allen, M. G., Dopita, M. A., \& Tsvetanov, Z. I. 1998, ApJ, 493, 571

Allen, M. G., Dopita, M. A., Tsvetanov, Z. I., \& Sutherland, R. S. 1999, ApJ, 511,686

Allende Prieto, C., Lambert, D. L., \& Asplund, M. 2001, ApJ, 556, L63

. 2002, ApJ, 573, L137

Anders, E., \& Grevesse, N. 1989, Geochim. Cosmochim. Acta, 53, 197

Asplund, M. 2000, A\&A, 359, 755

2003, preprint (astro-ph/0302409)

Asplund, M., Nordlund, A., Trampedach, R., \& Stein, R. F. 2000, A\&A, 359,743

Binette, L., Wilson, A. S., Raga, A., \& Storchi-Bergmann, T. 1997, A\&A, 327,909

Binette, L., Wilson, A. S., \& Storchi-Bergmann, T. 1996, A\&A, 312, 365

Borkowski, K. J., \& Harrington, J. P. 1991, ApJ, 379, 168

Bower, G., Wilson, A., Morse, J. A., Gelderman, R., Whittle, M., \& Mulchaey, J. 1995, ApJ, 454, 106

Bradley, L. D., Kaiser, M. E., \& Baan, W. A. 2004, ApJ, 603, 463

Brinkman, A. C., Kaastra, J. S., vanderMeer, R. L. J., Kinkhabwala, A., Behar, E., Kahn, S. M., Paerels, F. B. S., \& Sako, M. 2002, A\&A, 396, 761

Cecil, G., Dopita, M. A., Groves, B., Wilson, A. S., Ferruit, P., Pécontal, E., \& Binette, L. 2002, ApJ, 568, 627

Crenshaw, D. M., \& Kraemer, S. B. 2000, ApJ, 532, L101

Dopita, M. A., Binette, L., \& Schwartz, R. D. 1982, ApJ, 261, 183

Dopita, M. A., Groves, B. A., Sutherland, R. S., Binette, L., \& Cecil, G. 2002, ApJ, 572, 753 (DG02)

Dopita, M. A., Kewley, L. J., Heisler, C. A., \& Sutherland, R. S. 2000, ApJ, 542,224

Dopita, M. A., \& Sutherland, R. S. 1995, ApJ, 455, 468

1996, ApJS, 102, 161

2000, ApJ, 539, 742

Draine, B. T. 1978 , ApJS, 36, 595

2003, ARA\&A, 41, 241

Draine, B. T., \& Lazarian, A. 1998, ApJ, 508, 157
Draine, B. T., \& Lee, H. M. 1984, ApJ, 285, 89

Ferguson, J. W., Korista, K. T., Baldwin, J. A., \& Ferland, G. J. 1997, ApJ, 487,122

Grevesse, N., \& Sauval, A. J. 1998, Space Sci. Rev., 85, 161

Groves, B. A., Dopita, M. A., \& Sutherland, R. S. 2004, ApJS, 153, 75 (Paper II)

Honda, M., Kataza, H., Okamoto, Y. K., Miyata, T., Yamashita, T., Sako, S., Takubo, S., \& Onaka, T. 2003, ApJ, 585, L59

Hony, S., Bouwman, J., Keller, L. P., \& Waters, L. B. F. M. 2002, A\&A, 393, L103

Hony, S., Tielens, A. G. G. M., Waters, L. B. F. M., \& de Koter, A. 2003, A\&A, 402, 211

Jäger, C., Fabian, D., Schrempel, F., Dorschner, J., Henning, T., \& Wesch, W. 2003, A\&A, 401, 57

Jenkins, E. B. 1987, in ASSL Vol. 134: Interstellar Processes (Dordrecht: Reidel), 533

Jones, A. P., Tielens, A. G. G. M., \& Hollenbach, D. J. 1996, ApJ, 469, 740 Kaiser, M. E., et al. 2000, ApJ, 528, 260

Kennicutt, R. C., Bresolin, F., \& Garnett, D. R. 2003, ApJ, 591, 801

Komossa, S., \& Schulz, H. 1997, A\&A, 323, 31

Koski, A. T. 1978, ApJ, 223, 56

Kraemer, S. B., \& Crenshaw, D. M. 2000, ApJ, 532, 256

Kraemer, S. B., Crenshaw, D. M., Hutchings, J. B., Gull, T. R., Kaiser, M. E., Nelson, C. H., \& Weistrop, D. 2000, ApJ, 531, 278

Kraemer, S. B., Wu, C., Crenshaw, D. M., \& Harrington, J. P. 1994, ApJ, 435,171

Laor, A., \& Draine, B. T. 1993, ApJ, 402, 441

Laor, A., Fiore, F., Elvis, M., Wilkes, B. J., \& McDowell, J. C. 1997, ApJ, 477,93

Maciel, W. J., \& Pottasch, S. R. 1982, A\&A, 106, 1

Mathis, J. S., Rumpl, W., \& Nordsieck, K. H. 1977, ApJ, 217, 425 (MRN)

Moorwood, A. F. M., Lutz, D., Oliva, E., Marconi, A., Netzer, H., Genzel, R., Sturm, E., \& de Graauw, T. 1996, A\&A, 315, L109

Mouhcine, M., \& Contini, T. 2002, A\&A, 389, 106

Netzer, H., \& Laor, A. 1993, ApJ, 404, L51

Ogle, P. M., Brookings, T., Canizares, C. R., Lee, J. C., \& Marshall, H. L. 2003 , A\&A, 402, 849 
Oliva, E., Salvati, M., Moorwood, A. F. M., \& Marconi, A. 1994, A\&A, 288,457

Onaka, T., \& Okada, Y. 2003, ApJ, 585, 872

Osterbrock, D. E. 1989, Astrophysics of Gaseous Nebulae and Active Galactic Nuclei (Mill Valley: University Science Books)

Osterbrock, D. E., Tran, H. D., \& Veilleux, S. 1992, ApJ, 389, 196

Pagel, B. E. J., Simonson, E. A., Terlevich, R. J., \& Edmunds, M. G. 1992, MNRAS, 255, 325

Pedlar, A., Meaburn, J., Axon, D. J., Unger, S. W., Whittle, D. M., Meurs, E. J. A., Guerrine, N., \& Ward, M. J. 1989, MNRAS, 238, 863

Pitman, K. M., Clayton, G. C., \& Gordon, K. D. 2000, PASP, 112, 537

Prieto, M. A., \& Viegas, S. M. 2000, ApJ, 532, 238

Radomski, J. T., Piña, R. K., Packham, C., Telesco, C. M., De Buizer, J. M., Fisher, R. S., \& Robinson, A. 2003, ApJ, 587, 117

Russell, S. C., \& Bessell, M. S. 1989, ApJS, 70, 865
Russell, S. C., \& Dopita, M. A. 1990, ApJS, 74, 93 1992, ApJ, 384, 508

Savage, B. D., \& Sembach, K. R. 1996, ARA\&A, 34, 279

Sazonov, S. Y., Ostriker, J. P., \& Sunyaev, R. A. 2004, MNRAS, 347, 144 Stasińska, G. 1984, A\&A, 135, 341

Sutherland, R. S., \& Dopita, M. A. 1993, ApJS, 88, 253

Tomono, D., Doi, Y., Usuda, T., \& Nishimura, T. 2001, ApJ, 557, 637

Van Kerckhoven, C., Tielens, A. G. G. M., \& Waelkens, C. 2002, A\&A, 384,568

Veilleux, S., \& Osterbrock, D. E. 1987, ApJS, 63, 295

Véron-Cetty, M. P., \& Véron, P. 2000, Astron. Astrophys. Rev., 10, 81

Weingartner, J. C., \& Draine, B. T. 2001a, ApJ, 548, 296 . 2001b, ApJS, 134, 263

Whittle, M. 1992, ApJS, 79, 49 